Comparative Effectiveness Review

Number 228

\title{
Nonopioid Pharmacologic Treatments for Chronic Pain
}




\section{Comparative Effectiveness Review}

Number 228

\section{Nonopioid Pharmacologic Treatments for Chronic Pain}

Prepared for:

Agency for Healthcare Research and Quality

U.S. Department of Health and Human Services

5600 Fishers Lane

Rockville, MD 20857

www.ahrq.gov

Contract No. 290-2015-00009-I

Prepared by:

Pacific Northwest Evidence-based Practice Center

Portland, OR

Investigators:

Marian S. McDonagh, Pharm.D.

Shelley S. Selph, M.D., M.P.H.

David I. Buckley, M.D., M.P.H.

Rebecca S. Holmes, M.D., M.S.

Kimberly Mauer, M.D.

Shaun Ramirez, M.P.H.

Frances C. Hsu, M.S.

Tracy Dana, M.L.S.

Rochelle Fu, Ph.D.

Roger Chou, M.D.

AHRQ Publication No. 20-EHC010

April 2020 


\section{Key Messages}

\section{Purpose of Review}

Evaluate the benefits and harms of nonopioid drugs in randomized controlled trials of patients with specific types of chronic pain, considering the effects on pain, function, quality of life, and adverse events.

\section{Key Messages}

- In the short term, improvement in pain and function was small with specific anticonvulsants, moderate with specific antidepressants in diabetic peripheral neuropathy/post-herpetic neuralgia and fibromyalgia, and small with nonsteroidal antiinflammatory drugs (NSAIDs) in osteoarthritis and inflammatory arthritis.

- In the intermediate term, evidence was limited, with evidence of benefit for memantine in fibromyalgia and for serotonin norepinephrine reuptake inhibitor (SNRI) antidepressants in low back pain and fibromyalgia.

- In the long term, evidence was too limited to draw conclusions. In general, evidence on quality of life was limited and no treatment achieved a large improvement in pain or function.

- Small to moderate, dose-dependent increases in withdrawal due to adverse events were found with SNRIs duloxetine and milnacipran, anticonvulsants pregabalin and gabapentin, and NSAIDs. Large increases were seen with oxcarbazepine. NSAIDs have increased risk of serious gastrointestinal, liver dysfunction, and cardiovascular adverse events. 
This report is based on research conducted by the Pacific Northwest Evidence-based Practice Center (EPC) under contract to the Agency for Healthcare Research and Quality (AHRQ), Rockville, MD (Contract No. 290-2015-00009-I). The findings and conclusions in this document are those of the authors, who are responsible for its contents; the findings and conclusions do not necessarily represent the views of AHRQ. Therefore, no statement in this report should be construed as an official position of AHRQ or of the U.S. Department of Health and Human Services.

\section{None of the investigators have any affiliations or financial involvement that conflicts with the material presented in this report.}

The information in this report is intended to help healthcare decision makers-patients and clinicians, health system leaders, and policymakers, among others-make well-informed decisions and thereby improve the quality of healthcare services. This report is not intended to be a substitute for the application of clinical judgment. Anyone who makes decisions concerning the provision of clinical care should consider this report in the same way as any medical reference and in conjunction with all other pertinent information, i.e., in the context of available resources and circumstances presented by individual patients.

This report is made available to the public under the terms of a licensing agreement between the author and the Agency for Healthcare Research and Quality. This report may be used and reprinted without permission except those copyrighted materials that are clearly noted in the report. Further reproduction of those copyrighted materials is prohibited without the express permission of copyright holders.

AHRQ or U.S. Department of Health and Human Services endorsement of any derivative products that may be developed from this report, such as clinical practice guidelines, other quality enhancement tools, or reimbursement or coverage policies, may not be stated or implied.

This report may periodically be assessed for the currency of conclusions. If an assessment is done, the resulting surveillance report describing the methodology and findings will be found on the Effective Health Care Program website at www.effectivehealthcare.ahrq.gov. Search on the title of the report.

People using assistive technology may not be able to fully access information in this report. For assistance contact EPC@ahrq.hhs.gov.

Suggested citation: McDonagh MS, Selph SS, Buckley DI, Holmes RS, Mauer K, Ramirez S, Hsu FC, Dana T, Fu R, Chou R. Nonopioid Pharmacologic Treatments for Chronic Pain.

Comparative Effectiveness Review No. 228. (Prepared by the Pacific Northwest Evidence-based Practice Center under Contract No. 290-2015-00009-I.) AHRQ Publication No. 20-EHC010. Rockville, MD: Agency for Healthcare Research and Quality; April 2020. DOI: https://doi.org/10.23970/AHRQEPCCER228. Posted final reports are located on the Effective Health Care Program search page. 


\section{Preface}

The Agency for Healthcare Research and Quality (AHRQ), through its Evidence-based Practice Centers (EPCs), sponsors the development of evidence reports and technology assessments to assist public- and private-sector organizations in their efforts to improve the quality of healthcare in the United States. The Centers for Disease Control and Prevention requested this report from the EPC Program at AHRQ. AHRQ assigned this report to the following EPC: Pacific Northwest Evidence-based Practice Center (Contract No. 290-201500009-I).

The reports and assessments provide organizations with comprehensive, evidence-based information on common medical conditions and new healthcare technologies and strategies. They also identify research gaps in the selected scientific area, identify methodological and scientific weaknesses, suggest research needs, and move the field forward through an unbiased, evidence-based assessment of the available literature. The EPCs systematically review the relevant scientific literature on topics assigned to them by AHRQ and conduct additional analyses when appropriate prior to developing their reports and assessments.

To bring the broadest range of experts into the development of evidence reports and health technology assessments, AHRQ encourages the EPCs to form partnerships and enter into collaborations with other medical and research organizations. The EPCs work with these partner organizations to ensure that the evidence reports and technology assessments they produce will become building blocks for healthcare quality improvement projects throughout the Nation. The reports undergo peer review and public comment prior to their release as a final report.

AHRQ expects that the EPC evidence reports and technology assessments, when appropriate, will inform individual health plans, providers, and purchasers as well as the healthcare system as a whole by providing important information to help improve healthcare quality.

If you have comments on this evidence report, they may be sent by mail to the Task Order Officer named below at: Agency for Healthcare Research and Quality, 5600 Fishers Lane, Rockville, MD 20857, or by email to epc@ahrq.hhs.gov.

Gopal Khanna, M.B.A.

Director

Agency for Healthcare Research and Quality

Stephanie Chang, M.D., M.P.H.

Director

Evidence-based Practice Center Program

Center for Evidence and Practice

Improvement

Agency for Healthcare Research and Quality
Arlene S. Bierman, M.D., M.S

Director

Center for Evidence and Practice

Improvement

Agency for Healthcare Research and Quality

Suchitra Iyer, Ph.D.

Task Order Officer

Center for Evidence and Practice

Improvement

Agency for Healthcare Research and Quality 


\section{Acknowledgments}

The authors gratefully acknowledge the following individuals for their contributions to this project: Research Assistants Katrina Murphy, B.S., and Shelby Kuyl, B.S., both from Oregon Health \& Science University; Associate Editor M. Hassan Murad, M.D., Mayo Clinic Evidencebased Practice Center; and Task Order Officer Suchitra Iyer, Ph.D., at AHRQ.

\section{Technical Expert Panel}

In designing the study questions and methodology at the outset of this report, the EPC consulted several technical and content experts. Broad expertise and perspectives were sought. Divergent and conflicted opinions are common and perceived as healthy scientific discourse that results in a thoughtful, relevant systematic review. Therefore, in the end, study questions, design, methodologic approaches, and/or conclusions do not necessarily represent the views of individual technical and content experts.

Technical Experts must disclose any financial conflicts of interest greater than $\$ 5,000$ and any other relevant business or professional conflicts of interest. Because of their unique clinical or content expertise, individuals with potential conflicts may be retained. The TOO and the EPC work to balance, manage, or mitigate any potential conflicts of interest identified.

The list of Technical Experts who provided input to this report follows:

Daniel Clauw, M.D.*

Professor, Anesthesiology, Rheumatology, and Psychiatry

University of Michigan

Ann Arbor, MI

Christopher Goodman, M.D.*

Clinical Assistant Professor of Internal

Medicine

University of South Carolina School of

Medicine

Columbia, SC

Sharon Hertz, M.D.

Division Director, Office of Neuroscience

Division of Anesthesia, Addiction Medicine, and Pain Medicine

U.S. Food and Drug Administration

Beltsville, MD

Jennifer Kawi, Ph.D., M.S.N., A.P.R.N., F.N.P.-B.C.*

*Provided input on Draft Report
Associate Professor, School of Nursing University of Nevada, Las Vegas

Las Vegas, NV

Kurt Kroenke, M.D., M.A.C.P.*

Professor of Medicine, Indiana University

School of Medicine and Regenstrief Institute Indianapolis, IN

Michelle Matthews, Pharm.D., B.C.A.C.P, C.P.E, FASHP

Vice Chair, Department of Pharmacy

Practice

Massachusetts College of Pharmacy and

Health Sciences

Boston, MA

Mark Wallace, M.D.

Chair, Division of Pain Medicine

University of California, San Diego

San Diego, CA 


\section{Peer Reviewers}

Prior to publication of the final evidence report, EPCs sought input from independent Peer Reviewers without financial conflicts of interest. However, the conclusions and synthesis of the scientific literature presented in this report do not necessarily represent the views of individual reviewers.

Peer Reviewers must disclose any financial conflicts of interest greater than $\$ 5,000$ and any other relevant business or professional conflicts of interest. Because of their unique clinical or content expertise, individuals with potential nonfinancial conflicts may be retained. The TOO and the EPC work to balance, manage, or mitigate any potential nonfinancial conflicts of interest identified.

The list of Peer Reviewers follows:

Wendy L. Bennett, M.D., M.P.H. Associate Professor of Medicine Division of General Internal Medicine Core Faculty, The Welch Center for Prevention, Epidemiology and Clinical Research

Johns Hopkins School of Medicine Baltimore, MD

Haitao Chu, Ph.D., M.D. Professor, Division of Biostatistics University of Minnesota Minneapolis, MN
James Rathmell, M.D.

Chair, Department of Anesthesia

Brigham and Women's Hospital

Boston, MA

Barbara St. Marie, Ph.D, A.G.P.C.N.P., FAAN.P

Assistant Professor, College of Nursing University of Iowa

Iowa City, IA

Jasvinder Singh, M.D., M.P.H

Endowed Professor, Musculoskeletal

Outcomes Research, Division of Clinical Immunology and Rheumatology

Professor of Medicine and Epidemiology, University of Alabama at Birmingham Director, Gout Clinic Staff Physician, University of Alabama Health Services Foundation, Birmingham Veterans Affairs Medical Center

Birmingham, AL 


\section{Nonopioid Pharmacologic Treatments for Chronic Pain}

\section{Structured Abstract}

Objectives. To evaluate the effectiveness and comparative effectiveness of nonopioid pharmacologic agents in patients with specific types of chronic pain, considering effects on pain, function, quality of life, and adverse events.

Data sources. Electronic databases $\left(\right.$ Ovid $^{\circledR}$ MEDLINE $^{\circledR}$, Embase $^{\circledR}$, PsycINFO $^{\circledR}$, CINAHL $^{\circledR}$, Cochrane Central Register of Controlled Trials, and Cochrane Database of Systematic Reviews) through September 10, 2019, reference lists, data requests, and previous reviews.

Review methods. Randomized controlled trials (RCTs) of nonopioid pharmacologic agents in patients with chronic pain were selected using predefined criteria and dual review. This review focused on seven common chronic pain conditions (neuropathic pain, fibromyalgia,

osteoarthritis, inflammatory arthritis, low back pain, chronic headache, sickle cell disease), with effects analyzed at short term ( 1 to $<6$ months following treatment completion), intermediate term ( $\geq 6$ to $<12$ months), and long term ( $\geq 12$ months). Magnitude of effects were described as small, moderate, or large using previously defined criteria, and strength of evidence was assessed. Meta-analyses were conducted where data allowed, stratified by duration within each intervention type, using random effects models. We evaluated effect modification through subgroup and sensitivity analyses, including specific drug, dose, study quality, and pain type.

Results. We included 185 RCTs in 221 publications and 5 systematic reviews. In the short term, anticonvulsants (pregabalin, gabapentin, and oxcarbazepine for neuropathic pain, pregabalin/gabapentin for fibromyalgia), serotonin-norepinephrine reuptake inhibitor (SNRI) antidepressants (duloxetine for neuropathic pain, fibromyalgia, osteoarthritis, and low back pain, milnacipran for fibromyalgia), and nonsteroidal anti-inflammatory drugs (NSAIDs) (for osteoarthritis and inflammatory arthritis) were associated with mostly small improvements (e.g., 5 to 20 points on a 0 to 100 scale) in pain and function. Function was not found to be improved with duloxetine for low back pain or pregabalin/gabapentin for neuropathic pain. Moderate improvement in quality of life was seen with duloxetine in patients with neuropathic pain, and small improvements in patients with osteoarthritis, but evidence was insufficient to draw conclusions for other drugs and conditions. While most comparisons of drugs and doses did not identify differences, diclofenac improved pain and function moderately more than celecoxib. In the intermediate term, limited evidence (1 RCT) showed memantine moderately improved pain, function, and quality of life in patients with fibromyalgia; improvements in pain, but not function, were maintained in the intermediate term with duloxetine and milnacipran for fibromyalgia. Other drugs studied, including acetaminophen (osteoarthritis), capsaicin (neuropathic pain), cannabis (neuropathic pain), amitriptyline (fibromyalgia, neuropathic pain), and cyclobenzaprine (fibromyalgia) had no clear effects. Withdrawal from study due to adverse events was significantly increased with nonopioid drugs, with the greatest increase over placebo seen with cannabis. Large increases in risk of adverse events were seen with pregabalin (blurred vision, cognitive effects, dizziness, peripheral edema, sedation, and weight gain), gabapentin (blurred vision, cognitive effects, sedation, weight gain), and cannabis (nausea, dizziness). Dose 
reductions reduced the risk of some adverse events with SNRI antidepressants. In the short term small increases in risk of major coronary events and moderate increases in serious gastrointestinal events (both short and long term) were found with NSAIDs.

Conclusions. In the short term, small improvements in pain and/or function were seen with SNRI antidepressants for neuropathic pain, fibromyalgia, osteoarthritis, and low back pain; pregabalin/gabapentin for neuropathic pain and fibromyalgia; oxcarbazepine for neuropathic pain; and NSAIDs for osteoarthritis and inflammatory arthritis. Improvement in function was not found with duloxetine for low back pain and pregabalin/gabapentin for neuropathic pain. Intermediate- and long-term outcomes were mostly not assessed. Increased incidence of drug class-specific adverse events led to withdrawal from treatment in some patients, suggesting that careful consideration of patient characteristics is needed in selecting nonopioid drug treatments. 


\section{Contents}

Evidence Summary ............................................................................................................................... ES-1

Background and Objectives ............................................................................................................... 1

Understanding Chronic Pain ............................................................................................... 1

Chronic Pain Management............................................................................................... 1

Rationale for Evidence Review and What This Review Adds ................................................... 2

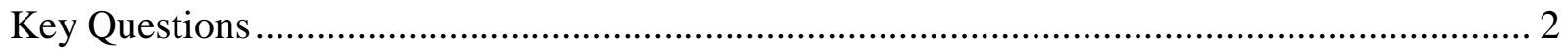

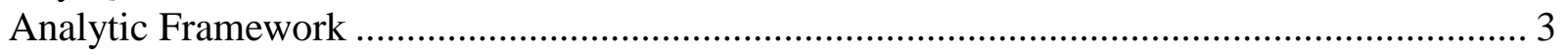

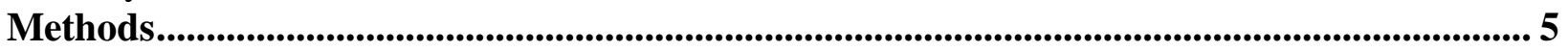

Criteria for Inclusion/Exclusion of Studies in the Review ………………............................. 5

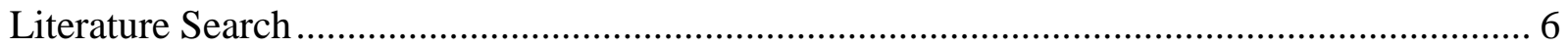

Assessment of Methodological Risk of Bias of Individual Studies.............................................. 7

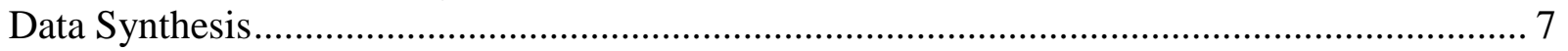

Grading the Strength of Evidence for Major Comparisons and Outcomes ................................. 8

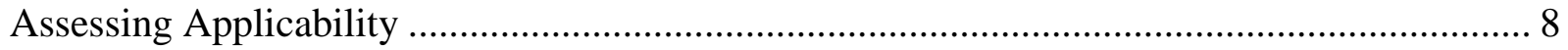

Peer Review and Public Commentary ................................................................................. 8

Results ........................................................................................................................................... 9

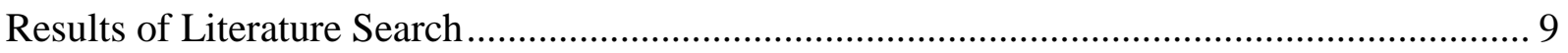

Key Question 1: Effectiveness and Comparative Effectiveness................................................ 10

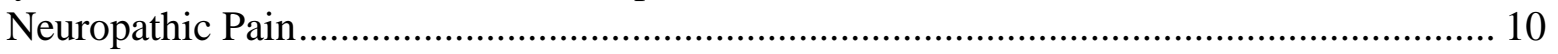

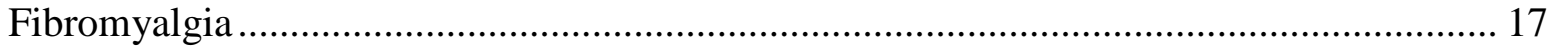

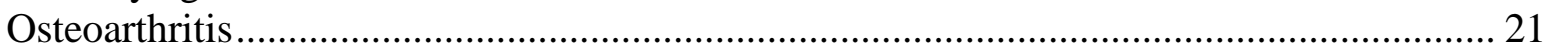

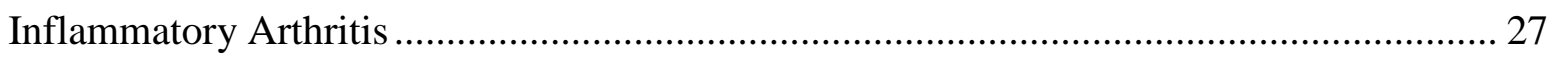

Low Back Pain/Neck Pain .................................................................................................. 31

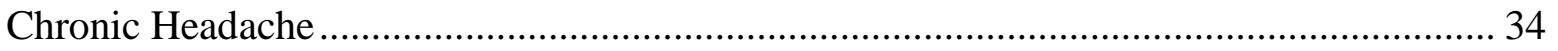

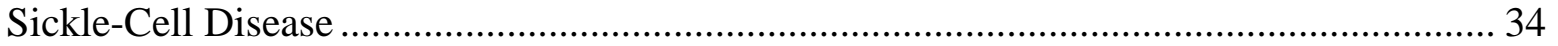

Key Question 2: Harms and Comparative Harms of Nonopioid Drugs for Chronic Pain......... 35

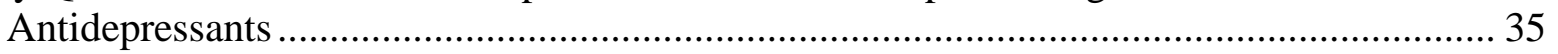

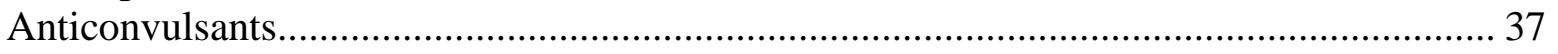

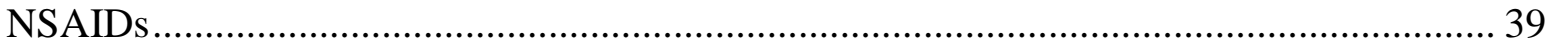

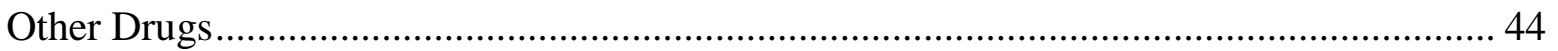

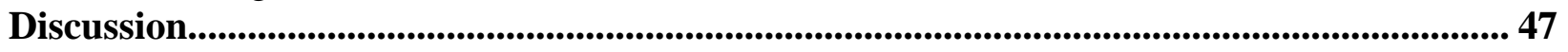

Key Findings and Strength of Evidence ………………........................................................ 47

Findings in Relationship to What Is Already Known.............................................................. 55

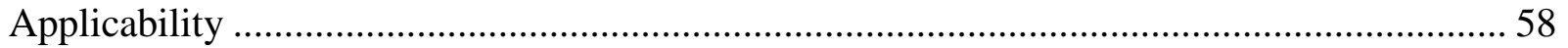

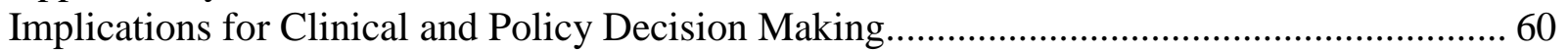

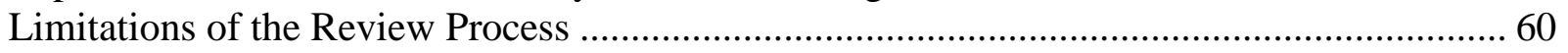

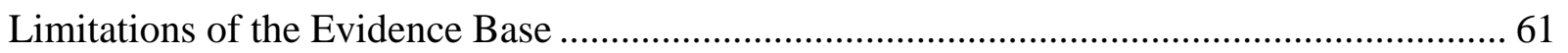

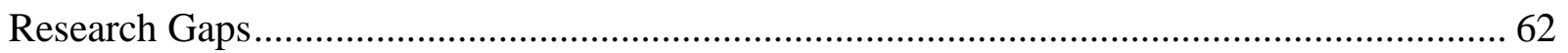

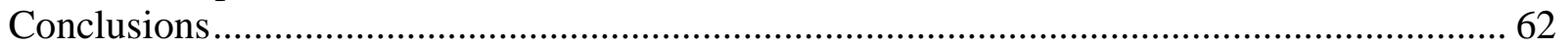

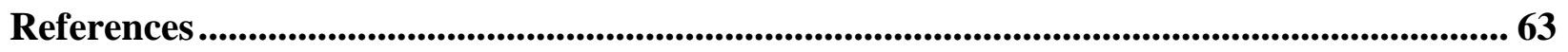

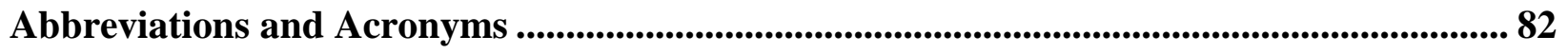

Tables

Table A. Description of the strength of evidence grades ................................................................

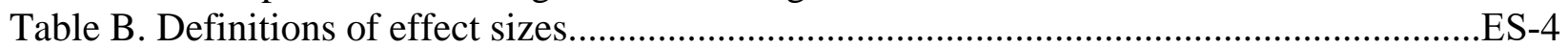


Table C. Effects of antidepressants in placebo-controlled and head-to-head trials ES-9

Table D. Effects of anticonvulsants in placebo-controlled and head-to-head trials ES-9

Table E. Effects of NSAIDs in placebo-controlled and head-to-head trials. ES-10

Table F. Effects of other drugs in placebo-controlled trials ES-11

Table G. Harms of antidepressants versus placebo ES-12

Table H. Harms of anticonvulsants versus placebo and active comparator ES-13

Table I. Harms of NSAIDs versus placebo and active comparators..... ES-14

Table J. SAEs and WAEs of other drugs versus placebo and active comparator ES-15

Table K. Summary of specific adverse events. ES-16

Table 1. PICOTS: Inclusion and exclusion criteria .............................................................. 5

Table 2. Definitions of effect sizes ........................................................................................ 7

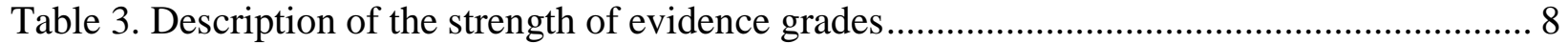

Table 4. Pregabalin/gabapentin pain improvement dose analysis .......................................... 13

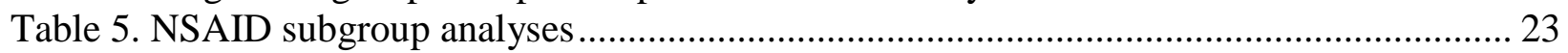

Table 6. Specific harms of pregabalin/gabapentin................................................................ 38

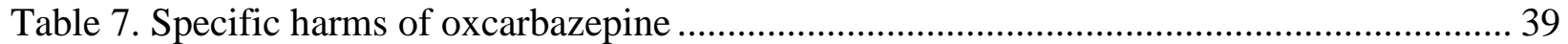

Table 8. Individual patient data meta-analysis of NSAID cardiovascular risks ........................ 41

Table 9. Risk of serious gastrointestinal events by NSAID drug versus placebo....................... 42

Table 10. Key Question 1 - Effectiveness and comparative effectiveness of nonopioid drugs for chronic pain: effects of antidepressants in placebo-controlled and head-to-head trials .............. 49 Table 11. Key Question 2 - Harms and adverse events of nonopioid drugs for chronic pain: harms of antidepressants versus placebo 50 Table 12. Key Question 1 - Effectiveness and comparative effectiveness of nonopioid drugs for chronic pain: effects of anticonvulsants in placebo-controlled and head-to-head trials .............. 50 Table 13. Key Question 2 - Harms and adverse events of nonopioid drugs for chronic pain: harms of anticonvulsants versus placebo and active comparator .......................................... 51 Table 14. Key Question 1 - Effectiveness and comparative effectiveness of nonopioid drugs for chronic pain: effects of NSAIDs in placebo-controlled and head-to-head trials ........................ 52 Table 15. Key Question 2 - Harms and adverse events of nonopioid drugs for chronic pain: harms of NSAIDs versus placebo and active comparators .................................................. 53 Table 16. Key Question 1 - Effectiveness and comparative effectiveness of nonopioid drugs for chronic pain: effects of other drugs in placebo-controlled trials 53 Table 17. Key Question 2 - Harms and adverse events of nonopioid drugs for chronic pain:

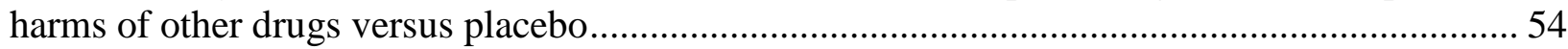

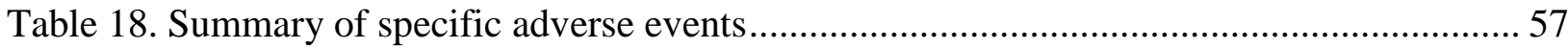

\section{Figures}

Figure 1. Analytic framework for nonopioid pharmacologic treatments for chronic pain ............. 3

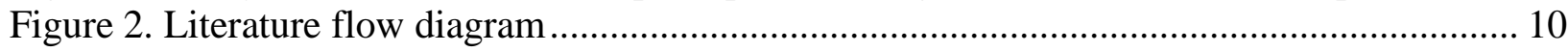

\section{Appendixes}

Appendix A. Literature Search Strategies

Appendix B. Methods

Appendix C. Included Studies

Appendix D. Excluded Studies

Appendix E. Study Characteristics Evidence Tables 
Appendix F. Meta-Analysis Evidence Tables

Appendix G. Quality Assessment

Appendix H. Strength of Evidence Tables

Appendix I. Forest Plots

Appendix J. Appendix References 


\section{Evidence Summary}

\section{Introduction}

Chronic pain is typically defined as pain lasting 3 to 6 months ${ }^{1}$ and can be the result of a wide array of issues, including underlying medical conditions or disease, inflammation of injured tissue, and neuropathic pain (which involves a lesion or disease of the somatosensory nervous system). Nearly 50 million adults in the United States live with chronic pain, garnering an estimated $\$ 560$ billion in annual healthcare costs ${ }^{1,2}$ and contributing to the economic burden on the healthcare system. ${ }^{2}$ Given the complexity of treating chronic pain and concerns regarding the safety and long-term effectiveness of opioids, there have been multiple initiatives in recent years to improve the evidence available to clinicians and patients for making treatment decisions.

These initiatives, along with the recent publication of the evidence-based guideline on opioid use for chronic pain by the Centers for Disease Control and Prevention, ${ }^{3}$ have prompted additional primary research on alternatives to opioids in managing chronic pain. There is a real need to fully understand the benefits and harms of nonopioid pharmacologic treatments for chronic pain. The most common forms of nonopioid pharmacologic treatment for pain are nonsteroidal antiinflammatory drugs (NSAIDs), acetaminophen, topical formulations such as capsaicin, and drugs used for other conditions such as anticonvulsants and antidepressants that can be implemented for pain moderation. Evidence is needed on common chronic pain conditions, including neuropathic pain, fibromyalgia, inflammatory arthritis (e.g., rheumatoid arthritis), osteoarthritis, low back pain, chronic headache, and sickle cell disease, comparing nonopioid drugs to placebo, to each other, and comparing different doses and with adequate durations of treatment to reflect real-life situations.

The purpose of this review is to evaluate the benefits and harms of nonopioid drugs in randomized controlled trials (RCTs) of patients with chronic pain, considering the effects on pain, function, quality of life, and adverse events.

\section{Scope and Key Questions}

This Comparative Effectiveness Review focused on nonopioid pharmacologic treatments for issues of chronic pain. Key Questions (KQs) focus on the following.

- KQ1. Effectiveness and comparative effectiveness:

o Of nonopioid pharmacologic agents versus placebo and versus other nonopioid pharmacologic agents.

o For outcomes related to pain, function, and quality of life.

o For treatment durations of 3 to 6 months (short-term), 6 to 12 months (intermediate), and $\geq 12$ months (long-term).

o How does this vary by pain condition, demographics, comorbidities, dose, duration, and titration?

- KQ2. Harms and adverse events:

o What are the risks of nonopioid pharmacologic agents for harms including overdose, misuse, dependence, withdrawals due to adverse events, and serious adverse events, and specific adverse events?

o How do these vary by pain condition, demographics, comorbidities, dose, duration, and titration? 
Pharmacologic interventions considered in this review include oral agents specifically used to treat pain such as NSAIDs, antidepressants, serotonin-norepinephrine reuptake inhibitors (SNRIs), tricyclic antidepressants (TCAs), anticonvulsants, acetaminophen, and muscle relaxants, and memantine. Some commonly used topical agents were included in this review, including diclofenac, capsaicin, and lidocaine. Medical cannabis is a broad category and was included in this study in all of its various forms.

\section{Methods}

This Comparative Effectiveness Review follows the methods suggested in the Agency for Healthcare Research and Quality (AHRQ) Methods Guide for Effectiveness and Comparative Effectiveness Reviews (hereafter “AHRQ Methods Guide”). ${ }^{4}$ All methods were determined $a$ priori, and a protocol was published on the AHRQ website (https://effectivehealthcare.ahrq.gov/topics/nonopioid-chronic-pain/protocol) and on the PROSPERO systematic reviews registry (registration no. CRD42019134249). Below is a summary of the specific methods used in this review, and a complete description is provided in Appendix B.

\section{Literature Search Strategy}

We conducted electronic searches in Ovid ${ }^{\circledR}$ MEDLINE $^{\circledR}$, Embase $^{\circledR}$, PsycINFO $^{\circledR}$, CINAHL $^{\circledR}$, Cochrane CENTRAL, and Cochrane Database of Systematic Reviews through September 10, 2019 (from database inception; see Appendix A for full strategies). Reference lists of included systematic reviews were screened for includable studies. Manufacturers of included drugs submitted potential relevant studies to include in this review using the Federal Register notification.

\section{Inclusion and Exclusion Criteria and Study Selection}

Criteria for study inclusion were developed prior to conducting our searches based on our KQs and the population, interventions, comparators, outcomes, timing, setting, and study design (PICOTS) detailed in Appendix B. For all KQs, we included and focused on RCTs with at least 3 months' duration. We recognized that by definition, chronic pain requires treatments that are effective in the long term, and short-term benefits may not persist. This duration threshold is similar to the duration used in the prior AHRQ systematic review on nonpharmacologic interventions for chronic pain, ${ }^{5}$ which included studies with greater than 1 month of followup after the end of treatment, with most studies involving 6 to 8 weeks of treatment. The Evidencebased Practice Center (EPC) evaluated the availability and quality of studies with 3 to 6 months duration and found adequate evidence, thus we did not include studies with shorter durations. However, existing systematic reviews were reviewed to summarize evidence where possible.

We evaluated the persistence of benefits or harms by evaluating the three periods identified in the KQs ( 3 to 6 months, 6 to 12 months, and $\geq 12$ months). We used existing systematic reviews primarily to screen their included studies to ensure we identified all relevant studies for this review. In the case where a systematic review is recent enough to cover the majority of the available evidence, and evaluates a cohesive group of interventions, outcomes and time frames included here, we included the review as the primary evidence and supplemented with any newer or excluded studies. 
We restricted to English-language articles, but reviewed English-language abstracts of nonEnglish language articles to identify studies that would otherwise meet inclusion criteria, in order to assess for the likelihood of language bias.

\section{Assessment of Methodological Risk of Bias of Individual Studies}

Study quality was independently assessed by two researchers using the predefined criteria below and based on methods recommended in the AHRQ Methods Guide. ${ }^{4}$ Studies were rated as "good," "fair," or "poor" (Appendix G of the full report). Studies rated "good" are considered to have the least risk of bias, and their results are considered valid. Studies rated "fair" are susceptible to some bias, though not enough to invalidate the results. Studies rated "poor" have significant flaws that imply biases of various types that may invalidate the results. We did not exclude studies rated as being poor in quality a priori, but poor-quality studies were considered to be less reliable than higher-quality studies when synthesizing the evidence, particularly if discrepancies between studies were present.

\section{Data Abstraction and Data Synthesis}

Data regarding general study characteristics, such as demographics, pain condition, country of trial, and baseline pain scores, were abstracted and dual-reviewed by independent investigators (Appendix E of the full report). For clarity, data used for meta-analysis were abstracted into separate forms, pooled, and synthesized (Appendix F of the full report). Methods for abstracting data for synthesis are detailed next. Data from studies included in a systematic review that met our inclusion criteria were abstracted from the published article with missing data supplemented by systematic reviews.

We preferentially abstracted pain assessed with the visual analog scale (VAS) or numerical rating scale (NRS) on a scale of 0 to 10 or 0 to 100 over other pain assessments (e.g., Western Ontario and McMaster Universities Osteoarthritis Index pain subscale). Primary pain response was defined as $\geq 30$ percent improvement (reduction) in pain score. Secondary pain response criteria included $>30$ percent improvement (e.g., $\geq 50 \%$ improvement), condition-specific composite measure (e.g., American College of Rheumatology 20 criteria [ACR20], Assessment in Spondyloarthritis International Society 20 criteria [ASAS20]), and improvement in physician's clinical global impression of change. For quality of life outcome, we preferentially abstracted the EuroQoL-5 Dimensions (EQ-5D) over Short Form-36 (SF-36) physical and mental components summary scores (PCS and MCS), and synthesized the two scales separately.

Pain outcomes were standardized to a scale of 0 to 10; standardized mean differences (SMD) were calculated for other outcomes (e.g., function, quality of life) unless all pertinent studies assessed the outcome using the same scale. Studies with multiple nonopioid arms were combined so each study was represented once in a meta-analysis in order to avoid overweighting and the issue of correlation within the same study. When reported, adjusted mean difference from analysis of covariance model or other appropriate regression models was used if reported by the study, followed by difference in change score and followup score.

\section{Strength of the Body of Evidence}

The strength of evidence (SOE) for each KQ was rated for each clinical outcome using the approach described in the AHRQ Methods Guide. ${ }^{4}$ To ensure consistency and validity of the evaluation, the grades were reviewed by a second reviewer. The domains assessed were study 
limitations (low, medium, or high), consistency (consistent, inconsistent, or unknown/not applicable), directness (direct or indirect), precision (precise or imprecise), and publication bias (suspected or undetected). The SOE was assigned an overall grade of high, moderate, low, or insufficient (Table A), reflecting our confidence in the effect estimates (Table B) and whether the findings are stable. Evidence is found to be insufficient to draw conclusions when we have no evidence available or the body of evidence has unacceptable deficiencies, precluding reaching a conclusion.

Table A. Description of the strength of evidence grades

\begin{tabular}{|l|l|}
\hline Strength of Evidence & Description \\
\hline High & $\begin{array}{l}\text { Very confident that the effect estimate lies close to the true effect for this outcome. The } \\
\text { body of evidence has few or no deficiencies. Findings are stable, i.e., inclusion of } \\
\text { additional studies would not change the conclusions. }\end{array}$ \\
\hline Moderate & $\begin{array}{l}\text { Moderately confident that the estimate of effect lies close to the true effect for this } \\
\text { outcome. The body of evidence has some deficiencies. We believe that the findings are } \\
\text { likely to be stable, but some doubt remains. }\end{array}$ \\
\hline Low & $\begin{array}{l}\text { Limited confidence that the effect estimate lies close to the true effect for this outcome. } \\
\text { The body of evidence has major or numerous deficiencies. Additional evidence is } \\
\text { needed before concluding that the findings are stable or that the estimate of effect is } \\
\text { close to the true effect. }\end{array}$ \\
\hline Insufficient & $\begin{array}{l}\text { No confidence in the estimate of effect for this outcome. No evidence is available or the } \\
\text { body of evidence has unacceptable deficiencies, precluding reaching a conclusion. }\end{array}$ \\
\hline
\end{tabular}

Table B. Definitions of effect sizes

\begin{tabular}{|l|l|}
\hline Effect Size & Definition \\
\hline Small effect & $\begin{array}{l}\text { - MD } 0.5 \text { to } 1.0 \text { points on a } 0 \text { to 10-point scale, } 5 \text { to } 10 \text { points on a } 0 \text { to 100-point scale } \\
\text { - SMD } 0.2 \text { to } 0.5 \\
\text { - RR/OR } 1.2 \text { to } 1.4\end{array}$ \\
\hline Moderate effect & $\begin{array}{l}\text { - MD }>1 \text { to } 2 \text { points on a } 0 \text { to10-point scale, }>10 \text { to } 20 \text { points on a } 0 \text { to 100-point scale } \\
\text { - SMD }>0.5 \text { to } 0.8 \\
\text { - RR/OR } 1.5 \text { to } 1.9\end{array}$ \\
\hline Large effect & $\begin{array}{l}\text { - MD }>2 \text { points on a } 0 \text { to10-point scale, }>20 \text { points on a } 0 \text { to 100-point scale } \\
\text { - SMD }>0.8\end{array}$ \\
\hline
\end{tabular}

$\mathrm{MD}=$ mean difference; $\mathrm{OR}$ = odds ratio; $\mathrm{RR}=$ relative risk; $\mathrm{SMD}=$ standardized mean difference

\section{Peer Review and Public Commentary}

Peer reviewers with expertise in primary care and management of the included chronic pain conditions were invited to provide written comments to the draft report. The AHRQ Task Order Officer and an EPC Associate Editor also provided comments and editorial review. Following this, the peer-reviewed draft report was posted on the AHRQ website for 4 weeks for public comment.

\section{Results}

Results for efficacy are shown by KQ and then by condition. Harms results are organized by drug class. Search results and selection of studies are summarized in the literature flow diagram (Figure 2 of the full report). After dual review of full-text articles, 184 RCTs (in 217 publications) were included in this review. In addition, we identified 5 systematic reviews that included 47 trials included in this review. Overall, 30 trials were rated poor quality, 129 fair quality, and 25 good quality (Appendix G of the full report). Of the good- and fair-quality trials, 
128 were classified as short duration ( 3 months to $<6$ months), 18 intermediate duration (6 months to $<1$ year), and 9 were long duration ( $\geq 1$ year). We included 32 RCTs in neuropathic pain, 26 RCTs in fibromyalgia, 59 RCTs in osteoarthritis, 21 RCTs in inflammatory arthritis, 7 RCTs in low back pain, and 1 trial each in chronic headache and sickle cell disease. An additional 7 trials of mixed osteoarthritis and inflammatory arthritis patients were included for harms outcomes. Most study participants were female (66.7\%) but proportion varied widely by condition with the highest seen in fibromyalgia trials. Mean age of participants was 59 years and mean pain duration was 7.9 years. Participants reported a weighted mean pain severity of 6 on a scale of 0 to10. Industry was the leading provider of funding for trials (82\%) while 15 trials (10\%) did not report funding source.

Data abstraction of study characteristics and results, and quality assessment for good- and fair-quality studies is available in Appendixes E, F, and G of the full report.

\section{Key Question 1. Benefits}

In patients with neuropathic pain (mainly diabetic peripheral neuropathy and/or postherpetic neuralgia), short-term RCTs $(n=31)$ of anticonvulsants (prodrug gabapentin enacarbil, pregabalin, and oxcarbazepine) found small improvement in pain, with no differences between drugs (SOE: Low to insufficient). The antidepressant duloxetine resulted in small improvements in pain, function, and quality of life in patients with diabetic peripheral neuropathy (SOE: Moderate to low). Tetrahydrocannabinol (THC) and cannabidiol (CBD) oral spray had inconsistent effects on pain in patients with multiple sclerosis or with allodynia (SOE: Low). Improvements in pain with topical capsaicin were not significant or did not reach the level of a small effect (SOE: Moderate).

In patients with fibromyalgia, RCTs $(n=24)$ show small short-term and intermediate-term improvements in pain and quality of life (function only short-term) with SNRI antidepressants milnacipran and duloxetine. Anticonvulsants pregabalin and gabapentin show short-term improvements in pain and function but not quality of life (SOE: Moderate). Dose comparisons did not find differences in pain results. Short and intermediate-term treatment with memantine resulted in moderate improvements in pain, function, and quality of life compared with placebo (SOE: Low).

In patients with osteoarthritis, treatment with nonsteroidal anti-inflammatory drugs (NSAIDs, $\mathrm{k}=26$ RCTs) in the short term ( $\mathrm{k}=44$ RCTS) resulted in small improvements in pain and function (SOE: Moderate for pain, High for function). Topical diclofenac led to a small improvement in average pain severity and patients reporting response. Few differences were found between drugs. Duloxetine resulted in a small improvement in pain severity, moderate improvement in pain response, and small improvements in function and quality of life (SOE: High). Acetaminophen did not show improvements in pain or function, across all doses (SOE: Low). In patients with inflammatory arthritis ( $\mathrm{k}=30 \mathrm{RCTs})$, NSAIDs resulted in small improvements in pain and function (SOE: Moderate). Differences were not found between drugs or doses. Patients with low-back pain (k=7 RCTs) had small improvement in pain and response, but improvements in function and quality of life did not meet the threshold for small improvement with duloxetine (SOE: Moderate).

\section{Key Question 2. Harms}

Across all classes, incidence of serious adverse event (SAEs) was low. Forty good- or fairquality trials evaluated harms of antidepressants. Antidepressants led to a moderate increase in 
withdrawal due to adverse events (WAE) in 27 short- and intermediate-term studies. SNRI antidepressants resulted in moderate to large increases in incidence of nausea (with no difference according to dose) and excessive sweating. Duloxetine resulted in a large, dose-dependent, increase in sedation (SOE: Moderate to Low).

Thirty-two trials evaluated harms in short-term treatment with anticonvulsants. Oxcarbazepine led to a large increased risk of WAEs. Pregabalin and gabapentin also led to a small increased risk of WAEs, with pregabalin risk being greater with higher doses. Pregabalin and gabapentin resulted in large increases in blurred vision, dizziness, weight gain, and cognitive effects (e.g., confusion). Gabapentin enacarbil may have lower risk of blurred vision, weight gain or cognitive effects. Additionally, pregabalin resulted in large increases in risk of peripheral edema and sedation (SOE: Moderate to Low).

Seventy-nine trials evaluated harms of NSAID treatment in the short term. WAEs were increased, specifically with ibuprofen and diclofenac (small increase) and naproxen (moderate increase). The risk of any cardiovascular event was not significantly elevated for NSAIDs as a group, but diclofenac had a small increase in risk, particularly in the first 6 months, and with higher doses. The risk of major coronary events was elevated with diclofenac and celecoxib (moderately) and with ibuprofen (large increase). There was no difference in cardiovascular events between celecoxib and nonselective NSAIDs in the intermediate and long term (SOE: Moderate). The risk of serious upper gastrointestinal events was increased with diclofenac (moderately) and ibuprofen or naproxen (large increase), particularly in the first 6 months of treatment. In the intermediate term, large increases in incidence of hepatic harms were found with diclofenac and naproxen (SOE: Moderate to Low).

In the short or intermediate term, acetaminophen did not increase WAEs (3 RCTs, SOE: Low). In the short term (3 RCTs), capsaicin 8 percent topical patch 60 minute application led to a moderate increase in SAEs compared with 30 minutes. Capsaicin resulted in a large increased risk of application site pain and a small increased risk of erythema (SOE: Moderate and Low). Cannabis showed large increases in incidence of dizziness with oral dronabinol solution, and in WAEs, dizziness, and nausea with tetrahydrocannabinol/cannabidiol oral spray (2 RCTs, SOE: Low).

\section{Discussion}

\section{Key Findings and Strength of Evidence}

The key findings of this review and effect size definitions are summarized below (Tables $\mathrm{C}$ through $\mathrm{K}$ ). (See the full report for a detailed discussion of our key findings and strength of evidence.) This review evaluated and synthesized the evidence on benefits and harms of nonopioid drugs in patients with chronic noncancer pain. The pain conditions included were neuropathic pain (diabetic peripheral neuropathy, post-herpetic neuralgia, other), fibromyalgia, osteoarthritis, inflammatory arthritis (rheumatoid arthritis or ankylosing spondylitis), spinal pain (neck or low back pain), chronic headache, and sickle cell disease. Drugs reviewed included antidepressants (SNRIs and TCAs), anticonvulsants (pregabalin, gabapentin, oxcarbazepine, and carbamazepine), NSAIDs, and other drugs such as acetaminophen, capsaicin, and cannabis. The findings are categorized in the paragraphs below according to pain condition. The magnitude of the findings and the strength of the evidence for each finding are categorized according to the 
methods described above. Interventions or comparisons for which all evidence was insufficient to draw conclusions are not included here.

In patients with neuropathic pain, in the short term, the anticonvulsant drugs gabapentin, pregabalin, and oxcarbazepine provided small improvement in pain outcomes in patients with diabetic peripheral neuropathy/post-herpetic neuralgia. Function did not improved with gabapentin and quality of life showed no improvements with the three anticonvulsants drugs. In patients with diabetic peripheral neuropathy, duloxetine resulted in small improvements in pain, function, and quality of life. Capsaicin patch had effects on pain severity short of small-effect in post-herpetic neuralgia and HIV-related neuralgia, and showed no improvement in pain response. Limited evidence on cannabis (dronabinol oral solution, tetrahydrocannabinol/cannabidiol oral spray) showed inconsistent effects on pain (depending on the measure) in patients with multiple sclerosis-associated neuropathy or allodynia in the short term, and no effect on function or quality of life in the short term,

In patients with fibromyalgia, in the short and intermediate term, SNRI antidepressants duloxetine and milnacipran resulted in small improvements in pain. Function improved to a small degree in the short term, but not in the intermediate term. Short-term treatment with the anticonvulsants pregabalin and gabapentin results in small improvements in pain and function, but not quality of life. Subgroup analyses showed no effect of specific drug, dose, or study quality on these results. Short- and intermediate-term treatment with memantine resulted in moderate improvements in pain, function, and quality of life. Evidence for cyclobenzaprine showed no effect on pain in the short term.

Oral NSAIDs improve pain and function in patients with osteoarthritis to a small degree in the short term, with evidence indicating these effects are maintained in the intermediate term for celecoxib. Subgroup analyses indicated that studies of patients with knee pain only and those of good quality had smaller effects, while patients with more severe pain at baseline experienced greater reduction in pain. Direct comparisons of NSAIDs with each other found few differences between drugs in pain or function in osteoarthritis patients in the short, intermediate, or long term. The exception was that diclofenac moderately improved pain and function more than celecoxib in the short term. Topical diclofenac showed small improvement in pain in the short term. The SNRI antidepressant duloxetine resulted in moderate improvement in pain response, and small effects on pain improvement, function, and quality of life. Subgroup analyses found that pain improvement was greater in older patients (>65 years) and patients with knee osteoarthritis. Acetaminophen did not improve pain significantly in the short or intermediate term. In patients with rheumatoid arthritis or ankylosing spondylitis, short-term treatment with oral NSAIDs resulted in small improvements in pain severity and function, and moderate improvements on pain response, but evidence on quality of life was inconsistent. Evidence on intermediate- and long-term outcomes was limited to one trial each, with improvements in pain but not function. Comparisons of different doses or between different NSAIDs did not find important differences. Subgroup analyses of specific drug, dose, year of publication, type of inflammatory arthritis, and study quality did not alter the findings meaningfully. The TCA amitriptyline did not improve pain severity. Evidence in patients with chronic headache or sickle cell disease was too limited to draw conclusions.

Adverse events categorized as "serious" were more often not reported with nonopioid drugs than placebo in patients with chronic pain, the exception being in neuropathic pain with longer duration capsaicin patch (compared with shorter duration, moderate effect). Withdrawal due to adverse events was increased with anticonvulsants, antidepressants (both moderately), NSAIDs 
(to a small degree), and cannabidiol oral spray (ranging from a small increase to large increases). SNRI antidepressants resulted in increased reports of nausea (dose did not alter these findings). Duloxetine also resulted in increased sedation, but lower doses did reduce the risk. Amitriptyline led to a moderate increase in reports of dry mouth, but other adverse events of interest were not reported or not different to placebo. There were no reports of serotonin syndrome in any included RCT of antidepressants. In the short term, pregabalin and gabapentin resulted in moderate to large increases in blurred vision, dizziness, weight gain, sedation, and cognitive effects (e.g., confusion). A prodrug of gabapentin, gabapentin enacarbil may have lower risk of blurred vision, weight gain, or cognitive effects. Additionally, pregabalin resulted in large increases in risk of peripheral edema and sedation. In the short term, the risk of any cardiovascular event was not significantly elevated for NSAIDs as a group, although there was a small increase in risk with diclofenac, particularly within the first 6 months, and with higher doses; risk was increased to a similar degree with ibuprofen and celecoxib but did not reach statistical significance. Although the absolute risk is low, there was a moderate relative increased risk of major coronary events with diclofenac and celecoxib and a large increase with ibuprofen. In the intermediate and long term, there was not a difference in cardiovascular events between drugs. In the short term, NSAIDs led to moderate to large increased risk of serious upper gastrointestinal events (largely bleeding), particularly in the first 6 months of treatment. In the intermediate term, although the incidence is low, large increases in hepatic harms were seen with diclofenac and naproxen. Dronabinol oral solution resulted in a large increase in dizziness and tetrahydrocannabinol/cannabidiol oral spray resulted in large increases in dizziness and nausea. Other adverse events of interest were not reported (cognitive effects, misuse, addiction, substance use disorder). 
Table C. Effects of antidepressants in placebo-controlled and head-to-head trials

\begin{tabular}{|c|c|c|c|c|c|c|c|}
\hline Condition & Drug & $\begin{array}{l}\text { Pain } \\
\text { Short Term } \\
\text { Effect Size } \\
\text { SOE }\end{array}$ & $\begin{array}{l}\text { Pain } \\
\text { Intermediate Term } \\
\text { Effect Size } \\
\text { SOE }\end{array}$ & $\begin{array}{l}\text { Function } \\
\text { Short Term } \\
\text { Effect Size } \\
\text { SOE }\end{array}$ & $\begin{array}{l}\text { Function } \\
\text { Intermediate Term } \\
\text { Effect Size } \\
\text { SOE }\end{array}$ & $\begin{array}{l}\text { QoL } \\
\text { Short Term } \\
\text { Effect Size } \\
\text { SOE }\end{array}$ & $\begin{array}{l}\text { QoL } \\
\text { Intermediate Term } \\
\text { Effect Size } \\
\text { SOE }\end{array}$ \\
\hline Neuropathic pain & Duloxetine vs. placebo & $\begin{array}{c}\text { Moderate } \\
++\end{array}$ & No evidence & $\begin{array}{c}\text { Small } \\
+\end{array}$ & No evidence & $\begin{array}{c}\text { Small } \\
++\end{array}$ & No evidence \\
\hline Fibromyalgia & $\begin{array}{l}\text { Duloxetine/milnacipran } \\
\text { vs. placebo }\end{array}$ & $\begin{array}{c}\text { Small } \\
++\end{array}$ & $\begin{array}{c}\text { Small } \\
++\end{array}$ & $\begin{array}{c}\text { Small } \\
++\end{array}$ & $\begin{array}{c}\text { None } \\
++\end{array}$ & $\begin{array}{c}\text { MCS: Small } \\
++ \\
\text { PCS: None } \\
++ \\
\end{array}$ & $\begin{array}{c}\text { Small } \\
++\end{array}$ \\
\hline Osteoarthritis & Duloxetine vs. placebo & $\begin{array}{c}\text { Small } \\
+++\end{array}$ & No evidence & $\begin{array}{c}\text { Small } \\
+++\end{array}$ & No evidence & $\begin{array}{c}\text { Small } \\
+++\end{array}$ & No evidence \\
\hline \multirow{3}{*}{ Low back pain } & Duloxetine vs. placebo & $\begin{array}{c}\text { Small } \\
++\end{array}$ & No evidence & $\begin{array}{c}\text { None } \\
++\end{array}$ & No evidence & $\begin{array}{c}\text { None } \\
++\end{array}$ & No evidence \\
\hline & Amitriptyline vs. placebo & No evidence & $\begin{array}{c}\text { None } \\
+\end{array}$ & No evidence & $\begin{array}{c}\text { None } \\
+\end{array}$ & No evidence & No evidence \\
\hline & $\begin{array}{l}\text { Amitriptyline vs. } \\
\text { pregabalin }\end{array}$ & $\begin{array}{c}\text { Small } \\
+\end{array}$ & No evidence & $\begin{array}{c}\text { None } \\
+\end{array}$ & No evidence & No evidence & No evidence \\
\hline
\end{tabular}

QoL = quality of life; SOE = strength of evidence; MCS = Mental Component Score; PCS = Physical Component Score

Effect size: none (i.e., no effect/no statistically significant effect), small, moderate, or large increased risk

SOE: $+=$ low,$++=$ moderate, $+++=$ high

Table D. Effects of anticonvulsants in placebo-controlled and head-to-head trials

\begin{tabular}{|c|c|c|c|c|}
\hline Condition & Drug & $\begin{array}{l}\text { Pain } \\
\text { Short Term } \\
\text { Effect Size } \\
\text { SOE }\end{array}$ & $\begin{array}{l}\text { Function } \\
\text { Short Term } \\
\text { Effect Size } \\
\text { SOE }\end{array}$ & $\begin{array}{l}\text { QoL } \\
\text { Short Term } \\
\text { Effect Size } \\
\text { SOE }\end{array}$ \\
\hline \multirow{4}{*}{ Neuropathic pain } & Pregabalin/gabapentin vs. placebo & $\begin{array}{l}\text { Small } \\
++\end{array}$ & $\begin{array}{l}\text { None } \\
+\end{array}$ & $\begin{array}{l}\text { None } \\
+ \\
\end{array}$ \\
\hline & Oxcarbazepine vs. placebo & $\begin{array}{l}\text { Small } \\
++\end{array}$ & No evidence & $\begin{array}{l}\text { None } \\
+\end{array}$ \\
\hline & Pregabalin vs. gabapentin & Insufficient & No evidence & No evidence \\
\hline & Pregabalin vs. gabapentin enacarbil a & $\begin{array}{l}\text { None } \\
+\end{array}$ & $\begin{array}{l}\text { None } \\
+\end{array}$ & $\begin{array}{l}\text { None } \\
+\end{array}$ \\
\hline Fibromyalgia & Pregabalin / gabapentin vs. placebo & $\begin{array}{l}\text { Small } \\
++\end{array}$ & $\begin{array}{l}\text { Small } \\
++\end{array}$ & $\begin{array}{l}\text { None } \\
++\end{array}$ \\
\hline
\end{tabular}

QoL = quality of life; SOE = strength of evidence

Effect size: none (i.e., no effect/no statistically significant effect), small, moderate, or large

SOE $:+=$ low,$++=$ moderate, $+++=$ high

${ }^{a}$ Gabapentin enacarbil is a prodrug of gabapentin 
Table E. Effects of NSAIDs in placebo-controlled and head-to-head trials

\begin{tabular}{|c|c|c|c|c|c|c|c|c|}
\hline Condition & Drug & $\begin{array}{l}\text { Pain } \\
\text { Short Term } \\
\text { Effect Size } \\
\text { SOE }\end{array}$ & $\begin{array}{l}\text { Pain } \\
\text { Intermediate } \\
\text { Term } \\
\text { Effect Size } \\
\text { SOE } \\
\end{array}$ & $\begin{array}{l}\text { Pain } \\
\text { Long Term } \\
\text { Effect Size } \\
\text { SOE }\end{array}$ & $\begin{array}{l}\text { Function } \\
\text { Short Term } \\
\text { Effect Size } \\
\text { SOE }\end{array}$ & $\begin{array}{l}\text { Function } \\
\text { Intermediate Term } \\
\text { Effect Size } \\
\text { SOE }\end{array}$ & $\begin{array}{l}\text { Function } \\
\text { Long Term } \\
\text { Effect Size } \\
\text { SOE }\end{array}$ & $\begin{array}{l}\text { QoL } \\
\text { Short Term } \\
\text { Effect Size } \\
\text { SOE }\end{array}$ \\
\hline \multirow{4}{*}{ Osteoarthritis } & NSAID vs. placebo & $\begin{array}{c}\text { Small } \\
++\end{array}$ & No evidence & $\begin{array}{c}\text { No } \\
\text { evidence }\end{array}$ & $\begin{array}{c}\text { Small } \\
+++\end{array}$ & No evidence & No evidence & $\begin{array}{c}\text { None } \\
++\end{array}$ \\
\hline & Diclofenac vs. celecoxib & $\begin{array}{c}\text { Moderate } \\
+\end{array}$ & No evidence & $\begin{array}{c}\text { No } \\
\text { evidence }\end{array}$ & $\begin{array}{c}\text { Moderate } \\
+\end{array}$ & No evidence & No evidence & $\begin{array}{c}\text { No } \\
\text { evidence }\end{array}$ \\
\hline & NSAID vs. NSAID & $\begin{array}{c}\text { None } \\
+\end{array}$ & $\begin{array}{c}\text { None } \\
+ \\
\end{array}$ & $\begin{array}{c}\text { None } \\
+\end{array}$ & $\begin{array}{c}\text { None } \\
+\end{array}$ & $\begin{array}{c}\text { None } \\
+\end{array}$ & No evidence & $\begin{array}{c}\text { No } \\
\text { evidence }\end{array}$ \\
\hline & $\begin{array}{l}\text { Topical diclofenac vs. } \\
\text { placebo }\end{array}$ & $\begin{array}{c}\text { Small } \\
++\end{array}$ & No evidence & $\begin{array}{c}\text { No } \\
\text { evidence }\end{array}$ & $\begin{array}{c}\text { None } \\
+ \\
\end{array}$ & No evidence & No evidence & $\begin{array}{c}\text { No } \\
\text { evidence }\end{array}$ \\
\hline \multirow{6}{*}{$\begin{array}{l}\text { Inflammatory } \\
\text { arthritis }\end{array}$} & NSAID vs. placebo & $\begin{array}{c}\text { Small/ } \\
\text { Moderate } \\
++ \\
\end{array}$ & $\begin{array}{l}\text { Small } \\
+\end{array}$ & $\begin{array}{l}\text { Large } \\
\quad+\end{array}$ & $\begin{array}{c}\text { Small } \\
++\end{array}$ & $\begin{array}{l}\text { Small } \\
+\end{array}$ & $\begin{array}{c}\text { None } \\
+\end{array}$ & Insufficient \\
\hline & Celecoxib vs. diclofenac & $\begin{array}{c}\text { None } \\
++\end{array}$ & No evidence & $\begin{array}{c}\text { No } \\
\text { evidence }\end{array}$ & $\begin{array}{c}\text { None } \\
++\end{array}$ & No evidence & No evidence & $\begin{array}{c}\text { No } \\
\text { evidence }\end{array}$ \\
\hline & Celecoxib vs. naproxen & $\begin{array}{c}\text { None } \\
+ \\
\end{array}$ & No evidence & $\begin{array}{c}\text { No } \\
\text { evidence }\end{array}$ & $\begin{array}{c}\text { None } \\
+ \\
\end{array}$ & No evidence & No evidence & $\begin{array}{c}\text { None } \\
+\end{array}$ \\
\hline & Diclofenac vs. meloxicam & $\begin{array}{c}\text { None } \\
+\end{array}$ & No evidence & $\begin{array}{c}\text { No } \\
\text { evidence } \\
\end{array}$ & $\begin{array}{c}\text { None } \\
+ \\
\end{array}$ & No evidence & No evidence & $\begin{array}{c}\text { No } \\
\text { evidence }\end{array}$ \\
\hline & Meloxicam vs. naproxen & $\begin{array}{c}\text { No } \\
\text { evidence }\end{array}$ & $\begin{array}{c}\text { None } \\
+\end{array}$ & $\begin{array}{c}\text { No } \\
\text { evidence }\end{array}$ & No evidence & No evidence & No evidence & $\begin{array}{c}\text { No } \\
\text { evidence }\end{array}$ \\
\hline & Nabumetone vs. naproxen & $\begin{array}{c}\text { None } \\
+\end{array}$ & $\begin{array}{c}\text { None } \\
+\end{array}$ & $\begin{array}{c}\text { No } \\
\text { evidence }\end{array}$ & $\begin{array}{c}\text { None } \\
+ \\
\end{array}$ & No evidence & No evidence & $\begin{array}{c}\text { No } \\
\text { evidence }\end{array}$ \\
\hline
\end{tabular}

NSAID = nonsteroidal anti-inflammatory drug; QoL = quality of life; SOE = strength of evidence

Effect size: none (i.e., no effect/no statistically significant effect), small, moderate, or large increased risk

SOE: $+=$ low,$++=$ moderate,$+++=$ high 
Table F. Effects of other drugs in placebo-controlled trials

\begin{tabular}{|c|c|c|c|c|c|c|c|}
\hline Condition & Drug & $\begin{array}{l}\text { Pain } \\
\text { Short Term } \\
\text { Effect Size } \\
\text { SOE }\end{array}$ & $\begin{array}{l}\text { Pain } \\
\text { Intermediate Term } \\
\text { Effect Size } \\
\text { SOE }\end{array}$ & $\begin{array}{l}\text { Function } \\
\text { Short Term } \\
\text { Effect Size } \\
\text { SOE }\end{array}$ & $\begin{array}{l}\text { Function } \\
\text { Intermediate Term } \\
\text { Effect Size } \\
\text { SOE }\end{array}$ & $\begin{array}{l}\text { QoL } \\
\text { Short Term } \\
\text { Effect Size } \\
\text { SOE }\end{array}$ & $\begin{array}{l}\text { QoL } \\
\text { Intermediate Term } \\
\text { Effect Size } \\
\text { SOE }\end{array}$ \\
\hline \multirow{2}{*}{ Neuropathic pain } & Capsaicin patch & $\begin{array}{c}\text { None } \\
++\end{array}$ & No evidence & No evidence & No evidence & No evidence & No evidence \\
\hline & Cannabis & $\begin{array}{c}\text { None } \\
+\end{array}$ & No evidence & $\begin{array}{c}\text { None } \\
+\end{array}$ & No evidence & $\begin{array}{c}\text { None } \\
+\end{array}$ & No evidence \\
\hline \multirow{2}{*}{ Fibromyalgia } & Memantine & No evidence & $\begin{array}{c}\text { Moderate } \\
+\end{array}$ & No evidence & $\begin{array}{c}\text { Moderate } \\
+ \\
\end{array}$ & No evidence & $\begin{array}{c}\text { Moderate } \\
+\end{array}$ \\
\hline & Cyclobenzaprine & No evidence & $\begin{array}{c}\text { None } \\
+\end{array}$ & No evidence & Insufficient & No evidence & No evidence \\
\hline Osteoarthritis & Acetaminophen & $\begin{array}{c}\text { None } \\
+\end{array}$ & $\begin{array}{c}\text { None } \\
+\end{array}$ & $\begin{array}{c}\text { None } \\
+\end{array}$ & $\begin{array}{c}\text { None } \\
+\end{array}$ & No evidence & No evidence \\
\hline
\end{tabular}

QoL = quality of life; SOE = strength of evidence

Effect size: none (i.e., no effect/no statistically significant effect), small, moderate, or large increased risk

SOE: $+=$ low,$++=$ moderate, $+++=$ high

KQ2 Harms and Adverse Events of Nonopioid Drugs for Chronic Pain 
Table G. Harms of antidepressants versus placebo

\begin{tabular}{|c|c|c|c|c|}
\hline Types of Adverse Events & $\begin{array}{l}\text { SNRIs (duloxetine/milnacipran) } \\
\text { Short Term } \\
\text { Effect Size } \\
\text { SOE }\end{array}$ & $\begin{array}{l}\text { SNRIs (duloxetine/milnacipran) } \\
\text { Intermediate Term } \\
\text { Effect Size } \\
\text { SOE }\end{array}$ & $\begin{array}{l}\text { TCAs } \\
\text { Short Term } \\
\text { Effect Size } \\
\text { SOE }\end{array}$ & $\begin{array}{l}\text { TCAs } \\
\text { Intermediate Term } \\
\text { Effect Size } \\
\text { SOE }\end{array}$ \\
\hline WAE & $\begin{array}{c}\text { Moderate } \\
++\end{array}$ & $\begin{array}{c}\text { Moderate } \\
++\end{array}$ & $\begin{array}{c}\text { None } \\
+\end{array}$ & Insufficient \\
\hline SAE & $\begin{array}{c}\text { None } \\
+\end{array}$ & $\begin{array}{c}\text { None } \\
+\end{array}$ & No evidence & No evidence \\
\hline Cognitive effects & $\begin{array}{c}\text { None } \\
+\end{array}$ & No evidence & No evidence & No evidence \\
\hline Nausea & $\begin{array}{c}\text { Large } \\
++\end{array}$ & $\begin{array}{c}\text { Moderate } \\
+\end{array}$ & NA & NA \\
\hline Sedation & $\begin{array}{c}\text { Large } \\
++\end{array}$ & $\begin{array}{c}\text { Large } \\
+\end{array}$ & NA & NA \\
\hline Serotonin syndrome & No evidence & No evidence & No evidence & No evidence \\
\hline Dry mouth & NA & NA & Insufficient & No evidence \\
\hline Cardiac rhythm abnormalities & NA & NA & No evidence & No evidence \\
\hline Urinary retention & NA & NA & No evidence & No evidence \\
\hline
\end{tabular}

NA = not applicable (i.e., specific adverse event not applicable to drug); SAE = serious adverse event; SNRI = serotonin-norepinephrine reuptake inhibitor; SOE = strength of evidence; TCA = tricyclic antidepressant; WAE = withdrawal due to adverse event

Effect size: none (i.e., no effect/no statistically significant effect), small, moderate, or large increased risk

SOE: $+=$ low,$++=$ moderate, $+++=$ high 
Table H. Harms of anticonvulsants versus placebo and active comparator

\begin{tabular}{|c|c|c|}
\hline Types of Adverse Events & $\begin{array}{l}\text { Pregabalin/Gabapentin } \\
\text { Short Term } \\
\text { Effect Size } \\
\text { SOE }\end{array}$ & $\begin{array}{l}\text { Oxcarbazepine } \\
\text { Short Term } \\
\text { Effect Size } \\
\text { SOE }\end{array}$ \\
\hline WAE & $\begin{array}{cc}\text { Moderate } \\
++\end{array}$ & $\begin{array}{c}\text { Large } \\
+ \\
\end{array}$ \\
\hline SAE & $\begin{array}{c}\text { None } \\
+\end{array}$ & $\begin{array}{c}\text { None } \\
+\end{array}$ \\
\hline Blurred vision & $\begin{array}{l}\text { Large } \\
+ \\
\end{array}$ & NA \\
\hline Cognitive effects & $\begin{array}{l}\text { Large } \\
+ \\
\end{array}$ & No evidence \\
\hline Dizziness & $\begin{array}{c}\text { Large } \\
++\end{array}$ & NA \\
\hline Peripheral edema & $\begin{array}{c}\text { Large } \\
++\end{array}$ & NA \\
\hline Sedation & $\begin{array}{c}\text { Large } \\
++\end{array}$ & $\begin{array}{c}\text { None } \\
+ \\
\end{array}$ \\
\hline Weight gain & $\begin{array}{c}\text { Large } \\
++\end{array}$ & NA \\
\hline Hyponatremia & NA & $\begin{array}{c}\text { None } \\
+\end{array}$ \\
\hline
\end{tabular}

NA = not applicable (i.e., specific adverse event not applicable to drug); SAE = serious adverse event; SOE = strength of evidence; WAE = withdrawal due to adverse event Effect size: none (i.e., no effect/no statistically significant effect), small, moderate, or large increased risk

SOE: $+=$ low,$++=$ moderate, $+++=$ high 
Table I. Harms of NSAIDs versus placebo and active comparators

\begin{tabular}{|c|c|c|c|c|c|c|}
\hline $\begin{array}{l}\text { Types of Adverse } \\
\text { Events }\end{array}$ & $\begin{array}{l}\text { NSAID } \\
\text { Short Term } \\
\text { Effect Size } \\
\text { SOE }\end{array}$ & $\begin{array}{l}\text { NSAID } \\
\text { Intermediate Term } \\
\text { Effect Size } \\
\text { SOE }\end{array}$ & $\begin{array}{l}\text { NSAID } \\
\text { Long Term } \\
\text { Effect Size } \\
\text { SOE }\end{array}$ & $\begin{array}{l}\text { Topical Diclofenac } \\
\text { Versus Placebo } \\
\text { Short Term } \\
\text { Effect Size } \\
\text { SOE }\end{array}$ & $\begin{array}{l}\text { nsNSAID Versus } \\
\text { Celecoxib } \\
\text { Intermediate Term } \\
\text { Effect Size } \\
\text { SOE }\end{array}$ & $\begin{array}{l}\text { nsNSAID Versus } \\
\text { Celecoxib } \\
\text { Long Term } \\
\text { Effect Size } \\
\text { SOE }\end{array}$ \\
\hline WAE & $\begin{array}{c}\text { Small } \\
++\end{array}$ & $\begin{array}{c}\text { None } \\
+\end{array}$ & Insufficient & $\begin{array}{c}\text { None } \\
+\end{array}$ & No evidence & No evidence \\
\hline SAE & $\begin{array}{c}\text { None } \\
+\end{array}$ & Insufficient & No evidence & $\begin{array}{c}\text { None } \\
+\end{array}$ & No evidence & No evidence \\
\hline Cardiovascular events & $\begin{array}{c}\text { Small } \\
++\end{array}$ & No evidence & No evidence & No evidence & $\begin{array}{c}\text { None } \\
++\end{array}$ & $\begin{array}{c}\text { None } \\
++\end{array}$ \\
\hline $\begin{array}{l}\text { Gastrointestinal } \\
\text { events }\end{array}$ & $\begin{array}{c}\text { Moderate } \\
+/++\end{array}$ & No evidence & No evidence & No evidence & $\begin{array}{c}\text { Moderate } \\
+\end{array}$ & No evidence \\
\hline Liver dysfunction & $\begin{array}{c}\text { Large } \\
+\end{array}$ & No evidence & No evidence & No evidence & No evidence & No evidence \\
\hline
\end{tabular}

NA = not applicable (i.e., specific AE not applicable to drug); NS = nonsteroidal anti-inflammatory drug; nsNSAID = nonselective nonsteroidal anti-inflammatory drug; SAE = serious adverse event; $\mathrm{SOE}$ = strength of evidence; WAE = withdrawal due to adverse event

Effect size: none (i.e., no effect/no statistically significant effect), small, moderate, or large increased risk

SOE: $+=$ low,$++=$ moderate, $+++=$ high 
Table J. SAEs and WAEs of other drugs versus placebo and active comparator

\begin{tabular}{|c|c|c|c|c|c|c|}
\hline Types of Adverse Events & $\begin{array}{l}\text { Capsaicin } \\
\text { Short Term } \\
\text { Effect Size } \\
\text { SOE }\end{array}$ & $\begin{array}{l}\text { Dronabinol } \\
\text { Short Term } \\
\text { Effect Size } \\
\text { SOE }\end{array}$ & $\begin{array}{l}\text { THC + CBD } \\
\text { Short Term } \\
\text { Effect Size } \\
\text { SOE }\end{array}$ & $\begin{array}{l}\text { Acetaminophen } \\
\text { Short Term } \\
\text { Effect Size } \\
\text { SOE }\end{array}$ & $\begin{array}{l}\text { Acetaminophen } \\
\text { Intermediate Term } \\
\text { Effect Size } \\
\text { SOE }\end{array}$ & $\begin{array}{l}\text { Cyclobenzaprine } \\
\text { Intermediate Term } \\
\text { Effect Size } \\
\text { SOE }\end{array}$ \\
\hline WAE & $\begin{array}{c}\text { None } \\
++ \\
\end{array}$ & $\begin{array}{c}\text { None } \\
+ \\
\end{array}$ & $\begin{array}{c}\text { Large } \\
+ \\
\end{array}$ & $\begin{array}{c}\text { None } \\
+ \\
\end{array}$ & $\begin{array}{c}\text { None } \\
+ \\
\end{array}$ & $\begin{array}{c}\text { None } \\
+ \\
\end{array}$ \\
\hline SAE & $\begin{array}{c}\text { None } \\
++\end{array}$ & $\begin{array}{c}\text { None } \\
+\end{array}$ & $\begin{array}{c}\text { None } \\
+\end{array}$ & $\begin{array}{c}\text { None } \\
+\end{array}$ & $\begin{array}{c}\text { None } \\
+\end{array}$ & No evidence \\
\hline Application site erythema & $\begin{array}{c}\text { Moderate } \\
++\end{array}$ & NA & NA & NA & NA & NA \\
\hline Application site pain & $\begin{array}{c}\text { Large } \\
++\end{array}$ & NA & NA & NA & NA & NA \\
\hline Application site pruritus & $\begin{array}{c}\text { None } \\
++\end{array}$ & NA & NA & NA & NA & NA \\
\hline Cognitive effects & NA & No evidence & No evidence & NA & NA & NA \\
\hline Hyperemesis & NA & No evidence & No evidence & NA & NA & NA \\
\hline Nausea & NA & $\begin{array}{c}\text { None } \\
+\end{array}$ & $\begin{array}{c}\text { Large } \\
+\end{array}$ & NA & NA & NA \\
\hline Dizziness & NA & $\begin{array}{c}\text { Large } \\
+\end{array}$ & $\begin{array}{c}\text { Large } \\
+\end{array}$ & NA & NA & Insufficient \\
\hline
\end{tabular}

CBD = cannabidiol; NA = not applicable; THC = tetrahydrocannabinol; SAE = serious adverse event; SOE = strength of evidence; WAE = withdrawal due to adverse event Effect size: none (i.e., no effect/no statistically significant effect), small, moderate, or large increased risk

SOE: $+=$ low,$++=$ moderate, $+++=$ high 
Table K. Summary of specific adverse events

\begin{tabular}{|c|c|c|c|c|}
\hline Drug Class & Drug & Outcomes of Interest & $\begin{array}{l}\text { Adverse Event Findings From RCTs in } \\
\text { Chronic Pain (Magnitude of Effect) }\end{array}$ & $\begin{array}{l}\text { Adverse Event Findings From Other } \\
\text { Sources (to Address Missing Evidence) }\end{array}$ \\
\hline \multirow[t]{2}{*}{ Antidepressants } & SNRIS & $\begin{array}{l}\text { Nausea, sedation, } \\
\text { serotonin syndrome }\end{array}$ & $\begin{array}{l}\text { Nausea (moderate-to-large, no dose effect), } \\
\text { sedation (duloxetine, dose-related), } \\
\text { serotonin syndrome symptoms (large) }\end{array}$ & No missing outcomes \\
\hline & TCAS & $\begin{array}{l}\text { Cardiac rhythm } \\
\text { abnormalities, dry mouth, } \\
\text { urinary retention, weight } \\
\text { gain, serotonin syndrome }\end{array}$ & Dry mouth (moderate) & $\begin{array}{l}\text { Cardiac arrhythmias and sinus tachycardia: } \\
\text { increases with higher dose and pre-existing } \\
\text { risk } \\
\text { Urinary retention: no estimate found } \\
\text { Weight gain: } 2-2.5 \mathrm{~kg} \text { over } 3 \text { months } \\
\text { Serotonin syndrome: very rare }{ }^{6}\end{array}$ \\
\hline \multirow[t]{2}{*}{$\begin{array}{l}\text { Antiepileptic } \\
\text { drugs }\end{array}$} & $\begin{array}{l}\text { Pregabalin, } \\
\text { gabapentin }\end{array}$ & $\begin{array}{l}\text { Blurred vision, cognitive } \\
\text { effects, dizziness, } \\
\text { peripheral edema, } \\
\text { sedation, weight gain }\end{array}$ & $\begin{array}{l}\text { Blurred vision, dizziness, weight gain, and } \\
\text { cognitive effects (moderate to large, lower } \\
\text { with the prodrug gabapentin enacarbil) } \\
\text { Peripheral edema (large with pregabalin) }\end{array}$ & No missing outcomes \\
\hline & Oxcarbazepine & $\begin{array}{l}\text { Cognitive effects, } \\
\text { hyponatremia, and } \\
\text { sedation }\end{array}$ & Hyponatremia - 1 RCT, no increased risk & $\begin{array}{l}\text { Significant hyponatremia: } 2.5 \% \text {, occurs in } \\
\text { first } 3 \text { months. } \\
\text { Cognitive effects: } 7-11 \% \\
\text { Somnolence: } 35 \% 7\end{array}$ \\
\hline NSAIDs & Oral NSAIDs & $\begin{array}{l}\mathrm{CV}, \mathrm{GI} \text {, renal, and } \\
\text { hepatic Events }\end{array}$ & $\begin{array}{l}\text { Short term: Increased CV risk - diclofenac } \\
\text { (small, dose-dependent); increased } \\
\text { coronary events - diclofenac, celecoxib } \\
\text { (moderate), ibuprofen (large); Increased GI } \\
\text { events - diclofenac (moderate), ibuprofen, } \\
\text { naproxen (large); } \\
\text { Intermediate term: Differences in CV risk } \\
\text { unclear; Increased hepatic harms- } \\
\text { diclofenac, naproxen (large, low incidence) }\end{array}$ & $\begin{array}{l}\text { Renal: Increased risk (moderate to large), } \\
\text { higher in older patients and those with } \\
\text { chronic kidney disease (evidence from } \\
\text { observational studies, includes short-term } \\
\text { use) No difference found between } \\
\text { NSAIDs. }{ }^{8,9}\end{array}$ \\
\hline \multirow[t]{3}{*}{ Other } & Acetaminophen & Hepatotoxicity & Not reported in included RCTs & $\begin{array}{l}\text { Increased risk with chronic use of }>3 \mathrm{gms} \\
\text { daily, effects often occur early in treatment; } \\
\text { dose-adjustment if hepatic or renal } \\
\text { dysfunction }{ }^{10,11}\end{array}$ \\
\hline & Cannabis & $\begin{array}{l}\text { Addiction/dependence, } \\
\text { cognitive effects, } \\
\text { hyperemesis, nausea, } \\
\text { sedation }\end{array}$ & $\begin{array}{l}\text { Dizziness (large) } \\
\text { Nausea (THC/CBD oral spray, large) }\end{array}$ & $\begin{array}{l}\text { Hyperemesis syndrome: Case reports (not } \\
\text { limited to medical uses), }>1 \times / \text { week for }>2 \\
\text { years. } \\
\text { Cognition: small negative impact with } \\
\text { chronic use } \\
\text { Addiction/dependence: not found }{ }^{12}\end{array}$ \\
\hline & Capsaicin & Application site reactions & $\begin{array}{l}\text { Pain (large), erythema (small) Greater with } \\
\text { longer application }\end{array}$ & No missing outcomes \\
\hline
\end{tabular}

CBD = cannabidiol; CV = cardiovascular; GI = gastrointestinal; $\mathrm{kg}=$ kilogram; NSAIDs = nonsteroidal anti-inflammatory drugs; RCTs = randomized controlled trials; SNRIs = serotonin-norepinephrine reuptake inhibitor; TCAs = tricyclic antidepressants; THC = tetrahydrocannabinol 


\section{Findings in Relationship to What Is Already Known}

This systematic review combines evidence across multiple pain conditions and multiple drug classes in a way that prior reviews have not. Prior reviews generally had dissimilar scope (e.g., limited to a single condition and/or drug class, included drugs or populations not included here), included very short duration studies ( $<12$ weeks), did not classify results according to treatment duration, and did not categorize effect sizes (small, moderate, large). Although our review includes more recent studies, other reviews of individual drugs, drug classes, or pain conditions have reviewed some of the evidence included here, and where comparisons of our results and prior findings are possible, they are generally consistent. For example, a 2015 systematic review with network meta-analysis of acetaminophen, NSAIDs, and injectable drugs for knee osteoarthritis found an SMD for acetaminophen of 0.18, and we found the mean difference (MD, 0 to 10 scale) was 0.34 . Both are less than a small magnitude of effect according to our system, and the prior review noted that the effect did not reach clinical significance in their system. ${ }^{13}$ Findings for NSAIDs were similar to ours, and our subgroup analysis of only knee osteoarthritis was also in a similar range of magnitude of effect to their findings. The exception was that they found a moderate-size effect with diclofenac, while our subgroup analysis of specific drug was not significant. For neuropathic pain, a 2017 systematic review of only diabetic peripheral neuropathy found duloxetine to have large effect (SMD -1.33), but when we added another study the magnitude was reduced to small (MD -0.79, 0 to 10 scale). ${ }^{14}$ This review and ours had similar findings for pregabalin (small effect). Both reviews found that the effect of gabapentin was not significant, but the effect was moderate in the older review, while in ours the effect was small after incorporating additional studies. In fibromyalgia, a 2016 systematic review with a network meta-analysis found a large magnitude of effect in pain response with SNRI antidepressants (odds ratio [OR] 1.61 to 2.33) while we found a moderate effect (relative risk [RR] 1.29 to 1.36), and the prior review found a moderate effect with pregabalin (OR 1.68) while we found a small effect with pregabalin and gabapentin combined (RR 1.41). ${ }^{15}$ Differences in magnitude could be due to the addition of 15 studies in our report, reporting relative risks rather than odds ratios, and using direct comparisons rather than network analysis. Our findings regarding the effects of nonopioid drugs on pain and function are also consistent with two related systematic reviews on opioids and nonpharmacologic treatments for chronic pain, which found similar small effects. ${ }^{16,17}$

In terms of evidence on the harms of the drugs included, because many of the drugs have been available for decades (e.g., acetaminophen), were initially approved for other indications (e.g., antidepressants and anticonvulsants), or primarily studied in acute pain and short-term treatment (e.g., acetaminophen, topical lidocaine), our findings on adverse events are not comprehensive relative to other, non-systematic review sources (e.g., product labels, large observational studies, Food and Drug Administration warnings, drug information texts). However, as Table $\mathrm{K}$ indicates, our analyses on adverse events are consistent with these other sources.

Table K provides a summary of the evidence on adverse events of interest that were identified in RCTs of patients with chronic pain meeting inclusion criteria for this review. Because the scope of this review focused on a specific patient population (chronic pain with specific conditions), a specific study design (RCTs), and study duration (12 weeks or more), it is unlikely that all important evidence on harms of these drugs would be identified. Where included evidence did not adequately address the prioritized harms, information from other sources is summarized. The evidence from other sources may have unclear applicability to patients with 
chronic pain, who may use these drugs for longer periods of time, possibly at higher doses, and who may be older (in some cases) or have more comorbidities than patients providing data for these sources.

\section{Applicability}

The applicability of the evidence-base for nonopioid drugs to treat chronic pain varies according to the pain population and intervention studied. In terms of patient populations studied, the participants were generally typical for each pain condition (with the possible exception of chronic headache). Because our definition of chronic headache was broad, and our criteria for treatments excluded use of nonopioids for prophylaxis, the result was a single, older, study of amitriptyline in patients with "chronic tension-type headache.” Headache classification has changed over the years such that the evidence identified may not be highly applicable to current patients or treatment strategies. While some RCTs excluded patients with mental illness, most did not report on baseline characteristics in relation to mental health, prior use of opioids, substance use disorder, etc.

Similarly, the specific interventions studied varied according to the pain condition. The medications studied in patients with neuropathic pain (predominantly peripheral diabetic neuropathy) and fibromyalgia were most often antidepressants (primarily duloxetine) and anticonvulsants (primarily pregabalin), with some evaluations of other categories such as capsaicin and cannabis in neuropathic pain and memantine in both conditions. In contrast, osteoarthritis and inflammatory arthritis studies involved primarily NSAIDs. In patients with osteoarthritis, a small number of studies evaluated topical diclofenac, duloxetine, and acetaminophen. As a result, we have little or no information on how some interventions that were found effective in one pain condition may affect another pain condition. An example is that the evidence on pregabalin and gabapentin is applicable mainly to patients with specific types of neuropathic pain and fibromyalgia; but not applicable to patients with osteoarthritis or rheumatoid arthritis, or other types of chronic pain. The reverse is true of NSAIDs in that the evidence is restricted to osteoarthritis or rheumatoid arthritis/ankylosing spondylitis. The use of comedications was rarely reported; acetaminophen use as a rescue medication in trials of NSAIDs was the only comedication reported. As such, it is unclear how applicable this evidence is to patients using comedications, including intermittent use of over-the-counter medications.

For all pain conditions, the most common comparator in the RCTs was placebo (117 out of 154 RCTs of good or fair quality), with limited head-to-head comparisons, especially across classes (7 RCTs). The most common head-to-head comparison was among different NSAIDs in patients with osteoarthritis (15 RCTs). The specific outcomes assessed in the included RCTs also varied according to the pain condition studied. The outcomes reported here apply mostly to the short term-12 to 24 months of treatment. The applicability of the study settings is very unclear, as few studies reported setting characteristics.

All of these elements affect how applicable the findings of this review are to a specific patient. The results apply mostly to addressing whether a drug is effective and/or harmful in comparison to no treatment, but less applicable to selecting among nonopioid treatments. However, the evidence base does provide some information on dose comparisons, such as higher and lower doses of SNRI antidepressants, pregabalin and gabapentin anticonvulsants, and some of the NSAIDs, where our analyses found little differences in efficacy, and a few cases of lower risk of adverse events with lower doses of antidepressants. 


\section{Implications and Conclusions}

Our findings show that nonopioid drugs (mainly SNRI antidepressants, pregabalin/gabapentin, and NSAIDs) result in small to moderate improvements in pain and function in the short term in patients with specific types of chronic pain, with few differences between drugs studied or doses of a drug. Drug class-specific adverse events can lead to withdrawal from treatment in some patients, and include serious cardiovascular or gastrointestinal effects with NSAIDs. Consideration of patient characteristics including comorbidities, is needed in selecting nonopioid drug treatments. These findings are mainly consistent with prior review findings, with our review finding smaller magnitude of effect in some cases.

Recent guidelines from the Centers for Disease Control and Prevention in the United States and the Canadian Guideline for Opioid Use in Chronic Non-Cancer Pain recommend nonopioid treatment as the preferred treatment for chronic pain. ${ }^{3,18}$ This review provides evidence that can be used to update these clinical practice guidelines on treating the specific, common, chronic pain conditions and can inform guideline producers on the balance of benefits and harms, in the short, intermediate, and longer term. Our report also reviewed evidence that may help inform decisions regarding prioritization of nonopioid drug therapies by clinicians and patients when selecting therapy.

Our ability to evaluate harms of included nonopioid drugs may have been limited by restricting the evidence to RCTs and to studies of patients with chronic pain, specifically. Restricting to studies of at least 12 weeks' duration may have limited the evidence for certain treatments (e.g., cannabis and topical agents) and favored interventions commonly studied in clinical trials, the majority coming from industry funding. In addition, the number of studies identified on chronic headache and sickle cell disease was low. Evidence on long-term treatment (>12 months) and for quality of life outcomes was sparse. 


\section{References}

1. Institute of Medicine Committee on

Advancing Pain Research, Care, and Education. Relieving Pain in America: A Blueprint for Transforming Prevention, Care, Education, and Research. Washington, DC: National Academies Press; 2011. doi: 10.17226/13172. PMID: 22553896.

2. Dahlhamer J, Lucas J, Zelaya C, et al. Prevalence of Chronic Pain and HighImpact Chronic Pain Among Adults - United States, 2016. MMWR. Morb Mortal Wkly Rep. 2018;67(36):1001-6. doi: 10.15585/mmwr.mm6736a2. PMID: 30212442 .

3. Dowell D, Haegerich TM, Chou R. CDC Guideline for Prescribing Opioids for Chronic Pain--United States, 2016. JAMA. 2016 Apr 19;315(15):1624-45. doi: 10.1001/jama.2016.1464. PMID: 26977696.

4. Methods Guide for Effectiveness and Comparative Effectiveness Reviews. AHRQ Publication No. 10(14)-EHC063-EF. Rockville, MD: Agency for Healthcare Research Quality; Jan 2014. https://effectivehealthcare.ahrq.gov/topics/ce r-methods-guide/overview Accessed February 1, 2019. PMID: 21433403.

5. Skelly AC, Chou R, Dettori JR, et al. AHRQ Comparative Effectiveness Reviews. Noninvasive Nonpharmacological Treatment for Chronic Pain: A Systematic Review. Rockville (MD): Agency for Healthcare Research and Quality (US); 2018.

6. Amitriptyline Hydrochloride. IBM Micromedex ${ }^{\circledR}$ (electronic version). Greenwood Village, CO: IBM Watson Health. https://www.micromedexsolutions.com/micr omedex2/librarian/PFDefaultActionId/evide ncexpert.DoIntegratedSearch?navitem=topH ome\&isToolPage=true\#. Accessed July 31, 2019.

7. Novartis. Trileptal (oxcarbazepine) [package insert]. U.S. Food and Drug Administration website. Revised Jan 4, 2019.

https://www.accessdata.fda.gov/scripts/cder/ daf/index.cfm?event=overview.process\&Ap plNo=021014. Accessed July 31, 2019.
8. Ungprasert P, Cheungpasitporn W, Crowson $\mathrm{CS}$, et al. Individual non-steroidal antiinflammatory drugs and risk of acute kidney injury: A systematic review and metaanalysis of observational studies. Eur J Intern Med. 2015 May;26(4):285-91. doi: 10.1016/j.ejim.2015.03.008. PMID: 25862494.

9. Zhang X, Donnan PT, Bell S, et al. Nonsteroidal anti-inflammatory drug induced acute kidney injury in the community dwelling general population and people with chronic kidney disease: systematic review and meta-analysis. BMC Nephrology. 2017 Aug 1;18(1):256. doi: 10.1186/s12882-0170673-8. PMID: 28764659.

10. J and J Consumer Inc. Tylenol (acetaminophen) [package insert]. U.S. Food and Drug Administration website. Revised Jan 2019. https://www.accessdata.fda.gov/scripts/cder/ daf/index.cfm?event=overview.process \&Ap plNo=019872. Accessed July 31, 2019.

11. Acetaminophen https://www.drugs.com/monograph/acetami nophen.html. Accessed July 31, 2019.

12. Kansagara D, O'Neil M, Nugent S, et al. VA Evidence-based Synthesis Program Reports. Benefits and Harms of Cannabis in Chronic Pain or Post-traumatic Stress Disorder: A Systematic Review. Washington, DC: Department of Veterans Affairs (US); 2017.

13. Bannuru RR, Schmid CH, Kent DM, et al. Comparative effectiveness of pharmacologic interventions for knee osteoarthritis: a systematic review and network metaanalysis. Ann Intern Med. 2015 Jan 06;162(1):46-54. doi: 10.7326/M14-1231. PMID: 25560713.

14. Waldfogel JM, Nesbit SA, Dy SM, et al. Pharmacotherapy for diabetic peripheral neuropathy pain and quality of life: A systematic review. Neurology. 2017 May 16;88(20):1958-67. doi: 10.1212/WNL.0000000000003882. PMID: 28341643. 
15. Lee YH, Song GG. Comparative efficacy and tolerability of duloxetine, pregabalin, and milnacipran for the treatment of fibromyalgia: a Bayesian network metaanalysis of randomized controlled trials. Rheumatol Int. 2016 May;36(5):663-72. doi: 10.1007/s00296-016-3468-5. PMID: 27000046.

16. Chou R, McDonagh M, Hartung D, et al. AHRQ Comparative Effecitveness Research. Opioid Treatments for Chronic Pain: A Systematic Review. Rockville MD: Agency for Healthcare and Quality Research (US); Forthcoming.
17. Skelly A, Chou R, Turner J, et al. AHRQ Comparative Effectiveness Review. Noninvasive Nonpharmacologic Treatments for Chronic Pain: An Update. Rockville, MD: Agency for Healthcare and Research Quality; Forthcoming.

18. Busse J, Guyatt G, Carrasco A. The 2017 Canadian Guideline for Opioids for Chronic Non-Cancer pain. Hamilton, ON: McMaster University; 2017. 


\section{Background and Objectives}

\section{Understanding Chronic Pain}

Chronic pain is typically defined as pain lasting at least 3 to 6 months or that which persists past the time for normal tissue healing. ${ }^{1}$ From a strictly biological perspective, pain is activation of the sensory nervous system's nociceptive and hypothalamic-pituitary-adrenal axis, ${ }^{2}$ and has been described as an aversive sensory and emotional experience typically caused by, or resembling that caused by, actual or potential tissue injury. ${ }^{3}$ Adding to the complexity of chronic pain are its diverse origins and the subjective experience of a sufferer. ${ }^{4}$ Chronic pain can be the result of several issues ranging from a potential underlying medical condition or disease to inflammation of injured tissue, to neuropathic pain involving a lesion or disease of the somatosensory nervous system. The manner in which pain is experienced is more than simply the biological output of an underlying issue. Attitudes, emotional disposition, and belief systems can shape the experience of pain. ${ }^{1}$ It is also heavily influenced by extrinsic psychosocial and socioeconomic factors and thus the biopsychosocial impact of chronic pain on the individual is as complex and varied as the condition itself. The physical deficits associated with chronic pain lead to reductions in function (disabilities) and quality of life, and increased medical costs. Psychological and social effects are also common and can manifest in a number of ways, including depression, anxiety, and an inability to fulfill social roles with family, friends, and employers. ${ }^{1}$ The Centers for Disease Control and Prevention (CDC) estimates that 20.4 percent of U.S. adults in 2016 had chronic pain, contributing to population morbidity and mortality and adding to the economic burden of the healthcare system. ${ }^{5}$ Annual healthcare costs due to chronic pain are estimated above $\$ 560$ billion, with 2008 costs to federal and state governments alone reaching $\$ 99$ billion. $^{1}$

\section{Chronic Pain Management}

Pain management is a dynamic process of care for an individual, with a goal of alleviating pain and dysfunction. ${ }^{6}$ Understanding pain from the biopsychosocial perspective, its management should be multimodal. The National Pain Strategy (NPS) report recommended a population-based approach which draws upon current scientific evidence. ${ }^{6}$ Self-management is often considered an important first step to alleviating chronic pain. ${ }^{1}$ While there exist numerous pharmacologic and nonpharmacologic interventions for the treatment of chronic pain, the overview below focuses on pharmacologic treatments.

The most common forms of pharmacologic treatment for pain include opioids, nonsteroidal anti-inflammatory drugs, acetaminophen, topical formulations of drugs such as lidocaine, and other drugs such as antiseizure/anticonvulsant medications and antidepressants that are used for moderating pain. Cannabis has also been used to treat chronic pain. Pharmacologic treatments can be used individually (monotherapy) or in combination, taking into consideration potential side effects and contraindications based on the patient's comorbidities.

Nationally, a concern regarding the appropriate use, misuse, and diversion of opioids, and development of substance use disorder (SUD) when opioids are used to treat chronic pain has been the subject of numerous scientific and news reports. Opioid prescriptions for chronic pain have increased substantially in the past 20 years; the number of opioid prescriptions dispensed rose from 76 million in 1999 to over 215 million in 2011, with approximately 35 percent of all opioid overdose deaths in 2017 being attributed to prescription medications. ${ }^{6,7}$ However, 
evidence shows only modest short-term benefits. ${ }^{8-12}$ Lack of evidence on long-term effectiveness ${ }^{10}$ and serious safety concerns ${ }^{9}$ speaks to the need to consider alternative treatments to opioids. The 2016 CDC Guideline for Prescribing Opioids for Chronic Pain recommended that nonopioid therapy is preferred for the treatment of chronic pain. ${ }^{13}$ To support, update, and expand such guidelines, synthesis of the current state of the science is required to guide clinicians and inform health policy.

\section{Rationale for Evidence Review and What This Review Adds}

The 2010 Patient Protection and Affordable Care Act mandated the Department of Health and Human Services to contract with the Institute of Medicine (IOM, now the National Academy of Medicine) to assess the state of the science on pain research, care, and education, and formulate recommendations in these key areas. ${ }^{1,6}$ Recommendations outlined in the 2011 IOM report have spawned a number of national initiatives to address gaps related to understanding the complexities of pain assessment and management, including the creation of the NPS under the oversight of the Interagency Pain Research Coordinating Committee (IPRCC), and creation of a federal portfolio of existing pain research to help inform additional research needs on pain. Concerns regarding the use of opioids for management of chronic pain are outlined in both the IOM report and the NPS. These initiatives, along with the recent publication of the evidencebased guideline on opioid use for chronic pain by the CDC, ${ }^{13}$ have prompted additional primary research on alternatives to opioids in managing chronic pain.

Given the complexity of treating chronic pain and concerns regarding the safety and longterm effectiveness of opioids, there is a need for a comprehensive understanding of the benefits and harms of nonopioid pharmacologic treatments for chronic pain. While there have been numerous systematic reviews on nonopioid drugs in chronic pain populations, ${ }^{14-20}$ many are outdated, focused on a single pain condition or a single drug/drug class, or reported on limited outcomes. An updated analysis that includes the main pain conditions and treatments is essential to respond to the current need to provide guidance on the use of nonopioid treatments in chronic pain.

The purpose of this report is to evaluate the effectiveness and comparative effectiveness of nonopioid pharmacologic agents, considering the effects on pain, function, quality of life, and adverse events. This review is one of three concurrent systematic reviews on treating chronic pain; other reviews address noninvasive nonpharmacologic treatments and opioids.

\section{Key Questions}

\section{Key Question 1. Effectiveness and Comparative Effectiveness}

a. In patients with chronic pain, what is the effectiveness of nonopioid pharmacologic agents versus placebo for outcomes related to pain, function, and quality of life after short-term treatment duration (3 to 6 months), intermediate-term treatment duration (6 to 12 months), and long-term treatment duration ( $\geq 12$ months)?

b. In patients with chronic pain, what is the comparative effectiveness of nonopioid pharmacologic agents compared to other nonopioid pharmacologic agents for outcomes related to pain, function, and 
quality of life after short-term treatment duration (3 to 6 months), intermediate-term treatment duration (6 to 12 months), and long-term treatment duration ( $\geq 12$ months)?

c. How does effectiveness or comparative effectiveness vary depending on: (1) the specific type or cause of pain, (2) patient demographics, (3) patient comorbidities, (4) dose of medication used, (5) duration of treatment, and (6) dose titration, including tapering?

\section{Key Question 2. Harms and Adverse Events}

a. In patients with chronic pain, what are the risks of nonopioid pharmacologic agents for harms, including overdose, misuse, dependence, substance use disorder, withdrawals due to adverse events, serious adverse events (including falls, fractures, motor vehicle accidents), and specific adverse events, according to drug class?

b. How do harms vary depending on: (1) the specific type or cause of pain, (2) patient demographics, (3) patient comorbidities, (4) dose of medication used, (5) duration of treatment, and (6) dose titration, including tapering?

\section{Analytic Framework}

The analytic framework (Figure 1) graphically describes the relationship between the Key Questions and the outcomes for this review. Inclusion criteria are provided in the Methods.

Figure 1. Analytic framework for nonopioid pharmacologic treatments for chronic pain

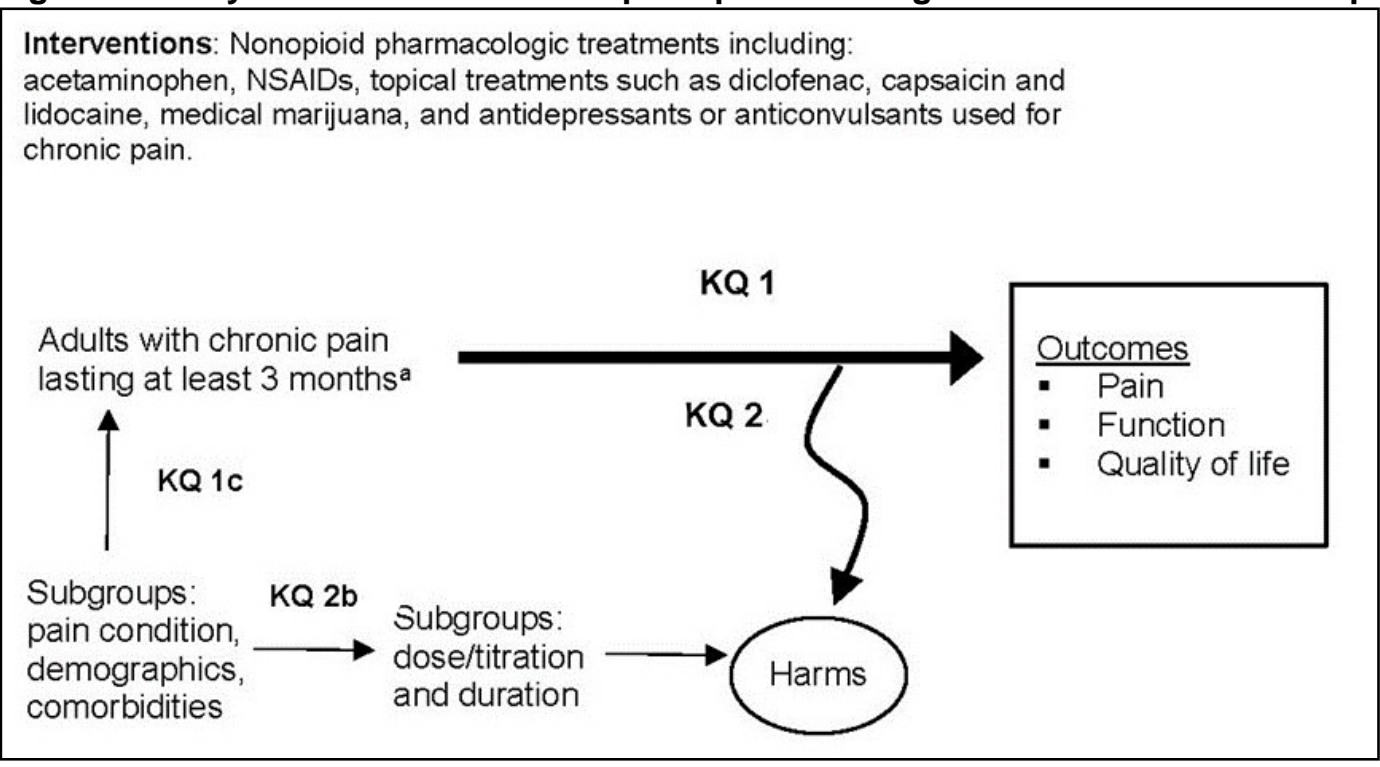

KQ = Key Question; NSAID = nonsteroidal anti-inflammatory drug 
${ }^{a}$ Includes acute exacerbations of chronic pain, pregnant/breastfeeding women, and patients treated with opioids for opioid use disorder 


\section{Methods}

This Comparative Effectiveness Review follows the methods suggested in the Agency for Healthcare Research and Quality (AHRQ) Methods Guide for Effectiveness and Comparative Effectiveness Reviews (hereafter “AHRQ Methods Guide”). ${ }^{21}$ All methods were determined $a$ priori, and a protocol was published on the AHRQ website (https://effectivehealthcare.ahrq.gov/topics/nonopioid-chronic-pain/protocol) and on PROSPERO systematic reviews registry (Registration No. CRD42019134249). Below is a summary of the specific methods used in this review. Appendix A presents the literature search strategy, and a detailed description of methods appears in Appendix B.

\section{Criteria for Inclusion/Exclusion of Studies in the Review}

The criteria for inclusion and exclusion of studies for this systematic review are based on the Key Questions and are described in Table 1. (See Appendix B for complete details.)

Table 1. PICOTS: Inclusion and exclusion criteria

\begin{tabular}{|c|c|c|}
\hline PICOTS & Inclusion Criteria & Exclusion Criteria \\
\hline $\begin{array}{l}\text { Populations } \\
\text { and } \\
\text { Conditions }\end{array}$ & $\begin{array}{l}\text { - For all KQs: Adults (age } \geq 18 \text { years) with chronic pain } \\
\text { (pain lasting }>3 \text { months). } \\
\text { - For KQs } 1 c, 2 \text { b specific chronic pain populations: } \\
\text { o Neuropathic } \\
\text { o Musculoskeletal (e.g., low back pain, osteoarthritis) } \\
\text { o Fibromyalgia (assessed using established criteria) } \\
\text { o Sickle cell disease } \\
\text { o Inflammatory arthritis (e.g., rheumatoid arthritis) } \\
\text { o Chronic headache }\end{array}$ & $\begin{array}{l}\text { - Pain at the end of life (life expectancy } \leq 6 \\
\text { months) } \\
\text { - } \text { Acute pain (<8 weeks duration), including } \\
\text { sickle cell crisis } \\
\text { - Pain due to active malignancy (e.g., } \\
\text { tumor-related pain while receiving active } \\
\text { treatment to reduce tumor size) } \\
\text { - Episodic migraine } \\
\text { - Undefined mixed pain conditions }\end{array}$ \\
\hline Interventions & $\begin{array}{l}\text { Nonopioid pharmacologic drugs for chronic pain: } \\
\text { - Oral pharmacologic agents specifically used to treat } \\
\text { chronic pain: } \\
\text { o NSAIDs (e.g., celecoxib, diclofenac, ibuprofen) } \\
\text { o Antidepressants SNRIs (i.e., duloxetine, } \\
\text { milnacipran) and TCAs (e.g., amitriptyline) } \\
\text { o Anticonvulsants: Carbamazepine, gabapentin, } \\
\text { oxcarbazepine, pregabalin } \\
\text { o Other: Acetaminophen, muscle relaxants (e.g., } \\
\text { cyclobenzaprine, diazepam), memantine } \\
\text { - Topical agents (diclofenac, capsaicin, and lidocaine) } \\
\text { - Medical cannabis in all forms, including } \\
\text { phytocannabinoids and synthetic cannabinoids }\end{array}$ & $\begin{array}{l}\text { - Injectable preparations, including biologic } \\
\text { drugs, corticosteroids, etc. } \\
\text { - Other antidepressants (e.g., SSRIs, } \\
\text { MAOIs) } \\
\text { - Other antiepileptics (e.g., topiramate, } \\
\text { lamotrigine, levetiracetam, phenytoin) } \\
\text { - Drugs used for migraine prophylaxis } \\
\text { (e.g., verapamil, beta-blockers) or } \\
\text { treating acute migraine (e.g., triptans) } \\
\text { - Salicylates (topical and oral) } \\
\text { - Topical menthol preparations } \\
\text { - Disease-modifying drugs for rheumatoid } \\
\text { arthritis (DMARDs, e.g., methotrexate) }\end{array}$ \\
\hline Comparators & $\begin{array}{l}\text { - For KQ } 1 \mathrm{a} / \mathrm{b} \text { and } 2 \mathrm{a} / \mathrm{b} \text { : Placebo } \\
\text { - For KQ } 1 \mathrm{c} \text { and } 2 \mathrm{a} / \mathrm{b} \text { : Another included nonopioid } \\
\text { pharmacologic agent, dose, or treatment duration }\end{array}$ & $\begin{array}{l}\text { - Nonpharmacologic treatment } \\
\text { (comparison to nonopioids included in } \\
\text { review of nonpharmacologic treatments) } \\
\text { - Opioid treatment }\end{array}$ \\
\hline
\end{tabular}




\begin{tabular}{|c|c|c|}
\hline PICOTS & Inclusion Criteria & Exclusion Criteria \\
\hline Outcomes & $\begin{array}{l}\text { - Pain, function, and quality of life using validated } \\
\text { outcome measures. } \\
\text { o Pain severity is the assessment of improvement in } \\
\text { pain from baseline as a continuous measure. Pain } \\
\text { response is the dichotomous assessment whether } \\
\text { patients' improvement meet an established } \\
\text { threshold (e.g., 30\% improvement). } \\
\text { o Patient-reported pain assessments are prioritized. } \\
\text { Pain response based on clinician assessments } \\
\text { was also acceptable and noted where they are } \\
\text { reported. } \\
\text { o Secondary outcomes include mood, sleep, and } \\
\text { global assessments using validated scales. } \\
\text { - All drug classes: Withdrawal from treatment due to } \\
\text { adverse events (any adverse event, not specifically } \\
\text { symptoms of withdrawal from an opioid or other } \\
\text { drug), incidence of serious adverse events, overdose, } \\
\text { misuse, addiction, and development of substance } \\
\text { use disorder. } \\
\text { Key specific adverse events according to drug class } \\
\text { (e.g., gastrointestinal and cardiovascular events, } \\
\text { kidney and liver-related harms with NSAIDs). }\end{array}$ & $\begin{array}{l}\text { - Intermediate outcomes (e.g., } \\
\text { pharmacokinetics/pharmacodynamics, } \\
\text { drug-drug interactions, dose } \\
\text { conversions) } \\
\text { - Indirect measurement of pain (e.g., } \\
\text { quantitative sensory testing). } \\
\end{array}$ \\
\hline Timing & $\begin{array}{l}\text { Short- ( } 3 \text { to }<6 \text { months), intermediate- ( } 6 \text { to }<12 \\
\text { months), and long-term }(\geq 12 \text { months) treatment duration }\end{array}$ & $\begin{array}{l}\text { Studies or outcomes reported with }<3- \\
\text { month duration of treatment }\end{array}$ \\
\hline Setting & $\begin{array}{l}\text { Outpatient settings (e.g., primary care, pain clinics, } \\
\text { emergency rooms, urgent care clinics) }\end{array}$ & $\begin{array}{l}\text { Addiction treatment settings, inpatient } \\
\text { settings }\end{array}$ \\
\hline $\begin{array}{l}\text { Study } \\
\text { Design }\end{array}$ & $\begin{array}{l}\text { - Randomized controlled trials } \\
\text { - High-quality, recent systematic reviews that best } \\
\text { match the scope of this review } \\
\text { - English language publications }\end{array}$ & $\begin{array}{l}\text { - Observational studies } \\
\text { - Outdated/out of scope systematic } \\
\text { reviews } \\
\text { - Non-English language publications }\end{array}$ \\
\hline
\end{tabular}

DMARDs = disease-modifying antirheumatic drug; KQ = Key Question; MAOI = monoamine oxidase inhibitor; NSAID = nonsteroidal anti-inflammatory drug; PICOTS = populations, interventions, comparators, outcomes, timing, setting, study design; SNRI = serotonin and norepinephrine reuptake inhibitor; SSRI = selective serotonin reuptake inhibitor; TCA = tricyclic antidepressant

${ }^{\text {a }}$ Chronic headache defined as (International Classification of Headache Disorders, 3rd edition definition ${ }^{22}$ ): Primary headaches attributed to the headache condition itself, not caused by another disease or medical condition. Chronic headache is defined as 15 or more days each month for at least 12 weeks or history of headache more than 180 days a year.

\section{Literature Search}

We conducted electronic searches in Ovid ${ }^{\circledR}$ MEDLINE ${ }^{\circledR}$, mbase $^{\circledR}$, PsycINFO $^{\circledR}$, CINAHL $^{\circledR}$, Cochrane CENTRAL, and Cochrane Database of Systematic Reviews through September 10, 2019 (from database inception, see Appendix A for full strategies). Reference lists of included systematic reviews were screened for includable studies. Manufacturers of included drugs submitted potential relevant studies to include in this review using the Federal Register notification. We screened citations identified through our searched using the pre-established criteria above to determine eligibility for full-text review, with any citation deemed not relevant by one reviewer screened by a second reviewer. ${ }^{21}$ Citations deemed potentially eligible were retrieved for full-text screening, with each article independently reviewed for eligibility by two reviewers. Any disagreements were resolved by consensus. 


\section{Assessment of Methodological Risk of Bias of Individual Studies}

We assessed the quality (or risk of bias) of included randomized controlled trials (RCTs) based on principles for appraisal as developed by the Cochrane Back and Neck Group, ${ }^{23}$ and outlined in the AHRQ Methods Guide chapter "Assessing the Risk of Bias of Individual Studies When Comparing Medical Interventions". ${ }^{21,24}$ Based on the risk of bias assessment, each included study was rated as "good," "fair," or "poor" quality. Assessments of RCTs included in good-quality systematic reviews that we included here were reviewed by a single reviewer, with the exception that any rated poor quality or high risk of bias were reassessed by our team using dual review.

\section{Data Synthesis}

Data were qualitatively summarized in tables. The magnitude of effects for pain, function, and quality of life were classified using the system in the 2018 AHRQ Noninvasive Nonpharmacological Treatment for Chronic Pain review (Table 2). ${ }^{25}$ Mean differences are based on a $0-10$ scale, unless otherwise noted.

Table 2. Definitions of effect sizes

\begin{tabular}{|l|l|}
\hline Effect Size & Definition \\
\hline Small effect & $\begin{array}{l}\text { - MD } 0.5 \text { to } 1.0 \text { points on a } 0 \text { to 10-point scale, } 5 \text { to } 10 \text { points on a } 0 \text { to 100-point scale } \\
\text { - SMD } 0.2 \text { to } 0.5 \\
\text { - RR/OR } 1.2 \text { to } 1.4\end{array}$ \\
\hline $\begin{array}{l}\text { Moderate } \\
\text { effect }\end{array}$ & $\begin{array}{l}\text { - MD }>1 \text { to } 2 \text { points on a } 0 \text { to10-point scale, }>10 \text { to } 20 \text { points on a } 0 \text { to 100-point scale } \\
\text { - } \mathrm{RR} / \mathrm{OR} 1.5 \text { to } 1.9\end{array}$ \\
\hline Large effect & $\begin{array}{l}\text { - MD }>2 \text { points on a } 0 \text { to10-point scale, }>20 \text { points on a } 0 \text { to 100-point scale } \\
\text { - SMD }>0.8\end{array}$ \\
& - RR/OR $\geq 2.0$
\end{tabular}

$\mathrm{MD}$ = mean difference; $\mathrm{OR}$ = odds ratio; $\mathrm{RR}$ = relative risk; SMD = standardized mean difference

Meta-analyses, using random effects model, were conducted to summarize data and obtain more precise estimates where there were at least two studies reporting outcomes homogeneous enough to provide a meaningful combined estimate. The Profile Likelihood model was used, unless the model failed to converge, then a DerSimonian and Laird model was used. To determine whether meta-analysis was meaningfully performed, we considered the quality of the studies and the heterogeneity among studies in design, patient population, interventions, and outcomes, and conducted sensitivity analyses. Poor-quality studies were not pooled with other studies. The Key Questions were designed to assess the comparative effectiveness and harms by patient demographics, comorbidities, pain types, treatment dosing strategies, and durations; we conducted subgroup and sensitivity analyses to explore the impact of these variables. In comparisons with placebo, we combined various dosing arms and drugs within the same pharmacologic class, exploring differences based on these factors in subgroup analyses. In metaanalysis findings below, $\mathrm{I}^{2}$ stands for Inconsistency (0\% to 100\%), reflecting statistical heterogeneity. See Appendix B for additional details on data synthesis. 


\section{Grading the Strength of Evidence for Major Comparisons and Outcomes}

The strength of evidence (SOE) was rated for priority clinical outcomes (pain, function, quality of life) for each pain condition-treatment pair, using the approach described in the AHRQ Methods Guide. ${ }^{21}$ To ensure consistency and validity of the evaluation, the grades were reviewed by a second reviewer. The domains assessed were study limitations (low, medium, or high), consistency (consistent, inconsistent, or unknown/not applicable), directness (direct or indirect), precision (precise or imprecise), and publication bias (suspected or undetected). The SOE was assigned an overall grade of high, moderate, low, or insufficient, reflecting our confidence in the effect estimates and whether the findings are stable (Table 3). Evidence is found to be insufficient to draw conclusions when we have no evidence available or the body of evidence has unacceptable deficiencies, precluding reaching a conclusion.

Table 3. Description of the strength of evidence grades

\begin{tabular}{|l|l|}
\hline Strength of Evidence & Description \\
\hline High & $\begin{array}{l}\text { Very confident that the effect estimate lies close to the true effect for this outcome. The } \\
\text { body of evidence has few or no deficiencies. Findings are stable, i.e., inclusion of } \\
\text { additional studies would not change the conclusions. }\end{array}$ \\
\hline Low & $\begin{array}{l}\text { Moderately confident that the estimate of effect lies close to the true effect for this } \\
\text { outcome. The body of evidence has some deficiencies. We believe that the findings are } \\
\text { likely to be stable, but some doubt remains. }\end{array}$ \\
\hline Insufficient & $\begin{array}{l}\text { Limited confidence that the effect estimate lies close to the true effect for this outcome. } \\
\text { needed body of evidence has major or numerous deficiencies. Additional evidence is } \\
\text { close to the true effect. }\end{array}$ \\
\hline & $\begin{array}{l}\text { No confidence in the estimate of effect for this outcome. No evidence is available or the } \\
\text { body of evidence has unacceptable deficiencies, precluding reaching a conclusion. }\end{array}$ \\
\hline
\end{tabular}

\section{Assessing Applicability}

Applicability of the bodies of evidence were assessed by examining the characteristics of the populations, interventions, comparators, outcomes, timing, setting, and study design (PICOTS) elements, such as patient population characteristics (e.g., demographic characteristics, duration or severity of pain, underlying pain condition, presence of medical co-morbidities), clinical settings (e.g., primary care, specialty setting), or countries (e.g., non-U.S.) in which the studies are performed. These characteristics indicate to whom the results are directly applicable; applicability to patients, interventions, outcomes, etc. outside of these may be limited and results may differ.

\section{Peer Review and Public Commentary}

Experts in the field of chronic pain conditions were invited to provide external peer review of this systematic review. Comments and editorial review were also provided by the AHRQ Task Order Officer and an associate editor. The draft report was posted on the AHRQ website for 4 weeks to elicit public comment. In response to reviewers' comments, we revised text as needed and addressed all relevant reviewer comments in an associated disposition of comments report with the authors' individual responses. This report will be posted after the publication of the final comparative effectiveness review on AHRQ’s website. 


\section{Results}

\section{Results of Literature Search}

A total of 8,488 references were reviewed, including 8,025 from electronic database searches and 463 from reviewing studies included in prior Evidence-based Practice Center reports and other systematic reviews. After dual review of titles and abstracts, 1,292 articles were selected for full-text review, of which 184 randomized controlled trials (RCTs) in 217 publications were included in this review (Appendix C) and 1,070 were excluded (Appendix D). In addition, we identified 5 systematic reviews that included 47 of the trials included in this review. Search results and selection of studies are summarized in the literature flow diagram (Figure 2). Results are shown by Key Question and then by condition for efficacy. Harms results are organized by drug class. Overall, 30 trials were rated poor quality, 129 fair quality, and 25 good quality. Of the good- and fair-quality trials, 128 were classified as short term ( 3 months to $<6$ months), 18 intermediate term ( 6 months to $<1$ year), and 8 were long term $(\geq 1$ year). We included 32 RCTs in neuropathic pain, 26 RCTs in fibromyalgia, 59 RCTs in osteoarthritis, 21 RCTs in inflammatory arthritis, 7 RCTs in low back pain, and 1 trial each in chronic headache and sickle cell disease. An additional seven trials of mixed osteoarthritis and inflammatory arthritis patients were included for harms outcomes. Most study participants were female (66.7\%) but proportion varied widely by condition with the highest seen in fibromyalgia trials. The median age of participants was 59 years and mean pain duration was 7.9 years. Participants reported a weighted mean pain severity of 6 on a scale of 0 to10. Industry was the leading provider of funding for trials (82\%) while 15 trials (10\%) did not report funding source. Data abstraction of study characteristics and results for good- and fair-quality studies, and quality assessment for all included studies, are available in Appendixes E, F, and G. Strength of evidence (SOE) grades for priority clinical outcomes (pain, function, quality of life) for each pain condition-treatment pair appear in Appendix H. Results of meta-analyses, including forest plots and subgroup analyses, appear in Appendix I. 
Figure 2. Literature flow diagram

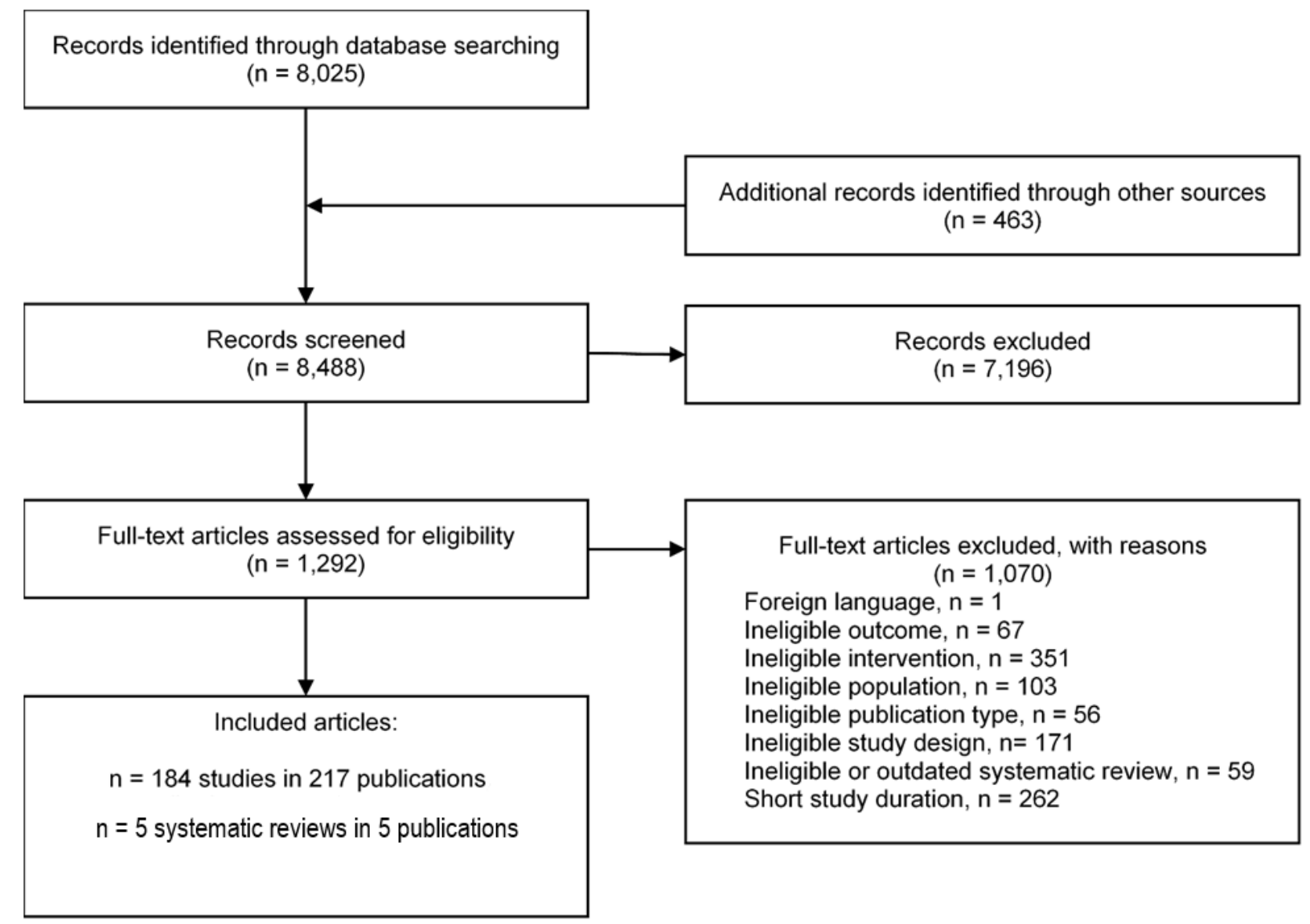

\section{Key Question 1: Effectiveness and Comparative Effectiveness}

\section{Neuropathic Pain}

\section{Key Points}

- In the short term, the anticonvulsant drugs pregabalin, the prodrug gabapentin enacarbil, and oxcarbazepine provided small improvement in pain (SOE: Moderate) and pain response (SOE: Moderate and Low) in patients with neuropathic pain mainly diabetic peripheral neuropathy and/or postherpetic neuralgia. Functional outcomes were not improved with gabapentin enacarbil in patients with postherpetic neuralgia, and quality of life was not improved with pregabalin, gabapentin enacarbil, or oxcarbazepine (SOE: Low).

- In the short term, the serotonin-norepinephrine reuptake inhibitor (SNRI) antidepressant duloxetine resulted in small improvements in pain, function, and quality of life in diabetic peripheral neuropathy (SOE: Moderate for pain and quality of life, Low for function).

- In the short term, topical capsaicin patch resulted in improvements in pain severity that did not reach the level of a small effect, and pain response was not significantly better 
than placebo in patients with postherpetic neuralgia and with HIV-associated neuropathy (SOE: Moderate).

- In the short term, cannabis (dronabinol oral solution, tetrahydrocannabinol/cannabidiol [THC/CBD] oral spray) had no effect on pain severity in multiple sclerosis or allodynia, but THC/CBD oral spray improved pain response to a moderate degree in patients with allodynia. Function and quality of life were not improved (SOE: Low).

- Comparisons of pregabalin with gabapentin (diabetic peripheral neuropathy and peripheral nerve injury), either drug with duloxetine (diabetic peripheral neuropathy), and memantine with placebo (HIV-related neuropathy) did not find significant differences (SOE: Low to insufficient).

\section{Detailed Assessment}

Thirty-two good- and fair-quality RCTs (in 36 publications) involving 9,392 patients evaluated nonopioid drugs to treat chronic neuropathic pain: 31 short-term (12 to 17 weeks) and 1 long-term trials (52 weeks). These included 29 placebo-controlled trials, 5 trials comparing multiple doses of duloxetine, 4 trials comparing multiple doses of pregabalin, 2 trials comparing multiple doses of the prodrug gabapentin enacarbil (with higher blood levels for longer periods than gabapentin), 1 trial comparing multiple doses of oxcarbazepine, and 3 head-to-head trials (gabapentin vs. pregabalin; gabapentin vs. pregabalin vs. duloxetine; and gabapentin enacarbil vs. pregabalin). Four trials met criteria for good quality, ${ }^{26-29} 28$ trials met criteria for fair quality, ${ }^{30-57}$ and 6 RCTs were rated poor quality (Appendix G). ${ }^{58-63}$ The poor-quality studies were deemed to have high risk of bias due to unclear randomization and allocation concealment techniques, baseline differences between randomized groups, lack of blinding, and high attrition. One of the poor-quality studies was the only RCT of carbamazepine found for this review. ${ }^{61}$

Studies were conducted most frequently in the United States (25\%) and in Asia (19\%); 34 percent were conducted in 4 or more countries. Most trials were funded by industry (91\%). The majority of studies enrolled patients with painful diabetic peripheral neuropathy (53\%) and/or with postherpetic neuralgia (16\%). Other conditions included neuropathic pain associated with HIV, spinal cord injury, peripheral nerve injury, stroke, and multiple sclerosis. Weighted mean age of enrolled participants across trials was 58 years (range 25 to 71 years) with 41 percent (weighted mean) being female (range $0 \%$ to $73 \%$ ) and 41 percent (weighted mean) nonwhite (range $0 \%$ to $100 \%)$. Weighted mean baseline pain score was 6.2 (0-10 numeric rating scale [NRS], range 5.3 to 7.0, 26 trials) and the weight mean visual analog scale (VAS) pain score was 70 (0-100, range 61 to 73, 4 trials). Few studies reported baseline function or quality of life. Weighted mean duration of neuropathic pain was 3.9 years (range 0.25 to 10.2 years, 26 trials). Complete descriptions of included study characteristics are in Appendix E.

\section{Anticonvulsants}

\section{Pregabalin and Gabapentin}

Fourteen RCTs compared pregabalin with placebo: six trials enrolled patients with diabetic peripheral neuropathy, ${ }^{30,39,44,45,50,51}$ one trial enrolled patients with postherpetic neuralgia, ${ }^{52}$ and one enrolled a mixed population of patients with either diabetic peripheral neuropathy or postherpetic neuralgia. ${ }^{35}$ Six RCTs enrolled patients with other types of neuropathic pain: spinal cord injury (2 studies), ${ }^{27,48}$ HIV (2 studies), ${ }^{29,49}$ and one study each in patients with neuropathic pain associated with stroke, ${ }^{41}$ and trauma. ${ }^{42}$ Study treatments were short term (range 12 to 17 
weeks) and involved flexible-dose pregabalin (e.g., $150 \mathrm{mg}$ to $600 \mathrm{mg}$ daily based on response and tolerability), ${ }^{29,35,39,41,42,48,49}$ or fixed-dose pregabalin (e.g., $150 \mathrm{mg}, 300 \mathrm{mg}, 600 \mathrm{mg}$ daily). ${ }^{27,30,45,50-52}$ One study compared flexible-dose pregabalin (150 mg to $600 \mathrm{mg}$ daily) with fixed-dose pregabalin (600 mg daily). ${ }^{35}$

A study of the prodrug gabapentin enacarbil in patients with postherpetic neuralgia randomized patients to $1200 \mathrm{mg}, 2400 \mathrm{mg}, 3600 \mathrm{mg}$ daily, or placebo, but combined data for the three drug arms after finding no difference in pain improvement between them. ${ }^{57} \mathrm{~A}$ study of gabapentin enacarbil, pregabalin, and placebo in patients with diabetic peripheral neuropathy also combined data for the drug arms for similar reasons. ${ }^{44}$

\section{Pain}

In the short term, meta-analysis of 15 trials found a small reduction in pain with pregabalin/gabapentin enacarbil compared with placebo ( $N=4,832$, mean difference [MD] -0.61 , $95 \%$ confidence interval [CI] -0.87 to $-0.36, \mathrm{I}^{2}=72 \%, 0-10$ scale; Appendix I). Treatment with pregabalin/gabapentin enacarbil also resulted in more patients achieving at least a 30 percent reduction in mean pain score (risk ratio [RR] 1.27, 95\% CI 1.12 to 1.50, $\mathrm{I}^{2}=72 \%$; Appendix I). Subgroup analyses on pain etiology, study drug, and trial quality did not alter these findings meaningfully (Appendix I).

Although the subgroup analysis of dose was not statistically significant, pregabalin $600 \mathrm{mg}$ daily resulted in a numerically larger, statistically significant, reduction in pain and more patients achieving response than lower doses (Table 4). ${ }^{45,51,52}$ Fixed-dose pregabalin $600 \mathrm{mg}$ daily and flexible-dose pregabalin (150 $\mathrm{mg}$ to $600 \mathrm{mg}$ daily) did not differ in the proportion who achieved response ( $\geq 30 \%$ decrease in pain score; $66.4 \%$ vs. $59.0 \%$, RR $1.13,95 \%$ CI 0.94 to 1.36 ). ${ }^{35}$ In the two trials of gabapentin enacarbil, there was little difference in pain score improvement among doses (Table 4). ${ }^{44,57}$ These findings are moderate strength of evidence. 
Table 4. Pregabalin/gabapentin pain improvement dose analysis

\begin{tabular}{|c|c|c|c|c|}
\hline $\begin{array}{l}\text { Outcome } \\
\text { Sample Size }\end{array}$ & Drug Dose & $\begin{array}{l}\text { N Studies } \\
\text { (Sample } \\
\text { Size) }\end{array}$ & Effect Size (95\% Cl) & $\begin{array}{l}\text { Treatment by Drug } \\
\text { Interaction p-value }\end{array}$ \\
\hline $\begin{array}{l}\text { Pain improvement } \\
15 \text { RCTs } \\
(n=4,576)\end{array}$ & $\begin{array}{l}\text { Pregabalin pooled } \\
150 \text { mg daily } \\
300 \text { mg daily } \\
600 \text { mg daily } \\
150-600 \text { mg daily } \\
300-600 \text { mg daily } \\
450-600 \text { mg daily } \\
\\
\text { Gabapentin pooled } \\
1200 \text { mg daily } \\
2400 \text { mg daily } \\
3600 \text { mg daily } \\
1200-3600 \text { mg } \\
\text { daily }\end{array}$ & $\begin{array}{l}14(3,971) \\
2(374) \\
5(1,035) \\
4(725) \\
10(2,715) \\
2(572) \\
1(373) \\
2(725) \\
2(384) \\
2(353) \\
2(418) \\
2(725)\end{array}$ & $\begin{array}{l}\text { MD }-0.61(-0.87 \text { to }-0.36) \\
\text { MD }-0.55(-1.31 \text { to } 0.17) \\
\text { MD }-0.36(-0.89 \text { to } 0.17) \\
\text { MD }-1.17(-1.69 \text { to }-0.67) \\
\text { MD }-0.75(-1.13 \text { to }-0.39) \\
\text { MD }-0.82(-1.48 \text { to }-0.18) \\
\text { MD }-0.02(-0.39 \text { to } 0.35) \\
\text { MD }-0.58(-1.26 \text { to } 0.10) \\
\text { MD }-0.66(-1.21 \text { to }-0.08) \\
\text { MD }-0.27(-1.33 \text { to } 0.82) \\
\text { MD }-0.74(-1.50 \text { to }-0.01)\end{array}$ & 0.90 \\
\hline $\begin{array}{l}\text { Pain response } \\
15 \text { RCTs } \\
(n=4,576)\end{array}$ & $\begin{array}{l}\text { Pregabalin pooled } \\
150 \text { mg daily } \\
300 \text { mg daily } \\
600 \text { mg daily } \\
150-600 \text { mg daily } \\
300-600 \text { mg daily } \\
450-600 \text { mg daily } \\
\\
\text { Gabapentin pooled } \\
1200 \text { mg daily } \\
2400 \text { mg daily } \\
3600 \text { mg daily } \\
1200-3600 \text { mg } \\
\text { daily }\end{array}$ & $\begin{array}{l}14(3,971) \\
2(369) \\
5(1,029) \\
4(719) \\
10(2,876) \\
2(572) \\
1(373) \\
\\
2(725) \\
2(384) \\
2(353) \\
2(418) \\
2(725)\end{array}$ & $\begin{array}{l}\text { RR } 1.28 \text { (1.09 to } 1.54) \\
\text { RR } 1.57 \text { (0.74 to } 3.58) \\
\text { RR } 1.21 \text { (0.89 to } 1.73) \\
\text { RR } 1.93 \text { (1.38 to } 2.79) \\
\text { RR } 1.35 \text { (1.13 to } 1.68) \\
\text { RR } 1.63 \text { (1.15 to } 2.26) \\
\text { RR } 0.94 \text { (0.77 to } 1.16) \\
\\
\text { RR } 1.20 \text { (0.94 to } 1.57) \\
\text { RR } 1.16(0.88 \text { to } 1.53) \\
\text { RR } 1.17(0.72 \text { to } 1.84) \\
\text { RR } 1.29(1.01 \text { to } 1.66) \\
\text { RR } 1.20(0.94 \text { to } 1.57)\end{array}$ & 0.82 \\
\hline
\end{tabular}

$\mathrm{CI}$ = confidence interval; $\mathrm{MD}$ = mean difference; RCTs = randomized controlled trials; $\mathrm{RR}$ = relative risk

\section{Function}

One short-term trial of gabapentin enacarbil $(\mathrm{N}=371)$ examined function using the Brief Pain Inventory (BPI) Interference scale in patients with postherpetic neuralgia and found no difference in function between pooled gabapentin enacarbil doses (1200 mg, $2400 \mathrm{mg}, 3600 \mathrm{mg}$ daily) versus placebo (MD - $0.23,95 \%$ CI -0.70 to 0.23$).{ }^{57}$ This is low strength of evidence.

\section{Quality of Life}

In the short term, three fair-quality pregabalin trials in patients with diabetic peripheral neuropathic pain found that treatment with pregabalin did not improve quality of life scores (standardized mean difference [SMD] 0.24, 95\% CI -0.07 to $0.54, \mathrm{I}^{2}=58 \%$ ) using the Euro Quality of Life (EQ-5D). ${ }^{39,41,51}$ Similarly, two RCTs of pregabalin (one each in diabetic peripheral neuropathy and HIV) and one of gabapentin enacarbil (in postherpetic neuralgia) found no difference between the drugs and placebo using the Short Form-36 (SF-36) scale (Appendix I). ${ }^{44,49,57}$ Subgroup analyses on study drug and drug dose did not show significant effects. This is low strength of evidence.

\section{Other Outcomes}

In the short term, meta-analysis of all RCTs of pregabalin and gabapentin enacarbil for neuropathic pain found a small magnitude of improvement in sleep compared with placebo (MD $-0.65,95 \%$ CI -0.89 to $-0.41, I^{2}=70 \%, 0-10$ scale; Appendix I). ${ }^{27,29,30,35,39,41,42,44,45,48-52,57}$ 
Six RCTs of pregabalin, ${ }^{27,29,39,41,48,49}$ one RCT of gabapentin enacarbil, ${ }^{57}$ and one of both pregabalin and gabapentin enacarbil ${ }^{44}$ found no short-term benefit on anxiety or depression as assessed with the Hospital Anxiety and Depression Scale (HADS; Appendix I). Subgroup analyses based on etiology of pain showed no significant effects for sleep, anxiety, or depression.

\section{Oxcarbazepine}

\section{Pain}

In the short term, in patients with diabetic peripheral neuropathic pain, oxcarbazepine resulted in a small improvement in pain severity (2 RCTs, N=493, MD -0.89, 95\% CI - 1.50 to $-0.37, \mathrm{I}^{2}=0 \%$, VAS $0-10$ scale; Appendix I). ${ }^{31,34}$ Doses ranged from $300 \mathrm{mg}$ to $1800 \mathrm{mg}$ daily flexible dose in one trial and $600 \mathrm{mg}, 1200 \mathrm{mg}$, or $1800 \mathrm{mg}$ daily fixed dose in a second trial. This is moderate strength of evidence. Treatment with higher dose oxcarbazepine (1200 mg and $1800 \mathrm{mg}$ daily) resulted in improved pain scores compared with placebo in one trial. ${ }^{31}$ A greater proportion of patients treated with oxcarbazepine achieved a greater than 30 percent improvement in pain response than patients given placebo ( $45.6 \%$ vs. $28.9 \%, \mathrm{p}=0.028){ }^{34}$ This is low strength of evidence.

\section{Quality of Life}

In patients with diabetic peripheral neuropathic pain, oxcarbazepine did not consistently improve quality of life, as measured on the SF-36 scale. Both trials reported similar SF-36 scale scores with oxcarbazepine and placebo, though one trial noted a statistically significant difference between groups in SF-36 mental component summary (MCS) scores (47.2 versus 50.2, $\mathrm{p}=0.03) .{ }^{34}$ This is low strength evidence.

\section{Other Outcomes}

One trial reported a lower incidence of sleep disruption due to pain in the oxcarbazepine group ( $\mathrm{p}=0.02$ ), while the other trial found no difference between groups in sleep. ${ }^{31}$

\section{Antidepressants, SNRI}

\section{Duloxetine}

Six short-term (12 week) RCTs compared duloxetine with placebo at doses from $20 \mathrm{mg}$ to 120 mg daily. ${ }^{36-38,43,54,56}$ All patients had peripheral neuropathic pain from diabetes. One longterm (52-week), open-label extension RCT compared duloxetine $40 \mathrm{mg}$ daily with $60 \mathrm{mg}$ daily. ${ }^{55}$

\section{Pain}

Pooled analysis of the six short-term trials found a small magnitude reduction in pain with duloxetine versus placebo (MD -0.79, 95\% CI - 1.10 to -0.49 , $\mathrm{I}^{2}=43 \%$, $0-10$ scale; Appendix I) ${ }^{36-}$ $38,43,54,56$ Patients were also more likely to achieve response ( $\geq 30 \%$ improvement in pain in 5 RCTs, $\geq 50 \%$ in 1 RCT) with duloxetine compared with placebo (RR 1.39, 95\% CI 1.22 to 1.62, $\left.\mathrm{I}^{2}=39 \%\right)$, a small magnitude effect. A long-term RCT $(\mathrm{N}=257)$ found that duloxetine $40 \mathrm{mg}$ daily versus $60 \mathrm{mg}$ daily produced similar reductions in pain at 52 weeks. ${ }^{55}$ This is moderate strength of evidence. 


\section{Function}

In the short term, based on a meta-analysis of six trials, function as assessed by the BPI Interference scale was improved to a small degree with duloxetine (SMD -0.31, 95\% CI -0.42 to -0.20, I ${ }^{2}=0 \%$; Appendix I). ${ }^{36-38,43,54,56}$ A long-term extension RCT (N=257) found no difference in function (BPI Interference) between duloxetine $40 \mathrm{mg}$ daily versus $60 \mathrm{mg}$ daily at 52 weeks, ${ }^{55}$ which was similar to the results at 12 weeks in another RCT. ${ }^{36-38,43,54,56}$ This is low strength of evidence.

\section{Quality of Life}

Meta-analysis of three trials finds that duloxetine improved quality of life to a small degree as measured on the EQ-5D (MD 0.22, 95\% CI 0.05 to 0.38, $\mathrm{I}^{2}=0 \%, 0-1$ scale; Appendix I). ${ }^{37,38,54}$ This is moderate strength of evidence.

\section{Other Outcomes}

In the short term, one trial $(\mathrm{N}=457)$ reported no difference in change from baseline on the Beck Anxiety Inventory (BAI) for duloxetine at daily doses of $20 \mathrm{mg}, 60 \mathrm{mg}$, and $120 \mathrm{mg}$ versus placebo. ${ }^{38}$ Three RCTs examined changes in depression symptoms as measured by the Hamilton Rating Scale for Depression (HAMD) and the Beck Depression Inventory (BDI) scales. ${ }^{38,43,54}$ Meta-analysis did not identify a significant difference between placebo and duloxetine (SMD -0.07, 95\% CI -0.34 to 0.20, $\mathrm{I}^{2}=44 \%$; Appendix I).

In the short term, meta-analysis based on five RCTs $(\mathrm{N}=2,478)$ found that sleep interference on the BDI subscale was improved more with duloxetine (40, 60 or $120 \mathrm{mg}$ daily) than placebo, but the difference was very small (MD $-0.60,95 \%$ CI -0.86 to $-0.34, \mathrm{I}^{2}=0 \%, 0-10$ scale). ${ }^{36,37,43,54,56}$ A long-term RCT ( $\left.\mathrm{N}=257\right)$ found no difference in sleep at 52 weeks between duloxetine $40 \mathrm{mg}$ daily and $60 \mathrm{mg}$ daily. ${ }^{55}$

\section{Subgroups}

A post-hoc analysis of three short-term RCTs in patients with diabetic peripheral neuropathic pain stratified patients based on age ( $<65$ years, $\geq 65$ years) and found no differences between the older subgroup and the younger subgroup on pain response (30\% and $50 \%$ reductions in pain) and function (BPI interference; Appendix I). ${ }^{55,64}$

\section{Other Drugs}

\section{Cannabis}

Cannabis (including derivatives and synthetic cannabinoids) was compared with placebo in two short-term trials $(\mathrm{N}=486)$ in those with neuropathic pain related to multiple sclerosis ${ }^{28}$ or with allodynia $^{47}$ (Appendix E). The trials utilized oral dronabinol solution (mean $13 \mathrm{mg}$ daily) and THC/CBD oromucosal spray (100 mL per spray, up to 24 sprays daily). One trial was rated good quality ${ }^{28}$ and the other fair quality. ${ }^{47} \mathrm{~A}$ third trial was rated poor quality due to unclear randomization and allocation concealment, between-group differences at baseline, and high rates of attrition; results from that trial are not included here. ${ }^{62}$

Both studies reported that change in mean pain score (NRS 0-10) from baseline to followup were similar for cannabis and placebo $\left(\mathrm{p}=0.68^{28}\right.$ and $\left.\mathrm{p}=0.14^{47}\right)$. Despite this, the trial of THC/CBD, conducted in a population with allodynia, found a moderate magnitude of effect on response ( $\geq 30 \%$ reduction in pain). Response was more likely with cannabis than placebo (28\% vs. 16\%; RR 1.70, 95\% CI 1.04 to 2.78). ${ }^{47}$ Response was not reported in the other trial. 
There was no difference between treatment groups in measures of function (1 trial), quality of life ( 2 trials), or sleep ( 1 trial). ${ }^{28,47}$ This is low strength of evidence.

\section{Capsaicin}

Three short-term trials $(\mathrm{N}=1,519)$ assessed the effect of an $8 \%$ topical capsaicin patch applied for either 30 or 60 minutes on HIV-related neuropathy ${ }^{32}$ or postherpetic neuralgia ${ }^{26,53}$ (Appendix F). A 0.04\% topical capsaicin patch was used as a control. One trial was goodquality ${ }^{26}$ and the other trials were fair quality.

Pooled analysis found that while topical capsaicin improved pain severity in the short term (MD -0.33, 95\% CI -0.60 to -0.004, $\mathrm{I}^{2}=0 \%, 0-10$ scale), the difference was less than a small magnitude as defined for this report (Appendix I). ${ }^{26,32,53}$ Meta-analysis of pain response ( $\geq 30 \%$ reduction in pain) resulted in a small, nonsignificant effect (RR 1.17, 95\% CI 0.98 to 1.37, $\mathrm{I}^{2}=0 \%$; Appendix I). Subgroup analyses of the impact of study quality and type of neuropathic pain did not alter these results meaningfully. This is moderate strength of evidence.

\section{Memantine}

A small short-term, fair-quality trial $(\mathrm{N}=45)$ compared the effect of memantine up to $40 \mathrm{mg}$ daily with placebo in patients with HIV-related neuropathy. ${ }^{46}$ After 16 weeks of treatment, memantine and placebo were associated with similar reductions in pain scores (mean change -1.82 [standard deviation (SD) 2.77] vs. -2.36 [SD 3.35], $\mathrm{p}=0.87,1-10$ scale). Due to study limitations, including size, lack of other studies, and imprecise estimates, this evidence is insufficient to draw conclusions.

\section{Head-to-Head Comparisons}

\section{Pregabalin Versus Gabapentin}

Three short-term head-to-head RCTs ( $\mathrm{N}=433)$ compared pregabalin (75 mg to $300 \mathrm{mg}$ daily) with gabapentin (300 mg to 2,400 mg daily) ${ }^{33,40}$ or gabapentin enacarbil (1200 mg to $3600 \mathrm{mg}$ daily) ${ }^{44}$ and found no difference between the drugs in pain relief, ${ }^{33,40,44}$ function (BPI Interference) ${ }^{44}$ quality of life (SF-36 physical component summary [PCS]/MCS), ${ }^{44}$ or sleep interference (Appendix E). ${ }^{33,44}$ This is low strength of evidence. Neuropathic pain was related to diabetic peripheral neuropathy ${ }^{33,44}$ and peripheral nerve injury. ${ }^{33,40}$

\section{Cross-Class Comparisons}

\section{Gabapentin Versus Pregabalin Versus Duloxetine}

One fair-quality, short-term trial $(\mathrm{N}=152)$ compared gabapentin, pregabalin, and duloxetine in participants with diabetic peripheral neuropathy (Appendix E). ${ }^{33}$ Gabapentin dose ranged from 300 to 1800, pregabalin 75 to 300, and duloxetine 20 to $120 \mathrm{mg}$ daily. At baseline, mean pain score was 61 (VAS scale 0-100). After 12 weeks of treatment, mean pain scores were reduced with all three interventions, ranging from 26.5 to 35.2, with no difference between groups ( $\mathrm{p}=\mathrm{not}$ reported). There was also no difference between groups in sleep interference score (scale 0-10; range 2.84 to 3.99). Due to study limitations, including size, lack of other studies, and imprecise estimates, this evidence is insufficient to draw conclusions. 


\section{Fibromyalgia}

\section{Key Points}

- In the short and intermediate term, SNRI antidepressants resulted in small improvements in pain. Function improved to a small degree in the short term, but not in the intermediate term. Based on the SF-36 MCS, quality of life improved to a small degree in the short and intermediate term, but no effect was seen on the PCS. (SOE Moderate for all, but Low for intermediate-term PCS). There was a small decrease in depression with short-term duloxetine treatment.

- Short-term treatment with anticonvulsants was associated with small improvements in pain and function, but not quality of life (SOE: Moderate). Subgroup analyses showed no effect of specific drug, dose, or study quality on these results. Small improvements in sleep were also seen.

- $\quad$ Short- and intermediate-term treatment with memantine resulted in moderate improvements in pain, function, and quality of life compared with placebo (SOE: Low).

\section{Detailed Assessment}

Twenty-six good- or fair-quality RCTs (in 32 publications) involving 12,744 patients meeting inclusion criteria evaluated nonopioid drugs to treat chronic pain in fibromyalgia. ${ }^{65-94}$ All studies used criteria defined in 1990 by the American College of Rheumatology (ACR) to identify patients with fibromyalgia; ${ }^{95}$ three studies in one publication ${ }^{74}$ also required patients to meet 2010 ACR criteria. Eighteen were short-term trials (range 12 to 16 weeks), six intermediate-term (26 to 28 weeks), and two long-term (each 52 weeks). These included 15 placebo-controlled trials; 3 trials comparing multiple doses of the SNRIs milnacipran or duloxetine, and 7 trials that included both placebo and dose comparisons for a single included drug. One additional trial had a head-to-head design, comparing cyclobenzaprine and amitriptyline, with a third arm comparing each drug to placebo. That trial ${ }^{78}$ and one other ${ }^{85}$ assessed the tricyclic antidepressant (TCA) amitriptyline; the 15 other trials of antidepressants in fibromyalgia all used SNRIs. Eight trials assessed anticonvulsants, and one the Alzheimer's drug memantine. Three RCTs met criteria for good quality, ${ }^{88-90} 23$ fair, ${ }^{65-67,69,71,72,74-81,84-87,92-94}$ and 1 poor $^{96}$ (Appendix G). The poor-quality study was deemed to have high risk of bias due to high attrition and unclear randomization and blinding methods, and was not synthesized with the other evidence. Thirteen studies (52\%) were conducted in the United States. ${ }^{65-67,69,71,72,80,81,84,86,87,93,94}$ Most were funded by industry (88\%, 23 of 26). One publication ${ }^{97}$ on fibromyalgia treatment in pregnancy did not meet inclusion criteria as a systematic review because it did not assess the quality of included studies or synthesize their results. The studies reviewed also did not meet our criteria for design (many were observational or case reports) or duration (up to 9 weeks).

The weighted mean age of enrolled patients across our 26 included studies was 49 years, a mean of 94 percent of patients were female, and a mean of 15 percent were nonwhite. Across the RCTs, the mean baseline pain severity (standardized to a 0-10 scale) was 6.7 (range 6.0 to 7.6). Duration of pain was reported in 11 of 26 studies; it was less than a year in three, while in the other eight it ranged from 5 to 13 years. The percent of participants with comorbid depression was reported in nine studies, with a weighted average across studies of 21 percent. Complete descriptions of included study characteristics are in Appendix E. 


\section{Antidepressants}

Sixteen RCTs (in 20 publications) assessed antidepressants to treat fibromyalgia, with comparisons to placebo and/or between doses: seven were of milnacipran, eight of duloxetine, and one of amitriptyline. ${ }^{65,66,68-71,73,75-77,79-81,83-86,88,93,94}$ Most were short-term studies, four were intermediate-term, ${ }^{79,84,86,93}$ and two were long-term. ${ }^{76,80}$ Pain was reported in all studies, and function in all but one, with moderate-strength evidence for pooled comparisons of SNRI antidepressants with placebo. Ten studies reported quality of life, with low to moderate SOE for pooled results (Appendix $\mathrm{H}$ and Appendix I).

\section{Pain}

Short-term results from 11 trials showed a small reduction in pain with an SNRI antidepressant compared with placebo (0-10 scale, MD -0.59, 95\% CI - 0.80 to -0.43 , $\mathrm{I}^{2}=26 \%$ ). Three studies showed similar intermediate-term results (0-10 scale, MD -0.67, 95\% CI -0.99 to $\left.-0.34, \mathrm{I}^{2}=0 \%\right)$. The proportion responding was also greater with SNRIs than placebo in the short term; 40 percent of patients given SNRIs had at least a 30 percent reduction in pain, compared with 31 percent of those given placebo (RR 1.36, 95\% CI 1.26 to $1.46, \mathrm{I}^{2}=0 \%$ ).

Intermediate-term response rates were also higher with treatment than placebo (34\% vs. 28\%, RR $1.29,95 \%$ CI 1.08 to $1.52, \mathrm{I}^{2}=0 \%$ ). Pooled subgroup analyses by specific drug (duloxetine or milnacipran), dose, and study quality showed no change in the effect of treatment on pain. This is moderate strength evidence.

Many individual trials also reported effects of baseline depression on pain response, but none found a statistically significant interaction between depression and treatment in effects on pain. ${ }^{66,69,71,75,79}$ Two trials ${ }^{88,94}$ stratified results and found that patients without baseline depression had a better response to SNRI than to placebo. However, these two trials did not assess whether the difference in response between patients with and without depression was statistically significant.

One fair-quality, short-term trial $(\mathrm{N}=87)^{85}$ randomized female patients with fibromyalgia to the tricyclic antidepressant amitriptyline or placebo. Patients assigned to amitriptyline had better response to treatment according to physicians' global assessments (74\% vs. 49\%, $\mathrm{p}=0.017)$, and lower pain severity at the 12-week endpoint (VAS 0-10, 4.5 vs. 5.2) than placebo. Using a VAS 0-10 scale, sleep problems were also rated lower at endpoint with amitriptyline than placebo (3.6 vs. 4.8), and the change from baseline was significant only with amitriptyline. This evidence is insufficient due to small sample size (imprecision), study limitations, and unknown consistency.

\section{Function}

Most studies of antidepressants in fibromyalgia measured function using the Fibromyalgia Impact Scale (FIQ, range either $0-80$ or $0-100)$; one study ${ }^{66}$ used the BPI Interference score (010). Pooled analysis of short-term results from 11 studies showed a small effect of SNRI antidepressants on function compared with placebo (SMD -0.24, 95\% CI -0.32 to -0.17, $\mathrm{I}^{2}=22 \%$ ), while intermediate-term results from 3 studies showed an effect less than that defined as small for this report (SMD $-0.13,95 \%$ CI -0.24 to $-0.02, \mathrm{I}^{2}=0 \%$ ). This is moderate strength evidence. Subgroup analyses by specific drug, dose, and study quality did not alter these results.

\section{Quality of Life}

Eight fair-quality trials reported effects of 3 to 12 months' SNRI treatment on quality of life. Short-term treatment with duloxetine or milnacipran was associated with small improvements in the SF-36 MCS (0-100 or not reported; SMD 0.19, 95\% CI 0.13 to $\left.0.27, \mathrm{I}^{2}=12 \%\right)$. Intermediate- 
term changes in the MCS reported in three trials were similar (SMD 0.18, 95\% CI 0.08 to 0.30, $\left.\mathrm{I}^{2}=0 \%\right)$. SF-36 PCS scores also improved with short-term treatment, but the difference was not clinically important as defined in this report, and intermediate-term treatment had no effect on physical wellbeing. Subgroup analyses did not show effects of specific drug or dose on these results. This is moderate strength evidence, except for intermediate-term results for the SF-36 PCS scale, which is low strength.

\section{Other Outcomes}

In the short term, SNRI antidepressants duloxetine and milnacipran improved depression symptoms in patients with fibromyalgia, but to a very small degree, based on meta-analysis of 10 RCTs that measured depression using the BDI, BDI-II, FIQ depression subscale, or HAMD (SMD -0.17, 95\% CI -0.24 to $-0.12, \mathrm{I}^{2}=0 \%$ ). ${ }^{65,66,69,71,75,77,81,86,88,94}$ Subgroup analyses by drug showed that only duloxetine had a clinically important effect on depression (SMD -0.28, 95\% CI -0.38 to $-0.18, \mathrm{I}^{2}=0 \%$ ). Seven short-term trials also measured anxiety, using several different instruments. ${ }^{65,66,69,75,77,88,94}$ Meta-analysis did not show a statistically significant effect and there was substantial heterogeneity across studies (SMD -0.08, 95\% CI -0.23 to $0.03, \mathrm{I}^{2}=56 \%$ ). Seven short-term trials measured effects on sleep using a variety of instruments. ${ }^{66,71,77,81,86,88,94}$ Metaanalysis found that milnacipran showed no effect on sleep (SMD 0.01, 95\% CI -0.16 to 0.08, $\mathrm{I}^{2}=0 \%$ ), and that duloxetine improved sleep compared with placebo, but the effect did was very small (SMD $-0.18,95 \%$ CI -0.33 to $-0.05, \mathrm{I}^{2}=0 \%$ ).

\section{Dose Comparisons}

Two fair-quality intermediate- or long-term studies compared different doses of milnacipran, ${ }^{76,84}$ and a third long-term study ${ }^{80}$ compared $60 \mathrm{mg}$ and $120 \mathrm{mg}$ daily of duloxetine. In the intermediate term (28 weeks), fibromyalgia patients treated with placebo in an earlier “lead-in" study ( $\mathrm{N}=129)$ were re-randomized to either $100 \mathrm{mg}$ or $200 \mathrm{mg}$ daily of milnacipran. ${ }^{84}$ Although pain decreased from lead-in study baseline to the end of the extension study with both milnacipran doses (VAS 0-100: -25.7 for $100 \mathrm{mg}$ daily and -29.1 for $200 \mathrm{mg}$ daily), the difference (-3.4 on a 0-100 scale) was below the threshold for a small effect for this report. Effects on physical function did not differ between doses. This evidence is insufficient to draw conclusions due to small sample size (imprecision), unknown consistency, and study limitations. The study also showed little or no difference between doses in effects on depression and sleep. In the long term (52 weeks), a similarly designed study ( $\mathrm{N}=270)$ re-randomized patients given placebo in a lead-in study to milnacipran $100 \mathrm{mg}, 150 \mathrm{mg}$, or $200 \mathrm{mg}$ daily, ${ }^{76}$ and did not show differences between doses in pain (VAS 0-100, change from extension baseline range -11.6 to -15.3), function, or quality of life, or a composite response measure including 30 percent improvement in pain and patient global impressions. This is low strength of evidence. Effects on sleep were also similar across doses (VAS 0-100, change from extension baseline range -6.6 to -13.6$)$.

A long-term study ( $\mathrm{N}=307)$ of the SNRI duloxetine $60 \mathrm{mg}$ or $120 \mathrm{mg}$ daily did not find differences in effects on pain. ${ }^{80}$ Function improved slightly for patients taking $60 \mathrm{mg}$ daily, while it deteriorated in those taking $120 \mathrm{mg}$ daily (FIQ total score, range not reported [NR], change from baseline: -0.69 vs. $3.49, \mathrm{p} \leq 0.05$ ), however on a $0-100$ scale this difference is below the threshold for a small magnitude of effect for this report (low strength of evidence). ${ }^{80}$ Improvements in sleep were greater with $60 \mathrm{mg}$ than $120 \mathrm{mg}$ of duloxetine, though the magnitude of effect was small (mean change in BPI sleep interference score of -1.16 vs. -0.23 on a scale of 1 to $10, \mathrm{p} \leq 0.01$ ). 


\section{Anticonvulsants}

Eight short-term RCTs (in 7 publications, N=4,821) compared an anticonvulsant to placebo in patients with fibromyalgia. ${ }^{67,72,74,82,87,89,92}$ One study met criteria for good quality, ${ }^{89}$ with the remainder being fair quality. One trial used gabapentin ${ }^{67}$ and the remaining trials used pregabalin. Pain and function outcomes were reported in all studies, and three studies provided evidence on quality of life; the strength of evidence on all outcomes was moderate. (Appendix $\mathrm{H}$ and Appendix I).

\section{Pain}

In the short term, anticonvulsants were associated with a small reduction in pain, based on meta-analysis of eight RCTs ( $0-10$ scale, MD $-0.57,95 \%$ CI -0.75 to $\left.-0.40, \mathrm{I}^{2}=30 \%\right)$. The proportion responding to anticonvulsants was also higher (41\% vs. 32\%, RR 1.30, 95\% CI 1.20 to $\left.1.43, \mathrm{I}^{2}=0 \%\right)$. Analyses of specific drug, pregabalin dose, and study quality did not alter results, with small but statistically significant pain reductions (and higher response rates) seen in each subgroup. One of the five trials assessed baseline depression as a subgroup, but found no statistically significant interaction with treatment in effects on pain. ${ }^{92}$

\section{Function}

Function as measured by the FIQ (range $0-80$ or 0 -100) improved with anticonvulsant treatment across eight short-term trials, but the difference compared with placebo was small (SMD -0.22 , 95\% CI -0.29 to $-0.15, \mathrm{I}^{2}=0 \%$ ). Subgroup analyses did not show significant effects of specific drug, pregabalin dose, or study quality.

\section{Quality of Life}

Four short-term fair quality RCTs of pregabalin (in 2 publications) reported the effect of anticonvulsants on quality of life. ${ }^{72,74}$ Results showed statistically significant improvements in the SF-36 with treatment, but differences were less than that defined as small for this report (SMD 0.13 for MCS and 0.17 for PCS). One study ${ }^{72}$ tested three doses of pregabalin (300 mg, $450 \mathrm{mg}$, or $600 \mathrm{mg}$ daily), but did not show differences in effects on quality of life.

\section{Other Outcomes}

Short-term pregabalin treatment improved depression symptoms measured by HADS-D, based on meta-analysis of five trials in three publications, but the effect was not clinically important (SMD -0.11, 95\% CI -0.18 to -0.03 , $\mathrm{I}^{2}=0 \%$ ). ${ }^{72,74,89}$ Effects on anxiety symptoms were similar in the same studies (HADS-A, SMD -0.11, 95\% CI -0.25 to $-0.02, \mathrm{I}^{2}=0 \%$ ). Seven studies in five publications measured sleep with various instruments, and found small improvements in sleep with short-term pregabalin compared with placebo (SMD -0.33, 95\% CI -0.40 to -0.25, $\left.\mathrm{I}^{2}=13 \%\right)^{72,74,87,89,92}$

\section{Other Drug Classes}

\section{Memantine}

A good-quality, 6-month RCT $(\mathrm{N}=63)$ randomized fibromyalgia patients to memantine, an $N$-Methyl-D-aspartic acid (NMDA) receptor antagonist approved for Alzheimer's dementia, or to placebo. Pain, function, and quality of life all improved moderately more with memantine than placebo. At 3 months (short term), results showed lower pain scores (VAS 0-10 scale, 5.06 vs. 6.85, $\mathrm{p}=0.001$ ), lower disability scores, (FIQ 0-10 scale, 49.91 vs. 59.67, $\mathrm{p}=0.011$ ), and better 
quality of life (EQ-5D 0-100 scale, 58.06 vs. 43.43, p=0.003) with memantine than placebo. Similar intermediate-term improvements were seen at 6 months (pain severity, VAS 0-10 scale 4.87 vs. 7.01, $\mathrm{p}=0.001$; FIQ 0 - 10 scale, 50.02 vs. 69.57, $\mathrm{p}<0.001$; EQ-5D quality of life scale 0 $100,60.48$ vs. $43.75, p=0.001) .{ }^{90,91}$ This evidence is low strength. Memantine had no effect on anxiety at 3 or 6 months, but treatment reduced symptoms of depression at both time points (HADS-D 0 to 21 scale, 7.87 vs. 10.46 at 6 months, $\mathrm{p}=0.002$ ).

\section{Cross-Class Comparisons}

A fair-quality RCT $(\mathrm{N}=208)$ compared the tricyclic antidepressant amitriptyline, the muscle relaxant cyclobenzaprine, and placebo for 6 months in fibromyalgia. ${ }^{78}$ Both short-term (3month) and intermediate-term (6-month) results were reported (treatment was for 24 weeks, but outcomes reported at 6 months). There were no differences at either time point for outcomes, pain, function, or a composite response measure including pain, sleep, fatigue, and global assessments. This is low strength evidence. No differences between treated groups and placebo were seen for depression, anxiety, or sleep at 3 or 6 months.

\section{Osteoarthritis}

\section{Key Points}

- Oral nonsteroidal anti-inflammatory drugs (NSAIDs) improved pain and function in patients with osteoarthritis (OA) to a small degree in the short term, with evidence indicating these effects are maintained in the intermediate term with celecoxib. Subgroup analyses indicated that studies of only patients with knee pain and those of good quality had smaller effects, while patients with more severe pain at baseline experienced greater reduction in pain. (SOE: Moderate for pain, quality of life, High for response and function).

- In the short term, topical diclofenac improved pain severity and response to a small degree (SOE: Moderate). There was no effect on improvement in function, but there was serious inconsistency among studies (SOE: Low).

- The SNRI antidepressant duloxetine resulted in small improvement in pain severity, moderate improvement in pain response, and small improvements in function and quality of life in OA patients in the short term. Subgroup analyses found that older patients ( $>65$ years) had better effects on pain, and studies of only patients with knee OA had larger effects on pain (SOE: High).

- Acetaminophen did not significantly improve pain or function in the short or intermediate term, across all doses (SOE: Low). Evidence from a single short-term study suggested that pain and function improve to a small degree at higher doses (3900 mg to $4000 \mathrm{mg}$ daily), but was insufficient to draw conclusions.

- Direct comparisons of NSAIDs with each other found few differences between drugs in pain or function in OA patients in the short, intermediate, or long term (SOE: Low). Cross-class comparisons were limited (3 RCTs) and insufficient to draw conclusions.

\section{Detailed Assessment}

Fifty-one fair- and good-quality RCTs (in 57 publications) involving 22,052 patients meeting inclusion criteria evaluated nonopioid drugs to treat chronic pain in osteoarthritis; 44 were short term (12 to 24 weeks), 6 intermediate term (26 weeks), and 1 long term (52 weeks). These 
included 40 placebo-controlled trials (8 of duloxetine, 4 of acetaminophen, 4 of topical diclofenac, and 26 of oral NSAIDs), 5 trials comparing multiple doses, 3 comparing different formulations of diclofenac (2 comparing oral and topical, 1 comparing oral formulations), 13 head-to-head trials comparing various NSAIDs, and 3 making cross-class comparisons (some trials included more than one of these categories). Fourteen RCTs met criteria for good quality, ${ }^{98-}$ ${ }^{110}$ seven were poor quality, ${ }^{111-117}$ and the remainder (37) were fair quality (Appendix G). Most studies were conducted in the United States (22 RCTs) and were funded by industry (84\%).

Studies included patients with osteoarthritis, but with varying and often unclear criteria for establishing the diagnosis. Mean age of enrolled patients ranged from 54 to 72 (weighted mean 63 years), a weighted mean of 68 percent were female, and a weighted mean of 14 percent were nonwhite. Across the RCTs, baseline pain severity ranged from 50 to 78 on a 0-100 VAS. Duration of pain was reported in 53 percent of trials, with a mean duration ranging from $<1$ year to 12 years. At baseline, function/disability ranged from 63 to 72 on a VAS scale, and 27 to 37 out of 68 on the Western Ontario and McMaster Universities Osteoarthritis Index (WOMAC) physical function subscale. Complete descriptions of included study characteristics are in Appendix E. Results of meta-analyses, including forest plots and subgroup analyses, can be found in Appendix I.

\section{Oral NSAIDs}

Twenty-seven RCTs (in 28 publications; $\mathrm{N}=13,808$ ) compared at least one NSAID versus placebo in patients with OA (5 had more than 2 treatment arms). ${ }^{101,103-108,118-138}$ Fifteen included the selective cyclooxygenase (COX)-2 inhibitor celecoxib (100 mg to $400 \mathrm{mg}$ daily), while 14 included nonselective NSAIDs (7 of naproxen $1000 \mathrm{mg}$ daily, 2 of meloxicam $3.75 \mathrm{mg}$ to $15 \mathrm{mg}$ daily, 2 of ibuprofen $2400 \mathrm{mg}$ daily, 3 of diclofenac $100 \mathrm{mg}$ to $150 \mathrm{mg}$ daily, and 1 of diclofenac submicron $70 \mathrm{mg}$ and $105 \mathrm{mg}$ daily). All of the RCTs evaluated pain at 12 to 13 weeks (short term), with one also evaluating at 26 weeks (intermediate term). ${ }^{131}$ Pain and function outcomes were reported in all studies, but quality of life only in three. ${ }^{125,127,132}$ The strength of evidence for NSAIDs on improvement in pain and quality of life is moderate, and for pain response and function is high.

\section{Pain}

In the short term, NSAIDs resulted in a small reduction in pain, based on meta-analysis of 27 RCTs (MD -0.73, 95\% CI -0.84 to $-0.62, \mathrm{I}^{2}=27 \%, 0-10$ scale; Appendix I). Similarly, the proportion responding to NSAIDs was significantly greater than placebo (15 RCTs, $56 \%$ vs. 46\%, RR 1.23, 95\% CI 1.18 to 1.31, $\mathrm{I}^{2}=0 \%$; Appendix I). At intermediate-term followup, celecoxib $200 \mathrm{mg}$ daily also resulted in a small improvement in pain (MD -0.63, 95\% CI -1.10 to -0.16, 0-10 scale), and a nonsignificant increase in response (RR 1.13, 95\% CI 0.94 to 1.35). ${ }^{131}$ Subgroup analyses of specific drug, dose (celecoxib), year of publication ( $\leq 2000$, $\geq 2001$ ), study quality (good and fair), and criteria used for response (30\% improvement, $50 \%$ improvement, Osteoarthritis Research Society International [OARSI]), did not alter the findings meaningfully, with no significant interactions found. Subgroup analyses of location of pain (hip, knee, either) was not significant for response, but was significant for improvement in pain $(\mathrm{p}=0.0021)$. In this subgroup analysis, studies that enrolled only patients with knee pain had a smaller pooled improvement in pain (MD - $0.57,95 \%$ CI -0.71 to $-0.46,0-10$ scale). Publication bias (small study bias) is possible, with the Egger's test being significant, but the funnel plot appearing balanced (Appendix I). 


\section{Function}

In the short term, NSAIDs resulted in a small improvement in function, based on metaanalysis of 28 RCTs (SMD $-0.32,95 \%$ CI -0.37 to $-0.28, \mathrm{I}^{2}=24 \%$ ), using mostly the WOMAC function subscale (Appendix I). At intermediate-term followup in one study, a similar improvement was maintained (SMD -0.25, 95\% CI -0.47 to -0.04). ${ }^{131}$ Subgroup analyses by specific drug, dose (celecoxib, diclofenac), location of pain (hip, knee, either), and year of publication ( $\leq 2000, \geq 2001)$ did not alter the findings meaningfully, with no significant interactions found. Good-quality studies found a smaller effect size (-0.35 for fair-quality studies, -0.26 for good-quality studies, p-value for interaction=0.052), but the magnitude of the effect was still in the range of a small effect (Table 5). Tests for publication bias (small-study bias) were not significant; evidence of this bias was not found (see funnel plot and Egger's test result, Appendix I).

Table 5. NSAID subgroup analyses

\begin{tabular}{|l|l|l|l|c|c|}
\hline Outcome & \multirow{2}{*}{$\begin{array}{l}\text { Variable } \\
\text { Pain }\end{array}$} & Subgroup & $\begin{array}{l}\text { N Studies (Sample } \\
\text { Size) }\end{array}$ & Effect Size (95\% CI) & $\begin{array}{l}\text { Interaction } \\
\text { p-value }\end{array}$ \\
\hline Funprovement & Pain & Knee & $14(7,352)$ & MD -0.57 (-0.71 to -0.46) & 0.0035 \\
& location & Hip & $4(2,617)$ & MD -0.88 (-1.12 to -0.62) & \\
& & Knee/Hip & $9(3,509)$ & MD -0.94 (-1.11 to -0.77) & 0.05 \\
& Study & Good & $8(4,212)$ & SMD -0.26 (-0.34 to -0.18) & $0.30)$ \\
& quality & Fair & $19(9,261)$ & SMD -0.35 (-0.41 to -0.30) & \\
\hline
\end{tabular}

$\mathrm{CI}=$ confidence interval; $\mathrm{MD}=$ mean difference; NSAID = nonsteroidal anti-inflammatory drug; SMD = standardized mean difference

\section{Quality of Life}

In the short term, NSAIDs improved quality of life as measured by the SF-36 PCS (MD 2.95, 95\% CI 1.79 to 4.18), but the difference was less than a small effect as defined for this report and also less than the 3-point minimal clinically important difference (MCID) used in OA studies. ${ }^{139}$ There was not a meaningful change in the MCS (MD 0.61, 95\% CI -0.50 to 1.79).

\section{Other Outcomes}

Sleep improved in the short term in one study of celecoxib $200 \mathrm{mg}$ daily (other arms included tramadol). ${ }^{125}$ Using the Chronic Pain Sleep Inventory (0-100 VAS), patients on celecoxib improved by 16.4 points (2.1 standard error of the mean [SEM]) compared with 8.6 (2.1 SEM) with placebo (analysis of covariance [ANCOVA] p-value across 5 study arms = 0.027, with the largest improvement in the celecoxib group).

\section{Subpopulations}

One study of naproxen $1000 \mathrm{mg}$ daily reported that subgroup analyses of age, gender, race, and ethnicity were consistent with the overall findings. ${ }^{132}$ Four studies analyzed impact of baseline pain, with two finding that improvement in pain with was greatest in patients whose pain was greater at baseline and least in those whose pain was lowest at baseline, ${ }^{101,102}$ but two others not finding a linear relationship. ${ }^{122,132}$ Two studies found that patients who had used or were using an NSAID prior to study enrollment responded better than those who had or were not. ${ }^{102,122}$ Because sample sizes varied and not all analyses were pre-planned, these findings are considered preliminary.

Based on the meta-analyses reported above, results of subgroup analyses on study quality, specific drug and dose, year of publication, and definition of pain response did not show statistically significant effects (Appendix I). As noted, subgroup analysis of improvement in pain 
by location of pain was significant, and improvement in function by study quality was significant (Appendix I).

\section{Topical NSAIDs: Diclofenac}

Four short-term trials ( $\mathrm{N}=1,541)$ evaluated topical formulations ( 2 of 1\% gel, 2 of 1.5\% solution) of diclofenac, used four times a day, compared with vehicle in patients with knee OA. ${ }^{99,108,140,141}$ Pain and function were reported in all four RCTs, with pain response also reported in two.

\section{Pain}

In the short term, topical diclofenac resulted in a small improvement in pain over vehicle (4 RCTs, MD -0.58 , 95\% CI -0.81 to $-0.35, \mathrm{I}^{2}=0 \%$ ). Based on meta-analysis of three RCTs, topical diclofenac resulted in a small magnitude of response to treatment, based on the OARSI criteria (65\% vs. 53\%, RR 1.20, 95\% CI 1.09 to $1.38, \mathrm{I}^{2}=0 \%$; Appendix I). ${ }^{140-142}$ The strength of this evidence is moderate.

\section{Function}

In the short term, based on meta-analysis of four RCTs, topical diclofenac did not improve function in patients with knee OA pain (WOMAC function subscale 0-68; MD -0.51, 95\% CI -1.06 to 0.04). However, one of the studies found a significant benefit favoring diclofenac, and the meta-analysis has high heterogeneity $\left(\mathrm{I}^{2}=94 \%\right) .{ }^{140}$ All of the studies used the same scale to measure function (WOMAC, 0-68). There were only small differences in baseline characteristics; this study had slightly younger patients (59 years vs. 62 to 64 years), and somewhat lower function scores (38 vs. 42 out of 68). Statistical heterogeneity was not found in analysis of pain (above) and other differences that may explain the heterogeneity were not identified, so the strength of this evidence is low.

\section{Subpopulations}

Subgroup analyses of age, gender, race or ethnicity, pain location, and dose were not conducted by individual studies or in our analyses (due to lack of variability).

\section{Head-to-Head Comparisons of NSAIDs}

Three RCTs of celecoxib versus naproxen, ${ }^{105,120,143}$ two of topical versus oral diclofenac, ${ }^{108,144}$ and two of nabumetone versus naproxen ${ }^{145,146}$ provided data for meta-analyses. Nine RCTs compared one NSAID to another, which could not be pooled in meta-analyses, with four short-term $(\mathrm{N}=2022),{ }^{102,137,147,148}$ two intermediate-term $(\mathrm{N}=921),{ }^{149,150}$ and one long-term (N=925). ${ }^{151}$ The most common comparator was diclofenac, with eight RCTs making comparisons with celecoxib (2), nabumetone (2), ibuprofen (1), meloxicam (2, multiple doses), and one comparing different formulations of diclofenac. All studies reported on pain, four studies reported on function, and none reported on quality of life. The strength of this evidence is low for all outcomes in this group of noncombinable studies.

\section{Pain}

In the short term, diclofenac resulted in moderate improvement over celecoxib (MD -12.2, 95\% CI -22.1 to -2.2) and small improvement over meloxicam $3.75 \mathrm{mg}$ daily, but no effect over meloxicam $7 \mathrm{mg}$ or $15 \mathrm{mg}$ daily. ${ }^{102,137}$ Pain improvement was not found to be different between NSAIDs for the remainder of comparisons. Meta-analyses of celecoxib and naproxen (3 RCTs, 
$\mathrm{N}=1,013$, MD $-0.37,95 \% \mathrm{CI}-0.76$ to $0.03, \mathrm{I}^{2=} 0 \%$ ) and of oral diclofenac (100 $\mathrm{mg}$ and $150 \mathrm{mg}$ daily) versus topical diclofenac $1.5 \%$ ( 2 RCTs, $\mathrm{N}=909$, MD $-0.27,95 \%$ CI -0.63 to $0.10, \mathrm{I}^{2}=0 \%$ ) and single studies of diclofenac and nabumetone, ibuprofen, different formulations of diclofenac or between ibuprofen and nabumetone did not find differences in pain between drugs. In two studies, the proportion of patients with response to treatment was not found different between ibuprofen and nabumetone or between dispersible and enteric coated diclofenac formulations. ${ }^{147,152}$ In the intermediate term, two studies found improvement in pain and response to treatment to not be different between celecoxib and naproxen (1 study) or between meloxicam and diclofenac (1 study). ${ }^{149,150}$ In the long term, one RCT found no significant differences between celecoxib and diclofenac at 12 months of treatment. ${ }^{151}$

\section{Function}

In the short term, meta-analysis of three RCTs $(\mathrm{N}=1,013)$ of celecoxib and naproxen did not find a difference in improvement in function, (MD -0.02, 95\% CI -0.21 to 0.16, $\mathrm{I}^{2}=16 \%$ ), and a meta-analysis of two RCTs ( $\mathrm{N}=909)$ of oral diclofenac (100 mg and $150 \mathrm{mg}$ daily) versus topical diclofenac $1.5 \%$ found a small difference that was on the border of being statistically significant (MD -0.18, 95\% CI -0.34 to $0.00, \mathrm{I}^{2=} 0 \%, \mathrm{p}=0.50$ ). A single RCT found that diclofenac had a moderate improvement in function over celecoxib when categorized as improved, no change, or worse (RR 2.06, 95\% CI 1.37 to 3.08). ${ }^{137}$ Another RCT found no difference in improvement in function between meloxicam $7 \mathrm{mg}$ or $15 \mathrm{mg}$ daily and diclofenac, but diclofenac had a small improvement over the $3.75 \mathrm{mg}$ daily dose of meloxicam. ${ }^{102}$ In the intermediate term, two studies found improvement in function to not be different between celecoxib and naproxen (1 study) or between meloxicam and diclofenac ( 1 study). ${ }^{149,150}$

\section{Antidepressants: SNRIs}

\section{Duloxetine}

Duloxetine was the only antidepressant with studies in OA patients that met inclusion criteria. All six included studies ( $\mathrm{N}=1,575$, 8 publications) were short term. ${ }^{98,100,110,153-157}$ Pain was reported in all studies, function in five, and quality of life in three, but none reported other secondary measures eligible for this review (e.g., sleep, depression). SOE for duloxetine versus placebo was high for pain, function outcomes, and quality of life.

\section{Pain}

In the short term, duloxetine resulted in a small reduction in pain, based on meta-analysis of 6 RCTs (MD -0.75, 95\% CI -1.05 to -0.53 , $\mathrm{I}^{2}=15 \%$, 0-10 scale). ${ }^{100,110,153,156-158}$ Similarly, duloxetine resulted in a moderate improvement in the proportion responding to treatment (4 RCTs, $65 \%$ vs. 47\%, RR 1.37, 95\% CI 1.24 to 1.52, $\mathrm{I}^{2}=0 \%$ ); in this set all RCTs used 30 percent improvement for a definition of response. Subgroup analyses of pain location (knee vs. hip or knee), dose (60 mg vs. 60 to $120 \mathrm{mg}$ daily), and study quality (good or fair) did not alter the findings meaningfully, with no significant interactions found.

\section{Function}

In the short term, duloxetine resulted in a small improvement in function, based on metaanalysis of five RCTs (SMD $-0.27,95 \%$ CI -0.41 to $-0.12, \mathrm{I}^{2}=27 \%$ ), using the WOMAC function subscale (3 RCTs), and the BPI Interference subscale (2 RCTs). ${ }^{98,100,110,153,157}$ 
Subgroup analyses of pain location (knee vs. hip or knee), dose (60 mg vs. 60 to $120 \mathrm{mg}$ daily), and study quality (good or fair) did not alter the findings meaningfully, with no significant interactions found.

\section{Quality of Life}

In the short term, duloxetine resulted in a small improvement in quality of life, based on meta-analysis of two RCTs (MD 0.05, 95\% CI 0.02 to $0.08, \mathrm{I}^{2}=0 \%$ ), using the EQ-5D. Subgroup analyses of pain location (knee vs. hip or knee), dose (60 mg vs. 60 to $120 \mathrm{mg}$ daily), and study quality (good or fair) did not alter the findings meaningfully, with no significant interactions found. A third fair-quality study reported the SF-36 PCS, with mean change from baseline of 7.8 (standard error $[\mathrm{SE}] 0.85$ ) with duloxetine and 4.41 (SE 0.81) with placebo $(\mathrm{p}<0.001) .{ }^{153}$

\section{Other Outcomes}

Sleep was improved with duloxetine $60 \mathrm{mg}$ daily in two studies, based on BDI sleep interference subscale, but the clinical meaning of the magnitude of difference seen $(-0.46$ and -0.22) is unclear. ${ }^{110,153}$ Changes in depression and anxiety scales were reported in one study, with no improvement over placebo seen. ${ }^{100}$

\section{Subpopulations}

Three studies reported subgroup analyses according to age, with one finding no effect of age, ${ }^{153}$ but two that analyzed age according to categories of $<65$ years and $\geq 65$ years found that a significant effect of duloxetine on pain was found in older patients, while the effect was similar to placebo in younger patients. ${ }^{100,110}$ Subgroup analyses of gender, race, and baseline pain scores were not significant. ${ }^{100,153}$ Based on the meta-analyses reported above, results of subgroup analyses on location of pain, study quality and dose did not show statistically significant effects for any outcome, although pain outcomes were better in studies of only patients with knee pain than in studies with a mix of patients with knee or hip pain (See Appendix I).

\section{Acetaminophen}

Three short-term RCTs ( $\mathrm{N}=1,107)$ and one intermediate-term study compared acetaminophen (1950 mg to $4000 \mathrm{mg}$ daily) with placebo in patients with OA. ${ }^{122,159-161}$ Pain and function outcomes were reported in all studies. The strength of evidence for acetaminophen is low for all outcomes.

\section{Pain}

In the short term, acetaminophen did not impact pain significantly (MD -0.34, 95\% CI -0.66 to $0.03, \mathrm{I}^{2}=0 \%$ ) based on meta-analysis of three trials (Appendix I). One of these RCTs included two doses of acetaminophen and found that, compared with placebo, pain improved significantly more with the higher dose (WOMAC pain subscale, least squares mean [LSM] change from baseline -25.9, -22.5, -19.8 for $3900 \mathrm{mg}$ daily, $1950 \mathrm{mg}$ daily, and placebo, respectively; p-value for $3900 \mathrm{mg}$ daily versus placebo=0.012). ${ }^{159}$ Comparisons of $1950 \mathrm{mg}$ daily with placebo were reported as not statistically significant. In the intermediate term, a single trial $(\mathrm{N}=212)$ also found no difference between acetaminophen and placebo in pain improvement (WOMAC pain subscale), or in the proportion of patients responding to treatment, using the OARSI criteria for response. ${ }^{160}$ 


\section{Function}

In the short term, acetaminophen did not impact function (SMD -0.14, 95\% CI -0.29 to 0.04, $\mathrm{I}^{2}=0 \%$ ) significantly based on meta-analysis of three trials (Appendix I). Similar to the findings on the impact of dose on pain, in a single RCT function was improved significantly with 3900 mg daily (WOMAC function subscale, LSM change from baseline -24.2, -19.0, and -18.2 for $3900 \mathrm{mg}$ daily, $1950 \mathrm{mg}$ daily, and placebo, respectively; p-value for $3900 \mathrm{mg}$ daily versus placebo=0.016). ${ }^{159}$ Comparisons of $1950 \mathrm{mg}$ daily with placebo were reported as not statistically significant. In the intermediate term, a single trial $(\mathrm{N}=212)$ found a slightly greater improvement in function with acetaminophen on the WOMAC function subscale (0-100; MD -3.7, 95\% CI -6.9 to -0.5), but the difference was less than the magnitude of effect defined as small for this report. ${ }^{160}$

\section{Subpopulations}

None of the four included RCTs conducted subgroup analyses by age, gender, race, or ethnicity. One evaluated baseline pain, but did not report results for acetaminophen other than to note that it was not different to placebo. ${ }^{122}$ Subgroup analyses could not be conducted based on study quality (all were fair) or on pain location (2 were knee, 1 was mixed knee/hip).

\section{Topical Lidocaine}

A single short-term study of lidocaine 5\% patch compared with celecoxib in patients with knee OA ( $\mathrm{N}=143)$ was poor-quality (unclear allocation concealment, no blinding, high attrition: $46 \%$ ), and terminated early due to the withdrawal of celecoxib from the market at that time. ${ }^{114}$

\section{Cross-Class Comparisons}

Evidence from two small, short-term RCTs comparing drugs across classes was insufficient to draw conclusions due to serious imprecision and inconsistency. One small $(\mathrm{N}=85)$ shortterm, fair-quality RCT compared diclofenac with acetaminophen over 12 weeks. ${ }^{122}$ A very small study of diclofenac $150 \mathrm{mg}$ daily and acetaminophen $4000 \mathrm{mg}$ daily found diclofenac to be superior in both pain and function improvement. ${ }^{122}$ In a small $(\mathrm{N}=65)$, good-quality RCT of patients with OA of the hand taking acetaminophen or an NSAID at baseline, pregabalin 1300 mg daily (MD -2.7, 95\% CI -3.5 to -1.9) and duloxetine $60 \mathrm{mg}$ daily (-2.3, 95\% CI -3.8 to -0.9) improved pain to a similar degree (NRS 0-10 scale), but a statistical comparison was not made. ${ }^{109}$

\section{Inflammatory Arthritis}

\section{Key Points}

- In the short term, oral NSAIDs resulted in small improvements in pain severity and function, and moderate improvement in pain response compared with placebo (SOE: Moderate). Evidence on quality of life is inconsistent, with one trial finding a moderate effect and one trial finding no effect (SOE: Insufficient). Evidence on intermediate-term outcomes is limited to one trial of naproxen, finding small improvements in pain severity and pain response and no improvement in function (SOE: Low). Evidence on long-term outcomes is limited to one trial of meloxicam, finding large improvements in pain severity and pain response and no improvement in function (SOE: Low). 
- Subgroup analyses of specific drug, dose, year of publication, type of inflammatory arthritis, and study quality did not alter the findings meaningfully.

- Comparisons of different doses of various NSAIDS and comparisons of different NSAIDs with one another found no meaningful differences in effectiveness for pain improvement, pain response, function, or quality of life (SOE: Low to Insufficient).

- The tricyclic antidepressant amitriptyline resulted in no improvement in pain severity compared with placebo in one trial (SOE: Low).

\section{Detailed Assessment}

Thirty RCTs (in 32 publications) evaluated nonopioid drugs to treat chronic pain due to inflammatory arthritis. ${ }^{162-193}$ One trial met criteria for good quality ${ }^{171}$ and 19 were fair quality. ${ }^{162,164,165,167,169,170,172,173,177-179,183-186,188,189,191,192}$ An additional 10 trials (in 11 publications) were rated as poor quality - deemed to have high risk of bias due to unclear randomization and allocation concealment techniques, baseline differences between randomized groups, lack of blinding, and/or high attrition - and are not synthesized with the other evidence (Appendix G). ${ }^{163,166,168,174-176,180,182,187,190}$ The 20 good- and fair-quality RCTs included 7,708 patients, with

15 studies (in 16 publications) of rheumatoid arthritis (RA) (N=6,218) ${ }^{164,165,169,170,172,173,177-179,183-}$ 185,189,192,194 and 6 studies of ankylosing spondylitis (AS) (N=1,869). ${ }^{162,167,171,186,188,191}$ Twenty trials evaluated various NSAIDs and one trial ${ }^{177}$ evaluated a TCA drug. Eighteen trials (13 in RA; 5 in AS) were short term (12 to 24 weeks); 2 trials in RA were intermediate term (26 weeks); and 1 trial in AS was long term (52 weeks). Thirteen placebo-controlled trials (9 in RA; 4 in AS) evaluated five different NSAIDs (celecoxib, diclofenac, etodolac, meloxicam, and naproxen) and one TCA (amitriptyline). Four trials (1 in RA; 3 in AS) compared multiple doses of celecoxib and two trials (1 in RA; 1 in AS) compared multiple doses of meloxicam. Ten trials included head-to-head comparisons of various NSAIDs: celecoxib vs. diclofenac; celecoxib vs. naproxen; diclofenac vs. etodolac; etodolac vs. naproxen; meloxicam vs. naproxen; and nabumetone vs. naproxen.

The good- and fair-quality studies were most often conducted in Europe (25\%) and the United States (35\%); 30 percent were conducted in 4 or more countries. Of the 16 good- and fairquality trials that reported the funding source, all but two (88\%) were funded by industry. The weighted mean age of enrolled participants across trials was 52 years (range 30 to 58 years, 19 trials), with a weighted mean proportion of female participants of 64 percent (range $22 \%$ to $87 \%$, 18 trials). The race of participants was reported in eight trials, with a weighted mean proportion of nonwhite participants of 19 percent (range $0.3 \%$ to $50 \%$ ). The weighted mean baseline pain severity was 65 (VAS scale 0-100, range 46 to 72, 9 trials). Six trials reported baseline pain using a variety of other measures and six trials did not report baseline pain. Fourteen trials reported mean baseline functional ability using a variety of measures, including Bath Ankylosing Spondylitis Functional Index (BASFI) 100-point scale (weighted mean $=50$, range 47 to 52, 2 trials), BASFI 10-point scale (weighted mean = 4, 2 trials), American Rheumatoid Association (ARA) Functional Class (weighted means: ARA I: 25\%, ARA II: 59\%, ARA III: 17\%, 3 trials), and the Modified Health Assessment Questionnaire (MHAQ; weighted mean =1.12, 2 trials). The weighted mean duration of pain at baseline was 122 months (range 61 to 147 months, 15 trials). Complete descriptions of included study characteristics are in Appendix E. Results of meta-analyses, including forest plots and subgroups analyses, are in Appendix I. 


\section{Nonsteroidal Anti-Inflammatory Drugs}

\section{Placebo-Controlled Trials}

\section{Pain}

At short-term followup, NSAIDs resulted in a small, statistically significant, reduction in pain compared with placebo, based on meta-analysis of nine RCTs (MD -0.97, 95\% CI -1.33 to $-0.74, \mathrm{I}^{2}=39 \%, 0-10$ scale; Appendix I). ${ }^{162,164,165,167,169-173,178,179,185,189,193}$ Similarly, the proportion of patients responding to treatment with NSAIDs was significantly higher than for placebo, with a moderate combined effect size (45\% vs. 32\%, RR 1.58, 95\% CI 1.34 to 2.06, 7 RCTs, I ${ }^{2}=52 \%$; Appendix I). ${ }^{162,164,171,173,185,189,193}$ These two meta-analyses combined studies of celecoxib, ${ }^{162,189,193}$ diclofenac, ${ }^{172}$ etodolac, ${ }^{178,179}$ meloxicam, ${ }^{172}$ and naproxen. ${ }^{162,164,171,173,185,189,193}$ The strength of evidence for NSAIDs on pain reduction and pain response in the short term is moderate. At intermediate-term followup in a single trial $(\mathrm{N}=563)$, naproxen $1000 \mathrm{mg}$ daily was associated with greater reduction in pain compared with placebo (MD -0.53, 95\% CI -0.93 to $-0.13,0-10$ scale) and a higher proportion responding to treatment (42\% vs. 32\%, RR 1.28, 95\% CI 1.03 to 1.60). ${ }^{173}$ At long-term followup in a single trial ( $\left.\mathrm{N}=365\right)$, meloxicam 15 to 22.5 mg daily was associated with a large and statistically significant greater reduction in pain compared with placebo (MD -2.10, 95\% CI -2.72 to -1.48 , 0-10 scale) and a significantly higher proportion responding to treatment (48\% vs. $16 \%$, RR 3.05, 95\% CI 1.98 to 4.71 ). ${ }^{167}$ The strength of evidence for NSAIDs on pain reduction and pain response in the intermediate and long term is low.

Subgroup analyses of specific drug, dose (celecoxib), year of publication $(\leq 2000, \geq 2001)$, and study quality (good and fair) did not alter the findings meaningfully, with no significant interactions found (Appendix I). Subgroup analysis of type of inflammatory arthritis (RA vs. AS) found improvement in pain was significantly greater in patients with AS (MD -2.02, 95\% CI -2.96 to $-1.07, \mathrm{I}^{2}=0 \%, 0-10$ scale), compared with those with RA (MD -0.88, 95\% CI -1.12 to $-0.65, \mathrm{I}^{2}=39 \%$, $0-10$ scale), with a statistically significant test for interaction ( $\mathrm{p}=0.03$; Appendix I). Pain response was also greater (RR 2.27, 95\% CI 1.72 to $3.84, \mathrm{I}^{2}=0 \%$ ) in AS patients than in RA patients (RR 1.41, 95\% CI 1.25 to 1.61 ) with a significant test for interaction ( $\mathrm{p}=0.03$ ). In addition, comparisons between different doses of celecoxib (200 mg daily vs. $400 \mathrm{mg}$ daily) ${ }^{162,188,189,191}$ and meloxicam (7.5 mg daily vs. $15 \mathrm{mg}$ daily vs. $22.5 \mathrm{mg}$ daily) ${ }^{167,172}$ found no meaningful differences between doses for pain reduction or pain response (Appendix I).

\section{Function}

At short-term followup, NSAIDs resulted in a small, statistically significant, improvement in function compared with placebo, based on meta-analysis of seven RCTs (SMD -0.34, 95\% CI -0.51 to $-0.20, \mathrm{I}^{2}=67 \%$ ), using the BASFI and the Health Assessment Questionnaire (HAQ). The meta-analysis combined studies of celecoxib, ${ }^{162,189,193}$ diclofenac, ${ }^{172}$ meloxicam, ${ }^{172}$ and naproxen (Appendix I). ${ }^{162,164,171,173,185,189,193}$ At intermediate-term followup in a single trial $(\mathrm{N}=563)$, naproxen $1000 \mathrm{mg}$ daily resulted in a small improvement in function compared with placebo (MD - $0.18,95 \%$ CI -0.35 to -0.02 , $0-3$ scale). ${ }^{173}$ At long-term followup in a single trial ( $\mathrm{N}=365)$, meloxicam 15 to $22.5 \mathrm{mg}$ daily did not improve function compared with placebo (MD $-0.63,95 \%$ CI -0.85 to $-0.40,0-40$ scale). ${ }^{167}$ The strength of evidence for NSAIDs on function in the short term is moderate; and for the intermediate term and long term it is low.

Subgroup analyses of specific drug, dose (celecoxib), year of publication $(<2000,>2001)$, type of inflammatory arthritis (RA vs. AS), and study quality (good and fair) did not alter the 
findings meaningfully, with no significant interactions found. In addition, comparisons between different doses of celecoxib (400 mg daily vs. $200 \mathrm{mg}$ daily), ${ }^{162,188,189,191}$ and meloxicam (7.5 mg daily vs. $15 \mathrm{mg}$ daily vs. $22.5 \mathrm{mg}$ daily) ${ }^{167,172}$ found no meaningful differences in function between doses.

\section{Quality of Life}

At short-term followup in one trial $(\mathrm{N}=55)$, naproxen $1000 \mathrm{mg}$ daily was associated with moderate improvement in quality of life compared with placebo, as measured by the Ankylosing Spondylitis Quality of Life (ASQoL) scale (MD -2.9, $\mathrm{p}=0.04,0-18$ scale). ${ }^{171}$ Another short-term trial in patients with RA $(\mathrm{N}=1,148)$ found improvement in quality of life, as measured by the SF36 PCS and MCS, for each of three different doses of celecoxib (200 mg, $400 \mathrm{mg}$, and $800 \mathrm{mg}$ daily) and for naproxen $1000 \mathrm{mg}$ daily. ${ }^{189}$ However, the effect sizes for the PCS (MD range: 1.6 to 3.5, $\mathrm{p}<0.01,0-100$ scale) and for the MCS (MD range: 2.5 to 3.5, $\mathrm{p}<0.05,0-100$ scale) were all less than a small effect as defined for this report. The mean differences for two doses of celecoxib (400 mg daily and $800 \mathrm{mg}$ daily) for the PCS (MD = 3.4 and 3.5, respectively, 0-100 scale) and one dose of celecoxib (400 mg daily) for the MCS (MD 3.5, 0-100 scale) were slightly higher than the 3-point MCID recommended for use with the SF-36, ${ }^{139}$ while the mean differences for naproxen and the other doses of celecoxib were less than the MCID. This evidence is insufficient to draw conclusions about quality of life, given the inconsistency in findings.

\section{Other Outcomes}

One trial $(\mathrm{N}=1,148)$ assessed changes in depression and/or anxiety, using the "role emotional" and "mental health" domains of the SF-36 in the short term. ${ }^{189}$ Three different doses of celecoxib and one dose of naproxen were associated with improvement in "role emotional" scores compared with placebo. The effect size was moderate for celecoxib $400 \mathrm{mg}$ daily (MD 10.3, $\mathrm{p}<0.05$ ) and small for celecoxib $200 \mathrm{mg}$ daily, celecoxib $800 \mathrm{mg}$ daily, and naproxen 1000 mg daily (MD 8.1, 7.5, and 8.4, respectively; $\mathrm{p}<0.05$ ). Although each dose of celecoxib and naproxen were also associated with improvement in "mental health" scores, all effect sizes were less than small as defined for this report (MD range: 2.8 to 4.6), with $\mathrm{p}<0.05$ for each dose except for celecoxib $400 \mathrm{mg}$ daily, which was not statistically significant. At long-term followup in another trial $(\mathrm{N}=365)$, meloxicam $15 \mathrm{mg}$ daily and $22.5 \mathrm{mg}$ daily were associated with large improvements in sleep disturbance due to pain compared with placebo (MD -26\% and -35\%, respectively, $\mathrm{p}<0.05){ }^{167}$

\section{Head-to-Head Comparisons of NSAIDs}

Three short-term, fair-quality RCTs of celecoxib versus diclofenac, ${ }^{170,188,191}$ two of celecoxib versus naproxen, ${ }^{162,189}$ and two of nabumetone versus naproxen ${ }^{169,183}$ provided data for metaanalyses. Five additional fair-quality RCTs, which could not be pooled in meta-analyses, compared one NSAID with another. These included short-term comparisons of diclofenac versus etodolac, ${ }^{184}$ diclofenac versus meloxicam, ${ }^{172}$ and etodolac versus naproxen, ${ }^{165}$ and intermediateterm comparisons of meloxicam versus naproxen ${ }^{192}$ and nabumetone versus naproxen. ${ }^{195}$

\section{Pain}

In short-term followup, no meaningful difference in pain improvement was found between any two NSAIDs, including: celecoxib versus diclofenac (3 trials),${ }^{170,188,191}$ celecoxib versus

naproxen (2 trials), ${ }^{162,189}$ diclofenac versus etodolac (1 trial) ${ }^{184}$ diclofenac versus meloxicam (1 
trial), ${ }^{172}$ etodolac versus naproxen (1 trial), ${ }^{165}$ and nabumetone versus naproxen (2 trials) $)^{169,183}$ (Appendix I). In intermediate-term followup, difference in pain improvement was not found between meloxicam versus naproxen ( 1 trial) or nabumetone versus naproxen $\left(1\right.$ trial). ${ }^{192,195}$ Similarly, in short-term followup, no difference was found in pain response between celecoxib versus diclofenac (3 trials) ${ }^{170,188,191}$ or celecoxib versus naproxen ( 2 trials). ${ }^{162,189}$ In the metaanalyses of celecoxib, subgroup analyses by year of publication $(<2000,>2001)$ and type of inflammatory arthritis (RA vs. AS) did not alter the findings meaningfully. This evidence is low strength, except for the small, single study comparisons of etodolac and diclofenac or naproxen, which was insufficient to draw conclusions.

\section{Function}

In short-term followup, no meaningful difference in function was found between any two NSAIDs, including: celecoxib versus diclofenac (3 trials), ${ }^{170,188,191}$ celecoxib versus naproxen (2 trials), ${ }^{162,189}$ diclofenac versus etodolac (1 trial), ${ }^{184}$ diclofenac versus meloxicam (1 trial), ${ }^{172}$ and nabumetone versus naproxen (2 trials) ${ }^{169,183}$ (Appendix I). In the meta-analyses of celecoxib, subgroup analyses by year of publication $(<2000,>2001)$ and type of inflammatory arthritis (RA vs. AS) did not alter the findings meaningfully. This evidence is low strength, except for the small, single study comparison of etodolac and diclofenac, which was insufficient to draw conclusions.

\section{Quality of Life}

In short-term followup in one trial ( $\mathrm{N}=917)$, no meaningful difference in quality of life was found between celecoxib (200 mg to $800 \mathrm{mg}$ daily) and naproxen $1000 \mathrm{mg}$ daily, as measured by the SF-36 PCS the MCS. ${ }^{189}$ This evidence is low strength.

\section{Antidepressants}

\section{Pain}

In short-term followup in one fair-quality trial $(\mathrm{N}=36)$, there was no meaningful difference between amitriptyline $50 \mathrm{mg}$ to $75 \mathrm{mg}$ daily and placebo for pain improvement (MD 0.12, p=not significant, 0-4 scale). ${ }^{177}$ The study did not assess pain response, function, or quality of life. This evidence was insufficient to draw conclusions due to study limitations and size.

\section{Low Back Pain/Neck Pain}

\section{Key Points}

- In patients with low back pain, short-term duloxetine use resulted in a small improvement in pain severity and response, but the improvements in function and quality of life did not meet the threshold for a small improvement, based on pooled analysis of three trials (SOE: Moderate).

- In the intermediate term, a single study of amitriptyline found no improvement in pain or function in patients with low back pain (SOE: Low).

\section{Detailed Assessment}

Seven RCTs (in 9 publications) involving 1,838 patients meeting inclusion criteria evaluated nonopioid drugs to treat chronic low back pain (Appendix E). ${ }^{196-204}$ Six were short-term studies 
(12 to 14 weeks) and one was intermediate-term (6 months). ${ }^{204}$ Six were placebo-controlled trials, ${ }^{196-201}$ two of which compared multiple doses of desipramine and/or duloxetine, and one head-to-head trial comparing amitriptyline and pregabalin. ${ }^{204}$ Two RCTs met criteria for good quality, ${ }^{199,204}$ and the other five RCTs were fair quality. Two studies were conducted in the United States, ${ }^{196,197}$ two studies were multinational, ${ }^{200,201}$ and one each was conducted in Australia, ${ }^{204}$ India, ${ }^{198}$ and Japan. ${ }^{199}$ Three studies were government-funded ${ }^{196,197,204}$ and three were industry-funded; ${ }^{199-201}$ one did not report the funding source. ${ }^{198}$

Mean age of enrolled patients ranged from 42 to 59 years and 23 to 61 percent were female. In four studies reporting race, less than 30 percent of participants were nonwhite. Four RCTs reported baseline pain severity ranging from 5 to 7 on a 0-10 VAS. ${ }^{198-201}$ In the remaining three trials, two reported baseline pain of 9 on a 0-20 VAS, ${ }^{196,197}$ and one reported baseline pain of 40 on a 0-100 VAS. ${ }^{204}$ Duration of pain across all studies ranged from 35 to 204 months (median 120). At baseline, function/disability ranged from 8 to 9 on the Roland Morris Disability Questionnaire (RMDQ) in three trials, ${ }^{199,201,204}$ and 42 on the Oswestry Disability Index (ODI) scale in one trial; ${ }^{198}$ baseline function/disability was unclear or not reported in the remaining three trials. ${ }^{196,197,200}$ Complete descriptions of included study characteristics are in Appendix E.

\section{Antidepressants: SNRIs}

\section{Duloxetine}

Duloxetine versus placebo was assessed in one good- and two fair-quality, short-term RCTs ( $\mathrm{N}=1,491)$ (Appendix E). ${ }^{199-201}$ Duloxetine dose ranged from 20 to $120 \mathrm{mg}$ daily. Pain, function, and quality of life were reported in all three publications. Strength of evidence for duloxetine versus placebo was moderate for pain, function outcomes, and quality of life.

\section{Pain}

In the short term, duloxetine resulted in a small reduction in pain, based on meta-analysis of three RCTs (BPI Pain Scale 0-10; MD -0.50, 95\% CI -0.71 to -0.29, I²=0\%; Appendix I). ${ }^{199-201}$ Similarly, the proportion responding to duloxetine was significantly greater than placebo (RR $1.25,95 \%$ CI 1.11 to $1.40, \mathrm{I}^{2}=0 \%$ ). Sensitivity analysis of study quality did not alter the findings meaningfully. Estimates were similar when stratified according to dose of duloxetine, though 20 mg daily was not associated with improvement in pain (MD 0.08, 95\% CI -0.66 to 0.82) or proportion responding to duloxetine (RR 0.95 , 95\% CI 0.65 to 1.38 ) based on one trial. ${ }^{200}$

\section{Function}

In the short term, duloxetine resulted in improvement in function that was below the threshold for a small magnitude of effect for this report, based on meta-analysis of three RCTs (BPI Interference Scale; MD -0.36, 95\% CI -0.73 to -0.04, I²=34\%; Appendix I). ${ }^{199-201}$ Sensitivity analysis of study quality did not alter the findings meaningfully, though only one study was good quality and the estimate was imprecise. Results were also consistent when stratified according to dose of duloxetine.

\section{Quality of Life}

Three short-term RCTs reported the effect of duloxetine on quality of life. ${ }^{199-201}$ All three trials reported small improvement in quality of life with duloxetine, but the effect estimate was only statistically significant in one trial that used a dose of $60 \mathrm{mg}$ daily. ${ }^{201}$ When pooled, the effect of duloxetine on quality of life was not statistically significant (SMD 0.18, 95\% CI -0.03 
to $0.39, \mathrm{I}^{2}=38 \%$; Appendix I). Results were consistent when studies were stratified according to study quality and dose of duloxetine.

\section{Tricyclic Antidepressants}

One short-term fair-quality trial $(\mathrm{N}=78)$ compared desipramine with placebo (Appendix E). ${ }^{196}$ Desipramine dose was not reported, rather the study focused on the effect of low ( $<60$ $\mathrm{mg} / \mathrm{ml}$ ) or high ( $>60 \mathrm{ng} / \mathrm{ml}$ ) plasma concentrations of desipramine. After 12 weeks of treatment, Descriptor Differential Scale (DDS) scores (scale 0-20) were not significantly different between all desipramine concentrations (6.0) and placebo (6.8) groups (MD -0.80, 95\% CI -2.64 to 1.04). Desipramine less than $60 \mathrm{mg} / \mathrm{ml}$ was more effective than placebo at reducing pain $(\mathrm{p}=0.05)$ with no such effect for higher plasma levels of desipramine. The proportion responding $(>75 \%$ reduction in pain) was similar for desipramine and placebo (23\% vs. 18\%, RR 1.28, 95\% CI 0.43 to 3.85), though low plasma concentration desipramine was associated with greater response than placebo (37\% vs. 18\%, RR 2.03, 95\% CI 0.70 to 5.87). Evidence on other outcomes for all desipramine concentrations was not reported, but low concentration desipramine improved function relative to placebo, based on RMDQ score (mean 2.3 vs. 4.1, $\mathrm{p}=0.05$ ). This evidence is insufficeint to draw conclusons, due to study quality, unknwn consistency, and imprecison.

One good-quality, intermediate-term trial ( $\mathrm{N}=146)$ comparing amitriptyline $25 \mathrm{mg}$ daily with placebo found a mean difference in pain score of -7.81 (VAS 0-100 scale) between groups after 6 months treatment; this difference was not statistically significant (95\% CI -15.7 to 0.10$){ }^{204}$ The mean difference (-0.98) between groups in function, measured using the RMDQ scale (024), also showed a nonsignificant effect favoring amitriptyline (95\% CI -2.42 to 0.46). This evidence is low strength.

\section{Anticonvulsants}

\section{Gabapentin}

A short-term, fair-quality trial $(\mathrm{N}=108)$ meeting inclusion criteria compared gabapentin up to $3600 \mathrm{mg}$ daily with placebo in patients with radicular and nonradicular back pain (Appendix E). ${ }^{197}$ After 12 weeks, both gabapentin and placebo were associated with similar reduction in DDS pain scores compared with baseline $(\mathrm{p}=0.42)$ and with similar proportions responding to treatment (36\% vs. 36\%, $\mathrm{p}=1.00$ ). Similar proportions of patients in both groups were rated as having at least "minimal improvement" on the physician-rated Clinical Global Impression of Change (37\% vs. 33\%, p=0.95). Quality of life, based on BDI-II scores, were also not different between groups following treatment $(\mathrm{p}=0.52)$. This evidence is insufficeint to draw conclusons due to study quality, unknwn consistency, and imprecison.

\section{Cross-Class Comparisons}

\section{Pregabalin Versus Amitriptyline}

One short-term trial $(\mathrm{N}=200)$ compared pregabalin $600 \mathrm{mg}$ daily versus amitriptyline $50 \mathrm{mg}$ daily in patients with low back pain (Appendix E). ${ }^{198}$ After 14 weeks, although both groups improved signficantly, a small greater improvement was seen with amitriptyline (-3.9 on VAS) compared with pregabalin ( -2.9 on VAS, $\mathrm{p}=0.03)$. The proportion of patients responding to treatment ( $>50 \%$ improvement in VAS score) was also significantly higher with amitriptyline (57\%) than pregabalin (39\%; RR 1.46, 95\% CI 1.08 to 1.97). Both interventions similarly 
improved function based on ODI scale score, with no difference between groups $(\mathrm{p}=0.09)$. This evidence is low strength.

\section{Chronic Headache}

\section{Key Points}

- Evidence from a single fair-quality RCT (N=197) did not find differences between amitriptyline 50 to $75 \mathrm{mg}$ daily and placebo in patients with "chronic tension-type headache” (SOE: Low).

\section{Detailed Assessment}

Although the classification of headache has changed over time, in order to capture any evidence relevant to treating chronic headache pain and being consistent with other similar reports, ${ }^{10,25}$ we defined chronic headache broadly using the International Headache Society 2013 definition: headache frequency of at least 15 days per month over a period of at least 6 months or headache more than 180 days per year. ${ }^{22}$ No other requirement was made in terms of defining chronic headache, although all the other inclusion criteria applied (e.g., 12 weeks duration minimum). Using this definition, three RCTs were found, ${ }^{205-207}$ but two were rated poor quality due to unclear randomization processes, differences at baseline in patient characteristics, and lack of blinding. ${ }^{206,207}$ One of these RCTs $(\mathrm{N}=41)$ compared pregabalin with placebo in patients with "chronic unilateral cervicogenic headache,"206 and the other $(\mathrm{N}=53)$ compared TCAs (amitriptyline or nortriptyline) with placebo, stress management, or a combination in patients with "chronic tension-type headache". 207

The fair-quality RCT (N=197) compared treatment with amitriptyline and placebo (and a drug studied in Germany, amitriptylinoxide-not reported here) in patients with "chronic tension-type headache.”205 Mean age of enrolled patients was 38 years, 56 percent were female, and mean baseline pain severity was 3.7 on a VAS of $0-8$. Dosing was adjusted for tolerability and ranged from 50 to $75 \mathrm{mg}$ of amitriptyline per day. In the short term (24 weeks), headache pain severity decreased in both amitriptyline and placebo groups (reduction of 0.9 with amitriptyline and 1.7 with placebo, on a scale of 0-8, no statistical analysis presented). Similarly, response (defined as 50\% reduction in duration and frequency of headache in weeks 13-16) was not different between groups (22.4\% vs. 21.9\%, calculated RR 1.024, 95\% CI 0.54 to 1.95). This is low strength of evidence.

\section{Sickle-Cell Disease}

\section{Key Points}

- Evidence from a single pilot study was insufficient to draw conclusions on the effect of pregabalin given over 3 months in patients with sickle cell disease and ongoing pain.

\section{Detailed Assessment}

A single fair-quality pilot study $(\mathrm{N}=22)$ compared pregabalin with placebo in patients with sickle cell disease and a history of pain that was not well controlled; at least a score of 4 on a 0 10 scale and requiring intermittent NSAIDs, acetaminophen, or opioids. Mean age of participants was 33 years, 73 percent were female, and nearly all were nonwhite (95\% African American). Mean pain score at baseline for pregabalin group was 3.8 versus 4.8 for placebo on the Average 
Pain Intensity (API) 0-10 scale; other pain measures showed similar differences at baseline. Mean SF-36 PCS at baseline was 64.3. Dosing of pregabalin was flexible based on tolerability with a range of 75 to $600 \mathrm{mg}$ daily, given for 3 months. In the short term, pregabalin led to a small reduction in API score (pregabalin -1.1, placebo -0.5 on a scale of $0-10$ ), but was not statistically significant given the small sample size. Differences on three other pain measures (the composite pain index, neuropathic pain symptom index, and the Leeds Assessment of Neuropathic Signs and Symptoms) were small and sometimes favored placebo. No difference was reported in SF-36 scores between groups. Due to the very small size, no corroborating evidence, and study limitations (e.g. differences in pain scores at baseline), this evidence is insufficient to draw conclusions.

\section{Key Question 2: Harms and Comparative Harms of Nonopioid Drugs for Chronic Pain}

We evaluated the harms of nonopioid drugs in patients with chronic pain, including (for comparison purposes) adverse events associated with opioid use (e.g., overdose, misuse, dependence, substance use disorder), over-arching adverse event outcomes that can be assessed across classes (i.e., withdrawals due to adverse events [WAE], and serious adverse events [SAE]), and adverse events that are specific to individual drug classes. We evaluated the impact of type of pain, patient demographics and comorbidities, and dose and duration of treatment. The evidence is limited to RCTs and systematic reviews of these drugs in patients with chronic pain, and is organized by drug classes.

\section{Antidepressants}

\section{Key Points}

- In the short and intermediate term, SNRI antidepressants (duloxetine and milnacipran) did not increase reports of SAEs (SOE: Low) but led to a moderate increases in risk of WAE (SOE: Moderate). In the short term, TCAs (amitriptyline and desipramine) did not increase the risk of WAE (SOE: Low).

- SNRI specific harms: in the short and intermediate term, reports of nausea were significantly increased with milnacipran (moderate increase) and duloxetine (large increase; SOE: Moderate). Dose did not affect the findings (SOE: Low). A large increase in sedation was reported with duloxetine in the short term (SOE: Moderate); $60 \mathrm{mg}$ daily resulted in lower risk than $120 \mathrm{mg}$ daily (SOE: Low). In the short term, cognitive adverse events were not significantly more frequent with SNRIs (SOE: Low).

- TCA specific harms: TCAs did not significantly increase the risk WAE (SOE: Low). Evidence on other adverse events of interest was not available or insufficient.

\section{Detailed Assessment}

Forty good- or fair-quality placebo-controlled trials (in 50 publications) ${ }^{33,36-38,43,54-56,64-66,68-}$ 71,73,75-77,79-81,83-86,88,93,94,98,100,109,110,153-157,177,196,198-205,208,209 involving 13,943 patients meeting inclusion criteria evaluated antidepressants to treat chronic pain; 32 were short-term studies, 5 intermediate-term, ${ }^{79,84,86,93,204}$ and 3 long-term. ${ }^{55,76,80}$ The large majority of evidence was for SNRIs, either milnacipran or duloxetine, with 34 trials including 13,156 participants, with 6 RCTs of TCAs (N=787). ${ }^{85,177,196,198,204,205}$ Seven trials met criteria for good 
quality. ${ }^{88,98,100,109,110,199,204}$ The patient population in 16 trials was fibromyalgia, 8 trials neuropathic pain, 8 trials osteoarthritis, 6 trials low back pain, and 1 trial each of rheumatoid arthritis and chronic headache. The specific adverse events of interest included nausea and sedation for SNRIs, cardiac rhythm abnormalities, dry mouth, urinary retention, and weight gain for TCAs, and cognitive effects and serotonin syndrome for both drug classes.

\section{Serious Adverse Events}

SAEs were infrequent; meta-analysis of 19 short-term trials of SNRI antidepressants (duloxetine and milnacipran) did not find a significant difference compared with placebo (1.5\% vs. $1.6 \%$, RR 0.88 , 95\% CI 0.62 to $\left.1.24, \mathrm{I}^{2}=0 \%\right)^{36,37,43,54,56,65,66,71,75,77,81,88,100,110,153,157,199-201}$ Subgroup analyses by pain population, study quality, specific drug, and dose within a single drug did not alter these results significantly. Two intermediate-term trials of SNRIs duloxetine or milnacipran also found no difference in the incidence of SAEs compared with placebo (2.2\% vs. $2.6 \%$, RR $0.86,95 \%$ CI 0.35 to $2.24, \mathrm{I}^{2}=0 \%$ ). These findings are low strength of evidence. Evidence on SAEs of TCAs was limited, with one trial of five reporting this outcome.

\section{Withdrawals Due to Adverse Events}

There was a moderate increase in WAEs with antidepressants (duloxetine and milnacipran) in 24 short-term studies (15.2\% vs. $7.5 \%$, RR 1.99 , 95\% CI 1.71 to $2.35, \mathrm{I}^{2}=18 \%$ ), and in 3 intermediate-term studies (21.9\% vs. $11.4 \%$, RR 1.83 , 95\% CI 1.23 to $\left.2.61, \mathrm{I}^{2}=4 \%\right)$. These findings are moderate strength of evidence. Subgroup analyses of pain population, study quality, or specific drug (duloxetine or milnacipran) did not significantly alter these results. Higher doses of duloxetine (60 mg or $120 \mathrm{mg}$ daily) and milnacipran (200 mg daily) resulted in significant increased risk of WAE (relative risks great than 2.0), while lower doses (20 mg and $40 \mathrm{mg}$ daily of duloxetine, $100 \mathrm{mg}$ daily of milnacipran) did not reach statistical significance and had lower relative risks (less than 2.0).

The risk of WAE with a TCA (amitriptyline or desipramine) was not significantly increased over placebo in the short term (5 RCTs, N=478, 19\% vs. 10\%, RR 1.49, 95\% CI 0.89 to 3.01, $\mathrm{I}^{2}=0 \%$ ) or the intermediate term ( 1 RCT, $\mathrm{N}=126,8 \%$ vs. 5\%, RR $1.7595 \%$ CI 0.38 to 8.06; Appendix I). The evidence in the short term is low strength, but the intermediate-term evidence is insufficient.

Subgroup analysis by study quality did not alter the findings. Analysis by specific drug found the risk with amitriptyline similar to the overall meta-analysis result (4 RCTs, N=400), and the risk with desipramine, based on a single small study $(\mathrm{N}=78)$, significantly increased (RR 8.50, 95\% CI 1.20 to 60.41 ).

\section{Specific Adverse Events}

\section{SNRIS}

\section{Nausea}

In the short term, 19 RCTs of SNRI antidepressants duloxetine and milnacipran $(\mathrm{N}=8,929)$ resulted in a large increased incidence of nausea (25\% vs. 9\%, RR 3.10, 95\% CI 2.50 to 4.06, $\mathrm{I}^{2}=60 \%$; Appendix I). ${ }^{36-38,54,56,65,66,71,75,77,81,88,100,110,153,157,199-201}$ This is moderate strength of evidence. Subgroup analyses by pain population, dose, and study quality did not alter these findings. Analysis by specific drug showed that duloxetine (16 RCTs, N=5,803) has a significantly greater risk of nausea (20\% vs. $4.8 \%$, RR 3.57, 95\% CI 2.97 to $4.50, \mathrm{I}^{2}=0 \%$; 
Appendix I) than milnacipran (3 RCTs, N=3,098, RR 1.90, 95\% CI 1.64 to 2.29, $\mathrm{I}^{2}=0 \%$, p-value for interaction=0.00). In the intermediate term, 3 RCTs $(\mathrm{N}=1,738)$ found a moderate increased risk of nausea (33\% vs. $15 \%$, RR $1.98,95 \%$ CI 1.57 to $2.82, \mathrm{I}^{2}=0 \%$ ). ${ }^{79,86,93}$ This is low-strength evidence.

\section{Sedation}

In the short term, 16 RCTs $(\mathrm{N}=5,831)$ of the SNRI duloxetine showed a large increased incidence of sedation compared with placebo (12\% vs. 4.6\%, RR 2.46, 95\% CI 2.00 to 3.01, $\left.\mathrm{I}^{2}=0 \%\right) .{ }^{36-38,54,56,66,71,75,88,100,110,153,157,199-201}$ This is moderate strength of evidence. In the intermediate term, 2 RCTs $(\mathrm{N}=850)$ found a large increased risk of sedation with duloxetine (11\% vs. $2.6 \%$, RR 3.51, 95\% CI 1.46 to $\left.11.05, \mathrm{I}^{2}=0 \%\right) .{ }^{79,93}$ This is low-strength evidence. Subgroup analyses of pain population and study quality did not alter these findings. Analyses by dose found significant increased risk and higher relative risks with $60 \mathrm{mg}$ and $120 \mathrm{mg}$ daily than with lower doses (20 mg and $30 \mathrm{mg}$ daily).

\section{Cognitive Effects}

Only two short-term RCTs of SNRI antidepressants reported cognitive adverse events, with the pooled estimate not reaching statistical significance $(0.8 \%$ vs. $0 \%$, RR 3.24, 95\% CI 0.26 to 40.17, $\left.\mathrm{I}^{2}=0 \%\right)$. This is low strength evidence.

\section{Serotonin Syndrome}

We found no RCTs reporting episodes of serotonin syndrome.

\section{TCAs}

\section{Dry Mouth}

Significantly more patients reported dry mouth with the TCA amitriptyline in a short-term RCT ( $\mathrm{N}=131)$ of patients with chronic tension-type headache (51\% vs. 28\%, RR 1.80, 95\% CI 1.14 to 2.85). ${ }^{205}$ No other trial of a TCA reported dry mouth as an adverse event, and this evidence is insufficient to draw conclusions.

\section{Other Specific Adverse Events}

No adverse events of interest, including cardiac rhythm abnormalities, were reported in the included studies.

\section{Anticonvulsants}

\section{Key Points}

- In the short term, oxcarbazepine led to a large increased risk of WAEs (SOE: Low).

- In the short term, pregabalin/gabapentin resulted in large increases in blurred vision, dizziness, weight gain, and cognitive effects (e.g., confusion).

- While the incidences of hyponatremia and sedation were greater with oxcarbazepine than placebo, the differences were not significant (SOE: Low).

\section{Detailed Assessment}

Twenty-eight RCTs provided evidence for harms in the short term: 21 RCTs provided information on SAEs, all 28 RCTs provide evidence on WAE, and 27 RCTs provided evidence 
on specific adverse events. Four studies met criteria for being good quality ${ }^{27,29,89,109}$ and the remainder were fair quality. Seventeen trials were of patients with neuropathic pain, eight of patients with fibromyalgia, one each of patients with low back pain, osteoarthritis, and sickle cell disease. Twenty-three RCTs involved pregabalin, and two each involved gabapentin, the prodrug gabapentin enacarbil, and oxcarbazepine (one included both pregabalin and gabapentin enacarbil). ${ }^{44}$ For this drug class, specific adverse events of interest included blurred vision, cognitive effects, dizziness, peripheral edema, sedation, weight gain for pregabalin, gabapentin, and gabapentin enacarbil, and cognitive effects, hyponatremia, neutropenia, and sedation for oxcarbazepine (there were no studies of carbamazepine).

\section{Serious Adverse Events}

Meta-analysis of 19 RCTs (N=7,982) of patients with fibromyalgia (6 RCTs) and neuropathic pain (13 RCTs) did not find a significant increase in risk of having an SAE with an anticonvulsant in the short term (2.3\% vs. $2.5 \%$, RR $0.90,95 \%$ CI 0.90 to $1.30, \mathrm{I}^{2}=0 \%$; Appendix I). Subgroup analyses by pain condition, specific drug, dose, and study quality did not alter these results. In the short term, oxcarbazepine did not significantly increase the risk of serious adverse events ( 2 RCTs, $\mathrm{N}=493$, 8.9\% vs. 4.8\%, RR 1.82, 95\% CI 0.74 to 5.05, $\mathrm{I}^{2}=0 \%$ ). This evidence is low strength.

\section{Withdrawal Due to Adverse Events}

Meta-analysis of 26 RCTs $(\mathrm{N}=9,754)$ of patients treated for chronic pain in the short term found a moderate increase in WAEs (14\% vs. 7.0\%, RR 1.73, 95\% CI 1.48 to 2.01, I²=5\%) with pregabalin/gabapentin. Subgroup analyses by pain condition, specific drug, dose, and study quality did not alter these results. This evidence is moderate strength. In the short term, oxcarbazepine led to a large increase in the risk of WAEs (2 RCTs, N=493, 26\% vs. 7\%, RR 3.64, $95 \%$ CI 1.86 to $7.12, \mathrm{I}^{2}=0 \%$ ). This is evidence is low strength.

\section{Specific Adverse Events}

\section{Pregabalin/Gabapentin}

Twenty-five RCTs provided data on specific harms of pregabalin and gabapentin. 27,29,30,35,39,41,42,44,45,48-52,57,67,72,74,87,89,92,109,197 There were large increases in the risk of blurred vision, cognitive effects, dizziness, peripheral edema, sedation, and weight gain (Table 6). This evidence is low to moderate strength.

Table 6. Specific harms of pregabalin/gabapentin

\begin{tabular}{|l|c|c|c|c|}
\hline Specific Harms & $\begin{array}{c}\text { N Studies } \\
\text { (n Patients) }\end{array}$ & Incidence & Relative Risk (95\% Cl) & $\begin{array}{c}\text { Magnitude of Effect } \\
\text { Strength of Evidence }\end{array}$ \\
\hline Cognitive effects & $8(\mathrm{~N}=3,801)$ & $4.8 \%$ vs. $1.3 \%$ & RR $3.15(1.86$ to 5.51$)$ & $\begin{array}{c}\text { Large effect } \\
\text { Low }\end{array}$ \\
\hline Dizziness & $25(\mathrm{~N}=9,696)$ & $25.6 \%$ vs. $7.4 \%$ & RR $2.97(2.53$ to 3.50$)$ & $\begin{array}{c}\text { Large effect } \\
\text { Moderate }\end{array}$ \\
\hline Peripheral edema & $22(\mathrm{~N}=9,005)$ & $8.8 \%$ vs. $3.7 \%$ & RR $2.32(1.80$ to 3.09$)$ & $\begin{array}{c}\text { Large effect } \\
\text { Moderate }\end{array}$ \\
\hline Sedation & $24(\mathrm{~N}=9,652)$ & $17 \%$ vs. $5.4 \%$ & RR $3.03(2.62$ to 3.67$)$ & $\begin{array}{c}\text { Large effect } \\
\text { Moderate }\end{array}$ \\
\hline Weight gain & $21(\mathrm{~N}=8,620)$ & $10.1 \%$ vs. $2.8 \%$ & RR $3.57(2.77$ to 4.91$)$ & $\begin{array}{c}\text { Large effect } \\
\text { Moderate }\end{array}$ \\
\hline
\end{tabular}

$\mathrm{CI}=$ confidence interval; $\mathrm{RR}=$ relative risk 


\section{Oxcarbazepine}

Two RCTs (N=490) provided evidence on sedation and hyponatremia. ${ }^{31,34}$ While the incidence of each was greater in the oxcarbazepine group, and the relative risks reflected large increased risk, the differences compared with placebo was not statistically significant. This is low-strength evidence (Table 7).

Table 7. Specific harms of oxcarbazepine

\begin{tabular}{|l|c|c|c|c|}
\hline Specific Harms & $\begin{array}{c}\text { N Studies } \\
\text { (n Patients) }\end{array}$ & Incidence & Relative Risk (95\% CI) & $\begin{array}{c}\text { Magnitude of Effect } \\
\text { Strength of Evidence }\end{array}$ \\
\hline Sedation & $2(\mathrm{~N}=490)$ & $8.6 \%$ vs. $3.0 \%$ & RR $3.13(0.74$ to 16.08$)$ & $\begin{array}{c}\text { No effect } \\
\text { Low }\end{array}$ \\
\hline Hyponatremia & $2(\mathrm{~N}=490)$ & $2.8 \%$ vs. $0.0 \%$ & RR $5.93(0.55$ to 63.8$)$ & $\begin{array}{c}\text { No effect } \\
\text { Low }\end{array}$ \\
\hline
\end{tabular}

$\mathrm{CI}=$ confidence interval; $\mathrm{RR}=$ relative risk

\section{NSAIDs}

\section{Key Points}

- In the short term, NSAIDs led to a small increase in WAEs, with ibuprofen, diclofenac, and naproxen having moderately-increased risk (SOE: Moderate). Reports of SAEs were not increased with NSAIDs and differences were not found between celecoxib and nonselective NSAIDs in SAEs or WAEs (SOE: Low).

- In the short term, the risk of any cardiovascular event was not significantly elevated for NSAIDs as a group, although there was a small increase in risk with diclofenac, particularly within the first 6 months, and with higher doses. There was a moderate increased risk of major coronary events with diclofenac and celecoxib and a large increase with ibuprofen. In the intermediate and long term, there was not a difference in cardiovascular events between celecoxib and nonselective NSAIDs (SOE: Moderate).

- In the short term, nonselective NSAIDs led to moderate to large increased risk of serious upper gastrointestinal events (largely bleeding), particularly in the first 6 months of treatment (SOE: Moderate). In the short-term, evidence on celecoxib versus nonselective NSAIDs was mixed and inconclusive; in the intermediate term, nonselective NSAIDs had a moderately greater risk of serious gastrointestinal events than celecoxib (SOE: Low).

- In the intermediate term, although the incidence was low, large increases in hepatic harms were seen with diclofenac and naproxen (SOE: Low). No evidence on renal harms met inclusion criteria.

\section{Detailed Assessment}

Ninety-six RCTs (in 114 publications) and three systematic reviews ${ }^{210-212}$ provided evidence on harms of NSAIDs. Twelve trials (in 16 publications) met criteria for good quality, ${ }^{99,101-}$ 108,124,171,213-216 21 were poor quality (in 25 publications), ${ }^{111-117,163,166,168,174-176,180-182,187,190,217-223 ~}$ and the remainder (63 trials in 74 publications) were fair quality (Appendix G). ${ }^{118-123,125-}$ 138,140,141,143-152,162-165,167,169,170,172,173,178,179,183-186,188,189,191-194,224-245 The poor-quality trials were deemed to have high risk of bias due to unclear randomization methods, important differences at baseline, and large amounts of missing data, and were not synthesized with the other evidence. Of the good- and fair-quality RCTs involving 89,063 patients, 60 were short term (12 to 24 weeks), 10 were intermediate term (26 weeks), and 5 long term (52 to 156 weeks). These 
included 46 placebo-controlled trials (17 of celecoxib 100 to $400 \mathrm{mg}$ daily, 6 of diclofenac 70 to $150 \mathrm{mg}$ daily, 5 of ibuprofen $2400 \mathrm{mg}$ daily, 4 of meloxicam 3.75 to $22.5 \mathrm{mg}$ daily, 16 of naproxen $1000 \mathrm{mg}$ daily, and 4 of topical diclofenac $1 \%$ to 1.5\%), 12 comparing various doses of a single NSAID, and 36 RCTs making head-to-head comparisons of NSAIDs (some trials included more than one of these categories). Most studies were conducted in the United States (37) and were funded by industry (83\%). Mean age of enrolled patients ranged from 30 to 72 years (weighted mean 61.8 years), 67 percent were female, and 18 percent were nonwhite. Two trials were conducted in older adults with mean age of 71 and 72 years. ${ }^{148,151}$

Two included systematic reviews were good quality. ${ }^{210,211}$ One evaluated cardiovascular and serious gastrointestinal harms using a mix of individual patient data (IPD) and published tabular data meta-analysis of 639 RCTs with a duration of at least 4 weeks and was published through $2001 .^{210}$ The other good-quality systematic review evaluated celecoxib in patients with OA, and included analyses of harms versus placebo and other NSAIDs. ${ }^{211}$ The fair-quality systematic review evaluating hepatic harms of NSAIDs included 64 RCTs of patients with OA or RA with duration of at least 4 weeks, and was published through $2004 .{ }^{212}$

Adverse events for NSAIDs selected for this review were WAE, SAEs, cardiovascular events (cardiovascular mortality, nonfatal myocardial infarction, nonfatal stroke), serious gastrointestinal events such as gastrointestinal bleeding or perforated ulcers, and renal or hepatic events. Results of meta-analyses of data from these trials, including Forest plots and subgroup analyses, can be found in Appendix I.

\section{Serious Adverse Events}

Based on meta-analysis of 23 short-term RCTs ( $\mathrm{N}=13,082)$, there was no increased risk of overall SAEs with NSAIDs (RR 0.96, 95\% CI 0.72 to $1.29, \mathrm{I}^{2}=0 \%$; Appendix I). Stratified analyses by subgroups indicated numerically greater risk in patients with RA, with ibuprofen and naproxen, and in good-quality studies, although it was not statically significant and analysis for interaction was also not statistically significant. This is low strength of evidence. In the intermediate term, a single RCT ( $\mathrm{N}=563)$ did not find an increased risk of SAEs with naproxen (RR $0.51,95 \%$ CI 0.05 to 5.58), but this evidence was insufficient to draw conclusions. A recent Cochrane review of celecoxib $200 \mathrm{mg}$ daily versus any nonselective NSAID or placebo in patients with OA found that compared with nonselective NSAIDs (9 RCTs, 6 versus naproxen, 3 versus diclofenac) or placebo (32 RCTs), there were no significant differences in the incidence of SAEs, although the authors rated this evidence as very low quality. ${ }^{211}$ Based on two RCTs $(\mathrm{N}=912)$, there was not a significant increase in SAEs with topical diclofenac compared with placebo (RR 1.03, 95\% CI 0.29 to $27.01, \mathrm{I}^{2}=0 \%$ ). ${ }^{99,140}$ This evidence is low strength.

\section{Withdrawals Due to Adverse Events}

Based on meta-analysis of 38 short-term RCTs ( $\mathrm{N}=20,060)$, WAEs were increased to a small degree with NSAIDs (RR 1.30, 95\% CI 1.14 to 1.49, $\mathrm{I}^{2}=13 \%$; Appendix I). This is moderate strength of evidence. Stratified analysis by population (RA or OA) or study quality did not meaningfully alter these results. However, the analysis by specific drug varied significantly; a moderate increase with diclofenac (6 RCTs, RR 1.71, 95\% CI 1.22 to 2.65), ibuprofen (5 RCTs, RR 1.96, 95\% CI 1.42 to 2.69), and naproxen (15 RCTs, RR 1.50, 95\% CI 1.23 to1.84), while celecoxib (16 RCTs, RR 1.05, 95\% CI 0.86 to 1.24) and meloxicam (3 RCTs, RR 1.16, 95\% CI 0.51 to 2.32 ) had no clear increased risk (p-value for interaction $=0.01$ ). Two intermediate-term RCTs ( $\mathrm{N}=941)^{131,173}$ and one long-term RCT $(\mathrm{N}=365)^{167}$ did not find significantly increased risk 
of WAE. This evidence is low strength and insufficient, respectively. A recent Cochrane review of celecoxib $200 \mathrm{mg}$ daily versus any nonselective NSAID or placebo in patients with OA found that compared with nonselective NSAIDs (9 RCTs, 6 vs. naproxen, 3 vs. diclofenac) or placebo (32 RCTs), there were no significant differences in the incidence of WAEs (rated moderate quality evidence by the authors). ${ }^{211}$ Based on four RCTs $(\mathrm{N}=1,549)$, there was not a significant increase in WAEs with topical diclofenac compared with placebo (RR 1.03, 95\% CI 0.29 to 27.01, $\left.\mathrm{I}^{2}=0 \%\right)^{99,108,140,141}$ This is low strength of evidence.

\section{Cardiovascular Adverse Events}

Evidence on cardiovascular risks of NSAIDs comes from a large number of RCTs, some with specific intent to study these harms. A good-quality systematic review of 639 RCTs evaluated cardiovascular harms using a combination of individual patient data and standard meta-analysis. ${ }^{210}$ The analyses combined data on four selective COX-2 inhibitor drugs (“coxibs”). This review found an increased risk in major vascular events with a coxib and diclofenac, and increased risk of vascular death with coxibs (Table 8). Major coronary events were increased with coxibs, diclofenac, and ibuprofen, and increased risk of hospitalization for heart failure was found with all NSAIDs. This analysis found that baseline risk did not alter the findings, that there may be increased risk of major vascular events in the first 6 months of treatment with diclofenac (but no evidence of increased risk over longer treatment periods for any NSAID or coxib studied), and that across the drugs higher doses were associated with greater risk.

Table 8. Individual patient data meta-analysis of NSAID cardiovascular risks ${ }^{210}$

\begin{tabular}{|c|c|c|c|c|}
\hline Event & $\begin{array}{l}\text { Diclofenac } \\
\text { Adjusted RR } \\
(95 \% \mathrm{Cl})\end{array}$ & $\begin{array}{l}\text { Ibuprofen } \\
\text { Adjusted RR } \\
\text { (95\% CI) }\end{array}$ & $\begin{array}{l}\text { Naproxen } \\
\text { Adjusted RR } \\
(95 \% \mathrm{CI})\end{array}$ & $\begin{array}{l}\text { Coxibs } \\
\text { Adjusted RR } \\
(95 \% \mathrm{Cl})\end{array}$ \\
\hline Major vascular events ${ }^{a}$ & 1.41 (1.12 to 1.78$)$ & 1.44 (0.89 to 2.33$)$ & 0.93 (0.69 to 1.27$)$ & $\begin{array}{c}1.37(1.14 \text { to } 1.66) \\
\text { Celecoxib } 1.36 \\
(1.00 \text { to } 1.84)\end{array}$ \\
\hline Vascular mortality & $1.65(0.95$ to 2.85$)$ & $1.90(0.56$ to 6.41$)$ & $1.08(0.48$ to 2.47$)$ & $1.58(1.00 \text { to } 2.49)^{\circ}$ \\
\hline Major coronary events ${ }^{b}$ & 1.70 (1.19 to 2.41$)$ & 2.22 (1.10 to 4.48$)$ & $0.84(0.52$ to 1.35$)$ & 1.76 (1.31 to 2.37$)$ \\
\hline $\begin{array}{l}\text { Heart failure } \\
\text { (hospitalization) }\end{array}$ & 1.85 (1.17 to 2.94$)$ & 2.59 (1.19 to 5.20$)$ & 1.87 (1.10 to 3.16$)$ & $2.28(1.62$ to 3.20$)$ \\
\hline
\end{tabular}

$\mathrm{CI}=$ confidence interval; NSAID = nonsteroidal anti-inflammatory drug; RR = risk ratio

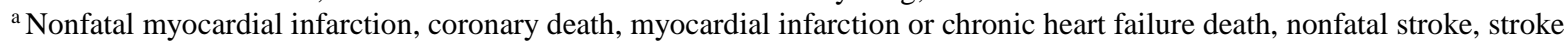
death, any stroke, other vascular death

${ }^{\mathrm{b}}$ Nonfatal myocardial infarction, coronary death, myocardial infarction or coronary heart disease death

c $99 \%$ CI calculated due to multiple comparisons

In the intermediate term, three RCTs compared the risk for cardiovascular events with celecoxib and nonselective NSAIDs, with none finding a significant difference. A large, goodquality RCT $(\mathrm{N}=24,081)$ evaluated cardiovascular harms in patients treated for OA or RA with celecoxib (mean 209 mg daily), ibuprofen (mean 2045 mg daily), and naproxen (mean 852 mg daily). ${ }^{236}$ Using a noninferiority analysis (on-treatment analysis), the incidence of cardiovascular death, nonfatal myocardial infarction, or nonfatal stroke was 1.7 percent with celecoxib, 1.9 percent with ibuprofen, and 1.8 percent with naproxen, with $\mathrm{p}<0.001$ for noninferiority between drugs. The second fair-quality RCT $(\mathrm{N}=916)$ also enrolled patients 60 years and older with OA and randomized to celecoxib or diclofenac (50 mg twice daily) and was included in a Cochrane review. ${ }^{151,211}$ This study also did not find a significant difference in cardiovascular events between the drugs (odds ratio [OR] 0.47, 95\% CI 0.17 to 1.25). A third fair-quality RCT 
$(\mathrm{N}=8,067)$ reported on adjudicated cardiovascular events, but did not conduct analyses comparing the event rates. The rate was very similar between celecoxib $(0.4 \%)$ and nonselective NSAIDs (0.3\%). ${ }^{226}$ This is moderate-strength evidence.

In the long term, a large good-quality RCT ( $\mathrm{N}=7297)$ randomized patients with OA or RA who were under the age of 60 years, had no known cardiovascular disease, and who were currently taking a nonselective NSAID, to celecoxib or continuing their nonselective NSAID. At followup (median 3 years), there was not a significant difference in the incidence of hospitalization for nonfatal myocardial infarction or other biomarker positive acute coronary syndrome, nonfatal stroke, or cardiovascular death (hazard ratio [HR] 1.12, 95\% CI 0.81 to 1.55). ${ }^{234}$ This study was designed as a noninferiority study, and noninferiority (equivalence) was achieved. This evidence is moderate strength.

\section{Serious Gastrointestinal Adverse Events}

In the short term, both study-level meta-analyses, and a published combination of individual patient data and standard meta-analysis found increased risk of serious gastrointestinal events with NSAIDs, with magnitude of risk varying by specific drug. A good-quality systematic review of 639 RCTs using a combination of individual patient data and standard meta-analysis found moderate to large increased risk of serious upper gastrointestinal harms compared with placebo (Table 9). ${ }^{210}$ The analyses combined data on four selective COX-2 inhibitor drugs ("coxibs"), and the authors reported no evidence of a difference in effect according to the specific coxib used. Most of the events were gastrointestinal bleeds, 2 percent were fatal, and the findings were not affected by lower or higher risk at baseline for gastrointestinal events. The risk was greater in the first 6 months for coxibs (RR 2.55, 99\% CI 1.49 to 4.35), diclofenac (RR 3.93, 99\% CI 2.16 to 7.13), ibuprofen (RR 5.73, 99\% CI 3.24 to 10.14), and naproxen (RR 6.31, 99\% CI 3.81 to 10.44$)$.

Our meta-analyses of study-level data from 13 RCTs are mostly consistent with these findings (Table 9), 101,104,107,126,129,162,172,189,194,229,232,233,241 with the main difference being that our analysis did not find celecoxib to have increased risk. Subgroup analyses did not indicate a difference based on the patient having OA or RA, study quality, or NSAID dose. This evidence is low strength due to high heterogeneity $\left(\mathrm{I}^{2}=73 \%\right.$, Appendix I).

Table 9. Risk of serious gastrointestinal events by NSAID drug versus placebo

\begin{tabular}{|c|c|c|c|c|}
\hline Drug & $\begin{array}{l}\text { Meta-Analysis of Study- } \\
\text { Level Events } \\
\text { Relative Risk }\left(95 \% \mathrm{Cl}, \mathrm{I}^{2}\right)\end{array}$ & $\begin{array}{l}\text { Magnitude } \\
\text { of Effect }\end{array}$ & $\begin{array}{l}\text { Individual Patient Data Meta- } \\
\text { Analysis } \\
\text { Relative Risk (95\% Cl) }\end{array}$ & $\begin{array}{l}\text { Magnitude } \\
\text { of Effect }\end{array}$ \\
\hline Coxibs & $\begin{array}{l}1.04\left(0.67 \text { to } 1.54, I^{2}=0 \%\right) \\
4 \text { RCTs, }{ }^{101,107,126} \mathrm{~N}=4,399\end{array}$ & None & $1.81(1.17$ to 2.81$)$ & Moderate \\
\hline Diclofenac & $\begin{array}{l}3.07\left(1.18 \text { to } 8.86, I^{2}=0 \%\right) \\
2 \text { RCTs, }{ }^{172,194} \mathrm{~N}=723,\end{array}$ & Large & 1.89 (1.16 to 3.09$)$ & Moderate \\
\hline Ibuprofen & $\begin{array}{l}3.60\left(2.27 \text { to } 6.19 .1^{2}=0 \%\right) \\
3 \text { RCTs, }{ }^{229,233,241} \mathrm{~N}=1,486\end{array}$ & Large & 3.97 (2.22 to 7.10$)$ & Large \\
\hline Meloxicam & $\begin{array}{l}1.65\left(0.19 \text { to } 14.04, I^{2}=N A\right) \\
1 \mathrm{RCT}, 167 \mathrm{~N}=713\end{array}$ & Moderate & No data & No data \\
\hline Naproxen & $\begin{array}{l}6.02(2.80 \text { to } 12.91 \\
\left.I^{2}=44 \%\right) \\
6 \text { RCTs, } 104,129,162,189,194,232 \\
N=2,097\end{array}$ & Large & $4.22(2.71$ to 6.56$)$ & Large \\
\hline
\end{tabular}

CI = confidence interval; NSAID = nonsteroidal anti-inflammatory drug; RCT = randomized controlled trial

Comparing the selective COX-2 inhibitor celecoxib with nonselective NSAIDs as a group, evidence is mixed. In the short term, a Cochrane review analyzed four RCTs $(\mathrm{N}=1,755)$ directly 
comparing celecoxib versus any nonselective NSAID in patients with OA with gastrointestinal perforation, obstruction, or bleeding. ${ }^{211}$ Their analysis found no difference between celecoxib and nonselective NSAIDs or placebo (OR $0.61,95 \%$ CI 0.15 to $2.43, \mathrm{I}^{2}=38 \%$ ). The authors rated this evidence as very low quality due to few events, concerns over missing data, and study limitations. Our meta-analysis of four short-term celecoxib versus placebo RCTs $(\mathrm{N}=4,399)$ resulted in a nonsignificant risk of serious gastrointestinal events (7.5\% vs. 6.7\%, RR 1.04, 95\% CI 0.67 to $1.54, \mathrm{I}^{2}=0 \%$ ) compared with the pooled analysis of diclofenac, ibuprofen, naproxen, and meloxicam (9 RCTs, $\mathrm{N}=4,448$ ), which found a large increased risk of serious gastrointestinal events (13\% vs. 3\%, RR 4.29, 95\% CI 2.75 to 6.93 , $\left.\mathrm{I}^{2}=46 \%\right)$. These estimates were significantly different to each other ( $p$-value for interaction $<0.001$ ). Because the evidence is inconsistent and imprecise, it is insufficient to draw conclusions. Finally, one additional fair-quality, intermediate-term RCT randomized 8,067 patients with OA to celecoxib or any nonselective NSAID for 6 months. ${ }^{226}$ Doses could be adjusted, and patients could switch nonselective NSAIDs. Nonselective NSAIDs had a moderately greater risk of clinically important gastrointestinal events than celecoxib (OR 1.82, 95\% CI 1.31 to 2.55). This evidence is low strength.

\section{Hepatic Adverse Events}

A fair-quality systematic review evaluated the hepatic harms of NSAIDs (specifically diclofenac, naproxen, ibuprofen, meloxicam, celecoxib, rofecoxib, and valdecoxib) in RCTs of patients with OA or RA with duration of at least 4-weeks, published through $2004 .^{212}$ This systematic review included 64 RCTs, primarily in patients with OA, and most were 6 months or longer in duration. Diclofenac was found to have a large increased incidence of elevated liver enzymes (aminotransferases more than three times the upper limit of normal) than placebo (3.55\%, 95\% CI 3.12 to 4.03 vs. $0.29 \%$, 95\% CI 0.17 to 0.51). Diclofenac also resulted in a large increase in liver-related discontinuations from treatment (2.17\%, 95\% CI 1.78 to 2.64) than placebo $(0.08 \%$, 95\% CI 0.02 to 0.29$)$. Liver enzyme elevations and liver-related discontinuations with diclofenac were elevated more with greater dose (>100 mg daily) and duration of treatment ( $>13$ weeks). Liver-related SAEs were infrequent, but naproxen resulted the highest incidence $(0.06 \%, 95 \%$ CI 0.02 to 0.15$)$ compared with placebo $(0.00 \%, 95 \%$ CI 0.00 to 0.08). One liver-related hospitalization and one liver-related death occurred, both with naproxen. A more recent systematic review with no criteria for study duration or population, but a composite outcome for hepatic injury, came to similar findings. ${ }^{246}$ This evidence is low strength.

\section{Renal Adverse Events}

No included study meeting inclusion criteria reported events of renal dysfunction or renal failure.

\section{Pruritus}

Three RCTs $(\mathrm{N}=1,129)$ reported on the incidence of pruritus with topical diclofenac, with the increase in risk not reaching statistical significance $(1.22 \%$ vs. $0.18 \%$, RR $3.84,95 \%$ CI 0.82 to $\left.18.09, \mathrm{I}^{2}=0 \%\right){ }^{108,140,141}$ 


\section{Other Drugs}

\section{Key Points}

- In the short or intermediate term, acetaminophen was not found to increase SAEs or WAEs, and no differences were found between doses (SOE: Low). No evidence on hepatic harms was found in studies eligible for this review.

- In the short term, capsaicin 8\% topical patch did not increase risk of SAEs or WAEs compared with an active placebo patch, but longer application duration (60 minutes) led to a moderate increase in SAEs compared with shorter duration (30 minutes). Capsaicin patch resulted in a large increased risk of application site pain and a moderate increased risk of erythema (no impact on pruritus) (SOE: Moderate for placebo comparisons; Low for dose comparisons).

- Cannabis: dronabinol oral solution did not increase SAEs, WAEs, or nausea, but dronabinol resulted in a large increase in dizziness. Oral THC/CBD spray resulted in large increases in WAEs, dizziness, and nausea, but no increase in SAEs or sedation (SOE: Low). Other adverse events of interest were not reported (cognitive effects, misuse, addiction, substance use disorder).

\section{Detailed Assessment}

\section{Acetaminophen}

In patients with chronic pain due to OA, three fair-quality RCTs $(\mathrm{N}=1,235)$ reported on adverse events from acetaminophen compared with placebo-two short-term and one intermediate-term. ${ }^{159-161}$ These trials were industry funded and conducted in the United States, Spain, and Portugal. The weighted mean age of participants was 62 years (range 62 to 64 years), and the weighted mean proportion of female participants was 73 percent (range $67 \%$ to $86 \%$ ). The race of participants was reported in two trials, each of which had a mean proportion of nonwhite participants of 18 percent. ${ }^{159,161}$ The strength of evidence for all outcomes is low.

\section{Serious Adverse Events}

At short-term followup, meta-analysis of two RCTs $(\mathrm{N}=1,023)$ found a higher incidence of SAEs with acetaminophen than placebo, an effect that was not statistically significant (2.4\% vs. 0.9\%, RR 2.57, 95\% CI 0.60 to $\left.10.8, \mathrm{I}^{2}=0 \%\right) .{ }^{159,161}$ One trial $(\mathrm{N}=318)$ found no meaningful difference in SAEs between $1950 \mathrm{mg}$ daily versus $3900 \mathrm{mg}$ daily of acetaminophen (1.9\% vs. 1.9\%, RR 1.01, 95\% CI 0.21 to 4.94). ${ }^{159}$ At intermediate-term followup in a single trial $(\mathrm{N}=212)$, there was no meaningful difference in SAEs between acetaminophen and placebo (4.6\% vs. $4.8 \%$, RR 0.96, $95 \%$ CI 0.29 to 3.23$).{ }^{160}$

\section{Withdrawals Due to Adverse Events}

Acetaminophen did not result in an increase in WAEs compared with placebo in the short or intermediate term. At short-term followup, meta-analysis of two RCTs $(\mathrm{N}=1,023)$ found no meaningful difference in WAEs between acetaminophen and placebo (7.4\% vs. 7.1\%, RR 1.14, 95\% CI 0.67 to $\left.1.95, \mathrm{I}^{2}=0 \%\right) .{ }^{159,161}$ One trial $(\mathrm{N}=318)$ found no meaningful difference in WAEs between $1950 \mathrm{mg}$ and $3900 \mathrm{mg}$ daily of acetaminophen (6.3\% vs. 5.0\%, RR 1.27, 95\% CI 0.51 to 3.12). ${ }^{159}$ At intermediate-term followup in a single trial $(\mathrm{N}=212)$, acetaminophen was 
associated with a slightly greater proportion of WAEs compared with placebo, a difference that was not statistically significant (11.1\% vs. $8.7 \%$, RR $1.28,95 \%$ CI 0.56 to 2.92). ${ }^{160}$

\section{Hepatic Events}

No evidence was found in studies eligible for this review.

\section{Topical Capsaicin}

In patients with chronic neuropathic pain, three short-term RCTs $(\mathrm{N}=1,051)$ reported on adverse events from capsaicin $8 \%$ topical patch compared with active placebo $(0.04 \%$ patch). ${ }^{26,32,53}$ These RCTs were industry funded and conducted in the United States, Canada, the United Kingdom, and Australia; one trial did not report where it was conducted. ${ }^{53}$ One trial was rated as good quality ${ }^{26}$ and two were rated as fair quality. ${ }^{32,53}$ The weighted mean age of participants was 61 years (range 50 to 71 years), the weighted mean proportion of female participants was 34 percent (range 13\% to 54\%), and the weighted mean proportion of nonwhite participants was 20 percent (range $8 \%$ to 30\%). The strength of evidence for all outcomes compared with placebo was moderate; evidence for dose comparisons is low.

\section{Serious Adverse Events}

At short-term followup, meta-analysis of three RCTs $(\mathrm{N}=1,051)$ found a greater proportion of SAEs reported in patients treated with capsaicin patch compared with placebo, an effect that was not statistically significant (5.6\% vs. 3.6\%, RR 1.32 , 95\% CI 0.71 to 3.47$).{ }^{26,32,53}$ One of these RCTs $(\mathrm{N}=332)$ compared two different durations of application of a capsaicin patch -60 minutes versus 30 minutes - and found the 60-minute application to result in a moderately increased risk of SAEs (24\% vs. $11.4 \%$, RR $1.76,95 \%$ CI 1.11 to 2.80$){ }^{32}$

\section{Withdrawals Due to Adverse Events}

At short-term followup, meta-analysis of two RCTs $(\mathrm{N}=896)$ found no difference in WAEs between capsaicin patch and placebo (0.4\% vs. $0.3 \%$, RR $1.04,95 \%$ CI 0.08 to 17.1$).{ }^{26,32}$ One of these RCTs $(\mathrm{N}=332)$ compared two different durations of application of a capsaicin patch - 60 minutes versus 30 minutes - and found no significant difference in WAEs $(0.6 \%$ vs. $0.0 \%$, RR 3.04, $95 \%$ CI 0.13 to 74.00$).^{32}$

\section{Specific Adverse Events}

Based on meta-analysis of three short-term RCTs, capsaicin patch resulted in a moderate increased risk of erythema (58\% vs. $45 \%$, RR 1.46, 95\% CI 1.29 to $1.66, \mathrm{I}^{2}=0 \%$ ). There was a large increase in pain at the application site with capsaicin (61\% vs. 26\%, RR 2.26, 95\% CI 1.81 to $\left.2.82, \mathrm{I}^{2}=0 \%\right)$. There was not a difference between groups in pruritus (6.1\% vs. $3.4 \%$, RR 1.70 , 95\% CI 0.92 to $\left.3.35, \mathrm{I}^{2}=0 \%\right){ }^{26,32,53}$

\section{Cannabis}

Cannabis (including derivatives and synthetic cannabinoids) was compared with placebo in two short-term trials ( $\mathrm{N}=486){ }^{28,47}$ The trials utilized oral dronabinol solution (mean $13 \mathrm{mg}$ daily) and THC/CBD oromucosal spray (100 mL per spray, up to 24 sprays daily). One trial was rated good quality ${ }^{28}$ and the other fair quality. ${ }^{47} \mathrm{~A}$ third trial was rated poor quality due to unclear randomization and allocation concealment, between-group differences at baseline, and high rates of attrition; results from that trial are not included here. ${ }^{62}$ The adverse event profiles for the two different formulations varied and are reported separately. 
In a good-quality study $(\mathrm{N}=240)$ there was no difference between dronabinol oral solution and placebo in the incidence of SAEs, WAEs, or nausea, but dronabinol had a large effect on the incidence of dizziness (20\% vs. $4.3 \%$, calculated RR 4.68, 95\% CI 1.85 to 11.8 ). ${ }^{28}$ In a fairquality study ( $\mathrm{N}=246)$, there was no difference between an oral spray with THC/CBD compared with placebo in SAEs or the incidence of sedation, but there were large differences in the incidence of WAEs (19\% vs. 6\%, calculated RR 3.16, 95\% CI 1.41 to 7.06), dizziness (39\% vs. 9\%, calculated RR 4.55, 95\% CI 2.48 to 8.32 ) and nausea (17\% vs. 8\%, calculated RR 2.25, 95\% CI 1.8 to 4.70). ${ }^{47}$ The strength of this evidence is low. Other adverse events of interest were not reported (cognitive effects, misuse, addiction, substance use disorder).

\section{Topical Lidocaine}

A single short-term study of lidocaine 5\% patch compared with celecoxib in patients with knee OA ( $\mathrm{N}=143$ ) was poor quality (unclear allocation concealment, no blinding, high attrition; $46 \%$ ), and stopped early due to the withdrawal of celecoxib from the market at that time. ${ }^{114}$ This evidence is insufficient to draw conclusions.

\section{Skeletal Muscle Relaxants}

A fair-quality, intermediate-term (6-month) RCT of fibromyalgia patients ( $\mathrm{N}=208)$ compared amitriptyline, the skeletal muscle relaxant cyclobenzaprine, and placebo. ${ }^{78}$ Thirteen of 82 patients (16\%) assigned to cyclobenzaprine withdrew from study due to adverse events, compared with 2 of 42 patients (5\%) taking placebo. Serious adverse events were not reported. Somnolence was the reason for discontinuing in three patients (3.7\%) with cyclobenzaprine, versus one patient (2.4\%) with placebo. Dizziness was reported in five (6.1\%) and one patient (2.4\%), respectively. Additional patients withdrew due to abdominal pain (3 patients, 3.7\%), rash, and headache (1 patient each, 1.2\%) with cyclobenzaprine. Due to study limitations, unknown consistency and limited events (imprecision), this evidence was insufficient to draw conclusions regarding adverse event outcomes.

\section{Memantine}

Two small RCTs included memantine, an NMDA receptor antagonist approved for Alzheimer's dementia, compared with placebo. ${ }^{46,90}$ A short-term fair-quality RCT $(\mathrm{N}=45)$ in patients with HIV-related neuropathy did not report adverse events in a specific way, noting only that there were no differences seen. ${ }^{46}$ A good-quality, intermediate-term (6-month) RCT (N=63) in patients with fibromyalgia also poorly reported adverse events. Two of 31 patients assigned to memantine (6\%) compared with 1 of 32 (3\%) withdrew from the study due to adverse events, and it was reported that there were no serious adverse events. Dizziness occurred in eight patients on memantine (25.8\%) versus four patients on placebo (12.5\%). Sedation (drowsiness) was reported in no patients taking memantine, and two taking placebo (6\%). None of these findings were statistically significantly different. This evidence was insufficient to draw conclusions as the studies were small (very imprecise findings) with unknown consistency or publication bias. 


\section{Discussion}

\section{Key Findings and Strength of Evidence}

The key findings of this review are summarized in Tables 10 to 17 and in Appendix H. (See Table 2 for definitions of effect sizes and Table 3 for descriptions of strength of evidence grades.) This review evaluates and synthesizes the evidence on benefits and harms of nonopioid drugs in patients with chronic noncancer pain. The pain conditions included were neuropathic pain, fibromyalgia, osteoarthritis, inflammatory arthritis, low back pain, chronic headache, and sickle cell disease. Drugs reviewed included antidepressants (serotonin-norepinephrine reuptake inhibitors [SNRIs] and tricyclic antidepressants [TCAs]), anticonvulsants (pregabalin, gabapentin, oxcarbazepine, and carbamazepine), nonsteroidal anti-inflammatory drugs (NSAIDs), and other drugs such as acetaminophen, capsaicin, and cannabis. The review included randomized controlled trials (RCTs) of at least 3 months duration, and categorizes findings according to duration of study, magnitude of the findings, and the strength of the evidence for each finding. Interventions or comparisons for which all evidence was insufficient to draw conclusions are not included in the tables below, but details can be found in the report results (above).

In patients with neuropathic pain, in the short term, the anticonvulsant drugs gabapentin, pregabalin, and oxcarbazepine provided small improvement in pain outcomes in patients with diabetic peripheral neuropathy/postherpetic neuralgia, but not function in postherpetic neuralgia or quality of life in HIV- or diabetes-associated neuropathy. In patients with diabetic peripheral neuropathy, duloxetine resulted in small improvements in pain, function, quality of life. Capsaicin patch did not have improvements in pain severity or response that were both significant and reached the level of a small effect in postherpetic neuralgia and HIV-related neuralgia. Cannabis (dronabinol oral solution, tetrahydrocannabinol/cannabidiol [THC/CBD] oral spray) had no effect on pain severity in multiple sclerosis-associated neuropathy or allodynia, but THC/CBD oral spray improved pain response to a moderate degree in patients with allodynia. Differences in pain improvement was not seen between drugs.

In patients with fibromyalgia, in the short and intermediate term, antidepressants resulted in small improvements in pain and mixed findings on quality of life. Function improved to a small degree in the short term, but not in the intermediate term. Short-term treatment with anticonvulsants (pregabalin and gabapentin) is associated with small improvements in pain and function, but not quality of life. Subgroup analyses showed no effect of specific drug, dose, or study quality on these results. Intermediate-term treatment with memantine resulted in moderate improvements in pain, function, and quality of life compared with placebo.

Oral NSAIDs improved pain and function in patients with osteoarthritis (OA) to a small degree in the short term, with evidence indicating these effects are maintained in the intermediate term for celecoxib. Subgroup analyses indicated that studies of only patients with knee pain and those of good quality had smaller effects, while patients with more severe pain at baseline experienced greater reduction in pain. Direct comparisons of NSAIDs with each other found few differences between drugs in pain or function in OA patients in the short, intermediate, or long term. Small improvements were seen in pain severity and response with topical diclofenac, but not function. The SNRI antidepressant duloxetine resulted in moderate effects on pain response, and small effects on pain severity, function, and quality of life. Subgroup analyses found that pain improvement was greater in older patients ( $>65$ years) and patients with knee osteoarthritis. Acetaminophen did not improve pain significantly in the short or intermediate term. In patients 
with rheumatoid arthritis or ankylosing spondylitis, short-term treatment with oral NSAIDs resulted in small improvements in pain severity, pain response, and function, but evidence on quality of life was inconsistent. Evidence on intermediate- and long-term outcomes was limited to one trial each, with improvements in pain but not function. Comparisons of different doses or between different NSAIDs did not find important differences. Subgroup analyses of specific drug, dose, year of publication, type of inflammatory arthritis, and study quality did not alter the findings meaningfully. The TCA amitriptyline did not improve pain outcomes. Evidence in patients with chronic headache or sickle cell disease was too limited to draw conclusions.

Serious adverse events were not reported more often with nonopioid drugs than placebo in patients with chronic pain, with the exception of oxcarbazepine and with longer duration capsaicin patch (compared with shorter duration). Withdrawal due to adverse events was increased significantly with anticonvulsants, antidepressants, NSAIDs, and cannabis oral spray, ranging from a small increase to large increases. SNRI antidepressants resulted in increased reports of nausea (dose did not alter these findings). Duloxetine also resulted in increased sedation, but lower doses did reduce the risk. Amitriptyline led to a moderate increase in reports of dry mouth, but other adverse events of interest were not reported or not different to placebo. There were no reports of serotonin syndrome in any included RCT of antidepressants. In the short term, pregabalin and gabapentin resulted in moderate to large increases in blurred vision, dizziness, weight gain, and cognitive effects (e.g., confusion). As a prodrug of gabapentin, gabapentin enacarbil may have lower risk of blurred vision, weight gain, or cognitive effects. Additionally, pregabalin resulted in large increases in risk of peripheral edema and sedation. In the short term, the risk of any cardiovascular event was not significantly elevated for NSAIDs as a group, although there was a small increase in risk with diclofenac, particularly within the first 6 months, and with higher doses; risk was increased to a similar degree with ibuprofen and celecoxib but did not reach statistical significance. Although the absolute risk is low, there was a moderate relative increased risk of major coronary events with diclofenac and celecoxib, and a large increase with ibuprofen. In the intermediate term, there was not a difference in cardiovascular events between drugs. NSAIDs led to moderate to large increased risk of serious upper gastrointestinal events (largely bleeding), particularly in the first 6 months of treatment. In the intermediate term, although the incidence was low, large increases in hepatic harms were seen with diclofenac and naproxen. Dronabinol oral solution resulted in a large increase in dizziness and THC/CBD oral spray resulted in large increases in dizziness and nausea. Other adverse events of interest were not reported (cognitive effects, misuse, addiction, substance use disorder). 
Table 10. Key Question 1 - Effectiveness and comparative effectiveness of nonopioid drugs for chronic pain: effects of antidepressants in placebo-controlled and head-to-head trials

\begin{tabular}{|c|c|c|c|c|c|c|c|}
\hline Condition & Drug & $\begin{array}{l}\text { Pain } \\
\text { Short Term } \\
\text { Effect Size } \\
\text { SOE }\end{array}$ & $\begin{array}{l}\text { Pain } \\
\text { Intermediate Term } \\
\text { Effect Size } \\
\text { SOE }\end{array}$ & $\begin{array}{l}\text { Function } \\
\text { Short Term } \\
\text { Effect Size } \\
\text { SOE }\end{array}$ & $\begin{array}{l}\text { Function } \\
\text { Intermediate Term } \\
\text { Effect Size } \\
\text { SOE }\end{array}$ & $\begin{array}{l}\text { QoL } \\
\text { Short Term } \\
\text { Effect Size } \\
\text { SOE }\end{array}$ & $\begin{array}{l}\text { QoL } \\
\text { Intermediate Term } \\
\text { Effect Size } \\
\text { SOE }\end{array}$ \\
\hline Neuropathic pain & Duloxetine vs. placebo & $\begin{array}{c}\text { Small } \\
++\end{array}$ & No evidence & $\underset{+}{\text { Small }}$ & No evidence & $\underset{++}{\text { Small }}$ & No evidence \\
\hline Fibromyalgia & $\begin{array}{l}\text { Duloxetine/milnacipran vs. } \\
\text { placebo }\end{array}$ & $\begin{array}{c}\text { Small } \\
++\end{array}$ & $\begin{array}{c}\text { Small } \\
++\end{array}$ & $\begin{array}{c}\text { Small } \\
++\end{array}$ & $\begin{array}{c}\text { None } \\
++\end{array}$ & $\begin{array}{c}\text { MCS: Small } \\
++ \\
\text { PCS: None } \\
++ \\
\end{array}$ & $\begin{array}{c}\text { Small } \\
++\end{array}$ \\
\hline Osteoarthritis & Duloxetine vs. placebo & $\begin{array}{c}\text { Small } \\
+++\end{array}$ & No evidence & $\begin{array}{c}\text { Small } \\
+++\end{array}$ & No evidence & $\begin{array}{c}\text { Small } \\
+++\end{array}$ & No evidence \\
\hline \multirow{3}{*}{ Low back pain } & Duloxetine vs. placebo & $\begin{array}{c}\text { Small } \\
++\end{array}$ & No evidence & $\begin{array}{c}\text { None } \\
++\end{array}$ & No evidence & $\begin{array}{c}\text { None } \\
++\end{array}$ & No evidence \\
\hline & Amitriptyline vs. placebo & No evidence & $\begin{array}{c}\text { None } \\
+\end{array}$ & No evidence & $\begin{array}{c}\text { None } \\
+\end{array}$ & $\begin{array}{c}\text { No } \\
\text { evidence }\end{array}$ & No evidence \\
\hline & Amitriptyline vs. pregabalin & $\begin{array}{c}\text { Small } \\
+\end{array}$ & No evidence & $\begin{array}{c}\text { None } \\
+\end{array}$ & No evidence & $\begin{array}{c}\text { No } \\
\text { evidence }\end{array}$ & No evidence \\
\hline
\end{tabular}

QoL = quality of life; SOE = strength of evidence

Effect size: none (i.e., no effect/no statistically significant effect), small, moderate, or large increased risk

SOE: $+=$ low, $++=$ moderate, $+++=$ high 
Table 11. Key Question 2 - Harms and adverse events of nonopioid drugs for chronic pain: harms of antidepressants versus placebo

\begin{tabular}{|c|c|c|c|c|}
\hline Types of Adverse Events & $\begin{array}{l}\text { SNRIs (Duloxetine/Milnacipran) } \\
\text { Short Term } \\
\text { Effect Size } \\
\text { SOE }\end{array}$ & $\begin{array}{l}\text { SNRIs (Duloxetine/Milnacipran) } \\
\text { Intermediate Term } \\
\text { Effect Size } \\
\text { SOE }\end{array}$ & $\begin{array}{l}\text { TCAs } \\
\text { Short Term } \\
\text { Effect Size } \\
\text { SOE }\end{array}$ & $\begin{array}{l}\text { TCAs } \\
\text { Intermediate Term } \\
\text { Effect Size } \\
\text { SOE }\end{array}$ \\
\hline WAE & $\begin{array}{c}\text { Moderate } \\
++\end{array}$ & $\begin{array}{c}\text { Moderate } \\
++\end{array}$ & $\begin{array}{c}\text { None } \\
+\end{array}$ & Insufficient \\
\hline SAE & $\begin{array}{c}\text { None } \\
+\end{array}$ & $\begin{array}{c}\text { None } \\
+\end{array}$ & No evidence & No evidence \\
\hline Cognitive effects & $\begin{array}{c}\text { None } \\
+\end{array}$ & No evidence & No evidence & No evidence \\
\hline Nausea & $\begin{array}{c}\text { Large } \\
++\end{array}$ & $\begin{array}{c}\text { Moderate } \\
+\end{array}$ & NA & NA \\
\hline Sedation & $\begin{array}{c}\text { Large } \\
++\end{array}$ & $\begin{array}{c}\text { Large } \\
+\end{array}$ & NA & NA \\
\hline Serotonin syndrome & No evidence & No evidence & No evidence & No evidence \\
\hline Dry mouth & NA & NA & Insufficient & No evidence \\
\hline Cardiac rhythm abnormalities & NA & NA & No evidence & No evidence \\
\hline Urinary retention & NA & NA & No evidence & No evidence \\
\hline
\end{tabular}

NA = not applicable (i.e., specific adverse event not applicable to drug); SAE = serious adverse event; SOE = strength of evidence; SNRI = serotonin-norepinephrine reuptake inhibitor; TCA = tricyclic antidepressant; WAE = withdrawal due to adverse event

Effect size: none (i.e., no effect/no statistically significant effect), small, moderate, or large increased risk

SOE: $+=$ low,$++=$ moderate, $+++=$ high

Table 12. Key Question 1 - Effectiveness and comparative effectiveness of nonopioid drugs for chronic pain: effects of anticonvulsants in placebo-controlled and head-to-head trials

\begin{tabular}{|c|c|c|c|c|}
\hline Condition & Drug & $\begin{array}{l}\text { Pain } \\
\text { Short Term } \\
\text { Effect Size } \\
\text { SOE }\end{array}$ & $\begin{array}{l}\text { Function } \\
\text { Short Term } \\
\text { Effect Size } \\
\text { SOE }\end{array}$ & $\begin{array}{l}\text { QoL } \\
\text { Short Term } \\
\text { Effect Size } \\
\text { SOE }\end{array}$ \\
\hline \multirow{4}{*}{ Neuropathic pain } & Pregabalin / Gabapentin vs. Placebo & $\begin{array}{c}\text { Small } \\
++\end{array}$ & $\begin{array}{c}\text { None } \\
+\end{array}$ & $\begin{array}{c}\text { None } \\
+\end{array}$ \\
\hline & Oxcarbazepine vs. Placebo & $\begin{array}{c}\text { Small } \\
++\end{array}$ & No evidence & $\begin{array}{c}\text { None } \\
+ \\
\end{array}$ \\
\hline & Pregabalin vs. Gabapentin & Insufficient & No evidence & No evidence \\
\hline & Pregabalin vs. Gabapentin Enacarbila & $\begin{array}{c}\text { None } \\
+\end{array}$ & $\begin{array}{c}\text { None } \\
+\end{array}$ & $\begin{array}{c}\text { None } \\
+\end{array}$ \\
\hline Fibromyalgia & Pregabalin / Gabapentin vs. Placebo & $\begin{array}{c}\text { Small } \\
++\end{array}$ & $\begin{array}{c}\text { Small } \\
++\end{array}$ & $\begin{array}{c}\text { None } \\
++\end{array}$ \\
\hline
\end{tabular}

QoL = quality of life; SOE = strength of evidence

Effect size: none (i.e., no effect/no statistically significant effect), small, moderate, or large. SOE: + = low, $++=$ moderate, $+++=$ high

${ }^{a}$ Gabapentin enacarbil is a prodrug of gabapentin. 
Table 13. Key Question 2 - Harms and adverse events of nonopioid drugs for chronic pain: harms of anticonvulsants versus placebo and active comparator

\begin{tabular}{|c|c|c|}
\hline Types of Adverse Events & $\begin{array}{l}\text { Pregabalin/Gabapentin } \\
\text { Short Term } \\
\text { Effect Size } \\
\text { SOE }\end{array}$ & $\begin{array}{l}\text { Oxcarbazepine } \\
\text { Short Term } \\
\text { Effect Size } \\
\text { SOE }\end{array}$ \\
\hline WAE & $\begin{array}{cc}\text { Moderate } \\
++\end{array}$ & $\begin{array}{c}\text { Large } \\
+ \\
\end{array}$ \\
\hline SAE & $\begin{array}{c}\text { None } \\
+\end{array}$ & $\begin{array}{c}\text { None } \\
+ \\
\end{array}$ \\
\hline Blurred vision & $\begin{array}{l}\text { Large } \\
+\end{array}$ & NA \\
\hline Cognitive effects & $\begin{array}{l}\text { Large } \\
+ \\
\end{array}$ & No evidence \\
\hline Dizziness & $\begin{array}{c}\text { Large } \\
++\end{array}$ & NA \\
\hline Peripheral edema & $\begin{array}{c}\text { Large } \\
++\end{array}$ & NA \\
\hline Sedation & $\begin{array}{c}\text { Large } \\
++\end{array}$ & $\begin{array}{c}\text { None } \\
+ \\
\end{array}$ \\
\hline Weight gain & $\begin{array}{c}\text { Large } \\
++\end{array}$ & NA \\
\hline Hyponatremia & NA & $\begin{array}{c}\text { None } \\
+\end{array}$ \\
\hline
\end{tabular}

NA = not applicable (i.e., specific adverse event not applicable to drug); SAE = serious adverse event; SOE = strength of evidence; WAE = withdrawal due to adverse event Effect size: none (i.e., no effect/no statistically significant effect), small, moderate, or large increased risk

SOE: + = low, ++ = moderate, $+++=$ high

${ }^{\text {a }}$ Gabapentin enacarbil is a prodrug of gabapentin. 
Table 14. Key Question 1 - Effectiveness and comparative effectiveness of nonopioid drugs for chronic pain: effects of NSAIDs in placebo-controlled and head-to-head trials

\begin{tabular}{|c|c|c|c|c|c|c|c|c|}
\hline Condition & Drug & $\begin{array}{l}\text { Pain } \\
\text { Short Term } \\
\text { Effect Size } \\
\text { SOE }\end{array}$ & $\begin{array}{l}\text { Pain } \\
\text { Intermediate Term } \\
\text { Effect Size } \\
\text { SOE }\end{array}$ & $\begin{array}{l}\text { Pain } \\
\text { Long Term } \\
\text { Effect Size } \\
\text { SOE }\end{array}$ & $\begin{array}{l}\text { Function } \\
\text { Short Term } \\
\text { Effect Size } \\
\text { SOE }\end{array}$ & $\begin{array}{l}\text { Function } \\
\text { Intermediate Term } \\
\text { Effect Size } \\
\text { SOE }\end{array}$ & $\begin{array}{l}\text { Function } \\
\text { Long Term } \\
\text { Effect Size } \\
\text { SOE }\end{array}$ & $\begin{array}{l}\text { QoL } \\
\text { Short Term } \\
\text { Effect Size } \\
\text { SOE }\end{array}$ \\
\hline \multirow{4}{*}{ Osteoarthritis } & NSAID vs. placebo & $\begin{array}{c}\text { Small } \\
++\end{array}$ & No evidence & No evidence & $\begin{array}{c}\text { Small } \\
+++\end{array}$ & No evidence & No evidence & $\begin{array}{c}\text { None } \\
++\end{array}$ \\
\hline & Diclofenac vs. celecoxib & $\begin{array}{c}\text { Moderate } \\
+\end{array}$ & No evidence & No evidence & $\begin{array}{c}\text { Moderate } \\
+\end{array}$ & No evidence & No evidence & No evidence \\
\hline & NSAID vs. NSAID & $\begin{array}{c}\text { None } \\
+\end{array}$ & $\begin{array}{c}\text { None } \\
+\end{array}$ & $\begin{array}{c}\text { None } \\
+\end{array}$ & $\begin{array}{c}\text { None } \\
+\end{array}$ & $\begin{array}{c}\text { None } \\
+\end{array}$ & No evidence & No evidence \\
\hline & $\begin{array}{l}\text { Topical diclofenac vs. } \\
\text { placebo }\end{array}$ & $\begin{array}{c}\text { Small } \\
++\end{array}$ & No evidence & No evidence & $\begin{array}{c}\text { None } \\
+\end{array}$ & No evidence & No evidence & No evidence \\
\hline \multirow{6}{*}{$\begin{array}{l}\text { Inflammatory } \\
\text { arthritis }\end{array}$} & NSAID vs. placebo & $\begin{array}{c}\text { Small/ } \\
\text { Moderate } \\
++ \\
\end{array}$ & $\begin{array}{l}\text { Small } \\
+\end{array}$ & $\begin{array}{l}\text { Large } \\
\quad+\end{array}$ & $\begin{array}{c}\text { Small } \\
++\end{array}$ & $\begin{array}{c}\text { Small } \\
+\end{array}$ & $\begin{array}{c}\text { None } \\
+\end{array}$ & Insufficient \\
\hline & Celecoxib vs. diclofenac & $\begin{array}{c}\text { None } \\
++\end{array}$ & No evidence & No evidence & $\begin{array}{c}\text { None } \\
++\end{array}$ & No evidence & No evidence & No evidence \\
\hline & Celecoxib vs. naproxen & $\begin{array}{c}\text { None } \\
+\end{array}$ & No evidence & No evidence & $\begin{array}{c}\text { None } \\
+\end{array}$ & No evidence & No evidence & $\begin{array}{c}\text { None } \\
+\end{array}$ \\
\hline & Diclofenac vs. meloxicam & $\begin{array}{c}\text { None } \\
+\end{array}$ & No evidence & No evidence & $\begin{array}{c}\text { None } \\
+\end{array}$ & No evidence & No evidence & No evidence \\
\hline & Meloxicam vs. naproxen & No evidence & $\begin{array}{c}\text { None } \\
+\end{array}$ & No evidence & No evidence & No evidence & No evidence & No evidence \\
\hline & Nabumetone vs. naproxen & $\begin{array}{c}\text { None } \\
+\end{array}$ & $\begin{array}{c}\text { None } \\
+\end{array}$ & No evidence & $\begin{array}{c}\text { None } \\
+\end{array}$ & No evidence & No evidence & No evidence \\
\hline
\end{tabular}

NSAID = nonsteroidal anti-inflammatory drug; QoL = quality of life; SOE = strength of evidence

Effect size: none (i.e., no effect/no statistically significant effect), small, moderate, or large increased risk

SOE: $+=$ low, $++=$ moderate, $+++=$ high 
Table 15. Key Question 2 - Harms and adverse events of nonopioid drugs for chronic pain: harms of NSAIDs versus placebo and active comparators

\begin{tabular}{|c|c|c|c|c|c|c|}
\hline $\begin{array}{l}\text { Types of Adverse } \\
\text { Events }\end{array}$ & $\begin{array}{l}\text { NSAID } \\
\text { Short Term } \\
\text { Effect Size } \\
\text { SOE }\end{array}$ & $\begin{array}{l}\text { NSAID } \\
\text { Intermediate Term } \\
\text { Effect Size } \\
\text { SOE }\end{array}$ & $\begin{array}{l}\text { NSAID } \\
\text { Long Term } \\
\text { Effect Size } \\
\text { SOE }\end{array}$ & $\begin{array}{l}\text { Topical Diclofenac } \\
\text { vs. Placebo } \\
\text { Short Term } \\
\text { Effect Size } \\
\text { SOE }\end{array}$ & $\begin{array}{l}\text { nsNSAID vs. } \\
\text { Celecoxib } \\
\text { Intermediate Term } \\
\text { Effect Size } \\
\text { SOE }\end{array}$ & $\begin{array}{l}\text { nsNSAID vs. } \\
\text { Celecoxib } \\
\text { Long Term } \\
\text { Effect Size } \\
\text { SOE }\end{array}$ \\
\hline WAE & $\begin{array}{c}\text { Small } \\
++\end{array}$ & $\begin{array}{c}\text { None } \\
+\end{array}$ & Insufficient & $\begin{array}{c}\text { None } \\
+\end{array}$ & No evidence & No evidence \\
\hline SAE & $\begin{array}{c}\text { None } \\
+\end{array}$ & Insufficient & No evidence & $\begin{array}{c}\text { None } \\
+\end{array}$ & No evidence & No evidence \\
\hline Cardiovascular events & $\underset{++}{\text { Small }}$ & No evidence & No evidence & No evidence & $\begin{array}{c}\text { None } \\
++\end{array}$ & $\begin{array}{c}\text { None } \\
++\end{array}$ \\
\hline Gastrointestinal events & $\begin{array}{c}\text { Moderate } \\
+/++\end{array}$ & No evidence & No evidence & No evidence & $\begin{array}{c}\text { Moderate } \\
+ \\
\end{array}$ & No evidence \\
\hline Liver dysfunction & $\begin{array}{c}\text { Large } \\
+\end{array}$ & No evidence & No evidence & No evidence & No evidence & No evidence \\
\hline
\end{tabular}

NSAID = nonsteroidal anti-inflammatory drug; nsNSAID = nonselective nonsteroidal anti-inflammatory drug; SAE = serious adverse event; SOE = strength of evidence; WAE = withdrawal due to adverse event

Effect size: none (i.e., no effect/no statistically significant effect), small, moderate, or large increased risk

SOE: $+=$ low,$++=$ moderate, $+++=$ high

Table 16. Key Question 1 - Effectiveness and comparative effectiveness of nonopioid drugs for chronic pain: effects of other drugs in placebo-controlled trials

\begin{tabular}{|c|c|c|c|c|c|c|c|}
\hline Condition & Drug & $\begin{array}{l}\text { Pain } \\
\text { Short Term } \\
\text { Effect Size } \\
\text { SOE }\end{array}$ & $\begin{array}{l}\text { Pain } \\
\text { Intermediate Term } \\
\text { Effect Size } \\
\text { SOE }\end{array}$ & $\begin{array}{l}\text { Function } \\
\text { Short Term } \\
\text { Effect Size } \\
\text { SOE }\end{array}$ & $\begin{array}{l}\text { Function } \\
\text { Intermediate Term } \\
\text { Effect Size } \\
\text { SOE }\end{array}$ & $\begin{array}{l}\text { QoL } \\
\text { Short Term } \\
\text { Effect Size } \\
\text { SOE }\end{array}$ & $\begin{array}{l}\text { QoL } \\
\text { Intermediate Term } \\
\text { Effect Size } \\
\text { SOE }\end{array}$ \\
\hline \multirow{2}{*}{ Neuropathic pain } & Capsaicin patch & $\begin{array}{c}\text { None } \\
++\end{array}$ & No evidence & No evidence & No evidence & No evidence & No evidence \\
\hline & Cannabis & $\begin{array}{c}\text { None } \\
+\end{array}$ & No evidence & $\begin{array}{c}\text { None } \\
+\end{array}$ & No evidence & $\begin{array}{c}\text { None } \\
+\end{array}$ & No evidence \\
\hline \multirow{2}{*}{ Fibromyalgia } & Memantine & No evidence & $\begin{array}{c}\text { Moderate } \\
+\end{array}$ & No evidence & $\begin{array}{c}\text { Moderate } \\
+\end{array}$ & No evidence & $\begin{array}{c}\text { Moderate } \\
+\end{array}$ \\
\hline & Cyclobenzaprine & No evidence & $\begin{array}{c}\text { None } \\
+\end{array}$ & No evidence & Insufficient & No evidence & No evidence \\
\hline Osteoarthritis & Acetaminophen & $\begin{array}{c}\text { None } \\
+\end{array}$ & $\begin{array}{c}\text { None } \\
+\end{array}$ & $\begin{array}{c}\text { None } \\
+\end{array}$ & $\begin{array}{c}\text { None } \\
+\end{array}$ & No evidence & No evidence \\
\hline
\end{tabular}

QoL = quality of life; SOE = strength of evidence

Effect size: none (i.e., no effect/no statistically significant effect), small, moderate, or large increased risk

SOE $:+=$ low,$++=$ moderate, $+++=$ high 
Table 17. Key Question 2 - Harms and adverse events of nonopioid drugs for chronic pain: harms of other drugs versus placebo

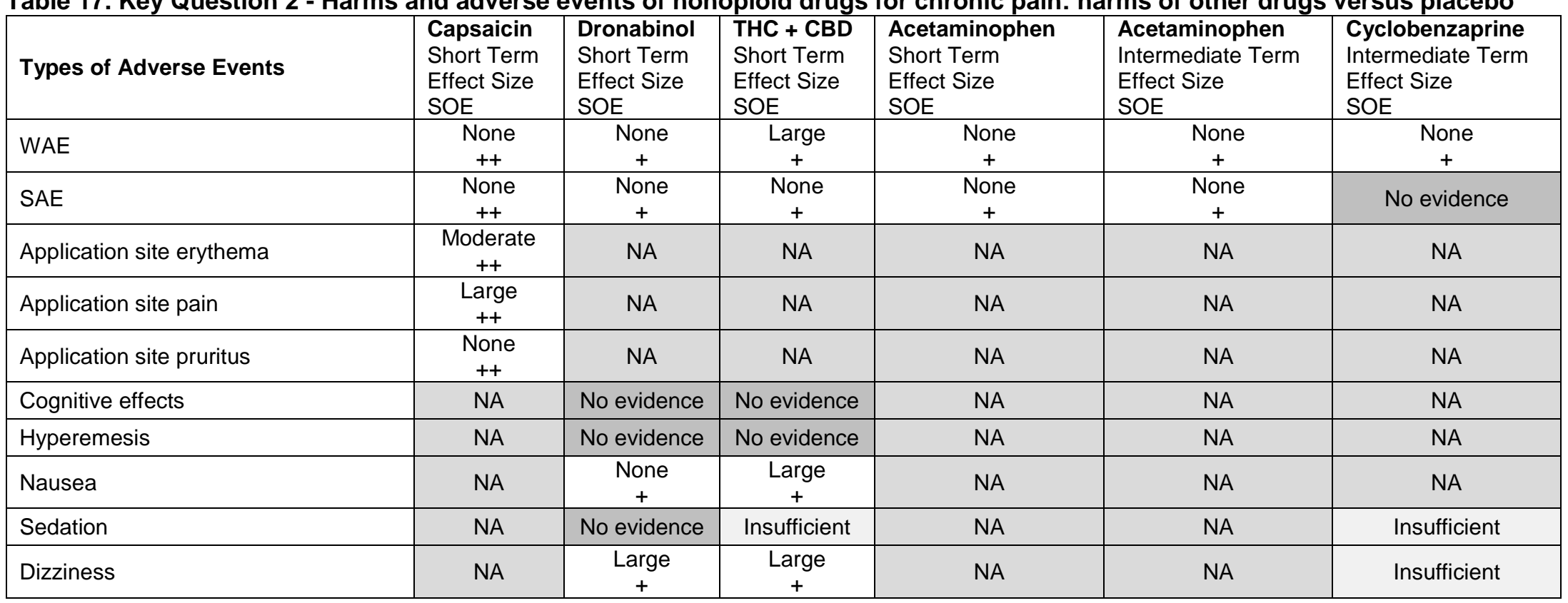

CBD = cannabidiol; SAE = serious adverse event; SOE = strength of evidence; THC = tetrahydrocannabinol; WAE = withdrawal due to adverse event

Effect size: none (i.e., no effect/no statistically significant effect), small, moderate, or large increased risk

SOE $:+=$ low,$++=$ moderate, $+++=$ high 


\section{Findings in Relationship to What Is Already Known}

This systematic review combines evidence across multiple pain conditions and multiple drug classes in a way that prior reviews have not. Prior reviews generally had dissimilar scope (e.g., limited to a single condition and/or drug class, included drugs or populations not included here), included very short duration studies ( $<12$ weeks), did not classify results according to treatment duration, and did not categorize effect sizes (small, moderate, large). Although our review includes more recent studies, other reviews of individual drugs, drug classes, or pain conditions have reviewed some of the evidence included here, and where comparisons of our results and prior findings are possible, they are generally consistent. For example, a 2015 systematic review with network meta-analysis of acetaminophen, NSAIDs, and injectable drugs for knee OA found a standardized mean difference (SMD) for acetaminophen of 0.18 , and we found the mean difference (MD, 0-10 scale) was 0.34. Both are less than a small magnitude of effect according to our system, and the prior review noted that the effect did not reach clinical significance in their system. ${ }^{247}$ Findings for NSAIDs were similar to ours, and our subgroup analysis of only knee OA was also in a similar range of magnitude of effect to their findings. The exception was that they found a moderate-size effect with diclofenac, while our subgroup analysis of specific drug was not significant. For neuropathic pain, a 2017 systematic review of only diabetic peripheral neuropathy found duloxetine to have large effect (SMD -1.33), but when we added another study the magnitude was reduced to small (MD -0.79, 0-10 scale). ${ }^{248}$ This review and ours had similar findings for pregabalin (small effect). Both reviews found that the effect of gabapentin was not significant, but the effect was moderate in the older review, while our effect was small after incorporating additional studies. In fibromyalgia, a 2016 systematic review with a network meta-analysis found a large magnitude of effect in pain response with SNRI antidepressants (odds ratio [OR] 1.61 to 2.33) while we found a moderate effect (relative risk [RR] 1.29 to 1.36), and the prior review found a moderate effect with pregabalin (OR 1.68) while we found a small effect with pregabalin and gabapentin combined (RR 1.41). ${ }^{249}$ Differences in magnitude could be due to the addition of 15 studies in our report, reporting relative risks rather than odds ratios, and using direct comparisons rather than network analysis. Our findings regarding the effects of nonopioid drugs on pain and function are also consistent with two related systematic reviews on opioids and nonpharmacologic treatments for chronic pain, which found similar small effects. ${ }^{250,251}$

In terms of evidence on the harms of the drugs included, because many of the drugs have been available for decades (e.g., acetaminophen), were initially approved for other indications (e.g., antidepressants and anticonvulsants), or primarily studied in acute pain and short-term treatment (e.g., acetaminophen, topical lidocaine), our findings on adverse events are not comprehensive relative to other, nonsystematic review sources (e.g., product labels, large observational studies, U.S. Food and Drug Administration [FDA] warnings, drug information texts). However, as Table 18 indicates, our analyses on adverse events are consistent with these other sources.

Table 18 provides a summary of the evidence on adverse events of interest that were identified in RCTs of patients with chronic pain meeting inclusion criteria for this review. Because the scope of this review focused on a specific patient population (chronic pain with specific conditions), a specific study design (RCTs), and study duration (12 weeks or more), it is unlikely that all important evidence on harms of these drugs would be identified. Where included evidence did not adequately address the prioritized harms, information from other sources is summarized. The evidence from other sources may have unclear applicability to patients with 
chronic pain, who may use these drugs for longer periods of time, possibly at higher doses, and who may be older (in some cases) or have more comorbidities than patients providing data for these sources. 
Table 18. Summary of specific adverse events

\begin{tabular}{|c|c|c|c|c|}
\hline Drug Class & Drug & Outcomes of Interest & $\begin{array}{l}\text { Adverse Event Findings From RCTs in } \\
\text { Chronic Pain (Magnitude of Effect) }\end{array}$ & $\begin{array}{l}\text { Adverse Event Findings From Other } \\
\text { Sources (To Address Missing Evidence) }\end{array}$ \\
\hline \multirow[t]{2}{*}{ Antidepressants } & SNRIS & $\begin{array}{l}\text { Nausea, sedation, } \\
\text { serotonin syndrome }\end{array}$ & $\begin{array}{l}\text { Nausea (moderate-to-large, no dose effect), } \\
\text { sedation (duloxetine, dose-related), } \\
\text { serotonin syndrome symptoms (large) }\end{array}$ & No missing outcomes \\
\hline & TCAs & $\begin{array}{l}\text { Cardiac rhythm } \\
\text { abnormalities, dry mouth, } \\
\text { urinary retention, weight } \\
\text { gain, serotonin syndrome }\end{array}$ & Dry mouth (moderate) & $\begin{array}{l}\text { Cardiac arrhythmias and sinus tachycardia: } \\
\text { increases with higher dose and pre-existing } \\
\text { risk } \\
\text { Urinary retention: no estimate found } \\
\text { Weight gain: } 2-2.5 \mathrm{~kg} \text { over } 3 \text { months } \\
\text { Serotonin syndrome: very rare } 252\end{array}$ \\
\hline \multirow[t]{2}{*}{$\begin{array}{l}\text { Antiepileptic } \\
\text { drugs }\end{array}$} & $\begin{array}{l}\text { Pregabalin, } \\
\text { gabapentin }\end{array}$ & $\begin{array}{l}\text { Blurred vision, cognitive } \\
\text { effects, dizziness, } \\
\text { peripheral edema, } \\
\text { sedation, weight gain }\end{array}$ & $\begin{array}{l}\text { Blurred vision, dizziness, weight gain, and } \\
\text { cognitive effects (moderate to large, lower } \\
\text { with the prodrug gabapentin enacarbil) } \\
\text { Peripheral edema (large with pregabalin) }\end{array}$ & No missing outcomes \\
\hline & Oxcarbazepine & $\begin{array}{l}\text { Cognitive effects, } \\
\text { hyponatremia, and } \\
\text { sedation }\end{array}$ & Hyponatremia - 1 RCT, no increased risk & $\begin{array}{l}\text { Significant hyponatremia: } 2.5 \% \text {, occurs in } \\
\text { first } 3 \text { months. } \\
\text { Cognitive effects: } 7-11 \% \\
\text { Somnolence: } 35 \%{ }^{253}\end{array}$ \\
\hline NSAIDs & Oral NSAIDs & $\begin{array}{l}\mathrm{CV}, \mathrm{GI} \text {, renal, and } \\
\text { hepatic events }\end{array}$ & $\begin{array}{l}\text { Short term: Increased CV risk - diclofenac } \\
\text { (small, dose-dependent); increased } \\
\text { coronary events - diclofenac, celecoxib } \\
\text { (moderate), ibuprofen (large); Increased GI } \\
\text { events - diclofenac (moderate), ibuprofen, } \\
\text { naproxen (large); } \\
\text { Intermediate term: Differences in CV risk } \\
\text { unclear; Increased hepatic harms- } \\
\text { diclofenac, naproxen (large, low incidence) }\end{array}$ & $\begin{array}{l}\text { Renal: Increased risk (moderate to large), } \\
\text { higher in older patients and those with } \\
\text { chronic kidney disease (evidence from } \\
\text { observational studies, includes short-term } \\
\text { use) No difference found between } \\
\text { NSAIDs. }{ }^{254,255}\end{array}$ \\
\hline \multirow[t]{3}{*}{ Other } & Acetaminophen & Hepatotoxicity & Not reported in included RCTs & $\begin{array}{l}\text { Increased risk with chronic use of }>3 \mathrm{gms} \\
\text { daily, effects often occur early in treatment; } \\
\text { dose-adjustment if hepatic or renal } \\
\text { dysfunction }{ }^{256,257}\end{array}$ \\
\hline & Cannabis & $\begin{array}{l}\text { Addiction/dependence, } \\
\text { cognitive effects, } \\
\text { hyperemesis, nausea, } \\
\text { sedation }\end{array}$ & $\begin{array}{l}\text { Dizziness (large) } \\
\text { Nausea (THC/CBD oral spray, large) }\end{array}$ & $\begin{array}{l}\text { Hyperemesis syndrome: Case reports (not } \\
\text { limited to medical uses), }>1 \times / \text { week for }>2 \\
\text { years. } \\
\text { Cognition: small negative impact with } \\
\text { chronic use } \\
\text { Addiction/dependence: not found }{ }^{258}\end{array}$ \\
\hline & Capsaicin & Application site reactions & $\begin{array}{l}\text { Pain (large), erythema (small) Greater with } \\
\text { longer application }\end{array}$ & No missing outcomes \\
\hline
\end{tabular}

CBD = cannabidiol; CV = cardiovascular; GI = gastrointestinal; kg = kilogram; NSAIDs = nonsteroidal anti-inflammatory drugs; RCTs = randomized controlled trials; SNRIs = serotonin-norepinephrine reuptake inhibitor; TCAs = tricyclic antidepressants; THC = tetrahydrocannabinol 
In relation to existing guidelines relating to treating chronic pain, our review findings differ in some respects. While the 2016 Center for Disease Control and Prevention (CDC) Guideline for Prescribing Opioids for Chronic Pain recommends nonopioid therapy for the treatment of chronic pain, specific recommendations were not within the scope of the guideline. ${ }^{13}$ Prior guidelines that made specific recommendations on nonopioid treatments commonly

recommended acetaminophen among the first-line treatments, ${ }^{259,260}$ while our review findings do not demonstrate that acetaminophen provided adequate pain relief to qualify as a small effect size. Similarly, guidelines on treating fibromyalgia recommended drugs we found to have insufficient evidence of effectiveness or to have inadequate pain relief (e.g., cyclobenzaprine, amitriptyline -although some are weak/low-level recommendations), and are either missing some drugs included in our review that have evidence of small or moderate effects (e.g., milnacipran) or recommended a class of drugs for which we found disparate results for specific drugs in the class (anticonvulsants). ${ }^{261}$ While guidelines on treating neuropathic pain do recommend drugs found effective in this review, they also include recommendations for medications not found to have evidence of effectiveness. ${ }^{262}$

\section{Applicability}

The applicability of the evidence base for nonopioid drugs to treat chronic pain varies according to the pain population and intervention studied. In terms of patient populations studied, the participants were generally typical for each pain condition (with the possible exception of chronic headache). For example, the mean age of participants with neuropathic pain was 58, most had painful diabetic peripheral neuropathy, 43 percent were female, and 34 percent were nonwhite and had a mean baseline pain of 6 to 7 (on a 0-10 scale) and a 4-year duration of pain. Fibromyalgia patients were younger, with a mean of 49 years, most (94\%) were female, and only 15 percent were nonwhite. Mean baseline pain was again 6 to 7 (on a 0-10 scale), with duration of pain ranging from less than a year in three RCTs, and 5 to 13 years in the rest. In osteoarthritis and inflammatory arthritis, mean age was 63 and 52 years, 68 percent and 63 percent were female, 24 percent and 12 percent were nonwhite, respectively. Mean baseline pain was 63 to 72 (osteoarthritis) and 65 (inflammatory arthritis) on a 0-100 visual analog scale (VAS), and duration of pain was typically not reported for patients with osteoarthritis, but a mean of 10 years was reported for inflammatory arthritis patients studied. Twenty-five percent of patients in the section on inflammatory arthritis had ankylosing spondylitis. Although there were few RCTs of patients with low back pain, mean age was 49 years, 42 percent were female, and 30 percent were nonwhite. Across 7 RCTs, baseline pain was lower than in other pain conditions, with a mean of 5 on a $0-10$ scale, and a median duration of 10 years. Because our definition of chronic headache was broad, and our criteria for treatments excluded use of nonopioids for prophylaxis, the result was a single, older, study of amitriptyline in patients with "chronic tension-type headache.” Headache classification has changed over the years such that the evidence identified may not be highly applicable to current patients or treatment strategies. While some RCTs excluded patients with mental illness, most did not report on baseline characteristics in relation to mental health, prior use of opioids, substance use disorder, etc.

Similarly, the specific interventions studied varied according to the pain condition. The medications studied in patients with neuropathic pain and fibromyalgia were most often antidepressants (primarily duloxetine) and anticonvulsants (primarily pregabalin), with some evaluations of other categories such as capsaicin and cannabis in neuropathic pain and memantine in both conditions. In contrast, osteoarthritis and inflammatory arthritis studies 
involved primarily NSAIDs. In patients with osteoarthritis, a small number of studies evaluated topical diclofenac, duloxetine, and acetaminophen. As a result, we have little or no information on how some interventions that were found effective in one pain condition may work in another pain condition. An example is that the evidence on pregabalin and gabapentin is applicable mainly to patients with specific types of neuropathic pain and fibromyalgia, but not applicable to patients with osteoarthritis or rheumatoid arthritis, or other type of chronic pain. The reverse is true of NSAIDs in that the evidence is restricted to osteoarthritis or rheumatoid arthritis/ankylosing spondylitis. The use of co-medications was rarely reported; acetaminophen use as a rescue medication in trials of NSAIDs was the only co-medication reported. As such, it is unclear how applicable this evidence is to patients using co-medications, including intermittent use of over-the-counter medications.

For all pain conditions, the most common comparator in the RCTs was placebo (114 out of 154 RCTs of good or fair quality), with limited head-to-head comparisons, especially across classes (7 RCTs). The most common head-to-head comparison was among different NSAIDs in patients with osteoarthritis (36 RCTs). The specific outcomes assessed in the included RCTs also varied according to the pain condition studied. Specific pain and function measures developed for specific conditions were used, for example the Fibromyalgia Impact Scale (FIQ) in fibromyalgia, the Western Ontario and McMaster Universities Osteoarthritis Index (WOMAC) in osteoarthritis, and the American College of Rheumatology (ACR) criteria for swollen and painful joints in rheumatoid arthritis. In our analyses, these were standardized where studies reported outcomes with scales of differing directions, ranges, etc. Other outcomes also varied according to pain condition, for example, sleep was reported most often for neuropathic pain, and depression was reported most often in studies of patients with fibromyalgia. To facilitate interpretation of results across trials and interventions, we categorized the magnitude of effects for function and pain outcomes using the system described in the Methods and used in two related systematic reviews. ${ }^{250,251}$ Using this system, beneficial effects identified were generally in the small or moderate range. We recognize that effects that we classified as small (e.g., 0.5 to 1.0 points on a 0 to 10 scale for pain or function) may be below some proposed thresholds for minimum clinically important differences for some measures and that there is variability across individual patients regarding what may constitute a clinically important effect, which is influenced by a number of factors such as preferences, duration and type of chronic pain, baseline symptom severity, harms, and costs. However, our classification provides some consistent and objective benchmarks to assess magnitude of smaller effects across trials and interventions. Interpretation of clinically important differences in mean change for continuous variables is challenging. If data were provided, we also evaluated the proportion of patients who experienced a clinically important improvement in pain or function (primarily at least a 30\% improvement from baseline). This provides valuable insight regarding clinically important improvement. The outcomes reported here apply mostly to the short term - 12 to 24 months of treatment. The applicability of the study settings is very unclear, as few studies reported setting characteristics. It was not apparent that the setting was specifically in pain clinics, but given the study design (RCT) and the high proportion with industry funding ( $>80 \%)$, it is likely that the setting was tertiary care clinics.

All of these elements affect how applicable the findings of this review are to a given, specific, patient. The evidence is less applicable to patients older than early 70's, those with severe pain, nonwhite patients, and for most conditions, patients with more recent onset of pain. The results apply mostly to addressing whether a drug is effective and/or harmful in comparison 
to no treatment, but less applicable to selecting among nonopioid treatments. However, the evidence base does provide some information on dose comparisons, such as higher and lower doses of SNRI antidepressants, pregabalin and gabapentin anticonvulsants, and some of the NSAIDs, where our analyses found little differences in efficacy, and a few cases of lower risk of adverse events with lower doses of antidepressants.

\section{Implications for Clinical and Policy Decision Making}

Recent guidelines from the CDC in the United States and the Canadian Guideline for Opioid Use in Chronic Non-Cancer Pain recommend nonopioid treatment as the preferred treatment for chronic pain. ${ }^{13,263}$ As noted above, many clinical practice guidelines recommend nonopioid treatments that may not provide adequate pain relief or improve functioning, while there are alternatives available. Our review provides evidence that can be used to update these clinical practice guidelines on treating the specific, common, chronic pain conditions included in this review. Given the need to offer nonopioid options to patients with chronic pain, especially in patients who wish to avoid an opioid, have or are at risk of developing opioid use disorder, this evidence is timely. Importantly, our review can inform guideline producers on the balance of benefits and harms, in the short, intermediate, and longer term.

Our report reviewed evidence that may also help inform decisions regarding prioritization of nonopioid drug therapies by clinicians and patients when selecting therapy. The evidence reviewed here may also help inform healthcare policy (including reimbursement policy) related to coverage of these nonopioid treatments, and inform policy decisions regarding funding priorities for future research.

\section{Limitations of the Review Process}

Limitations of our review process include that we excluded non-English language publications, and study results published only as abstract. We had limited ability to assess publication bias (small sample size bias), as most of our meta-analyses included fewer than 10 studies. We did not search clinical trial registries to identify unpublished trial results, but referred to study results reported in ClinicalTrials.gov when variance data were not reported in the trial publication. Another limitation was that we restricted inclusion to RCTs, limited to monotherapy, and limited the trials to those with at least 12 weeks of treatment. We could have missed effects reported only in shorter-term trials. This may have affected some older drugs (e.g., acetaminophen) more than others. Excluding observational studies may have meant not identifying serious harms of included drugs, or getting more precise estimates on these harms. We included information on such harms from other sources in Table 18 to complement our findings. For some of the drugs, there may be emerging concerns that were not prioritized here, such as misuse of, development of substance use disorder, or withdrawal symptoms associated with gabapentin or pregabalin, nonliver related harms of acetaminophen, and harms of drugs in older adults found in studies in other indications (not chronic pain). ${ }^{264-267}$ The effects of coprescribing gabapentin with opioids is not within the scope of this report, but is addressed in the related report on opioid use in chronic pain. ${ }^{250}$ We did not have access to individual patient data, which limited our ability to evaluate subgroup effects. Some meta-analyses were based on two or three trials; findings based on such meta-analyses must be interpreted with caution.

We did not include trials of patients with chronic pain conditions other than those specified. Our definition of chronic headache was broad, and may not align with currently used definitions of headache. Additionally, we excluded studies of prophylaxis of headache, which use many of 
the same drugs included in this review. Using these criteria, we included only one RCT, which did not find amitriptyline effective in reducing pain in "chronic tension-type headache." Therefore, our review is not adequate to address treatment of chronic forms of headache, which are now typically treated with medications such as onabotulinum toxin therapy, calcitonin generelated peptide (cGRP) antibody therapies, and cGRP receptor ligand blockers. We limited our analysis of NSAIDs to the nine most commonly prescribed in the United States, as identified using Centers for Medicare \& Medicaid Services data from 2018. We excluded combination therapies such as two included drugs (e.g., an NSAID plus and antidepressant). We also excluded specifically the combination of an NSAID and a proton-pump inhibitor. Given that most studies compared active drugs to placebo, we could have performed network meta-analyses to provide more information on how the drugs compare to each other. We did not perform such analyses due to time and resource limitations and concerns over validity of such analyses leading to a preference for direct comparisons.

\section{Limitations of the Evidence Base}

Important limitations of the evidence base include the small number of studies overall in most of the pain conditions, the small number of studies of individual drugs, and few studies of direct comparisons among the drugs. Most evidence on head-to-head comparisons of specific drugs is limited to one or two trials, making this evidence base not helpful in choosing among the nonopioid drug treatments. To address this latter limitation, we combined studies of within classes for meta-analyses compared with placebo. The clear majority $(>80 \%)$ of the trials were sponsored by industry, which might limit the evidence by increasing the likelihood of publication and/or other forms of bias. An unusually large proportion of the trials were poor-quality (16\%), largely due to poor reporting and reflecting that many studies were published prior to established guidance on reporting standards for RCTs. Since more of the studies of NSAIDs were older, and we were able to conduct meta-analyses of these studies, we evaluated the effect in studies published prior to 2000 versus those published later (after adoption of the CONSORT guidance), but did not find a significant interaction. Most studies (82\%) were short term (3 to $<6$ months), while only 13 percent were intermediate term ( 6 to $<12$ months), and 6 percent were long term ( $\geq 12$ months). Sample sizes of RCTs ranged from small $(<200)$ to medium $(<2000)$, but for some conditions/treatments the sample size was extremely small (e.g., an RCT of amitriptyline in sickle cell disease, $\mathrm{N}=22$ ).

Although the mean age of the populations studied is consistent with the age range of each pain condition, the evidence may be limited in not including a larger age range, or studies exclusively of older patients. Relatively few trials reported on the race of participants, and the evidence from trials that did report on race is limited to a largely White/Caucasian population. Assessment of primary outcomes were limited by trials that did not report on baseline pain or baseline function. Similarly, a very small proportion of trials (10\%) reported on quality of life and when reported, there was lack of consistency in the measures used, which limited our ability to combine results and draw conclusions. Inferences on effects for function are also limited by the heterogeneous variety of measures used for that outcome.

A major limitation of the evidence base is the inadequate reporting on harms for most of the included drugs, other than the NSAIDs. For example, cognitive effects were prioritized as an adverse event outcome of interest for multiple drug classes, but reporting varied widely (reported as confusion, "thinking abnormal," euphoric mood, disturbance in attention, etc.) leaving us to make decisions about which of these reflect cognition and should be combined. Specific serious 
harms were rarely reported in the included trials, in part because the trials were too short or too small to identify them, or because they were not specifically sought out.

\section{Research Gaps}

Although there are many studies included in this review, important gaps remain and future research should address these to better inform clinicians, patients, guideline developers and policymakers on the use of nonopioid pharmacologic treatments for chronic pain. Important gaps in the available research include a relative lack of:

- Comparative effectiveness trials - those that evaluate intermediate- and long-term treatment duration, long-term health outcomes (including quality of life), and make direct comparisons among key interventions both within- and across-classes;

- Good quality/low risk of bias studies - many trials suffered from poor reporting (e.g., unclear randomization and allocation concealment techniques), baseline differences between randomized groups, lack of blinding, and high attrition;

- Trials in older patients to better understand possible age-related difference in treatment effect and in patients of nonwhite race;

- Consistent use of recognized standard measures of pain and function to facilitate comparisons across trials;

- More trials in patients with chronic headache, low back pain, and sickle cell disease

\section{Conclusions}

Nonopioid drugs (mainly SNRI antidepressants, pregabalin/gabapentin, and NSAIDs) resulted in small to moderate improvements in pain and function outcomes in patients with specific types of noncancer chronic pain in the short term, with few differences between drugs in a class or doses of a drug. Evidence on intermediate- and long-term effects on pain, function, and quality of life is limited. Increased incidence of drug class-specific adverse events lead to withdrawal from treatment in some patients, suggesting that careful consideration of patient characteristics is needed in selecting nonopioid drug treatments. Additional research is needed on longer-term followup, quality of life, direct comparisons of nonopioid drugs, and in older patients, nonwhite patients, and patients with more severe pain and with comorbidities 


\section{References}

1. Institute of Medicine Committee on

Advancing Pain Research, Care, and

Education. Relieving Pain in America: A

Blueprint for Transforming Prevention,

Care, Education, and Research.

Washington, DC: National Academies

Press; 2011. doi: 10.17226/13172. PMID:

22553896.

2. Eller-Smith OC, Nicol AL, Christianson JA. Potential mechanisms underlying centralized pain and emerging therapeutic interventions. Front Cell Neurosci . 2018;12:35. PMID: 29487504.

3. IASP's Proposed New Definition of Pain Released for Comment. IASP; n.d.

https://www.iasp-

pain.org/PublicationsNews/NewsDetail.aspx

?ItemNumber=9218. Accessed October 4, 2019.

4. Gatchel RJ, McGeary DD, McGeary CA, et al. Interdisciplinary chronic pain management: past, present, and future. The Am Psychol. 2014 Feb-Mar;69(2):119-30. doi: 10.1037/a0035514. PMID: 24547798.

5. Dahlhamer J, Lucas J, Zelaya C, et al. Prevalence of Chronic Pain and HighImpact Chronic Pain Among Adults - United States, 2016. MMWR. Morb Mortal Wkly Rep. 2018;67(36):1001-6. doi: 10.15585/mmwr.mm6736a2. PMID: 30212442 .

6. National Pain Strategy Task Force, Interagency Pain Research Coordinating Committee. National Pain Strategy: A Comprehensive Population Health-Level Strategy for Pain. National Institutes of Health; 2015.

https://iprcc.nih.gov/sites/default/files/HHS

National_Pain_Strategy_508C.pdf.

Accessed February 1, 2019.

7. Centers for Disease Control and Prevention. Prescription Opioid Data. 2018.

https://www.cdc.gov/drugoverdose/data/pres cribing.html. Accessed February 1, 2019.

8. Boudreau D, Von Korff M, Rutter CM, et al. Trends in long-term opioid therapy for chronic non-cancer pain.

Pharmacoepidemiol Drug Saf. 2009;18(12):1166-75. doi: 10.1002/pds.1833. PMID: 19718704
9.

Centers for Disease Control and Prevention. Vital signs: overdoses of prescription opioid pain relievers---United States, 1999--2008. MMWR Morb Mortal Wkly Rep. 2011 Nov 4;60(43):1487-92. PMID: 22048730.

10. Chou R, Turner JA, Devine EB, et al. The effectiveness and risks of long-term opioid therapy for chronic pain: a systematic review for a National Institutes of Health Pathways to Prevention Workshop. Ann Intern Med. 2015;162(4):276-86. doi: 10.7326/M14-2559. PMID: 25581257.

11. Olsen Y, Daumit GL, Ford DE. Opioid prescriptions by U.S. primary care physicians from 1992 to 2001. J Pain. 2006 Apr;7(4):225-35. doi: 10.1016/j.jpain.2005.11.006. PMID: 16618466.

12. Sullivan MD, Edlund MJ, Fan M-Y, et al. Trends in use of opioids for non-cancer pain conditions 2000-2005 in commercial and Medicaid insurance plans: the TROUP study. Pain. 2008 Aug 31;138(2):440-9. doi: 10.1016/j.pain.2008.04.027. PMID: 18547726.

13. Dowell D, Haegerich TM, Chou R. CDC Guideline for Prescribing Opioids for Chronic Pain--United States, 2016. JAMA. 2016 Apr 19;315(15):1624-45. doi: 10.1001/jama.2016.1464. PMID: 26977696.

14. Yu X, Liu T, Zhao D, et al. Efficacy and safety of pregabalin in neuropathic pain followed spinal cord injury: A review and meta-analysis of randomized controlled trials. Clin J Pain. 2019 Mar;35(3):272-8. doi: 10.1097/AJP.0000000000000675. PMID: 30499836.

15. Rocco M, Rada G. Are cannabinoids effective for fibromyalgia? Medwave. 2018 Feb 14;18(1):e7154. doi: 10.5867/medwave.2018.01.7153. PMID: 29474351.

16. Mucke M, Phillips T, Radbruch L, et al. Cannabis-based medicines for chronic neuropathic pain in adults. Cochrane Database Syst Rev. 2018 Mar 7;3:CD012182. doi: 10.1002/14651858.CD012182.pub2. PMID: 29513392. 
17. Gregori D, Giacovelli G, Minto C, et al. Association of pharmacological treatments with long-term pain control in patients with knee osteoarthritis: a systematic review and meta-analysis. JAMA. 2018 Dec

25;320(24):2564-79. doi:

10.1001/jama.2018.19319. PMID:

30575881 .

18. Enke O, New HA, New $\mathrm{CH}$, et al.

Anticonvulsants in the treatment of low back pain and lumbar radicular pain: a systematic review and meta-analysis. CMAJ Canadian Medical Association Journal. 2018 Jul 3;190(26):E786-E93. doi: 10.1503/cmaj.171333. PMID: 29970367.

19. Arnold LM, Choy E, Clauw DJ, et al. An evidence-based review of pregabalin for the treatment of fibromyalgia. Curr Med Res Opin. 2018 Aug;34(8):1397-409. doi: 10.1080/03007995.2018.1450743. PMID: 29519159.

20. van Nooten F, Treur M, Pantiri K, et al. Capsaicin 8\% patch versus oral neuropathic pain medications for the treatment of painful diabetic peripheral neuropathy: A systematic literature review and network meta-analysis. Clin Ther. 2017 Apr;39(4):787-803.e18. doi: 10.1016/j.clinthera.2017.02.010. PMID: 28365034.

21. Methods Guide for Effectiveness and Comparative Effectiveness Reviews. AHRQ Publication No. 10(14)-EHC063-EF. Rockville, MD: Agency for Healthcare Research Quality; Jan 2014. https://effectivehealthcare.ahrq.gov/topics/ce r-methods-guide/overview. Accessed February 1, 2019. PMID: 21433403.

22. Headache Classification Committee of the International Headache Society (IHS). The International Classification of Headache Disorders, 3rd edition. Cephalalgia. 2013 Jan;38(1):1-211. doi: 10.1177/0333102417738202. PMID: 29368949.

23. Furlan AD, Malmivaara A, Chou R, et al. 2015 Updated Method Guideline for Systematic Reviews in the Cochrane Back and Neck Group. Spine (Phila Pa 1976). 2015 Nov;40(21):1660-73. doi: 10.1097/BRS.0000000000001061. PMID: 26208232.
24. Viswanathan M, Patnode CD, Berkman ND, et al. Assessing the risk of bias in systematic reviews of health care interventions. Methods Guide for Effectiveness and Comparative Effectiveness Reviews. Rockville, MD: Agency for Healthcare Research and Quality; 2017.

25. Skelly AC, Chou R, Dettori JR, et al. AHRQ Comparative Effectiveness Reviews. Noninvasive Nonpharmacological Treatment for Chronic Pain: A Systematic Review. Rockville, MD: Agency for Healthcare Research and Quality; 2018.

26. Backonja M, Wallace MS, Blonsky ER, et al. NGX-4010, a high-concentration capsaicin patch, for the treatment of postherpetic neuralgia: a randomised, double-blind study.[Erratum appears in Lancet Neurol. 2009 Jan;8(1):31]. Lancet Neurol. 2008 Dec;7(12):1106-12. doi: 10.1016/S1474-4422(08)70228-X. PMID: 18977178.

27. Cardenas DD, Nieshoff EC, Suda K, et al. A randomized trial of pregabalin in patients with neuropathic pain due to spinal cord injury. Neurology. 2013 Feb 5;80(6):533-9. doi: 10.1212/WNL.0b013e318281546b. PMID: 23345639.

28. Schimrigk S, Marziniak M, Neubauer C, et al. Dronabinol is a safe long-term treatment option for neuropathic pain patients. Eur Neurol. 2017;78(5-6):320-9. doi: 10.1159/000481089. PMID: 29073592.

29. Simpson DM, Schifitto G, Clifford DB, et al. Pregabalin for painful HIV neuropathy: a randomized, double-blind, placebocontrolled trial. Neurology. 2010 Feb 2;74(5):413-20. doi: 10.1212/WNL.0b013e3181ccc6ef. PMID: 20124207.

30. Arezzo JC, Rosenstock J, Lamoreaux L, et al. Efficacy and safety of pregabalin 600 $\mathrm{mg} / \mathrm{d}$ for treating painful diabetic peripheral neuropathy: a double-blind placebocontrolled trial. BMC Neurol. 2008 Sep 16;8(33)doi: 10.1186/1471-2377-8-33. PMID: 18796160. 
31. Beydoun A, Shaibani A, Hopwood M, et al. Oxcarbazepine in painful diabetic neuropathy: results of a dose-ranging study. Acta Neurol Scand. 2006 Jun;113(6):395404. doi: 10.1111/j.16000404.2006.00631.x. PMID: 16674606.

32. Clifford DB, Simpson DM, Brown S, et al. A randomized, double-blind, controlled study of NGX-4010, a capsaicin 8\% dermal patch, for the treatment of painful HIVassociated distal sensory polyneuropathy. J Acquir Immune Defic Syndr. 2012 Feb 1;59(2):126-33. doi: 10.1097/QAI.0b013e31823e31f7. PMID: 22067661.

33. Devi P, Madhu K, Ganapathy B, et al. Evaluation of efficacy and safety of gabapentin, duloxetine, and pregabalin in patients with painful diabetic peripheral neuropathy. Indian J Pharmacol. 2012 Jan;44(1):51-6. doi: 10.4103/02537613.91867. PMID: 22345870.

34. Dogra S, Beydoun S, Mazzola J, et al. Oxcarbazepine in painful diabetic neuropathy: a randomized, placebocontrolled study. Eur J Pain. 2005 Oct;9(5):543-54. doi: 10.1016/j.ejpain.2004.11.006. PMID: 16139183.

35. Freynhagen R, Strojek K, Griesing T, et al. Efficacy of pregabalin in neuropathic pain evaluated in a 12-week, randomised, doubleblind, multicentre, placebo-controlled trial of flexible- and fixed-dose regimens. Pain. 2005 Jun;115(3):254-63. PMID: 15911152.

36. Gao Y, Guo X, Han P, et al. Treatment of patients with diabetic peripheral neuropathic pain in China: a double-blind randomised trial of duloxetine vs. placebo. Int J Clin Pract. 2015 Sep;69(9):957-66. doi: 10.1111/ijcp.12641. PMID: 25939897.

37. Gao Y, Ning G, Jia WP, et al. Duloxetine versus placebo in the treatment of patients with diabetic neuropathic pain in China. Chin Med J. 2010 Nov;123(22):3184-92. PMID: 21163113.

38. Goldstein DJ, Lu Y, Detke MJ, et al. Duloxetine vs. placebo in patients with painful diabetic neuropathy. Pain. 2005 Jul;116(1-2):109-18. doi:

10.1016/j.pain.2005.03.029. PMID: 15927394.
39. Hoffman DL, Sadosky A, Dukes EM, et al. How do changes in pain severity levels correspond to changes in health status and function in patients with painful diabetic peripheral neuropathy? Pain. 2010;149(2):194-201. doi: 10.1016/j.pain.2009.09.017. PMID: 20303665.

40. Kelle B, Yavuz F, Yasar E, et al. The efficacy of gabapentin and pregabalin in the treatment of neuropathic pain due to peripheral nerve injury. J Musculoskelet Pain. 2012;20(4):300-5. doi: 10.3109/10582452.2012.733801.

41. Kim JS, Bashford G, Murphy TK, et al. Safety and efficacy of pregabalin in patients with central post-stroke pain. Pain. 2011 May;152(5):1018-23. doi: 10.1016/j.pain.2010.12.023. PMID: 21316855.

42. Markman J, Resnick M, Greenberg S, et al. Efficacy of pregabalin in post-traumatic peripheral neuropathic pain: a randomized, double-blind, placebo-controlled phase 3 trial. J Neurol. 2018 Dec;265(12):2815-24. doi: 10.1007/s00415-018-9063-9. PMID: 30242745.

43. Raskin J, Pritchett YL, Wang F, et al. A double-blind, randomized multicenter trial comparing duloxetine with placebo in the management of diabetic peripheral neuropathic pain. Pain Med. 2005 SepOct;6(5):346-56. doi: 10.1111/j.15264637.2005.00061.x. PMID: 16266355.

44. Rauck R, Makumi CW, Schwartz S, et al. A randomized, controlled trial of gabapentin enacarbil in subjects with neuropathic pain associated with diabetic peripheral neuropathy. Pain pract. 2013 Jul;13(6):48596. doi: 10.1111/papr.12014. PMID: 23186035.

45. Satoh J, Yagihashi S, Baba M, et al. Efficacy and safety of pregabalin for treating neuropathic pain associated with diabetic peripheral neuropathy: a 14 week, randomized, double-blind, placebocontrolled trial. Diabet Med. 2011 Jan;28(1):109-16. doi: 10.1111/j.14645491.2010.03152.x. PMID: 21166852. 
46. Schifitto G, Yiannoutsos CT, Simpson DM, et al. A placebo-controlled study of memantine for the treatment of human immunodeficiency virus-associated sensory neuropathy. J Neurovirol. 2006

Aug;12(4):328-31. doi: 10.1080/13550280600873835. PMID: 16966223.

47. Serpell M, Ratcliffe S, Hovorka J, et al. A double-blind, randomized, placebocontrolled, parallel group study of THC/CBD spray in peripheral neuropathic pain treatment. Eur J Pain. 2014 Aug;18(7):999-1012. doi: 10.1002/j.15322149.2013.00445.x. PMID: 24420962.

48. Siddall PJ, Cousins MJ, Otte A, et al. Pregabalin in central neuropathic pain associated with spinal cord injury: a placebo-controlled trial. Neurology. 2006 Nov 28;67(10):1792-800. doi: 10.1212/01.wnl.0000244422.45278.ff. PMID: 17130411.

49. Simpson DM, Rice AS, Emir B, et al. A randomized, double-blind, placebocontrolled trial and open-label extension study to evaluate the efficacy and safety of pregabalin in the treatment of neuropathic pain associated with human immunodeficiency virus neuropathy. Pain. 2014 Oct;155(10):1943-54. doi: 10.1016/j.pain.2014.05.027. PMID: 24907403.

50. Smith T, DiBernardo A, Shi Y, et al. Efficacy and safety of carisbamate in patients with diabetic neuropathy or postherpetic neuralgia: results from 3 randomized, double-blind placebocontrolled trials. Pain Pract. 2014 Apr;14(4):332-42. doi: 10.1111/papr.12080. PMID: 23692321.

51. Tolle T, Freynhagen R, Versavel M, et al. Pregabalin for relief of neuropathic pain associated with diabetic neuropathy: a randomized, double-blind study. Eur J Pain. 2008 Feb;12(2):203-13. doi: 10.1016/j.ejpain.2007.05.003. PMID: 17631400 .
52. van Seventer R, Feister HA, Young JP, Jr., et al. Efficacy and tolerability of twice-daily pregabalin for treating pain and related sleep interference in postherpetic neuralgia: a 13week, randomized trial. Curr Med Res Opin. 2006 Feb;22(2):375-84. doi: 10.1185/030079906X80404. PMID: 16466610.

53. Webster LR, Tark M, Rauck R, et al. Effect of duration of postherpetic neuralgia on efficacy analyses in a multicenter, randomized, controlled study of NGX-4010, an $8 \%$ capsaicin patch evaluated for the treatment of postherpetic neuralgia. BMC Neurol. 2010 Oct 11;10:92. doi: 10.1186/1471-2377-10-92. PMID: 20937130.

54. Wernicke JF, Pritchett YL, D'Souza DN, et al. A randomized controlled trial of duloxetine in diabetic peripheral neuropathic pain. Neurology. 2006a Oct 24;67(8):141120. doi:

10.1212/01.wnl.0000240225.04000.1a. PMID: 17060567.

55. Yasuda H, Hotta N, Kasuga M, et al. Efficacy and safety of $40 \mathrm{mg}$ or $60 \mathrm{mg}$ duloxetine in Japanese adults with diabetic neuropathic pain: Results from a randomized, 52-week, open-label study. J Diabetes Investig. 2016 Jan;7(1):100-8. doi: 10.1111/jdi.12361. PMID: 26816607.

56. Yasuda H, Hotta N, Nakao K, et al. Superiority of duloxetine to placebo in improving diabetic neuropathic pain: results of a randomized controlled trial in Japan. J Diabetes Investig. 2011 Apr 7;2(2):132-9. doi: 10.1111/j.2040-1124.2010.00073.x. PMID: 24843472.

57. Zhang L, Rainka M, Freeman R, et al. A randomized, double-blind, placebocontrolled trial to assess the efficacy and safety of gabapentin enacarbil in subjects with neuropathic pain associated with postherpetic neuralgia (PXN110748). J Pain. 2013 Jun;14(6):590-603. doi: 10.1016/j.jpain.2013.01.768. PMID: 23602345.

58. Dallocchio C, Buffa C, Mazzarello P, et al. Gabapentin vs. amitriptyline in painful diabetic neuropathy: an open-label pilot study. J Pain Symptom Manage. 2000 Oct;20(4):280-5. PMID: 11027910. 
59. Grosskopf J, Mazzola J, Wan Y, et al. A randomized, placebo-controlled study of oxcarbazepine in painful diabetic neuropathy. Acta Neurol Scand. 2006 Sep;114(3):177-80. doi: 10.1111/j.16000404.2005.00559.x. PMID: 16911345.

60. Joharchi K, Memari M, Azargashb E, et al. Efficacy and safety of duloxetine and Pregabalin in Iranian patients with diabetic peripheral neuropathic pain: a double-blind, randomized clinical trial. J Diabetes Metab Disord. 2019 Sep 13;18(2):575-582. doi: 10.1007/s40200-019-00427-w. PMID: 31890684.

61. Mahmood R, Jawed I, Khan MI, et al. Comparative role of pregabalin and carbamazepine regarding efficacy in painful diabetic neuropathy. Pak J Pharm Sci. 2017 Jul;30(4):1275-8. PMID: 29039325.

62. Selvarajah D, Gandhi R, Emery CJ, et al. Randomized placebo-controlled doubleblind clinical trial of cannabis-based medicinal product (Sativex) in painful diabetic neuropathy: depression is a major confounding factor. Diabetes Care. 2010 Jan;33(1):128-30. doi: 10.2337/dc09-1029. PMID: 19808912.

63. Tanenberg RJ, Irving G, Risser RC, et al. An open-label, randomized comparison of duloxetine, pregabalin, and the combination of duloxetine and gabapentin among patients with inadequate response to gabapentin for the management of diabetic peripheral neuropathic pain. Poster presentation, American Diabetes Association, June 25-29, 2010 Orlando, Florida. 2010.

64. Wasan AD, Ossanna MJ, Raskin J, et al. Safety and efficacy of duloxetine in the treatment of diabetic peripheral neuropathic pain in older patients. Curr Drug Saf. 2009 Jan;4(1):22-9. PMID: 19149522.

65. Arnold L, Gendreau R, Palmer R, et al. Efficacy and safety of milnacipran 100 $\mathrm{mg} /$ day in patients with fibromyalgia: results of a randomized, double-blind, placebocontrolled trial. Arthritis Rheum. 2010b Sep;62(9):2745-56. doi: 10.1002/art.27559. PMID: 20496365.
66. Arnold LM, Clauw D, Wang F, et al. Flexible dosed duloxetine in the treatment of fibromyalgia: a randomized, double-blind, placebo-controlled trial. J Rheumatol. 2010a Dec;37(12):2578-86. doi: 10.3899/jrheum.100365. PMID: 20843911.

67. Arnold LM, Goldenberg DL, Stanford SB, et al. Gabapentin in the treatment of fibromyalgia: a randomized, double-blind, placebo-controlled, multicenter trial. Arthritis Rheum. 2007a Apr;56(4):1336-44. doi: 10.1002/art.22457. PMID: 17393438.

68. Arnold LM, Hudson JI, Wang F, et al. Comparisons of the efficacy and safety of duloxetine for the treatment of fibromyalgia in patients with versus without major depressive disorder. Clin J Pain. 2009 JulAug;25(6):461-8. doi: 10.1097/AJP.0b013e318197d4e4. PMID: 19542792.

69. Arnold LM, Lu Y, Crofford LJ, et al. A double-blind, multicenter trial comparing duloxetine with placebo in the treatment of fibromyalgia patients with or without major depressive disorder. Arthritis Rheum. 2004 Sep;50(9):2974-84. doi: 10.1002/art.20485. PMID: 15457467.

70. Arnold LM, Palmer RH, Gendreau RM, et al. Relationships among pain, depressed mood, and global status in fibromyalgia patients: post hoc analyses of a randomized, placebo-controlled trial of milnacipran. Psychosomatics. 2012b Jul-Aug;53(4):3719. doi: 10.1016/j.psym.2012.02.005. PMID: 22677218.

71. Arnold LM, Rosen A, Pritchett YL, et al. A randomized, double-blind, placebocontrolled trial of duloxetine in the treatment of women with fibromyalgia with or without major depressive disorder. Pain. 2005 Dec 15;119(1-3):5-15. doi: 10.1016/j.pain.2005.06.031. PMID: 16298061.

72. Arnold LM, Russell IJ, Diri EW, et al. A 14week, randomized, double-blinded, placebocontrolled monotherapy trial of pregabalin in patients with fibromyalgia. J Pain. 2008 Sep;9(9):792-805. doi: 10.1016/j.jpain.2008.03.013. PMID: 18524684. 
73. Arnold LM, Wang F, Ahl J, et al. Improvement in multiple dimensions of fatigue in patients with fibromyalgia treated with duloxetine: secondary analysis of a randomized, placebo-controlled trial. Arthritis Res Ther. 2011 Jun 13;13(3):R86. doi: 10.1186/ar3359. PMID: 21668963.

74. Arnold LM, Whitaker S, Hsu C, et al. Efficacy and safety of mirogabalin for the treatment of fibromyalgia: results from three 13-week randomized, double-blind, placeboand active-controlled, parallel-group studies and a 52-week open-label extension study. Curr Med Res Opin. 2019 Jul 9:1-11. doi: 10.1080/03007995.2019.1629757. PMID: 31284771 .

75. Arnold LM, Zhang S, Pangallo BA. Efficacy and safety of duloxetine $30 \mathrm{mg} / \mathrm{d}$ in patients with fibromyalgia: a randomized, doubleblind, placebo-controlled study. Clin J Pain. 2012a Nov-Dec;28(9):775-81. doi: 10.1097/AJP.0b013e3182510295. PMID: 22971669.

76. Branco JC, Cherin P, Montagne A, et al. Longterm therapeutic response to milnacipran treatment for fibromyalgia. A European 1-year extension study following a 3-month study. J Rheumatol. 2011 Jul;38(7):1403-12. doi: 10.3899/jrheum.101025. PMID: 21459941.

77. Branco JC, Zachrisson O, Perrot S, et al. A European multicenter randomized doubleblind placebo-controlled monotherapy clinical trial of milnacipran in treatment of fibromyalgia. J Rheumatol. 2010

Apr;37(4):851-9. doi: 10.3899/jrheum.090884. PMID: 20156949.

78. Carette S, Bell MJ, Reynolds WJ, et al. Comparison of amitriptyline, cyclobenzaprine, and placebo in the treatment of fibromyalgia. A randomized, double-blind clinical trial. Arthritis Rheum. 1994 Jan;37(1):32-40. doi: 10.1002/art.1780370106. PMID: 8129762.

79. Chappell AS, Bradley LA, Wiltse C, et al. A six-month double blind placebo-controlled, randomized clinical trial of duloxetine for the treatment of fibromyalgia. Int J Gen Med. 2008 Nov 30;1:91-102. PMID: 20428412.
80. Chappell AS, Littlejohn G, Kajdasz DK, et al. A 1-year safety and efficacy study of duloxetine in patients with fibromyalgia. Clin J Pain. 2009b Jun;25(5):365-75. doi: 10.1097/AJP.0b013e31819be587. PMID: 19454869.

81. Clauw DJ, Mease P, Palmer RH, et al. Milnacipran for the treatment of fibromyalgia in adults: a 15-week, multicenter, randomized, double-blind, placebo-controlled, multiple-dose clinical trial. Clin Ther. 2008 Nov;30(11):19882004. doi: 10.1016/j.clinthera.2008.11.009. PMID: 19108787.

82. Emir B, Murphy TK, Petersel DL, et al. Treatment response to pregabalin in fibromyalgia pain: effect of patient baseline characteristics. Expert Opin Pharmacother. 2010 Oct;11(14):2275-80. doi: 10.1517/14656566.2010.509717. PMID: 20812879.

83. Gendreau RM, Thorn MD, Gendreau JF, et al. Efficacy of milnacipran in patients with fibromyalgia. J Rheumatol. 2005 Oct;32(10):1975-85. PMID: 16206355.

84. Goldenberg DL, Clauw DJ, Palmer RH, et al. Durability of therapeutic response to milnacipran treatment for fibromyalgia. Results of a randomized, double-blind, monotherapy 6-month extension study. Pain Med. 2010 Feb;11(2):180-94. doi: 10.1111/j.1526-4637.2009.00755.x. PMID: 20002596.

85. Hannonen P, Malminiemi K, Yli-Kerttula U, et al. A randomized, double-blind, placebocontrolled study of moclobemide and amitriptyline in the treatment of fibromyalgia in females without psychiatric disorder. Br J Rheumatol. 1998 Dec;37(12):1279-86. PMID: 9973149.

86. Mease PJ, Clauw DJ, Gendreau RM, et al. The efficacy and safety of milnacipran for treatment of fibromyalgia. a randomized, double-blind, placebo-controlled trial.[Erratum appears in J Rheumatol. 2009 Mar;36(3):661]. J Rheumatol. 2009 Feb;36(2):398-409. doi: 10.3899/jrheum.080734. PMID: 19132781. 
87. Mease PJ, Russell IJ, Arnold LM, et al. A randomized, double-blind, placebocontrolled, phase III trial of pregabalin in the treatment of patients with fibromyalgia. J Rheumatol. 2008 Mar;35(3):502-14. PMID: 18278830.

88. Murakami M, Osada K, Mizuno H, et al. A randomized, double-blind, placebocontrolled phase III trial of duloxetine in Japanese fibromyalgia patients. Arthritis Res Ther. 2015 Aug 22;17:224. doi: 10.1186/s13075-015-0718-y. PMID: 26296539.

89. Ohta H, Oka H, Usui C, et al. A randomized, double-blind, multicenter, placebocontrolled phase III trial to evaluate the efficacy and safety of pregabalin in Japanese patients with fibromyalgia. Arthritis Res Ther. 2012 Oct 12;14(5):R217. doi: 10.1186/ar4056. PMID: 23062189.

90. Olivan-Blazquez B, Herrera-Mercadal P, Puebla-Guedea M, et al. Efficacy of memantine in the treatment of fibromyalgia: a double-blind, randomised, controlled trial with 6-month follow-up. Pain. 2014 Dec;155(12):2517-25. doi: 10.1016/j.pain.2014.09.004. PMID: 25218600.

91. Olivan-Blazquez B, Puebla M, Masluk B, et al. Evaluation of the efficacy of memantine in the treatment of fibromyalgia: study protocol for a doubled-blind randomized controlled trial with six-month follow-up. Trials. 2013 Jan 3;14:3. doi: 10.1186/17456215-14-3. PMID: 23286311.

92. Pauer L, Winkelmann A, Arsenault P, et al. An international, randomized, double-blind, placebo-controlled, phase III trial of pregabalin monotherapy in treatment of patients with fibromyalgia. J Rheumatol. 2011 Dec;38(12):2643-52. doi: 10.3899/jrheum.110569. PMID: 21965636.

93. Russell IJ, Mease PJ, Smith TR, et al. Efficacy and safety of duloxetine for treatment of fibromyalgia in patients with or without major depressive disorder: results from a 6-month, randomized, double-blind, placebo-controlled, fixed-dose trial. Pain. 2008 Jun;136(3):432-44. doi: 10.1016/j.pain.2008.02.024. PMID: 18395345.
94. Vitton O, Gendreau M, Gendreau J, et al. A double-blind placebo-controlled trial of milnacipran in the treatment of fibromyalgia. Hum Psychopharmacol. 2004 Oct;19 (Suppl 1):S27-35. doi: 10.1002/hup.622. PMID: 15378666.

95. Wolfe F, Smythe HA, Yunus MB, et al. The American College of Rheumatology 1990 Criteria for the Classification of Fibromyalgia. Report of the Multicenter Criteria Committee. Arthritis Rheum. 1990 Feb;33(2):160-72. doi: 10.1002/art.1780330203. PMID: 2306288.

96. Allen R, Sharma U, Barlas S. Clinical experience with desvenlafaxine in treatment of patients with fibromyalgia syndrome. Clin Pharmacol Drug Dev. 2017 May;6(3):224-33. doi: 10.1002/cpdd.271. PMID: 27139158.

97. Gentile S, Fusco ML. Managing fibromyalgia syndrome in pregnancy no bridges between USA and EU. Arch Womens Ment Health. 2019 Jan 3doi: 10.1007/s00737-018-0933-z. PMID: 30607517.

98. Abou-Raya S, Abou-Raya A, Helmii M. Duloxetine for the management of pain in older adults with knee osteoarthritis: randomised placebo-controlled trial. Age Ageing. 2012 Sep;41(5):646-52. doi: 10.1093/ageing/afs072. PMID: 22743149.

99. Baraf HS, Gold MS, Clark MB, et al. Safety and efficacy of topical diclofenac sodium 1\% gel in knee osteoarthritis: a randomized controlled trial. Phys Sportsmed. 2010 Jun;38(2):19-28. doi: 10.3810/psm.2010.06.1779. PMID: 20631460.

100. Chappell AS, Ossanna MJ, Liu-Seifert H, et al. Duloxetine, a centrally acting analgesic, in the treatment of patients with osteoarthritis knee pain: a 13-week, randomized, placebo-controlled trial. Pain. 2009a Dec;146(3):253-60. doi: 10.1016/j.pain.2009.06.024. PMID: 19625125.

101. Clegg DO, Reda DJ, Harris CL, et al. Glucosamine, chondroitin sulfate, and the two in combination for painful knee osteoarthritis. N Engl J Med. 2006 Feb 23;354(8):795-808. doi: 10.1056/NEJMoa052771. PMID: 16495392. 
102. Emery P, Koncz T, Pan S, et al. Analgesic effectiveness of celecoxib and diclofenac in patients with osteoarthritis of the hip requiring joint replacement surgery: a 12week, multicenter, randomized, doubleblind, parallel-group, double-dummy, noninferiority study. Clin Ther. 2008 Jan;30(1):70-83. doi: 10.1016/j.clinthera.2008.01.016. PMID: 18343244.

103. Hochberg MC, Fort JG, Svensson O, et al. Fixed-dose combination of enteric-coated naproxen and immediate-release esomeprazole has comparable efficacy to celecoxib for knee osteoarthritis: two randomized trials. Curr Med Res Opin. 2011 Jun;27(6):1243-53. doi: 10.1185/03007995.2011.580340. PMID: 21524238.

104. Kivitz A, Eisen G, Zhao WW, et al. Randomized placebo-controlled trial comparing efficacy and safety of valdecoxib with naproxen in patients with osteoarthritis. J Fam Pract. 2002 Jun;51(6):530-7. PMID: 12100776.

105. Kivitz AJ, Moskowitz RW, Woods E, et al. Comparative efficacy and safety of celecoxib and naproxen in the treatment of osteoarthritis of the hip. J Int Med Res. 2001 Nov-Dec;29(6):467-79. doi: 10.1177/147323000102900602. PMID: 11803730.

106. Puopolo A, Boice JA, Fidelholtz JL, et al. A randomized placebo-controlled trial comparing the efficacy of etoricoxib $30 \mathrm{mg}$ and ibuprofen $2400 \mathrm{mg}$ for the treatment of patients with osteoarthritis. Osteoarthritis Cartilage. 2007 Dec;15(12):1348-56. doi: 10.1016/j.joca.2007.05.022. PMID: 17631392.

107. Schnitzer TJ, Dattani ID, Seriolo B, et al. A 13-week, multicenter, randomized, doubleblind study of lumiracoxib in hip osteoarthritis. Clin Rheumatol. 2011a Nov;30(11):1433-46. doi: 10.1007/s10067011-1776-4. PMID: 21607551.
108. Simon LS, Grierson LM, Naseer Z, et al. Efficacy and safety of topical diclofenac containing dimethyl sulfoxide (DMSO) compared with those of topical placebo, DMSO vehicle and oral diclofenac for knee osteoarthritis. Pain. 2009 Jun;143(3):238-45. doi: 10.1016/j.pain.2009.03.008. PMID: 19380203.

109. Sofat N, Harrison A, Russell MD, et al. The effect of pregabalin or duloxetine on arthritis pain: a clinical and mechanistic study in people with hand osteoarthritis.[Erratum appears in J Pain Res. 2017 Dec 15;10:2843]. J Pain Res. 2017;10:2437-49. PMID: 29066930.

110. Uchio Y, Enomoto H, Alev L, et al. A randomized, double-blind, placebocontrolled phase III trial of duloxetine in Japanese patients with knee pain due to osteoarthritis. J Pain Res. 2018;11:809-21. doi: 10.2147/JPR.S164128. PMID: 29713194.

111. Andelman SY. Etodolac, aspirin, and placebo in patients with degenerative joint disease: a twelve-week study. Clin Ther. 1983;5(6):651-61. PMID: 6226358.

112. Bond M, Davis A, Lohmander S, et al. Responsiveness of the OARSI-OMERACT osteoarthritis pain and function measures. Osteoarthritis Cartilage. 2012 Jun;20(6):541-7. doi: 10.1016/j.joca.2012.03.001. PMID: 22425883.

113. Garg Y, Singh J, Sohal HS, et al. Comparison of clinical effectiveness and safety of newer nonsteroidal antiinflammatory drugs in patients of osteoarthritis of knee joint: a randomized, prospective, open-label parallel-group study. Indian J Pharmacol. 2017 Sep-

Oct;49(5):383-9. doi: 10.4103/ijp.IJP_245_16. PMID: 29515279.

114. Kivitz A, Fairfax M, Sheldon EA, et al. Comparison of the effectiveness and tolerability of lidocaine patch $5 \%$ versus celecoxib for osteoarthritis-related knee pain: post hoc analysis of a 12 week, prospective, randomized, active-controlled, open-label, parallel-group trial in adults. Clin Ther. 2008 Dec;30(12):2366-77. doi: 10.1016/j.clinthera.2008.12.015. PMID: 19167595. 
115. Schnitzer TJ, Hochberg MC, Marrero CE, et al. Efficacy and safety of naproxcinod in patients with osteoarthritis of the knee: a 53week prospective randomized multicenter study. Semin Arthritis Rheum. 2011b Feb;40(4):285-97. doi: 10.1016/j.semarthrit.2010.06.002. PMID: 20828790.

116. Scott DL, Palmer RH. Safety and efficacy of nabumetone in osteoarthritis: emphasis on gastrointestinal safety. Aliment Pharmacol Ther. 2000 Apr;14(4):443-52. PMID: 10759624.

117. Vetter G. A comparison of naproxen and diclofenac sodium in the treatment of osteoarthritis in elderly patients. Br J Clin Pract. 1985 Jul;39(7):276-81. PMID: 3896286.

118. Altman R, Hochberg M, Gibofsky A, et al. Efficacy and safety of low-dose SoluMatrix meloxicam in the treatment of osteoarthritis pain: a 12-week, phase 3 study. Curr Med Res Opin. 2015 Dec;31(12):2331-43. doi: 10.1185/03007995.2015.1112772. PMID: 26503347.

119. Baerwald C, Verdecchia P, Duquesroix B, et al. Efficacy, safety, and effects on blood pressure of naproxcinod 750 mg twice daily compared with placebo and naproxen 500 mg twice daily in patients with osteoarthritis of the hip: a randomized, double-blind, parallel-group, multicenter study. Arthritis Rheum. 2010 Dec;62(12):3635-44. doi: 10.1002/art.27694. PMID: 20722026.

120. Bensen WG, Fiechtner JJ, McMillen JI, et al. Treatment of osteoarthritis with celecoxib, a cyclooxygenase-2 inhibitor: a randomized controlled trial. Mayo Clin Proc. 1999 Nov;74(11):1095-105. doi: 10.4065/74.11.1095. PMID: 10560596.

121. Bingham CO, 3rd, Sebba AI, Rubin BR, et al. Efficacy and safety of etoricoxib $30 \mathrm{mg}$ and celecoxib $200 \mathrm{mg}$ in the treatment of osteoarthritis in two identically designed, randomized, placebo-controlled, noninferiority studies. Rheumatology (Oxford). 2007 Mar;46(3):496-507. doi: 10.1093/rheumatology/kel296. PMID: 16936327.
122. Case JP, Baliunas AJ, Block JA. Lack of efficacy of acetaminophen in treating symptomatic knee osteoarthritis: a randomized, double-blind, placebocontrolled comparison trial with diclofenac sodium. Arch Intern Med. 2003 Jan 27;163(2):169-78. PMID: 12546607.

123. Conaghan PG, Dickson J, Bolten W, et al. A multicentre, randomized, placebo- and active-controlled trial comparing the efficacy and safety of topical ketoprofen in Transfersome gel (IDEA-033) with ketoprofen-free vehicle (TDT 064) and oral celecoxib for knee pain associated with osteoarthritis. Rheumatology (Oxford). 2013 Jul;52(7):1303-12. doi: 10.1093/rheumatology/ket133. PMID: 23542612.

124. Cryer BL, Sostek MB, Fort JG, et al. A fixed-dose combination of naproxen and esomeprazole magnesium has comparable upper gastrointestinal tolerability to celecoxib in patients with osteoarthritis of the knee: results from two randomized, parallel-group, placebo-controlled trials. Ann Med. 2011 Dec;43(8):594-605. doi: 10.3109/07853890.2011.625971. PMID: 22017620.

125. DeLemos BP, Xiang J, Benson C, et al. Tramadol hydrochloride extended-release once-daily in the treatment of osteoarthritis of the knee and/or hip: a double-blind, randomized, dose-ranging trial. Am J Ther. 2011 May;18(3):216-26. doi: 10.1097/MJT.0b013e3181сес307. PMID: 20215961.

126. Fleischmann R, Sheldon E, MaldonadoCocco $\mathrm{J}$, et al. Lumiracoxib is effective in the treatment of osteoarthritis of the knee: a prospective randomized 13-week study versus placebo and celecoxib. Clin Rheumatol. 2006 Feb;25(1):42-53. PMID: 16132165.

127. Gibofsky A, Hochberg MC, Jaros MJ, et al. Efficacy and safety of low-dose submicron diclofenac for the treatment of osteoarthritis pain: a 12 week, phase 3 study. Curr Med Res Opin. 2014 Sep;30(9):1883-93. doi: 10.1185/03007995.2014.946123. PMID: 25050589. 
128. Lehmann R, Brzosko M, Kopsa P, et al. Efficacy and tolerability of lumiracoxib 100 mg once daily in knee osteoarthritis: a 13week, randomized, double-blind study vs. placebo and celecoxib. Curr Med Res Opin. 2005 Apr;21(4):517-26. doi: 10.1185/030079905X38196. PMID: 15899100 .

129. Leung AT, Malmstrom K, Gallacher AE, et al. Efficacy and tolerability profile of etoricoxib in patients with osteoarthritis: a randomized, double-blind, placebo and active-comparator controlled 12-week efficacy trial. Curr Med Res Opin. 2002;18(2):49-58. doi: 10.1185/030079902125000282. PMID: 12017209.

130. Makarowski W, Zhao WW, Bevirt T, et al. Efficacy and safety of the COX-2 specific inhibitor valdecoxib in the management of osteoarthritis of the hip: a randomized, double-blind, placebo-controlled comparison with naproxen. Osteoarthritis Cartilage. 2002 Apr;10(4):290-6. doi: 10.1053/joca.2001.0510. PMID: 11950252.

131. Reginster JY, Dudler J, Blicharski T, et al. Pharmaceutical-grade Chondroitin sulfate is as effective as celecoxib and superior to placebo in symptomatic knee osteoarthritis: the ChONdroitin versus CElecoxib versus Placebo Trial (CONCEPT). Ann Rheum Dis. 2017 Sep;76(9):1537-43. doi: 10.1136/annrheumdis-2016-210860. PMID: 28533290.

132. Schnitzer TJ, Kivitz A, Frayssinet H, et al. Efficacy and safety of naproxcinod in the treatment of patients with osteoarthritis of the knee: a 13-week prospective, randomized, multicenter study.

Osteoarthritis Cartilage. 2010

May;18(5):629-39. doi: 10.1016/j.joca.2009.12.013. PMID: 20202489.

133. Sheldon E, Beaulieu A, Paster Z, et al. Efficacy and tolerability of lumiracoxib in the treatment of osteoarthritis of the knee: a 13-week, randomized, double-blind comparison with celecoxib and placebo. Clin Ther. 2005 Jan;27(1):64-77. doi: 10.1016/j.clinthera.2005.01.002. PMID: 15763607.
134. Strand V, Bergman M, Singh JA, et al. Lowdose SoluMatrix diclofenac in patients with osteoarthritis pain: impact on quality of life in a controlled trial. Clin Rheumatol. 2017 Jun;36(6):1357-67. doi: 10.1007/s10067017-3569-x. PMID: 28194525.

135. Tannenbaum H, Berenbaum F, Reginster $\mathrm{JY}$, et al. Lumiracoxib is effective in the treatment of osteoarthritis of the knee: a 13 week, randomised, double blind study versus placebo and celecoxib. Ann Rheum Dis. 2004 Nov;63(11):1419-26. doi: 10.1136/ard.2003.015974. PMID: 15020310 .

136. Wiesenhutter CW, Boice JA, Ko A, et al. Evaluation of the comparative efficacy of etoricoxib and ibuprofen for treatment of patients with osteoarthritis: a randomized, double-blind, placebo-controlled trial. Mayo Clin Proc. 2005 Apr;80(4):470-9. PMID: 15819283.

137. Yocum D, Fleischmann R, Dalgin P, et al. Safety and efficacy of meloxicam in the treatment of osteoarthritis: a 12-week, double-blind, multiple-dose, placebocontrolled trial. The Meloxicam Osteoarthritis Investigators. Arch Intern Med. 2000 Oct 23;160(19):2947-54. PMID: 11041902.

138. Zhao SZ, McMillen JI, Markenson JA, et al. Evaluation of the functional status aspects of health-related quality of life of patients with osteoarthritis treated with celecoxib. Pharmacotherapy. 1999 Nov;19(11):126978. PMID: 10555933.

139. Frendl DM, Ware JE, Jr. Patient-reported functional health and well-being outcomes with drug therapy: a systematic review of randomized trials using the SF-36 health survey. Med Care. 2014 May;52(5):439-45. doi: $10.1097 / \mathrm{mlr} .000000000000010311$. PMID: 24714581.

140. Barthel HR, Haselwood D, Longley S, 3rd, et al. Randomized controlled trial of diclofenac sodium gel in knee osteoarthritis.[Erratum appears in Semin Arthritis Rheum. 2010 Aug;40(1):95].

Semin Arthritis Rheum. 2009 Dec;39(3):203-12. doi: https://dx.doi.org/10.1016/j.semarthrit.2009. 09.002. PMID: 19932833. 
141. Roth SH, Shainhouse JZ. Efficacy and safety of a topical diclofenac solution (pennsaid) in the treatment of primary osteoarthritis of the knee: a randomized, double-blind, vehicle-controlled clinical trial. Arch Intern Med. 2004 Oct 11;164(18):2017-23. doi: 10.1001/archinte.164.18.2017. PMID: 15477437.

142. Simon LS, Yocum D. New and future drug therapies for rheumatoid arthritis.

Rheumatology (Oxford). 2000 Jun;39 Suppl 1:36-42. PMID: 11001378.

143. Sowers JR, White WB, Pitt B, et al. The effects of cyclooxygenase-2 inhibitors and nonsteroidal anti-inflammatory therapy on 24-hour blood pressure in patients with hypertension, osteoarthritis, and type 2 diabetes mellitus.[Erratum appears in Arch Intern Med. 2005 Mar 14;165(5):551]. Arch Intern Med. 2005 Jan 24;165(2):161-8. doi: 10.1001/archinte.165.2.161. PMID: 15668361.

144. Tugwell PS, Wells GA, Shainhouse JZ. Equivalence study of a topical diclofenac solution (pennsaid) compared with oral diclofenac in symptomatic treatment of osteoarthritis of the knee: a randomized controlled trial. J Rheumatol. 2004 Oct;31(10):2002-12. PMID: 15468367.

145. Pisko EJ, Bockow BI, Box P, et al. Sixmonth multi-center study comparing nabumetone with naproxen in the treatment of osteoarthritis. Am J Med. 1987a Oct 30;83(4B):86-91. PMID: 3318436.

146. Poiley JE. Nabumetone versus naproxen in the treatment of osteoarthritis. A six-month trial. Am J Med. 1987 Oct 30;83(4B):82-5. PMID: 3318435.

147. Bakshi R, Ezzet N, Frey L, et al. Efficacy and tolerability of diclofenac dispersible in painful osteoarthrosis. Clin Rheumatol. 1993 Mar;12(1):57-61. PMID: 7682167.

148. Morgan GJ, Jr., Kaine J, DeLapp R, et al. Treatment of elderly patients with nabumetone or diclofenac: gastrointestinal safety profile. J Clin Gastroenterol. 2001 Apr;32(4):310-4. PMID: 11276273.
149. Essex MN, Bhadra P, Sands GH. Efficacy and tolerability of celecoxib versus naproxen in patients with osteoarthritis of the knee: a randomized, double-blind, double-dummy trial. J Int Med Res. 2012;40(4):1357-70. doi: 10.1177/147323001204000414. PMID: 22971487.

150. Hosie J, Distel M, Bluhmki E. Meloxicam in osteoarthritis: a 6-month, double-blind comparison with diclofenac sodium. Br J Rheumatol. 1996 Apr;35 Suppl 1:39-43. PMID: 8630635.

151. Dahlberg LE, Holme I, Hoye K, et al. A randomized, multicentre, double-blind, parallel-group study to assess the adverse event-related discontinuation rate with celecoxib and diclofenac in elderly patients with osteoarthritis. Scand J Rheumatol. 2009 Mar-Apr;38(2):133-43. doi: 10.1080/03009740802419065. PMID: 19165648.

152. Roth SH, Tindall EA, Jain AK, et al. A controlled study comparing the effects of nabumetone, ibuprofen, and ibuprofen plus misoprostol on the upper gastrointestinal tract mucosa. Arch Intern Med. 1993 Nov 22;153(22):2565-71. PMID: 8239849.

153. Chappell AS, Desaiah D, Liu-Seifert H, et al. A double-blind, randomized, placebocontrolled study of the efficacy and safety of duloxetine for the treatment of chronic pain due to osteoarthritis of the knee. Pain Pract. 2011 Jan-Feb;11(1):33-41. doi: 10.1111/j.1533-2500.2010.00401.x. PMID: 20602715.

154. Enomoto H, Fujikoshi S, Tsuji T, et al. Efficacy of duloxetine by prior NSAID use in the treatment of chronic osteoarthritis knee pain: a post hoc subgroup analysis of a randomized, placebo-controlled, phase 3 study in Japan. J Orthop Sci. 2018 Nov;23(6):1019-26. doi: 10.1016/j.jos.2018.07.008. PMID: 30126675.

155. Tetreault P, Baliki MN, Baria AT, et al. Inferring distinct mechanisms in the absence of subjective differences: placebo and centrally acting analgesic underlie unique brain adaptations. Hum Brain Mapp. 2018 May;39(5):2210-23. doi: 10.1002/hbm.23999. PMID: 29417694. 
156. Tetreault P, Mansour A, Vachon-Presseau E, et al. Brain connectivity predicts placebo response across chronic pain clinical trials. PLoS Biol. 2016 Oct 27;14(10):e1002570. doi: 10.1371/journal.pbio.1002570. PMID: 27788130.

157. Wang G, Bi L, Li X, et al. Efficacy and safety of duloxetine in Chinese patients with chronic pain due to osteoarthritis: a randomized, double-blind, placebocontrolled study. Osteoarthritis Cartilage. 2017 Jun;25(6):832-8. doi: 10.1016/j.joca.2016.12.025. PMID: 28043937.

158. Abou-Raya A, Abou-Raya S, El Khadrawy T. Efficacy of rebamipide in prevention of COX-2 inhibition induced gastrointestinal injuries (gastropathy) in older adults with knee osteoarthritis: A randomized placebocontrolled study. Ann Rheum Dis. 2012;71(6) PMID: CN-01058571 NEW.

159. Altman RD, Zinsenheim JR, Temple AR, et al. Three-month efficacy and safety of acetaminophen extended-release for osteoarthritis pain of the hip or knee: a randomized, double-blind, placebocontrolled study. Osteoarthritis Cartilage. 2007 Apr;15(4):454-61. doi: 10.1016/j.joca.2006.10.008. PMID: 17142063.

160. Herrero-Beaumont G, Ivorra JA, Del Carmen Trabado M, et al. Glucosamine sulfate in the treatment of knee osteoarthritis symptoms: a randomized, double-blind, placebo-controlled study using acetaminophen as a side comparator. Arthritis Rheum. 2007 Feb;56(2):555-67. doi: 10.1002/art.22371. PMID: 17265490.

161. Prior MJ, Harrison DD, Frustaci ME. A randomized, double-blind, placebocontrolled 12 week trial of acetaminophen extended release for the treatment of signs and symptoms of osteoarthritis. Curr Med Res Opin. 2014 Nov;30(11):2377-87. doi: 10.1185/03007995.2014.949646. PMID: 25121804.

162. Barkhuizen A, Steinfeld S, Robbins J, et al. Celecoxib is efficacious and well tolerated in treating signs and symptoms of ankylosing spondylitis. J Rheumatol. 2006 Sep;33(9):1805-12. PMID: 16960941.
163. Bensen W, Weaver A, Espinoza L, et al. Efficacy and safety of valdecoxib in treating the signs and symptoms of rheumatoid arthritis: a randomized, controlled comparison with placebo and naproxen. Rheumatology (Oxford). 2002 Sep;41(9):1008-16. doi: 10.1093/rheumatology/41.9.1008. PMID: 12209034.

164. Collantes E, Curtis SP, Lee KW, et al. A multinational randomized, controlled, clinical trial of etoricoxib in the treatment of rheumatoid arthritis [ISRCTN25142273]. BMC Fam Pract. 2002 May 22;3:10. PMID: 12033987.

165. de Queiros MF. Double-blind comparison of etodolac and naproxen in the treatment of rheumatoid arthritis. Clin Ther. 1991 JanFeb;13(1):38-46. PMID: 1827613.

166. del Toro RA, Concepcion R. Twelve-week study of etodolac, aspirin, and placebo in patients with rheumatoid arthritis. Clin Ther. 1983;5(4):436-44. PMID: 6223699.

167. Dougados M, Gueguen A, Nakache JP, et al. Ankylosing spondylitis: what is the optimum duration of a clinical study? A one year versus a 6 weeks non-steroidal antiinflammatory drug trial. Rheumatology (Oxford). 1999 Mar;38(3):235-44. doi: 10.1093/rheumatology/38.3.235. PMID: 10325662.

168. Edwards W. Etodolac, aspirin, and placebo in patients with rheumatoid arthritis: a 12week study. Clin Ther. 1983;5(5):495-503. PMID: 6225520.

169. Emery P, Clarke A, Williams P, et al. Nabumetone compared with naproxen in the treatment of rheumatoid arthritis: a multicenter, double blind, randomized, parallel group trial in hospital outpatients.[Erratum appears in J Rheumatol 1993 May;20(5):924]. J Rheumatol Suppl. 1992 Nov;36:41-7. PMID: 1474534.

170. Emery P, Zeidler H, Kvien TK, et al. Celecoxib versus diclofenac in long-term management of rheumatoid arthritis: randomised double-blind comparison. Lancet. 1999;354(9196):2106-11. doi: 10.1016/S0140-6736(99)02332-6. PMID: 10609815. 
171. Fattahi MJ, Jamshidi AR, Mahmoudi M, et al. Evaluation of the efficacy and safety of beta-d-mannuronic acid in patients with ankylosing spondylitis: A 12-week randomized, placebo-controlled, phase I/II clinical trial. Int Immunopharmacol. 2018 Jan;54:112-7. doi: https://dx.doi.org/10.1016/j.intimp.2017.11. 003. PMID: 29127910.

172. Furst DE, Kolba KS, Fleischmann R, et al. Dose response and safety study of meloxicam up to $22.5 \mathrm{mg}$ daily in rheumatoid arthritis: a 12 week multicenter, double blind, dose response study versus placebo and diclofenac. J Rheumatol. 2002 Mar;29(3):436-46. PMID: 11908554.

173. Geusens P, Alten R, Rovensky J, et al. Efficacy, safety and tolerability of lumiracoxib in patients with rheumatoid arthritis. Int J Clin Pract. 2004 Nov;58(11):1033-41. PMID: 15605667.

174. Geusens PP, Truitt K, Sfikakis P, et al. A placebo and active comparator-controlled trial of rofecoxib for the treatment of rheumatoid arthritis. Scand J Rheumatol. 2002;31(4):230-8. PMID: 12369656.

175. Gibofsky A, Rodrigues J, Fiechtner J, et al. Efficacy and tolerability of valdecoxib in treating the signs and symptoms of severe rheumatoid arthritis: a 12-week, multicenter, randomized, double-blind, placebocontrolled study. Clin Ther. 2007 Jun;29(6):1071-85. doi: 10.1016/j.clinthera.2007.06.016. PMID: 17692722.

176. Gordon GV, Polsky BG. Three-month trial of etodolac (Ultradol $\AA$ ) compared with aspirin and placebo in patients with rheumatoid arthritis. Curr Ther Res Clin Exp. 1983;33(1):89-99.

177. Grace EM, Bellamy N, Kassam Y, et al. Controlled, double-blind, randomized trial of amitriptyline in relieving articular pain and tenderness in patients with rheumatoid arthritis. Curr Med Res Opin. 1985;9(6):426-9. doi: 10.1185/03007998509109614. PMID: 3886308.

178. Jacob G, Messina M, Caperton E. Safety and efficacy of etodolac, once or twice a day, in the treatment of active rheumatoid arthritis. Curr Ther Res Clin Exp. 1985;37(6):1124-9.
179. Jacob GB, Hart KK, Mullane JF. Placebocontrolled study of etodolac and aspirin in the treatment of rheumatoid arthritis. Curr Ther Res Clin Exp. 1983;33(4):703-13.

180. Khan MA. Diclofenac in the treatment of ankylosing spondylitis: review of worldwide clinical experience and report of a doubleblind comparison with indomethacin. Semin Arthritis Rheum. 1985 Nov;15(2 Suppl 1):80-4. PMID: 4081795.

181. Khan MA. A double blind comparison of diclofenac and indomethacin in the treatment of ankylosing spondylitis. J Rheumatol. 1987 Feb;14(1):118-23. PMID: 3553586.

182. Kivitz AJ, Nayiager S, Schimansky T, et al. Reduced incidence of gastroduodenal ulcers associated with lumiracoxib compared with ibuprofen in patients with rheumatoid arthritis. Aliment Pharmacol Ther. 2004 Jun 1;19(11):1189-98. doi: 10.1111/j.13652036.2004.01956.x. PMID: 15153172.

183. Krug H, Broadwell LK, Berry M, et al. Tolerability and efficacy of nabumetone and naproxen in the treatment of rheumatoid arthritis. Clin Ther. 2000 Jan;22(1):40-52. PMID: 10688389.

184. Lonauer G, Tisscher JR, Lim HG, et al. Double-blind comparison of etodolac and diclofenac in patients with rheumatoid arthritis. Curr Med Res Opin. 1993;13(2):70-7. doi: 10.1185/03007999309111535. PMID: 8325044.

185. Matsumoto AK, Melian A, Mandel DR, et al. A randomized, controlled, clinical trial of etoricoxib in the treatment of rheumatoid arthritis. J Rheumatol. 2002 Aug;29(8):1623-30. PMID: 12180720.

186. Nazeri S, Jamshidi AR, Mahmoudi M, et al. The safety and efficacy of Guluronic acid (G2013) in ankylosing spondylitis: A randomized controlled parallel clinical trial. Pharmacol Rep. 2019 Jun;71(3):393-8. doi: 10.1016/j.pharep.2019.02.002. PMID: 31003148.

187. Palferman TG, Webley M. A comparative study of nabumetone and indomethacin in ankylosing spondylitis. Eur J Rheumatol Inflamm. 1991;11(2):23-9. PMID: 1365469. 
188. Sieper J, Klopsch T, Richter M, et al. Comparison of two different dosages of celecoxib with diclofenac for the treatment of active ankylosing spondylitis: results of a 12-week randomised, double-blind, controlled study. Ann Rheum Dis. 2008 Mar;67(3):323-9. doi: 10.1136/ard.2007.075309. PMID: 17616556.

189. Simon L, Weaver AL, Graham DY, et al. Anti-inflammatory and upper gastrointestinal effects of celecoxib in rheumatoid arthritis: a randomized controlled trial. JAMA. 1999 Nov 24;282(20):1921-8. PMID: 10580457.

190. Vasey FB, Germain BF, Espinoza LR, et al. Controlled evaluation of nabumetone in the treatment of active adult rheumatoid arthritis. Nabumetone versus naproxen double-blind parallel study. Am J Med. 1987 Oct 30;83(4B):55-9. PMID: 3318430.

191. Walker C, Essex MN, Li C, et al. Celecoxib versus diclofenac for the treatment of ankylosing spondylitis: 12-week randomized study in Norwegian patients. J Int Med Res. 2016 Jun;44(3):483-95. doi: 10.1177/0300060516628704. PMID: 26980340.

192. Wojtulewski JA, Schattenkirchner M, Barcelo P, et al. A six-month double-blind trial to compare the efficacy and safety of meloxicam $7.5 \mathrm{mg}$ daily and naproxen 750 mg daily in patients with rheumatoid arthritis. Br J Rheumatol. 1996 Apr;35(Suppl 1):22-8. PMID: 8630632.

193. Zhao SZ, Fiechtner JI, Tindall EA, et al. Evaluation of health-related quality of life of rheumatoid arthritis patients treated with celecoxib. Arthritis Care Res (Hoboken). 2000 Apr;13(2):112-21. doi: 10.1002/15290131\%28200004\%2913:2\%3C112::AIDANR5\%3E3.0.CO;2-L. PMID: 14635284.

194. Hawkey CJ, Laine L, Simon T, et al. Incidence of gastroduodenal ulcers in patients with rheumatoid arthritis after 12 weeks of rofecoxib, naproxen, or placebo: a multicentre, randomised, double blind study.[Erratum appears in Gut. 2003 Dec;52(12):1800]. Gut. 2003 Dec;52(6):820-6. doi: 10.1136/gut.52.6.820. PMID: 12740337.
195. Hazleman BL, Thomas PP. Single-blind comparative study of nabumetone (Relafen) versus naproxen in the treatment of rheumatoid arthritis. Am J Med. 1987 Oct 30;83(4B):60-4. PMID: 3318431.

196. Atkinson J, Slater MA, Capparelli EV, et al. Efficacy of noradrenergic and serotonergic antidepressants in chronic back pain: A preliminary concentration-controlled trial. J Clin Psychopharmacol. 2007 Apr;27(2):13542. doi: 10.1097/jcp.0b013e3180333ed5. PMID: 17414235.

197. Atkinson JH, Slater MA, Capparelli EV, et al. A randomized controlled trial of gabapentin for chronic low back pain with and without a radiating component. Pain. 2016 Jul;157(7):1499-507. doi: 10.1097/j.pain.0000000000000554. PMID: 26963844.

198. Kalita J, Kohat AK, Misra UK, et al. An open labeled randomized controlled trial of pregabalin versus amitriptyline in chronic low backache. J Neurol Sci. 2014 Jul 15;342(1-2):127-32. doi: 10.1016/j.jns.2014.05.002. PMID: 24857356.

199. Konno S, Oda N, Ochiai T, et al. Randomized, double-blind, placebocontrolled phase III trial of duloxetine monotherapy in Japanese patients with chronic low back pain. Spine (Phila $\mathrm{Pa}$ 1976). 2016 Nov 15;41(22):1709-17. doi: 10.1097/BRS.0000000000001707. PMID: 27831985.

200. Skljarevski V, Ossanna M, Liu-Seifert H, et al. A double-blind, randomized trial of duloxetine versus placebo in the management of chronic low back pain. Eur J Neurol. 2009 Sep;16(9):1041-8. doi: 10.1111/j.1468-1331.2009.02648.x. PMID: 19469829.

201. Skljarevski V, Zhang S, Desaiah D, et al. Duloxetine versus placebo in patients with chronic low back pain: a 12-week, fixeddose, randomized, double-blind trial. J Pain. 2010 Dec;11(12):1282-90. doi: 10.1016/j.jpain.2010.03.002. PMID: 20472510. 
202. Tsuji T, Itoh N, Ishida M, et al. Response to duloxetine in chronic low back pain: exploratory post hoc analysis of a Japanese phase III randomized study. J Pain Res. 2017 Sep 4;10:2157-68. doi: 10.2147/JPR.S138172. PMID: 28919811.

203. Urquhart DM, Wluka AE, Sim MR, et al. Is low-dose amitriptyline effective in the management of chronic low back pain? Study protocol for a randomised controlled trial. Trials. 2016 Oct 22;17(1):514. doi: 10.1186/s13063-016-1637-1. PMID: 27770809.

204. Urquhart DM, Wluka AE, Van Tulder M, et al. Efficacy of low-dose amitriptyline for chronic low back pain: a randomized clinical trial. JAMA Intern Med. 2018 Nov 1;178(11):1474-81. doi: 10.1001/jamainternmed.2018.4222. PMID: 30285054.

205. Pfaffenrath V, Diener HC, Isler H, et al. Efficacy and tolerability of amitriptylinoxide in the treatment of chronic tension-type headache: a multi-centre controlled study. Cephalalgia. 1994 Apr;14(2):149-55. doi: 10.1046/j.1468-2982.1994.1402149.x. PMID: 8062354.

206. Boudreau GP, Marchand L. Pregabalin for the management of cervicogenic headache: a double blind study. Can J Neurol Sci. 2014 Sep;41(5):603-10. doi: 10.1017/cjn.2014.2. PMID: 25373811.

207. Holroyd KA, O'Donnell FJ, Stensland M, et al. Management of chronic tension-type headache with tricyclic antidepressant medication, stress management therapy, and their combination: a randomized controlled trial. JAMA. 2001 May 2;285(17):2208-15. doi: 10.1001/jama.285.17.2208. PMID: 11325322.

208. Enteshari-Moghaddam A, Azami A, Isazadehfar K, et al. Efficacy of duloxetine and gabapentin in pain reduction in patients with knee osteoarthritis. Clin Rheumatol. 2019 May 6doi: 10.1007/s10067-01904573-7. PMID: 31062253.
209. Wernicke JF, Raskin J, Rosen A, et al. Duloxetine in the long-term management of diabetic peripheral neuropathic pain: an open-label, 52-week extension of a randomized controlled clinical trial. Curr Ther Res Clin Exp. 2006b Sep;67(5):283304. doi: 10.1016/j.curtheres.2006.10.001. PMID: 24678103.

210. Bhala N, Emberson J, Merhi A, et al. Vascular and upper gastrointestinal effects of non-steroidal anti-inflammatory drugs: meta-analyses of individual participant data from randomised trials. Lancet. 2013 Aug 31;382(9894):769-79. doi: 10.1016/s01406736(13)60900-9. PMID: 23726390.

211. Puljak L, Marin A, Vrdoljak D, et al. Celecoxib for osteoarthritis. Cochrane Database Syst Rev. 2017 May 22;5:CD009865. doi: 10.1002/14651858.CD009865.pub2. PMID: 28530031.

212. Rostom A, Goldkind L, Laine L. Nonsteroidal anti-inflammatory drugs and hepatic toxicity: a systematic review of randomized controlled trials in arthritis patients. Clin Gastroenterol Hepatol. 2005 May;3(5):489-98. PMID: 15880319.

213. Goldstein JL, Eisen GM, Burke TA, et al. Dyspepsia tolerability from the patients' perspective: a comparison of celecoxib with diclofenac. Aliment Pharmacol Ther. 2002 Apr;16(4):819-27. PMID: 11929402.

214. Silverstein FE, Faich G, Goldstein JL, et al. Gastrointestinal toxicity with celecoxib vs nonsteroidal anti-inflammatory drugs for osteoarthritis and rheumatoid arthritis: the CLASS study: a randomized controlled trial. Celecoxib long-term arthritis safety study. JAMA. 2000 Sep 13;284(10):1247-55. PMID: 10979111.

215. Whelton A, Lefkowith JL, West CR, et al. Cardiorenal effects of celecoxib as compared with the nonsteroidal antiinflammatory drugs diclofenac and ibuprofen. Kidney Int. 2006 Oct;70(8):1495502. doi: 10.1038/sj.ki.5001766. PMID: 16941030. 
216. White WB, Faich G, Whelton A, et al. Comparison of thromboembolic events in patients treated with celecoxib, a cyclooxygenase-2 specific inhibitor, versus ibuprofen or diclofenac. Am J Cardiol. 2002 Feb 15;89(4):425-30. PMID: 11835924.

217. Becvar R, Urbanova Z, Vlasakova V, et al. Nabumetone induces less gastrointestinal mucosal changes than diclofenac retard. Clin Rheumatol. 1999;18(4):273-8. PMID: 10468165.

218. Eversmeyer W, Poland M, DeLapp RE, et al. Safety experience with nabumetone versus diclofenac, naproxen, ibuprofen, and piroxicam in osteoarthritis and rheumatoid arthritis. Am J Med. 1993 Aug 9;95(2A):10S-8S. PMID: 8356997.

219. Laine L, Harper S, Simon T, et al. A randomized trial comparing the effect of rofecoxib, a cyclooxygenase 2-specific inhibitor, with that of ibuprofen on the gastroduodenal mucosa of patients with osteoarthritis. Rofecoxib osteoarthritis endoscopy study group. Gastroenterology. 1999 Oct;117(4):776-83. PMID: 10500058.

220. Lister BJ, Poland M, DeLapp RE. Efficacy of nabumetone versus diclofenac, naproxen, ibuprofen, and piroxicam in osteoarthritis and rheumatoid arthritis. Am J Med. 1993 Aug 9;95(2A):2S-9S. PMID: 8356999.

221. Morgan GJ, Poland M, DeLapp RE. Efficacy and safety of nabumetone versus diclofenac, naproxen, ibuprofen, and piroxicam in the elderly. Am J Med. 1993 Aug 9;95(2A):19S-27S. PMID: 8356998.

222. Roth SH. Endoscopy-controlled study of the safety of nabumetone compared with naproxen in arthritis therapy. Am J Med. 1987 Oct 30;83(4B):25-30. PMID: 3318424.

223. Roth SH, Bennett R, Caldron P, et al. A longterm endoscopic evaluation of patients with arthritis treated with nabumetone vs naproxen. J Rheumatol. 1994 Jun;21(6):1118-23. PMID: 7932425.
224. Becker MC, Wang TH, Wisniewski L, et al. Rationale, design, and governance of Prospective Randomized Evaluation of Celecoxib Integrated Safety versus Ibuprofen Or Naproxen (PRECISION), a cardiovascular end point trial of nonsteroidal antiinflammatory agents in patients with arthritis. Am Heart J. 2009 Apr;157(4):60612. doi: 10.1016/j.ahj.2008.12.014. PMID: 19332185.

225. Cheung R, Cheng TT, Dong Y, et al. Incidence of gastroduodenal ulcers during treatment with celecoxib or diclofenac: pooled results from three 12-week trials in Chinese patients with osteoarthritis or rheumatoid arthritis. Int J Rheum Dis. 2010 May;13(2):151-7. doi: 10.1111/j.1756185X.2010.01463.x. PMID: 20536600.

226. Cryer B, Li C, Simon LS, et al. GIREASONS: a novel 6-month, prospective, randomized, open-label, blinded endpoint (PROBE) trial. Am J Gastroenterol. 2013 Mar;108(3):392-400. doi: 10.1038/ajg.2012.467. PMID: 23399552.

227. Farkouh ME, Kirshner H, Harrington RA, et al. Comparison of lumiracoxib with naproxen and ibuprofen in the Therapeutic Arthritis Research and Gastrointestinal Event Trial (TARGET), cardiovascular outcomes: randomised controlled trial. Lancet. 2004 Aug;364(9435):675-84. doi: 10.1016/S0140-6736(04)16894-3. PMID: 15325832.

228. Goldstein JL, Correa P, Zhao WW, et al. Reduced incidence of gastroduodenal ulcers with celecoxib, a novel cyclooxygenase-2 inhibitor, compared to naproxen in patients with arthritis. Am J Gastroenterol. 2001 Apr;96(4):1019-27. doi: 10.1111/j.15720241.2001.03740.x. PMID: CN-00347393.

229. Hawkey C, Laine L, Simon T, et al. Comparison of the effect of rofecoxib (a cyclooxygenase 2 inhibitor), ibuprofen, and placebo on the gastroduodenal mucosa of patients with osteoarthritis: a randomized, double-blind, placebo-controlled trial. The Rofecoxib osteoarthritis endoscopy multinational study group. Arthritis Rheum. 2000 Feb;43(2):370-7. doi: 10.1002/15290131(200002)43:2<370::AIDANR17>3.0.CO;2-D. PMID: 10693877. 
230. Hawkey CC, Svoboda P, FiedorowiczFabrycy IF, et al. Gastroduodenal safety and tolerability of lumiracoxib compared with Ibuprofen and celecoxib in patients with osteoarthritis. J Rheumatol. 2004a Sep;31(9):1804-10. PMID: 15338504.

231. Hawkey CJ, Farkouh M, Gitton X, et al. Therapeutic arthritis research and gastrointestinal event trial of lumiracoxib study design and patient demographics. Aliment Pharmacol Ther. 2004b Jul 01;20(1):51-63. PMID: 15225171.

232. Hunt RH, Harper S, Callegari P, et al. Complementary studies of the gastrointestinal safety of the cyclooxygenase-2-selective inhibitor etoricoxib. Aliment Pharmacol Ther. 2003 Jan;17(2):201-10. PMID: 12534404.

233. Laine L, Maller ES, Yu C, et al. Ulcer formation with low-dose enteric-coated aspirin and the effect of COX-2 selective inhibition: a double-blind trial. Gastroenterology. 2004 Aug;127(2):395402. PMID: 15300570.

234. MacDonald TM, Hawkey CJ, Ford I, et al. Randomized trial of switching from prescribed non-selective non-steroidal antiinflammatory drugs to prescribed celecoxib: the Standard care vs. Celecoxib Outcome Trial (SCOT).[Erratum appears in Eur Heart J. 2016 Dec 24;:; PMID: 28025195]. Eur Heart J. 2017 Jun 14;38(23):1843-50. doi: 10.1093/eurheartj/ehw387. PMID: 27705888.

235. MacDonald TM, Mackenzie IS, Wei L, et al. Methodology of a large prospective, randomised, open, blinded endpoint streamlined safety study of celecoxib versus traditional non-steroidal anti-inflammatory drugs in patients with osteoarthritis or rheumatoid arthritis: protocol of the standard care versus celecoxib outcome trial (SCOT). BMJ Open. 2013 Jan 29;3(1):e002295. doi: 10.1136/bmjopen-2012-002295. PMID: 23364320.

236. Nissen SE, Yeomans ND, Solomon DH, et al. Cardiovascular safety of celecoxib, naproxen, or ibuprofen for arthritis. N Engl J Med. 2016 Dec 29;375(26):2519-29. doi: 10.1056/NEJMoa1611593. PMID: 27959716.
237. Pisko EJ, Strader K, Rice D, et al. A 6month, double-blind study comparing nabumetone to naproxen in the treatment of osteoarthritis. Pharmatherapeutica. 1987b;5(2):90-8. PMID: 3310020.

238. Reed GW, Abdallah MS, Shao M, et al. Effect of aspirin coadministration on the safety of celecoxib, naproxen, or ibuprofen. J Am Coll Cardiol. 2018;71(16):1741-51. doi: 10.1016/j.jacc.2018.02.036. PMID: 29673465.

239. Roth SH, Bennett RE, Caldron PH, et al. Endoscopic evaluation of the long term effects of diclofenac sodium and naproxen in elderly patients with arthritis. Clin Drug Investig. 1995 Mar;9(3):171-9.

240. Schnitzer TJ, Burmester GR, Mysler E, et al. Comparison of lumiracoxib with naproxen and ibuprofen in the Therapeutic Arthritis Research and Gastrointestinal Event Trial (TARGET), reduction in ulcer complications: randomised controlled trial. Lancet. 2004 Aug 21-27;364(9435):665-74. PMID: 15325831.

241. Sikes DH, Agrawal NM, Zhao WW, et al. Incidence of gastroduodenal ulcers associated with valdecoxib compared with that of ibuprofen and diclofenac in patients with osteoarthritis. Eur J Gastroenterol Hepatol. 2002 Oct;14(10):1101-11. PMID: 12362101.

242. Solomon DH, Husni ME, Libby PA, et al. The risk of major NSAID toxicity with celecoxib, ibuprofen, or naproxen: a secondary analysis of the PRECISION Trial. Am J Med. 2017 Dec;130(12):1415-22.e4. doi: 10.1016/j.amjmed.2017.06.028. PMID: 28756267.

243. Solomon DH, Husni ME, Wolski KE, et al. Differences in safety of nonsteroidal antiinflammatory drugs in patients with osteoarthritis and patients with rheumatoid arthritis: a randomized clinical trial. Arthritis Rheumatol. 2018 Apr;70(4):537-46. doi: 10.1002/art.40400. PMID: 29266879. 
244. Temple AR, Benson GD, Zinsenheim JR, et al. Multicenter, randomized, double-blind, active-controlled, parallel-group trial of the long-term (6-12 months) safety of acetaminophen in adult patients with osteoarthritis. Clin Ther. 2006

Feb;28(2):222-35. doi:

10.1016/j.clinthera.2006.02.004. PMID: 16678643.

245. Yeomans ND, Graham DY, Husni ME, et al. Randomised clinical trial: gastrointestinal events in arthritis patients treated with celecoxib, ibuprofen or naproxen in the PRECISION trial. Aliment Pharmacol Ther. 2018 Jun;47(11):1453-63. doi:

10.1111/apt.14610. PMID: 29667211.

246. Sriuttha P, Sirichanchuen B, Permsuwan U. Hepatotoxicity of nonsteroidal antiinflammatory drugs: a systematic review of randomized controlled trials. Int J Hepatol. 2018 Jan 15;2018:5253623. doi: 10.1155/2018/5253623. PMID: 29568654.

247. Bannuru RR, Schmid CH, Kent DM, et al. Comparative effectiveness of pharmacologic interventions for knee osteoarthritis: a systematic review and network metaanalysis. Ann Intern Med. 2015 Jan 06;162(1):46-54. doi: 10.7326/M14-1231. PMID: 25560713.

248. Waldfogel JM, Nesbit SA, Dy SM, et al. Pharmacotherapy for diabetic peripheral neuropathy pain and quality of life: A systematic review. Neurology. 2017 May 16;88(20):1958-67. doi: 10.1212/WNL.0000000000003882. PMID: 28341643.

249. Lee YH, Song GG. Comparative efficacy and tolerability of duloxetine, pregabalin, and milnacipran for the treatment of fibromyalgia: a Bayesian network metaanalysis of randomized controlled trials. Rheumatol Int. 2016 May;36(5):663-72. doi: 10.1007/s00296-016-3468-5. PMID: 27000046.

250. Chou R, McDonagh M, Hartung D, et al. AHRQ Comparative Effectiveness Research. Opioid Treatments for Chronic Pain: A Systematic Review. Rockville, MD: Agency for Healthcare and Quality Research; Forthcoming.
251. Skelly A, Chou R, Turner J, et al. AHRQ Comparative Effectiveness Review. Noninvasive Nonpharmacologic Treatments for Chronic Pain: An Update. Rockville, MD: Agency for Healthcare and Research Quality; Forthcoming.

252. Amitripytline Hydrochloride. Micromedex ${ }^{\circledR}$ (electronic version). IBM Watson Health, Greenwood Village, Colorado.

https://www.micromedexsolutions.com/micr omedex2/librarian/PFDefaultActionId/evide ncexpert.DoIntegratedSearch?navitem=topH ome\&isToolPage=true\#. Accessed July 31, 2019.

253. Novartis. Trileptal (oxcarbazepine) [package insert] U.S. Food and Drug Administration website. Revised Jan 4, 2019.

https://www.accessdata.fda.gov/scripts/cder/ daf/index.cfm?event=overview.process\&Ap plNo=021014 Accessed July 31, 2019.

254. Ungprasert P, Cheungpasitporn W, Crowson $\mathrm{CS}$, et al. Individual non-steroidal antiinflammatory drugs and risk of acute kidney injury: A systematic review and metaanalysis of observational studies. Eur J Intern Med. 2015 May;26(4):285-91. doi: 10.1016/j.ejim.2015.03.008. PMID: 25862494.

255. Zhang X, Donnan PT, Bell S, et al. Nonsteroidal anti-inflammatory drug induced acute kidney injury in the community dwelling general population and people with chronic kidney disease: systematic review and meta-analysis. BMC nephrology. 2017 Aug 1;18(1):256. doi: 10.1186/s12882-0170673-8. PMID: 28764659.

256. J J and J Consumer Inc. Tylenol (acetaminophen) [package insert] U.S. Food and Drug Administration website. Revised Jan 2019.

https://www.accessdata.fda.gov/scripts/cder/ daf/index.cfm?event=overview.process\&Ap plNo=019872 Accessed July 31, 2019.

257. Acetaminophen.

https://www.drugs.com/monograph/acetami nophen.html. Accessed July 31, 2019.

258. Kansagara D, O'Neil M, Nugent S, et al. VA Evidence-based Synthesis Program Reports. Benefits and Harms of Cannabis in Chronic Pain or Post-traumatic Stress Disorder: A Systematic Review. Washington, DC: Department of Veterans Affairs; 2017. 
259. Chou R, Qaseem A, Snow V, et al.

Diagnosis and treatment of low back pain: a joint clinical practice guideline from the American College of Physicians and the American Pain Society. Ann Intern Med. 2007 Oct 2;147(7):478-91. doi: 10.7326/0003-4819-147-7-20071002000006. PMID: 17909209.

260. Schnitzer TJ. Update on guidelines for the treatment of chronic musculoskeletal pain. Clin Rheumatol. 2006;25 Suppl 1:S22-9. doi: 10.1007/s10067-006-0203-8. PMID: 16741783.

261. Hauser W, Thieme K, Turk DC. Guidelines on the management of fibromyalgia syndrome - a systematic review. Eur J Pain. 2010 Jan;14(1):5-10. doi:

10.1016/j.ejpain.2009.01.006. PMID: 19264521.

262. Cruccu G, Nurmikko TJ, Ernault E, et al. Superiority of capsaicin $8 \%$ patch versus oral pregabalin on dynamic mechanical allodynia in patients with peripheral neuropathic pain. Eur J Pain. 2017(pagination) PMID: CN-01442858 NEW.

263. Busse J, Guyatt G, Carrasco A. The 2017 Canadian Guideline for Opioids for Chronic Non-Cancer Pain. Hamilton, ON: McMaster University. 2017.
264. Peckham AM, Fairman KA, Sclar DA. AllCause and Drug-Related Medical Events Associated with Overuse of Gabapentin and/or Opioid Medications: A Retrospective Cohort Analysis of a Commercially Insured US Population. Drug Saf. 2018

Feb;41(2):213-28. doi: 10.1007/s40264-0170595-1. PMID: 28956286.

265. Coupland C, Dhiman P, Morriss R, et al. Antidepressant use and risk of adverse outcomes in older people: population based cohort study. BMJ. 2011 Aug 2;343:d4551. doi: 10.1136/bmj.d4551. PMID: 21810886.

266. McCrae JC, Morrison EE, MacIntyre IM, et al. Long-term adverse effects of paracetamol - a review. Br J Clin Pharmacol. 2018 Oct;84(10):2218-30. doi: 10.1111/bcp.13656. PMID: 29863746.

267. Roberts E, Delgado Nunes V, Buckner S, et al. Paracetamol: not as safe as we thought? A systematic literature review of observational studies. Ann Rheum Dis. 2016;75(3):552-9. doi: 10.1136/annrheumdis-2014-206914. PMID: 25732175. 


\section{Abbreviations and Acronyms}

\begin{tabular}{|c|c|}
\hline Acronym or Abbreviation & Definition \\
\hline ACR & American College of Rheumatology \\
\hline AHRQ & Agency for Healthcare Research and Quality \\
\hline ANCOVA & Analysis of Covariance \\
\hline API & Average pain intensity \\
\hline ARA & American Rheumatism Association \\
\hline AS & Ankylosing spondylitis \\
\hline ASQoL & Ankylosing Spondylitis Quality of Life \\
\hline BAl & Beck Anxiety Index \\
\hline BASFI & Bath Ankylosing Spondylitis Functional Index \\
\hline $\mathrm{BDI}$ & Beck Depression Inventory \\
\hline BID & Twice daily \\
\hline $\mathrm{BPI}$ & Brief Pain Inventory \\
\hline CBD & Cannabidiol \\
\hline CDC & Centers for Disease Control and Prevention \\
\hline CER & Comparative Effectiveness Review \\
\hline cGRP & calcitonin Gene-Related Peptide \\
\hline $\mathrm{Cl}$ & Confidence interval \\
\hline CMS & Centers for Medicare \& Medicaid Services \\
\hline CONSORT & Consolidated Standards of Reporting Trials \\
\hline cox-2 & Cyclooxygenase-2 \\
\hline $\mathrm{CV}$ & Cardiovascular \\
\hline DDS & Descriptor Differential Scale \\
\hline DMARD & Disease-Modifying Antirheumatic Drug \\
\hline EPC & Evidence-based Practice Center \\
\hline EQ-5D & Euro Quality of Life five-dimension \\
\hline FDA & Food and Drug Administration \\
\hline FIQ & Fibromyalgia Impact Questionnaire \\
\hline $\mathrm{Gl}$ & Gastrointestinal \\
\hline HAMD & Hamilton Rating Scale for Depression \\
\hline HAQ & Health Assessment Questionnaire \\
\hline IOM & Institute of Medicine \\
\hline IPD & Individual Patient Data \\
\hline IPRCC & Interagency Pain Research Coordinating Committee \\
\hline KQ & Key Question \\
\hline LSM & Least squares mean \\
\hline MAOI & Monoamine Oxidase Inhibitor \\
\hline MCID & Minimal Clinically Important Difference \\
\hline MD & Mean difference \\
\hline MHAQ & Modified Health Assessment Questionnaire \\
\hline MI & Myocardial infarction \\
\hline
\end{tabular}




\begin{tabular}{|c|c|}
\hline Acronym or Abbreviation & Definition \\
\hline NA & Not applicable \\
\hline NMDA & N-Methyl-D-aspartic acid \\
\hline NPS & National Pain Strategy \\
\hline NR & Not reported \\
\hline NRS & Numeric rating scale \\
\hline NSAID & Nonsteroidal anti-inflammatory drug \\
\hline $\mathrm{OA}$ & Osteoarthritis \\
\hline OARSI & Osteoarthritis Research Society International \\
\hline ODI & Oswestry Disability Index \\
\hline PICOTS & Population, Intervention, Comparison, Outcome, Time, Setting, Study design \\
\hline PROSPERO & International Prospective Register of Systematic Reviews \\
\hline QoL & Quality of life \\
\hline RA & Rheumatoid arthritis \\
\hline RCT & Randomized controlled trial \\
\hline RMDQ (RDQ) & Roland-Morris Disability Questionnaire \\
\hline $\mathrm{RR}$ & Relative risk; risk ratio \\
\hline SAE & Serious adverse event \\
\hline SD & Standard deviation \\
\hline SE & Standard error \\
\hline SEM & Standard error of the mean \\
\hline SF-36 (MCS, PCS) & Short Form-36 (Mental Component Summary, Physical Component Summary) \\
\hline SMD & Standardized mean difference \\
\hline SNRI & Serotonin-norepinephrine reuptake inhibitor \\
\hline SOE & Strength of evidence \\
\hline SR & Systematic review \\
\hline SSRI & Selective serotonin reuptake inhibitor \\
\hline TCA & Tricyclic antidepressant \\
\hline THC & Tetrahydrocannabinol \\
\hline TID & Three times daily \\
\hline US & United States \\
\hline VAS & Visual analog scale \\
\hline WAE & Withdrawal due to adverse event \\
\hline WOMAC & Western Ontario and McMaster Universities Osteoarthritis Index \\
\hline
\end{tabular}




\section{Appendix A. Literature Search Strategies}

\section{Randomized Controlled Trials}

\section{Database: Ovid MEDLINE(R) and Epub Ahead of Print, In-Process \& Other Non-Indexed Citations, Daily and Versions(R)}

1 (celecoxib or diclofenac or diflunisal or etodolac or fenoprofen or flurbiprofen or ibuprofen or indomethacin or ketoprofen or ketorolac or meclofenamate or "mefenamic acid" or meloxicam or nabumetone or naproxen or oxaprozin or piroxicam or salsalate or sulindac or tenoxicam or "tiaprofenic acid" or tolmetin).ab,kw,sh,ti.

2 (carbamazepine or gabapentin or oxcarbazepine or pregabalin).ab,kw,sh,ti.

3 (desvenlafaxine or duloxetine or levomilnacipran or milnacipran or venlafaxine).ab,kw,sh,ti.

4 (amitriptyline or desipramine or doxepin or imipramine or nortriptyline or alprazolam or chlordiazepoxide or clobazam or clonazepam or clorazepate or diazepam or estazolam or flurazepam or lorazepam or oxazepam or temazepam or triazolam or baclofen or carisoprodol or cyclobenzaprine or metaxalone or methocarboamol or tizanidine).ab,kw,sh,ti.

5 (acetaminophen or paracetamol or capsaicin or methocarbamol or cannabis or marijuana or cannabidiol or phytocannabinoid* or dronabinol or nabilone or memantine).ab,kw,sh,ti.

6 (topical adj2 lidocaine).ab,kw,ti.

7 or $1-6$

8 exp Neuralgia/

9 Fibromyalgia/

10 exp Anemia, Sickle Cell/

11 Headache/

12 exp Headache Disorders/

13 Musculoskeletal Pain/

14 exp Osteoarthritis/

15 Low Back Pain/

16 Neck Pain/

17 exp Arthritis, Rheumatoid/

18 Spondylitis, Ankylosing/

19 ("ankylosing spondylitis" or "neuropathic pain" or neuralgia or neuropathy or fibromyalgia or "sickle cell" or headache or "musculoskeletal pain" or osteoarthritis or "low back pain" or "neck pain" or "inflammatory pain" or "rheumatoid arthritis").ab,kw,ti.

20 or/8-19

$21 \quad 7$ and 20

22 randomized controlled trial.pt.

23 controlled clinical trial.pt.

24 clinical trials as topic.sh.

25 (random* or trial or placebo).ti,ab.

26 clinical trials as topic.sh.

27 exp animals/ not humans.sh.

28 or/22-26

2928 not 27

$30 \quad 21$ and 29 
31 limit 21 to randomized controlled trial

3230 or 31

33 limit 32 to (english language and humans)

\section{Database: EBM Reviews - Cochrane Central Register of Controlled Trials}

1 (celecoxib or diclofenac or diflunisal or etodolac or fenoprofen or flurbiprofen or ibuprofen or indomethacin or ketoprofen or ketorolac or meclofenamate or "mefenamic acid" or meloxicam or nabumetone or naproxen or oxaprozin or piroxicam or salsalate or sulindac or tenoxicam or "tiaprofenic acid" or tolmetin).ab,kw,sh,ti.

2 (carbamazepine or gabapentin or oxcarbazepine or pregabalin).ab,kw,sh,ti.

3 (desvenlafaxine or duloxetine or levomilnacipran or milnacipran or venlafaxine).ab,kw,sh,ti.

4 (amitriptyline or desipramine or doxepin or imipramine or nortriptyline or alprazolam or chlordiazepoxide or clobazam or clonazepam or clorazepate or diazepam or estazolam or flurazepam or lorazepam or oxazepam or temazepam or triazolam or baclofen or carisoprodol or cyclobenzaprine or metaxalone or methocarboamol or tizanidine).ab,kw,sh,ti.

5 (acetaminophen or paracetamol or capsaicin or methocarbamol or cannabis or marijuana or cannabidiol or phytocannabinoid* or dronabinol or nabilone or memantine).ab,kw,sh,ti.

6 (topical adj2 lidocaine).ab,kw,ti.

7 or/1-6

8 exp Neuralgia/

9 Fibromyalgia/

10 exp Anemia, Sickle Cell/

11 Headache/

12 exp Headache Disorders/

13 Musculoskeletal Pain/

14 exp Osteoarthritis/

15 Low Back Pain/

16 Neck Pain/

17 exp Arthritis, Rheumatoid/

18 Spondylitis, Ankylosing/

19 ("ankylosing spondylitis" or "neuropathic pain" or neuralgia or neuropathy or fibromyalgia or "sickle cell" or headache or "musculoskeletal pain" or osteoarthritis or "low back pain" or "neck pain" or "inflammatory pain" or "rheumatoid arthritis").ab,kw,ti.

20 or/8-19

$21 \quad 7$ and 20

$22 \quad 21$ not acute.ti.

23 limit 22 to english language

\section{Database: PsycINFO}

1 (celecoxib or diclofenac or diflunisal or etodolac or fenoprofen or flurbiprofen or ibuprofen or indomethacin or ketoprofen or ketorolac or meclofenamate or "mefenamic acid" or meloxicam or nabumetone or naproxen or oxaprozin or piroxicam or salsalate or sulindac or tenoxicam or "tiaprofenic acid" or tolmetin).ab,kw,sh,ti.

2 (carbamazepine or gabapentin or oxcarbazepine or pregabalin).ab,kw,sh,ti.

3 (desvenlafaxine or duloxetine or levomilnacipran or milnacipran or venlafaxine).ab,kw,sh,ti. 
4 (amitriptyline or desipramine or doxepin or imipramine or nortriptyline or alprazolam or chlordiazepoxide or clobazam or clonazepam or clorazepate or diazepam or estazolam or flurazepam or lorazepam or oxazepam or temazepam or triazolam or baclofen or carisoprodol or cyclobenzaprine or metaxalone or methocarboamol or tizanidine).ab,kw,sh,ti.

5 (acetaminophen or paracetamol or capsaicin or methocarbamol or cannabis or marijuana or cannabidiol or phytocannabinoid* or dronabinol or nabilone or memantine).ab,kw,sh,ti.

6 (topical adj2 lidocaine).ab,kw,ti.

7 or $1-6$

8 exp NEURALGIA/

9 exp chronic pain/

10 exp headache/

11 exp Back Pain/

12 sickle cell disease/

13 exp ARTHRITIS/

14 fibromyalgia/

15 ("ankylosing spondylitis" or "neuropathic pain" or neuralgia or neuropathy or fibromyalgia or "sickle cell" or headache or "musculoskeletal pain" or osteoarthritis or "low back pain" or "neck pain" or "inflammatory pain" or "rheumatoid arthritis").ab,hw,ti.

16 or/8-15

$17 \quad 7$ and 16

1817 and (random* or control* or trial).ti,ab.

19 limit 18 to english language

\section{Elsevier Embase}

(celecoxib:ti OR diclofenac:ti OR diflunisal:ti OR etodolac:ti OR fenoprofen:ti OR flurbiprofen:ti OR ibuprofen:ti OR indomethacin:ti OR ketoprofen:ti OR ketorolac:ti OR meclofenamate:ti OR 'mefenamic acid':ti OR meloxicam:ti OR nabumetone:ti OR naproxen:ti OR oxaprozin:ti OR piroxicam:ti OR salsalate:ti OR sulindac:ti OR tenoxicam:ti OR 'tiaprofenic acid':ti OR tolmetin:ti OR celecoxib:ab OR diclofenac:ab OR diflunisal:ab OR etodolac:ab OR fenoprofen:ab OR flurbiprofen:ab OR ibuprofen:ab OR indomethacin:ab OR ketoprofen:ab OR ketorolac:ab OR meclofenamate:ab OR 'mefenamic acid':ab OR meloxicam:ab OR nabumetone:ab OR naproxen:ab OR oxaprozin:ab OR piroxicam:ab OR salsalate:ab OR sulindac:ab OR tenoxicam:ab OR 'tiaprofenic acid':ab OR tolmetin:ab OR carbamazepine:ti OR gabapentin:ti OR oxcarbazepine:ti OR pregabalin:ti OR carbamazepine:ab OR gabapentin:ab OR oxcarbazepine:ab OR pregabalin:ab OR desvenlafaxine:ti OR duloxetine:ti OR

levomilnacipran:ti OR milnacipran:ti OR venlafaxine:ti OR desvenlafaxine:ab OR duloxetine:ab OR levomilnacipran:ab OR milnacipran:ab OR venlafaxine:ab OR amitriptyline:ti OR desipramine:ti OR doxepin:ti OR imipramine:ti OR nortriptyline:ti OR amitriptyline:ab OR desipramine:ab OR doxepin:ab OR imipramine:ab OR nortriptyline:ab OR alprazolam:ti OR chlordiazepoxide:ti OR clobazam:ti OR clonazepam:ti OR clorazepate:ti OR diazepam:ti OR estazolam:ti OR flurazepam:ti OR lorazepam:ti OR oxazepam:ti OR temazepam:ti OR triazolam:ti OR baclofen:ti OR carisoprodol:ti OR cyclobenzaprine:ti OR metaxalone:ti OR methocarboamol:ti OR tizanidine:ti OR alprazolam:ab OR chlordiazepoxide:ab OR clobazam:ab OR clonazepam:ab OR clorazepate:ab OR diazepam:ab OR estazolam:ab OR flurazepam:ab OR lorazepam:ab OR oxazepam:ab OR temazepam:ab OR triazolam:ab OR baclofen:ab OR carisoprodol:ab OR cyclobenzaprine:ab OR metaxalone:ab OR methocarboamol:ab OR 
tizanidine:ab OR acetaminophen:ti OR paracetamol:ti OR acetaminophen:ab OR paracetamol:ab OR capsaicin:ti OR capsaicin:ab OR methocarbamol;ti OR methocarbamol:ab OR marijuana:ti OR cannabidiol:ti OR phytocannabinoid:ti OR dronabinol:ti OR nabilone:ti OR marijuana:ab OR cannabidiol:ab OR phytocannabinoid:ab OR dronabinol:ab OR nabilone:ab OR memantine:ti OR memantine:ab OR (lidocaine:ti AND topical) OR (lidocaine:ab AND topical)) AND ('neuropathic pain':ti OR fibromyalgia:ti OR 'sickle cell':ti OR headache:ti OR 'musculoskeletal pain':ti OR osteoarthrtis:ti OR 'low back pain':ti OR 'neck pain':ti OR 'inflammatory pain':ti OR 'rheumatoid arthritis':ti OR 'neuropathic pain':ab OR fibromyalgia:ab OR 'sickle cell':ab OR headache:ab OR 'musculoskeletal pain':ab OR osteoarthrtis:ab OR 'low back pain':ab OR 'neck pain':ab OR 'inflammatory pain':ab OR 'rheumatoid arthritis':ab) AND ('clinical trial'/de OR 'randomized controlled trial'/de OR 'randomization'/de OR 'single blind procedure'/de OR 'double blind procedure'/de OR 'crossover procedure'/de OR 'placebo'/de OR 'prospective study'/de OR ('randomi?ed controlled' NEXT/1 trial*) OR rct OR 'randomly allocated' OR 'allocated randomly' OR 'random allocation' OR (allocated NEAR/2 random) OR (single NEXT/1 blind*) OR (double NEXT/1 blind*) OR ((treble OR triple) NEAR/1 blind*) OR placebo*) AND [humans]/lim AND [english]/lim AND [embase]/lim NOT ([embase]/lim AND [medline]/lim)

\section{Systematic Reviews}

Database: Ovid MEDLINE(R) and Epub Ahead of Print, In-Process \& Other Non-Indexed Citations, Daily and Versions(R)

1 (celecoxib or diclofenac or diflunisal or etodolac or fenoprofen or flurbiprofen or ibuprofen or indomethacin or ketoprofen or ketorolac or meclofenamate or "mefenamic acid" or meloxicam or nabumetone or naproxen or oxaprozin or piroxicam or salsalate or sulindac or tenoxicam or "tiaprofenic acid" or tolmetin).ab,kw,sh,ti.

2 (carbamazepine or gabapentin or oxcarbazepine or pregabalin).ab,kw,sh,ti.

3 (desvenlafaxine or duloxetine or levomilnacipran or milnacipran or venlafaxine).ab,kw,sh,ti.

4 (amitriptyline or desipramine or doxepin or imipramine or nortriptyline or alprazolam or chlordiazepoxide or clobazam or clonazepam or clorazepate or diazepam or estazolam or flurazepam or lorazepam or oxazepam or temazepam or triazolam or baclofen or carisoprodol or cyclobenzaprine or metaxalone or methocarboamol or tizanidine).ab,kw,sh,ti.

5 (acetaminophen or paracetamol or capsaicin or methocarbamol or cannabis or marijuana or cannabidiol or phytocannabinoid* or dronabinol or nabilone or memantine).ab,kw,sh,ti.

6 (topical adj2 lidocaine).ab,kw,ti.

7 or $1-6$

8 exp Neuralgia/

9 Fibromyalgia/

10 exp Anemia, Sickle Cell/

11 Headache/

12 exp Headache Disorders/

13 Musculoskeletal Pain/

14 exp Osteoarthritis/

15 Low Back Pain/

16 Neck Pain/

17 exp Arthritis, Rheumatoid/ 
18 ("ankylosing spondylitis" or "neuropathic pain" or neuralgia or neuropathy or fibromyalgia or "sickle cell" or headache or "musculoskeletal pain" or osteoarthrtis or "low back pain" or "neck pain" or "inflammatory pain" or "rheumatoid arthritis").ab,kw,ti.

19 or/8-18

$20 \quad 7$ and 19

21 meta-analysis.pt.

22 meta-analysis/ or systematic review/ or meta-analysis as topic/ or "meta analysis (topic)"/ or "systematic review (topic)"/ or exp technology assessment, biomedical/

23 ((systematic* adj3 (review* or overview*)) or (methodologic* adj3 (review* or overview*))).ti,ab.

24 ((quantitative adj3 (review* or overview* or synthes*)) or (research adj3 (integrati* or overview*))).ti,ab.

25 ((integrative adj3 (review* or overview*)) or (collaborative adj3 (review* or overview*)) or (pool* adj3 analy*)).ti,ab.

26 (data synthes* or data extraction* or data abstraction*).ti,ab.

27 (handsearch* or hand search*).ti,ab.

28 (mantel haenszel or peto or der simonian or dersimonian or fixed effect* or latin square*).ti,ab.

29 (met analy* or metanaly* or technology assessment* or HTA or HTAs or technology overview* or technology appraisal*).ti,ab.

30 (meta regression* or metaregression*).ti,ab.

31 (meta-analy* or metaanaly* or systematic review* or biomedical technology assessment* or bio-medical technology assessment*).mp,hw.

32 (medline or cochrane or pubmed or medlars or embase or cinahl).ti,ab,hw.

33 (cochrane or (health adj2 technology assessment) or evidence report).jw.

34 (meta-analysis or systematic review).ti,ab.

35 (comparative adj3 (efficacy or effectiveness)).ti,ab.

36 (outcomes research or relative effectiveness).ti,ab.

37 ((indirect or indirect treatment or mixed-treatment) adj comparison*).ti,ab.

38 or/21-37

$39 \quad 20$ and 38

40 limit 20 to (meta analysis or systematic reviews)

4139 or 40

42 limit 41 to yr="2008 -Current"

43 limit 42 to english language

\section{Database: EBM Reviews - Cochrane Database of Systematic Reviews}

1 (celecoxib or diclofenac or diflunisal or etodolac or fenoprofen or flurbiprofen or ibuprofen or indomethacin or ketoprofen or ketorolac or meclofenamate or "mefenamic acid" or meloxicam or nabumetone or naproxen or oxaprozin or piroxicam or salsalate or sulindac or tenoxicam or "tiaprofenic acid" or tolmetin).ab,ti.

2 (brivaracetam or carbamazepine or divalproex or "eslicarbazepine acetate" or ethotoin or gabapentin or lacosamide or lamotrigine or levetiracetam or oxcarbazepine or perampanel or phenytoin or pregabalin or tiagabine or topiramate or "valproic acid" or zonisamide).ab,ti. 
3 (bupropion or citalopram or desvenlafaxine or duloxetine or escitalopram or fluoxetine or fluvoxamine or levomilnacipran or mirtazapine or nefazodone or paroxetine or sertraline or trazodone or venlafaxine or vilazodone or vortioxetine).ab,ti.

4 (amitriptyline or desipramine or imipramine or nortriptyline or baclofen or carisoprodol or cyclobenzaprine or metaxalone or methocarboamol or tizanidine or alprazolam or chlordiazepoxide or clobazam or clonazepam or clorazepate or diazepam or estazolam or flurazepam or lorazepam or oxazepam or temazepam or triazolam).ab,ti.

5 (acetaminophen or paracetamol or capsaicin or methocarbamol or cannabis or marijuana or cannabidiol or phytocannabinoid* or dronabinol or nabilone or memantine).ab,ti.

6 (topical adj2 lidocaine).ab,ti.

7 or/1-6

8 ("ankylosing spondylitis" or "neuropathic pain" or neuralgia or neuropathy or fibromyalgia or "sickle cell" or headache or "musculoskeletal pain" or osteoarthrtis or "low back pain" or "neck pain" or "inflammatory pain" or "rheumatoid arthritis").ab,ti.

97 and 8

\section{Elsevier Embase}

(celecoxib:ti OR diclofenac:ti OR diflunisal:ti OR etodolac:ti OR fenoprofen:ti OR flurbiprofen:ti OR ibuprofen:ti OR indomethacin:ti OR ketoprofen:ti OR ketorolac:ti OR meclofenamate:ti OR 'mefenamic acid':ti OR meloxicam:ti OR nabumetone:ti OR naproxen:ti OR oxaprozin:ti OR piroxicam:ti OR salsalate:ti OR sulindac:ti OR tenoxicam:ti OR 'tiaprofenic acid':ti OR tolmetin:ti OR celecoxib:ab OR diclofenac:ab OR diflunisal:ab OR etodolac:ab OR fenoprofen:ab OR flurbiprofen:ab OR ibuprofen:ab OR indomethacin:ab OR ketoprofen:ab OR ketorolac:ab OR meclofenamate:ab OR 'mefenamic acid':ab OR meloxicam:ab OR nabumetone:ab OR naproxen:ab OR oxaprozin:ab OR piroxicam:ab OR salsalate:ab OR sulindac:ab OR tenoxicam:ab OR 'tiaprofenic acid':ab OR tolmetin:ab OR carbamazepine:ti OR gabapentin:ti OR oxcarbazepine:ti OR pregabalin:ti OR carbamazepine:ab OR gabapentin:ab OR oxcarbazepine:ab OR pregabalin:ab OR desvenlafaxine:ti OR duloxetine:ti OR levomilnacipran:ti OR milnacipran:ti OR venlafaxine:ti OR desvenlafaxine:ab OR duloxetine:ab OR levomilnacipran:ab OR milnacipran:ab OR venlafaxine:ab OR amitriptyline:ti OR desipramine:ti OR doxepin:ti OR imipramine:ti OR nortriptyline:ti OR amitriptyline:ab OR desipramine:ab OR doxepin:ab OR imipramine:ab OR nortriptyline:ab OR alprazolam:ti OR chlordiazepoxide:ti OR clobazam:ti OR clonazepam:ti OR clorazepate:ti OR diazepam:ti OR estazolam:ti OR flurazepam:ti OR lorazepam:ti OR oxazepam:ti OR temazepam:ti OR triazolam:ti OR baclofen:ti OR carisoprodol:ti OR cyclobenzaprine:ti OR metaxalone:ti OR methocarboamol:ti OR tizanidine:ti OR alprazolam:ab OR chlordiazepoxide:ab OR clobazam:ab OR clonazepam:ab OR clorazepate:ab OR diazepam:ab OR estazolam:ab OR flurazepam:ab OR lorazepam:ab OR oxazepam:ab OR temazepam:ab OR triazolam:ab OR baclofen:ab OR carisoprodol:ab OR cyclobenzaprine:ab OR metaxalone:ab OR methocarboamol:ab OR tizanidine:ab OR acetaminophen:ti OR paracetamol:ti OR acetaminophen:ab OR paracetamol:ab OR capsaicin:ti OR capsaicin:ab OR methocarbamol;ti OR methocarbamol:ab OR marijuana:ti OR cannabidiol:ti OR phytocannabinoid:ti OR dronabinol:ti OR nabilone:ti OR marijuana:ab OR cannabidiol:ab OR phytocannabinoid:ab OR dronabinol:ab OR nabilone:ab OR memantine:ti OR memantine:ab OR (lidocaine:ti AND topical) OR (lidocaine:ab AND topical)) AND ('neuropathic pain':ti OR fibromyalgia:ti OR 'sickle cell':ti OR headache:ti OR 'musculoskeletal pain':ti OR osteoarthrtis:ti OR 'low back pain':ti OR 'neck pain':ti OR 'inflammatory pain':ti OR 
'rheumatoid arthritis':ti OR 'neuropathic pain':ab OR fibromyalgia:ab OR 'sickle cell':ab OR headache:ab OR 'musculoskeletal pain':ab OR osteoarthrtis:ab OR 'low back pain':ab OR 'neck pain':ab OR 'inflammatory pain':ab OR 'rheumatoid arthritis':ab) AND ('systematic review' OR 'meta analysis') AND (2008:py OR 2009:py OR 2010:py OR 2011:py OR 2012:py OR 2013:py OR 2014:py OR 2015:py OR 2016:py OR 2017:py OR 2018:py) AND [embase]/lim NOT ([embase]/lim AND [medline]/lim 


\section{Appendix B. Methods}

Citations for Appendix B references appear in Appendix $\mathrm{J}$.

This Comparative Effectiveness Review (CER) follows the methods suggested in the Agency for Healthcare Research and Quality (AHRQ) Methods Guide for Effectiveness and Comparative Effectiveness Reviews (hereafter "AHRQ Methods Guide"). ${ }^{1}$ All methods were determined $a$ priori, and a protocol was published on the AHRQ website (https://effectivehealthcare.ahrq.gov/topics/nonopioid-chronic-pain/protocol) and on PROSPERO systematic reviews registry (registration no. CRD42019134249).

\section{Key Questions}

\section{Key Question 1. Effectiveness and Comparative Effectiveness}

a. In patients with chronic pain, what is the effectiveness of nonopioid pharmacologic agents versus placebo for outcomes related to pain, function, and quality of life, after short-term treatment duration (3 to 6 months), intermediate-term treatment duration (6 to 12 months), and long-term treatment duration ( $\geq 12$ months)?

b. In patients with chronic pain, what is the comparative effectiveness of nonopioid pharmacologic agents compared to other nonopioid pharmacologic agents for outcomes related to pain, function, and quality of life, after short-term treatment duration (3 to 6 months), intermediate-term treatment duration (6 to 12 months), and long-term treatment duration ( $\geq 12$ months)?

c. How does effectiveness or comparative effectiveness vary depending on: (1) the specific type or cause of pain, (2) patient demographics, (3) patient comorbidities, (4) the dose of medication used, (5) the duration of treatment, and (6) dose titration, including tapering.

\section{Key Question 2. Harms and Adverse Events}

a. In patients with chronic pain, what are the risks of nonopioid pharmacologic agents for harms including overdose, misuse, dependence, withdrawals due to adverse events, and serious adverse events (including falls, fractures, motor vehicle accidents), and specific adverse events, according to drug class?

b. How do harms vary depending on: (1) the specific type or cause of pain, (2) patient demographics, (3) patient comorbidities, (4) the dose of medication used, (5) the duration of treatment, and (6) dose titration, including tapering.

\section{Criteria for Inclusion/Exclusion of Studies in the Review}

\section{Population(s):}

- For all Key Questions (KQs): Adults (age $\geq 18$ years) with various types of chronic pain (defined as pain lasting >3 months), including patients with acute exacerbations of chronic pain, pregnant/breastfeeding women, and patients with opioid use disorder

- For KQs 1c, 2b: Subgroups of the above patient populations as defined by specific pain condition (neuropathic pain, musculoskeletal pain, fibromyalgia, inflammatory arthritis, 
and chronic headache), patient demographics (e.g., age, race, ethnicity, and sex), comorbidities and degree of nociplasticity/central sensitization.

\section{Interventions:}

- Oral pharmacologic agents: nonsteroidal anti-inflammatory drugs, acetaminophen, muscle relaxants (including benzodiazepines), antidepressants, and anticonvulsants

- Topical pharmacologic agents: diclofenac, capsaicin, and lidocaine

- Medical cannabis (any formulation)

\section{Comparators:}

- For KQ 1a/c and KQ2: Placebo (effectiveness)

- For KQ 1b/c and KQ2: Another included nonopioid pharmacologic agent, different doses, or treatment durations (comparative effectiveness)

\section{Outcomes:}

- KQ 1: Pain (intensity, severity, bothersomeness), function (physical disability, activity limitations, activity interference, work function), and quality of life (including depression)

o Only validated scales for assessments of pain, function, and quality of life

- KQ 2: For all drug classes: overdose, misuse, dependence, withdrawals due to adverse events, and serious adverse events. Specific adverse events for each drug class, such as gastrointestinal events, cardiovascular events, and liver or kidney-related harms for nonsteroidal anti-inflammatory drugs; weight gain, sedation, and cognitive effects for gabapentin and pregabalin, etc.

\section{Timing:}

- Short-term treatment duration (3 to 6 months), intermediate-term treatment duration (6 to 12 months), and long-term treatment duration ( $\geq 12$ months)

- We will assess available literature to ensure that adequate evidence exists from studies of $\geq 3$ months' treatment duration. If adequate evidence is not available for this shorterduration, we will consider adding shorter-duration studies. If high-quality systematic reviews are available covering the scope of the review for shorter duration studies, we will summarize these in this case.

\section{Settings:}

- Outpatient settings (e.g., primary care, pain clinics, other specialty clinics) 
Table B-1. PICOTS: Inclusion and exclusion criteria

\begin{tabular}{|c|c|c|}
\hline PICOTS & Inclusion Criteria & Exclusion Criteria \\
\hline $\begin{array}{l}\text { Populations } \\
\text { and } \\
\text { Conditions }\end{array}$ & $\begin{array}{l}\text { - For all KQs: Adults (age } \geq 18 \text { years) with chronic } \\
\text { pain (pain lasting }>3 \text { months). } \\
\text { - For KQs } 1 \text { b, } 2 \text { b Specific chronic pain populations: } \\
\text { - Neuropathic } \\
\text { - Musculoskeletal (e.g. low back pain, } \\
\text { osteoarthritis) } \\
\text { - Fibromyalgia (assessed using established } \\
\text { criteria) } \\
\text { - } \text { Sickle cell disease } \\
\text { - } \text { Inflammatory arthritis (e.g., rheumatoid arthritis) }\end{array}$ & $\begin{array}{l}\text { - Pain at the end of life (life expectancy } \leq 6 \\
\text { months) } \\
\text { - Acute pain (<8 weeks duration), } \\
\text { including sickle cell crisis } \\
\text { - Pain due to active malignancy (e.g. } \\
\text { tumor-related pain while receiving active } \\
\text { treatment to reduce tumor size) } \\
\text { - Episodic migraine } \\
\text { - Undefined mixed pain conditions }\end{array}$ \\
\hline Interventions & $\begin{array}{l}\text { Nonopioid pharmacologic drugs for chronic pain: } \\
\text { - Oral pharmacologic agents specifically used to treat } \\
\text { chronic pain: } \\
\text { - NSAIDs (e.g., celecoxib, diclofenac, ibuprofen) } \\
\text { - Antidepressants SNRIs (i.e., duloxetine, } \\
\text { milnacipran) and TCAs (e.g., amitriptyline) } \\
\text { - Anticonvulsants: carbamazepine, gabapentin, } \\
\text { oxcarbazepine, pregabalin } \\
\text { - Other: Acetaminophen, muscle relaxants (e.g., } \\
\text { cyclobenzaprine, diazepam), memantine } \\
\text { - Topical agents (diclofenac, capsaicin, and lidocaine) } \\
\text { - Medical cannabis in all forms, including } \\
\text { phytocannabinoids and synthetic cannabinoids }\end{array}$ & $\begin{array}{l}\text { - Injectable preparations, including } \\
\text { biologic drugs, corticosteroids, etc. } \\
\text { - Other antidepressants (e.g. SSRIs, } \\
\text { MAOIs) } \\
\text { - Other antiepileptics (e.g. topiramate, } \\
\text { lamotrigine, levetiracetam, phenytoin) } \\
\text { - Drugs used for migraine prophylaxis } \\
\text { (e.g., verapamil, beta-blockers) or } \\
\text { treating acute migraine (e.g., triptans) } \\
\text { - Salicylates (topical and oral) } \\
\text { - Topical menthol preparations } \\
\text { - Disease-modifying drugs for rheumatoid } \\
\text { arthritis (DMARDs, e.g. methotrexate) }\end{array}$ \\
\hline Comparators & $\begin{array}{l}\text { - For KQ } 1 \mathrm{a} / \mathrm{b} \text { and } 2 \mathrm{a} / \mathrm{b} \text { : Placebo } \\
\text { - For KQ } 1 \mathrm{c} \text { and } 2 \mathrm{a} / \mathrm{b} \text { : Another included nonopioid } \\
\text { pharmacologic agent, dose, or treatment duration }\end{array}$ & $\begin{array}{l}\text { - Nonpharmacologic treatment } \\
\text { (comparison to nonopioids included in } \\
\text { review of nonpharmacologic treatments) } \\
\text { - Opioid treatment }\end{array}$ \\
\hline Outcomes & $\begin{array}{l}\text { - Pain, function, and quality of life using validated } \\
\text { outcome measures. } \\
\text { - Pain severity is the measure of improvement in } \\
\text { pain from baseline, as a continuous measure. } \\
\text { Pain response is a categorical value (yes/no) of } \\
\text { significant improvement, e.g. 30\% improvement } \\
\text { on a 0-100 scale. } \\
\text { - Pain assessments are patient-reported. Clinician } \\
\text { assessments were also acceptable, and noted } \\
\text { where they are reported. } \\
\text { - Secondary outcomes include mood, sleep, and } \\
\text { global assessments using validated scales } \\
\text { - All drug classes: Withdrawal from treatment due to } \\
\text { adverse events (any adverse event, not specifically } \\
\text { symptoms of withdrawal from an opioid or other } \\
\text { drug), incidence of serious adverse events, } \\
\text { overdose, misuse, addiction, development of SUD. } \\
\text { - Key specific adverse events according to drug class } \\
\text { (e.g., gastrointestinal and cardiovascular events, } \\
\text { kidney and liver-related harms with NSAIDs). }\end{array}$ & $\begin{array}{l}\text { - Intermediate outcomes (e.g., } \\
\text { pharmacokinetics/pharmacodynamics, } \\
\text { drug-drug interactions, dose } \\
\text { conversions) } \\
\text { - Indirect measurement of pain, e.g. } \\
\text { quantitative sensory testing. }\end{array}$ \\
\hline Timing & $\begin{array}{l}\text { - Short- ( } 3 \text { to } 6 \text { months), intermediate- (6 to } 12 \\
\text { months), and long-term ( } \geq 12 \text { months) treatment } \\
\text { duration }\end{array}$ & $\begin{array}{l}\text { - Studies or outcomes reported with }<3 \text { - } \\
\text { month duration of treatment }\end{array}$ \\
\hline Setting & $\begin{array}{l}\text { - Outpatient settings (e.g., primary care, pain clinics, } \\
\text { emergency rooms, urgent care clinics) }\end{array}$ & $\begin{array}{l}\text { - Addiction treatment settings, inpatient } \\
\text { settings }\end{array}$ \\
\hline
\end{tabular}




\begin{tabular}{|l|l|l|}
\hline PICOTS & Inclusion Criteria & Exclusion Criteria \\
\hline $\begin{array}{l}\text { Study } \\
\text { Design }\end{array}$ & $\begin{array}{l}\text { Randomized controlled trials } \\
\text { High-quality, recent systematic reviews that best } \\
\text { match the scope of this review } \\
\text { English language publications }\end{array}$ & $\begin{array}{l}\bullet \text { Observational studies } \\
\text { Outdated/out of scope systematic } \\
\text { reviews }\end{array}$ \\
\hline
\end{tabular}

$\mathrm{CBD}$ = cannabidiol; KQ = Key Question; MAOI = monoamine oxidase inhibitor; NSAID = nonsteroidal anti-inflammatory drug; SNRI = serotonin and norepinephrine reuptake inhibitor; SSRI = selective serotonin reuptake inhibitor; TCA = tricyclic antidepressant; THC = tetrahydrocannabinol

${ }^{a}$ Chronic headache defined as (International Classification of Headache Disorders, 3rd edition definition²):

Primary headaches attributed to the headache condition itself, not caused by another disease or medical condition. Chronic headache is defined as 15 or more days each month for at least 12 weeks or history of headache more than 180 days a year.

\section{Literature Search}

We conducted electronic searches in Ovid ${ }^{\circledR}$ MEDLINE $^{\circledR}$, Embase $^{\circledR}$, PsycINFO $^{\circledR}$, CINAHL $^{\circledR}$, Cochrane CENTRAL, and Cochrane Database of Systematic Reviews in January 2019 (from database inception, see Appendix A for full strategies). Reference lists of included systematic reviews were screened for includable studies. Manufacturers of included drugs submitted potential relevant studies to include in this review using a Federal Register notification. We screened citations identified through our searched using the pre-established criteria above to determine eligibility for full-text review, with any citation deemed not relevant by one reviewer screened by a second reviewer. ${ }^{1}$ Citations deemed potentially eligible were retrieved for full-text screening, with each article independently reviewed for eligibility by two reviewers. Any disagreements were resolved by consensus. Prior to the final report, we will update these searches and incorporate any new eligible studies into the report.

\section{Study Design}

For all Key Questions, we included and focused on randomized controlled trials (RCTs) with at least 3 months duration to maintain a manageable scope for this review, recognizing that by definition, chronic pain requires treatments that are effective in the long term, and short-term benefits may not persist. This duration threshold is similar to the duration used in the prior AHRQ systematic review on nonpharmacologic interventions for chronic pain, ${ }^{3}$ which included studies with greater than 1 month of followup after the end of treatment, with most studies involving 6 to 8 weeks of treatment. The Evidence-based Practice Center (EPC) evaluated the availability and quality of studies with 3 to 6 months duration to determine if an evaluation of studies with shorter durations was needed. It was deemed that adequate evidence was found in this window of duration and thus we did not include studies with shorter durations. However, existing systematic reviews to summarize evidence where possible.

We evaluated the persistence of benefits or harms by evaluating the three periods identified in the Key Questions ( 3 to 6 months, 6 to 12 months, and $\geq 12$ months). We used existing systematic reviews primarily to screen their included studies to insure we have identified all relevant studies for this review. In the case where a systematic review is recent enough to cover the majority of the available evidence, and evaluates a cohesive group of interventions, outcomes and time frames included here, we included the review as the primary evidence and supplement with any newer or excluded studies. 


\section{Non-English Language Studies}

We restricted to English-language articles, but reviewed English-language abstracts of nonEnglish language articles to identify studies that would otherwise meet inclusion criteria, in order to assess for the likelihood of language bias.

\section{Data Abstraction and Data Management}

For studies meeting inclusion criteria, data were abstracted and dual-reviewed by independent investigators in multiple-parts. Data regarding general study characteristics were abstracted into forms as seen in Appendix E. Information abstracted in these evidence tables included author, year of publication, country, study quality, pain condition, age, sex, race/ethnicity, duration of chronic pain, severity of pain and function at baseline, presence of psychiatric, total randomized and analyzed, intervention characteristics (including the specific drug class and dose), duration of treatment, and funding source. For clarity, data used for metaanalysis were abstracted into separate forms, pooled and synthesized (Appendix F). Methods regarding abstracting data for synthesis are detailed below. Data from studies included in a systematic review that met our inclusion criteria were abstracted from the published article with missing data supplemented by systematic reviews.

Effectiveness and comparative effectiveness data were organized by pain condition with effects on pain abstracted as mean difference in pain intensity (continuous) and pain improvement (dichotomous) based on meeting specified thresholds ("pain response”). Pain conditions were categorized as neuropathic (diabetic peripheral neuropathy, post-herpetic neuralgia, other), fibromyalgia, osteoarthritis, inflammatory arthritis (e.g., rheumatoid arthritis or ankylosing spondylitis), spinal pain (neck or low-back pain), sickle cell disease, or chronic headache. We preferentially abstracted pain assessed with the visual analog scale (VAS) or numerical rating scale (NRS) on a scale of 0-10 or 0-100 over other pain assessments (e.g., Western Ontario and McMaster Universities Osteoarthritis Index pain subscale). Mean differences in effects on pain from baseline to followup, unadjusted differences in change from baseline, and differences (both within- and between-group) in followup scores were abstracted for meta-analysis. Primary pain response was defined as $\geq 30 \%$ improvement (reduction) in pain score. Secondary pain response criteria included $>30 \%$ improvement (e.g., $\geq 50 \%$ improvement), condition-specific composite measure (e.g., American College of Rheumatology 20 criteria [ACR20], Assessment in Spondyloarthritis International Society 20 criteria [ASAS20]), and improvement in physician's clinical global impression of change. For quality of life outcome, we preferentially abstracted the EuroQoL-5 Dimensions (EQ-5D) over Short Form-36 (SF-36) physical and mental components summary scores (PCS and MCS), and synthesized the two scales separately.

Effects on harms were organized by drug class and based on the proportion of patients experiencing adverse events. Generalized adverse events (e.g., serious adverse events, withdrawal due to adverse events, overdose and misuse), were abstracted for all classes of drugs. In addition, harms specific to each drug class were abstracted when available but limited to the items found in Table B-1. While the scope of this review limited the number of harms we could capture and report for each drug we recognize that other adverse events specific to an individual drug class do exist. For more information on these please refer to Table 21 in the main text. Nonopioid drugs considered in this review were categorized as, anticonvulsant drugs, benzodiazepines, nonsteroidal anti-inflammatory drugs (NSAIDs), skeletal muscle relaxants, 
antidepressants (e.g., SNRIs and Tricyclics), topical drugs (e.g., lidocaine or capsaicin), acetaminophen, memantine, and all forms of cannabis.

Table B-2. Harms

\begin{tabular}{|l|l|}
\hline Drug(s)/Drug Class & Harms by Drug Class \\
\hline All drugs & $\begin{array}{l}\text { Withdrawal due to adverse events, serious adverse events, overdose, } \\
\text { misuse, and dependence }\end{array}$ \\
\hline $\begin{array}{l}\text { Serotonin-norepinephrine reuptake } \\
\text { inhibitor antidepressants }\end{array}$ & Cognitive effects, nausea, sedation \\
\hline Tricyclic antidepressants & $\begin{array}{l}\text { Cardiac rhythm abnormalities, cognitive effects, dry mouth, urinary } \\
\text { retention, weight gain }\end{array}$ \\
\hline Pregabalin/gabapentin anticonvulsants & $\begin{array}{l}\text { Blurred vision, cognitive effects, dizziness, peripheral edema, } \\
\text { sedation, weight gain }\end{array}$ \\
\hline $\begin{array}{l}\text { Oxcarbazepine/carbamazepine } \\
\text { anticonvulsants }\end{array}$ & Cognitive effects, hyponatremia, neutropenia, sedation \\
\hline NSAIDs & CV events, GI, liver dysfunction, renal dysfunction \\
\hline Skeletal muscle relaxants & Dry mouth, sedation, urinary retention \\
\hline Acetaminophen & Liver toxicity \\
\hline Memantine & Cardiac rhythm abnormalities, cognitive effects, dizziness, sedation \\
\hline Topical (any) & Application site reactions \\
\hline Topical lidocaine & Cardiotoxicity, cognitive effects \\
\hline Topical diclofenac & CV events, Gl, liver dysfunction, renal dysfunction \\
\hline Cannabis & $\begin{array}{l}\text { Addiction/dependence, cognitive effects, hyperemesis, nausea, } \\
\text { sedation }\end{array}$ \\
\hline
\end{tabular}

\section{Assessment of Methodological Risk of Bias of Individual Studies}

Risk of bias was used to assess the quality of included RCTs based on principles for appraisal as developed by the Cochrane Back and Neck Group, ${ }^{4}$ and outlined in the AHRQ EPC Methods Guide chapter "Assessing the Risk of Bias of Individual Studies When Comparing Medical Interventions". 1,5 Given the risk of bias assessment, each included study was rated as "good," "fair," or "poor" quality. Assessments of RCTs included in good-quality systematic reviews that we included here were reviewed by a single reviewer, with the exception that any rated poor quality or high risk of bias were re-assessed by our team using dual review.

Studies rated "good" are considered to have the least risk of bias, and their results are considered valid. Good-quality studies include clear descriptions of the population, setting, interventions, and comparison groups; a valid method for allocation of patients to treatment; low dropout rates and clear reporting of dropouts; appropriate means for preventing bias; and appropriate measurement of outcomes.

Studies rated "fair" are susceptible to some bias, though not enough to invalidate the results. These studies may not meet all the criteria for a rating of good quality, but no flaw is likely to cause major bias. The study may be missing information, making it difficult to assess limitations and potential problems. The fair-quality category is broad, and studies with this rating will vary in their strengths and weaknesses. The results of some fair-quality studies are likely to be valid, while others may be only possibly valid.

Studies rated "poor" have significant flaws that imply biases of various types that may invalidate the results. They have a serious or "fatal" flaw in design, analysis, or reporting; large amounts of missing information; discrepancies in reporting; or serious problems in the delivery of the intervention. The results of these studies are least as likely to reflect flaws in the study design as the true difference between the compared interventions. We did not exclude studies 
rated as being poor in quality a priori, but poor-quality studies were considered to be less reliable than higher-quality studies when synthesizing the evidence, particularly if discrepancies between studies were present.

\section{Data Analysis and Synthesis}

Data were synthesized qualitatively (e.g., ranges and descriptive analysis) and quantitatively using meta-analysis where appropriate. Results are organized by Key Question (i.e., Effectiveness and Comparative Effectiveness and Harms and Adverse Events). Subquestions are then organized by pain condition and again by intervention within that condition (e.g., Osteoarthritis, duloxetine vs. placebo). Outcomes for effectiveness and comparative effectiveness were pain, function, and quality of life given treatment durations of 1 to $<6$ months (short term), 6 to 12 months (intermediate term), and more than 12 months (long term) ( $\geq 12$ months).

Using Stata $@ /$ IC 12.1 (StatsCorp, College Station, TX), meta-analyses were conducted using the profile-likelihood model ${ }^{6,7}$ or Dersimonian-Laird model, ${ }^{8}$ when profile-likelihood model did not converge. Statistical heterogeneity among studies was assessed using the standard Cochran's chi-square test and the $\mathrm{I}^{2}$ statistic. ${ }^{9}$ Pooled mean differences (MD) or standardized mean differences (SMD) were calculated for continuous pain assessment, function, quality of life, anxiety, depression, and sleep disturbances. Pooled relative risks (RR) were calculated for pain response and harms (e.g., withdrawals due to adverse events, serious adverse events). In addition, we performed subgroup and sensitivity analyses based on specific drug, drug dose, pain condition (e.g., rheumatoid arthritis vs. ankylosing spondylitis, diabetic polyneuropathy vs. postherpetic neuralgia vs. other neuropathic pain, osteoarthritis of the knee vs. hip vs. knee/hip vs. all sites), study quality (good or fair), and publication year ( $\leq 2000$ or $\geq 2001$ ). For analyses with more than 10 studies that were sufficiently homogeneous with regard to populations, interventions, and outcomes, publication bias was assessed using Funnel plot. Meta-analyses of harms outcomes included all treatment duration and were stratified by pain condition.

Pain outcomes were standardized to a scale of 0-10; SMD was calculated for other outcomes (e.g., function, quality of life) unless all pertinent studies assessed the outcome using the same scale. Studies with multiple nonopioid arms were combined so each study was represented once in a meta-analysis in order to avoid overweighting and the issue of correlation within the same study. When reported, adjusted MD from analysis of covariance model or other appropriate regression models was used if reported by the study, followed by difference in change score and followup score. Below lists hierarchical order for calculating missing standard deviations (SD):

- For followup score, missing SD was imputed by assuming constant coefficient of variation across included studies.

- For change score, it is not appropriate to assume constant coefficient of variation to impute missing SD given variability in treatment effects among studies. Instead,

o If baseline mean and SD were available, we imputed followup SD assuming constant coefficient of variation and calculated SD for change score assuming rho $=0.5$

o If baseline mean was available and SD was not, we imputed followup SD assuming constant coefficient of variation and used it as change score SD (This is equivalent to assuming the same baseline and followup SD, and calculating SD for change score assuming rho $=0.5$.) 
o If both baseline mean and SD were not available, we imputed change score SD as the average of follow up SD of other studies for the same outcome.

- The imputed values were based on all available data from the same outcome, which did not appear to vary much by type of pain or opioid.

- If the study publication was missing crucial data (e.g., SD, standard error [SE], change score), we searched www.ClinicalTrials.gov and abstracted the data when available.

\section{Grading the Strength of Evidence for Major Comparisons and Outcomes}

Regardless of whether evidence was synthesized quantitatively or qualitatively, the strength of evidence (SOE) was assessed for priority clinical outcomes (pain, function, quality of life) on each pain condition-treatment pair, using the approach described in the Methods Guide for Effectiveness and Comparative Effectiveness Reviews. ${ }^{1}$ To ensure consistency and validity of the evaluation, the grades were reviewed by a second reviewer. The domains assessed were study limitations (low, medium, or high), consistency (consistent, inconsistent, or unknown/not applicable), directness (direct or indirect), precision (precise or imprecise), and publication bias (suspected or undetected). Details of how each domain was assessed can be found in the AHRQ guidance. In general, study limitations were determined by the quality or risk of bias rating of the majority of studies reporting a given outcome. Consistency was evaluated based on the consistency of the point estimates (direction and magnitude) in a body of evidence, and the $\mathrm{I}^{2}$ statistic if a meta-analysis was conducted. Directness was determined based on the comparison, population, and outcome being those that were identified in the Key Questions. Precision was determined first based on whether the body of evidence met the optimal information size (OIS) for a given outcome, and then based on interpretation of the 95\% confidence intervals. For precision, we followed the guidance of the GRADE organization. The SOE was assigned an overall grade of high, moderate, low, or insufficient, reflecting our confidence in the effect estimates and whether the findings are stable. Evidence is found to be insufficient to draw conclusions when we have no evidence available or the body of evidence has unacceptable deficiencies, precluding reaching a conclusion. A definition of the SOE grades is as follows:

- High - Very confident that the effect estimate lies close to the true effect for this outcome. The body of evidence has few or no deficiencies. Findings are stable, i.e., inclusion of additional studies would not change the conclusions.

- Moderate - Moderately confident that the estimate of effect lies close to the true effect for this outcome. The body of evidence has some deficiencies. We believe that the findings are likely to be stable, but some doubt remains.

- Low - Limited confidence that the effect estimate lies close to the true effect for this outcome. The body of evidence has major or numerous deficiencies. Additional evidence is needed before concluding that the findings are stable or that the estimate of effect is close to the true effect.

- Insufficient - No confidence in the estimate of effect for this outcome. No evidence is available or the body of evidence has unacceptable deficiencies, precluding reaching a conclusion. 


\section{Appendix C. Included Studies}

1. Abou-Raya S, Abou-Raya A, Helmii M. Duloxetine for the management of pain in older adults with knee osteoarthritis: randomised placebo-controlled trial. Age Ageing. 2012 Sep;41(5):646-52. doi: 10.1093/ageing/afs072. PMID: 22743149.

2. Allen R, Sharma U, Barlas S. Clinical experience with desvenlafaxine in treatment of patients with fibromyalgia syndrome. Clin Pharmacol Drug Dev. 2017 May;6(3):224-33. doi: 10.1002/cpdd.271. PMID: 27139158.

3. Altman R, Hochberg M, Gibofsky A, et al. Efficacy and safety of low-dose SoluMatrix meloxicam in the treatment of osteoarthritis pain: a 12-week, phase 3 study. Curr Med Res Opin. 2015 Dec;31(12):2331-43. doi: 10.1185/03007995.2015.1112772. PMID: 26503347.

4. Altman RD, Zinsenheim JR, Temple AR, et al. Three-month efficacy and safety of acetaminophen extended-release for osteoarthritis pain of the hip or knee: a randomized, double-blind, placebocontrolled study. Osteoarthritis Cartilage. 2007 Apr;15(4):454-61. doi: 10.1016/j.joca.2006.10.008. PMID: 17142063.

5. Andelman SY. Etodolac, aspirin, and placebo in patients with degenerative joint disease: a twelve-week study. Clin Ther. 1983;5(6):651-61. PMID: 6226358.

6. Arezzo JC, Rosenstock J, Lamoreaux L, et al. Efficacy and safety of pregabalin 600 $\mathrm{mg} / \mathrm{d}$ for treating painful diabetic peripheral neuropathy: a double-blind placebocontrolled trial. BMC Neurol. 2008 Sep 16;8(33)doi: 10.1186/1471-2377-8-33. PMID: 18796160.

7. Arnold L, Gendreau R, Palmer R, et al. Efficacy and safety of milnacipran 100 $\mathrm{mg} /$ day in patients with fibromyalgia: results of a randomized, double-blind, placebocontrolled trial. Arthritis Rheum. 2010b Sep;62(9):2745-56. doi: 10.1002/art.27559. PMID: 20496365.
8. Arnold LM, Clauw D, Wang F, et al. Flexible dosed duloxetine in the treatment of fibromyalgia: a randomized, double-blind, placebo-controlled trial. J Rheumatol. 2010a Dec;37(12):2578-86. doi: 10.3899/jrheum.100365. PMID: 20843911.

9. Arnold LM, Goldenberg DL, Stanford SB, et al. Gabapentin in the treatment of fibromyalgia: a randomized, double-blind, placebo-controlled, multicenter trial. Arthritis Rheum. 2007a Apr;56(4):1336-44. doi: 10.1002/art.22457. PMID: 17393438.

10. Arnold LM, Hudson JI, Wang F, et al. Comparisons of the efficacy and safety of duloxetine for the treatment of fibromyalgia in patients with versus without major depressive disorder. Clin J Pain. 2009 JulAug;25(6):461-8. doi: 10.1097/AJP.0b013e318197d4e4. PMID: 19542792.

11. Arnold LM, Lu Y, Crofford LJ, et al. A double-blind, multicenter trial comparing duloxetine with placebo in the treatment of fibromyalgia patients with or without major depressive disorder. Arthritis Rheum. 2004 Sep;50(9):2974-84. doi: 10.1002/art.20485. PMID: 15457467.

12. Arnold LM, Palmer RH, Gendreau RM, et al. Relationships among pain, depressed mood, and global status in fibromyalgia patients: post hoc analyses of a randomized, placebo-controlled trial of milnacipran. Psychosomatics. 2012b Jul-Aug;53(4):3719. doi: 10.1016/j.psym.2012.02.005. PMID: 22677218.

13. Arnold LM, Rosen A, Pritchett YL, et al. A randomized, double-blind, placebocontrolled trial of duloxetine in the treatment of women with fibromyalgia with or without major depressive disorder. Pain. 2005 Dec 15;119(1-3):5-15. doi: 10.1016/j.pain.2005.06.031. PMID: 16298061. 
14. Arnold LM, Russell IJ, Diri EW, et al. A 14week, randomized, double-blinded, placebocontrolled monotherapy trial of pregabalin in patients with fibromyalgia. J Pain. 2008

Sep;9(9):792-805. doi: 10.1016/j.jpain.2008.03.013. PMID: 18524684.

15. Arnold LM, Wang F, Ahl J, et al. Improvement in multiple dimensions of fatigue in patients with fibromyalgia treated with duloxetine: secondary analysis of a randomized, placebo-controlled trial. Arthritis Res Ther. 2011 Jun 13;13(3):R86. doi: 10.1186/ar3359. PMID: 21668963.

16. Arnold LM, Whitaker S, Hsu C, et al. Efficacy and safety of mirogabalin for the treatment of fibromyalgia: results from three 13-week randomized, double-blind, placeboand active-controlled, parallel-group studies and a 52-week open-label extension study. Curr Med Res Opin. 2019 Jul 9:1-11. doi: 10.1080/03007995.2019.1629757. PMID: 31284771.

17. Arnold LM, Zhang S, Pangallo BA. Efficacy and safety of duloxetine $30 \mathrm{mg} / \mathrm{d}$ in patients with fibromyalgia: a randomized, doubleblind, placebo-controlled study. Clin J Pain. 2012a Nov-Dec;28(9):775-81. doi: 10.1097/AJP.0b013e3182510295. PMID: 22971669.

18. Atkinson J, Slater MA, Capparelli EV, et al. Efficacy of noradrenergic and serotonergic antidepressants in chronic back pain: A preliminary concentration-controlled trial. J Clin Psychopharmacol. 2007 Apr;27(2):13542. doi: 10.1097/jcp.0b013e3180333ed5. PMID: 17414235.

19. Atkinson JH, Slater MA, Capparelli EV, et al. A randomized controlled trial of gabapentin for chronic low back pain with and without a radiating component. Pain. 2016 Jul;157(7):1499-507. doi: 10.1097/j.pain.0000000000000554. PMID: 26963844.

20. Backonja M, Wallace MS, Blonsky ER, et al. NGX-4010, a high-concentration capsaicin patch, for the treatment of postherpetic neuralgia: a randomised, double-blind study.[Erratum appears in Lancet Neurol. 2009 Jan;8(1):31]. Lancet Neurol. 2008 Dec;7(12):1106-12. doi: 10.1016/S1474-4422(08)70228-X. PMID: 18977178.
21. Baerwald C, Verdecchia P, Duquesroix B, et al. Efficacy, safety, and effects on blood pressure of naproxcinod $750 \mathrm{mg}$ twice daily compared with placebo and naproxen 500 mg twice daily in patients with osteoarthritis of the hip: a randomized, double-blind, parallel-group, multicenter study. Arthritis Rheum. 2010 Dec;62(12):3635-44. doi: 10.1002/art.27694. PMID: 20722026.

22. Bakshi R, Ezzet N, Frey L, et al. Efficacy and tolerability of diclofenac dispersible in painful osteoarthrosis. Clin Rheumatol. 1993 Mar;12(1):57-61. PMID: 7682167.

23. Baraf HS, Gold MS, Clark MB, et al. Safety and efficacy of topical diclofenac sodium $1 \%$ gel in knee osteoarthritis: a randomized controlled trial. Phys Sportsmed. 2010 Jun;38(2):19-28. doi: 10.3810/psm.2010.06.1779. PMID: 20631460.

24. Barkhuizen A, Steinfeld S, Robbins J, et al. Celecoxib is efficacious and well tolerated in treating signs and symptoms of ankylosing spondylitis. J Rheumatol. 2006 Sep;33(9):1805-12. PMID: 16960941.

25. Barthel HR, Haselwood D, Longley S, 3rd, et al. Randomized controlled trial of diclofenac sodium gel in knee osteoarthritis.[Erratum appears in Semin Arthritis Rheum. 2010 Aug;40(1):95].

Semin Arthritis Rheum. 2009

Dec;39(3):203-12. doi: https://dx.doi.org/10.1016/j.semarthrit.2009. 09.002. PMID: 19932833.

26. Becker MC, Wang TH, Wisniewski L, et al. Rationale, design, and governance of Prospective Randomized Evaluation of Celecoxib Integrated Safety versus Ibuprofen Or Naproxen (PRECISION), a cardiovascular end point trial of nonsteroidal antiinflammatory agents in patients with arthritis. Am Heart J. 2009 Apr;157(4):60612. doi: 10.1016/j.ahj.2008.12.014. PMID: 19332185.

27. Becvar R, Urbanova Z, Vlasakova V, et al. Nabumetone induces less gastrointestinal mucosal changes than diclofenac retard. Clin Rheumatol. 1999;18(4):273-8. PMID: 10468165. 
28. Bensen W, Weaver A, Espinoza L, et al. Efficacy and safety of valdecoxib in treating the signs and symptoms of rheumatoid arthritis: a randomized, controlled comparison with placebo and naproxen. Rheumatology (Oxford). 2002

Sep;41(9):1008-16. doi: 10.1093/rheumatology/41.9.1008. PMID: 12209034.

29. Bensen WG, Fiechtner JJ, McMillen JI, et al. Treatment of osteoarthritis with celecoxib, a cyclooxygenase-2 inhibitor: a randomized controlled trial. Mayo Clin Proc. 1999 Nov;74(11):1095-105. doi: 10.4065/74.11.1095. PMID: 10560596.

30. Beydoun A, Shaibani A, Hopwood M, et al. Oxcarbazepine in painful diabetic neuropathy: results of a dose-ranging study. Acta Neurol Scand. 2006 Jun;113(6):395404. doi: 10.1111/j.16000404.2006.00631.x. PMID: 16674606.

31. Bhala N, Emberson J, Merhi A, et al. Vascular and upper gastrointestinal effects of non-steroidal anti-inflammatory drugs: meta-analyses of individual participant data from randomised trials. Lancet. 2013 Aug 31;382(9894):769-79. doi: 10.1016/s01406736(13)60900-9. PMID: 23726390.

32. Bingham CO, 3rd, Sebba AI, Rubin BR, et al. Efficacy and safety of etoricoxib $30 \mathrm{mg}$ and celecoxib $200 \mathrm{mg}$ in the treatment of osteoarthritis in two identically designed, randomized, placebo-controlled, noninferiority studies. Rheumatology (Oxford). 2007 Mar;46(3):496-507. doi: 10.1093/rheumatology/kel296. PMID: 16936327.

33. Bond M, Davis A, Lohmander S, et al. Responsiveness of the OARSI-OMERACT osteoarthritis pain and function measures. Osteoarthritis Cartilage. 2012

Jun;20(6):541-7. doi: 10.1016/j.joca.2012.03.001. PMID: 22425883.

34. Boudreau GP, Marchand L. Pregabalin for the management of cervicogenic headache: a double blind study. Can J Neurol Sci. 2014 Sep;41(5):603-10. doi: 10.1017/cjn.2014.2. PMID: 25373811.
35. Branco JC, Cherin P, Montagne A, et al. Longterm therapeutic response to milnacipran treatment for fibromyalgia. A European 1-year extension study following a 3-month study. J Rheumatol. 2011 Jul;38(7):1403-12. doi: 10.3899/jrheum.101025. PMID: 21459941.

36. Branco JC, Zachrisson O, Perrot S, et al. A European multicenter randomized doubleblind placebo-controlled monotherapy clinical trial of milnacipran in treatment of fibromyalgia. J Rheumatol. 2010 Apr;37(4):851-9. doi: 10.3899/jrheum.090884. PMID: 20156949.

37. Calkins AM, Gudin J, Gidal B, et al. Impact of data imputation methodology on pain assessment over 24 Hours in a randomized, placebo-controlled study of gabapentin enacarbil in patients with neuropathic pain associated with postherpetic neuralgia. Pain Med. 2016 Apr;17(4):728-36. doi: 10.1093/pm/pnv072. PMID: 26814307.

38. Cardenas DD, Nieshoff EC, Suda K, et al. A randomized trial of pregabalin in patients with neuropathic pain due to spinal cord injury. Neurology. 2013 Feb 5;80(6):533-9. doi: 10.1212/WNL.0b013e318281546b. PMID: 23345639.

39. Carette S, Bell MJ, Reynolds WJ, et al. Comparison of amitriptyline, cyclobenzaprine, and placebo in the treatment of fibromyalgia. A randomized, double-blind clinical trial. Arthritis Rheum. 1994 Jan;37(1):32-40. doi: 10.1002/art.1780370106. PMID: 8129762.

40. Case JP, Baliunas AJ, Block JA. Lack of efficacy of acetaminophen in treating symptomatic knee osteoarthritis: a randomized, double-blind, placebocontrolled comparison trial with diclofenac sodium. Arch Intern Med. 2003 Jan 27;163(2):169-78. PMID: 12546607.

41. Chappell AS, Bradley LA, Wiltse C, et al. A six-month double blind placebo-controlled, randomized clinical trial of duloxetine for the treatment of fibromyalgia. Int J Gen Med. 2008 Nov 30;1:91-102. PMID: 20428412. 
42. Chappell AS, Desaiah D, Liu-Seifert H, et al. A double-blind, randomized, placebocontrolled study of the efficacy and safety of duloxetine for the treatment of chronic pain due to osteoarthritis of the knee. Pain pract. 2011 Jan-Feb;11(1):33-41. doi: 10.1111/j.1533-2500.2010.00401.x. PMID: 20602715.

43. Chappell AS, Littlejohn G, Kajdasz DK, et al. A 1-year safety and efficacy study of duloxetine in patients with fibromyalgia. Clin J Pain. 2009b Jun;25(5):365-75. doi: 10.1097/AJP.0b013e31819be587. PMID: 19454869.

44. Chappell AS, Ossanna MJ, Liu-Seifert H, et al. Duloxetine, a centrally acting analgesic, in the treatment of patients with osteoarthritis knee pain: a 13-week, randomized, placebo-controlled trial. Pain. 2009a Dec;146(3):253-60. doi: 10.1016/j.pain.2009.06.024. PMID: 19625125.

45. Cheung R, Cheng TT, Dong Y, et al. Incidence of gastroduodenal ulcers during treatment with celecoxib or diclofenac: pooled results from three 12-week trials in Chinese patients with osteoarthritis or rheumatoid arthritis. Int J Rheum Dis. 2010 May;13(2):151-7. doi: 10.1111/j.1756185X.2010.01463.x. PMID: 20536600.

46. Clauw DJ, Mease P, Palmer RH, et al. Milnacipran for the treatment of fibromyalgia in adults: a 15-week, multicenter, randomized, double-blind, placebo-controlled, multiple-dose clinical trial. Clin Ther. 2008 Nov;30(11):19882004. doi: 10.1016/j.clinthera.2008.11.009. PMID: 19108787.

47. Clegg DO, Reda DJ, Harris CL, et al. Glucosamine, chondroitin sulfate, and the two in combination for painful knee osteoarthritis. N Engl J Med. 2006 Feb 23;354(8):795-808. doi:

10.1056/NEJMoa052771. PMID: 16495392.

48. Clifford DB, Simpson DM, Brown S, et al. A randomized, double-blind, controlled study of NGX-4010, a capsaicin 8\% dermal patch, for the treatment of painful HIVassociated distal sensory polyneuropathy. J Acquir Immune Defic Syndr. 2012 Feb 1;59(2):126-33. doi: 10.1097/QAI.0b013e31823e31f7. PMID: 22067661.
49. Collantes E, Curtis SP, Lee KW, et al. A multinational randomized, controlled, clinical trial of etoricoxib in the treatment of rheumatoid arthritis [ISRCTN25142273]. BMC Fam Pract. 2002 May 22;3:10. PMID: 12033987.

50. Conaghan PG, Dickson J, Bolten W, et al. A multicentre, randomized, placebo- and active-controlled trial comparing the efficacy and safety of topical ketoprofen in Transfersome gel (IDEA-033) with ketoprofen-free vehicle (TDT 064) and oral celecoxib for knee pain associated with osteoarthritis. Rheumatology (Oxford). 2013 Jul;52(7):1303-12. doi:

10.1093/rheumatology/ket133. PMID: 23542612.

51. Cryer B, Li C, Simon LS, et al. GIREASONS: a novel 6-month, prospective, randomized, open-label, blinded endpoint (PROBE) trial. Am J Gastroenterol. 2013 Mar;108(3):392-400. doi: 10.1038/ajg.2012.467. PMID: 23399552.

52. Cryer BL, Sostek MB, Fort JG, et al. A fixed-dose combination of naproxen and esomeprazole magnesium has comparable upper gastrointestinal tolerability to celecoxib in patients with osteoarthritis of the knee: results from two randomized, parallel-group, placebo-controlled trials. Ann Med. 2011 Dec;43(8):594-605. doi: 10.3109/07853890.2011.625971. PMID: 22017620.

53. Dahlberg LE, Holme I, Hoye K, et al. A randomized, multicentre, double-blind, parallel-group study to assess the adverse event-related discontinuation rate with celecoxib and diclofenac in elderly patients with osteoarthritis. Scand J Rheumatol. 2009 Mar-Apr;38(2):133-43. doi: 10.1080/03009740802419065. PMID: 19165648.

54. Dallocchio C, Buffa C, Mazzarello P, et al. Gabapentin vs. amitriptyline in painful diabetic neuropathy: an open-label pilot study. J Pain Symptom Manage. 2000 Oct;20(4):280-5. PMID: 11027910.

55. de Queiros MF. Double-blind comparison of etodolac and naproxen in the treatment of rheumatoid arthritis. Clin Ther. 1991 JanFeb;13(1):38-46. PMID: 1827613. 
56. del Toro RA, Concepcion R. Twelve-week study of etodolac, aspirin, and placebo in patients with rheumatoid arthritis. Clin Ther. 1983;5(4):436-44. PMID: 6223699.

57. DeLemos BP, Xiang J, Benson C, et al. Tramadol hydrochloride extended-release once-daily in the treatment of osteoarthritis of the knee and/or hip: a double-blind, randomized, dose-ranging trial. Am J Ther. 2011 May;18(3):216-26. doi: 10.1097/MJT.0b013e3181cec307. PMID: 20215961.

58. Derry S, Conaghan P, Da Silva JA, et al. Topical NSAIDs for chronic musculoskeletal pain in adults. Cochrane Database Syst Rev. 2016 Apr

22;4:CD007400. doi: 10.1002/14651858.CD007400.pub3. PMID: 27103611.

59. Devi P, Madhu K, Ganapathy B, et al. Evaluation of efficacy and safety of gabapentin, duloxetine, and pregabalin in patients with painful diabetic peripheral neuropathy. Indian J Pharmacol. 2012 Jan;44(1):51-6. doi: 10.4103/02537613.91867. PMID: 22345870.

60. Dogra S, Beydoun S, Mazzola J, et al. Oxcarbazepine in painful diabetic neuropathy: a randomized, placebocontrolled study. Eur J Pain. 2005 Oct;9(5):543-54. doi: 10.1016/j.ejpain.2004.11.006. PMID: 16139183.

61. Dougados M, Gueguen A, Nakache JP, et al. Ankylosing spondylitis: what is the optimum duration of a clinical study? A one year versus a 6 weeks non-steroidal antiinflammatory drug trial. Rheumatology (Oxford). 1999 Mar;38(3):235-44. doi: 10.1093/rheumatology/38.3.235. PMID: 10325662.

62. Edwards W. Etodolac, aspirin, and placebo in patients with rheumatoid arthritis: a 12week study. Clin Ther. 1983;5(5):495-503. PMID: 6225520.
63. Emery P, Clarke A, Williams P, et al. Nabumetone compared with naproxen in the treatment of rheumatoid arthritis: a multicenter, double blind, randomized, parallel group trial in hospital outpatients.[Erratum appears in J Rheumatol 1993 May;20(5):924]. J Rheumatol Suppl. 1992 Nov;36:41-7. PMID: 1474534.

64. Emery P, Koncz T, Pan S, et al. Analgesic effectiveness of celecoxib and diclofenac in patients with osteoarthritis of the hip requiring joint replacement surgery: a 12week, multicenter, randomized, doubleblind, parallel-group, double-dummy, noninferiority study. Clin Ther. 2008 Jan;30(1):70-83. doi: 10.1016/j.clinthera.2008.01.016. PMID: 18343244

65. Emery P, Zeidler H, Kvien TK, et al. Celecoxib versus diclofenac in long-term management of rheumatoid arthritis: randomised double-blind comparison. Lancet. 1999;354(9196):2106-11. doi: 10.1016/S0140-6736(99)02332-6. PMID: 10609815.

66. Emir B, Murphy TK, Petersel DL, et al. Treatment response to pregabalin in fibromyalgia pain: effect of patient baseline characteristics. Expert Opin Pharmacother. 2010 Oct;11(14):2275-80. doi: 10.1517/14656566.2010.509717. PMID: 20812879.

67. Enomoto H, Fujikoshi S, Tsuji T, et al. Efficacy of duloxetine by prior NSAID use in the treatment of chronic osteoarthritis knee pain: a post hoc subgroup analysis of a randomized, placebo-controlled, phase 3 study in Japan. J Orthop Sci. 2018 Nov;23(6):1019-26. doi: 10.1016/j.jos.2018.07.008. PMID: 30126675.

68. Enteshari-Moghaddam A, Azami A, Isazadehfar K, et al. Efficacy of duloxetine and gabapentin in pain reduction in patients with knee osteoarthritis. Clin Rheumatol. 2019 May 6doi: 10.1007/s10067-01904573-7. PMID: 31062253. 
69. Essex MN, Bhadra P, Sands GH. Efficacy and tolerability of celecoxib versus naproxen in patients with osteoarthritis of the knee: a randomized, double-blind, double-dummy trial. J Int Med Res. 2012;40(4):1357-70. doi: 10.1177/147323001204000414. PMID: 22971487.

70. Eversmeyer W, Poland M, DeLapp RE, et al. Safety experience with nabumetone versus diclofenac, naproxen, ibuprofen, and piroxicam in osteoarthritis and rheumatoid arthritis. Am J Med. 1993 Aug 9;95(2A):10S-8S. PMID: 8356997.

71. Farkouh ME, Kirshner H, Harrington RA, et al. Comparison of lumiracoxib with naproxen and ibuprofen in the Therapeutic Arthritis Research and Gastrointestinal Event Trial (TARGET), cardiovascular outcomes: randomised controlled trial. Lancet. 2004 Aug;364(9435):675-84. doi: 10.1016/S0140-6736(04)16894-3. PMID: 15325832.

72. Fattahi MJ, Jamshidi AR, Mahmoudi M, et al. Evaluation of the efficacy and safety of beta-d-mannuronic acid in patients with ankylosing spondylitis: A 12-week randomized, placebo-controlled, phase I/II clinical trial. Int Immunopharmacol. 2018 Jan;54:112-7. doi: https://dx.doi.org/10.1016/j.intimp.2017.11. 003. PMID: 29127910.

73. Fleischmann R, Sheldon E, MaldonadoCocco J, et al. Lumiracoxib is effective in the treatment of osteoarthritis of the knee: a prospective randomized 13-week study versus placebo and celecoxib. Clin Rheumatol. 2006 Feb;25(1):42-53. PMID: 16132165.

74. Freynhagen R, Strojek K, Griesing T, et al. Efficacy of pregabalin in neuropathic pain evaluated in a 12-week, randomised, doubleblind, multicentre, placebo-controlled trial of flexible- and fixed-dose regimens. Pain. 2005 Jun;115(3):254-63. PMID: 15911152.

75. Furst DE, Kolba KS, Fleischmann R, et al. Dose response and safety study of meloxicam up to $22.5 \mathrm{mg}$ daily in rheumatoid arthritis: a 12 week multicenter, double blind, dose response study versus placebo and diclofenac. J Rheumatol. 2002 Mar;29(3):436-46. PMID: 11908554.
76. Gao Y, Guo X, Han P, et al. Treatment of patients with diabetic peripheral neuropathic pain in China: a double-blind randomised trial of duloxetine vs. placebo. Int J Clin Pract. 2015 Sep;69(9):957-66. doi: 10.1111/ijcp.12641. PMID: 25939897.

77. Gao Y, Ning G, Jia WP, et al. Duloxetine versus placebo in the treatment of patients with diabetic neuropathic pain in China. Chin Med J. 2010 Nov;123(22):3184-92. PMID: 21163113.

78. Garg Y, Singh J, Sohal HS, et al. Comparison of clinical effectiveness and safety of newer nonsteroidal antiinflammatory drugs in patients of osteoarthritis of knee joint: a randomized, prospective, open-label parallel-group study. Indian J Pharmacol. 2017 SepOct;49(5):383-9. doi: 10.4103/ijp.IJP_245_16. PMID: 29515279.

79. Gendreau RM, Thorn MD, Gendreau JF, et al. Efficacy of milnacipran in patients with fibromyalgia. J Rheumatol. 2005 Oct;32(10):1975-85. PMID: 16206355.

80. Geusens P, Alten R, Rovensky J, et al. Efficacy, safety and tolerability of lumiracoxib in patients with rheumatoid arthritis. Int J Clin Pract. 2004 Nov;58(11):1033-41. PMID: 15605667.

81. Geusens PP, Truitt K, Sfikakis P, et al. A placebo and active comparator-controlled trial of rofecoxib for the treatment of rheumatoid arthritis. Scand J Rheumatol. 2002;31(4):230-8. PMID: 12369656.

82. Gibofsky A, Hochberg MC, Jaros MJ, et al. Efficacy and safety of low-dose submicron diclofenac for the treatment of osteoarthritis pain: a 12 week, phase 3 study. Curr Med Res Opin. 2014 Sep;30(9):1883-93. doi: 10.1185/03007995.2014.946123. PMID: 25050589.

83. Gibofsky A, Rodrigues J, Fiechtner J, et al. Efficacy and tolerability of valdecoxib in treating the signs and symptoms of severe rheumatoid arthritis: a 12-week, multicenter, randomized, double-blind, placebocontrolled study. Clin Ther. 2007 Jun;29(6):1071-85. doi: 10.1016/j.clinthera.2007.06.016. PMID: 17692722. 
84. Goldenberg DL, Clauw DJ, Palmer RH, et al. Durability of therapeutic response to milnacipran treatment for fibromyalgia. Results of a randomized, double-blind, monotherapy 6-month extension study. Pain Med. 2010 Feb;11(2):180-94. doi: 10.1111/j.1526-4637.2009.00755.x. PMID: 20002596.

85. Goldstein DJ, Lu Y, Detke MJ, et al. Duloxetine vs. placebo in patients with painful diabetic neuropathy. Pain. 2005 Jul;116(1-2):109-18. doi: 10.1016/j.pain.2005.03.029. PMID: 15927394.

86. Goldstein JL, Correa P, Zhao WW, et al. Reduced incidence of gastroduodenal ulcers with celecoxib, a novel cyclooxygenase-2 inhibitor, compared to naproxen in patients with arthritis. Am J Gastroenterol. 2001 Apr;96(4):1019-27. doi: 10.1111/j.15720241.2001.03740.x. PMID: CN-00347393.

87. Goldstein JL, Eisen GM, Burke TA, et al. Dyspepsia tolerability from the patients' perspective: a comparison of celecoxib with diclofenac. Aliment Pharmacol Ther. 2002 Apr;16(4):819-27. PMID: 11929402.

88. Gordon GV, Polsky BG. Three-month trial of etodolac (Ultradol®) compared with aspirin and placebo in patients with rheumatoid arthritis. Curr Ther Res Clin Exp. 1983;33(1):89-99.

89. Grace EM, Bellamy N, Kassam Y, et al. Controlled, double-blind, randomized trial of amitriptyline in relieving articular pain and tenderness in patients with rheumatoid arthritis. Curr Med Res Opin.

1985;9(6):426-9. doi: 10.1185/03007998509109614. PMID: 3886308.

90. Grosskopf J, Mazzola J, Wan Y, et al. A randomized, placebo-controlled study of oxcarbazepine in painful diabetic neuropathy. Acta Neurol Scand. 2006 Sep;114(3):177-80. doi: 10.1111/j.16000404.2005.00559.x. PMID: 16911345.

91. Hannonen P, Malminiemi K, Yli-Kerttula U, et al. A randomized, double-blind, placebocontrolled study of moclobemide and amitriptyline in the treatment of fibromyalgia in females without psychiatric disorder. Br J Rheumatol. 1998 Dec;37(12):1279-86. PMID: 9973149.
92. Hawkey C, Laine L, Simon T, et al. Comparison of the effect of rofecoxib (a cyclooxygenase 2 inhibitor), ibuprofen, and placebo on the gastroduodenal mucosa of patients with osteoarthritis: a randomized, double-blind, placebo-controlled trial. The Rofecoxib osteoarthritis endoscopy multinational study group. Arthritis Rheum. 2000 Feb;43(2):370-7. doi: 10.1002/15290131(200002)43:2<370::AIDANR17>3.0.CO;2-D. PMID: 10693877.

93. Hawkey CC, Svoboda P, FiedorowiczFabrycy IF, et al. Gastroduodenal safety and tolerability of lumiracoxib compared with Ibuprofen and celecoxib in patients with osteoarthritis. J Rheumatol. 2004a Sep;31(9):1804-10. PMID: 15338504.

94. Hawkey CJ, Farkouh M, Gitton X, et al. Therapeutic arthritis research and gastrointestinal event trial of lumiracoxib study design and patient demographics. Aliment Pharmacol Ther. 2004b Jul 01;20(1):51-63. PMID: 15225171.

95. Hawkey CJ, Laine L, Simon T, et al. Incidence of gastroduodenal ulcers in patients with rheumatoid arthritis after 12 weeks of rofecoxib, naproxen, or placebo: a multicentre, randomised, double blind study.[Erratum appears in Gut. 2003 Dec;52(12):1800]. Gut. 2003 Dec;52(6):820-6. doi: 10.1136/gut.52.6.820. PMID: 12740337.

96. Herrero-Beaumont G, Ivorra JA, Del Carmen Trabado M, et al. Glucosamine sulfate in the treatment of knee osteoarthritis symptoms: a randomized, double-blind, placebo-controlled study using acetaminophen as a side comparator. Arthritis Rheum. 2007 Feb;56(2):555-67. doi: 10.1002/art.22371. PMID: 17265490.

97. Hochberg MC, Fort JG, Svensson O, et al. Fixed-dose combination of enteric-coated naproxen and immediate-release esomeprazole has comparable efficacy to celecoxib for knee osteoarthritis: two randomized trials. Curr Med Res Opin. 2011 Jun;27(6):1243-53. doi: 10.1185/03007995.2011.580340. PMID: 21524238. 
98. Hoffman DL, Sadosky A, Dukes EM, et al. How do changes in pain severity levels correspond to changes in health status and function in patients with painful diabetic peripheral neuropathy? Pain.

2010;149(2):194-201. doi:

10.1016/j.pain.2009.09.017. PMID: 20303665.

99. Holroyd KA, O'Donnell FJ, Stensland M, et al. Management of chronic tension-type headache with tricyclic antidepressant medication, stress management therapy, and their combination: a randomized controlled trial. JAMA. 2001 May 2;285(17):2208-15. doi: 10.1001/jama.285.17.2208. PMID: 11325322 .

100. Hosie J, Distel M, Bluhmki E. Meloxicam in osteoarthritis: a 6-month, double-blind comparison with diclofenac sodium. Br J Rheumatol. 1996 Apr;35 Suppl 1:39-43. PMID: 8630635.

101. Hunt RH, Harper S, Callegari P, et al. Complementary studies of the gastrointestinal safety of the cyclooxygenase-2-selective inhibitor etoricoxib. Aliment Pharmacol Ther. 2003 Jan;17(2):201-10. PMID: 12534404.

102. Irving G, Tanenberg RJ, Raskin J, et al. Comparative safety and tolerability of duloxetine vs. pregabalin vs. duloxetine plus gabapentin in patients with diabetic peripheral neuropathic pain. Int J Clin Pract. 2014 Sep;68(9):1130-40. doi: 10.1111/ijcp.12452. PMID: 24837444.

103. Jacob G, Messina M, Caperton E. Safety and efficacy of etodolac, once or twice a day, in the treatment of active rheumatoid arthritis. Curr Ther Res Clin Exp. 1985;37(6):1124-9.

104. Jacob GB, Hart KK, Mullane JF. Placebocontrolled study of etodolac and aspirin in the treatment of rheumatoid arthritis. Curr Ther Res Clin Exp. 1983;33(4):703-13.

105. Joharchi K, Memari M, Azargashb E, et al. Efficacy and safety of duloxetine and Pregabalin in Iranian patients with diabetic peripheral neuropathic pain: a double-blind, randomized clinical trial. Journal of Diabetes and Metabolic Disorders. 2019doi: 10.1007/s40200-019-00427-w.
106. Kalita J, Kohat AK, Misra UK, et al. An open labeled randomized controlled trial of pregabalin versus amitriptyline in chronic low backache. J Neurol Sci. 2014 Jul 15;342(1-2):127-32. doi: 10.1016/j.jns.2014.05.002. PMID: 24857356.

107. Kelle B, Yavuz F, Yasar E, et al. The efficacy of gabapentin and pregabalin in the treatment of neuropathic pain due to peripheral nerve injury. J Musculoskelet Pain. 2012;20(4):300-5. doi: 10.3109/10582452.2012.733801.

108. Khan MA. Diclofenac in the treatment of ankylosing spondylitis: review of worldwide clinical experience and report of a doubleblind comparison with indomethacin. Semin Arthritis Rheum. 1985 Nov;15(2 Suppl 1):80-4. PMID: 4081795.

109. Khan MA. A double blind comparison of diclofenac and indomethacin in the treatment of ankylosing spondylitis. J Rheumatol. 1987 Feb;14(1):118-23. PMID: 3553586.

110. Kim JS, Bashford G, Murphy TK, et al. Safety and efficacy of pregabalin in patients with central post-stroke pain. Pain. 2011 May;152(5):1018-23. doi: 10.1016/j.pain.2010.12.023. PMID: 21316855.

111. Kivitz A, Eisen G, Zhao WW, et al. Randomized placebo-controlled trial comparing efficacy and safety of valdecoxib with naproxen in patients with osteoarthritis. J Fam Pract. 2002 Jun;51(6):530-7. PMID: 12100776.

112. Kivitz A, Fairfax M, Sheldon EA, et al. Comparison of the effectiveness and tolerability of lidocaine patch $5 \%$ versus celecoxib for osteoarthritis-related knee pain: post hoc analysis of a 12 week, prospective, randomized, active-controlled, open-label, parallel-group trial in adults. Clin Ther. 2008 Dec;30(12):2366-77. doi: 10.1016/j.clinthera.2008.12.015. PMID: 19167595. 
113. Kivitz AJ, Moskowitz RW, Woods E, et al. Comparative efficacy and safety of celecoxib and naproxen in the treatment of osteoarthritis of the hip. J Int Med Res. 2001 Nov-Dec;29(6):467-79. doi: 10.1177/147323000102900602. PMID: 11803730 .

114. Kivitz AJ, Nayiager S, Schimansky T, et al. Reduced incidence of gastroduodenal ulcers associated with lumiracoxib compared with ibuprofen in patients with rheumatoid arthritis. Aliment Pharmacol Ther. 2004 Jun 1;19(11):1189-98. doi: 10.1111/j.13652036.2004.01956.x. PMID: 15153172.

115. Konno S, Oda N, Ochiai T, et al. Randomized, double-blind, placebocontrolled phase III trial of duloxetine monotherapy in Japanese patients with chronic low back pain. Spine (Phila Pa 1976). 2016 Nov 15;41(22):1709-17. doi: 10.1097/BRS.0000000000001707. PMID: 27831985.

116. Krug H, Broadwell LK, Berry M, et al. Tolerability and efficacy of nabumetone and naproxen in the treatment of rheumatoid arthritis. Clin Ther. 2000 Jan;22(1):40-52. PMID: 10688389.

117. Laine L, Harper S, Simon T, et al. A randomized trial comparing the effect of rofecoxib, a cyclooxygenase 2-specific inhibitor, with that of ibuprofen on the gastroduodenal mucosa of patients with osteoarthritis. Rofecoxib osteoarthritis endoscopy study group. Gastroenterology. 1999 Oct;117(4):776-83. PMID: 10500058.

118. Laine L, Maller ES, Yu C, et al. Ulcer formation with low-dose enteric-coated aspirin and the effect of COX-2 selective inhibition: a double-blind trial. Gastroenterology. 2004 Aug;127(2):395402. PMID: 15300570.

119. Lehmann R, Brzosko M, Kopsa P, et al. Efficacy and tolerability of lumiracoxib 100 mg once daily in knee osteoarthritis: a 13week, randomized, double-blind study vs. placebo and celecoxib. Curr Med Res Opin. 2005 Apr;21(4):517-26. doi:

10.1185/030079905X38196. PMID: 15899100 .
120. Leung AT, Malmstrom K, Gallacher AE, et al. Efficacy and tolerability profile of etoricoxib in patients with osteoarthritis: a randomized, double-blind, placebo and active-comparator controlled 12-week efficacy trial. Curr Med Res Opin. 2002;18(2):49-58. doi: 10.1185/030079902125000282. PMID: 12017209.

121. Lister BJ, Poland M, DeLapp RE. Efficacy of nabumetone versus diclofenac, naproxen, ibuprofen, and piroxicam in osteoarthritis and rheumatoid arthritis. Am J Med. 1993 Aug 9;95(2A):2S-9S. PMID: 8356999.

122. Lonauer G, Tisscher JR, Lim HG, et al. Double-blind comparison of etodolac and diclofenac in patients with rheumatoid arthritis. Curr Med Res Opin. 1993;13(2):70-7. doi: 10.1185/03007999309111535. PMID: 8325044.

123. MacDonald TM, Hawkey CJ, Ford I, et al. Randomized trial of switching from prescribed non-selective non-steroidal antiinflammatory drugs to prescribed celecoxib: the Standard care vs. Celecoxib Outcome Trial (SCOT).[Erratum appears in Eur Heart J. 2016 Dec 24;:; PMID: 28025195]. Eur Heart J. 2017 Jun 14;38(23):1843-50. doi: 10.1093/eurheartj/ehw387. PMID: 27705888.

124. MacDonald TM, Mackenzie IS, Wei L, et al. Methodology of a large prospective, randomised, open, blinded endpoint streamlined safety study of celecoxib versus traditional non-steroidal anti-inflammatory drugs in patients with osteoarthritis or rheumatoid arthritis: protocol of the standard care versus celecoxib outcome trial (SCOT). BMJ Open. 2013 Jan 29;3(1):e002295. doi: 10.1136/bmjopen-2012-002295. PMID: 23364320.

125. Mahmood R, Jawed I, Khan MI, et al. Comparative role of pregabalin and carbamazepine regarding efficacy in painful diabetic neuropathy. Pak J Pharm Sci. 2017 Jul;30(4):1275-8. PMID: 29039325. 
126. Makarowski W, Zhao WW, Bevirt T, et al. Efficacy and safety of the COX-2 specific inhibitor valdecoxib in the management of osteoarthritis of the hip: a randomized, double-blind, placebo-controlled comparison with naproxen. Osteoarthritis Cartilage. 2002 Apr;10(4):290-6. doi: 10.1053/joca.2001.0510. PMID: 11950252.

127. Markman J, Resnick M, Greenberg S, et al. Efficacy of pregabalin in post-traumatic peripheral neuropathic pain: a randomized, double-blind, placebo-controlled phase 3 trial. J Neurol. 2018 Dec;265(12):2815-24. doi: 10.1007/s00415-018-9063-9. PMID: 30242745 .

128. Matsumoto AK, Melian A, Mandel DR, et al. A randomized, controlled, clinical trial of etoricoxib in the treatment of rheumatoid arthritis. J Rheumatol. 2002

Aug;29(8):1623-30. PMID: 12180720.

129. Mease PJ, Clauw DJ, Gendreau RM, et al. The efficacy and safety of milnacipran for treatment of fibromyalgia. a randomized, double-blind, placebo-controlled trial.[Erratum appears in J Rheumatol. 2009 Mar;36(3):661]. J Rheumatol. 2009 Feb;36(2):398-409. doi: 10.3899/jrheum.080734. PMID: 19132781.

130. Mease PJ, Russell IJ, Arnold LM, et al. A randomized, double-blind, placebocontrolled, phase III trial of pregabalin in the treatment of patients with fibromyalgia. $\mathrm{J}$ Rheumatol. 2008 Mar;35(3):502-14. PMID: 18278830 .

131. Morgan GJ, Jr., Kaine J, DeLapp R, et al. Treatment of elderly patients with nabumetone or diclofenac: gastrointestinal safety profile. J Clin Gastroenterol. 2001 Apr;32(4):310-4. PMID: 11276273.

132. Morgan GJ, Poland M, DeLapp RE. Efficacy and safety of nabumetone versus diclofenac, naproxen, ibuprofen, and piroxicam in the elderly. Am J Med. 1993 Aug 9;95(2A):19S-27S. PMID: 8356998.

133. Murakami M, Osada K, Mizuno H, et al. A randomized, double-blind, placebocontrolled phase III trial of duloxetine in Japanese fibromyalgia patients. Arthritis Res Ther. 2015 Aug 22;17:224. doi: 10.1186/s13075-015-0718-y. PMID: 26296539 .
134. Nazeri S, Jamshidi AR, Mahmoudi M, et al. The safety and efficacy of Guluronic acid (G2013) in ankylosing spondylitis: A randomized controlled parallel clinical trial. Pharmacol Rep. 2019 Jun;71(3):393-8. doi: 10.1016/j.pharep.2019.02.002. PMID: 31003148.

135. Nissen SE, Yeomans ND, Solomon DH, et al. Cardiovascular safety of celecoxib, naproxen, or ibuprofen for arthritis. N Engl J Med. 2016 Dec 29;375(26):2519-29. doi: 10.1056/NEJMoa1611593. PMID: 27959716.

136. Ohta H, Oka H, Usui C, et al. A randomized, double-blind, multicenter, placebocontrolled phase III trial to evaluate the efficacy and safety of pregabalin in Japanese patients with fibromyalgia. Arthritis Res Ther. 2012 Oct 12;14(5):R217. doi: 10.1186/ar4056. PMID: 23062189.

137. Olivan-Blazquez B, Herrera-Mercadal P, Puebla-Guedea M, et al. Efficacy of memantine in the treatment of fibromyalgia: a double-blind, randomised, controlled trial with 6-month follow-up. Pain. 2014 Dec;155(12):2517-25. doi: 10.1016/j.pain.2014.09.004. PMID: 25218600.

138. Olivan-Blazquez B, Puebla M, Masluk B, et al. Evaluation of the efficacy of memantine in the treatment of fibromyalgia: study protocol for a doubled-blind randomized controlled trial with six-month follow-up. Trials. 2013 Jan 3;14:3. doi: 10.1186/17456215-14-3. PMID: 23286311.

139. Palferman TG, Webley M. A comparative study of nabumetone and indomethacin in ankylosing spondylitis. Eur J Rheumatol Inflamm. 1991;11(2):23-9. PMID: 1365469.

140. Pauer L, Winkelmann A, Arsenault P, et al. An international, randomized, double-blind, placebo-controlled, phase III trial of pregabalin monotherapy in treatment of patients with fibromyalgia. J Rheumatol. 2011 Dec;38(12):2643-52. doi: 10.3899/jrheum.110569. PMID: 21965636. 
141. Pfaffenrath V, Diener HC, Isler H, et al. Efficacy and tolerability of amitriptylinoxide in the treatment of chronic tension-type headache: a multi-centre controlled study. Cephalalgia. 1994 Apr;14(2):149-55. doi: 10.1046/j.1468-2982.1994.1402149.x. PMID: 8062354.

142. Pfizer. A 14 week, double-blind, randomized, placebo-controlled, multicenter study to evaluate the safety and efficacy of pregabalin (150 mg - 600 $\mathrm{mg} /$ day) using a flexible, optimized dose schedule in patients with painful diabetic peripheral neuropathy (DPN) PhRMA Web Synopsis. 2007; Protocol A0081030:NCT00156078.

143. Pisko EJ, Bockow BI, Box P, et al. Sixmonth multi-center study comparing nabumetone with naproxen in the treatment of osteoarthritis. Am J Med. 1987a Oct 30;83(4B):86-91. PMID: 3318436.

144. Pisko EJ, Strader K, Rice D, et al. A 6month, double-blind study comparing nabumetone to naproxen in the treatment of osteoarthritis. Pharmatherapeutica. 1987b;5(2):90-8. PMID: 3310020.

145. Poiley JE. Nabumetone versus naproxen in the treatment of osteoarthritis. A six-month trial. Am J Med. 1987 Oct 30;83(4B):82-5. PMID: 3318435.

146. Prior MJ, Harrison DD, Frustaci ME. A randomized, double-blind, placebocontrolled 12 week trial of acetaminophen extended release for the treatment of signs and symptoms of osteoarthritis. Curr Med Res Opin. 2014 Nov;30(11):2377-87. doi: 10.1185/03007995.2014.949646. PMID: 25121804.

147. Puljak L, Marin A, Vrdoljak D, et al. Celecoxib for osteoarthritis. Cochrane Database Syst Rev. 2017 May

22;5:CD009865. doi: 10.1002/14651858.CD009865.pub2. PMID: 28530031.

148. Puopolo A, Boice JA, Fidelholtz JL, et al. A randomized placebo-controlled trial comparing the efficacy of etoricoxib $30 \mathrm{mg}$ and ibuprofen $2400 \mathrm{mg}$ for the treatment of patients with osteoarthritis. Osteoarthritis Cartilage. 2007 Dec;15(12):1348-56. doi: 10.1016/j.joca.2007.05.022. PMID: 17631392.
149. Raskin J, Pritchett YL, Wang F, et al. A double-blind, randomized multicenter trial comparing duloxetine with placebo in the management of diabetic peripheral neuropathic pain. Pain Med. 2005 SepOct;6(5):346-56. doi: 10.1111/j.15264637.2005.00061.x. PMID: 16266355.

150. Rauck R, Makumi CW, Schwartz S, et al. A randomized, controlled trial of gabapentin enacarbil in subjects with neuropathic pain associated with diabetic peripheral neuropathy. Pain pract. 2013 Jul;13(6):48596. doi: 10.1111/papr.12014. PMID: 23186035.

151. Reed GW, Abdallah MS, Shao M, et al. Effect of aspirin coadministration on the safety of celecoxib, naproxen, or ibuprofen. J Am Coll Cardiol. 2018;71(16):1741-51. doi: 10.1016/j.jacc.2018.02.036. PMID: 29673465.

152. Reginster JY, Dudler J, Blicharski T, et al. Pharmaceutical-grade Chondroitin sulfate is as effective as celecoxib and superior to placebo in symptomatic knee osteoarthritis: the ChONdroitin versus CElecoxib versus Placebo Trial (CONCEPT). Ann Rheum Dis. 2017 Sep;76(9):1537-43. doi: 10.1136/annrheumdis-2016-210860. PMID: 28533290.

153. Rostom A, Goldkind L, Laine L. Nonsteroidal anti-inflammatory drugs and hepatic toxicity: a systematic review of randomized controlled trials in arthritis patients. Clin Gastroenterol Hepatol. 2005 May;3(5):489-98. PMID: 15880319.

154. Roth SH. Endoscopy-controlled study of the safety of nabumetone compared with naproxen in arthritis therapy. Am J Med. 1987 Oct 30;83(4B):25-30. PMID: 3318424.

155. Roth SH, Bennett R, Caldron P, et al. A longterm endoscopic evaluation of patients with arthritis treated with nabumetone vs naproxen. J Rheumatol. 1994 Jun;21(6):1118-23. PMID: 7932425.

156. Roth SH, Bennett RE, Caldron PH, et al. Endoscopic evaluation of the long term effects of diclofenac sodium and naproxen in elderly patients with arthritis. Clin Drug Investig. 1995 Mar;9(3):171-9. 
157. Roth SH, Shainhouse JZ. Efficacy and safety of a topical diclofenac solution (pennsaid) in the treatment of primary osteoarthritis of the knee: a randomized, double-blind, vehicle-controlled clinical trial. Arch Intern Med. 2004 Oct 11;164(18):2017-23. doi: 10.1001/archinte.164.18.2017. PMID: 15477437.

158. Roth SH, Tindall EA, Jain AK, et al. A controlled study comparing the effects of nabumetone, ibuprofen, and ibuprofen plus misoprostol on the upper gastrointestinal tract mucosa. Arch Intern Med. $1993 \mathrm{Nov}$ 22;153(22):2565-71. PMID: 8239849.

159. Russell IJ, Mease PJ, Smith TR, et al. Efficacy and safety of duloxetine for treatment of fibromyalgia in patients with or without major depressive disorder: results from a 6-month, randomized, double-blind, placebo-controlled, fixed-dose trial. Pain. 2008 Jun;136(3):432-44. doi: 10.1016/j.pain.2008.02.024. PMID: 18395345.

160. Satoh J, Yagihashi S, Baba M, et al. Efficacy and safety of pregabalin for treating neuropathic pain associated with diabetic peripheral neuropathy: a 14 week, randomized, double-blind, placebocontrolled trial. Diabet Med. 2011 Jan;28(1):109-16. doi: 10.1111/j.14645491.2010.03152.x. PMID: 21166852.

161. Schifitto G, Yiannoutsos CT, Simpson DM, et al. A placebo-controlled study of memantine for the treatment of human immunodeficiency virus-associated sensory neuropathy. J Neurovirol. 2006 Aug;12(4):328-31. doi: 10.1080/13550280600873835. PMID: 16966223.

162. Schimrigk S, Marziniak M, Neubauer C, et al. Dronabinol is a safe long-term treatment option for neuropathic pain patients. Eur Neurol. 2017;78(5-6):320-9. doi: 10.1159/000481089. PMID: 29073592.

163. Schlaeger JM, Molokie RE, Yao Y, et al. Management of sickle cell pain using pregabalin: a pilot study. Pain Manag Nurs. 2017 Dec;18(6):391-400. doi: 10.1016/j.pmn.2017.07.003. PMID: 28843636.
164. Schnitzer TJ, Burmester GR, Mysler E, et al. Comparison of lumiracoxib with naproxen and ibuprofen in the Therapeutic Arthritis Research and Gastrointestinal Event Trial (TARGET), reduction in ulcer complications: randomised controlled trial. Lancet. 2004 Aug 21-27;364(9435):665-74. PMID: 15325831.

165. Schnitzer TJ, Dattani ID, Seriolo B, et al. A 13-week, multicenter, randomized, doubleblind study of lumiracoxib in hip osteoarthritis. Clin Rheumatol. 2011a Nov;30(11):1433-46. doi: 10.1007/s10067011-1776-4. PMID: 21607551.

166. Schnitzer TJ, Hochberg MC, Marrero CE, et al. Efficacy and safety of naproxcinod in patients with osteoarthritis of the knee: a 53week prospective randomized multicenter study. Semin Arthritis Rheum. 2011b Feb;40(4):285-97. doi: 10.1016/j.semarthrit.2010.06.002. PMID: 20828790.

167. Schnitzer TJ, Kivitz A, Frayssinet H, et al. Efficacy and safety of naproxcinod in the treatment of patients with osteoarthritis of the knee: a 13-week prospective, randomized, multicenter study. Osteoarthritis Cartilage. 2010 May;18(5):629-39. doi: 10.1016/j.joca.2009.12.013. PMID: 20202489.

168. Scott DL, Palmer RH. Safety and efficacy of nabumetone in osteoarthritis: emphasis on gastrointestinal safety. Aliment Pharmacol Ther. 2000 Apr;14(4):443-52. PMID: 10759624.

169. Selvarajah D, Gandhi R, Emery CJ, et al. Randomized placebo-controlled doubleblind clinical trial of cannabis-based medicinal product (Sativex) in painful diabetic neuropathy: depression is a major confounding factor. Diabetes Care. 2010 Jan;33(1):128-30. doi: 10.2337/dc09-1029. PMID: 19808912.

170. Serpell M, Ratcliffe S, Hovorka J, et al. A double-blind, randomized, placebocontrolled, parallel group study of THC/CBD spray in peripheral neuropathic pain treatment. Eur J Pain. 2014 Aug;18(7):999-1012. doi: 10.1002/j.15322149.2013.00445.x. PMID: 24420962. 
171. Sheldon E, Beaulieu A, Paster Z, et al. Efficacy and tolerability of lumiracoxib in the treatment of osteoarthritis of the knee: a 13-week, randomized, double-blind comparison with celecoxib and placebo. Clin Ther. 2005 Jan;27(1):64-77. doi: 10.1016/j.clinthera.2005.01.002. PMID: 15763607.

172. Siddall PJ, Cousins MJ, Otte A, et al. Pregabalin in central neuropathic pain associated with spinal cord injury: a placebo-controlled trial. Neurology. 2006 Nov 28;67(10):1792-800. doi: 10.1212/01.wnl.0000244422.45278.ff. PMID: 17130411.

173. Sieper J, Klopsch T, Richter M, et al. Comparison of two different dosages of celecoxib with diclofenac for the treatment of active ankylosing spondylitis: results of a 12-week randomised, double-blind, controlled study. Ann Rheum Dis. 2008 Mar;67(3):323-9. doi: 10.1136/ard.2007.075309. PMID: 17616556.

174. Sikes DH, Agrawal NM, Zhao WW, et al. Incidence of gastroduodenal ulcers associated with valdecoxib compared with that of ibuprofen and diclofenac in patients with osteoarthritis. Eur J Gastroenterol Hepatol. 2002 Oct;14(10):1101-11. PMID: 12362101.

175. Silverstein FE, Faich G, Goldstein JL, et al. Gastrointestinal toxicity with celecoxib vs nonsteroidal anti-inflammatory drugs for osteoarthritis and rheumatoid arthritis: the CLASS study: a randomized controlled trial. Celecoxib long-term arthritis safety study. JAMA. 2000 Sep 13;284(10):1247-55. PMID: 10979111.

176. Simon L, Weaver AL, Graham DY, et al. Anti-inflammatory and upper gastrointestinal effects of celecoxib in rheumatoid arthritis: a randomized controlled trial. JAMA. 1999 Nov 24;282(20):1921-8. PMID: 10580457.

177. Simon LS, Grierson LM, Naseer Z, et al. Efficacy and safety of topical diclofenac containing dimethyl sulfoxide (DMSO) compared with those of topical placebo, DMSO vehicle and oral diclofenac for knee osteoarthritis. Pain. 2009 Jun;143(3):238-45. doi: 10.1016/j.pain.2009.03.008. PMID: 19380203.
178. Simpson DM, Rice AS, Emir B, et al. A randomized, double-blind, placebocontrolled trial and open-label extension study to evaluate the efficacy and safety of pregabalin in the treatment of neuropathic pain associated with human immunodeficiency virus neuropathy. Pain. 2014 Oct;155(10):1943-54. doi: 10.1016/j.pain.2014.05.027. PMID: 24907403.

179. Simpson DM, Schifitto G, Clifford DB, et al. Pregabalin for painful HIV neuropathy: a randomized, double-blind, placebocontrolled trial. Neurology. 2010 Feb 2;74(5):413-20. doi: 10.1212/WNL.0b013e3181ccc6ef. PMID: 20124207.

180. Skljarevski V, Ossanna M, Liu-Seifert H, et al. A double-blind, randomized trial of duloxetine versus placebo in the management of chronic low back pain. Eur J Neurol. 2009 Sep;16(9):1041-8. doi: 10.1111/j.1468-1331.2009.02648.x. PMID: 19469829.

181. Skljarevski V, Zhang S, Desaiah D, et al. Duloxetine versus placebo in patients with chronic low back pain: a 12-week, fixeddose, randomized, double-blind trial. J Pain. 2010 Dec;11(12):1282-90. doi: 10.1016/j.jpain.2010.03.002. PMID: 20472510.

182. Smith T, DiBernardo A, Shi Y, et al. Efficacy and safety of carisbamate in patients with diabetic neuropathy or postherpetic neuralgia: results from 3 randomized, double-blind placebocontrolled trials. Pain pract. 2014 Apr;14(4):332-42. doi: 10.1111/papr.12080. PMID: 23692321.

183. Sofat N, Harrison A, Russell MD, et al. The effect of pregabalin or duloxetine on arthritis pain: a clinical and mechanistic study in people with hand osteoarthritis.[Erratum appears in J Pain Res. 2017 Dec 15;10:2843]. J Pain Res. 2017;10:2437-49. PMID: 29066930.

184. Solomon DH, Husni ME, Libby PA, et al. The risk of major NSAID toxicity with celecoxib, ibuprofen, or naproxen: a secondary analysis of the PRECISION Trial. Am J Med. 2017 Dec;130(12):1415-22.e4. doi: 10.1016/j.amjmed.2017.06.028. PMID: 28756267. 
185. Solomon DH, Husni ME, Wolski KE, et al. Differences in safety of nonsteroidal antiinflammatory drugs in patients with osteoarthritis and patients with rheumatoid arthritis: a randomized clinical trial. Arthritis Rheumatol. 2018 Apr;70(4):537-46. doi: 10.1002/art.40400. PMID: 29266879.

186. Sowers JR, White WB, Pitt B, et al. The effects of cyclooxygenase-2 inhibitors and nonsteroidal anti-inflammatory therapy on 24-hour blood pressure in patients with hypertension, osteoarthritis, and type 2 diabetes mellitus.[Erratum appears in Arch Intern Med. 2005 Mar 14;165(5):551]. Arch Intern Med. 2005 Jan 24;165(2):161-8. doi: 10.1001/archinte.165.2.161. PMID: 15668361.

187. Strand V, Bergman M, Singh JA, et al. Lowdose SoluMatrix diclofenac in patients with osteoarthritis pain: impact on quality of life in a controlled trial. Clin Rheumatol. 2017 Jun;36(6):1357-67. doi: 10.1007/s10067017-3569-х. PMID: 28194525.

188. Tanenberg RJ, Clemow DB, Giaconia JM, et al. Duloxetine compared with pregabalin for diabetic peripheral neuropathic pain management in patients with suboptimal pain response to gabapentin and treated with or without antidepressants: a post hoc analysis. Pain pract. 2014 Sep;14(7):640-8. doi: 10.1111/papr.12121. PMID: 24152185.

189. Tanenberg RJ, Irving G, Risser RC, et al. An open-label, randomized comparison of duloxetine, pregabalin, and the combination of duloxetine and gabapentin among patients with inadequate response to gabapentin for the management of diabetic peripheral neuropathic pain. Poster presentation, American Diabetes Association, June 25-29, 2010 Orlando, Florida. 2010.

190. Tanenberg RJ, Irving GA, Risser RC, et al. Duloxetine, pregabalin, and duloxetine plus gabapentin for diabetic peripheral neuropathic pain management in patients with inadequate pain response to gabapentin: an open-label, randomized, noninferiority comparison. Mayo Clin Proc. 2011 Jul;86(7):615-26. doi: 10.4065/mcp.2010.0681. PMID: 21719618.
191. Tannenbaum H, Berenbaum F, Reginster $\mathrm{JY}$, et al. Lumiracoxib is effective in the treatment of osteoarthritis of the knee: a 13 week, randomised, double blind study versus placebo and celecoxib. Ann Rheum Dis. 2004 Nov;63(11):1419-26. doi: 10.1136/ard.2003.015974. PMID: 15020310 .

192. Temple AR, Benson GD, Zinsenheim JR, et al. Multicenter, randomized, double-blind, active-controlled, parallel-group trial of the long-term (6-12 months) safety of acetaminophen in adult patients with osteoarthritis. Clin Ther. 2006

Feb;28(2):222-35. doi: 10.1016/j.clinthera.2006.02.004. PMID: 16678643.

193. Tetreault P, Baliki MN, Baria AT, et al. Inferring distinct mechanisms in the absence of subjective differences: placebo and centrally acting analgesic underlie unique brain adaptations. Hum Brain Mapp. 2018 May;39(5):2210-23. doi: 10.1002/hbm.23999. PMID: 29417694.

194. Tetreault P, Mansour A, Vachon-Presseau E, et al. Brain connectivity predicts placebo response across chronic pain clinical trials. PLoS Biol. 2016 Oct 27;14(10):e1002570. doi: 10.1371/journal.pbio.1002570. PMID: 27788130.

195. Tolle T, Freynhagen R, Versavel M, et al. Pregabalin for relief of neuropathic pain associated with diabetic neuropathy: a randomized, double-blind study. Eur J Pain. 2008 Feb;12(2):203-13. doi: 10.1016/j.ejpain.2007.05.003. PMID: 17631400 .

196. Tsuji T, Itoh N, Ishida M, et al. Response to duloxetine in chronic low back pain: exploratory post hoc analysis of a Japanese phase III randomized study. J Pain Res. 2017 Sep 4;10:2157-68. doi: 10.2147/JPR.S138172. PMID: 28919811.

197. Tugwell PS, Wells GA, Shainhouse JZ. Equivalence study of a topical diclofenac solution (pennsaid) compared with oral diclofenac in symptomatic treatment of osteoarthritis of the knee: a randomized controlled trial. J Rheumatol. 2004 Oct;31(10):2002-12. PMID: 15468367. 
198. Uchio Y, Enomoto H, Alev L, et al. A randomized, double-blind, placebocontrolled phase III trial of duloxetine in Japanese patients with knee pain due to osteoarthritis. J Pain Res. 2018;11:809-21. doi: 10.2147/JPR.S164128. PMID: 29713194.

199. Urquhart DM, Wluka AE, Sim MR, et al. Is low-dose amitriptyline effective in the management of chronic low back pain? Study protocol for a randomised controlled trial. Trials. 2016 Oct 22;17(1):514. doi: 10.1186/s13063-016-1637-1. PMID: 27770809 .

200. Urquhart DM, Wluka AE, Van Tulder M, et al. Efficacy of low-dose amitriptyline for chronic low back pain: a randomized clinical trial. JAMA Intern Med. 2018 Nov 1;178(11):1474-81. doi: 10.1001/jamainternmed.2018.4222. PMID: 30285054.

201. van Seventer R, Feister HA, Young JP, Jr., et al. Efficacy and tolerability of twice-daily pregabalin for treating pain and related sleep interference in postherpetic neuralgia: a 13week, randomized trial. Curr Med Res Opin. 2006 Feb;22(2):375-84. doi: 10.1185/030079906X80404. PMID: 16466610 .

202. Vasey FB, Germain BF, Espinoza LR, et al. Controlled evaluation of nabumetone in the treatment of active adult rheumatoid arthritis. Nabumetone versus naproxen double-blind parallel study. Am J Med. 1987 Oct 30;83(4B):55-9. PMID: 3318430.

203. Vetter G. A comparison of naproxen and diclofenac sodium in the treatment of osteoarthritis in elderly patients. Br J Clin Pract. 1985 Jul;39(7):276-81. PMID: 3896286.

204. Vitton O, Gendreau M, Gendreau J, et al. A double-blind placebo-controlled trial of milnacipran in the treatment of fibromyalgia. Hum Psychopharmacol. 2004 Oct;19 (Suppl 1):S27-35. doi: 10.1002/hup.622. PMID: 15378666.
205. Walker C, Essex MN, Li C, et al. Celecoxib versus diclofenac for the treatment of ankylosing spondylitis: 12-week randomized study in Norwegian patients. J Int Med Res. 2016 Jun;44(3):483-95. doi: 10.1177/0300060516628704. PMID: 26980340.

206. Wang G, Bi L, Li X, et al. Efficacy and safety of duloxetine in Chinese patients with chronic pain due to osteoarthritis: a randomized, double-blind, placebocontrolled study. Osteoarthritis Cartilage. 2017 Jun;25(6):832-8. doi: 10.1016/j.joca.2016.12.025. PMID: 28043937.

207. Wasan AD, Ossanna MJ, Raskin J, et al. Safety and efficacy of duloxetine in the treatment of diabetic peripheral neuropathic pain in older patients. Curr Drug Saf. 2009 Jan;4(1):22-9. PMID: 19149522.

208. Webster LR, Tark M, Rauck R, et al. Effect of duration of postherpetic neuralgia on efficacy analyses in a multicenter, randomized, controlled study of NGX-4010, an $8 \%$ capsaicin patch evaluated for the treatment of postherpetic neuralgia. BMC Neurol. 2010 Oct 11;10:92. doi: 10.1186/1471-2377-10-92. PMID: 20937130.

209. Welsch P, Uceyler N, Klose P, et al. Serotonin and noradrenaline reuptake inhibitors (SNRIs) for fibromyalgia. Cochrane Database Syst Rev. 2018 Feb 28;2:CD010292. doi: 10.1002/14651858.CD010292.pub2. PMID: 29489029.

210. Wernicke JF, Pritchett YL, D'Souza DN, et al. A randomized controlled trial of duloxetine in diabetic peripheral neuropathic pain. Neurology. 2006a Oct 24;67(8):141120. doi: 10.1212/01.wnl.0000240225.04000.1a. PMID: 17060567.

211. Wernicke JF, Raskin J, Rosen A, et al. Duloxetine in the long-term management of diabetic peripheral neuropathic pain: an open-label, 52-week extension of a randomized controlled clinical trial. Curr Ther Res Clin Exp. 2006b Sep;67(5):283304. doi: 10.1016/j.curtheres.2006.10.001. PMID: 24678103. 
212. Whelton A, Lefkowith JL, West CR, et al. Cardiorenal effects of celecoxib as compared with the nonsteroidal antiinflammatory drugs diclofenac and ibuprofen. Kidney Int. 2006 Oct;70(8):1495502. doi: 10.1038/sj.ki.5001766. PMID: 16941030 .

213. White WB, Faich G, Whelton A, et al. Comparison of thromboembolic events in patients treated with celecoxib, a cyclooxygenase-2 specific inhibitor, versus ibuprofen or diclofenac. Am J Cardiol. 2002 Feb 15;89(4):425-30. PMID: 11835924.

214. Wiesenhutter CW, Boice JA, Ko A, et al. Evaluation of the comparative efficacy of etoricoxib and ibuprofen for treatment of patients with osteoarthritis: a randomized, double-blind, placebo-controlled trial. Mayo Clin Proc. 2005 Apr;80(4):470-9. PMID: 15819283.

215. Wojtulewski JA, Schattenkirchner M, Barcelo P, et al. A six-month double-blind trial to compare the efficacy and safety of meloxicam $7.5 \mathrm{mg}$ daily and naproxen 750 $\mathrm{mg}$ daily in patients with rheumatoid arthritis. Br J Rheumatol. 1996 Apr;35(Suppl 1):22-8. PMID: 8630632.

216. Yasuda H, Hotta N, Kasuga M, et al. Efficacy and safety of $40 \mathrm{mg}$ or $60 \mathrm{mg}$ duloxetine in Japanese adults with diabetic neuropathic pain: Results from a randomized, 52-week, open-label study. J Diabetes Investig. 2016 Jan;7(1):100-8. doi: 10.1111/jdi.12361. PMID: 26816607.

217. Yasuda H, Hotta N, Nakao K, et al. Superiority of duloxetine to placebo in improving diabetic neuropathic pain: results of a randomized controlled trial in Japan. $\mathrm{J}$ Diabetes Investig. 2011 Apr 7;2(2):132-9. doi: 10.1111/j.2040-1124.2010.00073.x. PMID: 24843472.
218. Yeomans ND, Graham DY, Husni ME, et al. Randomised clinical trial: gastrointestinal events in arthritis patients treated with celecoxib, ibuprofen or naproxen in the PRECISION trial. Aliment Pharmacol Ther. 2018 Jun;47(11):1453-63. doi: 10.1111/apt.14610. PMID: 29667211.

219. Yocum D, Fleischmann R, Dalgin P, et al. Safety and efficacy of meloxicam in the treatment of osteoarthritis: a 12-week, double-blind, multiple-dose, placebocontrolled trial. The Meloxicam Osteoarthritis Investigators. Arch Intern Med. 2000 Oct 23;160(19):2947-54. PMID: 11041902.

220. Zhang L, Rainka M, Freeman R, et al. A randomized, double-blind, placebocontrolled trial to assess the efficacy and safety of gabapentin enacarbil in subjects with neuropathic pain associated with postherpetic neuralgia (PXN110748). J Pain. 2013 Jun;14(6):590-603. doi: 10.1016/j.jpain.2013.01.768. PMID: 23602345.

221. Zhao SZ, Fiechtner JI, Tindall EA, et al. Evaluation of health-related quality of life of rheumatoid arthritis patients treated with celecoxib. Arthritis Care Res (Hoboken). 2000 Apr;13(2):112-21. doi: 10.1002/15290131\%28200004\%2913:2\%3C112::AIDANR5\%3E3.0.CO;2-L. PMID: 14635284.

222. Zhao SZ, McMillen JI, Markenson JA, et al. Evaluation of the functional status aspects of health-related quality of life of patients with osteoarthritis treated with celecoxib. Pharmacotherapy. 1999 Nov;19(11):126978. PMID: 10555933. 


\section{Appendix D. Excluded Studies}

Table D-1. Key to exclusion codes

\begin{tabular}{|c|l|}
\hline $\begin{array}{l}\text { Exclusion } \\
\text { Code }\end{array}$ & Exclusion Reason \\
\hline 1 & Included primary or secondary publication \\
\hline 2 & Foreign language \\
\hline 3 & Ineligible intervention (including comparator) \\
\hline 4 & Ineligible population \\
\hline 5 & Ineligible publication type \\
\hline 6 & Ineligible study design \\
\hline 7 & Study not obtainable \\
\hline 8 & Outdated or ineligible systematic review \\
\hline 9 & Study duration <12 weeks \\
\hline
\end{tabular}

1. Duloxetine effective for fibromyalgia in some women. J Fam Pract. 2006 May;55(5):382. PMID: 16722009. Exclusion: 5

2. Accardo S, Seriolo B, Samanta E, et al. Controlled-release naproxen in rheumatoid arthritis. Curr Ther Res Clin Exp. 1991;49(6):936-42. Exclusion: 3

3. Acevedo E, Castaneda O, Ugaz M, et al. Tolerability profiles of rofecoxib (Vioxx) and Arthrotec. A comparison of six weeks treatment in patients with osteoarthritis. Scand J Rheumatol. 2001;30(1):19-24. doi: 10.1080/030097401750065274 PMID: 11252687. Exclusion: 3

4. Admani AK, Verma S. A study of sulindac versus ibuprofen in elderly patients with osteoarthritis. Curr Med Res Opin. 1983;8(5):315-20. doi: 10.1185/03007998309112390. PMID: 6839797. Exclusion: 3

5. Agrawal N, Van Kerckhove HE, Erhardt LJ, et al. Misoprostol coadministered with diclofenac for prevention of gastroduodenal ulcers. A one-year study. Dig Dis Sci. 1995 May;40(5):1125-31. doi: 10.1007/BF02064210. PMID: 7729275. Exclusion: 3

6. Agrawal NM, Roth S, Graham DY, et al. Misoprostol compared with sucralfate in the prevention of nonsteroidal antiinflammatory drug-induced gastric ulcer. A randomized, controlled trial. Ann Intern Med. 1991 Aug 1;115(3):195-200. doi: 10.7326/0003-4819-115-3-195. PMID: 1905501. Exclusion: 3
7. Ahern MJ, Wetherall M, Leslie A, et al. A comparison of ketoprofen SR and sulindac in the elderly with rheumatoid arthritis. Br J Clin Pract. 1992 Winter;46(4):229-33. PMID: 1290729. Exclusion: 3

8. Ahmed M, Aamir R, Jishi Z, et al. The effects of milnacipran on sleep disturbance in fibromyalgia: a randomized, double-blind, placebo-controlled, two-way crossover study. J Clin Sleep Med. 2016 Jan;12(1):7986. doi: 10.5664/jcsm.5400. PMID: 26414990. Exclusion: 9

9. Aicher B, Peil H, Peil B, et al. Pain measurement: Visual Analogue Scale (VAS) and Verbal Rating Scale (VRS) in clinical trials with OTC analgesics in headache. Cephalalgia. 2012 Feb;32(3):185-97. doi: 10.1177/0333102411430856. PMID: 22332207. Exclusion: 3

10. Al MJ, Maniadakis N, Grijseels EW, et al. Costs and effects of various analgesic treatments for patients with rheumatoid arthritis and osteoarthritis in the Netherlands. Value Health. 2008 JulAug;11(4):589-99. doi: 10.1111/j.15244733.2007.00303.x. PMID: 18194404. Exclusion: 6

11. Alballa S, Al-Arfaj H, Al-Sugair S, et al. Randomized, double-blind, short-term trial of nabumetone versus diclofenac in osteoarthritis of the knee. Curr Ther Res Clin Exp. 1992;52(4):581-6. doi: 10.1016/S0011-393X(05)80465-5. Exclusion: 10 
12. Alballa S, AlNasser A, AlArfaj H, et al. A randomized double-blind short-term trial of tenoxicam versus piroxicam in osteoarthritis of the knee. Curr Ther Res Clin Exp. 1990;47(3):591-6. Exclusion: 10

13. Alcoff J, Jones E, Rust P, et al. Controlled trial of imipramine for chronic low back pain. J Fam Pract. 1982 May;14(5):841-6. PMID: 6210751. Exclusion: 9

14. Alda M, Luciano JV, Andres E, et al. Effectiveness of cognitive behaviour therapy for the treatment of catastrophisation in patients with fibromyalgia: a randomised controlled trial. Arthritis Res Ther. 2011;13(5):R173. doi: 10.1186/ar3496. PMID: 22018333. Exclusion: 3

15. Aldrete JA, Aldrete VT, Williams SK, et al. Reduction of neuropathic pain in patients with arachnoiditis: crossover study of gabapentin versus phenytoin. Pain digest. 2000;10(2):64-7. Exclusion: 6

16. Alexander SJ. Clinical experience with naproxen in rheumatoid arthritis. Arch Intern Med. 1975 Nov;135(11):1429-35. PMID: 1103765. Exclusion: 6

17. Alharbi GS, Chen LC, Knaggs R. Efficacy of anticonvulsant, antidepressant and opioid in treating neuropathic pain - A systematic review and meta-analysis.

Pharmacoepidemiol Drug Saf.

2016;25(Suppl 3):583-4. doi:

10.1002/pds.4070. Exclusion: 8

18. Alho A, Jaer O, Slungaard U, et al. Piroxicam and naproxen in patients with osteoarthritis of the hip waiting for total hip replacement. Clin Rheumatol. 1988 Jun;7(2):208-13. PMID: 3046826. Exclusion: 9

19. al-Quorain AA, Satti MB, Marwah S, et al. Non-steroidal anti-inflammatory druginduced gastropathy: a comparative endoscopic and histopathological evaluation of the effects of tenoxicam and diclofenac. $\mathrm{J}$ Int Med Res. 1993 Mar-Apr;21(2):89-97. doi: 10.1177/030006059302100204. PMID: 8243794. Exclusion: 2
20. Al-Sharkawi MS. A multicentre study of diclofenac sodium slow-release (Voltaren Retard) in the treatment of rheumatic disorders in the Kingdom of Saudi Arabia. J Int Med Res. 1984;12(4):244-9. doi: 10.1177/030006058401200404. PMID: 6381168. Exclusion: 6

21. Altman RD, Dreiser R-L, Fisher CL, et al. Diclofenac sodium gel in patients with primary hand osteoarthritis: a randomized, double-blind, placebo-controlled trial. J Rheumatol. 2009 Sep;36(9):1991-9. doi: 10.3899/jrheum.081316. PMID: 19648310. Exclusion: 9

22. Altman RD, Moskowitz R. Intraarticular sodium hyaluronate (Hyalgan) in the treatment of patients with osteoarthritis of the knee: a randomized clinical trial. Hyalgan Study Group.[Erratum appears in J Rheumatol 1999 May;26(5):1216]. J Rheumatol. 1998 Nov;25(11):2203-12. PMID: 9818665. Exclusion: 3

23. Altman RD, Strand V, Hochberg MC, et al. Low-dose SoluMatrix diclofenac in the treatment of osteoarthritis: a 1-year, openlabel, phase III safety study. Postgrad Med. 2015 Jun;127(5):517-28. doi: 10.1080/00325481.2015.1040716. PMID: 25913498. Exclusion: 6

24. Amante CM. Randomized comparative study on programmed released ketoprofen vs. piroxicam in the treatment of rheumatoid arthritis. Philippine Journal of Internal Medicine. 1988;26(2):69-73. Exclusion: 3

25. Ambanelli U, Nervetti A, Colombo B, et al. Piroxicam-beta-cyclodextrin in the treatment of rheumatic diseases: a prospective study. Curr Ther Res Clin Exp. 1990;48(1):58-68. Exclusion: 3

26. Ambanelli U, Nervetti A, Colombo B, et al. $\beta$-cyclodextrin-piroxicam in the treatment of rheumatic diseases: a prospective study. J Drug Development. 1991;4(1):55-66. Exclusion: 3

27. Ammitzboll F. Fenbufen and indomethacin in the treatment of rheumatoid arthritis. A comparative double-blind, crossover study. Scand J Rheumatol Suppl. 1979(23):5-10. PMID: 373083. Exclusion: 3 
28. Anderberg UM, Marteinsdottir I, von Knorring L. Citalopram in patients with fibromyalgia--a randomized, double-blind, placebo-controlled study. Eur J Pain. 2000;4(1):27-35. doi: 10.1053/eujp.1999.0148. PMID: 10833553. Exclusion: 3

29. Anderson JA, Lee P, Webb J, et al. Evaluation of the therapeutic potential of ketoprofen in rheumatoid arthritis. Curr Med Res Opin. 1974;2(4):189-97. doi: 10.1185/03007997409112306. PMID: 4601567. Exclusion: 3

30. Anderson JJ, Firschein HE, Meenan RF. Sensitivity of a health status measure to short-term clinical changes in arthritis. Arthritis Rheum. 1989 Jul;32(7):844-50. PMID: 2665756. Exclusion: 6

31. Andrew A, Rodda B, Verhaest L, et al. Diflunisal: six-month experience in osteoarthritis. Br J Clin Pharmacol. 1977 Feb;4 Suppl 1:45S-52S. doi: 10.1111/j.1365-2125.1977.tb04514.x. PMID: 328034. Exclusion: 3

32. Anonymous. Clinical evaluation of ketoprofen in rheumatoid arthritis--early phase II study by multi-clinical trial. New drug research group. Ryumachi. 1973 Jul;13(3):256-60. PMID: 4755623. Exclusion: 9

33. Anonymous. Migraine treated with an antihistamine-analgesic combination. Practitioner. 1973 Sep;211(263):357-61. PMID: 4148490. Exclusion: 3

34. Anonymous. The simultaneous assessment of four nonsteroidal antiinflammatory drugs in rheumatoid arthritis using a simple and rapid trial design. Australasia Multicentre Trial Group. J Rheumatol. 1980 NovDec;7(6):857-64. PMID: 7009858. Exclusion: 6

35. Anonymous. Piroxicam vs indomethacin: a double blind multicenter comparative study in osteoarthritis. A Canadian Multicenter Study. J Rheumatol. 1985 Apr;12(2):328-36. PMID: 3897535. Exclusion: 3

36. Anonymous. A double blind comparison of piroxicam and enteric coated ASA in rheumatoid arthritis. A cooperative multicenter Canadian trial. J Rheumatol. 1985 Feb;12(1):68-77. PMID: 3884806. Exclusion: 3
37. Anonymous. Nabumetone: new preparation. Just another NSAID. Prescrire Int. 2000 Apr;9(46):43-6. PMID: 11503785. Exclusion: 5

38. Ansell BM, Carter ME, Gumpel JM, et al. Clinical studies with tolmetin: open study and a double-blind cross-over comparison with aspirin in rheumatoid arthritis. Rheumatol Rehabil. 1978 Aug;17(3):150-4. PMID: 360360. Exclusion: 9

39. Anthony M, Lance JW. Indomethacin in migraine. Med J Aust. 1968 Jan 13;1(2):567. PMID: 4867513. Exclusion: 4

40. Arai Y-CP, Matsubara T, Shimo K, et al. Low-dose gabapentin as useful adjuvant to opioids for neuropathic cancer pain when combined with low-dose imipramine. J Anesth. 2010 Jun;24(3):407-10. doi: 10.1007/s00540-010-0913-6 PMID: 20217150. Exclusion: 6

41. Argoff CE. Review of current guidelines on the care of postherpetic neuralgia. Postgrad Med. 2011 Sep;123(5):134-42. doi: 10.3810/pgm.2011.09.2469. PMID: 21904096. Exclusion: 6

42. Argyriou AA, Chroni E, Polychronopoulos $\mathrm{P}$, et al. Efficacy of oxcarbazepine for prophylaxis against cumulative oxaliplatininduced neuropathy. Neurology. 2006 Dec 26;67(12):2253-5. doi: 10.1212/01.wnl.0000249344.99671.d4. PMID: 17190958. Exclusion: 3

43. Armstrong DG, Chappell AS, Le TK, et al. Duloxetine for the management of diabetic peripheral neuropathic pain: evaluation of functional outcomes.[Erratum appears in Pain Med. 2007 Nov-Dec;8(8):690]. Pain Med. 2007 Jul-Aug;8(5):410-8. doi: 10.1111/j.1526-4637.2007.00276.x. PMID: 17661854. Exclusion: 6

44. Arnold LM. Duloxetine and other antidepressants in the treatment of patients with fibromyalgia. Pain Med. 2007 Sep;8(Suppl 2):S63-74. doi: 10.1111/j.15264637.2006.00178.x. PMID: 17714117. Exclusion: 6 
45. Arnold LM, Arsenault P, Huffman C, et al. Corrections to: once-daily controlled-release pregabalin in the treatment of patients with fibromyalgia: a phase III, double-blind, randomized withdrawal, placebo-controlled study. Curr Med Res Opin. 2017

Apr;33(4):795-6. doi: 10.1080/03007995.2017.1292446. PMID: 28276874. Exclusion: 6

46. Arnold LM, Arsenault P, Huffman C, et al. Once daily controlled-release pregabalin in the treatment of patients with fibromyalgia: a phase III, double-blind, randomized withdrawal, placebo-controlled study.[Erratum appears in Curr Med Res Opin. 2017 Apr;33(4):795-796; PMID: 28276874]. Curr Med Res Opin. 2014 Oct;30(10):2069-83. doi: 10.1185/03007995.2014.928275. PMID: 24867298. Exclusion: 6

47. Arnold LM, Bateman L, Palmer RH, et al. Preliminary experience using milnacipran in patients with juvenile fibromyalgia: lessons from a clinical trial program. Pediatr Rheumatol Online J. 2015 Jun 26;13:27. doi: 10.1186/s12969-015-0025-9. PMID: 26112278. Exclusion: 4

48. Arnold LM, Choy E, Clauw DJ, et al. An evidence-based review of pregabalin for the treatment of fibromyalgia. Curr Med Res Opin. 2018 Aug;34(8):1397-409. doi: 10.1080/03007995.2018.1450743. PMID: 29519159. Exclusion: 8

49. Arnold LM, Crofford LJ, Martin SA, et al. The effect of anxiety and depression on improvements in pain in a randomized, controlled trial of pregabalin for treatment of fibromyalgia. Pain Med. 2007 NovDec;8(8):633-8. doi: 10.1111/j.15264637.2007.00332.x. PMID: 18028041. Exclusion: 9

50. Arnold LM, Emir B, Pauer L, et al. Time to improvement of pain and sleep quality in clinical trials of pregabalin for the treatment of fibromyalgia. Pain Med. 2015 Jan;16(1):176-85. doi: 10.1111/pme.12636. PMID: 25529830. Exclusion: 6
51. Arnold LM, Hess EV, Hudson JI, et al. A randomized, placebo-controlled, doubleblind, flexible-dose study of fluoxetine in the treatment of women with fibromyalgia. Am J Med. 2002 Feb 15;112(3):191-7. doi: 10.1016/S0002-9343(01)01089-0. PMID: 11893345. Exclusion: 3

52. Arnold LM, Pritchett YL, D'Souza DN, et al. Duloxetine for the treatment of fibromyalgia in women: pooled results from two randomized, placebo-controlled clinical trials. J Womens Health (Larchmt). 2007 Oct;16(8):1145-56. doi: 10.1089/jwh.2006.0213. PMID: 17937567. Exclusion: 6

53. Arnold LM, Sarzi-Puttini P, Arsenault P, et al. Efficacy and safety of pregabalin in patients with fibromyalgia and comorbid depression taking concurrent antidepressant medication: a randomized, placebocontrolled study. J Rheumatol. 2015 Jul;42(7):1237-44. doi: 10.3899/jrheum.141196. PMID: 26034150. Exclusion: 9

54. Ashina S, Bendtsen L, Jensen R. Analgesic effect of amitriptyline in chronic tensiontype headache is not directly related to serotonin reuptake inhibition. Pain. 2004 Mar;108(1-2):108-14. doi: 10.1016/j.pain.2003.12.012. PMID: 15109513. Exclusion: 2

55. Astorga G. Double-blind, parallel clinical trial of tenoxicam (Ro 12-0068) versus piroxicam in patients with ankylosing spondylitis. Eur J Rheumatol Inflamm. 1987;9(2):70-3. PMID: 3329111. Exclusion: 3

56. Atalay H, Solak Y, Biyik Z, et al. Crossover, open-label trial of the effects of gabapentin versus pregabalin on painful peripheral neuropathy and health-related quality of life in haemodialysis patients. Clin Drug Investig. 2013 Jun;33(6):401-8. doi: 10.1007/s40261-013-0080-2. PMID: 23572323. Exclusion: 9

57. Ataoglu S, Ataoglu A, Erdogan F, et al. Comparison of paroxetine, amitriptyline in the treatment of fibromyalgia. Turk J Med Sci. 1997;27(6):535-9. Exclusion: 3 
58. Atkinson M, Germain G, Lee P. The efficacy and safety of sulindac (400 mg vs $600 \mathrm{mg}$ daily) in rheumatoid arthritis. A Canadian multicentre study. J Rheumatol. 1988 Jun;15(6):1001-4. PMID: 3047380. Exclusion: 9

59. Atra E, Metz CA, Brown BL, et al. Flurbiprofen versus diclofenac for the treatment of osteoarthritis of the knee. DICP. 1990 Oct;24(10):920-3. PMID: 2244403. Exclusion: 9

60. Averbuch M, Katzper M. Assessment of visual analog versus categorical scale for measurement of osteoarthritis pain. J Clin Pharmacol. 2004 Apr;44(4):368-72. doi: 10.1177/0091270004263995. PMID: 15051743. Exclusion: 2

61. Aylward M, Maddock J, Lewis PA, et al. Mefenamic acid and diclofenac sodium in osteoarthritis of the weight bearing joints: a double blind comparison. Br J Clin Pract. 1985 Apr;39(4):135-9. PMID: 3893502. Exclusion: 3

62. Aylward M, Maddock J, Parker RJ, et al. Evaluation of tolmetin in the treatment of arthritis: open and controlled double-blind studies. Curr Med Res Opin. 1976;4(2):15869. doi: 10.1185/03007997609109297. PMID: 776539. Exclusion: 6

63. Aylward M, Parker RJ, Holly F, et al. Longterm study of indomethacin and alclofenac in treatment of rheumatoid arthritis. Br Med J. 1975 Apr 5;2(5961):7-9. doi: 10.1136/bmj.2.5961.7. PMID: 236805. Exclusion: 3

64. Backonja M, Beydoun A, Edwards KR, et al. Gabapentin for the symptomatic treatment of painful neuropathy in patients with diabetes mellitus: a randomized controlled trial. JAMA. 1998 Dec 2;280(21):1831-6. doi:

10.1001/jama.280.21.1831. PMID: 9846777. Exclusion: 9

65. Backonja MM. Gabapentin monotherapy for the symptomatic treatment of painful neuropathy: a multicenter, double-blind, placebo-controlled trial in patients with diabetes mellitus. Epilepsia. 1999;40 Suppl 6:S57-9; discussion S73-4. doi: 10.1111/j.1528-1157.1999.tb00934.x. PMID: 10530684. Exclusion: 9
66. Bacon PA. Safety profile of etodolac in the elderly population. Eur J Rheumatol Inflamm. 1994;14(1):19-22. PMID: 7744124. Exclusion: 5

67. Bacon PA, Davies J, Ring FJ. Benoxaprofen--dose-range studies using quantitative thermography. J Rheumatol Suppl. 1980;6:48-53. PMID: 6993672. Exclusion: 3

68. Baer PA, Thomas LM, Shainhouse Z. Treatment of osteoarthritis of the knee with a topical diclofenac solution: a randomised controlled, 6-week trial

[ISRCTN53366886]. BMC Musculoskelet Disord. 2005 Aug 8;6(44)doi:

10.1186/1471-2474-6-44. PMID: 16086839. Exclusion: 9

69. Bahous I. Double-blind multicentre, between patients comparison of two different doses of indoprofen and one of indomethacin in osteoarthritis. Eur $\mathrm{J}$ Rheumatol Inflamm. 1981;4(1):118-25. PMID: 7042355. Exclusion: 3

70. Bain LS, el-Ghobarey AF, Collins RM, et al. Tolmetin: an evaluation of a new preparation in the treatment of rheumatoid arthritis. Br J Clin Pract. 1975 Aug;29(8):208-10. PMID: 1100086. Exclusion: 3

71. Bakshi R. Comparative efficacy and tolerability of two diclofenac formulations in the treatment of painful osteoarthritis. $\mathrm{Br}$ J Clin Pract. 1996 Sep;50(6):294-7. PMID: 8983316. Exclusion: 3

72. Balme HW, Wojtulewski JA, Huskisson EC. Clinical experience with tolmetin sodium. Curr Med Res Opin. 1980;7(2):127-30. doi: 10.1185/03007998009112039. PMID: 7002482. Exclusion: 5

73. Banerjee M, Pal S, Bhattacharya B, et al. A comparative study of efficacy and safety of gabapentin versus amitriptyline as coanalgesics in patients receiving opioid analgesics for neuropathic pain in malignancy. Indian J Pharmacol. 2013 JulAug;45(4):334-8. doi: 10.4103/02537613.115000. PMID: 24014906. Exclusion: 4

74. Bank J. A comparative study of amitriptyline and fluvoxamine in migraine prophylaxis. Headache. 1994 Sep;34(8):4768. PMID: 7960733. Exclusion: 3 
75. Bannuru RR, Schmid CH, Kent DM, et al. Comparative effectiveness of pharmacologic interventions for knee osteoarthritis: a systematic review and network metaanalysis. Ann Intern Med. 2015 Jan 06;162(1):46-54. doi: 10.7326/M14-1231. PMID: 25560713. Exclusion: 8

76. Bannwarth B. Safety of the nonselective NSAID nabumetone : focus on gastrointestinal tolerability. Drug Saf. 2008;31(6):485-503. doi: 10.2165/00002018-200831060-00004. PMID: 18484783. Exclusion: 8

77. Bansal D, Bhansali A, Hota D, et al. Amitriptyline vs. pregabalin in painful diabetic neuropathy: a randomized double blind clinical trial. Diabet Med. 2009 Oct;26(10):1019-26. doi: 10.1111/j.14645491.2009.02806.x. PMID: 19900234. Exclusion: 9

78. Baraf HS, Gloth FM, Barthel HR, et al. Safety and efficacy of topical diclofenac sodium gel for knee osteoarthritis in elderly and younger patients: pooled data from three randomized, double-blind, parallel-group, placebo-controlled, multicentre trials. Drugs Aging. 2011 Jan 1;28(1):27-40. doi: 10.2165/11584880-000000000-00000. PMID: 21174485. Exclusion: 6

79. Baraf HSB, Fuentealba C, Greenwald M, et al. Tolerability and effectiveness of etoricoxib compared to diclofenac sodium in patients with osteoarthritis: a randomized controlled study (EDGE trial). Arthritis Rheum. 2004;50(9):S346-7. Exclusion: 3

80. Barnard-Jones K, Davies RW, Lalla O, et al. Mefenamic acid versus ibuprofen in osteoarthritis--a double-blind cross-over study. Br J Clin Pract. 1986 Dec;40(12):52831. PMID: 3307863. Exclusion: 9

81. Baron R, Mayoral V, Leijon G, et al. 5\% lidocaine medicated plaster versus pregabalin in post-herpetic neuralgia and diabetic polyneuropathy: an open-label, noninferiority two-stage RCT study. Curr Med Res Opin. 2009 Jul;25(7):1663-76. doi: 10.1185/03007990903047880. PMID: 19485723. Exclusion: 9
82. Baron R, Mayoral V, Leijon G, et al. Efficacy and safety of combination therapy with $5 \%$ lidocaine medicated plaster and pregabalin in post-herpetic neuralgia and diabetic polyneuropathy. Curr Med Res Opin. 2009 Jul;25(7):1677-87. doi: 10.1185/03007990903048078. PMID: 19480610. Exclusion: 9

83. Barraclough DR, Lenaghan E, Muirden KD. A comparison of flurbiprofen and aspirin in the treatment of rheumatoid arthritis. Med J Aust. 1974 Dec 28;2(26):925-7. PMID: 4616159. Exclusion: 3

84. Battagliotti CA, Baetti EA. Clinical, doubleblind long-term study of tenoxicam $20 \mathrm{mg}$ (Ro 12-0068) versus piroxicam $20 \mathrm{mg}$ in patients with gonarthrosis. Eur J Rheumatol Inflamm. 1987;9(2):74-6. PMID: 3329112. Exclusion: 3

85. Baumgartner H, Hohmeister R, Blumenberg-Novoselac N. An observerblind crossover study to compare the efficacies of flurbiprofen, indomethacin and naproxen given orally and rectally in the relief of night pain and morning stiffness due to rheumatoid arthritis. J Int Med Res. 1988 May-Jun;16(3):189-96. doi: 10.1177/030006058801600304. PMID: 2457523. Exclusion: 3

86. Bayley TR, Haslock I. Night medication in rheumatoid arthritis. J R Coll Gen Pract. 1976 Aug;26(169):591-4. PMID: 787518. Exclusion: 4

87. Bedaiwi MK, Sari I, Wallis D, et al. Clinical Efficacy of Celecoxib Compared to Acetaminophen in Chronic Nonspecific Low Back Pain: Results of a Randomized Controlled Trial. Arthritis Care Res (Hoboken). 2016 Jun;68(6):845-52. doi: 10.1002/acr.22753. PMID: 26474041. Exclusion: 9

88. Beesdo K, Hartford J, Russell J, et al. The short- and long-term effect of duloxetine on painful physical symptoms in patients with generalized anxiety disorder: results from three clinical trials. J Anxiety Disord. 2009 Dec;23(8):1064-71. doi: 10.1016/j.janxdis.2009.07.008. PMID: 19643572. Exclusion: 4 
89. Beghi E. The use of anticonvulsants in neurological conditions other than epilepsy: A review of the evidence from randomized controlled trials. CNS Drugs. 1999;11(1):6182. Exclusion: 6

90. Bellamy N, Beaulieu A, Bombardier C, et al. Efficacy and tolerability of enteric-coated naproxen in the treatment of osteoarthritis and rheumatoid arthritis: a double-blind comparison with standard naproxen followed by an open-label trial. Curr Med Res Opin. 1992;12(10):640-51. doi: 10.1185/03007999209111531. PMID: 1633722. Exclusion: 6

91. Bellamy N, Bensen WG, Beaulieu A, et al. A multicenter study of nabumetone and diclofenac SR in patients with osteoarthritis. J Rheumatol. 1995 May;22(5):915-20. PMID: 8587082. Exclusion: 2

92. Bellamy N, Buchanan WW, Goldsmith $\mathrm{CH}$, et al. Validation study of WOMAC: a health status instrument for measuring clinically important patient relevant outcomes to antirheumatic drug therapy in patients with osteoarthritis of the hip or knee. J

Rheumatol. 1988 Dec;15(12):1833-40. PMID: 3068365. Exclusion: 3

93. Bellamy N, Wells G, Campbell J. Relationship between severity and clinical importance of symptoms in osteoarthritis. Clin Rheumatol. 1991 Jun;10(2):138-43. PMID: 1914413. Exclusion: 9

94. Bellavance AJ, Meloche JP. A comparative study of naproxen sodium, pizotyline and placebo in migraine prophylaxis. Headache. 1990 Nov;30(11):710-5. PMID: 2074163.

Exclusion: 2

95. Bendtsen L, Jensen R. Amitriptyline reduces myofascial tenderness in patients with chronic tension-type headache. Cephalalgia. 2000 Jul;20(6):603-10. doi: 10.1046/j.14682982.2000.00087.x. PMID: 11075846.

Exclusion: 9

96. Bendtsen L, Jensen R, Olesen J. A nonselective (amitriptyline), but not a selective (citalopram), serotonin reuptake inhibitor is effective in the prophylactic treatment of chronic tension-type headache. J Neurol Neurosurg Psychiatry. 1996 Sep;61(3):28590. doi: 10.1136/jnnp.61.3.285. PMID: 8795600. Exclusion: 4
97. Benhamou M, Gossec L, Dougados M. Clinical relevance of C-reactive protein in ankylosing spondylitis and evaluation of the NSAIDs/coxibs' treatment effect on Creactive protein. Rheumatology (Oxford). 2010 Mar;49(3):536-41. doi: 10.1093/rheumatology/kep393. PMID: 20028728. Exclusion: 6

98. Bennett RM. A 12-week, double-blind, multicenter study comparing diflunisal twice daily and ibuprofen four times daily in the treatment of rheumatoid arthritis. Clin Ther. 1986;9(Suppl C):27-36. PMID: 3548984. Exclusion: 3

99. Bennett RM. Antidepressants do not have better results than placebo in the treatment of fibromyalgia in Brazil. Curr Rheumatol Rep. 2002 Aug;4(4):284-5. PMID: 12166412. Exclusion: 5

100. Bennett RM, Gatter RA, Campbell SM, et al. A comparison of cyclobenzaprine and placebo in the management of fibrositis. A double-blind controlled study. Arthritis Rheum. 1988 Dec;31(12):1535-42. doi: 10.1002/art.1780311210. PMID: 3058130. Exclusion: 4

101. Bennett RM, Kamin M, Karim R, et al. Tramadol and acetaminophen combination tablets in the treatment of fibromyalgia pain: a double-blind, randomized, placebocontrolled study. Am J Med. 2003 May;114(7):537-45. PMID: 12753877. Exclusion: 3

102. Bensen WG, Zhao SZ, Burke TA, et al. Upper gastrointestinal tolerability of celecoxib, a COX-2 specific inhibitor, compared to naproxen and placebo. J Rheumatol. 2000 Aug;27(8):1876-83. PMID: 10955327. Exclusion: 5

103. Benson M, Marangou A, Russo MA, et al. Patient preference for sustained-release versus standard paracetamol (acetaminophen): a multicentre, randomized, open-label, two-way crossover study in subjects with knee osteoarthritis. J Int Med Res. 2009 Sep-Oct;37(5):1321-35. doi: 10.1177/147323000903700507. PMID: 19930837. Exclusion: 9 
104. Benvenuti C, Longoni L. Multicentre study on effectiveness and safety of flurbiprofen versus alternative therapy in 738 rheumatic patients. Curr Ther Res Clin Exp.

1983;34(1):30-44. Exclusion: 9

105. Berkowitz J, Rogenes P, Sharp J, et al. Ranitidine protects against gastroduodenal mucosal damage associated with chronic aspirin therapy. Arch Intern Med. 1987 Dec;147(12):2137-9. doi: 10.1001/archinte.1987.00370120073014. PMID: 3689065. Exclusion: 4

106. Berkowitz SS, Bernhard G, Bilka PJ, et al. Tolmetin versus placebo for the treatment of rheumatoid arthritis: a sequential doubleblind clinical trial. Curr Ther Res Clin Exp. 1974 May;16(5):442-51. PMID: 4209061. Exclusion: 9

107. Berry H, Bloom B, Mace BEW, et al. Comparison of indoprofen (Flosint) and diclofenac in rheumatoid arthritis. A placebo controlled trial. Clinical Trials Journal. 1982;19(4):248-59. Exclusion: 6

108. Berry H, Swinson D, Jones J, et al. Indomethacin and naproxen suppositories in the treatment of rheumatoid arthritis. Ann Rheum Dis. 1978 Aug;37(4):370-2. doi: 10.1136/ard.37.4.370. PMID: 356764. Exclusion: 3

109. Bertagnolli MM, Eagle CJ, Zauber AG, et al. Celecoxib for the prevention of sporadic colorectal adenomas. N Engl J Med. 2006 Aug 31;355(9):873-84. doi: 10.1056/NEJMoa061355. PMID: 16943400. Exclusion: 4

110. Bertin P, Behier JM, Noel E, et al. Celecoxib is as efficacious as naproxen in the management of acute shoulder pain. J Int Med Res. 2003 Mar-Apr;31(2):102-12. doi: 10.1177/147323000303100206. PMID: 12760313. Exclusion: 4

111. Bestard JA, Toth CC. An open-label comparison of nabilone and gabapentin as adjuvant therapy or monotherapy in the management of neuropathic pain in patients with peripheral neuropathy. Pain pract. 2011 Jul-Aug;11(4):353-68. doi: 10.1111/j.15332500.2010.00427.x. PMID: 21087411.

Exclusion: 6
112. Bianchi M, Broggini M. A randomised, double-blind, clinical trial comparing the efficacy of nimesulide, celecoxib and rofecoxib in osteoarthritis of the knee. Drugs. 2003;63(Suppl 1):37-46. doi: 10.2165/00003495-200363001-00006. PMID: 14506910. Exclusion: 3

113. Bidari A, Moazen-Zadeh E, Ghavidel-Parsa $\mathrm{B}$, et al. Comparing duloxetine and pregabalin for treatment of pain and depression in women with fibromyalgia: an open-label randomized clinical trial. Daru. 2019 Jun;27(1):149-58. doi: 10.1007/s40199-019-00257-4. PMID: 30877484. Exclusion: 9

114. Biegert C, Wagner I, Ludtke R, et al. Efficacy and safety of willow bark extract in the treatment of osteoarthritis and rheumatoid arthritis: results of 2 randomized double-blind controlled trials. J Rheumatol. 2004 Nov;31(11):2121-30. PMID: 15517622. Exclusion: 9

115. Bijlsma A. The long-term efficacy and tolerability of Voltaren (diclofenac sodium) and indomethacin in rheumatoid arthritis. Scand J Rheumatol Suppl. 1978;7(22):7480. doi: 10.3109/03009747809097221. PMID: 356250. Exclusion: 2

116. Bingham CO, 3rd, Bird SR, Smugar SS, et al. Responder analysis and correlation of outcome measures: pooled results from two identical studies comparing etoricoxib, celecoxib, and placebo in osteoarthritis. Osteoarthritis Cartilage. 2008

Nov;16(11):1289-93. doi: 10.1016/j.joca.2008.04.009. PMID: 18514551. Exclusion: 6

117. Bingham CO, 3rd, Smugar SS, Wang H, et al. Predictors of response to cyclooxygenase-2 inhibitors in osteoarthritis: pooled results from two identical trials comparing etoricoxib, celecoxib, and placebo. Pain Med. 2011 Mar;12(3):352-61. doi: 10.1111/j.1526-4637.2011.01060.x. PMID: 21332932. Exclusion: 6

118. Bird HA, Clarke AK, Fowler PD, et al. An assessment of tenoxicam, a nonsteroidal anti-inflammatory drug of long half-life, in patients with impaired renal function suffering from osteoarthritis or rheumatoid arthritis. Clin Rheumatol. 1989

Dec;8(4):453-60. PMID: 2692947. Exclusion: 3 
119. Blechman W, Willkens R, Boncaldo GL, et al. Naproxen in osteoarthrosis. Double-blind crossover trial. Ann Rheum Dis. 1978 Feb;37(1):80-4. doi: 10.1136/ard.37.1.80. PMID: 343726. Exclusion: 9

120. Blechman WJ, Zane S. Fenoprofen calcium in steroid treated rheumatoid arthritis: efficacy, safety, and steroid-sparing effect. J Rheumatol. 1976;2:38-42. PMID: 781229. Exclusion: 9

121. Bluhm GB, MacIlwain HH, Offen WW. Enteric-coated fenoprofen in large-joint osteoarthritis. Henry Ford Hosp Med J. 1988;36(1):56-60. PMID: 3294214. Exclusion: 3

122. Blumenthal DE, Malemud CJ. Recent strategies for drug development in fibromyalgia syndrome. Expert Rev Neurother. 2016 Dec;16(12):1407-11. doi: 10.1080/14737175.2016.1207531. PMID: 27362466. Exclusion: 5

123. Bobrove AM, Calin A. Efficacy and tolerance of a novel precision-dose formulation of indomethacin: double-blind trials in rheumatoid arthritis and osteoarthritis. Curr Med Res Opin. 1983;8 Suppl 2:55-61. doi: 10.1185/03007998309109824. PMID: 6342962. Exclusion: 9

124. Bocanegra T, Weaver AL, Tindall EA, et al. Diclofenac/misoprostol compared with diclofenac in the treatment of osteoarthritis of the knee or hip: a randomized, placebo controlled trial. Arthrotec Osteoarthritis Study Group. J Rheumatol. 1998 Aug;25(8):1602-11. PMID: 9712107. Exclusion: 9

125. Boey ML, Rae S, Feng PH. Comparison of efficacy of and tolerance to ketoprofen and diclofenac sodium in the treatment of rheumatoid arthritis. Singapore Med J. 1988 Jun;29(3):240-5. PMID: 3187575.

Exclusion: 3

126. Bohlooli S, Jastan M, Nakhostin-Roohi B, et al. A pilot double-blinded, randomized, clinical trial of topical virgin olive oil versus piroxicam gel in osteoarthritis of the knee. $\mathrm{J}$ Clin Rheumatol. 2012 Mar;18(2):99-101. doi: 10.1097/RHU.0b013e31824a47b5. PMID: 22334264. Exclusion: 3
127. Bolten W, Gomes JA, Stead H, et al. The gastroduodenal safety and efficacy of the fixed combination of diclofenac and misoprostol in the treatment of osteoarthritis. Br J Rheumatol. 1992 Nov;31(11):753-8. doi: 10.1093/rheumatology/31.11.753. PMID: 1450797. Exclusion: 10

128. Bombardier C, Laine L, Reicin A, et al. Comparison of upper gastrointestinal toxicity of rofecoxib and naproxen in patients with rheumatoid arthritis. VIGOR Study Group. N Engl J Med. 2000 Nov 23;343(21):1520-8. doi:

10.1056/NEJM200011233432103. PMID: 11087881. Exclusion: 3

129. Bombardier C, Peloso PM, Goldsmith CH, et al. Salsalate, a nonacetylated salicylate, is as efficacious as diclofenac in patients with rheumatoid arthritis. J Rheumatol. 1995 Apr;22(4):617-24. PMID: 7791151. Exclusion: 9

130. Bone M, Critchley P, Buggy DJ. Gabapentin in postamputation phantom limb pain: a randomized, double-blind, placebocontrolled, cross-over study. Reg Anesth Pain Med. 2002 Sep-Oct;27(5):481-6. doi: 10.1053/rapm.2002.35169. PMID: 12373695. Exclusion: 9

131. Bonney SL, Northington RS, Hedrich DA, et al. Renal safety of two analgesics used over the counter: ibuprofen and aspirin. Clin Pharmacol Ther. 1986 Oct;40(4):373-7. doi: 10.1038/clpt.1986.193. PMID: 3530585. Exclusion: 6

132. Bookman AA, Williams KS, Shainhouse JZ. Effect of a topical diclofenac solution for relieving symptoms of primary osteoarthritis of the knee: a randomized controlled trial. CMAJ. 2004 Aug 17;171(4):333-8. doi: 10.1503/cmaj.1031793. PMID: 15313991. Exclusion: 10

133. Boomershine CS. First pregabalin and now duloxetine for fibromyalgia syndrome: Closer to a brave new world? Nat Clin Pract Rheumatol. 2008 Dec;4(12):636-7. doi: 10.1038/ncprheum0938. PMID: 18982001. Exclusion: 5 
134. Borms T. Comparison of injectable formulations of tiaprofenic acid and ketoprofen in acute lumbar sciatica. Singleblind randomised trial. Drugs. 1988;35 Suppl 1:85-7. doi: 10.2165/00003495198800351-00019. PMID: 3282869.

Exclusion: 3

135. Bouhassira D, Wilhelm S, Schacht A, et al. Neuropathic pain phenotyping as a predictor of treatment response in painful diabetic neuropathy: data from the randomized, double-blind, COMBO-DN study. Pain. 2014 Oct;155(10):2171-9. doi: 10.1016/j.pain.2014.08.020. PMID: 25168665. Exclusion: 3

136. Bowen LL, McRae-Clark AL. Therapeutic benefit of smoked cannabis in randomized placebo-controlled studies.

Pharmacotherapy. 2018 Jan;38(1):80-5. doi: 10.1002/phar.2064. PMID: 29178487. Exclusion: 6

137. Bowsher D. The effects of pre-emptive treatment of postherpetic neuralgia with amitriptyline: a randomized, double-blind, placebo-controlled trial. J Pain Symptom Manage. 1997 Jun;13(6):327-31. PMID: 9204652. Exclusion: 4

138. Brackertz B, Busson M. Comparative study of sulindac (Clinoril) and ibuprofen (Brufen) in osteoarthrosis. Br J Clin Pract. 1978 Mar;32(3):77-80. PMID: 352382. Exclusion: 6

139. Bradley JD, Brandt KD, Katz BP, et al. Treatment of knee osteoarthritis: relationship of clinical features of joint inflammation to the response to a nonsteroidal antiinflammatory drug or pure analgesic. J Rheumatol. 1992

Dec;19(12):1950-4. PMID: 1294745.

Exclusion: 9

140. Bradley LA, Wohlreich MM, Wang F, et al. Pain response profile of patients with fibromyalgia treated with duloxetine. Clin J Pain. 2010 Jul-Aug;26(6):498-504. doi: 10.1097/AJP.0b013e3181dee80e. PMID: 20551724. Exclusion: 6

141. Bremova A, Pegrimova E, Rejholec V, et al. Double blind, multicenter study of diftalone and indomethacin in rheumatoid arthritis. Scand J Rheumatol Suppl. 1977;17:1-9. PMID: 897584. Exclusion: 3
142. Bresalier RS, Sandler RS, Quan H, et al. Cardiovascular events associated with rofecoxib in a colorectal adenoma chemoprevention trial. N Engl J Med. 2005 Mar 17;352(11):1092-102. doi: 10.1056/NEJMoa050493. PMID: 15713943. Exclusion: 4

143. Breuer B, Pappagallo M, Knotkova H, et al. A randomized, double-blind, placebocontrolled, two-period, crossover, pilot trial of lamotrigine in patients with central pain due to multiple sclerosis. Clin Ther. 2007 Sep;29(9):2022-30. doi: 10.1016/j.clinthera.2007.09.023. PMID: 18035201. Exclusion: 3

144. Briancon D, Peterschmitt J, Laviec G. Double-blind parallel-group evaluation of the safety and efficacy of etodolac capsules compared with piroxicam capsules in patients with rheumatoid arthritis. Acta Therapeutica. 1991;17(1):35-47. Exclusion: 3

145. Brix Finnerup N, Hein Sindrup S, Staehelin Jensen T. Management of painful neuropathies. Handb Clin Neurol. 2013;115:279-90. doi: 10.1016/B978-0-44452902-2.00017-5. PMID: 23931787.

Exclusion: 6

146. Brixner DI. A decision analysis model in the evaluation of NSAIDs in a managed care setting: a case study. Med Interface. 1994 Nov;7(11):145-50. PMID: 10138701. Exclusion: 5

147. Brobyn RD. Nabumetone in the treatment of active adult rheumatoid arthritis. Am J Med. 1987 Oct 30;83(4B):50-4. PMID: 3318429. Exclusion: 2

148. Bronfort G, Nilsson N, Haas M, et al. Noninvasive physical treatments for chronic/recurrent headache. Cochrane Database Syst Rev. 2004(3):CD001878. doi: 10.1002/14651858.CD001878.pub2. PMID: 15266458. Exclusion: 3

149. Brooke JW. Fenoprofen therapy in largejoint osteoarthritis: double-blind comparison with aspirin and longterm experience. $\mathrm{J}$ Rheumatol. 1976;2:71-5. PMID: 781234. Exclusion: 9

150. Brooks PM, Dougan MA, Thomas D, et al. Naproxen and diflunisal in osteoarthrosis. Rheumatol Rehabil. 1982 May;21(2):95-7. PMID: 7043712. Exclusion: 4 
151. Brown BL, Daenzer CL, Hearron MS, et al. Comparison of two dosing schedules of flurbiprofen for patients with rheumatoid arthritis. Twice-daily versus four-times-aday schedules. Am J Med. 1986 Mar 24;80(3A):19-22. PMID: 3515921. Exclusion: 3

152. Buchmann E. Mefenamic acid compared with indomethacin and placebo in osteoarthritis. Ann Phys Med. 1966(Suppl):119-25. PMID: 4895004. Exclusion: 3

153. Buckland-Wright JC, MacFarlane DG, Lynch JA, et al. Quantitative microfocal radiography detects changes in OA knee joint space width in patients in placebo controlled trial of NSAID therapy. $\mathrm{J}$ Rheumatol. 1995 May;22(5):937-43. PMID: 8587086. Exclusion: 2

154. Bulut S, Berilgen MS, Baran A, et al. Venlafaxine versus amitriptyline in the prophylactic treatment of migraine: randomized, double-blind, crossover study. Clin Neurol Neurosurg. 2004

Dec;107(1):44-8. doi: 10.1016/j.clineuro.2004.03.004. PMID: 15567552. Exclusion: 4

155. Burgos A, Busquier MP, Reino JG, et al. Double-blind, double-dummy comparative study of local action transcutaneous flurbiprofen (flurbiprofen LAT) versus piketoprofen cream in the treatment of extraarticular rheumatism. Clin Drug Investig. 2001 Feb;21(2):95-102. doi: 10.2165/00044011-200121020-00002. Exclusion: 3

156. Burry HC, Treadwell BL, Tweed JM. Piroxicam in the management of rheumatoid arthritis: a comparison with naproxen. N Z Med J. 1982 May 26;95(708):352-4. PMID: 7048162. Exclusion: 9

157. Burry HC, Witherington L. A comparison of sulindac with ibuprofen in the management of rheumatoid arthritis. N Z Med J. 1979 Apr 25;89(634):298-300. PMID: 377154. Exclusion: 6
158. Bushmakin AG, Cappelleri JC, Zlateva G, et al. Applying area-under-the-curve analysis to enhance interpretation of response profiles: an application to sleep quality scores in patients with fibromyalgia. Qual Life Res. 2011 May;20(4):491-8. doi: 10.1007/s11136-010-9776-4. PMID: 20978856. Exclusion: 2

159. Busson M. A long-term study of flurbiprofen in rheumatological disorders: II. Osteoarthrosis. J Int Med Res. 1986;14(1):712. PMID: 3514314. Exclusion: 6

160. Byon W, Ouellet D, Chew M, et al. Exposure-response analyses of the effects of pregabalin in patients with fibromyalgia using daily pain scores and patient global impression of change. J Clin Pharmacol. 2010 Jul;50(7):803-15. doi: 10.1177/0091270009352187. PMID: 20357295. Exclusion: 6

161. Cabrera J, Emir B, Dills D, et al. Characterizing and understanding body weight patterns in patients treated with pregabalin. Curr Med Res Opin. 2012 Jun;28(6):1027-37. doi: 10.1185/03007995.2012.684044. PMID: 22494020. Exclusion: 8

162. Calandre EP, Rico-Villademoros F, Galan J, et al. Quetiapine extended-release (Seroquel-XR) versus amitriptyline monotherapy for treating patients with fibromyalgia: a 16-week, randomized, flexible-dose, open-label trial. Psychopharmacology (Berl). 2014 Jun;231(12):2525-31. doi: 10.1007/s00213013-3422-0. PMID: 24398824. Exclusion: 3

163. Caldwell JR, Crain D, Hoffmeister RT, et al. Four-way, multicenter, crossover trial of ibuprofen, fenoprofen calcium, naproxen, and tolmetin sodium in osteoarthritis: comparative clinical profiles. South Med J. 1983 Jun;76(6):706-11. PMID: 6344227. Exclusion: 4

164. Caldwell JR, Germain BF, Lourie SH, et al. Ketoprofen versus indomethacin in patients with rheumatoid arthritis: a multicenter double blind comparative study. J Rheumatol. 1988 Oct;15(10):1476-9. PMID: 3060612. Exclusion: 3 
165. Caldwell JR, Roth SH. A double blind study comparing the efficacy and safety of enteric coated naproxen to naproxen in the management of NSAID intolerant patients with rheumatoid arthritis and osteoarthritis. Naproxen EC Study Group. J Rheumatol. 1994 Apr;21(4):689-95. PMID: 8035394. Exclusion: 9

166. Calin A, Bennett RM, Sukhupunyaraksa S, et al. Double-blind, multi-centre parallel trial of ketoprofen and ibuprofen in the treatment of rheumatoid arthritis. J Rheumatol. 1977 Summer;4(2):153-7. PMID: 328880. Exclusion: 2

167. Calin A, Britton M. Sulindac in ankylosing spondylitis. Double-blind evaluation of sulindac and indomethacin. JAMA. 1979 Oct 26;242(17):1885-6. PMID: 480622. Exclusion: 6

168. Campbell FG, Graham JG, Zilkha KJ. Clinical trial of carbazepine (tegretol) in trigeminal neuralgia. J Neurol Neurosurg Psychiatry. 1966 Jun;29(3):265-7. doi: 10.1136/jnnp.29.3.265. PMID: 5327969. Exclusion: 10

169. Cannon CP, Chen C, Curtis SP, et al. A comparison of cardiovascular biomarkers in patients treated for three months with etoricoxib, celecoxib, ibuprofen, and placebo. Arch Drug Inf. 2008 Jul;1(1):4-13. doi: 10.1111/j.1753-5174.2007.00002.x. PMID: 20157362. Exclusion: 2

170. Cannon CP, Curtis SP, Bolognese JA, et al. Clinical trial design and patient demographics of the Multinational Etoricoxib and Diclofenac Arthritis Longterm (MEDAL) study program: cardiovascular outcomes with etoricoxib versus diclofenac in patients with osteoarthritis and rheumatoid arthritis. Am Heart J. 2006 Aug;152(2):237-45. doi: 10.1016/j.ahj.2006.05.024. PMID: 16875903. Exclusion: 3

171. Cappelleri JC, Bushmakin AG, McDermott AM, et al. Measurement properties of the Medical Outcomes Study Sleep Scale in patients with fibromyalgia. Sleep Med. 2009 Aug;10(7):766-70. doi: 10.1016/j.sleep.2008.09.004. PMID: 19185539. Exclusion: 6
172. Cappelleri JC, Bushmakin AG, Zlateva G, et al. Pain responder analysis: use of area under the curve to enhance interpretation of clinical trial results. Pain pract. 2009 SepOct;9(5):348-53. doi: 10.1111/j.15332500.2009.00293.x. PMID: 19549060. Exclusion: 2

173. Car A, Jajic I, Krampac I, et al. A doubleblind multicentre comparison of diclofenac sodium and naproxen in osteoarthrosis of the hip. Scand J Rheumatol Suppl. 1978(22):638. PMID: 356248. Exclusion: 3

174. Cardenas DD, Warms CA, Turner JA, et al. Efficacy of amitriptyline for relief of pain in spinal cord injury: results of a randomized controlled trial. Pain. 2002 Apr;96(3):36573. doi: 10.1016/S03043959\%2801\%2900483-3. PMID: 11973011. Exclusion: 9

175. Cardoe N. A double-blind crossover study to compare the efficacy of three dosage levels of flurbiprofen in the treatment of rheumatoid disease and osteoarthrosis. Curr Med Res Opin. 1977;5(1):99-105. doi: 10.1185/03007997709108985. PMID: 71975. Exclusion: 4

176. Cardoe N, Fowler PD. Diclofenac sodium (Voltarol): a double-blind comparative study with ibuprofen in patients with rheumatoid arthritis. Rheumatol Rehabil. 1979;Suppl 2:89-99. PMID: 394282. Exclusion: 6

177. Carette S, McCain GA, Bell DA, et al. Evaluation of amitriptyline in primary fibrositis. A double-blind, placebocontrolled study. Arthritis Rheum. 1986 May;29(5):655-9. doi: 10.1002/art.1780290510. PMID: 3521612. Exclusion: 9

178. Carette S, Oakson G, Guimont C, et al. Sleep electroencephalography and the clinical response to amitriptyline in patients with fibromyalgia. Arthritis Rheum. 1995 Sep;38(9):1211-7. doi: 10.1002/art.1780380906. PMID: 7575714. Exclusion: 9

179. Carlsson KC, Hoem NO, Moberg ER, et al. Analgesic effect of dextromethorphan in neuropathic pain. Acta Anaesthesiol Scand. 2004 Mar;48(3):328-36. doi: 10.1111/j.0001-5172.2004.0325.x. PMID: 14982566. Exclusion: 3 
180. Carroll GJ, Bell MC, Laing BA, et al. Reduction of the concentration and total amount of keratan sulphate in synovial fluid from patients with osteoarthritis during treatment with piroxicam. Ann Rheum Dis. 1992 Jul;51(7):850-4. doi:

10.1136/ard.51.7.850. PMID: 1632658.

Exclusion: 9

181. Caruso I, Pietrogrande V. Italian doubleblind multicenter study comparing Sadenosylmethionine, naproxen, and placebo in the treatment of degenerative joint disease. Am J Med. 1987 Nov 20;83(5A):66-71. PMID: 3318442. Exclusion: 9

182. Castles JJ, Moore TL, Vaughan JH, et al. Multicenter comparison of naproxen and indomethacin in rheumatoid arthritis. Arch Intern Med. 1978 Mar;138(3):362-6. PMID: 343743. Exclusion: 9

183. Cates MJ, Beverly A. High dose aspirin versus ibuprofen for pain relief in rheumatoid arthritis: a systematic review. Ann Rheum Dis. 2013;72(Suppl 3):A895-6. doi: 10.1136/annrheumdis-2013-eular.2677. Exclusion: 8

184. Cazzola M, Montrone F, Azzolini V, et al. Analgesic activity of nabumetone versus naproxen in acute exacerbation of osteoarthritis. A short term study. Drugs. 1990;40 Suppl 5:78-9. doi: 10.2165/00003495-199000405-00020. PMID: 2081501. Exclusion: 6

185. Cevoli S, Giannini G, Favoni V, et al. Treatment of withdrawal headache in patients with medication overuse headache: a pilot study. J Headache Pain. 2017 Dec;18(1):56. doi: 10.1186/s10194-0170763-9. PMID: 28500492. Exclusion: 3

186. Chahade WH, Marques GC. Tenoxicam or diclofenac in the treatment of gonarthrosis. Eur J Rheumatol Inflamm. 1987;9(2):77-80. PMID: 3329113. Exclusion: 3

187. Chalmers IM, Cathcart BJ, Kumar EB, et al. Clinico-pharmacological studies and clinical evaluation of flurbiprofen. A new nonsteroidal antirheumatic agent. Ann Rheum Dis. 1972 Jul;31(4):319-24. doi: 10.1136/ard.31.4.319. PMID: 4558227. Exclusion: 9
188. Champion P. Re: Beaulieu AD, Peloso PM, Haraoui B, et al. Once-daily, controlledrelease tramadol and sustained-release diclofenac relieve chronic pain to to osteoarthritis: a randomized controlled trial. Pain Res Manage 2008; 13:103-10, and Thorne C, Beaulieu AD, Callaghan DJ, et. al. A randomized, double-blind, crossover comparison of the efficacy and safety of oral controlled-release tramadol and placebo in patients with painful osteoarthritis. Pain Res Manage 2008; 13:93-102. Pain Res Manag. 2008 Jul-Aug;13(4):342. PMID: 18816899. Exclusion: 5

189. Chan F, Hung, LC, Suen, BY, et al. Celecoxib versus diclofenac and omeprazole in reducing the risk of recurrent ulcer bleeding in patients with arthritis. N Engl $\mathrm{J}$ Med. 2002 Dec 26;347(26):2104-10. doi: 10.1056/NEJMoa021907. PMID: 12501222. Exclusion: 3

190. Chan F, Lanas A, Scheiman J, et al. Celecoxib versus omeprazole and diclofenac in patients with osteoarthritis and rheumatoid arthritis (CONDOR): a randomised trial. Lancet. $2010 \mathrm{Jul}$ 17;376(9736):173-9. doi: 10.1016/S01406736(10)60673-3. PMID: 20638563. Exclusion: 3

191. Chan F, Sung J, Ching J, et al. Randomized trial of low dose misoprostol and naproxen vs nambumetone to prevent recurrent upper gastrointestinal hemorrhage in users on nonsteroidal anti-inflammatory drugs. Aliment Pharmacol Ther. 2001 Jan;15(1):19-24. doi: 10.1046/j.1365-2036.2001.00890.x. PMID: 11136274. Exclusion: 4

192. Chan FK, Wong VW, Suen BY, et al. Combination of a cyclo-oxygenase-2 inhibitor and a proton-pump inhibitor for prevention of recurrent ulcer bleeding in patients at very high risk: a double-blind, randomised trial. Lancet. 2007 May 12;369(9573):1621-6. doi: 10.1016/s01406736(07)60749-1. PMID: 17499604. Exclusion: 3

193. Chan FKL, Lanas A, Scheiman J, et al. Erratum: celecoxib versusomeprazole and diclofenac in patients with osteoarthritis and rheumatoid arthritis (CONDOR): a randomised trial (The Lancet (2010) 376 (173-179)). Lancet. 2011;378(9787):228. Exclusion: 3 
194. Chandra K, Shafiq N, Pandhi P, et al. Gabapentin versus nortriptyline in postherpetic neuralgia patients: a randomized, double-blind clinical trial--the GONIP Trial. Intl J Clin Pharmacol Ther. 2006 Aug;44(8):358-63. PMID: 16961166. Exclusion: 9

195. Chandrasekaran A, Sambandam P, Lal H, et al. Double blind, placebo controlled trial on the cytoprotective effect of misoprostol in subjects with rheumatoid arthritis, osteoarthritits and seronegative sp ondarthropathy on NSAIDs. J Assoc Physicians India. 1991 Dec;39(12):919-21. PMID: 1816217. Exclusion: 3

196. Chang DM, Young TH, Hsu CT, et al. Endoscopic comparison of the gastroduodenal safety and the effects on arachidonic acid products between meloxicam and piroxicam in the treatment of osteoarthritis. Clin Rheumatol. 2001;20(2):104-13. PMID: 11346221. Exclusion: 10

197. Chodock AL. Crossover comparison of benoxaprofen and indomethacin in rheumatoid arthritis and osteoarthritis. Eur J Rheumatol Inflamm. 1982;5(2):246-57. PMID: 7044800. Exclusion: 3

198. Chou R, Chan BK. Gabapentin versus tricyclics for neuropathic pain: Authors' reply. J Gen Intern Med. 2009 Jun;24(6):782. doi: 10.1007/s11606-0090959-z. Exclusion: 5

199. Chou R, Deyo R, Friedly J, et al. Systemic pharmacologic therapies for low back pain: a systematic review for an American College of Physicians clinical practice guideline. Ann Intern Med. 2017 Apr 4;166(7):480-92. doi: 10.7326/M16-2458. PMID: 28192790. Exclusion: 8

200. Chwieduk CM, McCormack PL. Milnacipran: in fibromyalgia. Drugs. 2010;70(1):99-108. doi: 10.2165/11202810000000000-00000. PMID: 20030428.

Exclusion: 5

201. Ciccolunghi SN, Chaudri HA, Schubiger BI. The value and results of long-term studies with diclofenac sodium (Voltarol).

Rheumatol Rehabil. 1979; Suppl 2:100-15. PMID: 394274. Exclusion: 6
202. Ciccolunghi SN, Chaudri HA, Schubiger BI. How long should long be? Long-term trials in rheumatic diseases. Ann Rheum Dis. 1979 Apr;38(2):155-60. doi: 10.1136/ard.38.2.155. PMID: 375851. Exclusion: 5

203. Clair A, Emir B. The safety and efficacy of pregabalin for treating subjects with fibromyalgia and moderate or severe baseline widespread pain. Curr Med Res Opin. 2016;32(3):601-9. doi: 10.1185/03007995.2015.1134463. PMID: 26694975. Exclusion: 6

204. Clarke AK. A Double-blind comparison of naproxen against indometacin in osteoarthrosis. Arzneimittelforschung. 1975 Feb;25(2A):302-4. PMID: 1096901. Exclusion: 9

205. Clarke AK, Macleod M, Steinberg VL, et al. A double-blind, cross-over study of naproxen sodium and soluble aspirin in rheumatoid arthritis. Br J Clin Pract. 1981 Apr;35(4):143-6. PMID: 7028063. Exclusion: 3

206. Clauw DJ, Mease P, Palmer RH, et al. Milnacipran for the treatment of fibromyalgia in adults: a 15-week, multicenter, randomized, double-blind, placebo-controlled, multiple-dose clinical trial.[Erratum appears in Clin Ther. 2009 Feb;31(2):446], [Erratum appears in Clin Ther. 2009 Jul;31(7):1617]. Clin Ther. 2008 Nov;30(11):1988-2004. doi: 10.1016/j.clinthera.2008.11.009. PMID: 19108787. Exclusion: 5

207. Clauw DJ, Mease PJ, Palmer RH, et al. Continuing efficacy of milnacipran following long-term treatment in fibromyalgia: a randomized trial. Arthritis Res Ther. 2013 Aug 16;15(4):R88. doi: 10.1186/ar4268. PMID: 23953493. Exclusion: 6

208. Cochrane GM. A double-blind comparison of naproxen with indomethacin in osteoarthritis. Scand J Rheumatol. 1973(Suppl 2):89-93. PMID: 4590058. Exclusion: 10

209. Cohen de Lara A, Gompel H. Two comparative studies of Dosmalfate vs Misoprostol in the prevention of NSAIDinduced gastric ulcers in rheumatic patients. Drugs of Today. 2000(36). Exclusion: 3 
210. Colebatch AN, Marks JL, Edwards CJ. Safety of non-steroidal anti-inflammatory drugs, including aspirin and paracetamol (acetaminophen) in people receiving methotrexate for inflammatory arthritis (rheumatoid arthritis, ankylosing spondylitis, psoriatic arthritis, other spondyloarthritis). Cochrane Database Syst Rev. 2011 Nov 9(11):CD008872. doi: 10.1002/14651858.CD008872.pub2. PMID: 22071858. Exclusion: 8

211. Cook ME, Wallin JD, Thakur VD, et al. Comparative effects of nabumetone, sulindac, and ibuprofen on renal function. J Rheumatol. 1997 Jun;24(6):1137-44. PMID: 9195523. Exclusion: 9

212. Cooper TE, Derry S, Wiffen PJ, et al. Gabapentin for fibromyalgia pain in adults. Cochrane Database Syst Rev. 2017 Jan 3;1:CD012188. doi: 10.1002/14651858.CD012188.pub2. PMID: 28045473. Exclusion: 8

213. Cordrey LJ. Tolmetin sodium, a new antiarthritis drug: double-blind and long-term studies. J Am Geriatr Soc. 1976 Oct;24(10):440-6. PMID: 61224. Exclusion: 6

214. Couch JR, Hassanein RS. Migraine and depression: effect of amitriptyline prophylaxis. Trans Am Neurol Assoc. 1976;101:234-7. PMID: 800690. Exclusion: 4

215. Crofford LJ, Mease PJ, Simpson SL, et al. Fibromyalgia relapse evaluation and efficacy for durability of meaningful relief (FREEDOM): a 6-month, double-blind, placebo-controlled trial with pregabalin. Pain. 2008 Jun;136(3):419-31. doi: 10.1016/j.pain.2008.02.027. PMID: 18400400. Exclusion: 6

216. Crofford LJ, Rowbotham MC, Mease PJ, et al. Pregabalin for the treatment of fibromyalgia syndrome: results of a randomized, double-blind, placebocontrolled trial. Arthritis Rheum. 2005 Apr;52(4):1264-73. doi: 10.1002/art.20983. PMID: 15818684. Exclusion: 9

217. Crook PR, Fowler PD, Hothersall TE, et al. A study of the efficacy and tolerability of diclofenac and ibuprofen in osteoarthritis of the hip. Br J Clin Pract. 1981 Sep;35(9):30912. PMID: 7034760. Exclusion: 5
218. Cross PL, Ashby D, Harding G, et al. TOIB Study. Are topical or oral ibuprofen equally effective for the treatment of chronic knee pain presenting in primary care: a randomised controlled trial with patient preference study. ISRCTN79353052. BMC Musculoskelet Disord. 2005 Nov 7;6:55. doi: 10.1186/1471-2474-6-55. PMID: 16274477. Exclusion: 3

219. Currie WJ, Vandenburg MJ, Janke PG, et al. Symptomatic osteoarthritis in the elderly: multicentre comparative study of sulindac and ibuprofen. Br J Clin Pract. 1984 May;38(5):176-81. PMID: 6733011. Exclusion: 3

220. Curtis SP, Bockow B, Fisher C, et al. Etoricoxib in the treatment of osteoarthritis over 52-weeks: a double-blind, activecomparator controlled trial [NCT00242489]. BMC Musculoskelet Disord. 2005 Dec 1;6:58. doi: 10.1186/1471-2474-6-58. PMID: 16321158. Exclusion: 3

221. Dalal KL, Felix ER, Cardenas DD. Pregabalin for the management of neuropathic pain in spinal cord injury. Pain manag. 2013 Sep;3(5):359-67. doi: 10.2217/pmt.13.35. PMID: 24654869. Exclusion: 5

222. Das KV, Sundaram P. Comparative study of the clinical effects of ibuprofen and indomethacin in rheumatoid arthritis. J Indian Med Assoc. 1973 Aug 16;61(4):1703. PMID: 4590356. Exclusion: 9

223. Datto C, Hellmund R, Siddiqui MK. Efficacy and tolerability of naproxen/esomeprazole magnesium tablets compared with non-specific NSAIDs and COX-2 inhibitors: a systematic review and network analyses. Open access rheumatol. 2013 Feb 26;5:1-19. doi: 10.2147/OARRR.S41420. PMID: 27790020. Exclusion: 8 
224. Davies GM, Watson DJ, Bellamy N.

Comparison of the responsiveness and relative effect size of the western Ontario and McMaster Universities osteoarthritis index and the short-form medical outcomes study survey in a randomized, clinical trial of osteoarthritis patients. Arthritis Care Res (Hoboken). 1999 Jun;12(3):172-9. doi: 10.1002/15290131\%28199906\%2912:3\%3C172::AIDART4\%3E3.0.CO;2-Y. PMID: 10513507. Exclusion: 9

225. Day R, Morrison B, Luza A, et al. A randomized trial of the efficacy and tolerability of the COX-2 inhibitor rofecoxib vs ibuprofen in patients with osteoarthritis. Rofecoxib/Ibuprofen Comparator Study Group. Arch Intern Med. 2000 Jun 26;160(12):1781-7. PMID: 10871971. Exclusion: 9

226. De Almeida Coimbra F, De Araujo Filho NC. A double-blind study comparing the associations chlormezanone-acetaminophen and thiocolchicoside-glafenin in patients with low back pain. Folha Medica. 1980;81(1):113-6. Exclusion: 3

227. de Andrade DC, Jacobsen Teixeira M, Galhardoni R, et al. Pregabalin for the prevention of oxaliplatin-induced painful neuropathy: a randomized, double-blind trial. Oncologist. 2017 Oct;22(10):1154e105. doi: 10.1634/theoncologist.2017-0235. PMID: 28652279. Exclusion: 4

228. De Bock GH, Hermans J, Mulder JD. Randomized double-blind study of nabumetone and piroxicam in the treatment of osteoarthritis in Dutch general practice: efficacy and tolerability. Pharm World Sci. 1993 Jun 18;15(3):132-8. PMID: 8348110. Exclusion: 9

229. de Bock GH, Hermans J, van Marwijk HW, et al. Health-related quality of life assessments in osteoarthritis during NSAID treatment. Pharm World Sci. 1996 Aug;18(4):130-6. PMID: 8873228. Exclusion: 9

230. De Bono GG, Pearlgood M. General practice trials: a reappraisal in the light of the findings of a general practice study of oruvail. Br J Clin Pract. 1986 Oct;40(10):421-8. PMID: 3307857. Exclusion: 3
231. de Heer EW, Dekker J, Beekman ATF, et al. Comparative effect of collaborative care, pain medication, and duloxetine in the treatment of major depressive disorder and comorbid (sub)chronic pain: results of an exploratory randomized, placebo-controlled, multicenter trial (CC: PAINDIP). Front Psychiatry. 2018 Apr 5;9:118. doi: 10.3389/fpsyt.2018.00118. PMID: 29674981. Exclusion: 3

232. De Jaeger M, Goudman L, Van Schuerbeek $P$, et al. Cerebral biochemical effect of pregabalin in patients with painful diabetic neuropathy: a randomized controlled trial. Diabetes Ther. 2018 Aug;9(4):1591-604. doi: 10.1007/s13300-018-0460-y. PMID: 29951977. Exclusion: 2

233. de Melo Gomes JA. The safety of Arthrotec in patients with rheumatoid arthritis or osteoarthritis: an assessment of the upper gastrointestinal tract by endoscopy. Scand J Rheumatol Suppl. 1992;96:23-31. PMID: 1439621. Exclusion: 3

234. Deal CL, Moskowitz RW. Efficacy of diflunisal versus naproxen in osteoarthritis of the knee: an open study. Clin Ther. 1986;9(Suppl C):1-14. PMID: 3548982. Exclusion: 6

235. DeHaan MN, Guzman J, Bayley MT, et al. Knee osteoarthritis clinical practice guidelines -- how are we doing? J Rheumatol. 2007 Oct;34(10):2099-105. PMID: 17722223. Exclusion: 6

236. Delmas P, Lambert R, Capron MH. Misoprostol for preventing gastric erosions induced by nonsteroidal antiinflammatory drugs in patients with rheumatic diseases. Rev Rhum Engl Ed. 1994;61(2):115-20. Exclusion: 3

237. Demant DT, Lund K, Vollert J, et al. The effect of oxcarbazepine in peripheral neuropathic pain depends on pain phenotype: a randomised, double-blind, placebo-controlled phenotype-stratified study. Pain. 2014 Nov;155(11):2263-73. doi: 10.1016/j.pain.2014.08.014. PMID: 25139589. Exclusion: 9 
238. Dentali F, Douketis JD, Woods K, et al. Does celecoxib potentiate the anticoagulant effect of warfarin? A randomized, doubleblind, controlled trial. Ann Pharmacother. 2006 Jul-Aug;40(7-8):1241-7. doi: 10.1345/aph.1G733. PMID: 16804099. Exclusion: 3

239. Dequeker J, Hawkey C, Kahan A, et al. Improvement in gastrointestinal tolerability of the selective cyclooxygenase (COX)-2 inhibitor, meloxicam, compared with piroxicam: results of the Safety and Efficacy Large-scale Evaluation of COX-inhibiting Therapies (SELECT) trial in osteoarthritis. Br J Rheumatol. 1998 Sep;37(9):946-51. PMID: 9783758. Exclusion: 10

240. Derry S, Wiffen PJ, Hauser W, et al. Oral nonsteroidal anti-inflammatory drugs for fibromyalgia in adults. Cochrane Database Syst Rev. 2017 Mar 27;3:CD012332. doi: 10.1002/14651858.CD012332.pub2. PMID: 28349517. Exclusion: 8

241. Derry S, Wiffen PJ, Kalso EA, et al. Topical analgesics for acute and chronic pain in adults - an overview of Cochrane Reviews. Cochrane Database Syst Rev. 2017 May 12;5:CD008609. doi: 10.1002/14651858.CD008609.pub2. PMID: 28497473. Exclusion: 8

242. Desai MM, Shetty RN. Double-blind crossover comparison of naproxen and indomethacin in rheumatoid arthritis. J Assoc Physicians India. 1977 Oct;25(10):703-7. PMID: 353025.

Exclusion: 9

243. Detrembleur C, De Nayer J, van den Hecke A. Celecoxib improves the efficiency of the locomotor mechanism in patients with knee osteoarthritis. A randomised, placebo, double-blind and cross-over trial. Osteoarthritis Cartilage. 2005

Mar;13(3):206-10. doi: 10.1016/j.joca.2004.11.002. PMID: 15727886. Exclusion: 9

244. Dexter SL, Graham AN, Johnston ES, et al. Double-blind controlled study of paramax in the acute treatment of common and classical migraine. Br J Clin Pract. 1985 Oct;39(10):388-92. PMID: 3907680.

Exclusion: 3
245. Di Trapani G, Mei D, Marra C, et al. Gabapentin in the prophylaxis of migraine: a double-blind randomized placebo-controlled study. Clin Ter. 2000 May-Jun;151(3):1458. PMID: 10958046. Exclusion: 4

246. Diamond S. Ibuprofen versus aspirin and placebo in the treatment of muscle contraction headache. Headache. 1983 Sep;23(5):206-10. PMID: 6355010. Exclusion: 4

247. Diamond S. Caffeine as an analgesic adjuvant in the treatment of headache. Headache Quarterly. 1999;10(2):119-25. Exclusion: 6

248. Diamond S, Baltes BJ. Chronic tension headache--treated with amitriptyline--a double-blind study. Headache. 1971 Oct;11(3):110-6. PMID: 4940167. Exclusion: 4

249. Diamond S, Solomon GD, Freitag FG, et al. Fenoprofen in the prophylaxis of migraine: a double-blind, placebo controlled study. Headache. 1987 May;27(5):246-9. PMID: 3298164. Exclusion: 2

250. Dick WC, Franchimont P, Veys E. Doubleblind comparison of etodolac and piroxicam in the treatment of rheumatoid arthritis. Clin Ther. 1993 Jan-Feb;15(1):148-59. PMID: 8458044. Exclusion: 3

251. Dick-Smith JB. Ibuprofen, aspirin and placebo in the treatment of rheumatoid arthritis--a double-blind clinical trial. Med J Aust. 1969 Oct 25;2(17):853-9. PMID: 4900939. Exclusion: 6

252. Dickson D. A double-blind evaluation of topical piroxicam gel with oral ibuprofen in osteoarthritis of the knee. Curr Ther Res Clin Exp. 1991;49:199-207. Exclusion: 3

253. Diener HC, Davidai G. Dipyridamole and headache. Future Neurol. 2007;2(3):279-83. doi: 10.2217/14796708.2.3.279. Exclusion: 3 
254. Diener HC, Pfaffenrath V, Pageler L, et al. The fixed combination of acetylsalicylic acid, paracetamol and caffeine is more effective than single substances and dual combination for the treatment of headache: a multicentre, randomized, double-blind, single-dose, placebo-controlled parallel group study. Cephalalgia. 2005 Oct;25(10):776-87. doi: 10.1111/j.14682982.2005.00948.x. PMID: 16162254. Exclusion: 4

255. Dieppe P, Cushnaghan J, Jasani MK, et al. A two-year, placebo-controlled trial of nonsteroidal anti-inflammatory therapy in osteoarthritis of the knee joint. Br J Rheumatol. 1993 Jul;32(7):595-600. PMID: 8339132. Exclusion: 2

256. Digby JW, Wehner S. A double-blind crossover comparison of Orudis (ketoprofen) and indomethacin in the treatment of rheumatoid arthritis. J Int Med Res. 1976;4(6):427-31. doi: 10.1177/030006057600400608. PMID: 800382. Exclusion: 9

257. Dimova V, Oertel BG, Kabakci G, et al. A more pessimistic life orientation is associated with experimental inducibility of a neuropathy-like pain pattern in healthy individuals. J Pain. 2015 Aug;16(8):791800. doi: 10.1016/j.jpain.2015.05.004. PMID: 26056011. Exclusion: 4

258. Doherty M, Hawkey C, Goulder M, et al. A randomised controlled trial of ibuprofen, paracetamol or a combination tablet of ibuprofen/paracetamol in communityderived people with knee pain. Ann Rheum Dis. 2011 Sep;70(9):1534-41. doi: 10.1136/ard.2011.154047. PMID: 21804100. Exclusion: 4

259. Domingues RB, Silva AL, Domingues SA, et al. A double-blind randomized controlled trial of low doses of propranolol, nortriptyline, and the combination of propranolol and nortriptyline for the preventive treatment of migraine. Arq Neuropsiquiatr. 2009 Dec;67(4):973-7. PMID: 20069203. Exclusion: 3

260. Donnelly P, Lloyd K, Campbell H. Indomethacin in rheumatoid arthritis: an evaluation of its anti-inflammatory and side effects. Br Med J. 1967 Jan 14;1(5532):6975. doi: 10.1136/bmj.1.5532.69. PMID: 5333306. Exclusion: 9
261. Dosenovic S, Jelicic Kadic A, Miljanovic $\mathrm{M}$, et al. Interventions for neuropathic pain: an overview of systematic reviews. Anesth Analg. 2017 Aug;125(2):643-52. doi: 10.1213/ANE.0000000000001998. PMID: 28731977. Exclusion: 8

262. Dotti F, Ongari R, Carazzi R, et al. Clinical study of a new anti-inflammatory drug: 4prenyl-1,2-diphenyl-3,5-pyrazolidinedione (DA 2370). Arzneimittelforschung. 1972 Jan;22(1):Suppl 1a:265-8. PMID: 4553235. Exclusion: 9

263. Dougados M, Behier JM, Jolchine I, et al. Efficacy of celecoxib, a cyclooxygenase 2specific inhibitor, in the treatment of ankylosing spondylitis: a six-week controlled study with comparison against placebo and against a conventional nonsteroidal antiinflammatory drug. Arthritis Rheum. 2001 Jan;44(1):180-5. doi: 10.1002/1529-

0131(200101)44:1<180::AID-

ANR24>3.0.CO;2-K. PMID: 11212158.

Exclusion: 9

264. Dreiser RL. A comparison of the efficacy of etodolac SR (Lodine SR) and etodolac (Lodine) in patients with rheumatoid arthritis or osteoarthritis. Rheumatol Int. 1993;13(2 Suppl):S13-8. PMID: 8210919. Exclusion: 9

265. Dreiser RL, Dropsy R. A double-blind, multicentric study of etodolac $300 \mathrm{mg}$ twice daily verus $200 \mathrm{mg}$ three times daily in patients with osteoarthritis of the knee. Today's therapeutic trends. 1992;10(2):87100. Exclusion: 3

266. Dreiser RL, Tisne-Camus M. DHEP plasters as a topical treatment of knee osteoarthritis-a double-blind placebo-controlled study. Drugs Exp Clin Res. 1993;19(3):117-23. PMID: 8112200. Exclusion: 3

267. Duff IF, Neukom JE, Himes JE. Fenoprofen in patients with rheumatoid arthritis receiving maintenance gold therapy. J Rheumatol. 1976;2:32-7. PMID: 781228. Exclusion: 9

268. Dunky A, Mattern H. European doubleblind multicenter study comparing meclofenamate sodium and indomethacin in the treatment of rheumatoid arthritis. Arzneimittelforschung. 1983;33(4A):63640. PMID: 6349647. Exclusion: 9 
269. Dworkin RH, Corbin AE, Young JP, Jr., et al. Pregabalin for the treatment of postherpetic neuralgia: a randomized, placebo-controlled trial. Neurology. 2003 Apr 22;60(8):1274-83. doi: 10.1212/01.wnl.0000055433.55136.55. PMID: 12707429. Exclusion: 9

270. Dy SM, Bennett WL, Sharma R, et al. Preventing Complications and Treating Symptoms of Diabetic Peripheral Neuropathy. Comparative Effectiveness Review No. 187. (Prepared by the Johns Hopkins University Evidence-based Practice Center under Contract No. 290-2015-00006I.) AHRQ Publication No. 17-EHC005-EF. Rockville, MD: Agency for Healthcare Research Quality; March 2017. doi: 10.23970/AHRQEPCCER187. PMID: 28749633. Exclusion: 8

271. Ehmann L, Tyberghein JM. Diclofenac suppositories in osteoarthrosis. A doubleblind comparison with indomethacin. Practitioner. 1981 May;225(1355):757-8. PMID: 7027231. Exclusion: 3

272. Ehrich E, Davies GM, Watson DJ, et al. Minimal perceptible clinical improvement with the Western Ontario and McMaster Universities osteoarthritis index questionnaire and global assessments in patients with osteoarthritis. J Rheumatol. 2000 Nov;27(11):2635-41. PMID: 11093446. Exclusion: 3

273. Ehsanullah R, Page MC, Tildesley G, et al. Prevention of gastroduodenal damage induced by non-steroidal anti-inflammatory drugs: controlled trial of ranitidine. BMJ. 1988 Oct 22;297(6655):1017-21. doi: 10.1136/bmj.297.6655.1017. PMID: 3142593. Exclusion: 3

274. Eisenberg E, Lurie Y, Braker C, et al. Lamotrigine reduces painful diabetic neuropathy: a randomized, controlled study. Neurology. 2001 Aug 14;57(3):505-9. doi: 10.1212/wnl.57.3.505. PMID: 11502921. Exclusion: 3

275. Ekman EF, Gimbel JS, Bello AE, et al. Efficacy and safety of intravenous tanezumab for the symptomatic treatment of osteoarthritis: 2 randomized controlled trials versus naproxen. J Rheumatol. 2014 Nov;41(11):2249-59. doi: 10.3899/jrheum.131294. PMID: 25274899. Exclusion: 3
276. Elliott S, Yeomans ND, Buchanan RRC, et al. Efficacy of 12 months' misoprostol as prophylaxis against NSAID- induced gastric ulcers. Scand J Rheumatol. 1994;23(4):1716. PMID: 8091141. Exclusion: 3

277. Engel ER, Kudrow D, Rapoport AM. A prospective, open-label study of milnacipran in the prevention of headache in patients with episodic or chronic migraine. Neurol Sci. 2014 Mar;35(3):429-35. doi: 10.1007/s10072-013-1536-0. PMID: 24030685. Exclusion: 4

278. Enggaard TP, Klitgaard NA, Gram LF, et al. Specific effect of venlafaxine on single and repetitive experimental painful stimuli in humans. Clin Pharmacol Ther. 2001 Apr;69(4):245-51. doi: 10.1067/mcp.2001.114873. PMID: 11309553. Exclusion: 4

279. Enke O, New HA, New CH, et al. Anticonvulsants in the treatment of low back pain and lumbar radicular pain: a systematic review and meta-analysis. CMAJ Canadian Medical Association Journal. $2018 \mathrm{Jul}$ 3;190(26):E786-E93. doi: 10.1503/cmaj.171333. PMID: 29970367. Exclusion: 8

280. Enomoto H, Fujikoshi S, Funai J, et al. Assessment of direct analgesic effect of duloxetine for chronic low back pain: Post hoc path analysis of double-blind, placebocontrolled studies. J Pain Res. 2017 Jun 1;10:1357-68. doi: 10.2147/JPR.S133396. PMID: 28615967. Exclusion: 6

281. Enthoven WT, Roelofs PD, Deyo RA, et al. Non-steroidal anti-inflammatory drugs for chronic low back pain. Cochrane Database Syst Rev. 2016 Feb 10;2:CD012087. doi: 10.1002/14651858.CD012087. PMID: 26863524. Exclusion: 8

282. Esdaile J, Rothwell R, MacLaughlin K, et al. Double-blind comparison of tolmetin sodium and indomethacin in ankylosing spondylitis. J Rheumatol. 1982 JanFeb;9(1):69-74. PMID: 7045363. Exclusion: 6 
283. Essex MN, Behar R, O'Connell MA, et al. Efficacy and tolerability of celecoxib and naproxen versus placebo in Hispanic patients with knee osteoarthritis. Int J Gen Med. 2014 May 16;7:227-35. doi: 10.2147/IJGM.S61297. PMID: 24876792. Exclusion: 9

284. Essex MN, Brown PB, Sands GH. The efficacy of continuous versus intermittent celecoxib treatment in osteoarthritis patients aged $<60$ and $>60$ years. Int J Clin Rheumtol. 2014 Feb;9(1):13-20. doi: 10.2217/ijr.13.71. Exclusion: 6

285. Famaey JP, Colinet E. A double-blind trial of ketoprofen in the treatment of osteoarthritis of the hip. Scand J Rheumatol Suppl. 1976;1976(0):129-32. PMID: 790549. Exclusion: 2

286. Farkouh ME, Greenberg JD, Jeger RV, et al. Cardiovascular outcomes in high risk patients with osteoarthritis treated with ibuprofen, naproxen or lumiracoxib. Ann Rheum Dis. 2007 Jun;66(6):764-70. doi: 10.1136/ard.2006.066001. PMID: 17412741. Exclusion: 3

287. Fayed N, Olivan-Blazquez B, HerreraMercadal P, et al. Changes in metabolites after treatment with memantine in fibromyalgia. A double-blind randomized controlled trial with magnetic resonance spectroscopy with a 6-month follow-up. CNS Neurosci Ther. 2014 Nov;20(11):9991007. doi: 10.1111/cns.12314. PMID: 25230216. Exclusion: 2

288. Fenton SF, Ryan JP, Bensen WG. A doubleblind, crossover, multicenter study of piroxicam and naproxen in rheumatoid arthritis. Curr Ther Res Clin Exp. 1988;44(6):1058-70. Exclusion: 9

289. Fidahic M, Jelicic Kadic A, Radic M, et al. Celecoxib for rheumatoid arthritis. Cochrane Database Syst Rev. 2017 Jun 9;6:CD012095. doi: 10.1002/14651858.CD012095.pub2. PMID: 28597983. Exclusion: 8

290. Fine PG. The role of rofecoxib, a cyclooxygenase-2-specific inhibitor, for the treatment of non-cancer pain: a review. J Pain. 2002 Aug;3(4):272-83. doi: 10.1054/jpai.2002.125957. PMID: 14622751. Exclusion: 5
291. Finnerup NB, Grydehoj J, Bing J, et al. Levetiracetam in spinal cord injury pain: a randomized controlled trial. Spinal Cord. 2009 Dec;47(12):861-7. doi: 10.1038/sc.2009.55. PMID: 19506571. Exclusion: 3

292. Finnerup NB, Sindrup SH, Bach FW, et al. Lamotrigine in spinal cord injury pain: a randomized controlled trial. Pain. 2002 Apr;96(3):375-83. PMID: 11973012. Exclusion: 3

293. Finstad R. A double-blind, crossover, multicentre study of piroxicam and indomethacin in the treatment of rheumatoid arthritis. Br J Clin Pract. 1981 Jan;35(1):359. PMID: 7020731. Exclusion: 9

294. Fishbain DA, Hall J, Meyers AL, et al. Does pain mediate the pain interference with sleep problem in chronic pain? Findings from studies for management of diabetic peripheral neuropathic pain with duloxetine. J Pain Symptom Manage. 2008

Dec;36(6):639-47. doi: 10.1016/j.jpainsymman.2007.12.012. PMID: 18504092. Exclusion: 6

295. Fishbain DA, Hall JA, Risser RC, et al. Does pain cause the perception of fatigue in patients with chronic pain? Findings from studies for management of diabetic peripheral neuropathic pain with duloxetine. Pain pract. 2009 Sep-Oct;9(5):354-62. doi: 10.1111/j.1533-2500.2009.00294.x. PMID: 19500272. Exclusion: 6

296. Fiszman P, Perpetuo JB, Sidi A. Long-term study with tenoxicam in rheumatoid arthritis. Eur J Rheumatol Inflamm. 1987;9(2):86-90. PMID: 3329115. Exclusion: 3

297. Flavell Matts S, Hazleman B, Houben H, et al. Controlled study of once-daily, sustained-release ibuprofen in osteoarthritis. Curr Ther Res Clin Exp. 1993 Apr;53(4):394-400. doi: 10.1016/S0011393X\%2805\%2980199-7. Exclusion: 9

298. Fogelholm R, Murros K. Tizanidine in chronic tension-type headache: a placebo controlled double-blind cross-over study. Headache. 1992 Nov;32(10):509-13. doi: 10.1111/j.1526-4610.1992.hed3210509.x. PMID: 1468911. Exclusion: 9 
299. Fostiropoulos G, Croydon EA. A comparative study of the effectiveness and safety of two non-steroidal antiinflammatory agents, nabumetone and naproxen in rheumatoid arthritis. J Int Med Res. 1982;10(4):204-8. doi: 10.1177/030006058201000402. PMID: 6749576. Exclusion: 9

300. Franchi R, Viara M, Liverta C, et al. Ketoprofen and indomethacin in the treatment of rheumatoid arthritis: a doubleblind cross-over trial. Eur J Clin Pharmacol. 1975 Apr 4;8(3-4):205-8. PMID: 786679. Exclusion: 9

301. Frank RG, Kashani JH, Parker JC, et al. Antidepressant analgesia in rheumatoid arthritis. J Rheumatol. 1988

Nov;15(11):1632-8. PMID: 3236298. Exclusion: 3

302. Franssen MJ, Gribnau FW, van de Putte LB. A comparison of diflunisal and phenylbutazone in the treatment of ankylosing spondylitis. Clin Rheumatol. 1986 Jun;5(2):210-20. PMID: 3524970. Exclusion: 3

303. Freitas GG. A double-blind comparison of etodolac and piroxicam in the treatment of osteoarthritis. Curr Med Res Opin. 1990;12(4):255-62. doi: 10.1185/03007999009111655. PMID: 2150187. Exclusion: 9

304. Furst D, Blocka K, Cassell S, et al. A controlled study of concurrent therapy with a nonacetylated salicylate and naproxen in rheumatoid arthritis. Arthritis Rheum. 1987 Feb;30(2):146-54. PMID: 3548732.

Exclusion: 3

305. Furst DE. Meloxicam: selective COX-2 inhibition in clinical practice. Semin Arthritis Rheum. 1997 Jun;26(6 Suppl 1):21-7. PMID: 9219316. Exclusion: 6

306. Fusco BM, Marabini S, Maggi CA, et al. Preventative effect of repeated nasal applications of capsaicin in cluster headache. Pain. 1994 Dec;59(3):321-5. PMID: 7708405. Exclusion: 3

307. Gahimer J, Wernicke J, Yalcin I, et al. A retrospective pooled analysis of duloxetine safety in 23,983 subjects. Curr Med Res Opin. 2007 Jan;23(1):175-84. doi: 10.1185/030079906X162719. PMID: 17257478. Exclusion: 6
308. Galeazzi M, Marcolongo R. A placebocontrolled study of the efficacy and tolerability of a nonsteroidal antiinflammatory drug, DHEP plaster, in inflammatory peri- and extra-articular rheumatological diseases. Drugs Exp Clin Res. 1993;19(3):107-15. PMID: 8112199. Exclusion: 3

309. Galer BS, Jensen MP, Ma T, et al. The lidocaine patch $5 \%$ effectively treats all neuropathic pain qualities: results of a randomized, double-blind, vehiclecontrolled, 3-week efficacy study with use of the neuropathic pain scale. Clin J Pain. 2002 Sep-Oct;18(5):297-301. PMID: 12218500. Exclusion: 10

310. Garcia J, Simon MA, Duran M, et al. Differential efficacy of a cognitivebehavioral intervention versus pharmacological treatment in the management of fibromyalgic syndrome. Psychol Health Med. 2006 Nov;11(4):498506. doi: 10.1080/13548500600745286. PMID: 17129925. Exclusion: 3

311. Garcia-Borreguero D, Larrosa O, Williams AM, et al. Treatment of restless legs syndrome with pregabalin: a double-blind, placebo-controlled study. Neurology. 2010 Jun 8;74(23):1897-904. doi: 10.1212/WNL.0b013e3181e1ce73. PMID: 20427750. Exclusion: 4

312. Gawel MJ, Szalai JF, Stiglick A, et al. Evaluation of analgesic agents in recurring headache compared with other clinical pain models. Clin Pharmacol Ther. 1990 Apr;47(4):504-8. doi: 10.1038/clpt.1990.64. PMID: 2328558. Exclusion: 3

313. Geba GP, Weaver AL, Polis AB, et al. Efficacy of rofecoxib, celecoxib, and acetaminophen in osteoarthritis of the knee: a randomized trial. JAMA. 2002 Jan 2;287(1):64-71. PMID: 11754710. Exclusion: 9

314. Geis GS. Overall safety of Arthrotec. Scand J Rheumatol Suppl. 1992;96:33-6. PMID: 1439622. Exclusion: 3

315. Geis GS. Efficacy and upper GI safety of diclofenac/misoprostol, piroxicam and naproxen in patients with osteoarthritis. Drugs. 1993;45(Suppl 1):15; discussion -6. doi: 10.2165/00003495-199300451-00005. PMID: 7685683. Exclusion: 10 
316. Geisser ME, Clauw DJ, Strand V, et al. Contributions of change in clinical status parameters to Patient Global Impression of Change (PGIC) scores among persons with fibromyalgia treated with milnacipran. Pain. 2010 May;149(2):373-8. doi: 10.1016/j.pain.2010.02.043. PMID: 20332060. Exclusion: 2

317. Geisser ME, Palmer RH, Gendreau RM, et al. A pooled analysis of two randomized, double-blind, placebo-controlled trials of milnacipran monotherapy in the treatment of fibromyalgia. Pain pract. 2011 MarApr;11(2):120-31. doi: 10.1111/j.15332500.2010.00403.x. PMID: 20642487. Exclusion: 6

318. Gennery BA, Koch IM, Woodcock KR. Multicentre osteoarthritis trial. Eur J Rheumatol Inflamm. 1982;5(2):270-6. PMID: 7044802. Exclusion: 9

319. Gentiletti AA. Tenoxicam, a new nonsteroidal anti-inflammatory drug in the prolonged treatment of rheumatoid arthritis. Eur J Rheumatol Inflamm. 1987;9(2):91-4. PMID: 3329116. Exclusion: 6

320. Gerecz-Simon E, Soper WY, Kean WF, et al. A controlled comparison of piroxicam and diclofenac in patients with osteoarthritis. Clin Rheumatol. 1990 Jun;9(2):229-34.

PMID: 2202544. Exclusion: 3

321. Gerson GR, Jones RB, Luscombe DK. Studies on the concomitant use of carbamazepine and clomipramine for the relief of post-herpetic neuralgia. Postgrad Med J. 1977;53(Suppl 4):104-9. PMID: 304576. Exclusion: 3

322. Gewandter JS, Frazer ME, Cai X, et al. Extended-release gabapentin for failed back surgery syndrome: results from a randomized double-blind cross-over study. Pain. 2019 May;160(5):1029-36. doi: 10.1097/j.pain.0000000000001478. PMID: 30624344. Exclusion: 9

323. Ghosh AK, Rastogi AK. A randomized comparison between sulindac and ibuprofen in osteoarthritis of the aged. Curr Med Res Opin. 1981;7(7):482-7. doi: 10.1185/03007998109114288. PMID: 7261665. Exclusion: 3
324. Ghosh AK, Rastogi AK. Symptomatic osteoarthritis in the elderly: a comparative study of sulindac and ibuprofen. Curr Med Res Opin. 1982;7(Suppl 1):33-40. PMID: 7105794. Exclusion: 3

325. Ghosh S, Paul S, Das N, et al. A study on the effects of diclofenac sodium and etoricoxib in the treatment of osteoarthritis. J Indian Med Assoc. 2007 May;105(5):2602. PMID: 17915794. Exclusion: 9

326. Giacovazzo M. Clinical evaluation of a new NSAID applied topically (BPAA gel) vs. diclofenac emulgel in elderly osteoarthritic patients. Drugs Exp Clin Res. 1992;18(5):201-3. PMID: 1490436. Exclusion: 3

327. Giannopoulos S, Kosmidou M, Sarmas I, et al. Patient compliance with SSRIs and gabapentin in painful diabetic neuropathy. Clin J Pain. 2007 Mar-Apr;23(3):267-9. doi: 10.1097/AJP.0b013e31802fc14a. PMID: 17314587. Exclusion: 3

328. Gibofsky A, Williams GW, McKenna F, et al. Comparing the efficacy of cyclooxygenase 2-specific inhibitors in treating osteoarthritis: appropriate trial design considerations and results of a randomized, placebo-controlled trial. Arthritis Rheum. 2003 Nov;48(11):3102-11. doi: 10.1002/art.11330. PMID: 14613272. Exclusion: 9

329. Gibson T, Laurent R. Sulindac and indomethacin in the treatment of ankylosing spondylitis: a double-blind cross-over study. Rheumatol Rehabil. 1980 Aug;19(3):18992. PMID: 7414189. Exclusion: 9

330. Gilron I, Bailey JM, Tu D, et al. Nortriptyline and gabapentin, alone and in combination for neuropathic pain: a doubleblind, randomised controlled crossover trial. Lancet. 2009 Oct 10;374(9697):1252-61. doi: 10.1016/S0140-6736(09)61081-3. PMID: 19796802. Exclusion: 9

331. Gilron I, Bailey JM, Tu D, et al. Morphine, gabapentin, or their combination for neuropathic pain. N Engl J Med. 2005 Mar 31;352(13):1324-34. doi: 10.1056/NEJMoa042580. PMID: 15800228. Exclusion: 9 
332. Gilron I, Booher SL, Rowan JS, et al. Topiramate in trigeminal neuralgia: a randomized, placebo-controlled multiple crossover pilot study. Clin Neuropharmacol. 2001 Mar-Apr;24(2):109-12. PMID: 11307048. Exclusion: 3

333. Gilron I, Chaparro LE, Tu D, et al. Combination of pregabalin with duloxetine for fibromyalgia: a randomized controlled trial. Pain. 2016 Jul;157(7):1532-40. doi: 10.1097/j.pain.0000000000000558. PMID: 26982602. Exclusion: 9

334. Gimenez M, Pujol J, Ali Z, et al. Naproxen effects on brain response to painful pressure stimulation in patients with knee osteoarthritis: a double-blind, randomized, placebo-controlled, single-dose study. J Rheumatol. 2014 Nov;41(11):2240-8. doi: 10.3899/jrheum.131367. PMID: 25274897. Exclusion: 6

335. Ginsberg F, Mancaux A, Joos E, et al. A randomized placebo-controlled trial of sustained-release amitriptyline in primary fibromyalgia. J Musculoskelet Pain. 1996;4(3):37-47. doi: 10.1300/J094v04n03_05. Exclusion: 9

336. Giordano N, Geraci S, Santacroce C, et al. Efficacy and tolerability of paroxetine in patients with fibromyalgia syndrome: A single-blind study. Curr Ther Res Clin Exp. 1999 Dec;60(12):696-702. doi: 10.1016/S0011-393X(99)90008-5. Exclusion: 3

337. Glantz L, Godovic G, Lekar M, et al. Efficacy of transdermal nitroglycerin combined with etodolac for the treatment of chronic post-thoracotomy pain: an openlabel prospective clinical trial. J Pain Symptom Manage. 2004 Mar;27(3):277-81. PMID: 15038339. Exclusion: 4

338. GlaxoSmithKline. A Multicenter, Doubleblind, Randomized Study to Evaluate the Safety and Efficacy of Lamotrigine 200mg/day, 300mg/day, and 400mg/day Compared with Placebo in Subjects with Painful Diabetic Neuropathy (NPP30004). http://download.gskclinicalstudyregister.com/files/1626.pdf. 2005:Study no. NPP30004. Exclusion: 3
339. GlaxoSmithKline. A Multicenter, Doubleblind, Randomized Study to Evaluate the Safety and Efficacy of Lamotrigine 200mg/day, 300mg/day, and 400mg/day Compared with Placebo in Subjects with Painful Diabetic Neuropathy (NPP30005). 2005. p. Study no. NPP30005. Exclusion: 3

340. Glick EN. A clinical Trial of Tofranil in osteo-arthritis. J Int Med Res. 1976;4(2 Suppl):20-2. PMID: 799986. Exclusion: 3

341. Goei The HS, Lund B, Distel MR, et al. A double-blind, randomized trial to compare meloxicam $15 \mathrm{mg}$ with diclofenac $100 \mathrm{mg}$ in the treatment of osteoarthritis of the knee. Osteoarthritis Cartilage. 1997 Jul;5(4):2838. PMID: 9404473. Exclusion: 9

342. Goldenberg D, Mayskiy M, Mossey C, et al. A randomized, double-blind crossover trial of fluoxetine and amitriptyline in the treatment of fibromyalgia. Arthritis Rheum. 1996 Nov;39(11):1852-9. PMID: 8912507. Exclusion: 9

343. Goldenberg DL, Felson DT, Dinerman H. A randomized, controlled trial of amitriptyline and naproxen in the treatment of patients with fibromyalgia. Arthritis Rheum. 1986 Nov;29(11):1371-7. PMID: 3535811. Exclusion: 9

344. Goldie I. A clinical trial with indomethacin (indomee(R)) in low back pain and sciatica. Acta Orthop Scand. 1968;39(1):117-28. PMID: 4239771. Exclusion: 4

345. Goldie IF, Gunterberg B, Tiselius P. An objective evaluation of naproxen for the inflammatory reaction in the rheumatoid hand. Scand J Rheumatol. 1974;3(4):161-8. PMID: 4617299. Exclusion: 9

346. Goldstein J, Hagen M, Gold M. Results of a multicenter, double-blind, randomized, parallel-group, placebo-controlled, singledose study comparing the fixed combination of acetaminophen, acetylsalicylic acid, and caffeine with ibuprofen for acute treatment of patients with severe migraine. Cephalalgia. 2014 Nov;34(13):1070-8. doi: 10.1177/0333102414530527. PMID: 24733408. Exclusion: 4

347. Goldstein JL. Significant upper gastrointestinal events associated with conventional NSAID versus celecoxib. J Rheumatol Suppl. 2000 Oct;60:25-8. PMID: 11032099. Exclusion: 5 
348. Goldstein JL, Cryer B, Amer F, et al. Celecoxib plus aspirin versus naproxen and lansoprazole plus aspirin: a randomized, double-blind, endoscopic trial. Clin

Gastroenterol Hepatol. 2007

Oct;5(10):1167-74. doi:

10.1016/j.cgh.2007.06.009. PMID:

17916545. Exclusion: 3

349. Goldstein JL, Hochberg MC, Fort JG, et al. Clinical trial: the incidence of NSAIDassociated endoscopic gastric ulcers in patients treated with PN 400 (naproxen plus esomeprazole magnesium) vs. enteric-coated naproxen alone. Aliment Pharmacol Ther. 2010 Aug;32(3):401-13. doi: 10.1111/j.1365-2036.2010.04378.x. PMID: 20497139. Exclusion: 3

350. Goldstein JL, Silverstein FE, Agrawal NM, et al. Reduced risk of upper gastrointestinal ulcer complications with celecoxib, a novel COX-2 inhibitor. Am J Gastroenterol. 2000 Jul;95(7):1681-90. doi: 10.1111/j.15720241.2000.02194.x. PMID: 10925968. Exclusion: 6

351. Gomez G. Long-term safety study of ketoprofen. Rheumatol Rehabil. 1976;Suppl:85-6. PMID: 796955. Exclusion: 5

352. Goncalves AL, Martini Ferreira A, Ribeiro $\mathrm{RT}$, et al. Randomised clinical trial comparing melatonin $3 \mathrm{mg}$, amitriptyline 25 $\mathrm{mg}$ and placebo for migraine prevention. $\mathrm{J}$ Neurol Neurosurg Psychiatry. 2016 Oct;87(10):1127-32. doi: 10.1136/jnnp2016-313458. PMID: 27165014. Exclusion: 4

353. Gonzalez-Duarte A, Lem M, Diaz-Diaz E, et al. The Efficacy of Pregabalin in the Treatment of Prediabetic Neuropathic Pain. Clin J Pain. 2016 Nov;32(11):927-32. doi: 10.1097/AJP.0000000000000339. PMID: 26670614. Exclusion: 9

354. Gordh TE, Stubhaug A, Jensen TS, et al. Gabapentin in traumatic nerve injury pain: a randomized, double-blind, placebocontrolled, cross-over, multi-center study. Pain. 2008 Aug 31;138(2):255-66. doi: 10.1016/j.pain.2007.12.011. PMID: 18258368. Exclusion: 9
355. Gordin A, Karppinen I, Holttinen K. Comparison of slow-release indomethacin and diflunisal in patients with arthrosis. Curr Med Res Opin. 1984;9(4):275-9. doi: 10.1185/03007998409109591. PMID: 6510016. Exclusion: 9

356. Gorson KC, Schott C, Herman R, et al. Gabapentin in the treatment of painful diabetic neuropathy: a placebo controlled, double blind, crossover trial. J Neurol Neurosurg Psychiatry. 1999 Feb;66(2):2512. doi: 10.1136/jnnp.66.2.251. PMID: 10071116. Exclusion: 9

357. Grace EM, Mewa A, Rooney PJ, et al. Clinical therapeutic trial of sodium meclofenamate and naproxen in rheumatoid arthritis, with comments on the use of placebos in clinical trials. Curr Med Res Opin. 1983;8(6):417-24. doi: 10.1185/03007998309111748. PMID: 6342956. Exclusion: 3

358. Graham D, Agrawal NM, Roth SH. Prevention of NSAID-induced gastric ulcer with misoprostol: multicentre, double-blind, placebo-controlled trial. Lancet. 1988 Dec 3;2(8623):1277-80. PMID: 2904006. Exclusion: 3

359. Graham DY, Agrawal NM, Campbell DR, et al. Ulcer prevention in long-term users of nonsteroidal anti-inflammatory drugs: results of a double-blind, randomized, multicenter, active- and placebo-controlled study of misoprostol vs lansoprazole. Arch Intern Med. 2002 Jan 28;162(2):169-75. PMID: 11802750. Exclusion: 3

360. Graham DY, Jewell NP, Chan FK. Rofecoxib and clinically significant upper and lower gastrointestinal events revisited based on documents from recent litigation. Am J Med Sci. 2011 Nov;342(5):356-64. doi: 10.1097/MAJ.0b013e3182113658. PMID: 21986300. Exclusion: 8

361. Grayson MF. Knee joint temperatures during the use of anti-inflammatory drugs. Ann Clin Res. 1974 Jun;6(3):167-70. PMID: 4844129. Exclusion: 2

362. Grayson MF. A clinical trial of diflunisal against aspirin in osteoarthritis. Rheumatol Rehabil. 1978 Nov;17(4):265-9. PMID: 366723. Exclusion: 3 
363. Grayson MF, Huskisson EC. A sequential trial of indomethacin and sodium amylobarbitone at night in rheumatoid arthritis, with comments on the statistical method. Rheumatol Rehabil. 1974 Nov;13(4):176-8. PMID: 4614367. Exclusion: 3

364. Graziano FM. Once-daily or twice-daily administration of naproxen in patients with rheumatoid arthritis. Clin Ther. 1991;13(Suppl A):20-5. Exclusion: 3

365. Greb WH, von Schrader HW, Cerlek S, et al. Endoscopic studies of nabumetone in patients with rheumatoid arthritis. A comparative endoscopic and histologic evaluation. Am J Med. 1987 Oct 30;83(4B):19-24. PMID: 3318423. Exclusion: 2

366. Gregori D, Giacovelli G, Minto C, et al. Association of pharmacological treatments with long-term pain control in patients with knee osteoarthritis: a systematic review and meta-analysis. JAMA. 2018 Dec 25;320(24):2564-79. doi: 10.1001/jama.2018.19319. PMID: 30575881. Exclusion: 8

367. Grennan DM, Ferry DG, Ashworth ME, et al. The aspirin-ibuprofen interaction in rheumatoid arthritis. Br J Clin Pharmacol. 1979 Nov;8(5):497-503. doi: 10.1111/j.1365-2125.1979.tb01033.x. PMID: 389264. Exclusion: 3

368. Griffin AJ, Grahame R, Bloch M, et al. Ketoprofen: double-blind cross-over study with indomethacin administered as a combined suppository/capsule regime in patients with rheumatoid arthritis. A preliminary report. Rheumatol Rehabil. 1978;Suppl:84-9. PMID: 364616.

Exclusion: 3

369. Gross W. Long-term treatment of rheumatoid arthritis: results of a two-year study with tenoxicam. Eur J Rheumatol Inflamm. 1987;9(2):102-4. PMID: 3329098. Exclusion: 3

370. Group AR. Naproxen and celecoxib do not prevent $\mathrm{AD}$ in early results from a randomized controlled trial. Neurology. 2007 May 22;68(21):1800-8. doi: 10.1212/01.wnl.0000260269.93245.d2. PMID: 17460158. Exclusion: 4
371. Guan Y, Ding X, Cheng Y, et al. Efficacy of pregabalin for peripheral neuropathic pain: results of an 8-week, flexible-dose, doubleblind, placebo-controlled study conducted in China. Clin Ther. 2011 Feb;33(2):159-66. doi: 10.1016/j.clinthera.2011.02.007. PMID: 21444113. Exclusion: 9

372. Guiloff RJ. Carbamazepine in Morton's neuralgia. Br Med J. 1979 Oct

13;2(6195):904. doi:

10.1136/bmj.2.6195.904. PMID: 519226.

Exclusion: 5

373. Gulati PD, Sharma RK, Sood KC, et al.

Flurbiprofen--a new anti-prostaglandin antiinflammatory drug in rheumatoid arthritis. J Assoc Physicians India. 1981

Aug;29(8):603-7. PMID: 7040332.

Exclusion: 3

374. Gum OB. Long-term efficacy and safety of benoxaprofen: comparison with aspirin and ibuprofen in patients with active rheumatoid arthritis. J Rheumatol Suppl. 1980;6:76-88. PMID: 6993676. Exclusion: 3

375. Gunter BR, Butler KA, Wallace RL, et al. Non-steroidal anti-inflammatory druginduced cardiovascular adverse events: a meta-analysis. J Clin Pharm Ther. 2017 Feb;42(1):27-38. doi: 10.1111/jcpt.12484. PMID: 28019014. Exclusion: 8

376. Gupta SK, Mahajan A, Tandon V. Gabapentin for the treatment of neuropathic pain. JK Science. 2004;6(3):113-4. Exclusion: 5

377. Gutierrez T, Hohmann AG. Cannabinoids for the treatment of neuropathic pain: Are they safe and effective? Future Neurol. 2011;6(2):129-33. doi: 10.2217/fnl.11.6. Exclusion: 5

378. Guy W, Ban TA, McEvoy JP, et al. A collaborative study of a new antidepressant, viloxazine, in neurotic and endogenous depressives. Int Pharmacopsychiatry. 1982;17(1):36-42. PMID: 7045017. Exclusion: 3

379. Gyory AN, Bloch M, Burry HC, et al. Orudis in management of rheumatoid arthritis and osteoarthrosis of the hip: comparison with indomethacin. Br Med J. 1972 Nov 18;4(5837):398-400. doi: 10.1136/bmj.4.5837.398. PMID: 4564764. Exclusion: 3 
380. Haanpaa M. Controlled release oxycodoneAn evidence-based treatment for pain in acute herpes zoster. Pain. 2009

Apr;142(3):175-6. doi:

10.1016/j.pain.2009.01.025. PMID:

19233562. Exclusion: 4

381. Haanpaa M, Cruccu G, Nurmikko TJ, et al. Capsaicin 8\% patch versus oral pregabalin in patients with peripheral neuropathic pain. Eur J Pain. 2016 Feb;20(2):316-28. doi: 10.1002/ejp.731. PMID: 26581442.

Exclusion: 9

382. Hackett GI, Boddie HG, Harrison P. Chronic muscle contraction headache: the importance of depression and anxiety. J R Soc Med. 1987 Nov;80(11):689-91. PMID: 3320366. Exclusion: 4

383. Hahn K, Arendt G, Braun JS, et al. A placebo-controlled trial of gabapentin for painful HIV-associated sensory neuropathies. J Neurol. 2004 Oct;251(10):1260-6. doi: 10.1007/s00415004-0529-6. PMID: 15503108. Exclusion: 10

384. Hahn KJ. A comparison of phenylbutazone and indometacin in the treatment of rheumatoid arthritis. Arzneimittelforschung. 1973 Jun;23(6):851-4. PMID: 4579954. Exclusion: 3

385. Hamaty D, Valentine JL, Howard R, et al. The plasma endorphin, prostaglandin and catecholamine profile of patients with fibrositis treated with cyclobenzaprine and placebo: a 5-month study. J Rheumatol Suppl. 1989 Nov;19:164-8. PMID: 2532682. Exclusion: 4

386. Hammack JE, Michalak JC, Loprinzi CL, et al. Phase III evaluation of nortriptyline for alleviation of symptoms of cis-platinuminduced peripheral neuropathy. Pain. 2002 Jul;98(1-2):195-203. PMID: 12098632. Exclusion: 10

387. Hanly JG. Salsalate in the management of rheumatoid arthritis. Today's Therapeutic Trends. 1994;12(2):53-60. Exclusion: 5

388. Hannequin JR. Efficacy of Arthrotec in the treatment of rheumatoid arthritis. Scand J Rheumatol Suppl. 1992;96:7-14. PMID: 1439627. Exclusion: 3
389. Harden RN, Freeman R, Rainka M, et al. A phase 2a, randomized, crossover trial of gabapentin enacarbil for the treatment of postherpetic neuralgia in gabapentin inadequate responders. Pain Med. 2013 Dec;14(12):1918-32. doi: 10.1111/pme.12227. PMID: 24102928. Exclusion: 9

390. Hardy T, Sachson R, Shen S, et al. Does treatment with duloxetine for neuropathic pain impact glycemic control? Diabetes Care. 2007 Jan;30(1):21-6. doi: 10.2337/dc06-0947. PMID: 17192327. Exclusion: 6

391. Harris RE, Williams DA, McLean SA, et al. Characterization and consequences of pain variability in individuals with fibromyalgia. Arthritis Rheum. 2005 Nov;52(11):3670-4. doi: 10.1002/art.21407. PMID: 16258905.

Exclusion: 2

392. Harrison T, Miyahara S, Lee A, et al. Experience and challenges presented by a multicenter crossover study of combination analgesic therapy for the treatment of painful HIV-associated polyneuropathies. Pain Med. 2013 Jul;14(7):1039-47. doi: 10.1111/pme.12084. PMID: 23565581. Exclusion: 9

393. Hart CB, Rhymer AR, Miola SR. INDOCIN® SR (indomethacin, MSD) in the treatment of moderate to severe rheumatoid arthritis. Seminars in Arthritis and Rheumatism. 1982 Nov;12(2 Suppl 1):14751. Exclusion: 9

394. Hart CB, Tempero KF, Sromovsky JA, et al. 'Osmosin' (sodium indomethacin trihydrate) in the treatment of elderly patients with osteoarthritis. Curr Med Res Opin. 1983;8 Suppl 2:72-82. doi: 10.1185/03007998309109826. PMID: 6342964. Exclusion: 3

395. Hart FD, Boardman PL. Indomethacin and phenylbutazone: a comparison. Br Med J. 1965 Nov 27;2(5473):1281-4. doi: 10.1136/bmj.2.5473.1281. PMID: 5321595. Exclusion: 3

396. Harth M, Bondy DC. Indomethacin and acetylsalicylic acid in the treatment of osteoarthritis of the hips and knees. Can Med Assoc J. 1969 Sep 20;101(6):311-6. PMID: 4898391. Exclusion: 3 
397. Hauser W, Petzke F, Fitzcharles MA. Efficacy, tolerability and safety of cannabisbased medicines for chronic pain management - An overview of systematic reviews. Eur J Pain. 2018 Mar;22(3):455-70. doi: 10.1002/ejp.1118. PMID: 29034533. Exclusion: 8

398. Havranek H. Double-blind study of tenoxicam $20 \mathrm{mg}$ versus piroxicam $20 \mathrm{mg}$ in rheumatoid arthritis. Eur J Rheumatol Inflamm. 1987;9(2):105-8. PMID: 3329099. Exclusion: 3

399. Hawkey C, Kahan A, Steinbruck K, et al. Gastrointestinal tolerability of meloxicam compared to diclofenac in osteoarthritis patients. International MELISSA Study Group. Meloxicam Large-scale International Study Safety Assessment.[Erratum appears in Br J Rheumatol 1998 Oct;37(10):1142]. Br J Rheumatol. 1998 Sep;37(9):937-45. PMID: 9783757. Exclusion: 10

400. Hawkey CJ, Karrasch JA, Szczepanski L, et al. Omeprazole compared with misoprostol for ulcers associated with nonsteroidal antiinflammatory drugs. Omeprazole versus Misoprostol for NSAID-induced Ulcer Management (OMNIUM) Study Group. N Engl J Med. 1998 Mar 12;338(11):727-34. doi: 10.1056/nejm199803123381105. PMID: 9494149. Exclusion: 4

401. Hawkey CJ, Laine L, Harper SE, et al. Influence of risk factors on endoscopic and clinical ulcers in patients taking rofecoxib or ibuprofen in two randomized controlled trials. Aliment Pharmacol Ther. 2001 Oct;15(10):1593-601. PMID: 11563999. Exclusion: 6

402. Hawkey CJ, Weinstein WM, Smalley W, et al. Effect of risk factors on complicated and uncomplicated ulcers in the TARGET lumiracoxib outcomes study. Gastroenterology. 2007 Jul;133(1):57-64. doi: 10.1053/j.gastro.2007.04.045. PMID: 17631131. Exclusion: 3
403. Hawkey CJ, Weinstein WM, Stricker K, et al. Clinical trial: comparison of the gastrointestinal safety of lumiracoxib with traditional nonselective nonsteroidal antiinflammatory drugs early after the initiation of treatment--findings from the Therapeutic Arthritis Research and Gastrointestinal Event Trial. Aliment Pharmacol Ther. 2008 May;27(9):838-45. doi: 10.1111/j.13652036.2008.03622.x. PMID: 18221410. Exclusion: 2

404. Hays RD, Martin SA, Sesti AM, et al. Psychometric properties of the Medical Outcomes Study Sleep measure. Sleep Med. 2005 Jan;6(1):41-4. doi: 10.1016/j.sleep.2004.07.006. PMID: 15680294. Exclusion: 2

405. Hazleman BL, Bulgen DY. A comparative study of the long-term efficacy of flurbiprofen and indomethacin in the treatment of rheumatoid arthritis, with special reference to iron metabolism. Curr Med Res Opin. 1977;5(1):58-63. doi: 10.1185/03007997709108978. PMID: 334475. Exclusion: 6

406. Hazleman BL, Thomas PP. Single-blind comparative study of nabumetone (Relafen) versus naproxen in the treatment of rheumatoid arthritis. Am J Med. 1987 Oct 30;83(4B):60-4. PMID: 3318431. Exclusion: 2

407. Hazlewood G, van der Heijde DM, Bombardier C. Paracetamol for the management of pain in inflammatory arthritis: a systematic literature review. J Rheumatol Suppl. 2012 Sep;90:11-6. doi: 10.3899/jrheum.120336. PMID: 22942323. Exclusion: 8

408. Helby-Petersen P, Ibfelt H, Rossel I. A double-blind crossover comparison of naproxen and placebo in rheumatoid arthritis. Scand J Rheumatol Suppl. 1973;2:145-9. PMID: 4590040. Exclusion: 6 
409. Hendricks O, Andersen TE, Christiansen AA, et al. Efficacy and safety of cannabidiol followed by an open label add-on of tetrahydrocannabinol for the treatment of chronic pain in patients with rheumatoid arthritis or ankylosing spondylitis: protocol for a multicentre, randomised, placebocontrolled study. BMJ Open. 2019 Jun 4;9(6):e028197. doi: 10.1136/bmjopen2018-028197. PMID: 31167870. Exclusion: 5

410. Henry KA. Memantine for the prophylaxis of chronic tension-type headache. Curr Pain Headache Rep. 2009 Dec;13(6):423-4. PMID: 19889282. Exclusion: 5

411. Hering-Hanit R. Baclofen for prevention of migraine. Cephalalgia. 1999 Jul;19(6):58991. doi: 10.1046/j.1468-

2982.1999.019006589.x. PMID: 10448546. Exclusion: 4

412. Herrera JA, Millan A, Ramos R, et al. Evaluation of the effectiveness and tolerability of controlled-release diclofenacpotassium versus immediate-release diclofenac-potassium in the treatment of knee osteoarthritis. Curr Ther Res Clin Exp. 2007 Mar;68(2):82-93. doi:

10.1016/j.curtheres.2007.03.004. PMID: 24678122. Exclusion: 10

413. Heymann RE, Helfenstein M, Feldman D. A double-blind, randomized, controlled study of amitriptyline, nortriptyline and placebo in patients with fibromyalgia. An analysis of outcome measures. Clin Exp Rheumatol. 2001 Nov-Dec;19(6):697-702. PMID: 11791642. Exclusion: 9

414. Hickey RF. Chronic low back pain: a comparison of diflunisal with paracetamol. N Z Med J. 1982 May 12;95(707):312-4. PMID: 6212783. Exclusion: 9

415. Hill HF, Hill AG. Naproxen in ankylosing spondylitis. Scand J Rheumatol Suppl. 1973;2:121-6. PMID: 4590035. Exclusion: 6

416. Hill HF, Hill AG. Ankylosing spondylitis: open long-term and double-blind crossover studies with naproxen. J Clin Pharmacol. 1975 Apr;15(4 Pt. 2):355-62. PMID: 1092729. Exclusion: 9
417. Hill HF, Hill AG, Mowat AG, et al. Naproxen. A new non-hormonal antiinflammatory agent. Studies in rheumatoid arthritis. Ann Rheum Dis. 1974 Jan;33(1):12-9. doi: 10.1136/ard.33.1.12. PMID: 4595271. Exclusion: 9

418. Hill J, Bird HA, Fenn GC, et al. A doubleblind crossover study to compare lysine acetyl salicylate (Aspergesic) with ibuprofen in the treatment of rheumatoid arthritis. $\mathrm{J}$ Clin Pharm Ther. 1990 Jun;15(3):205-11. PMID: 2114414. Exclusion: 3

419. Hill KP. Medical Marijuana for Treatment of Chronic Pain and Other Medical and Psychiatric Problems: A Clinical Review. JAMA. 2015 Jun 23-30;313(24):2474-83. doi: 10.1001/jama.2015.6199. PMID: 26103031. Exclusion: 6

420. Hingorani K. Double-blind crossover trial comparing ibuprofen with flufenamic acid in rheumatoid arthritis. Rheumatol Phys Med. 1970;10(Suppl 10):76-82. PMID: 5524286. Exclusion: 9

421. Ho JM, Tricco AC, Perrier L, et al. Risk of heart failure and edema associated with the use of pregabalin: a systematic review. Syst. 2013 May 04;2:25. doi: 10.1186/2046-40532-25. PMID: 23641821. Exclusion: 8

422. Ho TW, Backonja M, Ma J, et al. Efficient assessment of neuropathic pain drugs in patients with small fiber sensory neuropathies. Pain. 2009 Jan;141(1-2):1924. doi: 10.1016/j.pain.2008.07.013. PMID: 19013718. Exclusion: 3

423. Hochberg MC. What have we learned from the large outcomes trials of COX-2 selective inhibitors? The rheumatologist's perspective. Clin Exp Rheumatol. 2001 Nov-Dec;19(6 Suppl 25):S15-22. PMID: 11695246. Exclusion: 5

424. Hochberg MC, Clegg DO. Potential effects of chondroitin sulfate on joint swelling: a GAIT report. Osteoarthritis Cartilage. 2008;16(Suppl 3):S22-4. doi: 10.1016/j.joca.2008.06.024. PMID: 18768335. Exclusion: 2

425. Hochberg MC, Dougados M. Pharmacological therapy of osteoarthritis. Best Pract Res Clin Rheumatol. 2001 Oct;15(4):583-93. doi: 10.1053/berh.2001.0175. PMID: 11567541. Exclusion: 5 
426. Hodge RH, Jr. Double-blind crossover comparison of piroxicam and naproxen in the treatment of active osteoarthritis. Semin Arthritis Rheum. 1985 Feb;14(3 Suppl 1):36. PMID: 3914702. Exclusion: 6

427. Hodgkinson R, Woolf D. A five-year clinical trial of indomethacin in osteoarthrosis of the hip. Practitioner. 1973 Mar;210(257):392-6. PMID: 4571359. Exclusion: 2

428. Holbech JV, Bach FW, Finnerup NB, et al. Imipramine and pregabalin combination for painful polyneuropathy: a randomized controlled trial. Pain. 2015 May;156(5):95866. doi: 10.1097/j.pain.0000000000000143. PMID: 25719617. Exclusion: 9

429. Holbech JV, Otto M, Bach FW, et al. The anticonvulsant levetiracetam for the treatment of pain in polyneuropathy: a randomized, placebo-controlled, cross-over trial. Eur J Pain. 2011 Jul;15(6):608-14. doi: 10.1016/j.ejpain.2010.11.007. PMID: 21183370. Exclusion: 3

430. Holman AJ. Pregabalin as treatment for fibromyalgia: the rest of the story? Curr Pain Headache Rep. 2009 Dec;13(6):420-2. PMID: 19889281. Exclusion: 5

431. Holmes EL. Experimental observations on flufenamic, mefenamic, and meclofenamic acids. IV. Toleration by normal human subjects. Ann Phys Med. 1966;Suppl:36-49. PMID: 4895006. Exclusion: 4

432. Holsgaard-Larsen A, Clausen B, Sondergaard J, et al. The effect of instruction in analgesic use compared with neuromuscular exercise on knee-joint load in patients with knee osteoarthritis: a randomized, single-blind, controlled trial. Osteoarthritis Cartilage. 2017

Apr;25(4):470-80. doi: 10.1016/j.joca.2016.10.022. PMID: 27836677. Exclusion: 3

433. Hosie J, Distel M, Bluhmki E. Efficacy and tolerability of meloxicam versus piroxicam in patients with osteoarthritis of the hip or knee. Clin Drug Investig. 1997 Apr;13(4):175-84. Exclusion: 3
434. Howard DL. A double-blind crossover evaluation of ketoprofen (Orudis) and placebo in rheumatoid arthritis with assessment of long-term tolerance. J Int Med Res. 1978;6(4):300-5. doi: 10.1177/030006057800600407. PMID: 357231. Exclusion: 9

435. $\mathrm{Hu} \mathrm{H}$, Chen L, Ma R, et al. Acupuncture for primary trigeminal neuralgia: A systematic review and PRISMA-compliant metaanalysis. Complement Ther Clin Pract. 2019 Feb;34:254-67. doi: 10.1016/j.ctcp.2018.12.013. PMID: 30712736. Exclusion: 8

436. Huang L, Bocek M, Jordan JK, et al. Memantine for the prevention of primary headache disorders. Ann Pharmacother. 2014 Nov;48(11):1507-11. doi: https://dx.doi.org/10.1177/10600280145488 72. PMID: 25159002. Exclusion: 8

437. Hubsher JA, Ballard IM, Walker BR, et al. A multicentre double-blind comparison of oxaprozin aspirin therapy on rheumatoid arthritis. J Int Med Res. 1979;7(1):69-76. doi: 10.1177/030006057900700111. PMID: 369925. Exclusion: 3

438. Hudson B, Williman JA, Stamp LK, et al. Nortriptyline in knee osteoarthritis (NortIKA Study): study protocol for a randomised controlled trial. Trials. 2015 Oct 9;16:448. doi: 10.1186/s13063-015-0961-1. PMID: 26453560. Exclusion: 2

439. Hudson N, Taha A, Russell R, et al. Famotidine for healing and maintenance in nonsteroidal anti-inflammatory drugassociated gastroduodeanl ulceration. Gastroenterology. 1997 Jun;112(6):1817-22. PMID: 9178671. Exclusion: 3

440. Huffman CL, Goldenberg JN, Weintraub J, et al. Efficacy and Safety of Once-Daily Controlled-Release Pregabalin for the Treatment of Patients With Postherpetic Neuralgia: A Double-Blind, Enriched Enrollment Randomized Withdrawal, Placebo-Controlled Trial. Clin J Pain. 2017 Jul;33(7):569-78. doi: 10.1097/AJP.0000000000000445. PMID: 27753650. Exclusion: 6 
441. Hughes J. Gabapentin and nortriptyline combined was better than either drug alone for relief of neuropathic pain. Ann Intern Med. 2010 Mar 16;152(6):JC3-6. doi: 10.7326/0003-4819-152-6-20100316002006. PMID: 20231560. Exclusion: 5

442. Humberto Lizarazo P, Pena Cortes M. Single-blind parallel study comparing naproxen with sulindac and with diclofenac in rheumatoid arthritis. Curr Ther Res Clin Exp. 1983;34(4):701-7. Exclusion: 6

443. Hunt RH, Harper S, Watson DJ, et al. The gastrointestinal safety of the COX-2 selective inhibitor etoricoxib assessed by both endoscopy and analysis of upper gastrointestinal events. Am J Gastroenterol. 2003 Aug;98(8):1725-33. doi: 10.1111/j.1572-0241.2003.07598.x. PMID: 12907325. Exclusion: 3

444. Hunter AM, Leuchter AF, Cook IA, et al. Brain functional changes and duloxetine treatment response in fibromyalgia: a pilot study. Pain Med. 2009 May-Jun;10(4):7308. doi: 10.1111/j.1526-4637.2009.00614.x. PMID: 19453962. Exclusion: 2

445. Husby G. The Norwegian multicenter study. Am J Med. 1986 Nov 28;81(5B):6-10. PMID: 3538870. Exclusion: 3

446. Husby G, Holme I, Rugstad HE, et al. A double-blind multicentre trial of piroxicam and naproxen in osteoarthritis. Clin Rheumatol. 1986 Jan;5(1):84-91. PMID: 3514082. Exclusion: 3

447. Huskisson EC. Long-term use of fenoprofen in rheumatoid arthritis: the therapeutic ratio. Curr Med Res Opin. 1974;2(9):545-50. doi: 10.1185/03007997409115255. PMID: 4615885. Exclusion: 3

448. Huskisson EC. Clinical trials of Sulindac in rheumatoid and osteoarthritis. Acta Rhumatol Belg. 1977 Jul-Dec;1(3-4):25761. PMID: 388989. Exclusion: 3

449. Huskisson EC, Bernstein RM, Coppock JS, et al. Enteric coated naproxen; a double blind trial comparing the tolerance of enteric coated and standard formulations. Eur J Rheumatol Inflamm. 1992;12(2):27-30. PMID: 1364935. Exclusion: 2
450. Huskisson EC, Bryans R, Scott J. Fluproquazone for osteoarthritis. Rheumatol Rehabil. 1981 May;20(2):122-4. PMID: 7025173. Exclusion: 9

451. Huskisson EC, Dieppe PA, Scott J, et al. Diclofenac sodium, diflunisal and naproxen: patient preferences for anti-inflammatory drugs in rheumatoid arthritis. Rheumatol Rehabil. 1982 Nov;21(4):238-42. PMID: 7134745. Exclusion: 9

452. Huskisson EC, Greenwood A. Naproxen and piroxicam. A comparative trial in rheumatoid arthritis. Eur J Rheumatol Inflamm. 1983;6(3):242-6. PMID: 6239778. Exclusion: 9

453. Huskisson EC, Scott J, Woolf DL. Three trials of indoprofen. Eur J Rheumatol Inflamm. 1981;4(1):97-102. PMID: 7042369. Exclusion: 6

454. Huskisson EC, Shenfield GM, Taylor RT, et al. A new look at ibuprofen. Rheumatol Phys Med. 1970;10(Suppl 10):88-98. PMID: 5524288. Exclusion: 5

455. Huskisson EC, Wojtulewski JA, Berry H, et al. Treatment of rheumatoid arthritis with fenoprofen: comparison with aspirin. $\mathrm{Br}$ Med J. 1974 Feb 2;1(5900):176-80. doi: 10.1136/bmj.1.5900.176. PMID: 4590669. Exclusion: 3

456. Hussar DA. New drugs: milnacipran hydrochloride, fesoterodine fumarate, and silodosin. J Am Pharm Assoc (2003). 2009 Mar-Apr;49(2):347-50. doi: 10.1331/JAPhA.2009.09509. PMID: 19289354. Exclusion: 5

457. Hybbinette CH. Piroxicam and naproxen in the treatment of osteoarthritis. Br J Clin Pract. 1981 Jan;35(1):30-4. PMID: 7020730. Exclusion: 9

458. Ibba G, Uras R, Perpignano G. Double-blind comparison of glucametacin and ketoprofen in chronic arthropathies. Curr Med Res Opin. 1983;8(5):321-6. doi: 10.1185/03007998309112391. PMID: 6340973. Exclusion: 3 
459. Indaco A, Carrieri PB. Amitriptyline in the treatment of headache in patients with Parkinson's disease: a double-blind placebocontrolled study. Neurology. 1988

Nov;38(11):1720-2. doi: 10.1212/wnl.38.11.1720. PMID: 3054626. Exclusion: 4

460. Irving G, Backonja M, Rauck R, et al. NGX4010, a capsaicin $8 \%$ dermal patch, administered alone or in combination with systemic neuropathic pain medications, reduces pain in patients with postherpetic neuralgia. Clin J Pain. 2012 Feb;28(2):1017. doi: 10.1097/AJP.0b013e318227403d. PMID: 21753727. Exclusion: 3

461. Irving GA, Sweeney M. Tolerability and safety of gastroretentive once-daily gabapentin tablets for the treatment of postherpetic neuralgia. J Pain Res. 2012;5:203-8. doi: 10.2147/JPR.S32562. PMID: 22792006. Exclusion: 6

462. Isomeri R, Mikkelsson M, Latikka P, et al. Effects of amitriptyline and cardiovascular fitness training on pain in patients with primary fibromyalgia. J Musculoskelet Pain. 1993;1(3-4):253-60. doi: 10.1300/J094v01n03_27. Exclusion: 3

463. Isuru A, Rodrigo A, Wijesinghe C, et al. A randomized, double-blind, placebocontrolled trial on the role of preemptive analgesia with acetaminophen [paracetamol] in reducing headache following electroconvulsive therapy [ECT]. BMC Psychiatry. 2017 Jul 28;17(1):275. doi: 10.1186/s12888-017-1444-6. PMID: 28754173. Exclusion: 4

464. Iversen O, Fowles M, Vlieg M, et al. Sustained release indomethacin: a double blind comparison with indomethacin suppositories. N Z Med J. 1981 Apr 22;93(682):261-2. PMID: 7019782. Exclusion: 3

465. Izhar M, Alausa T, Folker A, et al. Effects of COX inhibition on blood pressure and kidney function in ACE inhibitor-treated blacks and hispanics. Hypertension. 2004 Mar;43(3):573-7. doi: 10.1161/01.HYP.0000115921.55353.e0. PMID: 14744921. Exclusion: 10
466. Jacobs JH, Grayson MF. Trial of an antiinflammatory agent (indomethacin) in low back pain with and without radicular involvement. Br Med J. $1968 \mathrm{Jul}$ 20;3(5611):158-60. doi: 10.1136/bmj.3.5611.158. PMID: 4232743. Exclusion: 9

467. Jalali S, MacFarlane JG, Grace EM, et al. Frequency of administration of short halflife nonsteroidal anti-inflammatory analgesics (NSAID's): studies with ibuprofen. Clin Exp Rheumatol. 1986 JanMar;4(1):91-3. PMID: 3516497. Exclusion: 9

468. Jalava S. A double blind crossover study of carprofen and indomethacin in patients with rheumatoid arthritis. Scand J Rheumatol Suppl. 1983;48:5-9. PMID: 6340183. Exclusion: 3

469. Jamar R, Dequeker J. Oxaprozin versus aspirin in rheumatoid arthritis: a doubleblind trial. Curr Med Res Opin. 1978;5(6):433-8. doi: 10.1185/03007997809111911. PMID: 350495. Exclusion: 2

470. Janke PG, Diggins JB, Currie WJ, et al. A multi-centre study of sulindac versus naproxen in the treatment of elderly osteoarthritic patients. Pharmatherapeutica. 1984;3(10):663-7. PMID: 6379679. Exclusion: 3

471. Jasani MK, Downie WW, Samuels BM, et al. Ibuprofen in rheumatoid arthritis. Clinical study of analgesic and antiinflammatory activity. Ann Rheum Dis. 1968 Sep;27(5):457-62. doi: 10.1136/ard.27.5.457. PMID: 4283768. Exclusion: 3

472. Jayawardena S, Leyva R, Kellstein D. Safety of a novel formulation of ibuprofen sodium compared with standard ibuprofen and placebo. Postgrad Med. 2015 Jan;127(1):33-7. doi: 10.1080/00325481.2015.993268. PMID: 25526232. Exclusion: 8

473. Jennings M, Alfieri D. A controlled comparison of etodolac and naproxen in osteoarthritis of the foot. Lower Extremity. 1997;4:43-8. Exclusion: 9 
474. Jensen D, Ho S, Hamamah S, et al. A randomized study of omeprazole compared to misoprostol for prevention of recurrent ulcers and ulcer hemorrhage in high risk patients ingesting aspirin or NSAIDs. Gastroenterology. 2000 Apr;118(4 Pt 1):A892. doi: 10.1016/S00165085(00)85703-0. Exclusion: 3

475. Jensen KB, Petzke F, Carville S, et al. Segregating the cerebral mechanisms of antidepressants and placebo in fibromyalgia. J Pain. 2014 Dec;15(12):1328-37. doi: 10.1016/j.jpain.2014.09.011. PMID: 25283470. Exclusion: 2

476. Jensen MP, Chiang YK, Wu J. Assessment of pain quality in a clinical trial of gabapentin extended release for postherpetic neuralgia. Clin J Pain. 2009 May;25(4):28692. doi: 10.1097/AJP.0b013e318192bf87. PMID: 19590476. Exclusion: 9

477. Jensen MP, Molton IR. The reciprocal effects of pain intensity and activity limitations: implications for outcomes assessment in clinical trials. Clin J Pain. 2014 Jan;30(1):9-16. doi: 10.1097/AJP.0b013e318287a27d. PMID: 23446086. Exclusion: 9

478. Johnsen V, Bjerkhoel F, Bjorneboe O, et al. Duration of morning stiffness in rheumatic patients after medication with enteric-coated and plain naproxen tablets. Scand J Rheumatol. 1986;15(1):37-40. PMID: 3515526. Exclusion: 9

479. Johnson ES, Ratcliffe DM, Wilkinson M. Naproxen sodium in the treatment of migraine. Cephalalgia. 1985 Mar;5(1):5-10. doi: 10.1046/j.1468-2982.1985.0501005.x. PMID: 3886154. Exclusion: 4

480. Johnson RH, Hornabrook RW, Lambie DG. Comparison of mefenamic acid and propranolol with placebo in migraine prophylaxis. Acta Neurol Scand. 1986 May;73(5):490-2. PMID: 3524092. Exclusion: 4

481. Jose VM, Bhansali A, Hota D, et al. Randomized double-blind study comparing the efficacy and safety of lamotrigine and amitriptyline in painful diabetic neuropathy. Diabet Med. 2007 Apr;24(4):377-83. doi: 10.1111/j.1464-5491.2007.02093.x. PMID: 17335465. Exclusion: 3
482. Jung SY, Jang EJ, Nam SW, et al. Comparative effectiveness of oral pharmacologic interventions for knee osteoarthritis: A network meta-analysis. Mod Rheumatol. 2018 Nov;28(6):1021-8. doi: 10.1080/14397595.2018.1439694. PMID: 29429391. Exclusion: 8

483. Kaarela K, Lehtinen K, Makisara P, et al. Pharmacokinetics and tolerance of slowrelease indomethacin tablets in rheumatoid arthritis. Eur J Clin Pharmacol. 1982 Oct;23(4):349-51. PMID: 7173304. Exclusion: 9

484. Kageyama T. Clinical evaluation of naproxen in the treatment of osteoarthritis-double-blind, cross-over trial. Scand J Rheumatol Suppl. 1973;2:94-100. PMID: 4590059. Exclusion: 6

485. Kajdasz DK, Iyengar S, Desaiah D, et al. Duloxetine for the management of diabetic peripheral neuropathic pain: evidence-based findings from post hoc analysis of three multicenter, randomized, double-blind, placebo-controlled, parallel-group studies. Clin Ther. 2007;29 Suppl:2536-46. doi: 10.1016/j.clinthera.2007.12.002. PMID: 18164920. Exclusion: 6

486. Kalso E, Tasmuth T, Neuvonen PJ. Amitriptyline effectively relieves neuropathic pain following treatment of breast cancer. Pain. 1996 Feb;64(2):293302. PMID: 8740607. Exclusion: 10

487. Kanai A, Segawa Y, Okamoto T, et al. The analgesic effect of a metered-dose $8 \%$ lidocaine pump spray in posttraumatic peripheral neuropathy: a pilot study. Anesth Analg. 2009 Mar;108(3):987-91. doi: 10.1213/ane.0b013e31819431aa. PMID: 19224814. Exclusion: 9

488. Karbowski A. Double-blind, parallel comparison of etodolac and indomethacin in patients with osteoarthritis of the knee. Curr Med Res Opin. 1991;12(5):309-17. doi: 10.1185/03007999109112666. PMID: 1825972. Exclusion: 9 
489. Karmakar S, Rashidian H, Chan C, et al. Investigating the role of neuropathic pain relief in decreasing gait variability in diabetes mellitus patients with neuropathic pain: a randomized, double-blind crossover trial. J Neuroeng Rehabil. 2014 Aug 20;11:125. doi: 10.1186/1743-0003-11-125. PMID: 25139539. Exclusion: 9

490. Karp JF, Rollman BL, Reynolds CF, 3rd, et al. Addressing both depression and pain in late life: the methodology of the ADAPT study. Pain Med. 2012 Mar;13(3):405-18. doi: 10.1111/j.1526-4637.2011.01322.x. PMID: 22313547. Exclusion: 3

491. Karst M, Wippermann S, Ahrens J. Role of cannabinoids in the treatment of pain and (painful) spasticity. Drugs. 2010 Dec 24;70(18):2409-38. doi: 10.2165/11585260000000000-00000. PMID: 21142261. Exclusion: 5

492. Kater RM. Double-blind evaluation of the analgesic and toxic effects of flufenamic acid and mefenamic acid in patients with chronic pain. Med J Aust. 1968 May 18;1(20):848-51. PMID: 5652895. Exclusion: 4

493. Katona G, Vargas RB. Double-blind crossover study to evaluate the efficacy of a single daily dose of naproxen in rheumatoid arthritis. Eur J Rheumatol Inflamm. 1983;6(3):233-41. PMID: 6391928. Exclusion: 9

494. Katz N, Borenstein DG, Birbara C, et al. Efficacy and safety of tanezumab in the treatment of chronic low back pain. Pain. 2011 Oct;152(10):2248-58. doi: 10.1016/j.pain.2011.05.003. PMID: 21696889. Exclusion: 3

495. Kautio AL, Haanpaa M, Leminen A, et al. Amitriptyline in the prevention of chemotherapy-induced neuropathic symptoms. Anticancer Res. 2009 Jul;29(7):2601-6. PMID: 19596934. Exclusion: 6

496. Kautio AL, Haanpaa M, Saarto T, et al. Amitriptyline in the treatment of chemotherapy-induced neuropathic symptoms. J Pain Symptom Manage. 2008 Jan;35(1):31-9. doi: 10.1016/j.jpainsymman.2007.02.043. PMID: 17980550. Exclusion: 9
497. Kavirajan H. Memantine: a comprehensive review of safety and efficacy. Expert Opin Drug Saf. 2009 Jan;8(1):89-109. doi: 10.1517/14740330802528420. PMID: 19236221. Exclusion: 6

498. Kaydok E, Levendoglu F, Ozerbil MO, et al. Comparison of the efficacy of gabapentin and pregabalin for neuropathic pain in patients with spinal cord injury: a crossover study. Arch Sicil Med Chir 4 Acta Med Mediterr. 2014;30:1343-8. Exclusion: 9

499. Kellner HL, Li C, Essex MN. Celecoxib and diclofenac plus omeprazole are similarly effective in the treatment of arthritis in patients at high gi risk in the CONDOR trial. Open Rheumatol J. 2013 Nov 13;7:96-100. doi: 10.2174/1874312901307010096. PMID: 24358067. Exclusion: 3

500. Kennedy AC. Ketoprofen in the treatment of rheumatoid arthritis. Rheumatol Rehabil. 1976;Suppl:34-6. PMID: 796938. Exclusion: 9

501. Kerr MM. Amitriptyline in emotional states at the menopause. N Z Med J. 1970 Oct;72(461):243-5. PMID: 4922592. Exclusion: 4

502. Keskin EA, Onur O, Keskin HL, et al. Transcutaneous electrical nerve stimulation improves low back pain during pregnancy. Gynecol Obstet Invest. 2012;74(1):76-83. doi: 10.1159/000337720. PMID: 22722614. Exclusion: 3

503. Keskinbora K, Pekel AF, Aydinli I, et al. Gabapentin and an opioid combination versus opioid alone for the management of neuropathic cancer pain: a randomized open trial. J Pain Symptom Manage. 2007 Aug;34(2):183-9. doi: 10.1016/j.jpainsymman.2006.11.013. PMID: 17604592. Exclusion: 3

504. Khoromi S, Cui L, Nackers L, et al. Morphine, nortriptyline and their combination vs. placebo in patients with chronic lumbar root pain. Pain. 2007 Jul;130(1-2):66-75. doi: 10.1016/j.pain.2006.10.029. PMID: 17182183. Exclusion: 9

505. Khoromi S, Patsalides A, Parada S, et al. Topiramate in chronic lumbar radicular pain. J Pain. 2005 Dec;6(12):829-36. doi: 10.1016/j.jpain.2005.08.002. PMID: 16326371. Exclusion: 3 
506. Kieburtz K, Simpson D, Yiannoutsos C, et al. A randomized trial of amitriptyline and mexiletine for painful neuropathy in HIV infection. AIDS Clinical Trial Group 242 Protocol Team. Neurology. 1998

Dec;51(6):1682-8. doi: 10.1212/wnl.51.6.1682. PMID: 9855523. Exclusion: 9

507. Kimos P, Biggs C, Mah J, et al. Analgesic action of gabapentin on chronic pain in the masticatory muscles: a randomized controlled trial. Pain. 2007 Jan;127(12):151-60. doi: 10.1016/j.pain.2006.08.028. PMID: 17030096. Exclusion: 4

508. Kinsella TD, MacKenzie KR, Kim SO, et al. Evaluation of indomethacin by a controlled, cross-over technique in 30 patients with ankylosing spondylitis. Can Med Assoc J. 1967 Jun 3;96(22):1454-9. PMID: 4164930. Exclusion: 9

509. Kirchheiner B, Fossgreen J, Tophoj E, et al. Clinical evaluation of ketoprofen (Orudis) in rheumatoid arthritis: a double-blind crossover comparison with indomethacin. Rheumatol Rehabil. 1976;Suppl:50-1. PMID: 796942. Exclusion: 6

510. Kishore-Kumar R, Max MB, Schafer SC, et al. Desipramine relieves postherpetic neuralgia. Clin Pharmacol Ther. 1990 Mar;47(3):305-12. doi: 10.1038/clpt.1990.33. PMID: 2178851. Exclusion: 9

511. Kivitz AJ, Gimbel JS, Bramson C, et al. Efficacy and safety of tanezumab versus naproxen in the treatment of chronic low back pain. Pain. 2013 Jul;154(7):1009-21. doi: 10.1016/j.pain.2013.03.006. PMID: 23628600. Exclusion: 3

512. Kochar DK, Garg P, Bumb RA, et al. Divalproex sodium in the management of post-herpetic neuralgia: a randomized double-blind placebo-controlled study. QJM. 2005 Jan;98(1):29-34. doi: 10.1093/qjmed/hci005. PMID: 15625351. Exclusion: 3

513. Kochar DK, Jain N, Agarwal RP, et al. Sodium valproate in the management of painful neuropathy in type 2 diabetes - a randomized placebo controlled study. Acta Neurol Scand. 2002 Nov;106(5):248-52. PMID: 12371916. Exclusion: 3
514. Kochar DK, Rawat N, Agrawal RP, et al. Sodium valproate for painful diabetic neuropathy: a randomized double-blind placebo-controlled study. QJM. 2004 Jan;97(1):33-8. doi: 10.1093/qjmed/hch007. PMID: 14702509. Exclusion: 3

515. Kogstad O. A double-blind cross-over study of naproxen and indomethacin in patients with rheumatoid arthritis. Scand J Rheumatol Suppl. 1973;2:159-63. PMID: 4590043. Exclusion: 9

516. Kogstad O. Double blind crossover trial of piroxicam and naproxen in the treatment of osteoarthritis of hip and knee. Br J Clin Pract. 1981 Jan;35(1):45-50. PMID: 7020733. Exclusion: 9

517. Kolodny AL. Two double blind trials of diclofenac sodium with aspirin and with naproxen in the treatment of patients with rheumatoid arthritis. J Rheumatol. 1988 Aug;15(8):1205-11. PMID: 3054094. Exclusion: 2

518. Kosek E, Rosen A, Carville S, et al. Lower Placebo Responses After Long-Term Exposure to Fibromyalgia Pain. J Pain. 2017 Jul;18(7):835-43. doi: 10.1016/j.jpain.2017.02.434. PMID: 28279705. Exclusion: 6

519. Kosuwon W, Sirichatiwapee W, Wisanuyotin T, et al. Efficacy of symptomatic control of knee osteoarthritis with $0.0125 \%$ of capsaicin versus placebo. J Med Assoc Thai. 2010 Oct;93(10):1188-95. PMID: 20973322. Exclusion: 10

520. Kowanko IC, Pownall R, Knapp MS, et al. Circadian variations in the signs and symptoms of rheumatoid arthritis and in the therapeutic effectiveness of flurbiprofen at different times of day. Br J Clin Pharmacol. 1981 May;11(5):477-84. doi: 10.1111/j.1365-2125.1981.tb01153.x. PMID: 7272159. Exclusion: 6

521. Kravitz HM, Katz RS, Helmke N, et al. Alprazolam and ibuprofen in the treatment of fibromyalgia - Report of a double-blind placebo-controlled study. J Musculoskelet Pain. 1994;2(1):3-27. doi: 10.1300/J094v02n01_02. Exclusion: 9 
522. Kudoh A, Ishihara H, Matsuki A. Effect of carbamazepine on pain scores of unipolar depressed patients with chronic pain: A trial of off-on-off-on design. Clin J Pain. 1998

Mar;14(1):61-5. doi: 10.1097/00002508199803000-00009. PMID: 9535315.

Exclusion: 6

523. Kulkantrakorn K, Lorsuwansiri C, Meesawatsom P. 0.025\% capsaicin gel for the treatment of painful diabetic neuropathy: a randomized, double-blind, crossover, placebo-controlled trial. Pain pract. 2013 Jul;13(6):497-503. doi: 10.1111/papr.12013. PMID: 23228119. Exclusion: 5

524. Kumar U, Khan MA, Latha MS, et al. Choline magnesium trisalicylate vs naproxen in the symptomatic treatment of rheumatoid arthritis: A randomized clinical trial. Asian J Pharm Clin Res. 2012 JanMar;5(1):26-31. Exclusion: 3

525. Kvinesdal B, Molin J, Froland A, et al. Imipramine treatment of painful diabetic neuropathy. JAMA. 1984 Apr 6;251(13):1727-30. PMID: 6366276. Exclusion: 9

526. La Montagna G, Tirri G, Cacace E, et al. Quality of life assessment during six months of NSAID treatment [Gonarthrosis and Quality of Life (GOAL) Study]. Clin Exp Rheumatol. 1998 Jan-Feb;16(1):49-54. PMID: 9543561. Exclusion: 3

527. La Montagna G, Tirri G, Cacace E, et al. Quality of life assessment during six months of NSAID treatment. Clinical and experimental rheumatology. 1998;16(1):4954. Exclusion: 3

528. Lai KC, Chu KM, Hui WM, et al. Celecoxib compared with lansoprazole and naproxen to prevent gastrointestinal ulcer complications. Am J Med. 2005 Nov;118(11):1271-8. doi: 10.1016/j.amjmed.2005.04.031. PMID: 16271912. Exclusion: 3

529. Laine L, Bombardier C, Hawkey CJ, et al. Stratifying the risk of NSAID-related upper gastrointestinal clinical events: results of a double-blind outcomes study in patients with rheumatoid arthritis. Gastroenterology. 2002 Oct;123(4):1006-12. Exclusion: 3
530. Laine L, Connors LG, Reicin A, et al. Serious lower gastrointestinal clinical events with nonselective NSAID or coxib use. Gastroenterology. 2003 Feb;124(2):288-92. doi: 10.1053/gast.2003.50054. PMID: 12557133. Exclusion: 3

531. Laine L, Goldkind L, Curtis SP, et al. How common is diclofenac-associated liver injury? Analysis of 17,289 arthritis patients in a long-term prospective clinical trial. Am J Gastroenterol. 2009 Feb;104(2):356-62. doi: 10.1038/ajg.2008.149. PMID: 19174782. Exclusion: 3

532. Lambotte F. Therapeutic activity of 4allyloxy-3-chlorophenylacetic acid. Arzneimittelforschung. 1970 Apr;20(4):56971. PMID: 4911592. Exclusion: 3

533. Lance JW, Anthony M. Cyclobenzaprine in the treatment of chronic tension headache. Med J Aust. 1972 Dec 16;2(25):1409-11. PMID: 4568320. Exclusion: 9

534. Landmann G, Lustenberger C, Schleinzer $\mathrm{W}$, et al. Short lasting transient effects of a capsaicin 8\% patch on nociceptor activation in humans. Eur J Pain. 2016 10;20(9):144353. doi: 10.1002/ejp.867. PMID: 26988467. Exclusion: 4

535. Landy S, Hoagland R, Hoagland D, et al. Sumatriptan/naproxen sodium combination versus its components administered concomitantly for the acute treatment of migraine: A pragmatic, crossover, openlabel outcomes study. Ther Adv Neurol Disord. 2013;6(5):279-86. doi: 10.1177/1756285613499788. PMID: 23997813. Exclusion: 3

536. Lang E, Steger W. A comparative study of efficacy and tolerability of ketoprofen and piroxicam. Br J Clin Pract. 1981 JulAug;35(7-8):267. PMID: 7032566. Exclusion: 5

537. Larsen A. Piroxicam versus naproxen in rheumatoid arthritis: a double-blind, crossover study. Int J Clin Pharmacol Res. 1985;5(1):17-23. PMID: 3888863. Exclusion: 9 
538. Lee C, Straus WL, Balshaw R, et al. A comparison of the efficacy and safety of nonsteroidal antiinflammatory agents versus acetaminophen in the treatment of osteoarthritis: a meta-analysis. Arthritis Rheum. 2004 Oct 15;51(5):746-54. doi: 10.1002/art.20698. PMID: 15478167. Exclusion: 6

539. Lee KK, You JH, Ho JT, et al. Economic analysis of celecoxib versus diclofenac plus omeprazole for the treatment of arthritis in patients at risk of ulcer disease. Aliment Pharmacol Ther. 2003 Jul 15;18(2):217-22. PMID: 12869082. Exclusion: 3

540. Lee P, Davis P, Prat A. The efficacy of diflunisal in osteoarthritis of the knee: an extended study. J Rheumatol. 1986 Jun;13(3):666-7. PMID: 3525838. Exclusion: 6

541. Lee P, Kennedy AC, Anderson J, et al. Benefits of hospitalization in rheumatoid arthritis. Q J Med. 1974 Apr;43(170):20514. PMID: 4850737. Exclusion: 6

542. Lee P, Rose BS, Anderson JA, et al. Naproxen in the treatment of rheumatoid arthritis. N Z Med J. 1978 Jun 28;87(614):425-7. PMID: 355954. Exclusion: 5

543. Lee $\mathrm{P}$, Watson M, Webb J, et al. Therapeutic effectiveness of paracetamol in rheumatoid arthritis. Int J Clin Pharmacol Biopharm. 1975 Jan;11(1):68-75. PMID: 1171083. Exclusion: 9

544. Lee W, Weisman MH. Treating axial manifestations in ankylosing spondylitis in the presence of peripheral arthritis. Nat Clin Pract Rheumatol. 2006 Jun;2(6):298-9. doi: 10.1038/ncprheum0208. PMID: 16932706. Exclusion: 5

545. Lee YC, Massarotti E, Edwards RR, et al. Effect of Milnacipran on Pain in Patients with Rheumatoid Arthritis with Widespread Pain: A Randomized Blinded Crossover Trial. J Rheumatol. 2016 Jan;43(1):38-45. doi: 10.3899/jrheum.150550. PMID: 26628607. Exclusion: 9
546. Lee YH, Song GG. Comparative efficacy and tolerability of duloxetine, pregabalin, and milnacipran for the treatment of fibromyalgia: a Bayesian network metaanalysis of randomized controlled trials. Rheumatol Int. 2016 May;36(5):663-72. doi: 10.1007/s00296-016-3468-5. PMID: 27000046. Exclusion: 8

547. Lehtinen K, Kaarela K, Makisara P, et al. Tolerability and efficacy of a slow-release indomethacin tablet in ankylosing spondylitis. Br J Rheumatol. 1984 Feb;23(1):52-6. PMID: 6365231. Exclusion: 6

548. Leigh TJ, Bird HA, Hindmarch I, et al. The evaluation of a nonsteroidal antiinflammatory drug (tenoxicam) in the treatment of sleep disturbance in osteoarthritic patients. Clin Rheumatol. 1989 Dec;8(4):489-93. PMID: 2692948. Exclusion: 3

549. Leijon G, Boivie J. Central post-stroke pain-a controlled trial of amitriptyline and carbamazepine. Pain. 1989 Jan;36(1):27-36. PMID: 2465530. Exclusion: 10

550. Leslie RD. Mefenamic acid compared with ibuprofen in the treatment of rheumatoid arthritis. J Int Med Res. 1977;5(3):161-3. doi: 10.1177/030006057700500303. PMID: 330287. Exclusion: 6

551. Lesser H, Sharma U, LaMoreaux L, et al. Pregabalin relieves symptoms of painful diabetic neuropathy: a randomized controlled trial. Neurology. 2004 Dec 14;63(11):2104-10. doi: 10.1212/01.wnl.0000145767.36287.a1. PMID: 15596757. Exclusion: 9

552. Leuschen MP, Filipi M, Healey K. A randomized open label study of pain medications (naproxen, acetaminophen and ibuprofen) for controlling side effects during initiation of IFN beta-1a therapy and during its ongoing use for relapsing-remitting multiple sclerosis. Mult Scler. 2004 Dec;10(6):636-42. doi: 10.1191/1352458504ms1114oa. PMID: 15584488. Exclusion: 4 
553. Levendoglu F, Ogun CO, Ozerbil O, et al. Gabapentin is a first line drug for the treatment of neuropathic pain in spinal cord injury. Spine (Phila Pa 1976). 2004 Apr 1;29(7):743-51. PMID: 15087796.

Exclusion: 9

554. Levine L, Cloud M, Enas N. Nizatidine prevents peptic ulceration in high-risk patients taking nonsteroidal antiinflammatory drugs. $1993 \mathrm{Nov}$ 8;153(21):2449-54. PMID: 8215749. Exclusion: 3

555. Lewis T, Lain D, Baumgartner SW. Comparison of diflunisal and piroxicam in the management of patients with osteoarthritis. Clin Ther. 1986;9 Suppl C:1526. PMID: 3548983. Exclusion: 3

556. Liang TH, Hsu PN. Double-blind, randomised, comparative trial of etodolac SR versus diclofenac in the treatment of osteoarthritis of the knee. Curr Med Res Opin. 2003;19(4):336-41. doi: 10.1185/030079903125001866. PMID: 12841927. Exclusion: 10

557. Lightfoot R. Comparison of the efficacy and safety of etodolac and piroxicam in patients with rheumatoid arthritis. Etodolac Study 326 Rheumatoid Arthritis Investigators Group. J Rheumatol Suppl. 1997 Feb;47:106. PMID: 9035015. Exclusion: 3

558. Lindegaard KF, Ovrelid L, Sjaastad O. Naproxen in the prevention of migraine attacks. A double-blind placebo-controlled cross-over study. Headache. 1980

Mar;20(2):96-8. PMID: 6989789. Exclusion: 4

559. Linden B, Distel M, Bluhmki E. A doubleblind study to compare the efficacy and safety of meloxicam $15 \mathrm{mg}$ with piroxicam $20 \mathrm{mg}$ in patients with osteoarthritis of the hip. Br J Rheumatol. 1996 Apr;35(Suppl 1):35-8. PMID: 8630634. Exclusion: 9

560. Lipkovich IA, Choy EH, Van Wambeke P, et al. Typology of patients with fibromyalgia: cluster analysis of duloxetine study patients. BMC Musculoskelet Disord. 2014 Dec 23;15:450. doi: 10.1186/14712474-15-450. PMID: 25532971. Exclusion: 6
561. Lipton RB, Bigal ME, Kolodner KB, et al. Acetaminophen in the treatment of headaches associated with dipyridamoleaspirin combination. Neurology. 2004 Sep 28;63(6):1099-101. doi: 10.1212/01.wnl.0000138494.66691.07. PMID: 15452309. Exclusion: 4

562. Lisse J, Espinoza L, Zhao SZ, et al. Functional status and health-related quality of life of elderly osteoarthritic patients treated with celecoxib. J Gerontol A Biol Sci Med Sci. 2001 Mar;56(3):M167-75. doi: 10.1093/gerona/56.3.m167. PMID: 11253158. Exclusion: 6

563. Lisse JR. Clinical efficacy and safety of naprelan versus naprosyn in the treatment of rheumatoid arthritis. Am J Orthop (Belle Mead NJ). 1996 Sep;25(9 Suppl):21-9. PMID: 8886214. Exclusion: 2

564. Lisse JR, Perlman M, Johansson G, et al. Gastrointestinal tolerability and effectiveness of rofecoxib versus naproxen in the treatment of osteoarthritis: a randomized, controlled trial. Ann Intern Med. 2003 Oct 7;139(7):539-46. PMID: 14530224. Exclusion: 3

565. Liu Q, Chen H, Xi L, et al. A randomized, double-blind, placebo-controlled trial to evaluate the efficacy and safety of pregabalin for postherpetic neuralgia in a population of Chinese patients. Pain pract. 2017 Jan;17(1):62-9. doi:

10.1111/papr.12413. PMID: 26714731. Exclusion: 9

566. Liu WQ, Kanungo A, Toth C. Equivalency of tricyclic antidepressants in open-label neuropathic pain study. Acta Neurol Scand. 2014 Feb;129(2):132-41. doi:

10.1111/ane.12169. PMID: 23937282.

Exclusion: 6

567. Liyanage SP, Macauley DI, English JR. Naproxen and sulindac in the treatment of osteoarthritis of the hip and knee. Rheumatol Rehabil. 1981 Aug;20(3):184-7. PMID: 7025178. Exclusion: 9

568. Lomen PL, Lamborn KR, Porter GH, et al. Treatment of osteoarthritis of the knee. A comparison of flurbiprofen and aspirin. Am J Med. 1986 Mar 24;80(3A):97-102. PMID: 3963030. Exclusion: 3 
569. Lomen PL, Turner LF, Lamborn KR, et al. Flurbiprofen in the treatment of ankylosing spondylitis. A comparison with indomethacin. Am J Med. 1986 Mar 24;80(3A):127-32. PMID: 3963018. Exclusion: 3

570. Lomen PL, Turner LF, Lamborn KR, et al. Flurbiprofen in the treatment of ankylosing spondylitis. A comparison with phenylbutazone. Am J Med. 1986 Mar 24;80(3A):120-6. PMID: 3963017. Exclusion: 3

571. Lone AR, Wafai ZA, Buth BA, et al. Analgesic efficacy of transcutaneous electrical nerve stimulation compared with diclofenac sodium in osteo-arthritis of the knee. Physiotherapy. 2003 Aug;89(8):47885. doi: 10.1016/S0031-9406(05)60005-3. Exclusion: 3

572. Loose LD, Sipe JD, Kirby DS, et al. Reduction of acute-phase proteins with tenidap sodium, a cytokine-modulating antirheumatic drug. Br J Rheumatol. 1993 Jun;32 Suppl 3:19-25. PMID: 7685226. Exclusion: 3

573. Low PA, Opfer-Gehrking TL, Dyck PJ, et al. Double-blind, placebo-controlled study of the application of capsaicin cream in chronic distal painful polyneuropathy. Pain. 1995 Aug;62(2):163-8. PMID: 8545141. Exclusion: 9

574. Lucache B, Jaba I, Albu E, et al. Acupuncture and bee venom therapy vs. diclofenac in the chronic low back pain treatment. Therapeutics, Pharmacology and Clinical Toxicology. 2015 Sep;19(3):103-8. Exclusion: 3

575. Luciano JV, D'Amico F, Feliu-Soler A, et al. Cost-Utility of Group Acceptance and Commitment Therapy for Fibromyalgia Versus Recommended Drugs: An Economic Analysis Alongside a 6-Month Randomized Controlled Trial Conducted in Spain (EFFIGACT Study). J Pain. 2017 Jul;18(7):868-80. doi: 10.1016/j.jpain.2017.03.001. PMID: 28342891. Exclusion: 3
576. Lucker PW, Pawlowski C, Friedrich I, et al. Double-blind, randomised, multi-centre clinical study evaluating the efficacy and tolerability of nimesulide in comparison with etodalac in patients suffering from osteoarthritis of the knee. Eur J Rheumatol Inflamm. 1994;14(2):29-38. PMID: 7744131. Exclusion: 3

577. Luftschein S, Bienenstock H, Varady JC, et al. Increasing dose of naproxen in rheumatoid arthritis: use with and without corticosteroids. J Rheumatol. 1979 JulAug;6(4):397-404. PMID: 392094. Exclusion: 3

578. Lujan M, Lopez-Fiesco A, Lopez y Martinez $\mathrm{E}$, et al. Experimental tension headache in humans: a double blind comparison of the analgesic effect of dipyrone, naproxen plus paracetamol or placebo. Proc West Pharmacol Soc. 1992;35:201-5. PMID: 1502224. Exclusion: 3

579. Lund B, Distel M, Bluhmki E. A doubleblind, randomized, placebo-controlled study of efficacy and tolerance of meloxicam treatment in patients with osteoarthritis of the knee. Scand J Rheumatol. 1998;27(1):32-7. PMID: 9506875. Exclusion: 6

580. Lussier A. Long-term clinical assessment of naproxen on rheumatoid arthritis patients and 51-Cr gastrointestinal bleeding on volunteers. Arzneimittelforschung. 1975 Feb;25(2A):294-8. PMID: 807222. Exclusion: 9

581. Lussier A, Myhal D, Boost G, et al. Long term study of naproxen challenged by a short-term double blind cross-over study with placebo in rheumatoid patients. Scand J Rheumatol Suppl. 1973;2:113-20. PMID: 4590034. Exclusion: 6

582. Lussier A, Segre EJ, Multz CV, et al. Naproxen: a novel approach to dose-finding efficacy trails in rheumatoid arthritis. Clin Pharmacol Ther. 1973 May-Jun;14(3):43441. doi: 10.1002/cpt1973143434. PMID: 4572801. Exclusion: 9 
583. Luyten FP, Geusens P, Malaise M, et al. A prospective randomised multicentre study comparing continuous and intermittent treatment with celecoxib in patients with osteoarthritis of the knee or hip. Ann Rheum Dis. 2007 Jan;66(1):99-106. doi: 10.1136/ard.2006.052308. PMID: 16815864. Exclusion: 3

584. Lynch ME, Cesar-Rittenberg P, Hohmann AG. A double-blind, placebo-controlled, crossover pilot trial with extension using an oral mucosal cannabinoid extract for treatment of chemotherapy-induced neuropathic pain. J Pain Symptom Manage. 2014 Jan;47(1):166-73. doi: 10.1016/j.jpainsymman.2013.02.018. PMID: 23742737. Exclusion: 9

585. Machado GC, Maher CG, Ferreira PH, et al. Efficacy and safety of paracetamol for spinal pain and osteoarthritis: systematic review and meta-analysis of randomised placebo controlled trials. BMJ. 2015 Mar 31;350:h1225. doi: 10.1136/bmj.h1225. PMID: 25828856. Exclusion: 8

586. Mack A. Examination of the evidence for off-label use of gabapentin. J Manag Care Pharm. 2003 Nov-Dec;9(6):559-68. doi: 10.18553/jmcp.2003.9.6.559. PMID: 14664664. Exclusion: 6

587. MacNeill AL, Dick WC. Imipramine and rheumatoid factor. J Int Med Res. 1976;4(2 Suppl):23-7. PMID: 799987. Exclusion: 6

588. Maggioni F, Ruffatti S, Dainese F, et al. Weight variations in the prophylactic therapy of primary headaches: 6-month follow-up. J Headache Pain. 2005 Sep;6(4):322-4. doi: 10.1007/s10194-0050221-y. PMID: 2015-43744-047. Exclusion: 6

589. Maibach E. European experiences with tolmetin in the treatment of rheumatic diseases. Curr Ther Res Clin Exp. 1976 Mar;19(3):350-62. PMID: 817865. Exclusion: 5

590. Malinoski PT. Mediators and moderators of stress management and antidepressant treatment for chronic tension-type headache. Dissertation Abstracts International: Section B: The Sciences and Engineering. 2001 Sep;62(3-B):1587. Exclusion: 6
591. Mallen SR, Essex MN, Zhang R. Gastrointestinal tolerability of NSAIDs in elderly patients: a pooled analysis of 21 randomized clinical trials with celecoxib and nonselective NSAIDs. Curr Med Res Opin. 2011 Jul;27(7):1359-66. doi: 10.1185/03007995.2011.581274. PMID: 21561397. Exclusion: 8

592. Maneksha S. 'Safapryn' and benorylate--a comparative trial of two new preparations of aspirin and paracetamol in the treatment of rheumatoid arthritis and osteoarthritis. Curr Med Res Opin. 1973;1(9):563-8. doi: 10.1185/03007997309111722. PMID: 4591823. Exclusion: 3

593. Manitpisitkul P, Flores CM, Moyer JA, et al. A multiple-dose double-blind randomized study to evaluate the safety, pharmacokinetics, pharmacodynamics and analgesic efficacy of the TRPV1 antagonist JNJ-39439335 (mavatrep). Scand J Pain. 2018;18(2):151-64. doi: 10.1515/sjpain2017-0184. PMID: 29794306. Exclusion: 3

594. Manna V. Effects of piroxicam-betacyclodextrin in the treatment of spontaneous migraine attacks and reserpine-induced headache: Putative serotoninergic involvement in antinociceptive drug activity. Adv Ther. 1998;15(2):75-84. Exclusion: 3

595. Marble EL. Advances in headache therapy. Drug News Perspect. 1999 Jun;12(5):316-9. Exclusion: 5

596. Marchand DK, Loshak H. Nonsteriodal antiinflammatory drugs and acute kidney injury: safety. (CADTH rapid response report: summary of abstracts). Ottawa, Canada: Canadian Agency for Drugs and Technologies in Health; January 12, 2019. https://www.cadth.ca/sites/default/files/pdf/h tis/2019/RB1299\%20NSAIDs\%20and\%20A KI\%20Final.pdf. Exclusion: 8

597. Marchettini P, Wilhelm S, Petto H, et al. Are there different predictors of analgesic response between antidepressants and anticonvulsants in painful diabetic neuropathy? Eur J Pain. 2016 Mar;20(3):472-82. doi: 10.1002/ejp.763. PMID: 26311228. Exclusion: 3 
598. Marcolongo R, Giordano N, Bassi GP, et al. Double-blind preference and compliance multicentre study in osteoarthritis: once-aday treatment. Clin Rheumatol. 1985 Sep;4(3):267-77. PMID: 3905218. Exclusion: 6

599. Marcolongo R, Rubegni M, Provvedi D, et al. A double-blind, interpatient comparison of plain and slow-release ketoprofen in osteoarthritis. Int J Clin Pharmacol Ther Toxicol. 1984 Jul;22(7):377-81. PMID: 6381334. Exclusion: 3

600. Martin Arias LH, Martin Gonzalez A, Sanz Fadrique R, et al. Cardiovascular risk of nonsteroidal anti-inflammatory drugs and classical and selective cyclooxygenase- 2 Inhibitors: a meta-analysis of observational studies. J Clin Pharmacol. 2019 Jan;59(1):55-73. doi: 10.1002/jcph.1302. PMID: 30204233. Exclusion: 8

601. Martinez-Lavin M, Holman KI, Smyth CJ, et al. A comparison of naproxen, indomethacin and aspirin in osteoarthritis. J Rheumatol. 1980 Sep-Oct;7(5):711-6. PMID: 7441660. Exclusion: 6

602. Martio J, Uuspaa V. Ketoprofen and pethidine in the treatment of post-operative pain following synovectomy. A doubleblind trial on rheumatoid arthritis patients. Br J Clin Pract. 1981 Jul-Aug;35(7-8):265. PMID: 7032565. Exclusion: 3

603. Mathew NT. Indomethacin responsive headache syndromes. Headache. 1981 Jul;21(4):147-50. PMID: 7021474. Exclusion: 6

604. Mathew NT. Prophylaxis of migraine and mixed headache. A randomized controlled study. Headache. 1981 May;21(3):105-9. PMID: 7021472. Exclusion: 3

605. Mathew NT. Amelioration of ergotamine withdrawal symptoms with naproxen. Headache. 1987 Mar;27(3):130-3. PMID: 3298163. Exclusion: 4

606. Mathew NT, Rapoport A, Saper J, et al. Efficacy of gabapentin in migraine prophylaxis.[Expression of Concern in Headache. 2014 Apr;54(4):599-600; PMID: 24697222]. Headache. 2001 Feb;41(2):11928. PMID: 11251695. Exclusion: 4
607. Mathieson S, Billot L, Maher CG, et al. PRECISE--pregabalin in addition to usual care: statistical analysis plan. Trials. 2016 Jan 27;17:53. doi: 10.1186/s13063-0161174-y. PMID: 26818733. Exclusion: 9

608. Mathieson S, Maher CG, McLachlan AJ, et al. PRECISE - pregabalin in addition to usual care for sciatica: study protocol for a randomised controlled trial. Trials. $2013 \mathrm{Jul}$ 11;14:213. doi: 10.1186/1745-6215-14-213. PMID: 23845078. Exclusion: 9

609. Matsumoto A, Melian A, Shah A, et al. Etoricoxib versus naproxen in patients with rheumatoid arthritis: a prospective, randomized, comparator-controlled 121week trial. Curr Med Res Opin. 2007 Sep;23(9):2259-68. doi: 10.1185/030079907X219625. PMID: 17697449. Exclusion: 3

610. Matthey A, Cedraschi C, Piguet V, et al. Dual reuptake inhibitor milnacipran and spinal pain pathways in fibromyalgia patients: a randomized, double-blind, placebo-controlled trial. Pain Physician. 2013 Sep-Oct;16(5):E553-62. PMID: 24077206. Exclusion: 9

611. Matucci Cerinic M, McCarthy G, Lombardi A, et al. Neurogenic influences in arthritis: potential modification by capsaicin. $\mathrm{J}$ Rheumatol. 1995 Aug;22(8):1447-9. PMID: 7473463. Exclusion: 5

612. Mavrikakis ME, MacLeod M, Buchanan WW, et al. Mefenamic acid: an under-rated antirheumatic? Curr Med Res Opin. 1977;4(8):535-9. doi: 10.1185/03007997709115267. PMID: 326491. Exclusion: 6

613. Max MB, Culnane M, Schafer SC, et al. Amitriptyline relieves diabetic neuropathy pain in patients with normal or depressed mood. Neurology. 1987 Apr;37(4):589-96. doi: 10.1212/wnl.37.4.589. PMID: 2436092. Exclusion: 9

614. Max MB, Kishore-Kumar R, Schafer SC, et al. Efficacy of desipramine in painful diabetic neuropathy: a placebo-controlled trial. Pain. 1991 Apr;45(1):3-9; discussion 1-2. PMID: 1861872. Exclusion: 9 
615. Max MB, Lynch SA, Muir J, et al. Effects of desipramine, amitriptyline, and fluoxetine on pain in diabetic neuropathy. $\mathrm{N}$ Engl J Med. 1992 May 7;326(19):1250-6. doi: 10.1056/NEJM199205073261904. PMID: 1560801. Exclusion: 9

616. Max MB, Schafer SC, Culnane M, et al. Amitriptyline, but not lorazepam, relieves postherpetic neuralgia. Neurology. 1988 Sep;38(9):1427-32. doi: 10.1212/wnl.38.9.1427. PMID: 3412591. Exclusion: 9

617. Mayo-Wilson E, Li T, Fusco N, et al. Cherry-picking by trialists and meta-analysts can drive conclusions about intervention efficacy. J Clin Epidemiol. 2017 Nov;91:95110. doi: 10.1016/j.jclinepi.2017.07.014. PMID: 28842290. Exclusion: 8

618. Mbuyi-Muamba JM, Dequeker J. Piroxicam versus naproxen in rheumatoid arthritis. Curr Med Res Opin. 1983;8(9):612-7. doi: 10.1185/03007998309109806. PMID: 6607819. Exclusion: 3

619. McCabe CJ, Akehurst RL, Kirsch J, et al. Choice of NSAID and management strategy in rheumatoid arthritis and osteoarthritis. The impact on costs and outcomes in the UK. Pharmacoeconomics. 1998 Aug;14(2):191-9. doi: 10.2165/00019053199814020-00007. PMID: 10186459.

Exclusion: 6

620. McCleane G. $200 \mathrm{mg}$ daily of lamotrigine has no analgesic effect in neuropathic pain: a randomised, double-blind, placebo controlled trial. Pain. 1999 Oct;83(1):105-7. PMID: 10506679. Exclusion: 3

621. McGuinness BW, Lloyd-Jones M, Fowler PD. A double-blind comparative trial of 'parazolidin' and paracetamol. Br J Clin Pract. 1969 Nov;23(11):452-5. PMID: 4242567. Exclusion: 3

622. McGuire RJ, Wright V. A statistical approach to indices of disease activity in rheumatoid arthritis with reference to a trial of indomethacin. Proc R Soc Med. 1971 Jun;64(6):658. PMID: 4933090. Exclusion: 5

623. McKenna F. Diclofenac/misoprostol: the European clinical experience. J Rheumatol Suppl. 1998 May;51:21-30. PMID: 9596551. Exclusion: 5
624. McKenna F, Arguelles L, Burke T, et al. Upper gastrointestinal tolerability of celecoxib compared with diclofenac in the treatment of osteoarthritis and rheumatoid arthritis. Clin Exp Rheumatol. 2002 JanFeb;20(1):35-43. PMID: 11892706. Exclusion: 6

625. McKenna F, Weaver A, Fiechtner JJ, et al. COX-2 specific inhibitors in the management of osteoarthritis of the knee: a placebo-controlled, randomized, doubleblind study. J Clin Rheumatol. 2001 Jun;7(3):151-9. PMID: 17039120. Exclusion: 9

626. McLaughlin GE. A double-blind comparative study of piroxicam and ibuprofen in the treatment of rheumatoid arthritis. Semin Arthritis Rheum. 1985 Feb;14(3 Suppl 1):11-3. PMID: 3914701. Exclusion: 3

627. McQuay HJ, Carroll D, Jadad AR, et al. Dextromethorphan for the treatment of neuropathic pain: a double-blind randomised controlled crossover trial with integral n-of1 design. Pain. 1994 Oct;59(1):127-33. PMID: 7854793. Exclusion: 3

628. Mease P, Arnold L, Wang F, et al. The effect of duloxetine on cognition in patients with fibromyalgia. Arthritis and rheumatism [abstracts from the american college of rheumatology/association of rheumatology health professionals annual scientific meeting, ACR/ARHP. 2009;10:16-21. Exclusion: 5

629. Mease PJ, Clauw DJ, Trugman JM, et al. Efficacy of long-term milnacipran treatment in patients meeting different thresholds of clinically relevant pain relief: subgroup analysis of a randomized, double-blind, placebo-controlled withdrawal study. J Pain Res. 2014 Nov 21;7:679-87. doi: 10.2147/JPR.S70200. PMID: 25473309. Exclusion: 6

630. Mease PJ, Russell IJ, Kajdasz DK, et al. Long-term safety, tolerability, and efficacy of duloxetine in the treatment of fibromyalgia. Semin Arthritis Rheum. 2010 Jun;39(6):454-64. doi: 10.1016/j.semarthrit.2008.11.001. PMID: 19152958. Exclusion: 6 
631. Mease PJ, Spaeth M, Clauw DJ, et al. Estimation of minimum clinically important difference for pain in fibromyalgia. Arthritis Care Res (Hoboken). 2011 Jun;63(6):821-6. doi: 10.1002/acr.20449. PMID: 21312349.

Exclusion: 6

632. Mehlisch DR, Ardia A, Pallotta T. A controlled comparative study of ibuprofen arginate versus conventional ibuprofen in the treatment of postoperative dental pain. J Clin Pharmacol. 2002;42(8):904-11. PMID: CN-00409361. Exclusion: 4

633. Mehlisch DR, Weaver M, Fladung B. Ketoprofen, acetaminophen, and placebo in the treatment of tension headache. Headache. 1998 Sep;38(8):579-89. PMID: 11398300. Exclusion: 4

634. Mehta S, Dasarathy S, Tandon RK, et al. A prospective randomized study of the injurious effects of aspirin and naproxen on the gastroduodenal mucosa in patients with rheumatoid arthritis. Am J Gastroenterol. 1992 Aug;87(8):996-1000. PMID:

1642224. Exclusion: 9

635. Meisel AD, Germain BF. The efficacy of piroxicam in comparison with sulindac in the treatment of rheumatoid arthritis. Semin Arthritis Rheum. 1985 Feb;14(3 Suppl 1):710. PMID: 3914703. Exclusion: 3

636. Melo Gomes JA, Roth SH, Zeeh J, et al. Double-blind comparison of efficacy and gastroduodenal safety of diclofenac/misoprostol, piroxicam, and naproxen in the treatment of osteoarthritis. Ann Rheum Dis. 1993 Dec;52(12):881-5. doi: 10.1136/ard.52.12.881. PMID: 8311540. Exclusion: 10

637. Melton JW, 3rd, Lussier A, Ward JR, et al. Naproxen vs. aspirin in osteoarthritis of the hip and knee. J Rheumatol. 1978

Fall;5(3):338-46. PMID: 372525. Exclusion: 3

638. Mena HR, Caperton EM, Loebl DH, et al. High doses of ibuprofen in rheumatoid arthritis: a comparison with aspirin. J La State Med Soc. 1977 Dec;129(12):263-7. PMID: 340601. Exclusion: 3

639. Merren MD. Gabapentin for treatment of pain and tremor: a large case series. South Med J. 1998 Aug;91(8):739-44. PMID: 9715219. Exclusion: 6
640. Mett A, Tfelt-Hansen P. Acute migraine therapy: recent evidence from randomized comparative trials. Curr Opin Neurol. 2008 Jun;21(3):331-7. doi: 10.1097/WCO.0b013e3282fee843. PMID: 18451718. Exclusion: 6

641. Meyer W. Combined oral and rectal administration of ketoprofen. Rheumatol Rehabil. 1979;Suppl:116. PMID: 375368. Exclusion: 3

642. Meyers OL, Quantock OP, Joubert PG, et al. A multicentre trial of Voltaren in the treatment of rheumatoid Arthritis. S Afr Med J. 1974 Oct 5;48(48):2013-7. PMID: 4612752. Exclusion: 9

643. Micca JL, Ruff D, Ahl J, et al. Safety and efficacy of duloxetine treatment in older and younger patients with osteoarthritis knee pain: a post hoc, subgroup analysis of two randomized, placebo-controlled trials. BMC Musculoskelet Disord. 2013 Apr 17;14:137. doi: 10.1186/1471-2474-14-137. PMID: 23590727. Exclusion: 6

644. Miki K, Murakami M, Oka H, et al. Efficacy of mirtazapine for the treatment of fibromyalgia without concomitant depression: a randomized, double-blind, placebo-controlled phase IIa study in Japan. Pain. 2016 Sep;157(9):2089-96. doi: 10.1097/j.pain.0000000000000622. PMID: 27218868. Exclusion: 3

645. Mikulaschek WM, Ridolfo AS. Clinical experience with fenoprofen, a new antirheumatic agent. Curr Med Res Opin. 1974;2(9):556-62. doi: 10.1185/03007997409115257. PMID: 4615887. Exclusion: 5

646. Milgrom C, Finestone A, Lev B, et al. Overexertional lumbar and thoracic back pain among recruits: a prospective study of risk factors and treatment regimens. J Spinal Disord. 1993 Jun;6(3):187-93. PMID: 8347966. Exclusion: 3

647. Miller DS, Talbot CA, Simpson W, et al. A comparison of naproxen sodium, acetaminophen and placebo in the treatment of muscle contraction headache. Headache. 1987 Jul;27(7):392-6. PMID: 3308770. Exclusion: 4 
648. Mills SB, Bloch M, Bruckner FE. Doubleblind cross-over study of ketoprofen and ibuprofen in management of rheumatoid arthritis. Br Med J. 1973 Oct 13;4(5884):824. doi: 10.1136/bmj.4.5884.82. PMID: 4583184. Exclusion: 9

649. Min K, Oh Y, Lee SH, et al. SymptomBased Treatment of Neuropathic Pain in Spinal Cord-Injured Patients: A Randomized Crossover Clinical Trial. Am J Phys Med Rehabil. 2016 May;95(5):330-8. doi: 10.1097/PHM.0000000000000382. PMID: 26368836. Exclusion: 6

650. Mishra A, Singh Nar A, Bawa A, et al. Pregabalin in chronic post-thoracotomy pain. J Clin Diagn Res. 2013

Aug;7(8):1659-61. doi: 10.7860/JCDR/2013/5707.3239. PMID: 24086867. Exclusion: 4

651. Mishra S, Bhatnagar S, Goyal GN, et al. A comparative efficacy of amitriptyline, gabapentin, and pregabalin in neuropathic cancer pain: a prospective randomized double-blind placebo-controlled study. Am J Hosp Palliat Care. 2012 May;29(3):177-82. doi: 10.1177/1049909111412539. PMID: 21745832. Exclusion: 4

652. Mitchell RA, Woolf D, Murray-Leslie C, et al. Problems in assessing the possible disease-modifying effect of naproxen. $\mathrm{Br} \mathrm{J}$ Clin Pract. 1987 Jan;41(1):560-5. PMID: 3311106. Exclusion: 3

653. Mody S, Jolly M, Kwasny MJ, et al. Patient reported outcomes and analgesia use in osteoarthritis of the knee. Osteoarthritis Cartilage. 2008 Nov;16(11):1294-9. doi: 10.1016/j.joca.2008.03.023. PMID: 18456521. Exclusion: 6

654. Moen MD. Topical diclofenac solution. Drugs. 2009;69(18):2621-32. doi: 10.2165/11202850-000000000-00000. PMID: 19943711. Exclusion: 6

655. Mohs R, Mease P, Arnold LM, et al. The effect of duloxetine treatment on cognition in patients with fibromyalgia. Psychosom Med. 2012 Jul-Aug;74(6):628-34. doi: 10.1097/PSY.0b013e31825b9855. PMID: 22753629. Exclusion: 2
656. Molnar JP, Moxley TE. Ibuprofen (Motrin $\left.{ }^{\circledR}\right)$, a double blind comparison of two dosages, $2400 \mathrm{MC}$ and 3200 MG daily, for treating rheumatoid arthritis. Curr Ther Res Clin Exp. 1979;26(5):581-91. Exclusion: 2

657. Molony J, Pigott PV, Quill JO. A doubleblind trial of a new anti-inflammatory drug in the management of osteo-arthritis of the hip-joint. J Ir Med Assoc. 1971 Nov 18;64(424):605-10. PMID: 4940697. Exclusion: 3

658. Montrone F, Caruso I, Cazzola M. Salsalate in the treatment of rheumatoid arthritis: a double-blind clinical and gastroscopic trial versus piroxicam. I. Clinical trial. J Int Med Res. 1989 Jul-Aug;17(4):316-9. doi: 10.1177/030006058901700402. PMID: 2676647. Exclusion: 10

659. Moore ME. Amitriptyline or cyclobenzaprine provided short-term benefit for fibromyalgia. Ann Intern Med. 1994;121(1):10. Exclusion: 5

660. Moore N. Forty years of ibuprofen use. Int J Clin Pract. 2003 Apr(135):28-31. PMID: 12723744. Exclusion: 3

661. Moore N. Diclofenac potassium 12.5mg tablets for mild to moderate pain and fever: a review of its pharmacology, clinical efficacy and safety. Clin Drug Investig. 2007;27(3):163-95. doi: 10.2165/00044011200727030-00002. PMID: 17305413.

Exclusion: 6

662. Moore RA, Derry S, Aldington D, et al. Amitriptyline for neuropathic pain in adults. Cochrane Database Syst Rev. 2015 Jul 6(7):CD008242. doi: 10.1002/14651858.CD008242.pub3. PMID: 26146793. Exclusion: 8

663. Moore RA, Derry S, Aldington D, et al. Amitriptyline for fibromyalgia in adults. Cochrane Database Syst Rev.

2017(10):Cd011824. doi: 10.1002/14651858.CD011824. Exclusion: 8

664. Moore RA, Derry S, Makinson GT, et al. Tolerability and adverse events in clinical trials of celecoxib in osteoarthritis and rheumatoid arthritis: systematic review and meta-analysis of information from company clinical trial reports. Arthritis Res Ther. 2005;7(3):R644-65. doi: 10.1186/ar1704. PMID: 15899051. Exclusion: 6 
665. Morello CM, Leckband SG, Stoner CP, et al. Randomized double-blind study comparing the efficacy of gabapentin with amitriptyline on diabetic peripheral neuropathy pain. Arch Intern Med. 1999 Sep 13;159(16):1931-7. PMID: 10493324. Exclusion: 9

666. Morley KD, Bernstein RM, Hughes GR, et al. A comparative trial of a controlledrelease formulation of ketoprofen ('Oruvail') and a conventional capsule formulation of ketoprofen ('Orudis') in patients with osteoarthritis of the hip. Curr Med Res Opin. 1984;9(1):28-34. doi:

10.1185/03007998409109555. PMID: 6723349. Exclusion: 3

667. Morreale P, Manopulo R, Galati M, et al. Comparison of the antiinflammatory efficacy of chondroitin sulfate and diclofenac sodium in patients with knee osteoarthritis. J Rheumatol. 1996 Aug;23(8):1385-91. PMID: 8856618. Exclusion: 3

668. Moser U, Waldburger H, Schwarz HA, et al. A double-blind randomised multicentre study with tenoxicam, piroxicam and diclofenac sodium retard in the treatment of ambulant patients with osteoarthritis and extra-articular rheumatism. Scand J Rheumatol Suppl. 1989;80:71-80. PMID: 2688081. Exclusion: 3

669. Moskowitz RW, Sunshine A, Brugger A, et al. American pain society pain questionnaire and other pain measures in the assessment of osteoarthritis pain: a pooled analysis of three celecoxib pivotal studies. Am J Ther. 2003 Jan-Feb;10(1):12-20. PMID: 12522515. Exclusion: 6

670. Mowat AM, Mowat AG, Jones CW, et al. A comparison of once and twice daily naproxen in rheumatoid arthritis. Br J Clin Pract. 1984 Mar;38(3):95-8. PMID: 6370285. Exclusion: 3

671. Mucke M, Phillips T, Radbruch L, et al. Cannabis-based medicines for chronic neuropathic pain in adults. Cochrane Database Syst Rev. 2018 Mar 7;3:CD012182. doi: 10.1002/14651858.CD012182.pub2. PMID: 29513392. Exclusion: 8
672. Muehlbacher M, Nickel MK, Kettler C, et al. Topiramate in treatment of patients with chronic low back pain: a randomized, double-blind, placebo-controlled study. Clin J Pain. 2006 Jul-Aug;22(6):526-31. doi: 10.1097/.ajp.0000192516.58578.a4. PMID: 16788338. Exclusion: 3

673. Muller-Fassbender HH, Nagel E. Comparative study of piroxicam and indomethacin retard in osteoarthritis. Eur J Rheumatol Inflamm. 1983;6(1):106-12. PMID: 6861809. Exclusion: 9

674. Mullin GT. Sodium meclofenamate for rheumatoid arthritis: an early double-blind evaluation. Curr Ther Res Clin Exp. 1975 Dec;18(6):785-92. PMID: 813942.

Exclusion: 9

675. Muncie HL, Jr. Medical aspects of the multidisciplinary assessment and management of osteoarthritis. Clin Ther. 1986;9 Suppl B:4-13. PMID: 3829096. Exclusion: 5

676. Murray WJ. Evaluation of acetaminophensalicylamide combinations in treatment of headache. J Clin Pharmacol J New Drugs. 1967 May-Jun;7(3):150-5. PMID: 5231433. Exclusion: 3

677. Myles AB, Bacon PA, Williams KA. Mefenamic acid in rheumatoid arthritis. Ann Rheum Dis. 1967 Nov;26(6):494-8. doi: 10.1136/ard.26.6.494. PMID: 4294443. Exclusion: 9

678. Naccari C. FDA approves pregabalin for the treatment of fibromyalgia. CNS Spectr. 2007 Jul;12(7):510. doi: 10.1017/S1092852900021234. Exclusion: 5

679. Nahir AM, Scharf Y. A comparative study of diclofenac and sulindac in ankylosing spondylitis. Rheumatol Rehabil. 1980 Aug;19(3):193-8. PMID: 6997973. Exclusion: 9

680. Natelson BH, Vu D, Mao X, et al. Effect of Milnacipran Treatment on Ventricular Lactate in Fibromyalgia: A Randomized, Double-Blind, Placebo-Controlled Trial. J Pain. 2015 Nov;16(11):1211-9. doi: 10.1016/j.jpain.2015.08.004. PMID: 26335989. Exclusion: 9 
681. National Center for C, Alternative M. The NIH Glucosamine/Chondroitin Arthritis Intervention Trial (GAIT). J Pain Palliat Care Pharmacother. 2008;22(1):39-43. PMID: 19062354. Exclusion: 5

682. Nebe J, Heier M, Diener HC. Low-dose ibuprofen in self-medication of mild to moderate headache: a comparison with acetylsalicylic acid and placebo. Cephalalgia. 1995 Dec;15(6):531-5. doi: 10.1046/j.1468-2982.1995.1506531.x. PMID: 8706118. Exclusion: 4

683. Nelson KA, Park KM, Robinovitz E, et al. High-dose oral dextromethorphan versus placebo in painful diabetic neuropathy and postherpetic neuralgia. Neurology. 1997 May;48(5):1212-8. doi: 10.1212/wnl.48.5.1212. PMID: 9153445. Exclusion: 3

684. Nestvold K. Naproxen and naproxen sodium in acute migraine attacks. Cephalalgia. 1986;6 Suppl 4:81-4. doi: 10.1177/03331024860060S410. PMID: 3539361. Exclusion: 4

685. Neustadt DH. Double blind evaluation of the long-term effects of etodolac versus ibuprofen in patients with rheumatoid arthritis. J Rheumatol Suppl. 1997 Feb;47:17-22. PMID: 9035016. Exclusion: 2

686. Newton RE, Casten GP, Alms DR, et al. The side effect profile of buspirone in comparison to active controls and placebo. $\mathrm{J}$ Clin Psychiatry. 1982 Dec;43(12 Pt 2):1002. PMID: 6130065. Exclusion: 4

687. Nicol CF. A four year double-blind study of tegretol in facial pain. Headache. 1969 Apr;9(1):54-7. PMID: 4896266. Exclusion: 6

688. Niculescu L, Li C, Huang J, et al. Pooled analysis of GI tolerability of 21 randomized controlled trials of celecoxib and nonselective NSAIDs. Curr Med Res Opin. 2009 Mar;25(3):729-40. doi: 10.1185/03007990802714382. PMID: 19210159. Exclusion: 8

689. Nigam DK, Agarwal R, Pandey GS, et al. Efficacy and tolerability of 'NorvedanFentiazac' in rheumatoid arthritis: Double blind study versus ibuprofen. Clinician. 1987;51(13):443-8. Exclusion: 3
690. Norregaard J, Volkmann H, DanneskioldSamsoe B. A randomized controlled trial of citalopram in the treatment of fibromyalgia. Pain. 1995 Jun;61(3):445-9. PMID: 7478688. Exclusion: 3

691. Noruzzadeh R, Modabbernia A, Aghamollaii V, et al. Memantine for Prophylactic Treatment of Migraine Without Aura: A Randomized Double-Blind Placebo-Controlled Study. Headache. 2016 Jan;56(1):95-103. doi: 10.1111/head.12732. PMID: 26638119. Exclusion: 4

692. Nugent SM, Morasco BJ, O'Neil ME, et al. The effects of cannabis among adults with chronic pain and an overview of general harms: a systematic review. Ann Intern Med. 2017 Sep 5;167(5):319-31. doi: 10.7326/M17-0155. PMID: 28806817. Exclusion: 8

693. Nwidu LL, Eniojukan JF, Oparah AC. Patient-reported outcomes of therapy with two brands of ibuprofen. Pharmacy practice. 2008;6(7):142-7. Exclusion: 3

694. Nyfos L. Controlled clinical trial of 1-(2'methyl-2'-dimethyl amino-ethyl)-3-phenyl indole $\mathrm{HCl}$ (A28A) in rheumatoid arthritis compared with indomethacin (Confortid). Acta Rheumatol Scand. 1971;17(1-4):11524. doi: 10.3109/rhe1.1971.17.issue-1-4.17. Exclusion: 3

695. O'Connor TP, Anderson AM, Lennox B, et al. A novel sustained-release formulation of ibuprofen provides effective once-daily therapy in the treatment of rheumatoid arthritis and osteoarthritis. Br J Clin Pract. 1993 Jan-Feb;47(1):10-3. PMID: 8461240. Exclusion: 3

696. Odrcich M, Bailey JM, Cahill CM, et al. Chronobiological characteristics of painful diabetic neuropathy and postherpetic neuralgia: diurnal pain variation and effects of analgesic therapy. Pain. 2006 Jan;120(12):207-12. doi: 10.1016/j.pain.2005.10.007. PMID: 16359793. Exclusion: 3

697. Oguzhanoglu A, Sahiner T, Kurt T, et al. Use of amitriptyline and fluoxetine in prophylaxis of migraine and tension-type headaches. Cephalalgia. 1999 Jun;19(5):531-2. doi: 10.1046/j.14682982.1999.019005531.x. PMID: 10403070. Exclusion: 2 
698. Okasha A, Ghaleb HA, Sadek A. A double blind trial for the clinical management of psychogenic headache. Br J Psychiatry. 1973 Feb;122(567):181-3. doi: 10.1192/bjp.122.2.181. PMID: 4577014. Exclusion: 4

699. Okea R. Milnacipran (Savella) for fibromyalgia. Am Fam Physician. 2010 Oct 15;82(8):901-4. Exclusion: 5

700. Orbell G. Headaches and Migraine Associated with Eyestrain. Preliminary Report of a Trial of Chlordiazepoxide. Br J Ophthalmol. 1963 Apr;47:246-7. doi: 10.1136/bjo.47.4.246. PMID: 14188329. Exclusion: 4

701. Orozco-Alcala JJ, Barrera-Tenorio EF. Long-term treatment with tenoxicam in rheumatoid arthritis. Eur J Rheumatol Inflamm. 1987;9(2):118-21. PMID: 3329102. Exclusion: 3

702. Orozco-Alcala JJ, Barrera-Tenorio EF. A long-term, double-blind, comparative study of tenoxicam (Ro 12-0068) and piroxicam in gonarthrosis and coxarthrosis. Eur J Rheumatol Inflamm. 1987;9(2):109-13. PMID: 3329100. Exclusion: 3

703. Osborn TG, Parks RL, Moore TL, et al. Diflunisal versus naproxen in the management of rheumatoid arthritis. Clin Ther. 1989 Nov-Dec;11(6):736-43. PMID: 2692819. Exclusion: 3

704. Otto M, Bach FW, Jensen TS, et al. Valproic acid has no effect on pain in polyneuropathy: a randomized, controlled trial. Neurology. 2004 Jan 27;62(2):285-8. doi: 10.1212/wnl.62.2.285. PMID: 14745070. Exclusion: 3

705. Owen-Smith BD, Burry HC. Ibuprofen in the management of osteoarthrosis of the hip. Rheumatol Phys Med. 1972 May;11(6):2816. PMID: 4556920. Exclusion: 6

706. Oyemade GA, Onadeko BO. A controlled clinical study comparing sulindac with ibuprofen and aspirin in the treatment of musculo-skeletal diseases. J Int Med Res. 1979;7(6):556-9. doi: 10.1177/030006057900700615. PMID: 160348. Exclusion: 9
707. Ozyalcin SN, Talu GK, Kiziltan E, et al. The efficacy and safety of venlafaxine in the prophylaxis of migraine. Headache. 2005 Feb;45(2):144-52. doi: 10.1111/j.15264610.2005.05029.x. PMID: 15705120. Exclusion: 4

708. Pae CU, Masand P. Duloxetine: a new psychopharmacologic treatment option for fibromyalgia? Curr Psychiatry Rep. 2008 Jun;10(3):237-9. doi: 10.1007/s11920-0080039-9. PMID: 18652792. Exclusion: 5

709. Pae CU, Masand PS, Marks DM, et al. History of early abuse as a predictor of treatment response in patients with fibromyalgia: a post-hoc analysis of a 12week, randomized, double-blind, placebocontrolled trial of paroxetine controlled release. World J Biol Psychiatry. 2009;10(4 Pt 2):435-41. doi: 10.1080/15622970902789155. PMID: 19382010. Exclusion: 3

710. Pae C-U, Masand PS, Marks DM, et al. History of depressive and/or anxiety disorders as a predictor of treatment response: a post hoc analysis of a 12-week, randomized, double-blind, placebocontrolled trial of paroxetine controlled release in patients with fibromyalgia. Prog Neuropsychopharmacol Biol Psychiatry. 2009 Aug 31;33(6):996-1002. doi: 10.1016/j.pnpbp.2009.05.005. PMID: 19433129. Exclusion: 3

711. Paech MJ, Goy R, Chua S, et al. A randomized, placebo-controlled trial of preoperative oral pregabalin for postoperative pain relief after minor gynecological surgery. Anesth Analg. 2007 Nov;105(5):1449-53, table of contents. doi: 10.1213/01.ane.0000286227.13306.d7. PMID: 17959981. Exclusion: 4

712. Palmer M, Highton J, Palmer DG. A comparative study of fenbufen and diclofenac in osteoarthritis. Medicine and surgery. 1988;28(1):14-5. Exclusion: 3

713. Palmer RH. Correction: milnacipran for treatment of fibromyalgia. Ann Pharmacother. 2011 Jun;45(6):827-8; author reply. doi: 10.1345/aph.1P218a. PMID: 21815297. Exclusion: 5 
714. Panerai A, Monza G, Movilia P, et al. A randomized, within-patient, cross-over, placebo-controlled trial on the efficacy and tolerability of the tricyclic antidepressants chlorimipramine and nortriptyline in central pain. Acta Neurol Scand. 1990 Jul;82(1):348. doi: 10.1111/j.1600-0404.1990.tb01584.x. PMID: 2239134. Exclusion: 6

715. Panicheeva S, Ngsriwongse S, Mokkhavesa $\mathrm{C}$, et al. Original versus generic piroxicams, their cost-effective evaluation in rheumatoid arthritis (RA) patients. J Med Assoc Thai. 1992 Feb;75(2):104-9. PMID: 1645047. Exclusion: 9

716. Pappagallo M, Haldey EJ. Pharmacological management of postherpetic neuralgia. CNS Drugs. 2003;17(11):771-80. doi: 10.2165/00023210-200317110-00001. PMID: 12921490. Exclusion: 6

717. Parsons B, Argoff CE, Clair A, et al. Improvement in pain severity category in clinical trials of pregabalin. J Pain Res. 2016 Oct 7;9:779-85. doi: 10.2147/JPR.S102696. PMID: 27785092. Exclusion: 8

718. Parsons B, Fujii K, Nozawa K, et al. The efficacy of pregabalin for the treatment of neuropathic pain in Japanese subjects with moderate or severe baseline pain. J Pain Res. 2019 Mar 22;12:1061-8. doi: 10.2147/JPR.S181729. PMID: 30962707. Exclusion: 6

719. Patel BR. A comparative study of subsyde$\mathrm{CR}$ versus meloxicam in rheumatic disorders. J Indian Med Assoc. 2000 May;98(5):250-2. PMID: 11002624. Exclusion: 9

720. Pathy MS. Osteoarthritis and non-steroidal and anti-inflammatory drugs: a multi-centre comparative study. Curr Med Res Opin. 1982;7(Suppl 1):41-52. PMID: 7105795. Exclusion: 6

721. Patkar AA, Masand PS, Krulewicz S, et al. A randomized, controlled, trial of controlled release paroxetine in fibromyalgia. Am J Med. 2007 May;120(5):448-54. doi: 10.1016/j.amjmed.2006.06.006. PMID: 17466657. Exclusion: 3
722. Pauer L, Atkinson G, Murphy TK, et al. Long-term maintenance of response across multiple fibromyalgia symptom domains in a randomized withdrawal study of pregabalin. Clin J Pain. 2012 Sep;28(7):60914. doi: 10.1097/AJP.0b013e31823dd315. PMID: 22688598. Exclusion: 6

723. Paulus HE, Di Primeo D, Sharp JT, et al. Patient retention and hand-wrist radiograph progression of rheumatoid arthritis during a 3-year prospective study that prohibited disease modifying antirheumatic drugs. J Rheumatol. 2004 Mar;31(3):470-81. PMID: 14994390. Exclusion: 2

724. Pavelka K, Recker DP, Verburg KM. Valdecoxib is as effective as diclofenac in the management of rheumatoid arthritis with a lower incidence of gastroduodenal ulcers: results of a 26-week trial. Rheumatology (Oxford). 2003 Oct;42(10):1207-15. doi: 10.1093/rheumatology/keg359. PMID: 12810937. Exclusion: 3

725. Pavelka K, Sr., Trnavsky K. Pharmacotherapy of osteoarthritis with particular reference to night pain (double blind trial of pirprofen and piroxicam). $\mathrm{Z}$ Rheumatol. 1986 Mar-Apr;45(2):87-9. PMID: 3524067. Exclusion: 3

726. Paymaster NJ. Lorazepam (WY 4036) as a pre-operative medication. Anaesthesia. 1973;28(5):521-6. PMID: 4580513. Exclusion: 4

727. Pearce I, Frank GJ, Pearce JM. Ibuprofen compared with paracetamol in migraine. Practitioner. 1983 Mar;227(1377):465-7. PMID: 6351046. Exclusion: 4

728. Peatfield RC, Petty RG, Rose FC. Double blind comparison of mefenamic acid and acetaminophen (paracetamol) in migraine. Cephalalgia. 1983 Jun;3(2):129-34. doi: 10.1046/j.1468-2982.1983.0302129.x. PMID: 6347393. Exclusion: 4

729. Pelletier JP, Raynauld JP, Beaulieu AD, et al. Chondroitin sulfate efficacy versus celecoxib on knee osteoarthritis structural changes using magnetic resonance imaging: a 2-year multicentre exploratory study. Arthritis Res Ther. 2016 Nov 3;18(1):256. doi: 10.1186/s13075-016-1149-0. PMID: 27809891. Exclusion: 3 
730. Peniston JH, Gold MS, Alwine LK. An open-label, long-term safety and tolerability trial of diclofenac sodium $1 \%$ gel in patients with knee osteoarthritis. Phys Sportsmed. 2011 Sep;39(3):31-8. doi: 10.3810/psm.2011.09.1918. PMID: 22030938. Exclusion: 6

731. Peniston JH, Gold MS, Wieman MS, et al. Tolerability of diclofenac sodium $1 \%$ gel with concomitant medications known to interact with diclofenac. Ther Clin Risk Manag. 2013;9(1):153-9. doi: 10.2147/TCRM.S41931. PMID: 23620668. Exclusion: 2

732. Perez HE, Sanchez GF. Gabapentin therapy for diabetic neuropathic pain. Am J Med. 2000 Jun 01;108(8):689. PMID: 10896633. Exclusion: 6

733. Perlin E, Finke H, Castro O, et al. Enhancement of pain control with ketorolac tromethamine in patients with sickle cell vaso-occlusive crisis. Am J Hematol. 1994 May;46(1):43-7. PMID: 7514356.

Exclusion: 3

734. Perloff MD, Berlin RK, Gillette M, et al. Gabapentin in headache disorders: what is the evidence? Pain Med. 2016 Jan;17(1):162-71. doi: 10.1111/pme.12931. PMID: 26398728. Exclusion: 8

735. Perolo A, Calabi V. Report on a doubleblind, proctoscopic and clinical comparison of flurbiprofen suppositories and indomethacin suppositories, in osteoarthrosis. Panminerva Med. 1979 OctDec;21(4):181-9. PMID: 400181.

Exclusion: 3

736. Perpignano G, Bogliolo A, Puccetti L. Double-blind comparison of the efficacy and safety of etodolac SR $600 \mathrm{mg}$ u.i.d. and of tenoxicam $20 \mathrm{mg}$ u.i.d. in elderly patients with osteoarthritis of the hip and of the knee. Int J Clin Pharmacol Res. 1994;14(5-6):20316. PMID: 7672877. Exclusion: 9

737. Perras C. Sativex for the management of multiple sclerosis symptoms. Issues Emerg Health Technol. 2005 Sep(72):1-4. PMID: 16317825. Exclusion: 2
738. Persson LI, Ben-Menachem E, Bengtsson E, et al. Differences in side effects between a conventional carbamazepine preparation and a slow-release preparation of carbamazepine. Epilepsy Res. 1990 Jul;6(2):134-40. PMID: 2201541. Exclusion: 4

739. Peters BH, Fraim CJ, Masel BE. Comparison of $650 \mathrm{mg}$ aspirin and 1,000 mg acetaminophen with each other, and with placebo in moderately severe headache. Am J Med. 1983 Jun 14;74(6A):36-42. PMID: 6344625. Exclusion: 4

740. Petersen LJ, Lyngholm AM, Arendt-Nielsen L. A novel model of inflammatory pain in human skin involving topical application of sodium lauryl sulfate. Inflammation Research. 2010 Sep;59(9):775-81. doi: 10.1007/s00011-010-0189-1. PMID: 20358390. Exclusion: 4

741. Petersen SG, Beyer N, Hansen M, et al. Nonsteroidal anti-inflammatory drug or glucosamine reduced pain and improved muscle strength with resistance training in a randomized controlled trial of knee osteoarthritis patients. Arch Phys Med Rehabil. 2011 Aug;92(8):1185-93. doi: 10.1016/j.apmr.2011.03.009. PMID: 21807137. Exclusion: 6

742. Petersen SG, Saxne T, Heinegard D, et al. Glucosamine but not ibuprofen alters cartilage turnover in osteoarthritis patients in response to physical training. Osteoarthritis Cartilage. 2010 Jan;18(1):34-40. doi: 10.1016/j.joca.2009.07.004. PMID: 19679221. Exclusion: 2

743. Petrella RJ, DiSilvestro MD, Hildebrand C. Effects of hyaluronate sodium on pain and physical functioning in osteoarthritis of the knee: a randomized, double-blind, placebocontrolled clinical trial. Arch Intern Med. 2002 Feb 11;162(3):292-8. PMID: 11822921. Exclusion: 3

744. Petzke F, Jensen KB, Kosek E, et al. Using fMRI to evaluate the effects of milnacipran on central pain processing in patients with fibromyalgia. Scand J Pain. 2013;4(2):6574. doi: 10.1016/j.sjpain.2012.10.002. PMID: 29913906. Exclusion: 2 
745. Pfeifer MA, Ross DR, Schrage JP, et al. A highly successful and novel model for treatment of chronic painful diabetic peripheral neuropathy. Diabetes Care. 1993 Aug;16(8):1103-15. doi: 10.2337/diacare.16.8.1103. PMID: 8375240. Exclusion: 6

746. Pfizer. A Placebo-Controlled Trial of Pregabalin and Amitriptyline for Treatment of Painful Diabetic Peripheral Neuropathy. PhRMA Web Synopsis. 2007;Protocol 1008-040. Exclusion: 9

747. Pfizer. An 8 Week Multi-Center, Randomized, Double Blind, PlaceboControlled Study to Evaluate the Efficacy, Safety and Tolerability of Pregabalin (150mg-600mg/Day) Using A Flexible Dosing Schedule in the Treatment of Subjects with Symptoms of Neuropathic Pain. PhRMA Web Synopsis. 2008;Protocol A0081081 NCT00301223. Exclusion: 9

748. Pheasant H, Bursk A, Goldfarb J, et al. Amitriptyline and chronic low-back pain. A randomized double-blind crossover study. Spine (Phila Pa 1976). 1983 JulAug;8(5):552-7. PMID: 6228015. Exclusion: 9

749. Philip J, Joseph PP, Das KV. Anti-rheumatic efficacy of naproxen. J Assoc Physicians India. 1982 Sep;30(9):593-6. PMID: 6764215. Exclusion: 9

750. Pi ZB, Lin H, He GD, et al. Randomized and controlled prospective trials of Ultrasound-guided spinal nerve posterior ramus pulsed radiofrequency treatment for lower back post-herpetic neuralgia. Clin Ter. 2015;166(5):e301-5. doi: 10.7417/T.2015.1882. PMID: 26550813. Exclusion: 3

751. Pickering G, Macian N, Delage N, et al. Milnacipran poorly modulates pain in patients suffering from fibromyalgia: A randomized double-blind controlled study. Drug Des Devel Ther. 2018;12:2485-96. doi: 10.2147/DDDT.S162810. PMID: 30127596. Exclusion: 9

752. Pinals RS, Frank S. Relative efficacy of indomethacin and acetylsalicylic acid in rheumatoid arthritis. N Engl J Med. 1967 Mar 2;276(9):512-4. doi: 10.1056/NEJM196703022760906. PMID: 5335042. Exclusion: 3
753. Pincus T, Koch G, Lei H, et al. Patient Preference for Placebo, Acetaminophen (paracetamol) or Celecoxib Efficacy Studies (PACES): two randomised, double blind, placebo controlled, crossover clinical trials in patients with knee or hip osteoarthritis. Ann Rheum Dis. 2004 Aug;63(8):931-9. doi: 10.1136/ard.2003.020313. PMID: 15082468. Exclusion: 9

754. Pincus T, Koch G, Lei H, et al. Patient preference for Placebo, Acetaminophen (paracetamol) or Celecoxib Efficacy Studies (PACES): two randomised, double blind, placebo controlled, crossover clinical trials in patients with knee or hip osteoarthritis.[see comment]. Ann Rheum Dis. 2004 Aug;63(8):931-9. PMID: 15082468. Exclusion: 9

755. Porzio F, Schattenkirchner M. Double-blind comparison of etodolac sustained-release tablets and diclofenac sustained-release tablets in patients with rheumatoid arthritis: An interim report. Curr Ther Res Clin Exp. 1993 Feb;53(2):144-53. doi: 10.1016/S0011-393X\%2805\%2980239-5. Exclusion: 9

756. Pota V, Barbarisi M, Sansone P, et al. Combination therapy with transdermal buprenorphine and pregabalin for chronic low back pain. Pain manag. 2012;2(1):2331. doi: 10.2217/pmt.11.71. PMID: 24654615. Exclusion: 3

757. Pownall R, Pickvance NJ. Does treatment timing matter?--A double blind crossover study of ibuprofen $2400 \mathrm{mg}$ per day in different dosage schedules in treatment of chronic low back pain. Br J Clin Pract. 1985 Jul;39(7):267-75. PMID: 3161532. Exclusion: 3

758. Pownall R, Pickvance NJ. The optimum interval between flurbiprofen doses-scheduling for circadian rhythms in rheumatoid arthritis. Br J Clin Pract. 1987 Apr;41(4):689-93. PMID: 3689648. Exclusion: 6

759. Prabhu VV. A comparative clinical trial evaluating efficacy and safety of fixed dose combination of nimesulide (100 mg) and racemethionine (50 $\mathrm{mg}$ ) (namsafe) versus reference drug (nimesulide) and other NSAIDs in the treatment of osteoarthritis. $\mathrm{J}$ Indian Med Assoc. 2008 Jun;106(6):402-4. PMID: 18839654. Exclusion: 3 
760. Pritchett YL, McCarberg BH, Watkin JG, et al. Duloxetine for the management of diabetic peripheral neuropathic pain: response profile. Pain Med. 2007 JulAug;8(5):397-409. doi: 10.1111/j.15264637.2007.00305.x. PMID: 17661853. Exclusion: 6

761. Proft F, Muche B, Listing J, et al. Study protocol: COmparison of the effect of treatment with Nonsteroidal antiinflammatory drugs added to anti-tumour necrosis factor a therapy versus anti-tumour necrosis factor a therapy alone on progression of StrUctural damage in the spine over two years in patients with ankyLosing spondylitis (CONSUL) - an open-label randomized controlled multicenter trial. BMJ Open. 2017 Jun 10;7(6):e014591. doi: 10.1136/bmjopen2016-014591. PMID: 28601821. Exclusion: 3

762. Provenza JR, Shinjo SK, Silva JM, et al. Combined glucosamine and chondroitin sulfate, once or three times daily, provides clinically relevant analgesia in knee osteoarthritis. Clin Rheumatol. 2015 Aug;34(8):1455-62. doi: 10.1007/s10067014-2757-1. PMID: 25085275. Exclusion: 3

763. Puiu T, Kairys AE, Pauer L, et al. Association of alterations in gray matter volume with reduced evoked-pain connectivity following short-term administration of pregabalin in patients with fibromyalgia. Arthritis rheumatol. 2016 Jun;68(6):1511-21. doi: 10.1002/art.39600. PMID: 26816332. Exclusion: 6

764. Pujalte J, Valdez E, De La Paz R. Clinical comparison of the analgesic efficacy of suprofen and mefenamic acid in the treatment of musculoskeletal pain. Curr Ther Res Clin Exp. 1984;36(2):245-52.

Exclusion: 3

765. Quimby LG, Gratwick GM, Whitney CD, et al. A randomized trial of cyclobenzaprine for the treatment of fibromyalgia. J Rheumatol Suppl. 1989 Nov;19:140-3. PMID: 2481741. Exclusion: 9
766. Rad RE, Ghaffari F, Fotokian Z, et al. The effectiveness of ibuprofen and lorazepam combination therapy in treating the symptoms of acute Migraine: A randomized clinical trial. Electronic Physician [Electronic Resource]. 2017 Mar 25;9(3):3912-7. doi: 10.19082/3912. PMID: 28461864. Exclusion: 3

767. Ragan C, Batchelor WH, Baum J, et al. A three-month trial of indomethacin in rheumatoid arthritis, with special reference to analysis and inference. Clin Pharmacol Ther. 1967 Jan-Feb;8(1):11-37. doi: 10.1002/cpt196781part111. PMID: 5334081. Exclusion: 2

768. Raja SN, Haythornthwaite JA, Pappagallo $\mathrm{M}$, et al. Opioids versus antidepressants in postherpetic neuralgia: a randomized, placebo-controlled trial. Neurology. 2002 Oct 8;59(7):1015-21. doi: 10.1212/wnl.59.7.1015. PMID: 12370455. Exclusion: 9

769. Ramzy EA. Comparative Efficacy of Newer Antidepressants in Combination with Pregabalin for Fibromyalgia Syndrome: A Controlled, Randomized Study. Pain pract. 2017 Jan;17(1):32-40. doi: 10.1111/papr.12409. PMID: 26892006. Exclusion: 3

770. Rao RD, Flynn PJ, Sloan JA, et al. Efficacy of lamotrigine in the management of chemotherapy-induced peripheral neuropathy: a phase 3 randomized, doubleblind, placebo-controlled trial, N01C3. Cancer. 2008 Jun 15;112(12):2802-8. doi: 10.1002/cncr.23482. PMID: 18428211. Exclusion: 3

771. Rao RD, Michalak JC, Sloan JA, et al. Efficacy of gabapentin in the management of chemotherapy-induced peripheral neuropathy: a phase 3 randomized, doubleblind, placebo-controlled, crossover trial (N00C3). Cancer. 2007 Nov 1;110(9):21108. doi: 10.1002/cncr.23008. PMID: 17853395. Exclusion: 9

772. Rapoport AM. New acute treatments for headache. Neurol Sci. 2010 Jun;31 Suppl 1:S129-32. doi: 10.1007/s10072-010-03047. PMID: 20464603. Exclusion: 3 
773. Raskin J, Smith TR, Wong K, et al. Duloxetine versus routine care in the longterm management of diabetic peripheral neuropathic pain. J Palliat Med. 2006 Feb;9(1):29-40. doi: 10.1089/jpm.2006.9.29. PMID: 16430342. Exclusion: 3

774. Raskin J, Wang F, Pritchett YL, et al. Duloxetine for patients with diabetic peripheral neuropathic pain: a 6-month open-label safety study. Pain Med. 2006 Sep-Oct;7(5):373-85. doi: 10.1111/j.15264637.2006.00207.x. PMID: 17014595. Exclusion: 6

775. Raskin J, White R, jackson J, et al. Misoprostol dosage in the prevention of nonsteroidal anti-inflammatory druginduced gastric and duodenal ulcers: A comparison of three regimens. Ann Intern Med. 1995 Sep 1;123(5):344-50. PMID: 7625622. Exclusion: 3

776. Raskin J, White R, Jaszewski R, et al. Misoprostol and rantidine in the prevention of NSAID-induced ulcers: a prospective, double-blind, multicenter study. Am J Gastroenterol. 1996 Feb;91(2):223-7. PMID: 8607484. Exclusion: 3

777. Raskin P, Donofrio PD, Rosenthal NR, et al. Topiramate vs placebo in painful diabetic neuropathy: analgesic and metabolic effects. Neurology. 2004 Sep 14;63(5):865-73. doi: 10.1212/01.wnl.0000137341.89781.14. PMID: 15365138. Exclusion: 3

778. Raskin P, Huffman C, Toth C, et al. Pregabalin in patients with inadequately treated painful diabetic peripheral neuropathy: A randomized withdrawal trial. Clin J Pain. 2014 May;30(5):379-90. doi: 10.1097/AJP.0b013e31829ea1a1. PMID: 23887339. Exclusion: 4

779. Raskin P, Huffman C, Yurkewicz L, et al. Pregabalin in patients with painful diabetic peripheral neuropathy using an NSAID for other pain conditions: A double-blind crossover study. Clin J Pain. 2016 Mar;32(3):203-10. doi: 10.1097/AJP.0000000000000254. PMID: 25968451. Exclusion: 9

780. Rasmussen P, Riishede J. Facial pain treated with carbamazepin (Tegretol). Acta Neurol Scand. 1970;46(4):385-408. PMID: 4994082. Exclusion: 4
781. Rauck RL, Shaibani A, Biton V, et al. Lacosamide in painful diabetic peripheral neuropathy: a phase 2 double-blind placebocontrolled study. Clin J Pain. 2007

Feb;23(2):150-8. doi: 10.1097/01.ajp.0000210957.39621.b2. PMID: 17237664. Exclusion: 3

782. Reginster JY, Malmstrom K, Mehta A, et al. Evaluation of the efficacy and safety of etoricoxib compared with naproxen in two, 138-week randomised studies of patients with osteoarthritis. Ann Rheum Dis. 2007 Jul;66(7):945-51. doi: 10.1136/ard.2006.059162. PMID: 17142385. Exclusion: 6

783. Rejholec V, Vitulova V, Vachtenheim J. Preliminary observations from a double blind crossover study to evaluate the efficacy of flurbiprofen given at different times of day in the treatment of rheumatoid arthritis. Annu Rev Chronopharmacol. 1984;1:357-60. Exclusion: 6

784. Revill P, Bolós J, Serradell N, et al. Gabapentin enacarbil. Treatment of restless legs syndrome, treatment of postherpetic neuralgia, treatment of neuropathic pain. Drugs of the Future. 2006;31(9):771-7. doi: 10.1358/dof.2006.031.09.1034212. Exclusion: 3

785. Reynolds PM, Whorwell PJ. A single-blind crossover comparison of fenoprofen, ibuprofen and naproxen in rheumatoid arthritis. Curr Med Res Opin.

1974;2(8):461-4. doi: 10.1185/03007997409115243. PMID: 4614951. Exclusion: 6

786. Reynolds WJ, Moldofsky H, Saskin P, et al. The effects of cyclobenzaprine on sleep physiology and symptoms in patients with fibromyalgia. J Rheumatol. 1991 Mar;18(3):452-4. PMID: 1856814. Exclusion: 10

787. Rhymer AR, Hart CB, Daurio C. A doubleblind trial comparing indomethacin sustained release capsules (Indocid-R) with indomethacin capsules in patients with rheumatoid arthritis. Rheumatol Rehabil. 1982 May;21(2):101-6. PMID: 7043710. Exclusion: 9 
788. Rhymer AR, Sromovsky JA, Dicenta C, et al. 'Osmosin': a multi-centre evaluation of a technological advance in the treatment of osteoarthritis. Curr Med Res Opin. 1983;8 Suppl 2:62-71. doi: 10.1185/03007998309109825. PMID: 6342963. Exclusion: 3

789. Rice AS, Maton S, Postherpetic Neuralgia Study G. Gabapentin in postherpetic neuralgia: a randomised, double blind, placebo controlled study. Pain. 2001 Nov;94(2):215-24. PMID: 11690735. Exclusion: 9

790. Rice DM. Comparison of diflunisal and piroxicam in the management of patients with rheumatoid arthritis. Clin Ther. 1987;10(1):92-7. PMID: 3329968. Exclusion: 3

791. Richards AM, Burry HC, Treadwell BL, et al. Nabumetone, a new non-steroidal antiinflammatory drug: a comparison with naproxen. N Z Med J. 1983 Dec 14;96(745):1015-6. PMID: 6361628. Exclusion: 9

792. Richards JS, Bombardier CH, Wilson CS, et al. Efficacy of venlafaxine XR for the treatment of pain in patients with spinal cord injury and major depression: a randomized, controlled trial. Arch Phys Med Rehabil. 2015 Apr;96(4):680-9. doi: 10.1016/j.apmr.2014.11.024. PMID: 25527253. Exclusion: 4

793. Richter RW, Portenoy R, Sharma U, et al. Relief of painful diabetic peripheral neuropathy with pregabalin: a randomized, placebo-controlled trial. J Pain. 2005 Apr;6(4):253-60. doi: 10.1016/j.jpain.2004.12.007. PMID: 15820913. Exclusion: 9

794. Rintala DH, Fiess RN, Tan G, et al. Effect of dronabinol on central neuropathic pain after spinal cord injury: a pilot study. Am J Phys Med Rehabil. 2010 Oct;89(10):840-8. doi: 10.1097/PHM.0b013e3181f1c4ec. PMID: 20855984. Exclusion: 9
795. Rintala DH, Holmes SA, Courtade D, et al. Comparison of the effectiveness of amitriptyline and gabapentin on chronic neuropathic pain in persons with spinal cord injury. Arch Phys Med Rehabil. 2007 Dec;88(12):1547-60. doi: 10.1016/j.apmr.2007.07.038. PMID: 18047869. Exclusion: 9

796. Rintala DH, Holmes SA, Courtade D, et al. Comparison of the effectiveness of amitriptyline and gabapentin on chronic neuropathic pain in persons with spinal cord injury.[Erratum appears in Arch Phys Med Rehabil. 2008 Jun;89(6):1206]. Arch Phys Med Rehabil. 2007 Dec;88(12):1547-60. doi: 10.1016/j.apmr.2007.07.038. PMID: 18047869. Exclusion: 9

797. Rizzi B, Lodigiani F, Terni E, et al. A double-blind, proctoscopic and clinical comparison of flurbiprofen suppositories and ibuprofen suppositories in osteoarthrosis. Panminerva Med. 1979 OctDec;21(4):173-80. PMID: 400180. Exclusion: 4

798. Robinson H, Abruzzo JL, Miyara A, et al. Concomitant tolmetin and aspirin therapy for rheumatoid arthritis; 1975. Exclusion: 3

799. Robinson LR, Czerniecki JM, Ehde DM, et al. Trial of amitriptyline for relief of pain in amputees: results of a randomized controlled study. Arch Phys Med Rehabil. 2004 Jan;85(1):1-6. PMID: 14970960. Exclusion: 9

800. Robinson M, Griffin J, Bowers J. Effect of ranitidine on gastroduodenal mucosal damage induced by non-steroidal antiinflammatory drug therapy. Dig Dis Sci. 1989 Mar;34(3):424-8. doi: 10.1007/BF01536266. Exclusion: 3

801. Robinson M, Mills R, Euler A. Ranitidine prevents duodenal ulcers associated with non-steroidal anti-inflammatory drug therapy. Aliment Pharmacol Ther. 1991 Apr;5(2):143-50. PMID: 1832313. Exclusion: 3

802. Rocco M, Rada G. Are cannabinoids effective for fibromyalgia? Medwave. 2018 Feb 14;18(1):e7154. doi: 10.5867/medwave.2018.01.7153. PMID: 29474351. Exclusion: 8 
803. Rogind H, Bliddal H, Klokker D, et al.: a prospective, randomised, double-blind, controlled multicentre study. Clin Drug Invest. 1997;13(2):66-75. doi: 10.2165/00044011-199713020-00002. PMID: 18370453. Exclusion: 9

804. Rolan P. Potential drug interactions with the novel antimigraine compound zolmitriptan (Zomig, 311C90). Cephalalgia. 1997 Oct;17 Suppl 18:21-7. doi: 10.1177/0333102497017S1804. PMID: 9399014. Exclusion: 4

805. Romberg O. Tenoxicam (Ro 12-0068) in ankylosing spondylitis. Eur J Rheumatol Inflamm. 1987;9(2):122-5. PMID: 3329103. Exclusion: 6

806. Rompel H, Bauermeister PW. Aetiology of migraine and prevention with carbamazepine (Tegretol): results of a double-blind, cross-over study. S Afr Med J. 1970 Jan 24;44(4):75-80. PMID: 4905910. Exclusion: 9

807. Rooney PJ, Capell HA, Paterson S, et al. Continued use of non-steroidal antiinflammatory drugs: an index of clinical efficacy. Br J Clin Pharmacol. 1978 May;5(5):453-5. doi: 10.1111/j.13652125.1978.tb01654.x. PMID: 350250. Exclusion: 2

808. Rosenberg K. Melatonin: Safe And Effective for the Prevention of Migraine Headache. Am J Nurs. 2016 Sep;116(9):69. doi: 10.1097/01.NAJ.0000494704.01488.c1. PMID: 27560348. Exclusion: 3

809. Rosenstock J, Tuchman M, LaMoreaux L, et al. Pregabalin for the treatment of painful diabetic peripheral neuropathy: a doubleblind, placebo-controlled trial. Pain. 2004 Aug;110(3):628-38. doi: 10.1016/j.pain.2004.05.001. PMID: 15288403. Exclusion: 9

810. Rossi S, Mataluni G, Codeca C, et al. Effects of levetiracetam on chronic pain in multiple sclerosis: results of a pilot, randomized, placebo-controlled study. Eur J Neurol. 2009 Mar;16(3):360-6. doi: 10.1111/j.1468-1331.2008.02496.x. PMID: 19364364. Exclusion: 3
811. Roth S, Bennett R, Caldron P, et al. Reduced risk of NSAID gastropathy (GI mucosal toxicity) with nonacetylated salicylate (salsalate): an endoscopic study. Semin Arthritis Rheum. 1990 Feb;19(4 Suppl 2):11-9. PMID: 2181673. Exclusion: 3

812. Roth SH. A comparison of benoxaprofen and sulindac in patients with rheumatoid arthritis and osteoarthritis. Eur J Rheumatol Inflamm. 1982;5(2):258-69. PMID: 7044801. Exclusion: 3

813. Roth SH. New understandings of NSAID gastropathy. Scand J Rheumatol Suppl. 1989;78:24-9; discussion 30-2. PMID: 2660252. Exclusion: 2

814. Roth SH, Englund DW, Harris BK, et al. Tolmetin with acetaminophen in the treatment of rheumatoid arthritis; 1975. Exclusion: 3

815. Roth T, Bhadra-Brown P, Pitman VW, et al. Pregabalin improves fibromyalgia-related sleep disturbance. Clin J Pain. 2016 Apr;32(4):308-12. doi: 10.1097/AJP.0000000000000262. PMID: 26035523. Exclusion: 9

816. Rother M, Lavins BJ, Kneer W, et al. Efficacy and safety of epicutaneous ketoprofen in Transfersome (IDEA-033) versus oral celecoxib and placebo in osteoarthritis of the knee: multicentre randomised controlled trial. Ann Rheum Dis. 2007 Sep;66(9):1178-83. doi: 10.1136/ard.2006.065128. PMID: 17363401. Exclusion: 3

817. Rowbotham M, Harden N, Stacey B, et al. Gabapentin for the treatment of postherpetic neuralgia: a randomized controlled trial. JAMA. 1998 Dec 2;280(21):1837-42. PMID: 9846778. Exclusion: 9

818. Rowbotham MC, Goli V, Kunz NR, et al. Venlafaxine extended release in the treatment of painful diabetic neuropathy: a double-blind, placebo-controlled study.[erratum appears in Pain. 2005 Jan;113(1-2):248]. Pain. 2004 Aug;110(3):697-706. doi: 10.1016/j.pain.2004.05.010. PMID: 15288411. Exclusion: 9 
819. Royer GL, Jr., Moxley TE, Hearron MS, et al. A six-month double-blind trial of ibuprofen and indomethacin in osteoarthritis. Curr Ther Res Clin Exp. 1975 Mar;17(3):234-48. PMID: 805018. Exclusion: 2

820. Royer GL, Moxley TE, Hearron MS, et al. A long-term double-blind clinical trial of ibuprofen and indomethacin in rheumatoid arthritis. J Int Med Res. 1975;3(3):158-71. doi: 10.1177/030006057500300304. PMID: 162672. Exclusion: 2

821. Rubinstein J, Sidi A. Long-term study with tenoxicam (Ro 12-0068) in the treatment of gonarthrosis and coxarthrosis. Eur J Rheumatol Inflamm. 1987;9(2):126-8. PMID: 3329104. Exclusion: 3

822. Rudge SR, Lloyd-Jones JK, Hind ID. Interaction between flurbiprofen and indomethacin in rheumatoid arthritis. $\mathrm{Br} \mathrm{J}$ Clin Pharmacol. 1982 Mar;13(3):448-51. doi: 10.1111/j.1365-2125.1982.tb01401.x. PMID: 7037032. Exclusion: 6

823. Rull JA, Quibrera R, Gonzalez-Millan H, et al. Symptomatic treatment of peripheral diabetic neuropathy with carbamazepine (Tegretol): double blind crossover trial. Diabetologia. 1969 Aug;5(4):215-8. PMID: 4902717. Exclusion: 10

824. Russell AS, Labelle JL. A double-blind crossover study of ketoprofen enteric-coated and ketoprofen non-enteric-coated tablets in patients with ankylosing spondylitis, osteoarthritis and rheumatoid arthritis. Curr Ther Res Clin Exp. 1983;33(2):185-91. Exclusion: 9

825. Russell IJ, Crofford LJ, Leon T, et al. The effects of pregabalin on sleep disturbance symptoms among individuals with fibromyalgia syndrome. Sleep Med. 2009 Jun;10(6):604-10. doi: 10.1016/j.sleep.2009.01.009. PMID: 19410509. Exclusion: 6

826. Russell IJ, Fletcher EM, Michalek JE, et al. Treatment of primary fibrositis/fibromyalgia syndrome with ibuprofen and alprazolam. A double-blind, placebo-controlled study. Arthritis Rheum. 1991 May;34(5):552-60. PMID: 2025309. Exclusion: 9
827. Russell IJ, Perkins AT, Michalek JE, et al. Sodium oxybate relieves pain and improves function in fibromyalgia syndrome: a randomized, double-blind, placebocontrolled, multicenter clinical trial. Arthritis Rheum. 2009 Jan;60(1):299-309. doi: 10.1002/art.24142. PMID: 19116896. Exclusion: 3

828. Russell J, Kamin M, Bennett RM, et al. Efficacy of tramadol in treatment of pain in fibromyalgia. J Clin Rheumatol. 2000 Oct;6(5):250-7. PMID: 19078481. Exclusion: 3

829. Russell MD, Barrick TR, Howe FA, et al. Reduced anterior cingulate grey matter volume in painful hand osteoarthritis. Rheumatol Int. 2018 Aug;38(8):1429-35. doi: 10.1007/s00296-018-4085-2. PMID: 29936571. Exclusion: 2

830. Saag K, van der Heijde D, Fisher C, et al. Rofecoxib, a new cyclooxygenase 2 inhibitor, shows sustained efficacy, comparable with other nonsteroidal antiinflammatory drugs: a 6-week and a 1-year trial in patients with osteoarthritis. Osteoarthritis Studies Group. Arch Fam Med. 2000 Nov-Dec;9(10):1124-34. PMID: 11115219. Exclusion: 3

831. Sabatowski R, Galvez R, Cherry DA, et al. Pregabalin reduces pain and improves sleep and mood disturbances in patients with postherpetic neuralgia: results of a randomised, placebo-controlled clinical trial. Pain. 2004 May;109(1-2):26-35. doi: 10.1016/j.pain.2004.01.001. PMID: 15082123. Exclusion: 9

832. Sacks S. Diclophenac sodium in rheumatoid arthritis and osteo-arthritis. S Afr Med J. 1974 Feb 9;48(6):213-5. PMID: 4592319. Exclusion: 9

833. Sadosky A, Parsons B, Emir B, et al. Pain relief and functional improvement in patients with neuropathic pain associated with spinal cord injury: an exploratory analysis of pregabalin clinical trials. J Pain Res. 2016 Jun 15;9:405-16. doi: 10.2147/JPR.S97770. PMID: 27366103. Exclusion: 6 
834. Saggioro A, Alvisi V, Blasi A, et al. Misoprostol prevents NSAID-induced gastrodudenal lesions in patients with osteoarthritis and rheumatoid arthritis (published erratum appears in Ital J Gastroenterol 1991 Jun:23(5):273). Ital J Gastroenterol. 1991 Mar-Apr;23(3):119-23. PMID: 1742504. Exclusion: 3

835. Saldana MT, Perez C, Navarro A, et al. Pain alleviation and patient-reported health outcomes following switching to pregabalin in individuals with gabapentin-refractory neuropathic pain in routine medical practice. Clin Drug Investig. 2012 Jun 1;32(6):40112. doi: $10.2165 / 11599400-000000000-$ 00000. PMID: 22480279. Exclusion: 6

836. Salinas FA, Lugo LH, Garcia HI. Efficacy of early treatment with carbamazepine in prevention of neuropathic pain in patients with spinal cord injury. Am J Phys Med Rehabil. 2012 Dec;91(12):1020-7. doi: 10.1097/PHM.0b013e3182643c85. PMID: 22854901. Exclusion: 4

837. Sampalis JS, Brownell LA. A randomized, double blind, placebo and active comparator controlled pilot study of UP446, a novel dual pathway inhibitor anti-inflammatory agent of botanical origin. Nutr J. 2012 Apr 5;11:21. doi: 10.1186/1475-2891-11-21. PMID: 22480204. Exclusion: 2

838. Sances G, Martignoni E, Fioroni L, et al. Naproxen sodium in menstrual migraine prophylaxis: a double-blind placebo controlled study. Headache. 1990 Nov;30(11):705-9. PMID: 2074162. Exclusion: 4

839. Sandelin J, Harilainen A, Crone H, et al. Local NSAID gel (eltenac) in the treatment of osteoarthritis of the knee. A double blind study comparing eltenac with oral diclofenac and placebo gel. Scand J Rheumatol. 1997;26(4):287-92. PMID: 9310109. Exclusion: 10

840. Sanders D, Krause K, O'Muircheartaigh J, et al. Pharmacologic modulation of hand pain in osteoarthritis: a double-blind placebocontrolled functional magnetic resonance imaging study using naproxen. Arthritis rheumatol. 2015 Mar;67(3):741-51. doi: 10.1002/art.38987. PMID: 25533872. Exclusion: 6
841. Sandrini G, Cerbo R, Del Bene E, et al. Efficacy of dosing and re-dosing of two oral fixed combinations of indomethacin, prochlorperazine and caffeine compared with oral sumatriptan in the acute treatment of multiple migraine attacks: a double-blind, double-dummy, randomised, parallel group, multicentre study. Int J Clin Pract. 2007 Aug;61(8):1256-69. doi: 10.1111/j.17421241.2007.01458.x. PMID: 17627707. Exclusion: 3

842. Sands GH, Brown PB, Essex MN. The efficacy of continuous versus intermittent celecoxib treatment in osteoarthritis patients with body mass index $>30$ and $<30 \mathrm{~kg} / \mathrm{m} 2$. Open Rheumatol J. 2013 Jul 12;7:32-7. doi: 10.2174/1874312901307010032. PMID: 23919092. Exclusion: 6

843. Sang CN, Booher S, Gilron I, et al. Dextromethorphan and memantine in painful diabetic neuropathy and postherpetic neuralgia: efficacy and dose-response trials. Anesthesiology. 2002 May;96(5):1053-61. PMID: 11981142. Exclusion: 3

844. Sankar V, Oommen AE, Thomas A, et al. Efficacy, safety and cost effectiveness of amitriptyline and pregabalin in patients with diabetic peripheral neuropathy. Indian Journal of Pharmaceutical Sciences. 2017;79(4):646-50. doi: 10.4172/pharmaceutical-sciences.1000274. Exclusion: 6

845. Santandrea S, Montrone F, Sarzi-Puttini P, et al. A double-blind crossover study of two cyclobenzaprine regimens in primary fibromyalgia syndrome. J Int Med Res. 1993 Mar-Apr;21(2):74-80. doi: 10.1177/030006059302100202. PMID: 8243792. Exclusion: 3

846. Saper JR, Lake AE, Cantrell DT, et al. Chronic daily headache prophylaxis with tizanidine: a double-blind, placebocontrolled, multicenter outcome study. Headache. 2002 Jun;42(6):470-82. PMID: 12167135. Exclusion: 3

847. Saragiotto BT, Machado GC, Ferreira ML, et al. Paracetamol for low back pain. Cochrane Database Syst Rev. 2016 Jun 7(6):CD012230. doi: 10.1002/14651858.CD012230. PMID: 27271789. Exclusion: 8 
848. Sargent JD, Peters K, Goldstein J, et al. Naproxen sodium for muscle contraction headache treatment. Headache. 1988 Apr;28(3):180-2. PMID: 3290156. Exclusion: 4

849. Satoh J, Yagihashi S, Baba M, et al. Efficacy and safety evaluation of pregabalin treatment over 52weeks in patients with diabetic neuropathic pain extended after a double-blind placebo-controlled trial. J Diabetes Investig. 2011 Nov 30;2(6):457-63. doi: 10.1111/j.2040-1124.2011.00122.x. PMID: 24843530. Exclusion: 6

850. Saxe PA, Arnold LM, Palmer RH, et al. Short-term (2-week) effects of discontinuing milnacipran in patients with fibromyalgia. Curr Med Res Opin. 2012 May;28(5):81521. doi: 10.1185/03007995.2012.677418. PMID: 22429066. Exclusion: 6

851. Saxena RP, Saxena U. A comparative trial of ketoprofen and ibuprofen in patients with rheumatic disease. Curr Med Res Opin. 1978;5(6):484-8. doi: 10.1185/03007997809111919. PMID: 350500. Exclusion: 4

852. Schachtel BP, Thoden WR. Onset of action of ibuprofen in the treatment of musclecontraction headache. Headache. 1988 Aug;28(7):471-4. PMID: 3072316. Exclusion: 4

853. Scharf MB, Baumann M, Berkowitz DV. The effects of sodium oxybate on clinical symptoms and sleep patterns in patients with fibromyalgia. J Rheumatol. 2003 May;30(5):1070-4. PMID: 12734908. Exclusion: 3

854. Scharf Y, Nahir M, Schapira D, et al. A comparative study of naproxen with diclofenac sodium in osteoarthrosis of the knees. Rheumatol Rehabil. 1982 Aug;21(3):167-70. PMID: 7048497. Exclusion: 2

855. Schattenkirchner M. Double-blind comparison of etodolac and piroxicam in patients with rheumatoid arthritis. Curr Med Res Opin. 1991;12(8):497-506. doi: 10.1185/03007999109111660. PMID: 1837260. Exclusion: 3
856. Scheiman JM, Behler EM, Berardi RR, et al. Salicylsalicylic acid causes less gastroduodenal mucosal damage than enteric-coated aspirin. An endoscopic comparison. Dig Dis Sci. 1989 Feb;34(2):229-32. PMID: 2914543. Exclusion: 4

857. Scheiman JM, Yeomans ND, Talley NJ, et al. Prevention of ulcers by esomeprazole in at-risk patients using non-selective NSAIDs and COX-2 inhibitors. Am J Gastroenterol. 2006 Apr;101(4):701-10. doi: 10.1111/j.1572-0241.2006.00499.x. PMID: 16494585. Exclusion: 3

858. Schiff MH. A comparison of naprelan and naprosyn in the treatment of osteoarthritis of the knee. Am J Orthop (Belle Mead NJ). 1996 Sep;25(9 Suppl):14-20. PMID: 8886213. Exclusion: 2

859. Schmitt W, Walter K, Kurth HJ. Clinical trial on the efficacy and safety of different diclofenac formulations: multiple-unit formulations compared to enteric coated tablets in patients with activated osteoarthritis. Inflammopharmacology. 1999;7(4):363-75. doi: 10.1007/s10787-9990030-z. PMID: 17657439. Exclusion: 2

860. Schnitzer TJ, Ballard IM, Constantine G, et al. Double-blind, placebo-controlled comparison of the safety and efficacy of orally administered etodolac and nabumetone in patients with active osteoarthritis of the knee. Clin Ther. 1995 Jul-Aug;17(4):602-12. PMID: 8565024. Exclusion: 10

861. Schnitzer TJ, Ekman EF, Spierings EL, et al. Efficacy and safety of tanezumab monotherapy or combined with nonsteroidal anti-inflammatory drugs in the treatment of knee or hip osteoarthritis pain. Ann Rheum Dis. 2015 Jun;74(6):1202-11. doi: 10.1136/annrheumdis-2013-204905. PMID: 24625625. Exclusion: 3

862. Schorn D. Diflunisal in rheumatoid arthritis. A controlled trial. S Afr Med J. 1981 Dec 12;60(24):936-7. PMID: 7029742. Exclusion: 2

863. Schorn D. A comparative study of isoxicam and naproxen in rheumatoid arthritis. S Afr Med J. 1985 Mar 23;67(12):452-4. PMID: 3885424. Exclusion: 5 
864. Schorn D, Seymour MA. Indomethacin or sulindac at night in rheumatoid arthritis. S Afr Med J. 1981 Jun 13;59(25):913-4. PMID: 7015541. Exclusion: 9

865. Schreijenberg M, Luijsterburg PA, Van Trier YD, et al. Efficacy of paracetamol, diclofenac and advice for acute low back pain in general practice: design of a randomized controlled trial (PACE Plus). BMC Musculoskelet Disord. 2017 Feb 1;18(1):56. doi: 10.1186/s12891-017-14325. PMID: 28143496. Exclusion: 3

866. Scott WA. The relief of pain with an antidepressant in arthritis. Practitioner. 1969;202(212):802-7. PMID: 4894317. Exclusion: 9

867. Scudds RA, McCain GA, Rollman GB, et al. Improvements in pain responsiveness in patients with fibrositis after successful treatment with amitriptyline. J Rheumatol Suppl. 1989 Nov;19:98-103. PMID: 2481743. Exclusion: 10

868. Seidel EJ, Rother M, Regenspurger K, et al. A randomised trial comparing the efficacy and safety of topical ketoprofen in Transfersome() gel (IDEA-033) with oral ketoprofen and drug-free ultra-deformable SequessomeTM vesicles (TDT 064) for the treatment of muscle soreness following exercise. J Sports Sci. 2016;34(1):88-95. doi: 10.1080/02640414.2015.1035667. PMID: 25893979. Exclusion: 4

869. Seideman P, Samuelson P, Neander G. Naproxen and paracetamol compared with naproxen only in coxarthrosis. Increased effect of the combination in 18 patients. Acta Orthop Scand. 1993 Jun;64(3):285-8. PMID: 8322582. Exclusion: 9

870. Selvarajah D, Petrie J, White D, et al. Multicentre, double-blind, crossover trial to identify the Optimal Pathway for TreatIng neurOpathic paiN in Diabetes Mellitus (OPTION-DM): study protocol for a randomised controlled trial. Trials. 2018 Oct 22;19(1):578. doi: 10.1186/s13063-0182959-y. PMID: 30348206. Exclusion: 3

871. Sendra JM, Junyent TT, Pellicer MJ. Pregabalin-induced hepatotoxicity. Ann Pharmacother. 2011 Jun;45(6):e32. doi: 10.1345/aph.1Q032. PMID: 21652790. Exclusion: 8
872. Serni U. Rheumatic diseases--clinical experience with piroxicam-betacyclodextrin. Eur J Rheumatol Inflamm. 1993;12(4):47-54. PMID: 7805704. Exclusion: 3

873. Serpell M, Latymer M, Almas M, et al. Neuropathic pain responds better to increased doses of pregabalin: An in-depth analysis of flexible-dose clinical trials. J Pain Res. 2017 Jul 26;10:1769-76. doi: 10.2147/JPR.S129832. PMID: 28794656. Exclusion: 6

874. Serpell MG, Neuropathic pain study group. Gabapentin in neuropathic pain syndromes: a randomised, double-blind, placebocontrolled trial. Pain. 2002 Oct;99(3):55766. PMID: 12406532. Exclusion: 9

875. Sevelius H, Segre E, Bursick K. Comparative analgesic effects of naproxen sodium, aspirin, and placebo. J Clin Pharmacol. 1980 Jul;20(7):480-5. PMID: 7000856. Exclusion: 4

876. Shah R. Potentized, oral preparation of Capsaicin alkaloids and magnesium phosphoricum in treatment of chronic pain: A clinical trial. J Pain Manag. 2014 JanMar;7(1):63-73. Exclusion: 3

877. Shaibani A, Biton V, Rauck R, et al. Longterm oral lacosamide in painful diabetic neuropathy: a two-year open-label extension trial. Eur J Pain. 2009 May;13(5):458-63. doi: 10.1016/j.ejpain.2008.05.016. PMID: 18619874. Exclusion: 3

878. Shaibani A, Fares S, Selam JL, et al. Lacosamide in painful diabetic neuropathy: an 18-week double-blind placebo-controlled trial. J Pain. 2009 Aug;10(8):818-28. doi: 10.1016/j.jpain.2009.01.322. PMID: 19409861. Exclusion: 3

879. Shephard NW, Steele CE. Comparison of tolmetin sodium with indomethacin in osteoarthritis. Practitioner. 1981 Nov;225(1361):1696-7. PMID: 7038657. Exclusion: 9

880. Shi W, Wang YM, Li LS, et al. Safety and efficacy of oral nonsteroidal antiinflammatory drugs in patients with rheumatoid arthritis: a six-month randomised study. Clin Drug Investig. 2004;24(2):89-101. doi: 10.2165/00044011200424020-00004. PMID: 17516695.

Exclusion: 2 
881. Shichikawa K. A double blind evaluation of piroxicam in the treatment of rheumatoid arthritis - a standardized approach to new drug evaluation in Japan The management of rheumatic diseases. ASIA PAC. CONGR. SER. 1982;No. 10:34-45. Exclusion: 9

882. Shin S. Safety of celecoxib versus traditional nonsteroidal anti-inflammatory drugs in older patients with arthritis. J Pain Res. 2018 Dec 14;11:3211-9. doi: 10.2147/JPR.S186000. PMID: 30588073. Exclusion: 8

883. Shipley M, Berry H, Bloom B. A doubleblind cross-over trial of indomethacin, fenoprofen and placebo in ankylosing spondylitis, with comments on patient assessment. Rheumatol Rehabil. 1980 May;19(2):122-5. PMID: 6996071. Exclusion: 9

884. Shlay JC, Chaloner K, Max MB, et al. Acupuncture and amitriptyline for pain due to HIV-related peripheral neuropathy: a randomized controlled trial. Terry Beirn Community Programs for Clinical Research on AIDS. JAMA. 1998 Nov 11;280(18):1590-5. PMID: 9820261. Exclusion: 3

885. Shukla R, Nag D, Ahuja RC. Alprazolam in chronic tension type headache. J Assoc Physicians India. 1996 Sep;44(9):641-4. PMID: 9251377. Exclusion: 9

886. Sidell FB, Pless JE, Neitlich H, et al. Dimethylheptyl-delta 6a-10atetrahydrocannabinol: effects after parenteral administration to man. Proc Soc Exp Biol Med. 1973 Mar;142(3):867-73. PMID: 4571010. Exclusion: 4

887. Sieper J, Lenaerts J, Wollenhaupt J, et al. Maintenance of biologic-free remission with naproxen or no treatment in patients with early, active axial spondyloarthritis: results from a 6-month, randomised, open-label follow-up study, INFAST Part 2. Ann Rheum Dis. 2014 Jan;73(1):108-13. doi: 10.1136/annrheumdis-2013-203460. PMID: 23740231. Exclusion: 3
888. Sieper J, Listing J, Poddubnyy D, et al. Effect of continuous versus on-demand treatment of ankylosing spondylitis with diclofenac over 2 years on radiographic progression of the spine: results from a randomised multicentre trial (ENRADAS). Ann Rheum Dis. 2016 Aug;75(8):1438-43. doi: 10.1136/annrheumdis-2015-207897. PMID: 26242443. Exclusion: 6

889. Silberstein S, Goode-Sellers S, Twomey C, et al. Randomized, double-blind, placebocontrolled, phase II trial of gabapentin enacarbil for migraine prophylaxis. Cephalalgia. 2013 Jan;33(2):101-11. doi: 10.1177/0333102412466968. PMID: 23165696. Exclusion: 4

890. Silberstein S, Saper J, Berenson F, et al. Oxcarbazepine in migraine headache: a double-blind, randomized, placebocontrolled study. Neurology. 2008 Feb 12;70(7):548-55. doi: 10.1212/01.wnl.0000297551.27191.70. PMID: 18268247. Exclusion: 4

891. Silberstein SD, Armellino JJ, Hoffman HD, et al. Treatment of menstruation-associated migraine with the nonprescription combination of acetaminophen, aspirin, and caffeine: results from three randomized, placebo-controlled studies. Clin Ther. 1999 Mar;21(3):475-91. doi: 10.1016/S01492918(00)88303-4. PMID: 10321417. Exclusion: 4

892. Silver M, Blum D, Grainger J, et al. Doubleblind, placebo-controlled trial of lamotrigine in combination with other medications for neuropathic pain. J Pain Symptom Manage. 2007 Oct;34(4):446-54. doi: 10.1016/j.jpainsymman.2006.12.015. PMID: 17662571. Exclusion: 3

893. Silverstein F, Graham D, Senior J, et al. Misoprostol reduces gastrointestinal complications in patients with rheumatoid arthritis receiving nonsteroidal antiinflammatory drugs: A randomized, doubleblind, placebo-controlled trial. Ann Intern Med. 1995 Aug 15;123(4):241-9. PMID: 7611589. Exclusion: 3

894. Simpson DA. Gabapentin and venlafaxine for the treatment of painful diabetic neuropathy. J Clin Neuromuscul Dis. 2001 Dec;3(2):53-62. PMID: 19078655. Exclusion: 9 
895. Simpson DM. High-concentration capsaicin in HIV-associated neuropathy: Clinical evidence and cases. Eur J Pain Suppl. 2010 Aug;4(2):166-9. doi: 10.1016/S17543207(10)70528-9. Exclusion: 6

896. Simpson DM, Brown S, Tobias JK, et al. NGX-4010, a capsaicin 8\% dermal patch, for the treatment of painful HIV-associated distal sensory polyneuropathy: results of a 52-week open-label study. Clin J Pain. 2014 Feb;30(2):134-42. doi: 10.1097/AJP.0b013e318287a32f. PMID: 23446088. Exclusion: 6

897. Simpson DM, McArthur JC, Olney R, et al. Lamotrigine for HIV-associated painful sensory neuropathies: a placebo-controlled trial. Neurology. 2003 May 13;60(9):150814. doi: 10.1212/01.wnl.0000063304.88470.d9. PMID: 12743240. Exclusion: 3

898. Simpson DM, Olney R, McArthur JC, et al. A placebo-controlled trial of lamotrigine for painful HIV-associated neuropathy.

Neurology. 2000 Jun 13;54(11):2115-9. doi: 10.1212/wnl.54.11.2115. PMID: 10851374. Exclusion: 3

899. Sindrup SH, Bach FW, Madsen C, et al. Venlafaxine versus imipramine in painful polyneuropathy: a randomized, controlled trial. Neurology. 2003 Apr 22;60(8):1284-9. doi: 10.1212/01.wnl.0000058749.49264.bd. PMID: 12707430. Exclusion: 10

900. Sindrup SH, Holbech J, Demant D, et al. Impact of etiology and duration of pain on pharmacological treatment effects in painful polyneuropathy. Eur J Pain. 2017 Sep;21(8):1443-50. doi: 10.1002/ejp.1048. PMID: 28557178. Exclusion: 6

901. Sindrup SH, Jensen TS. Efficacy of pharmacological treatments of neuropathic pain: an update and effect related to mechanism of drug action. Pain. 1999 Dec;83(3):389-400. doi: 10.1016/S03043959\%2899\%2900154-2. PMID: 10568846. Exclusion: 6

902. Singer F, Bouda H, Broll H, et al. Efficacy and tolerability of tenoxicam versus diclofenac in rheumatoid arthritis. Results of a double-blind study. Drug Investigation. 1990;2(SUPPL. 3):54-5. Exclusion: 5
903. Singer F, Singer C, Oberleitner H. Phlogenzym versus diclofenac in the treatment of activated osteoarthritis of the knee. A double-blind prospective randomized study. International journal of immunotherapy. 2001;17(2):135-41. Exclusion: 6

904. Singh G, Fort JG, Goldstein JL, et al. Celecoxib versus naproxen and diclofenac in osteoarthritis patients: SUCCESS-I Study. Am J Med. 2006 Mar;119(3):255-66. doi: 10.1016/j.amjmed.2005.09.054. PMID: 16490472. Exclusion: 6

905. Singh G, Fort JG, Goldstein JL, et al. Celecoxib versus naproxen and diclofenac in osteoarthritis patients: SUCCESS-I Study.[Erratum appears in Am J Med. 2006 Sep;119(9):801]. Am J Med. 2006 Mar;119(3):255-66. PMID: 16490472. Exclusion: 3

906. Skljarevski V, Desaiah D, Liu-Seifert H, et al. Efficacy and safety of duloxetine in patients with chronic low back pain. Spine (Phila Pa 1976). 2010 Jun 1;35(13):E57885. doi: 10.1097/BRS.0b013e3181d3cef6. PMID: 20461028. Exclusion: 9

907. Smugar SS, Schnitzer TJ, Weaver AL, et al. Rofecoxib $12.5 \mathrm{mg}$, rofecoxib $25 \mathrm{mg}$, and celecoxib $200 \mathrm{mg}$ in the treatment of symptomatic osteoarthritis: results of two similarly designed studies. Curr Med Res Opin. 2006 Jul;22(7):1353-67. doi: 10.1185/030079906X104876. PMID: 16834834. Exclusion: 9

908. Snedecor SJ, Sudharshan L, Cappelleri JC, et al. Systematic review and meta-analysis of pharmacological therapies for painful diabetic peripheral neuropathy. Pain pract. 2014 Feb;14(2):167-84. doi: 10.1111/papr.12054. PMID: 23534696. Exclusion: 8

909. Solomon DH, Libby P, Yeomans ND, et al. The potential benefits of aspirin for primary cardiovascular prevention in rheumatoid arthritis: a secondary analysis of the PRECISION Trial. Rheumatology (Oxford). 2018 Aug 1;57(8):1364-9. doi: 10.1093/rheumatology/key105. PMID: 29688503. Exclusion: 3 
910. Solomon L, Abrams G. Orudis in the management of osteo-arthritis of the knee. A double-blind trial. S Afr Med J. 1974 Jul 27;48(36):1526-9. PMID: 4608409.

Exclusion: 6

911. Solomon L, Abrams G. Voltaren in the treatment of rheumatoid arthritis. S Afr Med J. 1974 May 11;48(22):949-52. PMID: 4599883. Exclusion: 9

912. Soni P, Shell B, Cawkwell G, et al. The hepatic safety and tolerability of the cyclooxygenase-2 selective NSAID celecoxib: pooled analysis of 41 randomized controlled trials. Curr Med Res Opin. 2009 Aug;25(8):1841-51. doi: 10.1185/03007990903018279. PMID: 19530981. Exclusion: 8

913. Stacey BR, Barrett JA, Whalen E, et al. Pregabalin for postherpetic neuralgia: placebo-controlled trial of fixed and flexible dosing regimens on allodynia and time to onset of pain relief. J Pain. 2008

Nov;9(11):1006-17. doi: 10.1016/j.jpain.2008.05.014. PMID: 18640074. Exclusion: 10

914. Stacey BR, Dworkin RH, Murphy K, et al. Pregabalin in the treatment of refractory neuropathic pain: results of a 15-month open-label trial. Pain Med. 2008 Nov;9(8):1202-8. doi: 10.1111/j.15264637.2008.00423.x. PMID: 18346060. Exclusion: 6

915. Steardo L, Leo A, Marano E. Efficacy of baclofen in trigeminal neuralgia and some other painful conditions. A clinical trial. Eur Neurol. 1984;23(1):51-5. doi: 10.1159/000115677. PMID: 6201366. Exclusion: 6

916. Steiner TJ, Lange R. Ketoprofen ( $25 \mathrm{mg}$ ) in the symptomatic treatment of episodic tension-type headache: double-blind placebo-controlled comparison with acetaminophen (1000 mg). Cephalalgia. 1998 Jan;18(1):38-43. doi: 10.1046/j.14682982.1998.1801038.x. PMID: 9601623. Exclusion: 4
917. Stengaard-Pedersen K, Ekesbo R, Karvonen $\mathrm{AL}$, et al. Celecoxib $200 \mathrm{mg}$ q.d. is efficacious in the management of osteoarthritis of the knee or hip regardless of the time of dosing. Rheumatology (Oxford). 2004 May;43(5):592-5. doi: 10.1093/rheumatology/keh121. PMID: 14762227. Exclusion: 3

918. Stensrud P, Sjaastad O. Clinical trial of a new anti-bradykinin, anti-inflammatory drug, ketoprofen (19.583 r.p.) in migraine prophylaxis. Headache. 1974 Jul;14(2):96100. PMID: 4604234. Exclusion: 3

919. Stewart I, Thomas A. Mefenamic acid compared with diclofenac sodium in elderly patients with osteoarthritis. Br J Clin Pract. 1988 Aug;42(8):316-20. PMID: 3061435. Exclusion: 9

920. Stewart M, Cibere J, Sayre EC, et al. Efficacy of commonly prescribed analgesics in the management of osteoarthritis: a systematic review and meta-analysis. Rheumatol Int. 2018 Nov;38(11):1985-97. doi: 10.1007/s00296-018-4132-z. PMID: 30120508. Exclusion: 8

921. Stockings E, Campbell G, Hall WD, et al. Cannabis and cannabinoids for the treatment of people with chronic noncancer pain conditions: a systematic review and metaanalysis of controlled and observational studies. Pain. 2018 Oct;159(10):1932-54. doi: 10.1097/j.pain.0000000000001293. PMID: 29847469. Exclusion: 8

922. Stockman A, Varigos GA, Muirden KD. Comparison of effectiveness of mefenamic acid and ibuprofen in treatment of rheumatoid arthritis. Med J Aust. 1976 Nov 27;2(22):819-21. PMID: 796645. Exclusion: 6

923. Sturrock RD, Hart FD. Double-blind crossover comparison of indomethacin, flurbiprofen, and placebo in ankylosing spondylitis. Ann Rheum Dis. 1974 Mar;33(2):129-31. doi: 10.1136/ard.33.2.129. PMID: 4595274. Exclusion: 9

924. Suarez-Otero R, Robles-San Roman M, Jaimes-Hernandez J, et al. Efficacy and safety of diclofenac-cholestyramine and celecoxib in osteoarthritis. Proc West Pharmacol Soc. 2002;45:26-8. PMID: 12434517. Exclusion: 3 
925. Sullivan MJ, Lynch ME, Clark A, et al. Catastrophizing and treatment outcome: Differential impact on response to placebo and active treatment outcome.

Contemporary Hypnosis. 2008 SepDec;25(3-4):129-40. doi: 10.1002/ch.365.

Exclusion: 2

926. Sweetman BJ, Baig A, Parsons DL. Mefenamic acid, chlormezanoneparacetamol, ethoheptazine-aspirinmeprobamate: a comparative study in acute low back pain. Br J Clin Pract. 1987 Feb;41(2):619-24. PMID: 2960369. Exclusion: 3

927. Swift G, Heneghan M, Williams G, et al. Effect of rantidine on gastroduodenal mucosal damage in patients on long-term non-steriodal anti-inflammatory drugs. Digestion. 1989;44(2):86-94. doi: 10.1159/000199896. PMID: 2693162. Exclusion: 3

928. Sydnes OA. Comparison of piroxicam with indomethacin in ankylosing spondylitis: a double-blind crossover trial. Br J Clin Pract. 1981 Jan;35(1):40-4. PMID: 7020732. Exclusion: 9

929. Sydnes OA. Comparison of piroxicam with naproxen in rheumatoid arthritis: a doubleblind, cross-over, multicentre study. Eur J Rheumatol Inflamm. 1981;4(3):318-22. PMID: 6242953. Exclusion: 6

930. Symonds T, Hughes B, Liao S, et al. Validation of the Chinese Western Ontario and McMaster Universities Osteoarthritis Index in patients from mainland China with osteoarthritis of the knee. Arthritis Care Res (Hoboken). 2015 Nov;67(11):1553-60. doi: 10.1002/acr.22631. PMID: 26018634.

Exclusion: 2

931. Szanto E. A double-blind comparison of naproxen and indomethacin in rheumatoid arthritis. Scand J Rheumatol. 1974;3(3):11820. PMID: 4610736. Exclusion: 9

932. Szekely B, Merryman S, Croft H, et al. Prophylactic effects of naproxen sodium on perimenstrual headache: A double-blind, placebo-controlled study. Cephalalgia. 1989 Oct 1;9(10_suppl):452-3. doi: 10.1177/0333102489009S10240. Exclusion: 4
933. Taborn J, Anderson S, Goldberg M, et al. Relief of morning stiffness: a comparative study of naproxen and ibuprofen. Curr Med Res Opin. 1985;9(6):359-65. doi: 10.1185/03007998509109604. PMID: 3886298. Exclusion: 6

934. Taha As, Hudson N, Hawkey CJ, et al. Famotidine for the prevention of gastric and duodenal ulcers caused by nonsteroidal antiinflammatory drugs. N Engl J Med. 1996 May 30;334(22):1435-9. doi: 10.1056/NEJM199605303342204. PMID: 8618582. Exclusion: 3

935. Tai Q, Kirshblum S, Chen B, et al. Gabapentin in the treatment of neuropathic pain after spinal cord injury: a prospective, randomized, double-blind, crossover trial. J Spinal Cord Med. 2002 Summer;25(2):1005. doi: 10.1080/10790268.2002.11753609. PMID: 12137213. Exclusion: 10

936. Tanaka S, Ito T, Mori E, et al. Double-blind study of Naproxen in osteoarthritis of the knee joint. J Rheumatol. 1976 Mar;3(1):2736. PMID: 775086. Exclusion: 6

937. Tannenbaum H, Esdaile J, Topp JR, et al. A double-blind, multicenter, controlled study on diclofenac (voltaren ${ }^{\circledR}$ ) and naproxen in patients with rheumatoid arthritis (R.A.). Curr Ther Res Clin Exp. 1984;35(3):357-62. Exclusion: 2

938. Tasleem RA, Buth BA, Koul PA, et al. Chronic low back pain - Comparative analysis of treatment response to drugs and different physical modalities. JK Practitioner. 2003;10(3):201-4. Exclusion: 3

939. Tasmuth T, Hartel B, Kalso E. Venlafaxine in neuropathic pain following treatment of breast cancer. Eur J Pain. 2002;6(1):17-24. doi: 10.1053/eujp.2001.0266. PMID: 11888224. Exclusion: 10

940. Tausch G. Time of onset and duration of activity of piroxicam in rheumatoid arthritis: a placebo controlled study. Eur J Rheumatol Inflamm. 1981;4(3):368-75. PMID: 6242959. Exclusion: 6

941. Telhag H, Bach-Andersen R, Persson B. A double-blind comparative evaluation of tolmetin versus naproxen in osteoarthritis. Curr Med Res Opin. 1981;7(6):392-400. PMID: 7016451. Exclusion: 3 
942. Tesfaye S, Wilhelm S, Lledo A, et al. Duloxetine and pregabalin: high-dose monotherapy or their combination? The "COMBO-DN study"--a multinational, randomized, double-blind, parallel-group study in patients with diabetic peripheral neuropathic pain. Pain. 2013

Dec;154(12):2616-25. doi: 10.1016/j.pain.2013.05.043. PMID: 23732189. Exclusion: 3

943. Tesfaye S, Wilhelm S, Lledo A, et al. Duloxetine and pregabalin: high-dose monotherapy or their combination? the "COMBO-DN study" - A multinational, randomized, double-blind, parallel-group study in patients with diabetic peripheral neuropathic pain. Pain. 2013

Dec;154(12):2616-25. doi: 10.1016/j.pain.2013.05.043. PMID: 23732189. Exclusion: 9

944. Teule M. Double-blind comparative multicenter study of the efficacy and safety of a slow-release tablet of 200-mg ketoprofen once daily and a 50-mg ketoprofen capsule four times daily. Curr Ther Res Clin Exp. 1986;40(6):1129-46. Exclusion: 6

945. Thapa AS. Amitriptyline versus tizanidine in the management of tension-type headache in Nepal. Emerg Med Australas. 2013 Dec;25(6):611-2. doi: 10.1111/17426723.12154. PMID: 24224929. Exclusion: 5

946. Thienel U, Neto W, Schwabe SK, et al. Topiramate in painful diabetic polyneuropathy: findings from three doubleblind placebo-controlled trials. Acta Neurol Scand. 2004 Oct;110(4):221-31. doi: 10.1111/j.1600-0404.2004.00338.x. PMID: 15355485. Exclusion: 3

947. Thomas J. Combined gabapentin and nortriptyline more efficacious for neuropathic pain. Australian Journal of Pharmacy. 2010;91(1076):59. Exclusion: 5

948. Thomas M, Eriksson SV, Lundeberg T. A comparative study of diazepam and acupuncture in patients with osteoarthritis pain: a placebo controlled study. Am J Chin Med. 1991;19(2):95-100. doi: 10.1142/S0192415X91000156. PMID: 1816730. Exclusion: 3
949. Thompson M, Daymond TJ, Essigman WK. Short-term efficacy and tolerance of tiaprofenic acid (surgam) in rheumatoid arthritis and osteoarthritis. Multicentre placebo-controlled trials. Arquivos de Reumatologia e Doencas Osteo-Articulares. 1982;4(Suppl.1):37-45. Exclusion: 3

950. Tilwe GH, Beria S, Turakhia NH, et al. Efficacy and tolerability of oral enzyme therapy as compared to diclofenac in active osteoarthrosis of knee joint: an open randomized controlled clinical trial. J Assoc Physicians India. 2001 Jun;49:617-21. PMID: 11584936. Exclusion: 3

951. Tindall EA, Sharp JT, Burr A, et al. A 12month, multicenter, prospective, open-label trial of radiographic analysis of disease progression in osteoarthritis of the knee or hip in patients receiving celecoxib. Clin Ther. 2002 Dec;24(12):2051-63. PMID: 12581544. Exclusion: 6

952. Tiso RL, Tong-Ngork S, Fredlund KL. Oral versus topical Ibuprofen for chronic knee pain: a prospective randomized pilot study. Pain Physician. 2010 Sep-Oct;13(5):457-67. PMID: 20859315. Exclusion: 3

953. Todorov AA, Kolchev CB, Todorov AB. Tiagabine and gabapentin for the management of chronic pain. Clin J Pain. 2005 Jul-Aug;21(4):358-61. PMID: 15951655. Exclusion: 4

954. Treadwell BL, Tweed JM. Ketoprofen (Orudis) in ankylosing spondylitis. N Z Med J. 1975 May 14;81(539):411-3. PMID: 1099489. Exclusion: 3

955. Treister R, Honigman L, Lawal OD, et al. A deeper look at pain variability and its relationship with the placebo response: results from a randomized, double-blind, placebo-controlled clinical trial of naproxen in osteoarthritis of the knee. Pain. 2019 Jul;160(7):1522-8. doi: 10.1097/j.pain.0000000000001538. PMID: 30817436. Exclusion: 2

956. Trelle S, Reichenbach S, Wandel S, et al. Cardiovascular safety of non-steroidal antiinflammatory drugs: network meta-analysis. BMJ. 2011 Jan 11;342:c7086. doi: 10.1136/bmj.c7086. PMID: 21224324. Exclusion: 8 
957. Trnavsky K, Fischer M, Vogtle-Junkert U, et al. Efficacy and safety of 5\% ibuprofen cream treatment in knee osteoarthritis. Results of a randomized, double-blind, placebo-controlled study. J Rheumatol. 2004 Mar;31(3):565-72. PMID: 14994406. Exclusion: 3

958. Trudeau J, Van Inwegen R, Eaton T, et al. Assessment of pain and activity using an electronic pain diary and actigraphy device in a randomized, placebo-controlled crossover trial of celecoxib in osteoarthritis of the knee. Pain pract. 2015 Mar;15(3):24755. doi: 10.1111/papr.12167. PMID: 24494935. Exclusion: 6

959. Turner JA, Jensen MP, Warms CA, et al. Blinding effectiveness and association of pretreatment expectations with pain improvement in a double-blind randomized controlled trial. Pain. 2002 Sep;99(1-2):919. doi: 10.1016/S03043959\%2802\%2900060-X. PMID: 12237187. Exclusion: 2

960. Turner RA, Whipple JP, Shackleford RW. Diflunisal 500-750 mg versus aspirin 2600$3900 \mathrm{mg}$ in the treatment of rheumatoid arthritis. Pharmacotherapy. 1984 MayJun;4(3):151-7. PMID: 6377249. Exclusion: 3

961. UCB Pharma SA. (Clinical Study Summary of SP874) A multi-center, randomized, double-blind, placebo-controlled trial to assess the efficacy and safety of $400 \mathrm{mg} /$ day lacosamide in subjects with painful distal diabetic neuropathy using two different titration schemes. ClinicalTrials.gov. 2008;NCT00350103. Exclusion: 3

962. Umbenhauer ER. Diflunisal in the treatment of the pain of osteoarthritis. Summary of clinical studies. Pharmacotherapy. 1983 Mar-Apr;3(2 Pt 2):55S-60S. PMID: 6344040. Exclusion: 5

963. Underwood M, Ashby D, Cross P, et al. Advice to use topical or oral ibuprofen for chronic knee pain in older people: randomised controlled trial and patient preference study. BMJ. 2008 Jan 19;336(7636):138-42. doi: 10.1136/bmj.39399.656331.25. PMID: 18056743. Exclusion: 3
964. Upasani SP, Mutalik GS, Nayak NJ, et al. Evaluation of ibuprofen in the treatment of rheumatoid arthritis assessed by sequential analysis. J Assoc Physicians India. 1973 Jul;21(7):575-8. PMID: 4283772.

Exclusion: 4

965. Vaishnava H, Dasgupta MK, Sachar MS. Treatment of articular and non-articular (rheumatic diseases by indomethacin suppositories). J Assoc Physicians India. 1971;19(2):157-65. PMID: 4934419.

Exclusion: 3

966. Valentini G, Tirri G, Longatti S. Relative efficacy and tolerance of tiaprofenic acid and ketoprofen in rheumatoid arthritis. Drugs. 1988;35(Suppl 1):87-9. doi: 10.2165/00003495-198800351-00020. PMID: 3359950. Exclusion: 3

967. Valentini M, Cannizzaro R, Poletti M, et al. Nonsteroidal antinflammatory drugs for cancer pain: comparison between misoprostol and ranitidine in prevention of upper gastrointestinal damage. J Clin Oncol. 1995 Oct;13(10):2637-42. doi: 10.1200/JCO.1995.13.10.2637. PMID: 7595718. Exclusion: 3

968. van den Driest JJ, Schiphof D, Luijsterburg PAJ, et al. Effectiveness and costeffectiveness of duloxetine added to usual care for patients with chronic pain due to hip or knee osteoarthritis: protocol of a pragmatic open-label cluster randomised trial (the DUO trial). BMJ Open. 2017 Sep 11;7(9):e018661. doi: 10.1136/bmjopen2017-018661. PMID: 28893757. Exclusion: 3

969. van der Heijde D, Baraf HSB, Ramos-

Remus C, et al. Evaluation of the efficacy of etoricoxib in ankylosing spondylitis: results of a fifty-two-week, randomized, controlled study. Arthritis Rheum. 2005 Apr;52(4):1205-15. doi: 10.1002/art.20985. PMID: 15818702. Exclusion: 9

970. Van Groenendael J, Markusse H, Dijkmans $B$, et al. The effect of ranitidine on NSAID related dyspeptic symptoms with and without peptic ulcer disease of patients with rheumatoid arthritis and osteoarthritis. Clin Rheumatol. 1996 Sep;15(5):450-6. PMID: 8894357. Exclusion: 3 
971. van Nooten F, Treur M, Pantiri K, et al. Capsaicin 8\% patch versus oral neuropathic pain medications for the treatment of painful diabetic peripheral neuropathy: A systematic literature review and network meta-analysis. Clin Ther. 2017 Apr;39(4):787-803.e18. doi: 10.1016/j.clinthera.2017.02.010. PMID: 28365034. Exclusion: 8

972. van Seventer R, Bach FW, Toth CC, et al. Pregabalin in the treatment of post-traumatic peripheral neuropathic pain: a randomized double-blind trial. Eur J Neurol. 2010 Aug;17(8):1082-9. doi: 10.1111/j.14681331.2010.02979.x. PMID: 20236172. Exclusion: 9

973. Vandenburg MJ, Currie WJ, Mann SG, et al. Differential effects of two non steroidal antiinflammatory drugs on the plasma urea of elderly patients with osteoarthritis. A multicentre study. Br J Clin Pract. 1984 Nov-Dec;38(11-12):403-6. PMID: 6397222. Exclusion: 2

974. Verdickt W, Moran C, Hantzschel H, et al. A double-blind comparison of the gastroduodenal safety and efficacy of diclofenac and a fixed dose combination of diclofenac and misoprostol in the treatment of rheumatoid arthritis. Scand J Rheumatol. 1992;21(2):85-91. PMID: 1570496.

Exclusion: 3

975. Verkleij SP, Luijsterburg PA, Koes BW, et al. Effectiveness of diclofenac versus acetaminophen in primary care patients with knee osteoarthritis: [NTR1485], DIPA-trial: design of a randomized clinical trial. BMC Musculoskelet Disord. 2010 Jan 12;11:7. doi: 10.1186/1471-2474-11-7. PMID: 20067607. Exclusion: 4

976. Verkleij SP, Luijsterburg PA, Willemsen SP, et al. Effectiveness of diclofenac versus paracetamol in knee osteoarthritis: a randomised controlled trial in primary care. Br J Gen Pract. 2015 Aug;65(637):e530-7. doi: 10.3399/bjgp15X686101. PMID: 26212849. Exclusion: 4

977. Verma V, Singh N, Singh Jaggi A. Pregabalin in Neuropathic Pain: Evidences and Possible Mechanisms. Curr Neuropharmacol. 2014 Jan;12(1):44-56. doi: 10.2174/1570159X1201140117162802.

PMID: 24533015. Exclusion: 5
978. Vernon H, Jansz G, Goldsmith CH, et al. A randomized, placebo-controlled clinical trial of chiropractic and medical prophylactic treatment of adults with tension-type headache: results from a stopped trial. J Manipulative Physiol Ther. 2009 Jun;32(5):344-51. doi: 10.1016/j.jmpt.2009.04.004. PMID: 19539116. Exclusion: 2

979. Vestergaard K, Andersen G, Gottrup H, et al. Lamotrigine for central poststroke pain: a randomized controlled trial. Neurology. 2001 Jan 23;56(2):184-90. doi: 10.1212/wnl.56.2.184. PMID: 11160953. Exclusion: 3

980. Veys EM, Verbruggen G, Suykens S, et al. Lymphocyte sub-population counts after a single $40 \mathrm{mg}$ administration of piroxicam in 20 patients with rheumatoid arthritis. A placebo-controlled study. Inflammation. 1984 Jun;8 Suppl:S115-22. PMID: 6237051. Exclusion: 3

981. Vickers ER, Cousins MJ, Walker S, et al. Analysis of 50 patients with atypical odontalgia. A preliminary report on pharmacological procedures for diagnosis and treatment. Oral Surg Oral Med Oral Pathol Oral Radiol Endod. 1998 Jan;85(1):24-32. PMID: 9474610. Exclusion: 4

982. Vijayalakshmi A, Chaitanya. Efficacy and safety of duloxetine in patients with neuropathic pain. International journal of pharmtech research. 2016;9(4):48-53. Exclusion: 9

983. Vilholm OJ, Cold S, Rasmussen L, et al. Effect of levetiracetam on the postmastectomy pain syndrome. Eur $\mathrm{J}$ Neurol. 2008 Aug;15(8):851-7. doi: 10.1111/j.1468-1331.2008.02206.x. PMID: 18565107. Exclusion: 10

984. Vince JD, Kremer D. Double-blind trial of diazepam in rheumatoid arthritis. Practitioner. 1973;210(256):264-7. PMID: 4570523. Exclusion: 6 
985. Vinik AI, Perrot S, Vinik EJ, et al. Capsaicin $8 \%$ patch repeat treatment plus standard of care (SOC) versus SOC alone in painful diabetic peripheral neuropathy: a randomised, 52-week, open-label, safety study. BMC Neurol. 2016 Dec 6;16(1):251. doi: 10.1186/s12883-016-0752-7. PMID: 27919222. Exclusion: 2

986. Vinik AI, Tuchman M, Safirstein B, et al. Lamotrigine for treatment of pain associated with diabetic neuropathy: results of two randomized, double-blind, placebocontrolled studies. Pain. 2007 Mar;128(12):169-79. doi: 10.1016/j.pain.2006.09.040. PMID: 17161535. Exclusion: 3

987. Vinje O, Fagertun HE, Laerum E, et al. Ketoprofen controlled release (CR) in the treatment of osteoarthrosis; a double blind, randomized multicentre study of single morning versus evening dose. Norwegian Study Group of General Practitioners. Scand J Prim Health Care. 1993 Jun;11(2):91-7. doi: 10.3109/02813439308994909. PMID: 8356371. Exclusion: 9

988. Vollmer TL, Robinson MJ, Risser RC, et al. A randomized, double-blind, placebocontrolled trial of duloxetine for the treatment of pain in patients with multiple sclerosis. Pain pract. 2014 Nov;14(8):73244. doi: 10.1111/papr.12127. PMID: 24152240. Exclusion: 9

989. von Delius S, Eckel F, Wagenpfeil S, et al. Carbamazepine for prevention of oxaliplatin-related neurotoxicity in patients with advanced colorectal cancer: final results of a randomised, controlled, multicenter phase II study. Invest New Drugs. 2007 Apr;25(2):173-80. doi: 10.1007/s10637-006-9010-y. PMID: 16983507. Exclusion: 9

990. von Heymann WJ, Schloemer P, Timm J, et al. Spinal high-velocity low amplitude manipulation in acute nonspecific low back pain: a double-blinded randomized controlled trial in comparison with diclofenac and placebo. Spine (Phila $\mathrm{Pa}$ 1976). 2013 Apr 01;38(7):540-8. doi: 10.1097/BRS.0b013e318275d09c. PMID: 23026869. Exclusion: 4
991. Vranken JH, Dijkgraaf MG, Kruis MR, et al. Pregabalin in patients with central neuropathic pain: a randomized, doubleblind, placebo-controlled trial of a flexibledose regimen. Pain. 2008 May;136(12):150-7. doi: 10.1016/j.pain.2007.06.033. PMID: 17703885. Exclusion: 10

992. Vreede PD, Harper FE, Sheldon WB. Use of oxaprozin in the treatment of aspirin failures in rheumatoid arthritis. Seminars in Arthritis and Rheumatism. 1986;15(3 Suppl 2):66-71. doi: 10.1016/S0049-0172(86)80010-5.

Exclusion: 3

993. Vrethem M, Boivie J, Arnqvist H, et al. A comparison of amitriptyline and maprotiline in the treatment of painful polyneuropathy in diabetics and nondiabetics. Clin J Pain. 1997 Dec;13(4):313-23. PMID: 9430812. Exclusion: 10

994. Waikakul S, Danputipong P, Soparat K. Topical analgesics, indomethacin plaster and diclofenac emulgel for low back pain: a parallel study. J Med Assoc Thai. 1996 Aug;79(8):486-90. PMID: 8855630. Exclusion: 3

995. Waikakul S, Penkitt iP, Soparat K, et al. Topical analgesics for knee arthrosis: a parallel study of ketoprofen gel and diclofenac emulgel. J Med Assoc Thai. 1997 Sep;80(9):593-7. PMID: 9347673. Exclusion: 3

996. Waldfogel JM, Nesbit SA, Dy SM, et al. Pharmacotherapy for diabetic peripheral neuropathy pain and quality of life: A systematic review. Neurology. 2017 May 16;88(20):1958-67. doi: 10.1212/WNL.0000000000003882. PMID: 28341643. Exclusion: 8

997. Walitt B, Klose P, Fitzcharles MA, et al. Cannabinoids for fibromyalgia. Cochrane Database Syst Rev. 2016 Jul 18;7:CD011694. doi: 10.1002/14651858.CD011694.pub2. PMID: 27428009. Exclusion: 8

998. Walitt B, Klose P, Uceyler N, et al. Antipsychotics for fibromyalgia in adults. Cochrane Database Syst Rev. 2016 Jun 2(6):CD011804. doi: 10.1002/14651858.CD011804.pub2. PMID: 27251337. Exclusion: 8 
999. Walker JS, Carmody JJ. Experimental pain in healthy human subjects: gender differences in nociception and in response to ibuprofen. Anesth Analg. 1998

Jun;86(6):1257-62. PMID: 9620515.

Exclusion: 4

1000. Walker JS, Sheather-Reid RB, Carmody JJ, et al. Nonsteroidal antiinflammatory drugs in rheumatoid arthritis and osteoarthritis: support for the concept of "responders" and "nonresponders". Arthritis Rheum. 1997 Nov;40(11):1944-54. doi: 10.1002/15290131(199711)40:11<1944::AIDART5>3.0.CO;2-H. PMID: 9365082. Exclusion: 9

1001. Walker Z, Walker RW, Robertson MM, et al. Antidepressant treatment of chronic tension-type headache: a comparison between fluoxetine and desipramine. Headache. 1998 Jul-Aug;38(7):523-8. doi: 10.1046/j.1526-4610.1998.3807523.x. PMID: 15613168. Exclusion: 3

1002. Wanders A, Heijde D, Landewe R, et al. Nonsteroidal antiinflammatory drugs reduce radiographic progression in patients with ankylosing spondylitis: a randomized clinical trial. Arthritis Rheum. 2005 Jun;52(6):1756-65. doi: 10.1002/art.21054. PMID: 15934081. Exclusion: 6

1003. Wang F, Ruberg SJ, Gaynor PJ, et al. Early improvement in pain predicts pain response at endpoint in patients with fibromyalgia. $\mathrm{J}$ Pain. 2011 Oct;12(10):1088-94. doi: 10.1016/j.jpain.2011.05.002. PMID: 21763211. Exclusion: 6

1004. Ward DE, Veys EM, Bowdler JM, et al. Comparison of aceclofenac with diclofenac in the treatment of osteoarthritis. Clin Rheumatol. 1995 Nov;14(6):656-62. PMID: 8608684. Exclusion: 3

1005. Ward NG. Tricyclic antidepressants for chronic low-back pain. Mechanisms of action and predictors of response. Spine (Phila Pa 1976). 1986 Sep;11(7):661-5. PMID: 2947334. Exclusion: 6

1006. Waterworth RF, Waterworth SM, Taylor KM. A comparison of tenoxicam and piroxicam in a long-term clinical study in patients with osteoarthritis of hip or knee joints. Eur J Rheumatol Inflamm. 1985;8(1):21-7. PMID: 3915884. Exclusion: 6
1007. Watson CP. Topical capsaicin as an adjuvant analgesic. J Pain Symptom Manage. 1994 Oct;9(7):425-33. PMID: 7822881. Exclusion: 6

1008. Watson CP, Vernich L, Chipman M, et al. Nortriptyline versus amitriptyline in postherpetic neuralgia: a randomized trial. Neurology. 1998 Oct;51(4):1166-71. doi: 10.1212/wnl.51.4.1166. PMID: 9781549. Exclusion: 9

1009. Weinberger A, Fischer DG, Rubinstein M. A crossover study comparing diflunisal to diclofenac sodium in rheumatoid arthritis. A correlation with serum interferon-like activity. Rhumatologie - Revue International de Rhumatologie. 1985;15(4 NO. 79):215-9. Exclusion: 9

1010. Weinbroum AA, Zur E. Patient-tailored combinations of systemic and topical preparations for localized peripheral neuropathic pain: A two-case report. J Pain Palliat Care Pharmacother. 2015 Mar;29(1):27-33. doi: 10.3109/15360288.2014.997852. PMID: 25594152. Exclusion: 6

1011. Weintraub M, Jacox RF, Angevine CD, et al. Piroxicam (CP 16171) in rheumatoid arthritis: a controlled clinical trial with novel assessment techniques. J Rheumatol. 1977;4(4):393-404. PMID: 342691. Exclusion: 3

1012. Welch KM. Naproxen sodium in the treatment of migraine. Cephalalgia. 1986;6 Suppl 4:85-92. doi: 10.1177/03331024860060S411. PMID: 3539362. Exclusion: 4

1013. Welsch P, Bernardy K, Derry S, et al. Mirtazapine for fibromyalgia in adults. Cochrane Database Syst Rev. 2018 Aug 6;8:CD012708. doi: 10.1002/14651858.CD012708.pub2. PMID: 30080242. Exclusion: 8

1014. Wernicke JF, Prakash A, Kajdasz DK, et al. Safety and tolerability of duloxetine treatment of diabetic peripheral neuropathic pain between patients with and without cardiovascular conditions. J Diabetes Complications. 2009 Sep-Oct;23(5):349-59. doi: 10.1016/j.jdiacomp.2008.07.004. PMID: 18768332. Exclusion: 6 
1015. Wernicke JF, Wang F, Pritchett YL, et al. An open-label 52-week clinical extension comparing duloxetine with routine care in patients with diabetic peripheral neuropathic pain. Pain Med. 2007 Sep;8(6):503-13. doi: 10.1111/j.1526-4637.2006.00258.x. PMID: 17716324. Exclusion: 3

1016. Wetzel L, Zadrazil M, Paternostro-Sluga T, et al. Intravenous nonopioid analgesic drugs in chronic low back pain patients on chronic opioid treatment: a crossover, randomised, double-blinded, placebo-controlled study.[Retraction in Eur J Anaesthesiol. 2015 Apr;32(4):287; PMID: 25747315]. Eur J Anaesthesiol. 2014 Jan;31(1):35-40. doi: 10.1097/EJA.0b013e328365ae28. PMID: 24141646. Exclusion: 3

1017. Wheatley D. Comparative trial of a new mono-amine oxidase inhibitor in depression. Br J Psychiatry. 1970 Nov;117(540):573-4. PMID: 4921178. Exclusion: 4

1018. Whelton A, Fort JG, Puma JA, et al. Cyclooxygenase-2-specific inhibitors and cardiorenal function: A randomized, controlled trial of celecoxib and rofecoxib in older hypertensive osteoarthritis patients. Am J Manag Care. 2002;8(15 SUPPL.):S371-S82. Exclusion: 3

1019. Whelton A, Fort JG, Puma JA, et al. Cyclooxygenase-2--specific inhibitors and cardiorenal function: a randomized, controlled trial of celecoxib and rofecoxib in older hypertensive osteoarthritis patients.[erratum appears in Am J Ther 2001 May-Jun;8(3):220]. Am J Ther. 2001 MarApr;8(2):85-95. PMID: 11304662.

Exclusion: 3

1020. Whelton A, White WB, Bello AE, et al. Effects of celecoxib and rofecoxib on blood pressure and edema in patients $>$ or $=65$ years of age with systemic hypertension and osteoarthritis. Am J Cardiol. 2002 Nov 1;90(9):959-63. PMID: 12398962. Exclusion: 3

1021. White AG, Martin VM. A comparison of alclofenac and indomethacin in the relief of rheumatoid morning stiffness. Curr Med Res Opin. 1975;3(5):329-32. doi: 10.1185/03007997509114783. PMID: 241602. Exclusion: 3
1022. White WB, Schnitzer TJ, Bakris GL, et al. Effects of naproxcinod on blood pressure in patients with osteoarthritis. Am J Cardiol. 2011 May 1;107(9):1338-45. doi: 10.1016/j.amjcard.2010.12.046. PMID: 21371681. Exclusion: 3

1023. White WB, Strand V, Roberts R, et al. Effects of the cyclooxygenase-2 specific inhibitor valdecoxib versus nonsteroidal antiinflammatory agents and placebo on cardiovascular thrombotic events in patients with arthritis. Am J Ther. 2004 JulAug;11(4):244-50. PMID: 15266215. Exclusion: 6

1024. Wibbenmeyer L, Eid A, Liao J, et al. Gabapentin is ineffective as an analgesic adjunct in the immediate postburn period. J Burn Care Res. 2014 Mar-Apr;35(2):136-42. doi: 10.1097/BCR.0b013e31828a4828. PMID: 23511293. Exclusion: 4

1025. Wiech K, Kiefer RT, Topfner S, et al. A placebo-controlled randomized crossover trial of the N-methyl-D-aspartic acid receptor antagonist, memantine, in patients with chronic phantom limb pain. Anesth Analg. 2004 Feb;98(2):408-13, table of contents. PMID: 14742379. Exclusion: 9

1026. Wiffen PJ, Derry S, Bell RF, et al. Gabapentin for chronic neuropathic pain in adults. Cochrane Database Syst Rev. 2017 Jun 9;6:CD007938. doi: 10.1002/14651858.CD007938.pub4. PMID: 28597471. Exclusion: 8

1027. Wijnands M, van Riel P, van 't Hof M, et al. Longterm treatment with nonsteroidal antiinflammatory drugs in rheumatoid arthritis: a prospective drug survival study. J Rheumatol. 1991 Feb;18(2):184-7. PMID: 2023212. Exclusion: 6

1028. Willans MJ, Wehner SE, Bourgouin J, et al. Double-blind study of ketoprofen capsules and enteric-coated tablets in the maintenance treatment of patients with rheumatoid arthritis. Curr Ther Res Clin Exp. 1985;38(6):870-9. Exclusion: 3

1029. Williams GW, Kivitz AJ, Brown MT, et al. A comparison of valdecoxib and naproxen in the treatment of rheumatoid arthritis symptoms. Clin Ther. 2006 Feb;28(2):20421. doi: 10.1016/j.clinthera.2006.01.016. PMID: 16678642. Exclusion: 2 
1030. Willkens RF. Double-blind crossover trial of naproxen and placebo in patients with rheumatoid arthritis. Scand J Rheumatol Suppl. 1973;2:132-6. PMID: 4590037. Exclusion: 9

1031. Willkens RF. Worldwide clinical safety experience with diclofenac. Semin Arthritis Rheum. 1985 Nov;15(2 Suppl 1):105-10. PMID: 3909410. Exclusion: 6

1032. Wilsey B, Marcotte T, Deutsch R, et al. Low-dose vaporized cannabis significantly improves neuropathic pain. J Pain. 2013 Feb;14(2):136-48. doi: 10.1016/j.jpain.2012.10.009. PMID: 23237736. Exclusion: 3

1033. Wilsey B, Marcotte TD, Deutsch R, et al. An Exploratory Human Laboratory Experiment Evaluating Vaporized Cannabis in the Treatment of Neuropathic Pain From Spinal Cord Injury and Disease. J Pain. 2016 09;17(9):982-1000. doi:

10.1016/j.jpain.2016.05.010. PMID: 27286745. Exclusion: 6

1034. Wilson A. Comparison of flurbiprofen and alprazolam in the management of chronic pain syndrome. Psychiatr J Univ Ott. 1990 Sep;15(3):144-9. PMID: 2243877. Exclusion: 3

1035. Wojtulewski J, Walter J, Thornton EJ. Tiaprofenic acid (surgam) in the treatment of osteoarthritis of the knee and hip. Rheumatol Rehabil. 1981 Aug;20(3):17780. PMID: 7025176. Exclusion: 3

1036. Wojtulewski JA. Fenoprofen in the treatment of osteoarthrosis. Curr Med Res Opin. 1974;2(9):551-5. doi: 10.1185/03007997409115256. PMID: 4452293. Exclusion: 3

1037. Wojtulewski JA, Hart FD, Huskisson EC. Fenoprofen in treatment of osteoarthrosis of hip and knee. Br Med J. 1974 Jun 1;2(5917):475-6. doi: 10.1136/bmj.2.5917.475. PMID: 4599766. Exclusion: 9

1038. Wolfe F, Cathey MA, Hawley DJ. A doubleblind placebo controlled trial of fluoxetine in fibromyalgia. Scand J Rheumatol. 1994;23(5):255-9. doi: 10.3109/03009749409103725. PMID: 7973479. Exclusion: 3
1039. Wolff RF, Bala MM, Westwood M, et al. $5 \%$ lidocaine-medicated plaster vs other relevant interventions and placebo for postherpetic neuralgia (PHN): a systematic review. Acta Neurol Scand. 2011 May;123(5):295-309. doi: 10.1111/j.16000404.2010.01433.x. PMID: 21039364. Exclusion: 8

1040. Worz R, Scherhag R. Treatment of chronic tension headache with doxepin or amitriptyline: Results of a double-blind study. Headache Quarterly. 1990;1(3):21623. Exclusion: 9

1041. Worz R, Scherhag R. Treatment of chronic tension headache with doxepin or amitriptylinle Results of a double blind study. Headache Quarterly. 1990;1(3):21623. Exclusion: 9

1042. Wright V, Walker WC, McGuire RJ. Indomethacin in the treatment of rheumatoid arthritis. A controlled trial comparing indomethacin, phenylbutazone, and placebo. Ann Rheum Dis. 1969;28(2):157-62. doi: 10.1136/ard.28.2.157. PMID: 4887768. Exclusion: 9

1043. Wu EQ, Birnbaum HG, Mareva MN, et al. Cost-effectiveness of duloxetine versus routine treatment for U.S. patients with diabetic peripheral neuropathic pain. J Pain. 2006 Jun;7(6):399-407. doi: 10.1016/j.jpain.2006.01.443. PMID: 16750796. Exclusion: 3

1044. Wu N, Chen S, Boulanger L, et al. Average daily dose, medication adherence, and healthcare costs among commerciallyinsured patients with fibromyalgia treated with duloxetine. Curr Med Res Opin. 2011 Jun;27(6):1131-9. doi: 10.1185/03007995.2011.570744. PMID: 21456939. Exclusion: 6

1045. Wymer JP, Simpson J, Sen D, et al. Efficacy and safety of lacosamide in diabetic neuropathic pain: an 18-week double-blind placebo-controlled trial of fixed-dose regimens. Clin J Pain. 2009 Jun;25(5):37685. doi: 10.1097/AJP.0b013e318196d2b6. PMID: 19454870. Exclusion: 3 
1046. Wysenbeek AJ, Mor F, Lurie Y, et al. Imipramine for the treatment of fibrositis: a therapeutic trial. Ann Rheum Dis. 1985 Nov;44(11):752-3. doi: 10.1136/ard.44.11.752. PMID: 3864398. Exclusion: 4

1047. Yeomans N, Tulassay Z, Juhasz L, et al. A comparison of omeprazole with ranitidine for ulcers associated with nonsteroidal antiinflammatory drugs. N Engl J Med. 1998;338:719-26. doi: 10.1056/NEJM199803123381104. Exclusion: 3

1048. Yildirim K, Şışecıoğlu M, Karatay S, et al. The effectiveness of gabapentin in patients with chronic radiculopathy. The Pain Clinic. 2003;15(3):213-8. doi: 10.1163/156856903767650718. Exclusion: 9

1049. Yilmaz B, Yasar E, Koroglu Omac O, et al. Gabapentin vs. pregabalin for the treatment of neuropathic pain in patients with spinal cord injury: A cross-over study. Eur J Pain Suppl. 2011;5(1):239. doi: 10.1016/S17543207(11)70824-0. Exclusion: 9

1050. Yilmaz B, Yasar E, Koroglu Omac O, et al. Gabapentin vs. pregabalin for the treatment of neuropathic pain in patients with spinal cord injury: A crossover study. Turkiye Fiziksel Tip ve Rehabilitasyon Dergisi. 2015;61:1-5. doi: 10.5152/tftrd.2015.79069. Exclusion: 9

1051. Young D, Peterson C, Basch C, et al. Effects of naproxen and nabumetone on serum cholesterol levels in patients with osteoarthritis. Clin Ther. 1995 MarApr;17(2):231-40. PMID: 7614523. Exclusion: 2

1052. Yucel A, Ozyalcin S, Koknel Talu G, et al. The effect of venlafaxine on ongoing and experimentally induced pain in neuropathic pain patients: a double blind, placebo controlled study. Eur J Pain. 2005 Aug;9(4):407-16. doi: 10.1016/j.ejpain.2004.09.009. PMID: 15979021. Exclusion: 9
1053. Yue L, Luo S, Wang Y, et al. Clinical meaningfulness of duloxetine's effect in Chinese patients with chronic pain due to osteoarthritis: post hoc analyses of a phase 3 randomized trial. Open access rheumatol. 2019 Mar 21;11:67-76. doi: 10.2147/OARRR.S193044. PMID: 30962729. Exclusion: 2

1054. Yue L, Luo S, Wang Y, et al. Clinical meaningfulness of duloxetinea[Euro sign][TM]s effect in chinese Patients with chronic pain due to osteoarthritis: post hoc analyses of a phase 3 randomized trial. Open access rheumatology: research and reviews. 2019;11:67-76. PMID: CN-01964281 NEW. Exclusion: 2

1055. Yuen E, Gueorguieva I, Bueno-Burgos L, et al. Population pharmacokinetic/pharmacodynamic models for duloxetine in the treatment of diabetic peripheral neuropathic pain. Eur J Pain. 2013 Mar;17(3):382-93. doi: 10.1002/j.1532-2149.2012.00209.x. PMID: 22893563. Exclusion: 6

1056. Zaccara G, Gangemi P, Perucca P, et al. The adverse event profile of pregabalin: a systematic review and meta-analysis of randomized controlled trials. Epilepsia. 2011 Apr;52(4):826-36. doi: 10.1111/j.15281167.2010.02966.x. PMID: 21320112. Exclusion: 8

1057. Zaccara G, Perucca P, Gangemi PF. The adverse event profile of pregabalin across different disorders: a meta-analysis. Eur J Clin Pharmacol. 2012 Jun;68(6):903-12. doi: 10.1007/s00228-012-1213-x. PMID: 22271298. Exclusion: 8

1058. Zacher J, Burger KL, Farber L, et al. Topical diclofenac versus oral ibuprofen: A double blind, randomized clinical trial to demonstrate efficacy and tolerability in patients with activated osteoarthritis of the finger joints. Aktuelle Rheumatologie. 2001;26:7-14. Exclusion: 1

1059. Zhao J, Wang Q, Wu J, et al. Therapeutic effects of low-frequency phonophoresis with a Chinese herbal medicine versus sodium diclofenac for treatment of knee osteoarthritis: a double-blind, randomized, placebo-controlled clinical trial. J Tradit Chin Med. 2016 10;36(5):613-7. PMID: 29933529. Exclusion: 9 
1060. Zhao SZ, Dedhiya SD, Bocanegra TS, et al. Health-related quality-of-life effects of oxaprozin and nabumetone in patients with osteoarthritis of the knee. Clin Ther. 1999 Jan;21(1):205-17. doi: 10.1016/S01492918(00)88279-X. PMID: 10090436. Exclusion: 9

1061. Zheng Y, Gu M, Shi D, et al. Tomographyguided palisade sacroiliac joint radiofrequency neurotomy versus celecoxib for ankylosing spondylitis: a open-label, randomized, and controlled trial. Rheumatol Int. 2014 Sep;34(9):1195-202. doi: 10.1007/s00296-014-2959-5. PMID: 24518967. Exclusion: 3

1062. Ziegler D, Hidvegi T, Gurieva I, et al. Efficacy and safety of lacosamide in painful diabetic neuropathy. Diabetes Care. 2010 Apr;33(4):839-41. doi: 10.2337/dc09-1578. PMID: 20067958. Exclusion: 3

1063. Ziegler D, Pritchett YL, Wang F, et al. Impact of disease characteristics on the efficacy of duloxetine in diabetic peripheral neuropathic pain. Diabetes Care. 2007 Mar;30(3):664-9. doi: 10.2337/dc06-2009. PMID: 17327338. Exclusion: 6

1064. Ziegler DK, Ellis DJ. Naproxen in prophylaxis of migraine. Arch Neurol. 1985 Jun;42(6):582-4. PMID: 4004602.

Exclusion: 4

1065. Ziegler DK, Hurwitz A, Preskorn S, et al. Propranolol and amitriptyline in prophylaxis of migraine. Pharmacokinetic and therapeutic effects. Arch Neurol. 1993 Aug;50(8):825-30. PMID: 8352668. Exclusion: 3

1066. Zingler G, Hermann B, Fischer T, et al. Cardiovascular adverse events by nonsteroidal anti-inflammatory drugs: when the benefits outweigh the risks. Expert Rev Clin Pharmacol. 2016 Nov;9(11):1479-92. doi: 10.1080/17512433.2016.1230495. PMID: 27575590. Exclusion: 8

1067. Zissis NP, Harmoussi S, Vlaikidis N, et al. A randomized, double-blind, placebocontrolled study of venlafaxine XR in outpatients with tension-type headache. Cephalalgia. 2007 Apr;27(4):315-24. doi: 10.1111/j.1468-2982.2007.01300.x. PMID: 17346304. Exclusion: 4
1068. Zizic TM, Stevens MB, Sutton JD. Piroxicam in osteoarthritis: a controlled long-term study. Postgrad Med. 1983 May;Spec No:32-40. PMID: 6348718. Exclusion: 6

1069. Zuccollo R, Mackinnon MJ, Fraser KM, et al. Oxaprozin and sulindac in rheumatoid arthritis: a double-blind comparative trial. Curr Med Res Opin. 1983;8(5):302-9. doi: 10.1185/03007998309112388. PMID: 6340971. Exclusion: 2

1070. Zutshi DW, Stern D, Bloch M, et al. Ketoprofen: double-blind cross-over study with indomethacin in patients with rheumatoid arthritis. Rheumatol Rehabil. 1974 Feb;13(1):10-6. PMID: 4604659. Exclusion: 6 


\section{Appendix E. Study Characteristics Evidence Tables}

Shown in associated Excel ${ }^{\circledR}$ files. 


\section{Appendix F. Meta-Analysis Evidence Tables}

Shown in associated Excel files. 


\section{Appendix G. Quality Assessment}

Shown in associated Excel file. 


\section{Appendix H. Strength of Evidence Tables}

\section{Table of Contents}

Efficacy

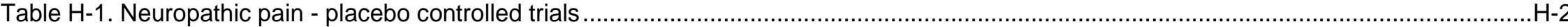

Table $\mathrm{H}$-2. Neuropathic pain - cross-class comparisons

Table H-3. Neuropathic pain - head-to-head comparisons .............

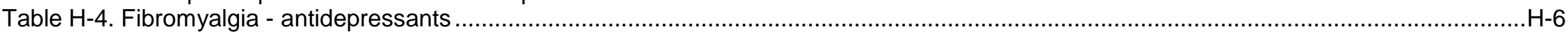

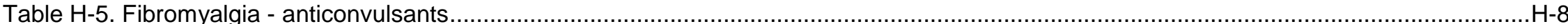

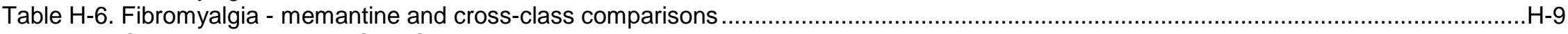

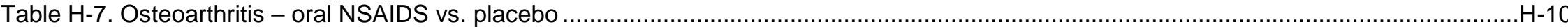

Table H-8. Osteoarthritis - topical diclofenac vs. placebo

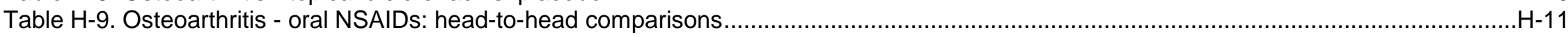

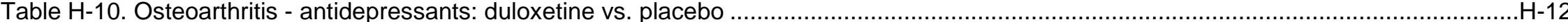

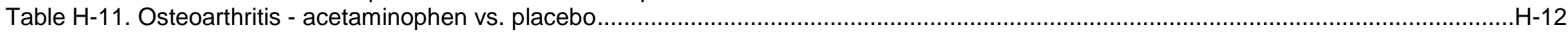

Table H-12. Osteoarthritis - anticonvulsants vs. antidepressants: duloxetine vs. pregabalin ...............................................................13

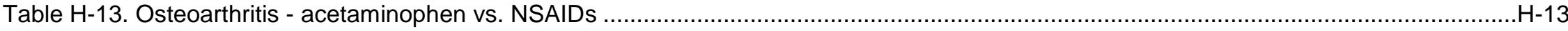

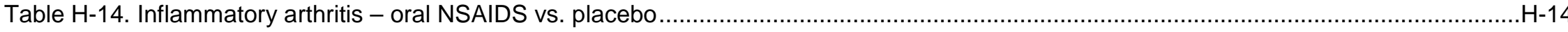

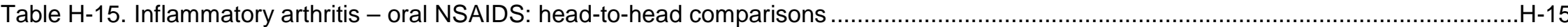

Table H-16. Inflammatory arthritis - antidepressants: placebo controlled trials ........................................................................................

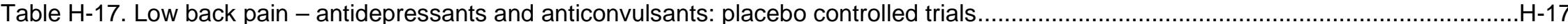

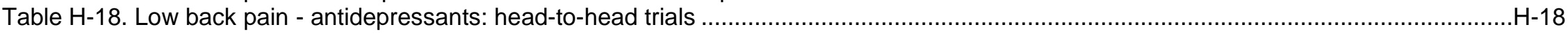

Harms

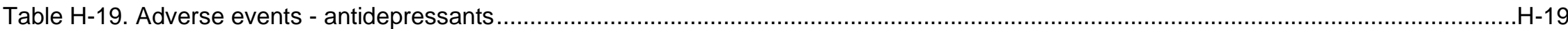

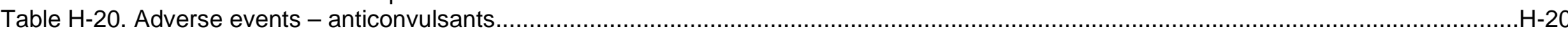

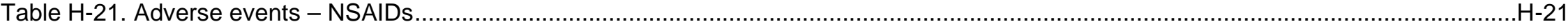

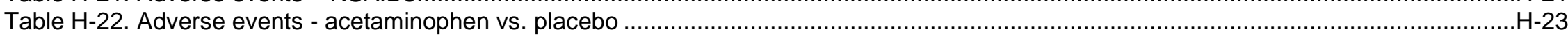

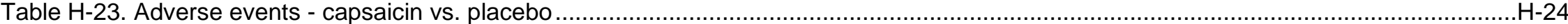

Table H-24. Adverse events - cannabis vs. placebo ……

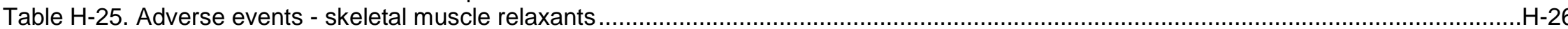

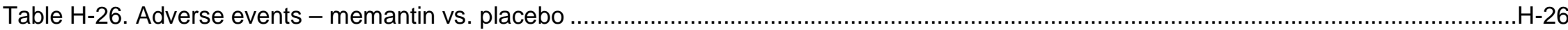




\section{Efficacy}

Table H-1. Neuropathic pain - placebo controlled trials

\begin{tabular}{|c|c|c|c|c|c|c|c|c|c|}
\hline Outcome & Duration & $\begin{array}{c}\text { Number of Studies } \\
\text { (n participants) }\end{array}$ & $\begin{array}{c}\text { Study } \\
\text { Qualityb }^{b}\end{array}$ & Directness & $\begin{array}{l}\text { Consistency } \\
\text { (1 study= } \\
\text { Unknown) }\end{array}$ & Precision & $\begin{array}{l}\text { Publication } \\
\text { Bias }\end{array}$ & $\begin{array}{c}\text { Main Findings } \\
\text { Effect Size }(95 \% \mathrm{Cl})\end{array}$ & $\begin{array}{l}\text { Strength of } \\
\text { Evidence } \\
\text { Grade }\end{array}$ \\
\hline \multirow{6}{*}{$\begin{array}{l}\text { Pain } \\
\text { Improvement } \\
\text { (Continuous) }\end{array}$} & \multirow{6}{*}{ Short } & $15(N=4,832)^{10-24}$ & Fair & Direct & Consistent & Precise & Undetected & $\begin{array}{c}\text { Small effect } \\
\text { (NRS) } \\
\text { Pregabalin/gabapentin vs. } \\
\text { placebo } \\
\text { MD -0.61 (-0.87 to -0.36), } \\
\left.\right|^{2}=72 \%\end{array}$ & Moderate \\
\hline & & $2(\mathrm{~N}=493)^{25,26}$ & Fair & Direct & Consistent & Imprecise & Unknown & $\begin{array}{c}\text { Small effect } \\
\text { (VAS) } \\
\text { Oxcarbazepine vs. placebo } \\
\text { MD -0.89 (-1.50 to }-0.37) \\
1^{2}=0 \%\end{array}$ & Moderate \\
\hline & & $6(\mathrm{~N}=2,082)^{27-32}$ & Fair & Direct & Consistent & Precise & Unknown & $\begin{array}{c}\text { Small effect } \\
\text { (NRS) } \\
\text { Duloxetine vs. placebo } \\
\text { MD -0.79 (-1.10 to }-0.49) \\
\mathrm{I}^{2}=43 \% \\
\end{array}$ & Moderate \\
\hline & & $2(\mathrm{~N}=486)^{33,34}$ & Fair & Direct & Consistent & Imprecise & Unknown & $\begin{array}{c}\text { No effect } \\
\text { (NRS) } \\
\text { Cannabis vs. placebo } \\
\text { no difference between } \\
\text { groups ( } \mathrm{p}=0.68 \text { and } 0.14 \text { ) }\end{array}$ & Low \\
\hline & & $3(\mathrm{~N}=1,519)^{35-37}$ & Fair & Direct & Consistent & Precise & Unknown & $\begin{array}{c}\text { No effect } \\
\text { (NRS) } \\
\text { Capsaicin vs. Placebo } \\
\text { MD -0.33 (-0.60 to }-0.004) \\
1^{2}=0 \%\end{array}$ & Moderate \\
\hline & & $1(\mathrm{~N}=45)^{38}$ & Fair & Direct & Unknown & Imprecise & Unknown & $\begin{array}{c}\text { VAS } \\
\text { Memantine vs. placebo } \\
\text { mean change } 1.82 \text { (SD 2.77) } \\
\text { vs. -2.36 (SD 3.35), } p=0.87\end{array}$ & Insufficient \\
\hline \multirow[t]{2}{*}{$\begin{array}{c}\text { Pain } \\
\text { Response } \\
\text { (Dichotomous) }\end{array}$} & \multirow[t]{2}{*}{ Short } & $15(N=4,576)^{10-24}$ & Fair & Direct & Consistent & Imprecise & Undetected & $\begin{array}{c}\text { Small effect } \\
(\geq 30 \%) \\
\text { Pregabalin/gabapentin vs. } \\
\text { placebo } \\
\text { RR 1.27 (1.12 to } 1.50) \\
\mathrm{I}^{2}=72 \%\end{array}$ & Moderate \\
\hline & & $1(N=144)^{25,26}$ & Fair & Direct & Unknown & Imprecise & Unknown & $\begin{array}{c}\text { Small effect } \\
\text { Oxcarbazepine vs. placebo } \\
45.6 \% \text { vs. } 28.9 \%, p=0.028\end{array}$ & Low \\
\hline
\end{tabular}




\begin{tabular}{|c|c|c|c|c|c|c|c|c|c|}
\hline Outcome & Duration & $\begin{array}{l}\text { Number of Studies } \\
\text { (n participants) }\end{array}$ & $\begin{array}{l}\text { Study } \\
\text { Quality }\end{array}$ & Directness & $\begin{array}{c}\text { Consistency } \\
\text { (1 study= } \\
\text { Unknown) }\end{array}$ & Precision & $\begin{array}{l}\text { Publication } \\
\text { Bias }\end{array}$ & $\begin{array}{c}\text { Main Findings } \\
\text { Effect Size }(95 \% \mathrm{Cl})\end{array}$ & $\begin{array}{l}\text { Strength of } \\
\text { Evidence } \\
\text { Grade }\end{array}$ \\
\hline & & $6(\mathrm{~N}=2,075)^{27-32}$ & Fair & Direct & Consistent & Imprecise & Unknown & $\begin{array}{c}\text { Small effect } \\
(\geq 30 \%) \\
\text { Duloxetine vs. placebo } \\
\text { RR 1.39 (1.22 to 1.62), } \\
\left.\right|^{2}=39 \%\end{array}$ & Moderate \\
\hline & & $1(\mathrm{~N}=246)^{34}$ & Fair & Direct & Unknown & Imprecise & Unknown & $\begin{array}{c}\text { Moderate effect } \\
\text { Cannabis vs. placebo } \\
28 \% \text { vs. } 16 \% ; \\
\text { RR } 1.70(1.04 \text { to } 2.78), \\
\text { p=0.03 }\end{array}$ & Low \\
\hline & & $3(\mathrm{~N}=1,519)^{35-37}$ & Fair & Direct & Consistent & Precise & Unknown & $\begin{array}{c}\text { No effect } \\
\text { Capsaicin vs. placebo } \\
\text { RR 1.17 (0.98 to 1.37), } \\
\mathrm{I}^{2}=0 \%\end{array}$ & Moderate \\
\hline \multirow{3}{*}{ Function } & \multirow{3}{*}{ Short } & $1(\mathrm{~N}=371)^{24}$ & Fair & Direct & Unknown & Imprecise & Unknown & $\begin{array}{c}\text { No effect } \\
\text { BPI Interference } \\
\text { Gabapentin enacarbil vs. } \\
\text { placebo } \\
\text { MD }-0.23(-0.70 \text { to } 0.23)\end{array}$ & Low \\
\hline & & $6(\mathrm{~N}=2,082)^{27-32}$ & Fair & Direct & Consistent & Imprecise & Unknown & $\begin{array}{c}\text { Small effect } \\
\text { (BPI Interference) } \\
\text { Duloxetine vs. placebo } \\
\text { SMD -0.31 (-0.42 to }-0.20), \\
\left.\right|^{2}=0 \%\end{array}$ & Low \\
\hline & & $1(\mathrm{~N}=303)^{34}$ & Fair & Direct & Unknown & Imprecise & Unknown & $\begin{array}{c}\text { No effect } \\
\text { (BPI) } \\
\text { Cannabis vs. placebo } \\
\mathrm{p}=0.18\end{array}$ & Low \\
\hline Quality of Life & Short & $\begin{array}{l}3(\mathrm{~N}=1,015)^{13,14,22} \\
3(\mathrm{~N}=1,400)^{16,19,24} \\
3(\mathrm{~N}=1,400)^{16,19,24}\end{array}$ & Fair & Direct & Consistent & Imprecise & Unknown & $\begin{array}{c}\text { No effect } \\
\text { (EQ-5D) } \\
\text { Pregabalin/gabapentin vs. } \\
\text { placebo } \\
\text { SMD 0.24 (-0.07 to 0.54), } \\
I^{2}=58 \% \\
\text { (SF-36 MCS) } \\
\text { Pregabalin/gabapentin vs. } \\
\text { placebo } \\
\text { MD 0.22 (-1.93 to 2.37) } \\
\text { (SF-36 PCS) } \\
\text { Pregabalin/gabapentin vs. } \\
\text { placebo } \\
\text { MD 0.80 (-0.29 to 2.07) }\end{array}$ & Low \\
\hline
\end{tabular}




\begin{tabular}{|c|c|c|c|c|c|c|c|c|c|}
\hline Outcome & Duration & $\begin{array}{l}\text { Number of Studies } \\
\text { (n participants) }\end{array}$ & $\begin{array}{l}\text { Study } \\
\text { Quality }^{\mathrm{b}}\end{array}$ & Directness & $\begin{array}{c}\text { Consistency } \\
\text { (1 study= } \\
\text { Unknown) }\end{array}$ & Precision & $\begin{array}{c}\text { Publication } \\
\text { Bias }\end{array}$ & $\begin{array}{c}\text { Main Findings } \\
\text { Effect Size }(95 \% \mathrm{Cl})\end{array}$ & $\begin{array}{l}\text { Strength of } \\
\text { Evidence } \\
\text { Grade }\end{array}$ \\
\hline & & $2(\mathrm{~N}=493)^{25,26}$ & Fair & Direct & Inconsistent & Precise & Unknown & $\begin{array}{l}\text { (SF-36 MCS) } \\
\text { Oxcarbazepine vs. placebo } \\
47.2 \text { vs. } 50.2 ; p=0.03(1 \\
\text { trial); No difference for other } \\
\text { SF-36 scales }\end{array}$ & Low \\
\hline & & $3(\mathrm{~N}=9,444)^{27,29,31}$ & Fair & Direct & Consistent & Precise & Unknown & $\begin{array}{c}\text { Small effect } \\
\text { (EQ-5D) } \\
\text { Duloxetine vs. placebo } \\
\text { MD 0.22 (0.05 to 0.38), } \\
\mathrm{I}^{2}=0 \%\end{array}$ & Moderate \\
\hline & & $2(\mathrm{~N}=486)^{33,34}$ & Fair & Direct & Consistent & Imprecise & Unknown & $\begin{array}{c}\text { No effect } \\
\text { (EQ-5D) } \\
\text { Cannabis vs. placebo } p=0.62 \\
\text { (SF-36) } \\
\text { Cannabis vs. placebo } p=\text { not } \\
\text { significant }\end{array}$ & Low \\
\hline
\end{tabular}

a Study references are in Appendix J.

b Study Quality: poo- quality studies not synthesized

${ }^{\mathrm{c}}$ Pain Response main findings, percentages represent threshold for Pain Response

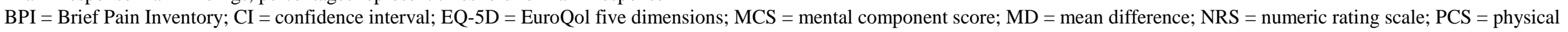
component score; RR = risk ratio; $\mathrm{SD}$ = standard deviation; SF-36 = Short Form-36; SMD = standard mean difference; VAS = visual analogue scale 
Table H-2. Neuropathic pain - cross-class comparisons

\begin{tabular}{|c|c|c|c|c|c|c|c|c|c|}
\hline Outcome & Duration & $\begin{array}{c}\text { Number of Studies } \\
\text { (n participants) }\end{array}$ & $\begin{array}{c}\text { Study } \\
\text { Quality }^{b}\end{array}$ & Directness & $\begin{array}{l}\text { Consistency } \\
\text { (1 study= } \\
\text { Unknown) } \\
\end{array}$ & Precision & $\begin{array}{c}\text { Publication } \\
\text { Bias }\end{array}$ & $\begin{array}{l}\text { Main Findings } \\
\text { Effect Size }\end{array}$ & $\begin{array}{l}\text { Strength of } \\
\text { Evidence } \\
\text { Grade } \\
\end{array}$ \\
\hline $\begin{array}{c}\text { Pain } \\
\text { Improvement } \\
\text { (Continuous) }\end{array}$ & Short & $1(\mathrm{~N}=152)^{39}$ & Fair & Direct & Unknown & Imprecise & Unknown & $\begin{array}{c}\text { (VAS) } \\
\text { Gabapentin vs. duloxetine } \\
\text { No difference between groups } \\
\text { ( } p=\text { not reported) }\end{array}$ & Insufficient \\
\hline
\end{tabular}

a Study references are in Appendix J.

b Study Quality: poor-quality studies not synthesized

VAS = visual analogue scale

Table H-3. Neuropathic pain - head-to-head comparisons

\begin{tabular}{|c|c|c|c|c|c|c|c|c|c|}
\hline+2 & Duration & $\begin{array}{c}\text { Number of } \\
\text { Studies }^{\mathrm{a}} \\
\text { (n participants) } \\
\end{array}$ & Study Quality & Directness & $\begin{array}{c}\text { Consistency } \\
\text { (1 study= } \\
\text { Unknown) } \\
\end{array}$ & Precision & $\begin{array}{c}\text { Publication } \\
\text { Bias }\end{array}$ & $\begin{array}{l}\text { Main Findings } \\
\text { Effect Size }\end{array}$ & $\begin{array}{c}\text { Strength of } \\
\text { Evidence } \\
\text { Grade } \\
\end{array}$ \\
\hline \multirow[t]{2}{*}{$\begin{array}{l}\text { Pain Improvement } \\
\text { (Continuous) }\end{array}$} & \multirow[t]{2}{*}{ Short } & $1(\mathrm{~N}=301)^{16}$ & Fair & Direct & Unknown & Imprecise & Unknown & $\begin{array}{c}\text { No effect } \\
\text { (NRS) } \\
\text { Pregabalin vs. gabapentin } \\
\text { enacarbil } \\
\text { (p-values NR) }\end{array}$ & Low \\
\hline & & $2(\mathrm{~N}=132)^{39,40}$ & Fair & Direct & Unknown & Imprecise & Unknown & $\begin{array}{c}\text { (VAS) } \\
\begin{array}{c}\text { Pregabalin vs. gabapentin } \\
(p-\text { value NR) }\end{array}\end{array}$ & Insufficient \\
\hline Function & Short & $1(\mathrm{~N}=301)^{16}$ & Fair & Direct & Unknown & Imprecise & Unknown & $\begin{array}{c}\text { No effect } \\
\text { (BPI Interference) } \\
\text { Pregabalin vs. gabapentin } \\
\text { enacarbil } \\
\text { (p-values NR) }\end{array}$ & Low \\
\hline Quality of Life & Short & $1(\mathrm{~N}=301)^{16}$ & Fair & Direct & Unknown & Imprecise & Unknown & $\begin{array}{c}\text { No differences between } \\
\text { Pregabalin vs. gabapentin } \\
\text { enacarbil } \\
\text { (p-values NR) }\end{array}$ & Low \\
\hline
\end{tabular}

a Study references are in Appendix J.

b Study Quality: poor-quality studies not synthesized

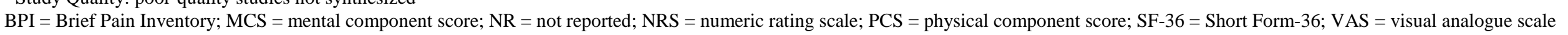


Table H-4. Fibromyalgia - antidepressants

\begin{tabular}{|c|c|c|c|c|c|c|c|c|c|}
\hline Outcome & Duration & $\begin{array}{c}\text { Number of } \\
\text { Studies }^{\mathrm{a}} \\
\text { (n participants) }\end{array}$ & Study Qualityb & Directness & $\begin{array}{l}\text { Consistency } \\
\text { (1 study= } \\
\text { Unknown) } \\
\end{array}$ & Precision & $\begin{array}{c}\text { Publication } \\
\text { Bias }\end{array}$ & $\begin{array}{c}\text { Main Findings } \\
\text { Effect Size }(95 \% \mathrm{Cl})\end{array}$ & $\begin{array}{l}\text { Strength } \\
\text { of } \\
\text { Evidence } \\
\text { Grade } \\
\end{array}$ \\
\hline \multirow{3}{*}{$\begin{array}{c}\text { Pain } \\
\text { Improvement } \\
\text { (Continuous) }\end{array}$} & \multirow{2}{*}{ Short } & $11\left(\begin{array}{c}\mathrm{N}=5,936) \\
\mathrm{d}, 41-51\end{array}\right.$ & Fair & Direct & Consistent & Precise & Undetected & $\begin{array}{c}\text { Small effect } \\
\text { (0-10 scale) } \\
\text { Antidepressants vs. placebo } \\
\text { MD -0.59 (-0.80 to -0.43), } \\
\mathrm{I}^{2}=26 \%\end{array}$ & Moderate \\
\hline & & $1(\mathrm{~N}=87)^{52}$ & Fair & Direct & Unknown & Imprecise & Unknown & $\begin{array}{c}\text { (VAS 0-10) } \\
\text { Amitriptyline vs. placebo } \\
\text { MD -0.7 (Endpoint VAS } 4.5 \text { vs. } \\
5.2, p=N R)\end{array}$ & Insufficient \\
\hline & Intermediate & $3\left(\begin{array}{c}N=1,357) \\
d, 45,46,53\end{array}\right.$ & Fair & Direct & Consistent & Precise & Unknown & $\begin{array}{c}\text { Small effect } \\
(0-10 \text { scale) } \\
\text { Antidepressants vs. placebo } \\
\text { MD -0.67 }(-0.99 \text { to }-0.34), I^{2}=0 \%\end{array}$ & Moderate \\
\hline \multirow{3}{*}{$\begin{array}{c}\text { Pain } \\
\text { Response } \\
\text { (Dichotomous) }\end{array}$} & \multirow{2}{*}{ Short } & $\begin{array}{c}10(\mathrm{~N}=5,853) \\
\mathrm{d}, 41-44,46-51\end{array}$ & Fair & Direct & Consistent & Precise & Undetected & $\begin{array}{c}\begin{array}{c}\text { Small effect } \\
(\geq 30 \%)\end{array} \\
\text { Antidepressants vs. placebo } \\
\text { RR } 1.36 \text { (1.26 to } 1.46), I^{2}=0 \%\end{array}$ & Moderate \\
\hline & & $1(\mathrm{~N}=87)^{52}$ & Fair & Direct & Unknown & Imprecise & Unknown & $\begin{array}{c}\text { (Physician's global assessment) } \\
\text { Amitriptyline vs. placebo } \\
74 \% \text { vs. } 49 \%, p=0.017\end{array}$ & Insufficient \\
\hline & Intermediate & $3\left(\begin{array}{c}\mathrm{N}=1,715) \\
\mathrm{d}, 45,46,53\end{array}\right.$ & Fair & Direct & Consistent & Precise & Unknown & $\begin{array}{c}\begin{array}{c}\text { Small effect } \\
(\geq 30 \%)\end{array} \\
\text { Antidepressants vs. placebo } \\
\text { RR } 1.29(1.08 \text { to } 1.52), I^{2}=0 \%\end{array}$ & Moderate \\
\hline \multirow{2}{*}{ Function } & Short & $11 \underset{d, 41-51}{(N=6,240)}$ & Fair & Direct & Consistent & Precise & Undetected & $\begin{array}{c}\text { Small effect } \\
\text { Antidepressants vs. placebo } \\
\text { SMD }-0.24(-0.32 \text { to }-0.17) \\
\mathrm{I}^{2}=22 \%\end{array}$ & Moderate \\
\hline & Intermediate & $3\left(\begin{array}{c}N=1,724) \\
d, 45,46,53\end{array}\right.$ & Fair & Direct & Consistent & Precise & Unknown & $\begin{array}{c}\text { No effect } \\
\text { Antidepressants vs. placebo } \\
\text { SMD }-0.13(-0.24 \text { to }-0.02) \\
1^{2}=0 \%\end{array}$ & Moderate \\
\hline $\begin{array}{l}\text { Quality of } \\
\text { Life }\end{array}$ & Short & $\begin{array}{c}8(\mathrm{~N}=5,487) \\
\mathrm{e}, 41,43,45-50\end{array}$ & Fair & Direct & Consistent & Precise & Undetected & $\begin{array}{c}\text { Small effect } \\
\text { (SF-36 MCS or PCS, } 0-100) \\
\text { Antidepressants vs. placebo } \\
\text { MCS: SMD } 0.19(0.13 \text { to } 0.27) \text {, } \\
I^{2}=12 \% \\
\text { PCS: SMD } 0.16(0.10 \text { to } 0.22) \\
\left.\right|^{2}=0 \%\end{array}$ & Moderate \\
\hline
\end{tabular}




\begin{tabular}{|c|c|c|c|c|c|c|c|c|c|}
\hline Outcome & Duration & $\begin{array}{c}\text { Number of } \\
\text { Studies }^{\mathrm{a}} \\
\text { (n participants) }\end{array}$ & Study Quality & Directness & $\begin{array}{c}\text { Consistency } \\
(1 \text { study= } \\
\text { Unknown) }\end{array}$ & Precision & $\begin{array}{c}\text { Publication } \\
\text { Bias }\end{array}$ & $\begin{array}{c}\text { Main Findings } \\
\text { Effect Size }(95 \% \mathrm{Cl})\end{array}$ & $\begin{array}{c}\text { Strength } \\
\text { of } \\
\text { Evidence } \\
\text { Grade } \\
\end{array}$ \\
\hline & Intermediate & $3 \underset{\mathrm{e}, 45,46,53}{(\mathrm{~N}=1,716)}$ & Fair & Direct & Consistent & Precise & Unknown & $\begin{array}{c}\text { Small effect } \\
\text { (SF-36 MCS or PCS, } 0-100) \\
\text { Antidepressants vs. placebo } \\
\text { MCS: SMD } 0.18(0.08 \text { to } 0.30) \\
I^{2}=0 \% \\
\text { PCS: SMD } 0.07(-0.10 \text { to } 0.24) \text {, } \\
I^{2}=0 \%\end{array}$ & Moderate \\
\hline
\end{tabular}

a Study references are in Appendix J.

b Study Quality: poor-quality studies not synthesized

${ }^{c}$ Pain Response main findings, percentages represent threshold for Pain Response

${ }^{\text {d }}$ Russell 2008 and Mease 2009 included both short-term and intermediate-term time points, and n's are included in both totals

e Russell 2008 included both short-term and intermediate-term time points, and n's are included in both totals

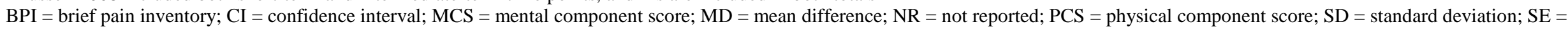
standard error; SEM = standard error of the mean; SF-36 = Short Form-36; SMD = standard mean difference 
Table H-5. Fibromyalgia - anticonvulsants

\begin{tabular}{|c|c|c|c|c|c|c|c|c|c|}
\hline Outcome & Duration & $\begin{array}{l}\text { Number of } \\
\text { Studies } \\
(\mathbf{n} \\
\text { participants) }\end{array}$ & Study Qualityb & Directness & $\begin{array}{l}\text { Consistency } \\
\text { (1 study= } \\
\text { Unknown) } \\
\end{array}$ & Precision & $\begin{array}{c}\text { Publication } \\
\text { Bias }\end{array}$ & $\begin{array}{c}\text { Main Findings } \\
\text { Effect Size }(95 \% \mathrm{Cl})\end{array}$ & $\begin{array}{c}\text { Strength of } \\
\text { Evidence } \\
\text { Grade } \\
\end{array}$ \\
\hline $\begin{array}{c}\text { Pain } \\
\text { Improvement } \\
\text { (Continuous) }\end{array}$ & Short & $8(\mathrm{~N}=4,747)^{54-59}$ & Fair & Direct & Consistent & Precise & Undetected & $\begin{array}{c}\text { Small effect } \\
(0-10 \text { scale }) \\
\text { MD }-0.57(-0.75 \text { to }-0.40), I^{2}=30 \%\end{array}$ & Moderate \\
\hline $\begin{array}{c}\text { Pain } \\
\text { Response }^{c} \\
\text { (Dichotomous) }\end{array}$ & Short & $8(\mathrm{~N}=4,773)^{54-59}$ & Fair & Direct & Consistent & Precise & Undetected & $\begin{array}{c}\text { Small effect } \\
(\geq 30 \%) \\
\text { RR } 1.30(1.20 \text { to } 1.43), I^{2}=0 \%\end{array}$ & Moderate \\
\hline Function & Short & $8(\mathrm{~N}=4,740)^{54-59}$ & Fair & Direct & Consistent & Precise & Undetected & $\begin{array}{c}\text { Small effect } \\
\text { (FIQ } 0-80 \text { or } 0-100) \\
\text { SMD }-0.22(-0.29 \text { to }-0.15), I^{2}=0 \%\end{array}$ & Moderate \\
\hline Quality of Life & Short & $4(\mathrm{~N}=2,520)^{55,56}$ & Fair & Direct & Consistent & Precise & Unknown & $\begin{array}{c}\text { No effect } \\
\text { (SF-36 MCS or PCS, } 0-100) \\
\text { Pregabalin vs. placebo } \\
\text { MCS: SMD } 0.13(0.04 \text { to } 0.22) \\
\mathrm{I}^{2}=0 \% \\
\text { PCS: SMD } 0.17(0.04 \text { to } 0.31) \\
\mathrm{I}^{2}=39 \%\end{array}$ & Moderate \\
\hline
\end{tabular}

${ }^{a}$ Study references are in Appendix J.

b Study Quality: poor-quality studies not synthesized

${ }^{\mathrm{c}}$ Pain Response main findings, percentages represent threshold for Pain Response

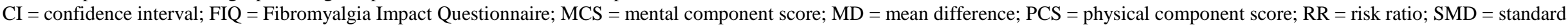
mean difference; SF-36 = short form 36 
Table H-6. Fibromyalgia - memantine and cross-class comparisons

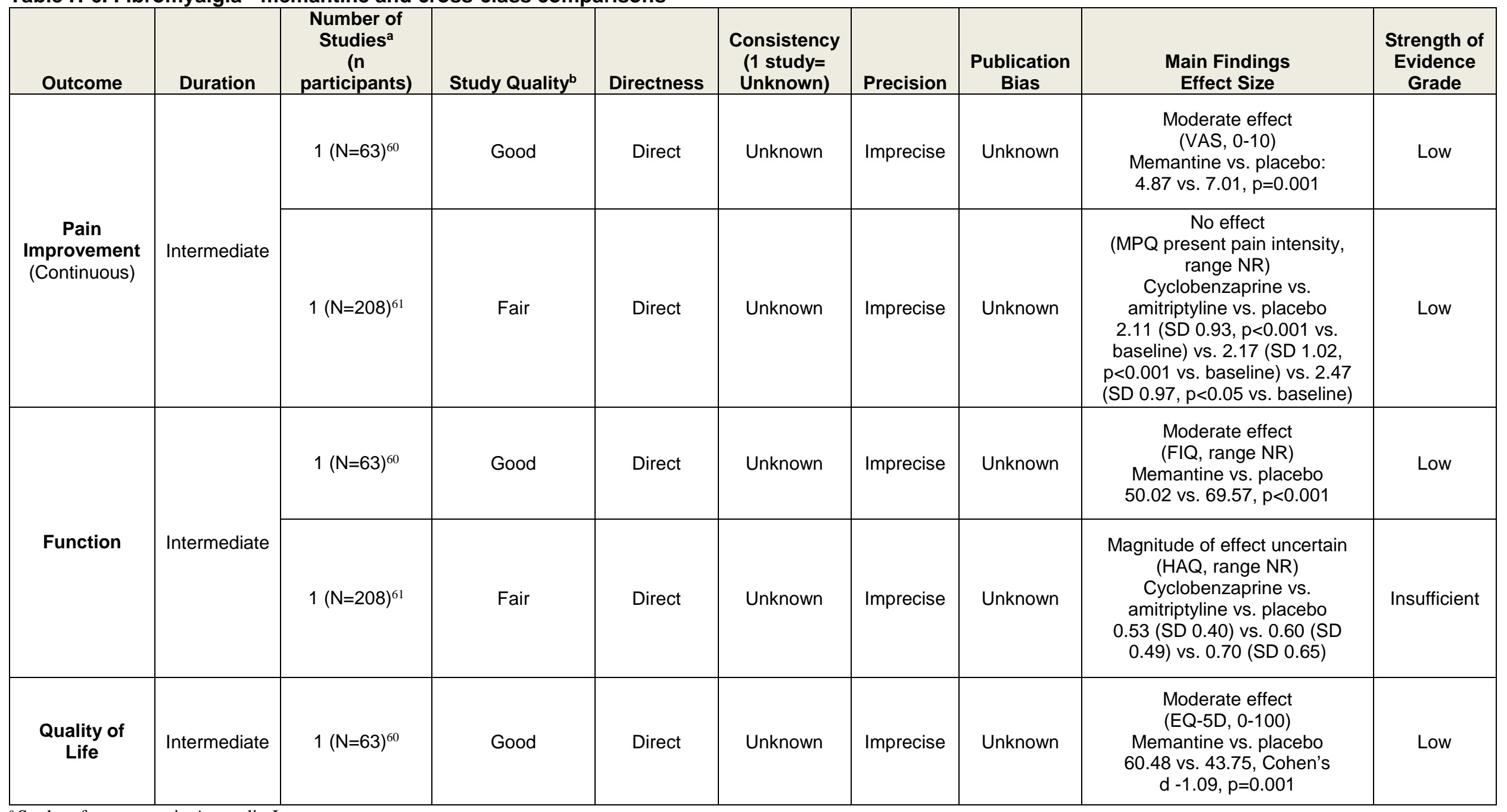

a Study references are in Appendix J.

b Study Quality: poor-quality studies not synthesized

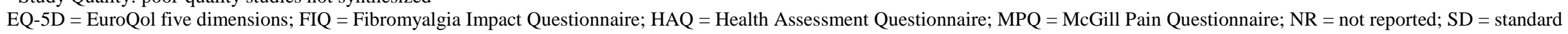
deviation; VAS = visual analogue scale 
Table H-7. Osteoarthritis - oral NSAIDS vs. placebo

\begin{tabular}{|c|c|c|c|c|c|c|c|c|c|}
\hline (n) & Duration & $\begin{array}{c}\text { Number of } \\
\text { Studies ( } n \\
\text { participants) } \\
\end{array}$ & Study Qualityb & Directness & $\begin{array}{l}\text { Consistency } \\
\text { (1 study= } \\
\text { Unknown) } \\
\end{array}$ & Precision & $\begin{array}{c}\text { Publication } \\
\text { Bias }\end{array}$ & $\begin{array}{c}\text { Main Findings } \\
\text { Effect Size (95\% Cl) }\end{array}$ & $\begin{array}{c}\text { Strength } \\
\text { of } \\
\text { Evidence } \\
\text { Grade } \\
\end{array}$ \\
\hline $\begin{array}{l}\text { Pain Improvement } \\
\text { (Continuous) }\end{array}$ & Short & $27(\mathrm{~N}=13,478)^{62-86}$ & Fair & Direct & Consistent & Precise & Possible & $\begin{array}{c}\text { Small effect } \\
\text { (NRS 0-10) } \\
\text { MD -0.73 (-0.84 to - } \\
0.62), I^{2}=27 \% \\
\end{array}$ & Moderate \\
\hline $\begin{array}{l}\text { Pain Response } \\
\text { (Dichotomous) }\end{array}$ & Short & $\begin{array}{c}15(\mathrm{~N}=8,253)^{62-} \\
65,67,68,70-72,75,79,80,82\end{array}$ & Fair & Direct & Consistent & Precise & Undetected & $\begin{array}{c}\text { Small effect } \\
\text { RR } 1.23(1.18 \text { to } 1.31) \\
I^{2}=0 \%\end{array}$ & High \\
\hline Function & Short & $28(N=13,473)^{62-86}$ & Fair & Direct & Consistent & Precise & Undetected & $\begin{array}{c}\text { Small effect } \\
\text { (WOMAC, LI) } \\
\text { SMD }-0.32(-0.37 \text { to - } \\
0.28), \mathrm{I}^{2}=24 \%\end{array}$ & High \\
\hline Quality of Life & Short & $3(\mathrm{~N}=1,027)^{69,71,81}$ & Fair & Direct & Consistent & Imprecise & Unknown & $\begin{array}{c}\text { No effect } \\
\text { (SF-36 MCS or PCS) } \\
\text { MCS: MD } 0.61 \text { (-0.50 to } \\
1.79) \\
\text { PCS: MD } 2.95 \text { (1.79 to } \\
4.18) \\
\end{array}$ & Moderate \\
\hline
\end{tabular}

a Study references are in Appendix J.

b Study Quality: poor-quality studies not synthesized

${ }^{\mathrm{c}}$ Pain Response main findings, percentages represent threshold for Pain Response

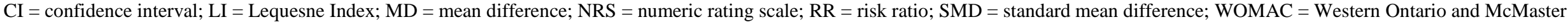
Universities Osteoarthritis Index

\section{Table H-8. Osteoarthritis - topical diclofenac vs. placebo}

\begin{tabular}{|c|c|c|c|c|c|c|c|c|c|}
\hline Outcome & Duration & $\begin{array}{l}\text { Number of } \\
\text { Studies }^{\mathrm{a}}(\mathbf{n} \\
\text { participants })\end{array}$ & Study Quality ${ }^{b}$ & Directness & $\begin{array}{l}\text { Consistency } \\
\text { (1 study= } \\
\text { Unknown) }\end{array}$ & Precision & $\begin{array}{l}\text { Publication } \\
\text { Bias }\end{array}$ & $\begin{array}{c}\text { Main Findings } \\
\text { Effect Size ( } 95 \% \text { Cl) }\end{array}$ & $\begin{array}{l}\text { Strength } \\
\text { of } \\
\text { Evidence } \\
\text { Grade }\end{array}$ \\
\hline $\begin{array}{l}\text { Pain Improvement } \\
\text { (Continuous) }\end{array}$ & Short & $\begin{array}{c}4(\mathrm{~N}=1,541)^{83,87-} \\
89\end{array}$ & Fair & Direct & Consistent & Precise & Unknown & $\begin{array}{c}\text { Small effect } \\
\text { (WOMAC) } \\
\text { MD -0.58 (-0.81 to -0.35), } \\
\left.\right|^{2}=0 \%\end{array}$ & Moderate \\
\hline $\begin{array}{l}\text { Pain Response } \\
\text { (Dichotomous) }\end{array}$ & Short & $3(N=1,232)^{87-89}$ & Good & Direct & Consistent & Imprecise & Unknown & $\begin{array}{c}\text { Small effect } \\
\text { RR } 1.20(1.09 \text { to } 1.38) \\
I^{2}=0 \%\end{array}$ & Moderate \\
\hline Function & Short & $\begin{array}{c}4(\mathrm{~N}=1,538)^{83,87-} \\
89\end{array}$ & Fair & Direct & Inconsistent & Precise & Unknown & $\begin{array}{c}\text { No effect } \\
\text { (WOMAC) } \\
\text { MD -0.51 (-1.06 to 0.04), } \\
\left.\right|^{2}=94 \%\end{array}$ & Low \\
\hline
\end{tabular}

a Study references are in Appendix J.

b Study Quality, poor-quality studies not synthesized

${ }^{\mathrm{c}}$ Pain Response main findings, percentages represent threshold for Pain Response

$\mathrm{CI}$ = confidence interval; $\mathrm{MD}$ = mean difference; $\mathrm{RR}$ = risk ratio; WOMAC = Western Ontario and McMaster Universities Osteoarthritis Index 
Table H-9. Osteoarthritis - oral NSAIDs: head-to-head comparisons

\begin{tabular}{|c|c|c|c|c|c|c|c|c|c|}
\hline Outcome & Duration & $\begin{array}{l}\text { Number of } \\
\text { Studies }^{\mathrm{a}}(\mathrm{n} \\
\text { participants) }^{\text {natipan }}\end{array}$ & Study Qualityb & Directness & $\begin{array}{l}\text { Consistency } \\
\text { (1 study= } \\
\text { Unknown) }\end{array}$ & Precision & $\begin{array}{l}\text { Publication } \\
\text { Bias }\end{array}$ & $\begin{array}{c}\text { Main Findings } \\
\text { Effect Size }(95 \% \mathrm{Cl})\end{array}$ & $\begin{array}{l}\text { Strength } \\
\text { of } \\
\text { Evidence } \\
\text { Grade }\end{array}$ \\
\hline \multirow[t]{3}{*}{$\begin{array}{l}\text { Pain Improvement } \\
\text { (Continuous) }\end{array}$} & Short & $\begin{array}{c}4(\mathrm{~N}=1,313) \\
86,90-92\end{array}$ & Fair & Direct & Consistent & Imprecise & Unknown & $\begin{array}{c}\text { Moderate effect } \\
\text { (VAS, WOMAC pain } \\
\text { Subscale) } \\
\text { Diclofenac vs. celecoxib } \\
\text { MD -12.2 (2.2 to } 22.1) \\
\text { Small effect } \\
\text { Diclofenac vs. } 3.75 \mathrm{mg} / \mathrm{d} \\
\text { meloxicam } \\
\text { No effect } 7 \text { mg vs. } 15 \mathrm{mg} \\
\text { No effect with other } \\
\text { comparisons }\end{array}$ & Low \\
\hline & Intermediate & $1(\mathrm{~N}=586)^{93}$ & Fair & Direct & Consistent & Imprecise & Unknown & $\begin{array}{c}\text { No effect } \\
\text { (VAS, WOMAC pain } \\
\text { subscale) } \\
\text { Celecoxib vs. naproxen }\end{array}$ & Low \\
\hline & Long & $1(\mathrm{~N}=916)^{94}$ & Fair & Direct & Unknown & Precise & Unknown & $\begin{array}{l}\text { No significant differences } \\
\text { between groups at } \\
\text { endpoint } \\
\text { (VAS) } \\
\text { Celecoxib vs. diclofenac }\end{array}$ & Low \\
\hline \multirow[t]{2}{*}{$\begin{array}{l}\text { Pain Response }^{c} \\
\text { (Dichotomous) }\end{array}$} & Short & $2(\mathrm{~N}=849)^{86,92}$ & Fair & Direct & Consistent & Imprecise & Unknown & $\begin{array}{c}\text { No effect } \\
\text { Diclofenac dispersible vs. } \\
\text { enteric coated: RR } 0.82 \\
\quad(0.73 \text { to } 1.09) \\
\\
\text { Ibuprofen vs. nabumetone: } \\
\text { RR } 1.2 \text { (0.88 to } 1.66) \\
\end{array}$ & Low \\
\hline & Intermediate & $1(N=586)^{93}$ & Fair & Direct & Consistent & Imprecise & Unknown & $\begin{array}{c}\text { No effect } \\
\text { Celecoxib vs. naproxen }\end{array}$ & Low \\
\hline \multirow[t]{2}{*}{ Function } & Short & $2(\mathrm{~N}=301)^{86,92}$ & Fair & Direct & Consistent & Imprecise & Unknown & $\begin{array}{c}\text { Moderate effect } \\
\text { (WOMAC 0-68) } \\
\text { Diclofenac vs. celecoxib } \\
\text { RR } 2.06 \text { (1.37 to 3.08) } \\
\\
\text { No effect: Diclofenac vs. } \\
\text { meloxicam } 7 \text { or } 15 \mathrm{mg} / \mathrm{d} \text {, } \\
\text { but small effect over } \\
\text { meloxicam } 3.75 \mathrm{mg} / \mathrm{d}\end{array}$ & Low \\
\hline & Intermediate & $2(\mathrm{~N}=921)^{93,95}$ & Fair & Direct & Consistent & Imprecise & Unknown & $\begin{array}{c}\text { No effect } \\
\text { (WOMAC) } \\
\text { Celecoxib vs. naproxen } \\
\text { Meloxicam vs. diclofenac }\end{array}$ & Low \\
\hline
\end{tabular}

a Study references are in Appendix J. 
b Study Quality: poor-quality studies not synthesized

${ }^{\mathrm{c}}$ Pain Response main findings, percentages represent threshold for Pain Response

$\mathrm{CI}$ = confidence interval; $\mathrm{MD}$ = mean difference; RR = risk ratio; VAS = visual analogue scale; WOMAC = Western Ontario and McMaster Universities Osteoarthritis Index

Table H-10. Osteoarthritis - antidepressants: duloxetine vs. placebo

\begin{tabular}{|c|c|c|c|c|c|c|c|c|c|}
\hline Outcome & Duration & $\begin{array}{l}\text { Number of } \\
\text { Studies }^{a}(n \\
\text { participants) }\end{array}$ & $\begin{array}{c}\text { Study } \\
\text { Quality }\end{array}$ & Directness & $\begin{array}{c}\text { Consistency } \\
\text { (1 study= } \\
\text { Unknown) }\end{array}$ & Precision & $\begin{array}{l}\text { Publication } \\
\text { Bias }\end{array}$ & $\begin{array}{c}\text { Main Findings } \\
\text { Effect Size }(95 \% \mathrm{Cl})\end{array}$ & $\begin{array}{c}\text { Strength of } \\
\text { Evidence } \\
\text { Grade }\end{array}$ \\
\hline $\begin{array}{c}\text { Pain } \\
\text { Improvement } \\
\text { (Continuous) }\end{array}$ & Short & $6(\mathrm{~N}=1,508)^{96-101}$ & Good & Direct & Consistent & Precise & Unknown & $\begin{array}{c}\text { Small effect } \\
(0 \text { to10 scale }) \\
\text { MD }-0.75(-1.05 \text { to }-0.53), I^{2}=15 \%\end{array}$ & High \\
\hline $\begin{array}{l}\text { Pain Response } \\
\text { (Dichotomous) }\end{array}$ & Short & $\begin{array}{c}4(\mathrm{~N}=1,247) \\
97,98,100,101\end{array}$ & Good & Direct & Consistent & Precise & Unknown & $\begin{array}{c}\text { Moderate effect } \\
\text { RR 1.37 (1.24 to } 1.52), l^{2}=0 \% \\
\end{array}$ & High \\
\hline Function & Short & $\begin{array}{l}5(\mathrm{~N}=1,480)^{96-} \\
98,100,101\end{array}$ & Good & Direct & Consistent & Precise & Unknown & $\begin{array}{c}\text { Small effect } \\
\text { (WOMAC, BPI) } \\
\text { SMD }-0.27(-0.41 \text { to }-0.12) \\
I^{2}=27 \% \\
\end{array}$ & High \\
\hline Quality of Life & Short & $2(\mathrm{~N}=570)^{98,100}$ & Good & Direct & Consistent & Precise & Unknown & $\begin{array}{c}\text { Small effect } \\
(E Q-5 D, 0 \text { to } 1 \text { scale }) \\
\text { MD } 0.05(0.02 \text { to } 0.08), I^{2}=0 \%\end{array}$ & High \\
\hline
\end{tabular}

${ }^{a}$ Study references are in Appendix J.

b Study Quality: poor-quality studies not synthesized

${ }^{c}$ Pain Response main findings, percentages represent threshold for Pain Response

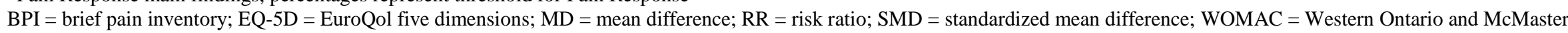
Universities Osteoarthritis Index

Table H-11. Osteoarthritis - acetaminophen vs. placebo

\begin{tabular}{|c|c|c|c|c|c|c|c|c|c|}
\hline Outcome & Duration & $\begin{array}{l}\text { Number of } \\
\text { Studies }^{a}(n \\
\text { participants) }\end{array}$ & Study Quality & Directness & $\begin{array}{l}\text { Consistency } \\
\text { (1 study= } \\
\text { Unknown) }\end{array}$ & Precision & $\begin{array}{l}\text { Publication } \\
\text { Bias }\end{array}$ & $\begin{array}{c}\text { Main Findings } \\
\text { Effect Size }(95 \% \mathrm{Cl})\end{array}$ & $\begin{array}{c}\text { Strength of } \\
\text { Evidence } \\
\text { Grade }\end{array}$ \\
\hline \multirow{2}{*}{$\begin{array}{c}\text { Pain } \\
\text { Improvement } \\
\text { (Continuous) }\end{array}$} & Short & $3 \underset{66,102,103}{(N=1,082)}$ & Fair & Direct & Consistent & Imprecise & Unknown & $\begin{array}{c}\text { No effect } \\
\text { MD }-0.34(-0.66 \text { to } 0.03) \\
1^{2}=0 \%\end{array}$ & Low \\
\hline & Intermediate & $1(\mathrm{~N}=212)^{104}$ & Fair & Direct & Unknown & Imprecise & Unknown & $\begin{array}{c}\text { No effect } \\
\text { (WOMAC subscale) } \\
\text { MD }-0.30(-0.77 \text { to } 0.17)\end{array}$ & Low \\
\hline \multirow[t]{2}{*}{ Function } & Short & $3 \underset{66,102,103}{(N=1,081)}$ & Fair & Direct & Consistent & Imprecise & Unknown & $\begin{array}{c}\text { No effect } \\
\text { (WOMAC) } \\
\text { SMD -0.14 (-0.29 to 0.04), } \\
1^{2}=0 \%\end{array}$ & Low \\
\hline & Intermediate & $1(\mathrm{~N}=212)^{104}$ & Fair & Direct & Unknown & Imprecise & Unknown & $\begin{array}{l}\text { No effect }(<\text { small) } \\
\text { (WOMAC, } 1 \text { to } 100) \\
\text { MD }-3.7(-6.9 \text { to }-0.5)\end{array}$ & Low \\
\hline
\end{tabular}

a Study references are in Appendix J.

b Study Quality: poor-quality studies not synthesized 
${ }^{\mathrm{c}}$ Pain Response main findings, percentages represent threshold for Pain Response

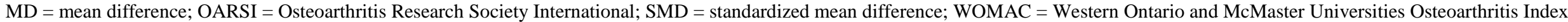

Table H-12. Osteoarthritis - anticonvulsants vs. antidepressants: duloxetine vs. pregabalin

\begin{tabular}{|c|c|c|c|c|c|c|c|c|c|}
\hline Outcome & Duration & $\begin{array}{c}\text { Number of } \\
\text { Studies }^{a}(n \\
\text { participants) }\end{array}$ & $\begin{array}{c}\text { Study } \\
\text { Qualityb }\end{array}$ & Directness & $\begin{array}{c}\text { Consistency } \\
(1 \text { study= } \\
\text { Unknown) }\end{array}$ & Precision & $\begin{array}{c}\text { Publication } \\
\text { Bias }\end{array}$ & $\begin{array}{c}\text { Main Findings } \\
\text { Effect Size }(95 \% \mathrm{Cl})\end{array}$ & $\begin{array}{c}\text { Strength of } \\
\text { Evidence } \\
\text { Grade } \\
\end{array}$ \\
\hline $\begin{array}{c}\text { Pain } \\
\text { Improvement } \\
\text { (Continuous) }\end{array}$ & Short & $1(\mathrm{~N}=65)^{105}$ & Good & Direct & Unknown & Imprecise & Unknown & $\begin{array}{l}\text { (NRS scale, } 0 \text { to } 10): \\
\text { Pregabalin } 300 \mathrm{mg} / \mathrm{d}:-2.7(-3.5 \text { to }-1.9) \\
\text { vs. duloxetine } 60 \mathrm{mg} / \mathrm{d}:-2.3(-3.8 \text { to }- \\
\text { 0.9) vs. placebo: }-0.9(-2.0 \text { to } 0.2) ; \\
\text { Pregabalin vs. placebo }=0.023 \text { and } 0.19\end{array}$ & Insufficient \\
\hline Function & Short & $1(\mathrm{~N}=65)^{105}$ & Good & Direct & Unknown & Imprecise & Unknown & $\begin{array}{l}\text { (AUSCAN Function scale, } 0 \text { to } 900): \\
\text { Pregabalin } 300 \mathrm{mg} / \mathrm{d}:-46.4(-341.7 \text { to - } \\
\text { 151.0) vs. duloxetine } 60 \mathrm{mg} / \mathrm{d}:-101.8(- \\
248.4 \text { to }-44.7) \text { vs. placebo: }-67.3(- \\
156.4 \text { to }-21.8) ; \text { Pregabalin vs. placebo } \\
=0.009 \text { and }>0.05\end{array}$ & Insufficient \\
\hline
\end{tabular}

a Study references are in Appendix J.

b Study Quality: poor-quality studies not synthesized

AUSCAN = Australian Canadian osteoarthritis hand index; CI = confidence interval; NRS = numeric rating scale

Table H-13. Osteoarthritis - acetaminophen vs. NSAIDs

\begin{tabular}{|c|c|c|c|c|c|c|c|c|c|}
\hline Outcome & Duration & $\begin{array}{l}\text { Number of } \\
\text { Studies }^{\text {a }}(\mathbf{n} \\
\text { participants) }\end{array}$ & $\begin{array}{l}\text { Study } \\
\text { Qualityb }\end{array}$ & Directness & $\begin{array}{c}\text { Consistency } \\
\text { (1 study= } \\
\text { Unknown) }\end{array}$ & Precision & $\begin{array}{c}\text { Publication } \\
\text { Bias }\end{array}$ & $\begin{array}{l}\text { Main Findings } \\
\text { Effect Size }\end{array}$ & $\begin{array}{l}\text { Strength of } \\
\text { Evidence } \\
\text { Grade }\end{array}$ \\
\hline $\begin{array}{c}\text { Pain } \\
\text { Improvement } \\
\text { (Continuous) }\end{array}$ & Short & $1(\mathrm{~N}=85)^{66}$ & Fair & Direct & Inconsistent & Imprecise & Unknown & $\begin{array}{c}\text { (WOMAC) } \\
\text { Diclofenac } 150 \mathrm{mg} / \mathrm{d} \text { vs. acetaminophen } \\
4000 \mathrm{mg} / \mathrm{d} \text {; diclofenac shows greater } \\
\text { pain improvement (-53.9 vs. }-23.8 \\
\text { WOMAC; } \mathrm{p}=0.003)\end{array}$ & Insufficient \\
\hline Function & Short & $1(\mathrm{~N}=25)^{66}$ & Fair & Direct & Unknown & Imprecise & Unknown & $\begin{array}{c}\text { (WOMAC) } \\
\text { Diclofenac } 150 \mathrm{mg} / \mathrm{d} \text { vs. acetaminophen } \\
4000 \mathrm{mg} / \mathrm{d} \text {; diclofenac shows greater } \\
\text { function improvement }(-163.0 \text { vs. }-41.8 \\
\text { WOMAC; } p<0.001)\end{array}$ & Insufficient \\
\hline
\end{tabular}

${ }^{\text {a }}$ Study references are in Appendix J.

b Study Quality: poor-quality studies not synthesized

WOMAC = Western Ontario and McMaster Universities Osteoarthritis Index 
Table H-14. Inflammatory arthritis - oral NSAIDS vS. placebo

\begin{tabular}{|c|c|c|c|c|c|c|c|c|c|}
\hline Outcome & Duration & $\begin{array}{c}\text { Number of } \\
\text { Studies }^{\mathbf{a}} \\
\text { (n participants) }\end{array}$ & $\begin{array}{c}\text { Study } \\
\text { Qualityb }\end{array}$ & Directness & $\begin{array}{l}\text { Consistency } \\
\text { (1 study= } \\
\text { Unknown) }\end{array}$ & Precision & $\begin{array}{c}\text { Publication } \\
\text { Bias }\end{array}$ & $\begin{array}{c}\text { Main Findings } \\
\text { Effect Size }(95 \% \mathrm{Cl})\end{array}$ & $\begin{array}{c}\text { Strength of } \\
\text { Evidence } \\
\text { Grade }\end{array}$ \\
\hline \multirow{3}{*}{$\begin{array}{l}\text { Pain } \\
\text { Improvement } \\
\text { (Continuous) }\end{array}$} & Short & $9(\mathrm{~N}=4,543)^{106-114}$ & Fair & Direct & Consistent & Precise & Undetected & $\begin{array}{c}\text { Small effect } \\
(0 \text { to } 10 \text { scale }) \\
\text { MD }-0.97(-1.33 \text { to }-0.74), I^{2}=39 \%\end{array}$ & Moderate \\
\hline & Intermediate & $1(\mathrm{~N}=563)^{110}$ & Fair & Direct & Unknown & Precise & Unknown & $\begin{array}{c}\text { Small effect } \\
(0 \text { to } 10 \text { scale }) \\
\text { Naproxen } 1000 \mathrm{mg} / \mathrm{d} \\
\text { MD }-0.53(-0.93 \text { to }-0.13)\end{array}$ & Low \\
\hline & Long & $1(\mathrm{~N}=365)^{115}$ & Fair & Direct & Unknown & Precise & Unknown & $\begin{array}{c}\text { Large effect } \\
(0 \text { to10 scale }) \\
\text { Meloxicam } 15-22.5 \mathrm{mg} / \mathrm{d} \\
\text { MD }-2.10(-2.72 \text { to }-1.48) \\
\end{array}$ & Low \\
\hline \multirow{3}{*}{$\begin{array}{c}\text { Pain } \\
\text { Response }^{c} \\
\text { (Dichotomous) }\end{array}$} & Short & $\begin{array}{c}7(\mathrm{~N}=3,434)^{106-} \\
108,110,113,114,116\end{array}$ & Fair & Direct & Consistent & Precise & Unknown & $\begin{array}{c}\text { Moderate effect } \\
\text { (ACR 20; ASAS 20) } \\
\text { RR 1.58 (1.34 to 2.06), } \mathrm{I}^{2}=52 \%\end{array}$ & Moderate \\
\hline & Intermediate & $1(\mathrm{~N}=563)^{110}$ & Fair & Direct & Unknown & Precise & Unknown & $\begin{array}{l}\text { Small effect (ACR 20) } \\
\text { Naproxen } 1000 \mathrm{mg} / \mathrm{d}: \\
\text { RR } 1.28(1.03 \text { to } 1.60) \\
\end{array}$ & Low \\
\hline & Long & $1(\mathrm{~N}=365)^{115}$ & Fair & Direct & Unknown & Precise & Unknown & $\begin{array}{l}\text { Large effect }(\geq 50 \%) \\
\text { Meloxicam 15- } 22.5 \mathrm{mg} / \mathrm{d} \text { : } \\
\text { RR } 3.05 \text { (1.98 to } 4.71)\end{array}$ & Low \\
\hline \multirow{3}{*}{ Function } & Short & $\begin{array}{c}7(\mathrm{~N}=4,284)^{106-} \\
110,113,114\end{array}$ & Fair & Direct & Consistent & Precise & Undetected & $\begin{array}{c}\text { Small effect } \\
\text { (HAQ; BASFI) } \\
\text { SMD -0.34 }(-0.51 \text { to }-0.20), I^{2}=67 \%\end{array}$ & Moderate \\
\hline & Intermediate & $1(\mathrm{~N}=563)^{110}$ & Fair & Direct & Unknown & Precise & Unknown & $\begin{array}{l}\text { Small effect (HAQ-DI, } 0-3) \\
\text { Naproxen } 1000 \mathrm{mg} / \mathrm{d}: \\
\text { MD }-0.18(-0.35 \text { to }-0.02)\end{array}$ & Low \\
\hline & Long & $1(\mathrm{~N}=365)^{115}$ & Fair & Direct & Unknown & Precise & Unknown & $\begin{array}{c}\text { No effect } \\
\left(\text { ASFl }^{117}, 0-40\right) \\
\text { Meloxicam 15-22.5 mg/d: } \\
\text { MD -0.63 (-0.85 to }-0.40)\end{array}$ & Low \\
\hline Quality of Life & Short & $2(\mathrm{~N}=1,204)^{108,114}$ & Fair & Direct & Inconsistent & Imprecise & Unknown & $\begin{array}{c}\text { (ASQoL, } 0 \text { to } 18) \\
\text { Naproxen } 1000 \mathrm{mg} / \mathrm{d}: \mathrm{MD}-2.9 \\
\mathrm{p}=0.04 \\
\text { (SF-36 PCS and MCS) } \\
\text { Celecoxib } 200-800 \mathrm{mg} / \mathrm{d} \text { or } \\
\text { Naproxen } 1000 \mathrm{mg} / \mathrm{d}\end{array}$ & Insufficient \\
\hline
\end{tabular}

${ }^{a}$ Study references are in Appendix J.

b Study Quality: poor-quality studies not synthesized

${ }^{c}$ Pain Response main findings, percentages represent threshold for Pain Response

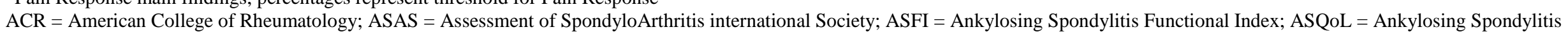

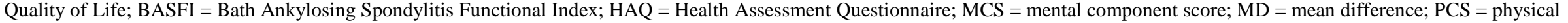

component score; $\mathrm{RR}$ = risk ratio; $\mathrm{SMD}=$ standardized mean difference 
Table H-15. Inflammatory arthritis - oral NSAIDS: head-to-head comparisons

\begin{tabular}{|c|c|c|c|c|c|c|c|c|c|}
\hline Outcome & Duration & $\begin{array}{c}\text { Number of } \\
\text { Studies }^{\mathrm{a}} \\
\text { (n participants) }\end{array}$ & $\begin{array}{c}\text { Study } \\
\text { Qualityb }^{b}\end{array}$ & Directness & $\begin{array}{l}\text { Consistency } \\
\text { (1 study= } \\
\text { Unknown) }\end{array}$ & Precision & $\begin{array}{c}\text { Publication } \\
\text { Bias }\end{array}$ & $\begin{array}{l}\text { Main Findings } \\
\text { Effect Size }\end{array}$ & $\begin{array}{c}\text { Strength of } \\
\text { Evidence } \\
\text { Grade }\end{array}$ \\
\hline \multirow{8}{*}{$\begin{array}{c}\text { Pain } \\
\text { Improvement } \\
\text { (Continuous) }\end{array}$} & \multirow{6}{*}{ Short } & $3(\mathrm{~N}=1,453)^{118-120}$ & Fair & Direct & Consistent & Precise & Unknown & $\begin{array}{c}\text { No effect } \\
(0-10 \text { scale }) \\
\text { Celecoxib } 200-400 \mathrm{mg} / \mathrm{d} \text { vs. } \\
\text { diclofenac } 150 \mathrm{mg} / \mathrm{d}: \mathrm{NS}\end{array}$ & Moderate \\
\hline & & $2(\mathrm{~N}=1,132)^{106,114}$ & Fair & Direct & Consistent & Imprecise & Unknown & $\begin{array}{c}\text { No effect } \\
(0-10 \text { scale }) \\
\text { Celecoxib } 200-400 \mathrm{mg} / \mathrm{d} \text { vs. } \\
\text { naproxen } 1000 \mathrm{mg} / \mathrm{d}: \mathrm{NS}\end{array}$ & Low \\
\hline & & $1(\mathrm{~N}=103)^{121}$ & Fair & Direct & Unknown & Imprecise & Unknown & $\begin{array}{l}\text { (Non-visual scale, 1-5) } \\
\text { Diclofenac vs. etodolac }\end{array}$ & Insufficient \\
\hline & & $1(\mathrm{~N}=717)^{109}$ & Fair & Direct & Unknown & Imprecise & Unknown & $\begin{array}{c}\text { No effect } \\
\text { (VAS, 0-100) } \\
\text { Diclofenac vs. meloxicam } \\
\end{array}$ & Low \\
\hline & & $1(\mathrm{~N}=39)^{122}$ & Fair & Direct & Unknown & Imprecise & Unknown & $\begin{array}{l}\text { (Non-visual scale, 1-5) } \\
\text { Etodolac vs. naproxen }\end{array}$ & Insufficient \\
\hline & & $2(\mathrm{~N}=621)^{123,124}$ & Fair & Direct & Consistent & Precise & Unknown & $\begin{array}{c}\text { No effect } \\
(0-10 \text { scale }) \\
\text { Nabumetone } 2000 \mathrm{mg} / \mathrm{d} \text { vs. } \\
\text { naproxen } 1000 \mathrm{mg} / \mathrm{d}: \mathrm{NS}\end{array}$ & Low \\
\hline & \multirow{2}{*}{ Intermediate } & $1(\mathrm{~N}=379)^{125}$ & Fair & Direct & Unknown & Precise & Unknown & $\begin{array}{c}\text { No effect } \\
\text { (VAS, 0-100) } \\
\text { Meloxicam vs. naproxen }\end{array}$ & Low \\
\hline & & $1(\mathrm{~N}=47)^{126}$ & Fair & Direct & Unknown & Imprecise & Unknown & $\begin{array}{c}\text { No effect } \\
\text { (\% better, \%same, \%worse) } \\
\text { Nabumetone vs. naproxen }\end{array}$ & Low \\
\hline \multirow{3}{*}{$\begin{array}{l}\text { Pain Response } \\
\text { (Dichotomous) }\end{array}$} & \multirow{3}{*}{ Short } & $3(\mathrm{~N}=1,443)^{118-120}$ & Fair & Direct & Consistent & Precise & Unknown & $\begin{array}{c}\text { No effect } \\
\text { (ACR 20; ASAS 20) } \\
\text { Celecoxib 200-400 mg/d vs. } \\
\text { diclofenac } 150 \mathrm{mg} / \mathrm{d}: \text { NS }\end{array}$ & Moderate \\
\hline & & $2(\mathrm{~N}=1,133)^{106,114}$ & Fair & Direct & Inconsistent & Imprecise & Unknown & $\begin{array}{c}\text { No effect } \\
\text { (ACR 20; ASAS 20) } \\
\text { Celecoxib 200-400 mg/d vs. } \\
\text { naproxen } 1000 \mathrm{mg} / \mathrm{d} \text { : NS }\end{array}$ & Low \\
\hline & & $1(\mathrm{~N}=344)^{124}$ & Fair & Direct & Unknown & Imprecise & Unknown & $\begin{array}{c}\text { No effect } \\
\text { (Pt global assess, \% improved) } \\
\text { Nabumetone } 2000 \mathrm{mg} / \mathrm{d} \text { vs. } \\
\text { naproxen } 1000 \mathrm{mg} / \mathrm{d} \text { : NS }\end{array}$ & Low \\
\hline Function & Short & $3(\mathrm{~N}=1,448)^{118-120}$ & Fair & Direct & Consistent & Precise & Unknown & $\begin{array}{c}\text { No effect } \\
\text { (mHAQ; BASFI) } \\
\text { Celecoxib 200-400 mg/d vs. } \\
\text { diclofenac } 150 \mathrm{mg} / \mathrm{d} \text { : NS }\end{array}$ & Moderate \\
\hline
\end{tabular}




\begin{tabular}{|c|c|c|c|c|c|c|c|c|c|}
\hline Outcome & Duration & $\begin{array}{c}\text { Number of } \\
\text { Studies }^{\mathrm{a}} \\
\text { (n participants) }\end{array}$ & $\begin{array}{l}\text { Study } \\
\text { Qualityb }^{b}\end{array}$ & Directness & $\begin{array}{l}\text { Consistency } \\
\text { (1 study= } \\
\text { Unknown) }\end{array}$ & Precision & $\begin{array}{l}\text { Publication } \\
\text { Bias }\end{array}$ & $\begin{array}{l}\text { Main Findings } \\
\text { Effect Size }\end{array}$ & $\begin{array}{l}\text { Strength of } \\
\text { Evidence } \\
\text { Grade }\end{array}$ \\
\hline & & $2(\mathrm{~N}=1,373)^{106,114}$ & Fair & Direct & Consistent & Imprecise & Unknown & $\begin{array}{c}\text { No effect } \\
\text { (HAQ; BASFI) } \\
\text { Celecoxib 200-400 mg/d vs. } \\
\text { naproxen } 1000 \mathrm{mg} / \mathrm{d} \text { : NS }\end{array}$ & Low \\
\hline & & $1(\mathrm{~N}=103)^{121}$ & Fair & Direct & Unknown & Imprecise & Unknown & $\begin{array}{l}\text { (Non-visual scale, 1-4) } \\
\text { Diclofenac vs. etodolac }\end{array}$ & Insufficient \\
\hline & & $1(\mathrm{~N}=717)^{109}$ & Fair & Direct & Unknown & Imprecise & Unknown & $\begin{array}{c}\text { No effect } \\
\text { (mHAQ, max 3) } \\
\text { Diclofenac vs. meloxicam }\end{array}$ & Low \\
\hline & & $1(\mathrm{~N}=346)^{124}$ & Fair & Direct & Unknown & Imprecise & Unknown & $\begin{array}{c}\text { No effect } \\
\text { (ACR class) } \\
\text { Nabumetone } 2000 \mathrm{mg} / \mathrm{d} \text { vs. } \\
\text { naproxen } 1000 \mathrm{mg} / \mathrm{d} \text { : NS }\end{array}$ & Low \\
\hline Quality of Life & Short & $1(\mathrm{~N}=917)^{114}$ & Fair & Direct & Unknown & Precise & Unknown & $\begin{array}{c}\text { No effect } \\
\text { (SF-36 PCS and MCS) } \\
\text { Celecoxib vs. naproxen }\end{array}$ & Low \\
\hline
\end{tabular}

a Study references are in Appendix J.

b Study Quality: poor-quality studies not synthesized

c Pain Response main findings, percentages represent threshold for Pain Response

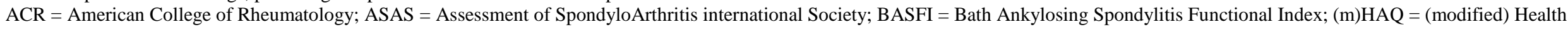

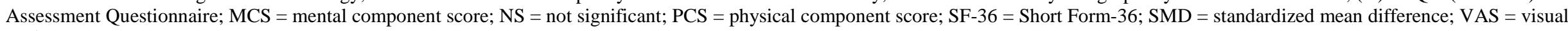
analogue score

Table H-46. Inflammatory arthritis - antidepressants: placebo controlled trials

\begin{tabular}{|c|c|c|c|c|c|c|c|c|c|}
\hline Outcome & Duration & $\begin{array}{c}\text { Number of } \\
\text { Studies }^{\mathrm{a}} \\
\text { (n participants) }\end{array}$ & $\begin{array}{l}\text { Study } \\
\text { Quality }\end{array}$ & Directness & $\begin{array}{l}\text { Consistency } \\
\text { (1 study= } \\
\text { Unknown) }\end{array}$ & Precision & $\begin{array}{l}\text { Publication } \\
\text { Bias }\end{array}$ & $\begin{array}{l}\text { Main Findings } \\
\text { Effect Size }\end{array}$ & $\begin{array}{l}\text { Strength of } \\
\text { Evidence } \\
\text { Grade }\end{array}$ \\
\hline $\begin{array}{l}\text { Pain Improvement } \\
\text { (Continuous) }\end{array}$ & Short & $1(\mathrm{~N}=36)^{127}$ & Fair & Direct & Unknown & Imprecise & Unknown & $\begin{array}{c}\text { (Non-visual scale, 0-4) } \\
\text { Amitriptyline } 50-75 \mathrm{mg} / \mathrm{d}\end{array}$ & Insufficient \\
\hline
\end{tabular}

a Study references are in Appendix J.

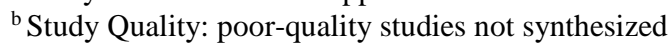


Table H-17. Low back pain - antidepressants and anticonvulsants: placebo controlled trials

\begin{tabular}{|c|c|c|c|c|c|c|c|c|c|}
\hline Outcome & Duration & $\begin{array}{c}\text { Number of } \\
\text { Studies }^{\mathrm{a}} \\
\text { (n participants) }\end{array}$ & $\begin{array}{c}\text { Study } \\
\text { Quality }\end{array}$ & Directness & $\begin{array}{l}\text { Consistency } \\
\text { (1 study= } \\
\text { Unknown) }\end{array}$ & Precision & $\begin{array}{c}\text { Publication } \\
\text { Bias }\end{array}$ & $\begin{array}{c}\text { Main Findings } \\
\text { Effect Size }(95 \% \mathrm{Cl})\end{array}$ & $\begin{array}{c}\text { Strength of } \\
\text { Evidence } \\
\text { Grade }\end{array}$ \\
\hline \multirow{5}{*}{$\begin{array}{l}\text { Pain } \\
\text { Improvement } \\
\text { (Continuous) }\end{array}$} & \multirow{4}{*}{ Short } & $3(\mathrm{~N}=1,491)^{128-130}$ & Fair & Direct & Consistent & Precise & Unknown & $\begin{array}{c}\text { Small effect } \\
\text { (BPI Pain Scale) } \\
\text { Duloxetine vs. placebo } \\
\text { MD }-0.50(-0.71 \text { to }-0.29), \mathrm{I}^{2}=0 \%\end{array}$ & Moderate \\
\hline & & $1(\mathrm{~N}=78)^{131}$ & Fair & Direct & Unknown & Imprecise & Unknown & $\begin{array}{l}\text { (DDS, } 0-20) \\
\text { Despiramine vs. placebo } \\
\text { MD -0.80 (-2.64 to } 1.04)\end{array}$ & Insufficient \\
\hline & & $1(\mathrm{~N}=86)^{131}$ & Fair & Direct & Unknown & Imprecise & Unknown & $\begin{array}{c}\text { (DDS, } 0-20) \\
\text { Fluoxetine vs. placebo } \\
\text { MD } 0.70(-1.40 \text { to } 2.80)\end{array}$ & Insufficient \\
\hline & & $1(\mathrm{~N}=108)^{132}$ & Fair & Direct & Unknown & Imprecise & Unknown & $\begin{array}{c}\text { (DDS, 0-20) } \\
\text { Gabapentin vs. placebo } \\
p=0.42\end{array}$ & Insufficient \\
\hline & Intermediate & $1(\mathrm{~N}=146)^{133}$ & Good & Direct & Unknown & Imprecise & Unknown & $\begin{array}{l}\text { No effect (VAS) } \\
\text { Amitriptyline vs. placebo } \\
\text { MD -7.81 (-15.7 to 0.10) }\end{array}$ & Low \\
\hline $\begin{array}{c}\text { Pain } \\
\text { Response } \\
\text { (Dichotomous) }\end{array}$ & Short & $3(\mathrm{~N}=1,235)^{128-130}$ & Fair & Direct & Consistent & Imprecise & Unknown & $\begin{array}{c}\text { Small effect } \\
\text { Duloxetine vs. placebo } \\
\text { RR } 1.25(1.11 \text { to } 1.40), \mathrm{I}^{2}=0 \%\end{array}$ & Low \\
\hline \multirow{4}{*}{ Function } & \multirow{3}{*}{ Short } & $3(\mathrm{~N}=1,214)^{128-130}$ & Fair & Direct & Consistent & Precise & Unknown & $\begin{array}{c}\text { No effect } \\
\text { (BPI Interference Scale) } \\
\text { Duloxetine vs. placebo } \\
\text { MD -0.36 (-0.73 to }-0.04), I^{2}=34 \%\end{array}$ & Moderate \\
\hline & & $1(\mathrm{~N}=78)^{131}$ & Fair & Direct & Unknown & Imprecise & Unknown & $\begin{array}{c}\text { (RMDQ score) } \\
\text { Despiramine vs. placebo } \\
2.3 \text { vs. } 4.1, p=0.05 \\
\text { (Physician-rated CGI) } \\
\text { Despiramine vs. placebo } \\
5.9 \text { vs. } 4.8, p=0.003\end{array}$ & Insufficient \\
\hline & & $1(\mathrm{~N}=108)^{132}$ & Fair & Direct & Unknown & Imprecise & Unknown & $\begin{array}{c}\text { (CGI-C "minimal improvement") } \\
\text { Gabapentin vs. placebo } \\
37 \% \text { vs. } 33 \%, p=0.95\end{array}$ & Insufficient \\
\hline & Intermediate & $1(\mathrm{~N}=146)^{133}$ & Good & Direct & Unknown & Imprecise & Unknown & $\begin{array}{c}\text { No effect } \\
\text { (RMDQ) } \\
\text { Amitriptyline vs. placebo } \\
\text { MD -0.98 (-2.42 to } 0.46)\end{array}$ & Low \\
\hline Quality of Life & Short & $3(\mathrm{~N}=1,198)^{128-130}$ & Fair & Direct & Consistent & Precise & Unknown & $\begin{array}{c}\text { No effect } \\
\text { Duloxetine vs. placebo } \\
\text { SMD } 0.18(-0.03 \text { to } 0.39), I^{2}=38 \%\end{array}$ & Moderate \\
\hline
\end{tabular}




\begin{tabular}{|c|c|c|c|c|c|c|c|c|c|}
\hline Outcome & Duration & $\begin{array}{c}\text { Number of } \\
\text { Studies }^{\mathrm{a}} \\
\text { (n participants) }\end{array}$ & $\begin{array}{l}\text { Study } \\
\text { Quality }^{\mathrm{b}}\end{array}$ & Directness & $\begin{array}{l}\text { Consistency } \\
\text { (1 study= } \\
\text { Unknown) }\end{array}$ & Precision & $\begin{array}{l}\text { Publication } \\
\text { Bias }\end{array}$ & $\begin{array}{c}\text { Main Findings } \\
\text { Effect Size }(95 \% \mathrm{Cl})\end{array}$ & $\begin{array}{l}\text { Strength of } \\
\text { Evidence } \\
\text { Grade }\end{array}$ \\
\hline & & $1(\mathrm{~N}=108)^{132}$ & Fair & Direct & Unknown & Imprecise & Unknown & $\begin{array}{c}\text { (BDI-II) } \\
\text { Gabapentin vs. placebo } \\
p=0.52\end{array}$ & Insufficient \\
\hline
\end{tabular}

a Study references are in Appendix $\mathrm{J}$.

b Study Quality: poor-quality studies not synthesized

${ }^{\mathrm{c}}$ Pain Response main findings, percentages represent threshold for Pain Response

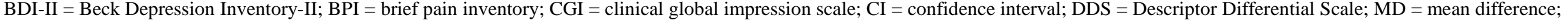

RMDQ = Roland-Morris Disability Questionnaire; SMD = standard mean difference; VAS = visual analogue scale

Table H-18. Low back pain - antidepressants: head-to-head trials

\begin{tabular}{|c|c|c|c|c|c|c|c|c|c|}
\hline Outcome & Duration & $\begin{array}{c}\text { Number of } \\
\text { Studies }^{\mathrm{a}} \\
\text { (n participants) }\end{array}$ & $\begin{array}{l}\text { Study } \\
\text { Quality }\end{array}$ & Directness & $\begin{array}{l}\text { Consistency } \\
\text { (1 study= } \\
\text { Unknown) } \\
\end{array}$ & Precision & $\begin{array}{l}\text { Publication } \\
\text { Bias }\end{array}$ & $\begin{array}{l}\text { Main Findings } \\
\text { Effect Size }\end{array}$ & $\begin{array}{l}\text { Strength of } \\
\text { Evidence } \\
\text { Grade }\end{array}$ \\
\hline $\begin{array}{l}\text { Pain } \\
\text { Improvement } \\
\text { (Continuous) }\end{array}$ & Short & $1(\mathrm{~N}=200)^{134}$ & Fair & Direct & Unknown & Imprecise & Unknown & $\begin{array}{c}\text { Small effect } \\
\text { (VAS) } \\
\text { Amitriptyline vs. pregabalin } \\
\text { Mean change from baseline: } 2.9 \text { vs. } 3.9 \\
p=0.03\end{array}$ & Low \\
\hline Function & Short & $1(\mathrm{~N}=200)^{134}$ & Fair & Direct & Unknown & Imprecise & Unknown & $\begin{array}{c}\text { No effect } \\
\text { (ODI) } \\
\text { Amitriptyline vs. pregabalin } \\
\text { Mean change from baseline: } p=0.09 \\
\end{array}$ & Low \\
\hline
\end{tabular}

a Study references are in Appendix J.

b Study Quality: poor-quality studies not synthesized

ODI = Oswestry Disability Index; VAS = visual analogue scale; 


\section{Harms}

Table H-19. Adverse events - antidepressants

\begin{tabular}{|c|c|c|c|c|c|c|c|c|c|c|}
\hline $\begin{array}{l}\text { Adverse } \\
\text { Effect }\end{array}$ & Comparison & Duration & $\begin{array}{c}\text { Number of } \\
\text { Studies }^{\mathrm{a}} \\
\text { (n participants) }\end{array}$ & $\begin{array}{c}\text { Study } \\
\text { Quality }^{\text {b }}\end{array}$ & Directness & $\begin{array}{l}\text { Consistency } \\
\text { (1 study= } \\
\text { Unknown) }\end{array}$ & Precision & $\begin{array}{l}\text { Publication } \\
\text { Bias }\end{array}$ & $\begin{array}{c}\text { Main Findings } \\
\text { Effect Size }(95 \% \mathrm{Cl})\end{array}$ & $\begin{array}{c}\text { Strength of } \\
\text { Evidence } \\
\text { Grade }\end{array}$ \\
\hline \multirow{2}{*}{ SAE } & \multirow{2}{*}{$\begin{array}{l}\text { SNRI } \\
\text { Antidepressants } \\
\text { vs. Placebo }\end{array}$} & Short & $\begin{array}{c}19(\mathrm{~N}=8,832)^{28-} \\
32,42,43,47- \\
51,97,98,100,101,128-130\end{array}$ & Fair & Direct & Consistent & Imprecise & Undetected & $\begin{array}{c}\text { No effect } \\
\text { RR } 0.88(0.62 \text { to } \\
1.24), \mathrm{I}^{2}=0 \%\end{array}$ & Low \\
\hline & & Intermediate & $2(\mathrm{~N}=1,218)^{46,53}$ & Fair & Direct & Consistent & Imprecise & Unknown & $\begin{array}{c}\text { No effect } \\
\text { RR } 0.86(0.35 \text { to } \\
2.24), \mathrm{I}^{2}=0 \%\end{array}$ & Low \\
\hline \multirow{4}{*}{ WAE } & \multirow{2}{*}{$\begin{array}{l}\text { SNRI } \\
\text { Antidepressants } \\
\text { vs. Placebo }\end{array}$} & Short & $\begin{array}{c}24(N=9,971)^{27-} \\
32,41-44,47-51,96- \\
98,100,101,105,128-130\end{array}$ & Fair & Direct & Consistent & Precise & Undetected & $\begin{array}{l}\text { Moderate effect } \\
\text { RR } 1.99(1.71 \text { to } \\
2.35), I^{2}=18 \%\end{array}$ & Moderate \\
\hline & & Intermediate & $3\left(\begin{array}{c}N=1,738) \\
45,46,53\end{array}\right.$ & Fair & Direct & Consistent & Precise & Unknown & $\begin{array}{l}\text { Moderate effect } \\
\text { RR } 1.83 \text { (1.23 to } \\
2.61), \mathrm{I}^{2}=4 \%\end{array}$ & Moderate \\
\hline & \multirow{2}{*}{$\begin{array}{l}\text { TCA } \\
\text { Antidepressants } \\
\text { vs. placebo }\end{array}$} & Short & $\begin{array}{c}5 \\
(\mathrm{~N}=478)^{52,127,131} \\
133,135\end{array}$ & Fair & Direct & Consistent & Imprecise & Unknown & $\begin{array}{c}\text { No effect } \\
\text { RR } 1.49(0.89 \text { to } \\
3.01)\end{array}$ & Low \\
\hline & & Intermediate & $1(\mathrm{~N}=126)^{61}$ & Far & Direct & Unknown & Imprecise & Unknown & $\begin{array}{c}\text { RR } 1.75(0.38 \text { to } \\
8.06)\end{array}$ & Insufficient \\
\hline \multirow{2}{*}{ Nausea } & \multirow{2}{*}{$\begin{array}{l}\text { SNRI } \\
\text { Antidepressants } \\
\text { vs. Placebo }\end{array}$} & Short & $\begin{array}{c}19(\mathrm{~N}=8,929)^{27-} \\
29,31,32,42,43,47- \\
51,97,98,100,101,128-130\end{array}$ & Fair & Direct & Consistent & Precise & Undetected & $\begin{array}{c}\text { Large effect } \\
\text { RR 3.10 ( } 2.50 \text { to } \\
4.06), \mathrm{I}^{2}=60 \%\end{array}$ & Moderate \\
\hline & & Intermediate & $3\left(\begin{array}{c}N=1,738) \\
45,46,53\end{array}\right.$ & Fair & Direct & Consistent & Imprecise & Unknown & $\begin{array}{l}\text { Moderate effect } \\
\text { RR } 1.98 \text { (1.57 to } \\
2.82), I^{2}=0 \%\end{array}$ & Low \\
\hline \multirow{2}{*}{ Sedation } & \multirow{2}{*}{$\begin{array}{l}\text { SNRI } \\
\text { Duloxetine vs. } \\
\text { Placebo }\end{array}$} & Short & $\begin{array}{c}16(\mathrm{~N}=5,831)^{27-} \\
29,31,32,42,47,50,51,97 \\
, 98,100,101,128-130\end{array}$ & Fair & Direct & Consistent & Precise & Undetected & $\begin{array}{c}\text { Large effect } \\
\text { RR } 2.46(2.00 \text { to } \\
3.01),\left.\right|^{2}=0 \%\end{array}$ & Moderate \\
\hline & & Intermediate & $2(\mathrm{~N}=850)^{45,53}$ & Fair & Direct & Consistent & Imprecise & Unknown & $\begin{array}{c}\text { Large effect } \\
\text { RR } 3.51 \text { (1.46 to } \\
11.05), \mathrm{I}^{2}=0 \%\end{array}$ & Low \\
\hline $\begin{array}{c}\text { Dry } \\
\text { Mouth }\end{array}$ & $\begin{array}{l}\text { TCA } \\
\text { Antidepressants } \\
\text { vs Placebo }\end{array}$ & Short & $1(\mathrm{~N}=131)^{135}$ & Fair & Direct & Unknown & Imprecise & Unknown & $\begin{array}{c}\text { RR } 1.80,(1.14 \text { to } \\
2.85)\end{array}$ & Insufficient \\
\hline $\begin{array}{l}\text { Cognitive } \\
\text { effects }\end{array}$ & $\begin{array}{l}\text { SNRI } \\
\text { Antidepressants } \\
\text { vs. Placebo }\end{array}$ & Short & $2(\mathrm{~N}=805)^{129,130}$ & Fair & Direct & Consistent & Imprecise & Unknown & $\begin{array}{c}\text { No effect } \\
\text { RR } 3.24(0.26 \text { to } \\
40.17), 12=0\end{array}$ & Low \\
\hline
\end{tabular}

a Study references are in Appendix J.

b Study Quality; poor quality studies not synthesized 


\section{Table H-20. Adverse events - anticonvulsants}

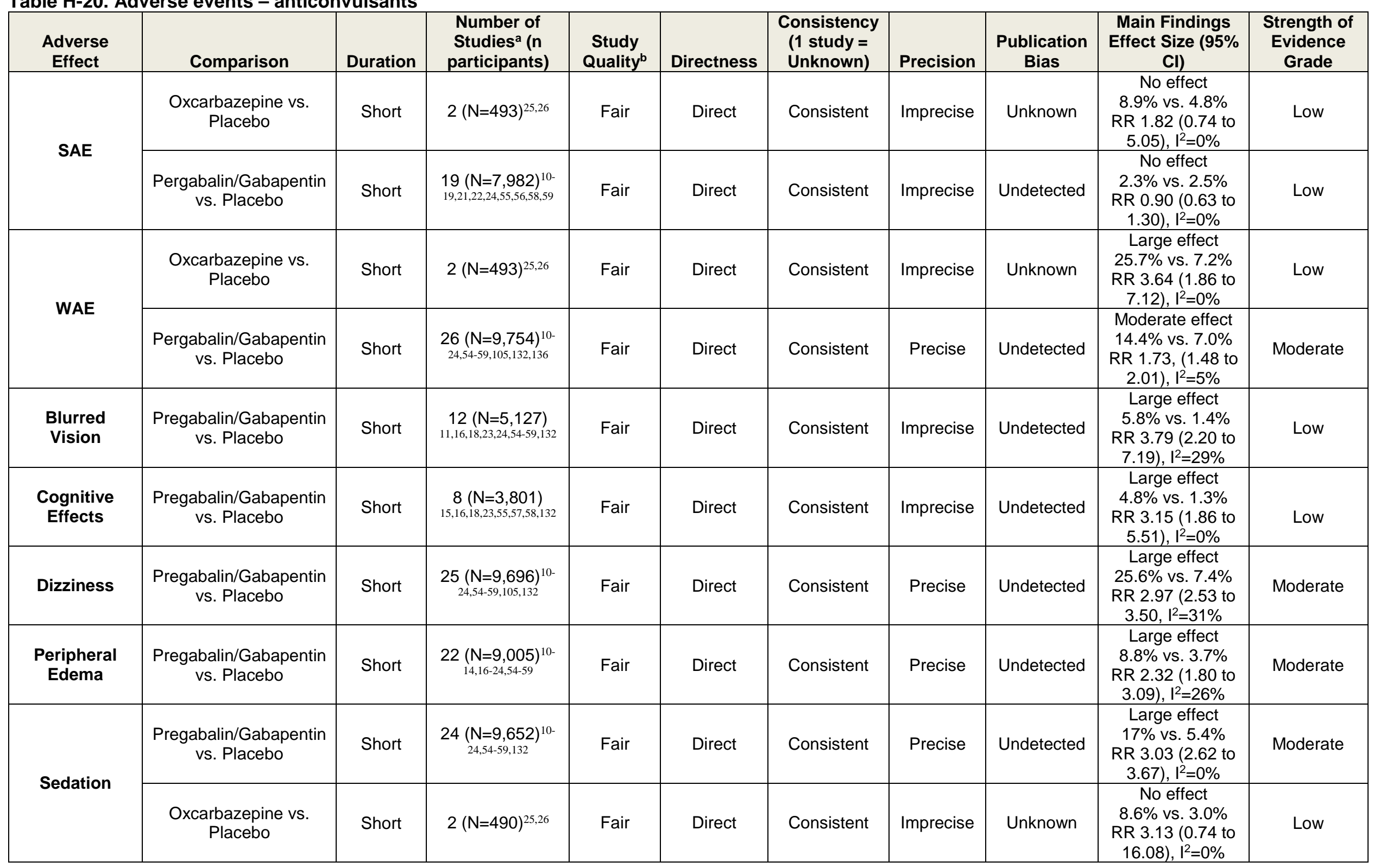




\begin{tabular}{|c|c|c|c|c|c|c|c|c|c|c|}
\hline $\begin{array}{c}\text { Adverse } \\
\text { Effect }\end{array}$ & Comparison & Duration & $\begin{array}{c}\text { Number of } \\
\text { Studies }^{a}(n \\
\text { participants) }\end{array}$ & $\begin{array}{l}\text { Study } \\
\text { Quality }^{b}\end{array}$ & Directness & $\begin{array}{l}\text { Consistency } \\
\text { (1 study = } \\
\text { Unknown) }\end{array}$ & Precision & $\begin{array}{l}\text { Publication } \\
\text { Bias }\end{array}$ & $\begin{array}{c}\text { Main Findings } \\
\text { Effect Size (95\% } \\
\text { Cl) }\end{array}$ & $\begin{array}{c}\text { Strength of } \\
\text { Evidence } \\
\text { Grade }\end{array}$ \\
\hline Weight Gain & $\begin{array}{l}\text { Pregabalin/Gabapentin } \\
\text { vs. Placebo }\end{array}$ & Short & $\begin{array}{c}21(\mathrm{~N}=8,620)^{10-} \\
14,16,18,1,1,22-24,54- \\
59,105,132\end{array}$ & Fair & Direct & Consistent & Precise & Undetected & $\begin{array}{c}\text { Large effect } \\
10.1 \% \text { vs. } 2.8 \% \\
\text { RR } 3.57(2.77 \text { to } \\
4.91), I^{2}=7 \%\end{array}$ & Moderate \\
\hline Hyponatremia & $\begin{array}{l}\text { Oxcarbazepine vs. } \\
\text { Placebo }\end{array}$ & Short & $2(\mathrm{~N}=490)^{25,26}$ & Fair & Direct & Consistent & Imprecise & Unknown & $\begin{array}{c}\text { No effect } \\
2.8 \% \text { vs. } 0.0 \% \\
\text { RR } 5.93(0.55 \text { to } \\
63.8), \mathrm{I}^{2}=0 \%\end{array}$ & Low \\
\hline
\end{tabular}

a Study references are in Appendix J.

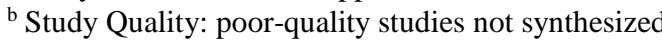

$\mathrm{CI}$ = confidence interval; $\mathrm{RR}$ = risk ratio; $\mathrm{SAE}$ = serious adverse event; $\mathrm{WAE}$ = withdrawal due to adverse event

\section{Table H-21. Adverse events - NSAIDs}

\begin{tabular}{|c|c|c|c|c|c|c|c|c|c|c|}
\hline $\begin{array}{l}\text { Adverse } \\
\text { Effect }\end{array}$ & Comparison & Duration & $\begin{array}{c}\text { Number of } \\
\text { Studies }^{\mathrm{a}} \\
\text { (n participants) }\end{array}$ & $\begin{array}{c}\text { Study } \\
\text { Qualityb }\end{array}$ & Directness & $\begin{array}{c}\text { Consistency } \\
\text { (1 study= } \\
\text { Unknown) }\end{array}$ & Precision & $\begin{array}{c}\text { Publication } \\
\text { Bias }\end{array}$ & $\begin{array}{c}\text { Main Findings } \\
\text { Effect Size }(95 \% \mathrm{Cl})\end{array}$ & $\begin{array}{c}\text { Strength of } \\
\text { Evidence } \\
\text { Grade }\end{array}$ \\
\hline \multirow{3}{*}{ SAE } & \multirow{2}{*}{$\begin{array}{l}\text { NSAIDs vs. } \\
\text { Placebo }\end{array}$} & Short & $\begin{array}{c}23(\mathrm{~N}=13,082)^{63-} \\
65,68,70-72,75,76,78,80- \\
82,84,85,106,107,109,113,114 \\
, 137\end{array}$ & Fair & Direct & Consistent & Imprecise & Undetected & $\begin{array}{c}\text { No effect } \\
\text { RR } 0.96(0.72 \text { to } 1.29), I^{2}=0 \%\end{array}$ & Low \\
\hline & & Intermediate & $1(\mathrm{~N}=563)^{110}$ & Fair & Direct & Unknown & Imprecise & Unknown & RR 0.51 (0.05 to 5.58) & Insufficient \\
\hline & $\begin{array}{l}\text { Topical } \\
\text { diclofenac } \\
\text { vs placebo }\end{array}$ & Short & $2(\mathrm{~N}=912)^{87,88}$ & Fair & Direct & Consistent & Imprecise & Unknown & $\begin{array}{c}\text { No effect } \\
\text { RR } 1.03(0.29 \text { to } 27.01), I^{2}=0 \%\end{array}$ & Low \\
\hline \multirow{4}{*}{ WAE } & \multirow{3}{*}{$\begin{array}{l}\text { NSAIDs vs. } \\
\text { Placebo }\end{array}$} & Short & $\begin{array}{c}38(\mathrm{~N}=20,060)^{62-} \\
78,80-86,106- \\
109,113,114,116,137-141\end{array}$ & Fair & Direct & Consistent & Precise & Undetected & $\begin{array}{c}\text { Small effect } \\
\text { RR } 1.30(1.14 \text { to } 1.49), I^{2}=13 \%\end{array}$ & Moderate \\
\hline & & Intermediate & $2(\mathrm{~N}=941)^{79,110}$ & Fair & Direct & Consistent & Imprecise & Unknown & $\begin{array}{c}\text { No effect } \\
\text { RR } 1.59(0.89 \text { to } 3.08), I^{2}=0 \%\end{array}$ & Low \\
\hline & & Long & $1(\mathrm{~N}=365)^{115}$ & Fair & Direct & Unknown & Imprecise & Unknown & RR 1.59 (0.81 to 3.12) & Insufficient \\
\hline & $\begin{array}{l}\text { Topical } \\
\text { diclofenac } \\
\text { vs placebo }\end{array}$ & Short & $4(\mathrm{~N}=1,549)^{83,87-89}$ & Fair & Direct & Consistent & Imprecise & Unknown & $\begin{array}{c}\text { No effect } \\
\text { RR } 1.03(0.29 \text { to } 27.01), I^{2}=0 \%\end{array}$ & Low \\
\hline
\end{tabular}




\begin{tabular}{|c|c|c|c|c|c|c|c|c|c|c|}
\hline $\begin{array}{l}\text { Adverse } \\
\text { Effect }\end{array}$ & Comparison & Duration & $\begin{array}{c}\text { Number of } \\
\text { Studies }^{\mathrm{a}} \\
\text { (n participants) }\end{array}$ & $\begin{array}{l}\text { Study } \\
\text { Quality }^{\mathrm{b}}\end{array}$ & Directness & $\begin{array}{l}\text { Consistency } \\
\text { (1 study= } \\
\text { Unknown) }\end{array}$ & Precision & $\begin{array}{l}\text { Publication } \\
\text { Bias }\end{array}$ & $\begin{array}{c}\text { Main Findings } \\
\text { Effect Size }(95 \% \mathrm{Cl})\end{array}$ & $\begin{array}{c}\text { Strength of } \\
\text { Evidence } \\
\text { Grade }\end{array}$ \\
\hline \multirow{3}{*}{$\begin{array}{c}\text { CV } \\
\text { Events }\end{array}$} & $\begin{array}{l}\text { NSAIDs vs. } \\
\text { Placebo }\end{array}$ & Short & 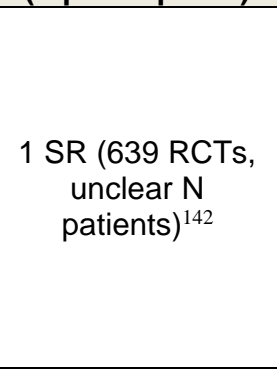 & Fair & Direct & Consistent & Precise & Unknown & $\begin{array}{c}\text { Small effect } \\
\text { Diclofenac RR } 1.41 \text { (1.12 to } \\
1.78 \text { ) } \\
\text { No effect } \\
\text { Ibuprofen RR } 1.44 \text { (0.89 to } \\
2.33 \text { ) } \\
\text { Naproxen RR } 0.93 \text { (0.69 to } \\
1.27 \text { ) } \\
\text { Celecoxib RR } 1.36 \text { (1.00 to } \\
1.84 \text { ) } \\
\end{array}$ & Moderate \\
\hline & \multirow{2}{*}{$\begin{array}{l}\text { Celecoxib } \\
\text { vs. } \\
\text { nonselective } \\
\text { NSAIDs }\end{array}$} & Intermediate & $\begin{array}{c}3 \text { RCTs } \\
(\mathrm{N}=33,064)^{94,143,144}\end{array}$ & Fair & Direct & Consistent & Precise & Unknown & $\begin{array}{c}\text { No effect } \\
\text { Cardiovascular death, nonfatal } \\
\text { myocardial infarction, or } \\
\text { nonfatal stroke: celecoxib 1.7\%; } \\
\text { ibuprofen 1.9\%; naproxen } \\
1.8 \% \text { ( } \begin{array}{c}\text { < } 0.001 \text { for noninferiority } \\
\text { between drugs) }\end{array}\end{array}$ & Moderate \\
\hline & & Long & $\begin{array}{c}1 \mathrm{RCT} \\
(\mathrm{N}=7,297)^{145}\end{array}$ & Good & Direct & Unknown & Precise & Unknown & $\begin{array}{l}\text { No effect } \\
\text { Hospitalization for non-fatal MI } \\
\text { or other biomarker positive } \\
\text { acute coronary syndrome, non- } \\
\text { fatal stroke or CV death } \\
\text { hazard ratio } 1.12 \text { ( } 0.81 \text { to } 1.55)\end{array}$ & Moderate \\
\hline $\begin{array}{l}\text { Serious } \\
\text { GI Events }\end{array}$ & $\begin{array}{l}\text { NSAIDs vs. } \\
\text { Placebo }\end{array}$ & Short & $\begin{array}{c}1 \text { SR }(639 \mathrm{RCTS}, \\
\text { unclear } \mathrm{N} \\
\text { patients })^{142} \\
13 \mathrm{RCTS} \\
(\mathrm{N}=7,262) \\
67,70,73,76,80,106,109,114,1 \\
37-141\end{array}$ & Fair & Direct & $\begin{array}{l}\text { Consistent/ } \\
\text { Inconsistent }\end{array}$ & Precise & Undetected & $\begin{array}{c}\text { Moderate effect } \\
\text { EPC meta-analysis } \\
\text { NSAIDs vs. placebo } \\
\text { RR 3.04 (1.73 to } 5.11), 1^{2}=73 \% \\
\\
\text { IPD meta-analysis } \\
\text { coxibs RR 1.81 (1.17 to } 2.81) ; \\
\text { Diclofenac RR } 1.89 \text { (1.16 to } \\
3.09) ; \\
\text { Ibuprofen RR } 3.97 \text { (2.22 to } \\
7.10) ; \\
\text { Naproxen RR 4.22 (2.71 to } \\
6.56) ; \\
\text { Celecoxib vs. placebo: } \\
\text { 1.02 ( } 0.47 \text { to } 1.56 ; \\
\text { 3 RCTs, N=1,877), }\left.\right|^{2}=0 \%\end{array}$ & $\begin{array}{c}\begin{array}{c}\text { Moderate } \\
\text { (non- } \\
\text { selectives) }\end{array} \\
\text { Low } \\
\text { (celecoxib) }\end{array}$ \\
\hline
\end{tabular}




\begin{tabular}{|c|c|c|c|c|c|c|c|c|c|c|}
\hline $\begin{array}{l}\text { Adverse } \\
\text { Effect }\end{array}$ & Comparison & Duration & $\begin{array}{c}\text { Number of } \\
\text { Studies } \\
\text { (n participants) }\end{array}$ & $\begin{array}{l}\text { Study } \\
\text { Quality }\end{array}$ & Directness & $\begin{array}{l}\text { Consistency } \\
\text { (1 study= } \\
\text { Unknown) }\end{array}$ & Precision & $\begin{array}{l}\text { Publication } \\
\text { Bias }\end{array}$ & $\begin{array}{c}\text { Main Findings } \\
\text { Effect Size }(95 \% \mathrm{Cl})\end{array}$ & $\begin{array}{c}\text { Strength of } \\
\text { Evidence } \\
\text { Grade }\end{array}$ \\
\hline & \multirow[t]{2}{*}{\begin{tabular}{|l} 
Coxibs \\
(celecoxib) \\
vs. \\
nonselective \\
NSAIDs
\end{tabular}} & Short & $\begin{array}{c}1 \text { SR }(639 \text { RCTs, } \\
\text { unclear N } \\
\text { patients })^{142} \text {; } \\
13 \text { RCTs } \\
(\mathrm{N}=7,262)^{67,70,73,76,8} \\
0,106,109,114,137-141\end{array}$ & Fair & $\begin{array}{l}\text { Direct and } \\
\text { Indirect }\end{array}$ & Inconsistent & Imprecise & Undetected & $\begin{array}{c}\text { No clear effect } \\
\text { SR (4 RCTs, N=1,755) OR } 0.61 \\
\text { (0.15 to } 2.43), I^{2}=38 \% \\
\text { Placebo trials: Celecoxib RR } \\
1.04 \text { (0.67 to } 1.54), I^{2}=0 \% \\
\\
\text { Nonselective NSAIDs RR } 4.29 \\
\text { (2.75 to 6.93), I I }=46 \% ; p<0.001 \\
\text { for interaction }\end{array}$ & Insufficient \\
\hline & & Intermediate & $\begin{array}{c}1 \mathrm{RCT} \\
(\mathrm{N}=8,067)^{146}\end{array}$ & Fair & Direct & Unknown & Precise & Unknown & $\begin{array}{c}\text { Moderate effect } \\
\text { OR } 1.82(1.31 \text { to } 2.55)\end{array}$ & Low \\
\hline $\begin{array}{l}\text { Serious } \\
\text { Hepatic } \\
\text { Events }\end{array}$ & $\begin{array}{l}\text { NSAIDs vs. } \\
\text { Placebo }\end{array}$ & Intermediate & $1 \mathrm{SR}(64 \mathrm{RCTs})^{147}$ & Fair & Direct & Unclear & Imprecise & Unknown & $\begin{array}{c}\text { Large effect } \\
\text { Aminotransferase }>3 x \text { upper } \\
\text { limit of normal: } \\
\text { Diclofenac } 3.55 \% \text { ( } 3.12 \% \text { to } \\
4.03 \%) \text { vs. } 0.29 \%(0.17 \% \text { to } \\
0.51 \%) \\
\text { Large effect } \\
\text { Liver-related discontinuations: } \\
\text { Diclofenac } 2.17 \%(1.78 \% \text { to } \\
2.64 \%) \text { vs. } 0.08 \%(0.02 \% \text { to } \\
0.29 \%) \\
\text { No effect } \\
\text { Liver-related SAE: } \\
\text { Naproxen } 0.06 \%(0.02 \% \text { to } \\
0.15 \%) \text { vs. } 0.00 \%(0.00 \% \text { to } \\
0.08 \%)\end{array}$ & Low \\
\hline
\end{tabular}

a Study references are in Appendix J.

b Study Quality: poor quality studies not synthesized

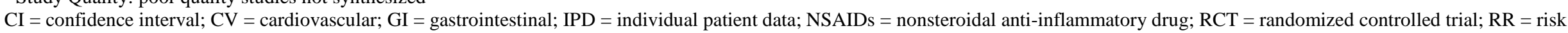
ratio; $\mathrm{SAE}$ = serious adverse event; $\mathrm{SR}$ = systematic review; WAE = withdrawal due to adverse event

\section{Table H-22. Adverse events - acetaminophen vs. placebo}

\begin{tabular}{|c|c|c|c|c|c|c|c|c|c|}
\hline & Duration & $\begin{array}{l}\text { Number of Studies } \\
\text { (n) participants }\end{array}$ & $\begin{array}{l}\text { Study } \\
\text { Quality }\end{array}$ & Directness & $\begin{array}{c}\text { Consistency } \\
\text { (1 study= } \\
\text { Unknown) }\end{array}$ & Precision & $\begin{array}{c}\text { Publication } \\
\text { Bias }\end{array}$ & $\begin{array}{c}\text { Main Findings } \\
\text { Effect Size }(95 \% \mathrm{Cl})\end{array}$ & $\begin{array}{c}\text { Strength of } \\
\text { Evidence } \\
\text { Grade }\end{array}$ \\
\hline \multirow{2}{*}{ SAE } & Short & $2(\mathrm{~N}=1,023)^{102,103}$ & Fair & Direct & Consistent & Imprecise & Unknown & $\begin{array}{c}\text { No effect } \\
\text { RR } 2.57(0.60 \text { to } 10.8) ; l^{2}=0 \%\end{array}$ & Low \\
\hline & Intermediate & $1(\mathrm{~N}=212)^{104}$ & Fair & Direct & Unknown & Imprecise & Unknown & $\begin{array}{c}\text { No effect } \\
\text { RR } 0.96(0.29 \text { to } 3.23)\end{array}$ & Low \\
\hline WAE & Short & $2(\mathrm{~N}=1,023)^{102,103}$ & Fair & Direct & Consistent & Imprecise & Unknown & $\begin{array}{c}\text { No effect } \\
\text { RR } 1.14(0.67 \text { to } 1.95) ; l^{2}=0 \%\end{array}$ & Low \\
\hline
\end{tabular}




\begin{tabular}{|c|c|c|c|c|c|c|c|c|}
\hline Duration & $\begin{array}{l}\text { Number of Studies } \\
\text { (n) participants }\end{array}$ & $\begin{array}{c}\text { Study } \\
\text { Quality }\end{array}$ & Directness & $\begin{array}{c}\text { Consistency } \\
\text { (1 study= } \\
\text { Unknown) }\end{array}$ & Precision & $\begin{array}{l}\text { Publication } \\
\text { Bias }\end{array}$ & $\begin{array}{c}\text { Main Findings } \\
\text { Effect Size (95\% Cl) }\end{array}$ & $\begin{array}{c}\text { Strength of } \\
\text { Evidence } \\
\text { Grade }\end{array}$ \\
\hline Intermediate & $1(\mathrm{~N}=212)^{104}$ & Fair & Direct & Unknown & Imprecise & Unknown & $\begin{array}{c}\text { No effect } \\
\text { RR } 1.28 \text { (0.56 to 2.92) }\end{array}$ & Low \\
\hline
\end{tabular}

a Study references are in Appendix J.

b Study Quality; poor quality studies not synthesized

$\mathrm{SAE}=$ serious adverse event; $\mathrm{WAE}$ = withdrawal due to adverse event; $\mathrm{RR}$ = risk ratio

Table H-53. Adverse events - capsaicin vs. placebo

\begin{tabular}{|c|c|c|c|c|c|c|c|c|c|}
\hline & Duration & $\begin{array}{l}\text { Number of } \\
\text { Studies }^{a} \\
\text { (n) participants }\end{array}$ & $\begin{array}{c}\text { Study } \\
\text { Qualityb }\end{array}$ & Directness & $\begin{array}{c}\text { Consistency } \\
\text { (1 study= } \\
\text { Unknown) } \\
\end{array}$ & Precision & $\begin{array}{l}\text { Publication } \\
\text { Bias }\end{array}$ & $\begin{array}{c}\text { Main Findings } \\
\text { Effect Size }(95 \% \mathrm{Cl}) \\
\end{array}$ & $\begin{array}{c}\text { Strength of } \\
\text { Evidence } \\
\text { Grade } \\
\end{array}$ \\
\hline SAE & Short & $3(N=1,051)^{35-37}$ & Good & Direct & Consistent & Imprecise & Unknown & $\begin{array}{c}\text { No effect } \\
\text { RR } 1.32 \text { (0.71 to } 3.47), I^{2}=0 \%\end{array}$ & Moderate \\
\hline WAE & Short & $2(\mathrm{~N}=896)^{35,36}$ & Good & Direct & Consistent & Imprecise & Unknown & $\begin{array}{c}\text { No effect } \\
\text { RR } 1.04(0.08 \text { to } 17.1), I^{2}=0 \%\end{array}$ & Moderate \\
\hline $\begin{array}{l}\text { Application } \\
\text { Site Erythema }\end{array}$ & Short & $3(\mathrm{~N}=1,051)^{35-37}$ & Good & Direct & Consistent & Imprecise & Unknown & $\begin{array}{c}\text { Moderate effect } \\
\text { RR } 1.46(1.29 \text { to } 1.66), \mathrm{I}^{2}=0 \%\end{array}$ & Moderate \\
\hline $\begin{array}{l}\text { Application } \\
\text { Site Pain }\end{array}$ & Short & $3(\mathrm{~N}=1,051)^{35-37}$ & Good & Direct & Consistent & Imprecise & Unknown & $\begin{array}{c}\text { Large effect } \\
\text { RR } 2.26(1.61 \text { to } 2.82), I^{2}=0 \%\end{array}$ & Moderate \\
\hline $\begin{array}{l}\text { Application } \\
\text { Site Pruritus }\end{array}$ & Short & $3(\mathrm{~N}=1,051)^{35-37}$ & Good & Direct & Consistent & Imprecise & Unknown & $\begin{array}{c}\text { No effect } \\
\text { RR } 1.70(0.92 \text { to } 3.35), I^{2}=0 \%\end{array}$ & Moderate \\
\hline
\end{tabular}

a Study references are in Appendix J.

b Study Quality; poor quality studies not synthesized

$\mathrm{SAE}=$ serious adverse event; WAE = withdrawal due to adverse event; RR = risk ratio 
Table H-24. Adverse events - cannabis vs. placebo

\begin{tabular}{|c|c|c|c|c|c|c|c|c|c|c|}
\hline $\begin{array}{l}\text { Adverse } \\
\text { Event }\end{array}$ & Cannabis type & Duration & $\begin{array}{l}\text { Number of } \\
\text { Studies } \\
\text { ( } \mathrm{n} \\
\text { participants) }\end{array}$ & $\begin{array}{c}\text { Study } \\
\text { Qualityb }\end{array}$ & Directness & $\begin{array}{l}\text { Consistency } \\
\text { (1 study= } \\
\text { Unknown) } \\
\end{array}$ & Precision & $\begin{array}{c}\text { Publication } \\
\text { Bias }\end{array}$ & $\begin{array}{c}\text { Main Findings } \\
\text { Effect Size }(95 \% \mathrm{Cl})\end{array}$ & $\begin{array}{l}\text { Strength of } \\
\text { Evidence } \\
\text { Grade }\end{array}$ \\
\hline \multirow{2}{*}{ SAE } & $\begin{array}{l}\text { Dronabinol 7.5- } \\
15 \mathrm{mg} / \mathrm{d}\end{array}$ & Short & $1(\mathrm{~N}=240)^{33}$ & Good & Direct & Unknown & Imprecise & Unknown & $\begin{array}{c}\text { No effect } \\
13.7 \% \text { vs. } 8.9 \% \\
\text { RR } 1.58(0.75 \text { to } 3.30)\end{array}$ & Low \\
\hline & $\begin{array}{l}\text { THC } 2.7 \mathrm{~m} / \mathrm{microL}+ \\
\text { CBD } 2.5 \mathrm{gm} / \mathrm{microL}\end{array}$ & Short & $1(\mathrm{~N}=246)^{34}$ & Fair & Direct & Unknown & Imprecise & Unknown & $\begin{array}{c}\text { No effect } \\
8 \% \text { vs. } 5 \% \\
\text { RR } 1.54 \text { (0.58 to } 4.10) \\
\end{array}$ & Low \\
\hline \multirow{2}{*}{ WAE } & $\begin{array}{l}\text { Dronabinol } 7.5- \\
15 \mathrm{mg} / \mathrm{d}\end{array}$ & Short & $1(\mathrm{~N}=240)^{33}$ & Good & Direct & Unknown & Imprecise & Unknown & $\begin{array}{c}\text { No effect } \\
14.5 \% \text { vs. } 14.0 \% \\
\text { RR } 1.05 \text { (0.56 to } 1.96) \\
\end{array}$ & Low \\
\hline & $\begin{array}{l}\text { THC } 2.7 \mathrm{~m} / \mathrm{microL}+ \\
\text { CBD } 2.5 \mathrm{gm} / \mathrm{microL}\end{array}$ & Short & $1(\mathrm{~N}=246)^{34}$ & Fair & Direct & Unknown & Imprecise & Unknown & $\begin{array}{c}\text { Large effect } \\
19 \% \text { vs. } 6 \% \\
\text { RR } 3.16(1.41 \text { to } 7.06)\end{array}$ & Low \\
\hline Dizziness & $\begin{array}{l}\text { Dronabinol } 7.5- \\
15 \mathrm{mg} / \mathrm{d}\end{array}$ & Short & $1(N=240)^{33}$ & Good & Direct & Unknown & Imprecise & Unknown & $\begin{array}{c}\text { Large effect } \\
20 \% \text { vs. } 4.3 \% \\
\text { RR } 4.68(1.85 \text { to } 11.8) \\
\end{array}$ & Low \\
\hline \multirow{2}{*}{ Nausea } & $\begin{array}{l}\text { Dronabinol } 7.5- \\
15 \mathrm{mg} / \mathrm{d}\end{array}$ & Short & $1(\mathrm{~N}=240)^{33}$ & Good & Direct & Unknown & Imprecise & Unknown & $\begin{array}{c}\text { No effect } \\
4.2 \% \text { vs. } 6.8 \% \\
\text { RR } 1.39(0.40 \text { to } 4.80) \\
\end{array}$ & Low \\
\hline & $\begin{array}{l}\text { THC } 2.7 \mathrm{~m} / \mathrm{microL}+ \\
\text { CBD } 2.5 \mathrm{gm} / \mathrm{microL}\end{array}$ & Short & $1(\mathrm{~N}=246)^{34}$ & Fair & Direct & Unknown & Imprecise & Unknown & $\begin{array}{c}\text { Large effect } \\
17 \% \text { vs. } 8 \% \\
\text { RR } 2.25(1.8 \text { to } 4.70)\end{array}$ & Low \\
\hline Sedation & $\begin{array}{l}\text { THC } 2.7 \mathrm{~m} / \mathrm{microL}+ \\
\text { CBD } 2.5 \mathrm{gm} / \mathrm{microL}\end{array}$ & Short & $1(\mathrm{~N}=246)^{34}$ & Fair & Direct & Unknown & Imprecise & Unknown & $\begin{array}{c}3 \% \text { vs. } 0 \% \\
\text { RR } 8.30(0.45 \text { to } 152.58)\end{array}$ & Insufficient \\
\hline
\end{tabular}

a Study references are in Appendix J.

b Study Quality; poor quality studies not synthesized

$\mathrm{SAE}$ = serious adverse event; $\mathrm{WAE}=$ withdrawal due to adverse event; $\mathrm{RR}=$ risk ratio; $\min =$ minute; $\mathrm{THC}=$ tetrahydrocannabinol; $\mathrm{CBD}=\mathrm{Cannabidiol}$ 
Table H-25. Adverse events - skeletal muscle relaxants

\begin{tabular}{|c|c|c|c|c|c|c|c|c|c|c|}
\hline $\begin{array}{l}\text { Adverse } \\
\text { Effect }\end{array}$ & Comparison & Duration & $\begin{array}{c}\text { Number of } \\
\text { Studies }^{\mathrm{a}} \\
\text { (n participants) }\end{array}$ & $\begin{array}{l}\text { Study } \\
\text { Qualityb }\end{array}$ & Directness & $\begin{array}{l}\text { Consistency } \\
\text { (1 study= } \\
\text { Unknown) }\end{array}$ & Precision & $\begin{array}{l}\text { Publication } \\
\text { Bias }\end{array}$ & $\begin{array}{c}\text { Main Findings } \\
\text { Effect Size }(95 \% \mathrm{Cl})\end{array}$ & $\begin{array}{c}\text { Strength of } \\
\text { Evidence } \\
\text { Grade }\end{array}$ \\
\hline \multirow{2}{*}{ WAE } & $\begin{array}{l}\text { Cyclobenzaprine } \\
\text { vs. Placebo }\end{array}$ & Intermediate & $1(\mathrm{~N}=208)^{61}$ & Fair & Direct & Unknown & Imprecise & Unknown & $\begin{array}{c}\text { No effect } \\
16 \% \text { vs. } 5 \% ; p=0.20 \\
\text { RR } 2.82(0.65 \text { to } 12.1) \\
\end{array}$ & Low \\
\hline & $\begin{array}{l}\text { Cyclobenzaprine } \\
\text { vs. Amitriptyline }\end{array}$ & Intermediate & $1(N=208)^{61}$ & Fair & Direct & Unknown & Imprecise & Unknown & $\begin{array}{c}\text { No effect } \\
16 \% \text { vs. } 8 \% \\
\text { RR } 2.25(0.82 \text { to } 6.20) \\
\end{array}$ & Low \\
\hline \multirow[b]{2}{*}{ Dizziness } & $\begin{array}{l}\text { Cyclobenzaprine } \\
\text { vs. Placebo }\end{array}$ & Intermediate & $1(N=208)^{61}$ & Fair & Direct & Unknown & Imprecise & Unknown & $\begin{array}{l}6.1 \% \text { vs. } 2.4 \% ; p=0.38 \\
\text { RR } 2.56(0.31 \text { to } 21.22)\end{array}$ & Insufficient \\
\hline & $\begin{array}{l}\text { Cyclobenzaprine } \\
\text { vs. Amitriptyline }\end{array}$ & Intermediate & $1(N=208)^{61}$ & Fair & Direct & Unknown & Imprecise & Unknown & $\begin{array}{c}61 . \% \text { vs. } 0 \% \\
\text { RR } 11.27(0.63 \text { to } \\
200.53)\end{array}$ & Insufficient \\
\hline \multirow{2}{*}{ Sedation } & $\begin{array}{l}\text { Cyclobenzaprine } \\
\text { vs. Placebo }\end{array}$ & Intermediate & $1(\mathrm{~N}=208)^{61}$ & Fair & Direct & Unknown & Imprecise & Unknown & $\begin{array}{l}3.7 \% \text { vs. } 2.4 \% ; p=0.23 \\
\text { RR } 2.00(023 \text { to } 17.34)\end{array}$ & Insufficient \\
\hline & $\begin{array}{l}\text { Cyclobenzaprine } \\
\text { vs. Amitriptyline }\end{array}$ & Intermediate & $1(\mathrm{~N}=208)^{61}$ & Fair & Direct & Unknown & Imprecise & Unknown & $\begin{aligned} & 3.7 \% \text { vs. } 4.8 \% \\
& \text { RR } 1.30(0.30 \text { to } 5.64)\end{aligned}$ & Insufficient \\
\hline
\end{tabular}

a Study references are in Appendix J.

b Study Quality: poor-quality studies not synthesized

$\mathrm{WAE}=$ withdrawal due to adverse event

Table H-26. Adverse events - memantine vs. placebo

\begin{tabular}{|c|c|c|c|c|c|c|c|c|c|c|}
\hline $\begin{array}{l}\text { Adverse } \\
\text { Effect }\end{array}$ & Comparison & Duration & $\begin{array}{c}\text { Number of } \\
\text { Studies }^{\mathrm{a}} \\
\text { (n participants) }\end{array}$ & $\begin{array}{c}\text { Study } \\
\text { Qualityb }\end{array}$ & Directness & $\begin{array}{c}\text { Consistency } \\
\text { (1 study= } \\
\text { Unknown) }\end{array}$ & Precision & $\begin{array}{l}\text { Publication } \\
\text { Bias }\end{array}$ & $\begin{array}{c}\text { Main Findings } \\
\text { Effect Size }(95 \% \mathrm{Cl})\end{array}$ & $\begin{array}{c}\text { Strength of } \\
\text { Evidence } \\
\text { Grade }\end{array}$ \\
\hline \multirow{2}{*}{ SAE } & $\begin{array}{l}\text { Neuropathic } \\
\text { Pain }\end{array}$ & Short & $1(\mathrm{~N}=45)^{38}$ & Fair & Direct & Unknown & Imprecise & Unknown & Reported as "no differences" & Insufficient \\
\hline & Fibromyalgia & Medium & $1(\mathrm{~N}=63)^{60}$ & Good & Direct & Unknown & Imprecise & Unknown & $\begin{array}{l}\text { Reported as "no serious } \\
\text { adverse events" }\end{array}$ & Insufficient \\
\hline \multirow{2}{*}{ WAE } & $\begin{array}{l}\text { Neuropathic } \\
\text { Pain }\end{array}$ & Short & $1(\mathrm{~N}=45)^{38}$ & Fair & Direct & Unknown & Imprecise & Unknown & Reported as "no differences" & Insufficient \\
\hline & Fibromyalgia & Medium & $1(\mathrm{~N}=63)^{60}$ & Good & Direct & Unknown & Imprecise & Unknown & $6 \%$ vs. $3 \% ; p=0.55$ & Insufficient \\
\hline Dizziness & Fibromyalgia & Medium & $1(\mathrm{~N}=63)^{60}$ & Good & Direct & Unknown & Imprecise & Unknown & $\begin{array}{c}25.8 \% \text { vs. } 12.5 \% ; \\
\text { RR } 2.06(0.69 \text { to } 6.16), p=0.22\end{array}$ & Insufficient \\
\hline Sedation & Fibromyalgia & Medium & $1(\mathrm{~N}=63)^{60}$ & Good & Direct & Unknown & Imprecise & Unknown & $\begin{array}{c}0 \% \text { vs. } 6 \% ; \\
\text { RR } 0.21(0.01 \text { to } 4.13), p=0.30\end{array}$ & Insufficient \\
\hline
\end{tabular}

a Study references are in Appendix J.

b Study Quality: poor-quality studies not synthesized

$\mathrm{CI}=$ confidence interval; $\mathrm{RR}=$ risk ratio; $\mathrm{SAE}=$ serious adverse event; $\mathrm{WAE}=$ withdrawal due to adverse event 


\section{Appendix I. Forest Plots}

\section{Table of Contents}

\section{Tables}

Table I-1. Pregabalin and gabapentin pain outcomes subgroup analyses .......................................... -3

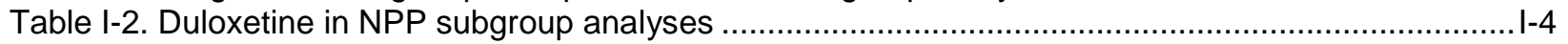

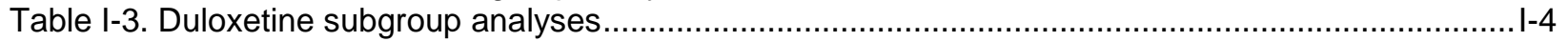

\section{Figures}

Key Question 1: Effectiveness and Comparative Effectiveness

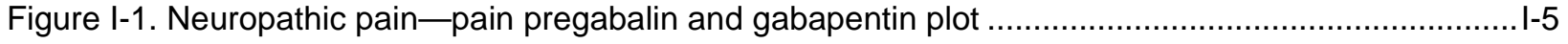

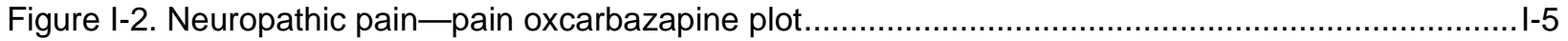

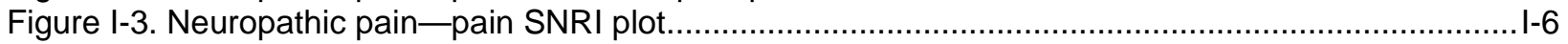

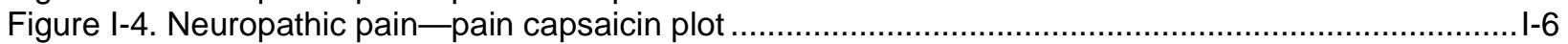

Figure 1-5. Neuropathic pain—pain response pregabalin and gabapentin plot ......................................

Figure I-6. Neuropathic pain—pain response SNRI plot.....................................................................

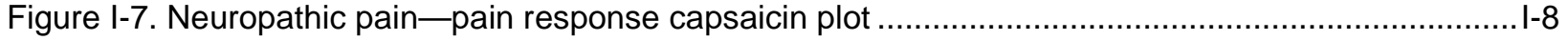

Figure I-8. Neuropathic pain-function pregabalin and gabapentin plot ............................................ I-9

Figure I-9. Neuropathic pain-function SNRI plot........................................................................

Figure I-10. Neuropathic pain-quality of life pregabalin and gabapentin EQ-5D plot ........................... -10

Figure I-11. Neuropathic pain-quality of life pregabalin and gabapentin SF-36 PCS plot..................... I-10

Figure I-12. Neuropathic pain-quality of life pregabalin and gabapentin SF-36 MCS plot .....................-11

Figure I-13. Neuropathic pain—quality of life SNRI plot .................................................................

Figure I-14. Neuropathic pain-anxiety pregabalin and gabapentin plot..............................................12

Figure I-15. Neuropathic pain—depression pregabalin and gabapentin plot .................................... -12

Figure I-16. Neuropathic pain-depression SNRI plot...................................................................

Figure I-17. Neuropathic pain-sleep pregabalin and gabapentin plot ............................................ 13

Figure I-18. Fibromyalgia_-pain pregabalin and gabapentin plot ....................................................

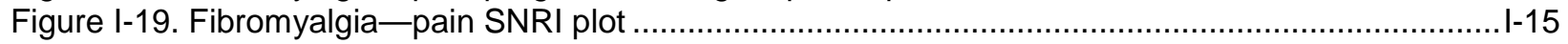

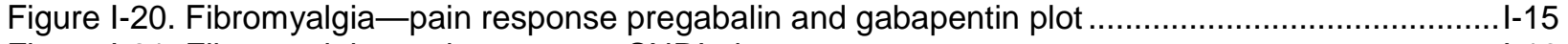

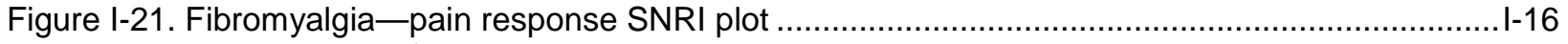

Figure I-22. Fibromyalgia_function pregabalin and gabapentin plot ..............................................16

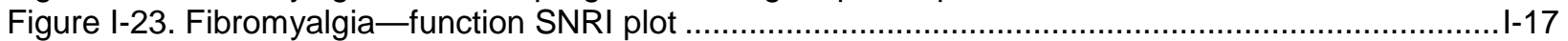

Figure I-24. Fibromyalgia — quality of life pregabalin and gabapentin plot...........................................

Figure I-25. Fibromyalgia—quality of life SNRI SF-36 PCS plot.......................................................

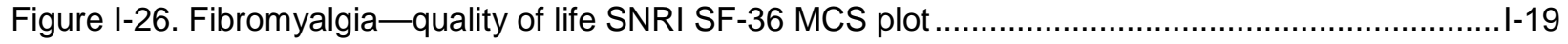

Figure I-27. Fibromyalgia_anxiety pregabalin and gabapentin plot .............................................. 19

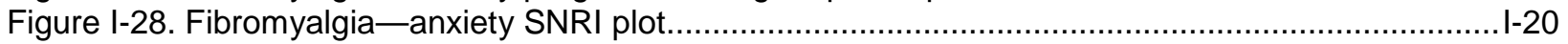

Figure I-29. Fibromyalgia—depression pregabalin and gabapentin plot .......................................... I-20

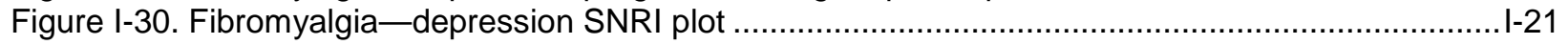

Figure I-31. Fibromyalgia_sleep pregabalin and gabapentin plot ................................................. -21

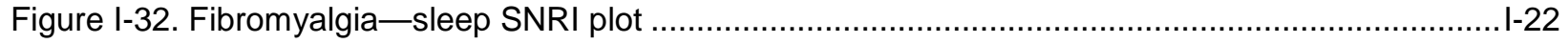

Key Question 2: Adverse Events

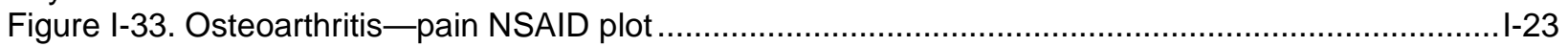

Figure I-34. Osteoarthritis_pain NSAID topical diclofenac plot ....................................................

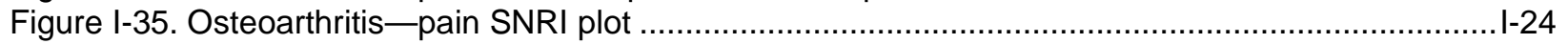

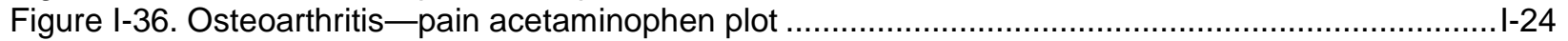

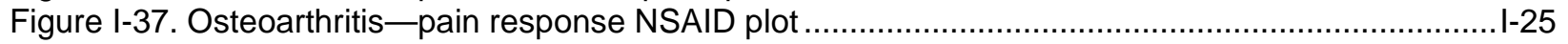

Figure I-38. Osteoarthritis_pain response NSAID topical diclofenac plot ........................................ I-25

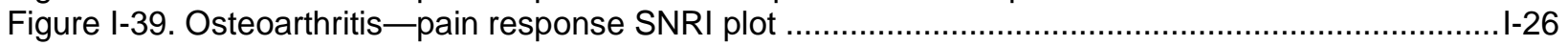

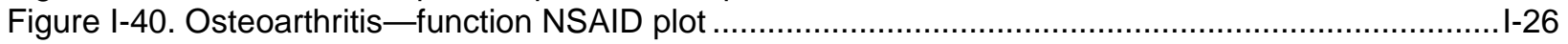

Figure I-41. Osteoarthritis—function topical diclofenac plot ......................................................

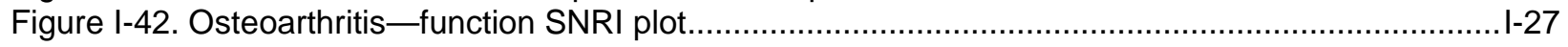

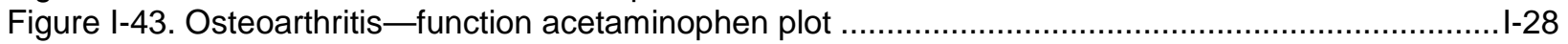




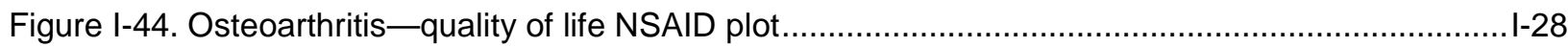

Figure I-45. Osteoarthritis—quality of life SNRI plot ..................................................................

Figure I-46. Osteoarthritis_pain naproxen vs. nabumetone NSAID plot .......................................... -29

Figure 1-47. Inflammatory arthritis - pain NSAID plot...................................................................

Figure I-48. Inflammatory arthritis_pain response NSAID plot ........................................................

Figure I-49. Inflammatory arthritis_function NSAID plot .................................................................

Figure I-50. Inflammatory arthritis - pain celecoxib $200 \mathrm{mg} / \mathrm{d}$ vs. naproxen plot....................................31

Figure 1-51. Inflammatory arthritis_pain celecoxib 400 mg/d vs. naproxen plot..................................-32

Figure I-52. Inflammatory arthritis_pain diclofenac vs. celecoxib $200 \mathrm{mg} / \mathrm{d}$ plot..................................

Figure I-53. Inflammatory arthritis_pain diclofenac vs. celecoxib $400 \mathrm{mg} / \mathrm{d}$ plot..................................

Figure I-54. Inflammatory arthritis_pain naproxen vs. nabumetone plot ........................................... -33

Figure I-55. Inflammatory arthritis_pain response celecoxib $200 \mathrm{mg} / \mathrm{d}$ vs. naproxen plot...................... -34

Figure I-56. Inflammatory arthritis_pain response celecoxib $400 \mathrm{mg} / \mathrm{d}$ vs. naproxen plot ...................... I-34

Figure 1-57. Inflammatory arthritis_pain response diclofenac vs celecoxib $200 \mathrm{mg} / \mathrm{d}$ plot..................... I-35

Figure I-58. Inflammatory arthritis - function celecoxib $200 \mathrm{mg} / \mathrm{d}$ vs. naproxen plot ............................... -35

Figure I-59. Inflammatory arthritis_function celecoxib $400 \mathrm{mg} / \mathrm{d}$ vs. naproxen plot ............................ I-36

Figure I-60. Inflammatory arthritis_function diclofenac vs. celecoxib $200 \mathrm{mg} / \mathrm{d}$ plot...............................36

Figure I-61. Inflammatory arthritis_function diclofenac vs. celecoxib $400 \mathrm{mg} / \mathrm{d}$ plot...............................-37

Figure I-62. Inflammatory arthritis_function naproxen vs. nabumetone plot .......................................

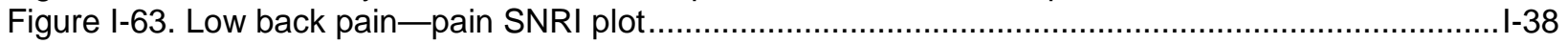

Figure I-64. Low back pain—pain response SNRI plot................................................................

Figure I-65. Low back pain-function SNRI plot ...........................................................................

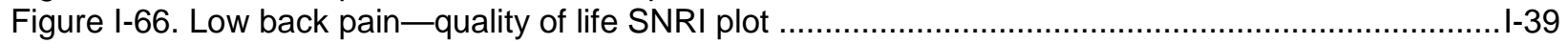

Figure I-67. Antidepressants-SNRI withdrawals due to adverse events plot .................................... -40

Figure I-68. Antidepressants—SNRI serious adverse events plot.....................................................

Figure I-69. Antidepressants_SNRI adverse cognitive effects plot .................................................

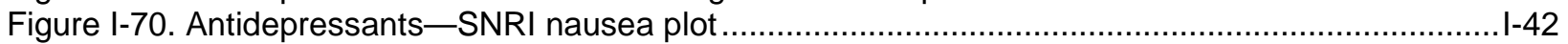

Figure I-71. Antidepressants-SNRI sedation plot ........................................................................

Figure I-72. Antidepressants-TCA withdrawals due to adverse events plot...................................... -43

Figure I-73. Anticonvulsants_-pregabalin and gabapentin withdrawals due to adverse events plot........ I-44

Figure I-74. Anticonvulsants_pregabalin and gabapentin serious adverse events plot ........................ I-45

Figure I-75. Anticonvulsants_pregabalin and gabapentin blurred vision plot.................................... -45

Figure I-76. Anticonvulsants_-pregabalin and gabapentin adverse cognitive effects plot ..................... I-46

Figure 1-77. Anticonvulsants_pregabalin and gabapentin dizziness plot ......................................... -46

Figure 1-78. Anticonvulsants_pregabalin and gabapentin peripheral edema plot .............................. -47

Figure I-79. Anticonvulsants_pregabalin and gabapentin sedation plot ......................................... -48

Figure I-80. Anticonvulsants_pregabalin and gabapentin weight gain plot ....................................... -49

Figure I-81. Anticonvulsants-oxcarbazepine withdrawals due to adverse events plot ......................... I-49

Figure I-82. Anticonvulsants—oxcarbazepine serious adverse events plot .......................................

Figure I-83. Anticonvulsants—oxcarbazepine hyponatremia plot..................................................... -50

Figure I-84. Anticonvulsants_oxcarbazepine sedation plot.........................................................

Figure I-85. NSAIDs—oral NSAIDs withdrawals due to adverse events plot ..................................... -51

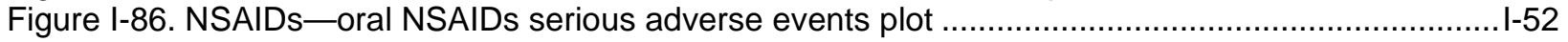

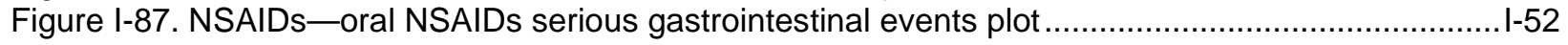

Figure I-88. NSAIDs—topical diclofenac withdrawals due to adverse events plot .............................. I-53

Figure I-89. NSAIDs_topical diclofenac serious adverse events plot .......................................... -53

Figure I-90. NSAIDs_topical diclofenac application site pruritus plot.........................................

Figure I-91. Acetaminophen—withdrawals due to adverse events plot........................................... -53

Figure I-92. Acetaminophen-serious adverse events plot ............................................................

Figure I-93. Capsaicin patch—withdrawals due to adverse events plot .......................................... -54

Figure I-94. Capsaicin patch—application site erythema plot......................................................... 54

Figure 1-95. Capsaicin patch-application site pain plot ..............................................................

Figure 1-96. Capsaicin patch—application site pruritus plot.........................................................

Figure I-97. Osteoarthritis_Egger's test for publication bias ........................................................ -55 
Table I-1. Pregabalin and gabapentin pain outcomes subgroup analyses

\begin{tabular}{|c|c|c|c|c|c|}
\hline $\begin{array}{l}\text { Outcome } \\
\text { Sample Size }\end{array}$ & Variable & Subgroup & $\begin{array}{l}\text { N studies } \\
\text { (sample size) }\end{array}$ & Effect Size (95\% Cl) & $\begin{array}{l}\text { Interaction } \\
\text { p-value }\end{array}$ \\
\hline \multirow[t]{3}{*}{$\begin{array}{l}\text { Pain } \\
\text { Improvement } \\
15 \text { RCTs } \\
(n=4,832)\end{array}$} & Pain type & $\begin{array}{l}\text { DPN } \\
\text { PHN } \\
\text { DPN/PHN } \\
\text { Other }\end{array}$ & $\begin{array}{l}7(2,424) \\
2(747) \\
1(338) \\
5(1,478)\end{array}$ & $\begin{array}{l}\text { MD }-0.45(-0.71 \text { to }-0.22) \\
M D-1.04(-1.55 \text { to }-0.54) \\
M D-1.30(-1.78 \text { to }-0.81) \\
M D-0.49(-1.07 \text { to } 0.04)\end{array}$ & 0.2087 \\
\hline & $\begin{array}{l}\text { Pregabalin } \\
\text { Dose } \\
\\
\text { Gabapentin } \\
\text { enacarbil } \\
\text { Dose }\end{array}$ & $\begin{array}{l}\text { Pregabalin pooled } \\
150 \mathrm{mg} / \text { day } \\
300 \mathrm{mg} / \text { day } \\
600 \mathrm{mg} / \text { day } \\
150-600 \mathrm{mg} / \mathrm{day} \\
300-600 \mathrm{mg} / \mathrm{day} \\
450-600 \mathrm{mg} / \text { day } \\
\\
\text { Gabapentin pooled } \\
1200 \mathrm{mg} / \mathrm{day} \\
2400 \mathrm{mg} / \mathrm{day} \\
3600 \mathrm{mg} / \mathrm{day} \\
1200-3600 \mathrm{mg} / \mathrm{day} \\
\end{array}$ & $\begin{array}{l}15(4,832) \\
2(375) \\
5(1,035) \\
4(735) \\
10(2,963) \\
2(511) \\
1(375) \\
2(725) \\
2(384) \\
2(353) \\
2(418) \\
2(725) \\
\end{array}$ & $\begin{array}{l}\text { MD }-0.63(-0.92 \text { to }-0.36) \\
\text { MD }-0.55(-1.31 \text { to } 0.17) \\
\text { MD }-0.36(-0.89 \text { to } 0.17) \\
\text { MD }-1.17(-1.69 \text { to }-0.67) \\
\text { MD }-0.75(-1.13 \text { to }-0.39) \\
\text { MD }-0.82(-1.48 \text { to }-0.18) \\
\text { MD }-0.02(-0.39 \text { to } 0.35) \\
\text { MD }-0.58(-1.26 \text { to } 0.10) \\
\text { MD }-0.66(-1.21 \text { to }-0.08) \\
\text { MD }-0.27(-1.33 \text { to } 0.82) \\
\text { MD }-0.74(-1.50 \text { to }-0.01) \\
\end{array}$ & $0.8967^{a}$ \\
\hline & $\begin{array}{l}\text { Study } \\
\text { quality }\end{array}$ & $\begin{array}{l}\text { Good } \\
\text { Fair }\end{array}$ & $\begin{array}{l}2(509) \\
13(4,323)\end{array}$ & $\begin{array}{l}\text { MD }-0.51(-1.04 \text { to } 0.08) \\
\text { MD }-0.63(-0.93 \text { to }-0.35)\end{array}$ & 0.7095 \\
\hline \multirow[t]{3}{*}{$\begin{array}{l}\text { Pain } \\
\text { Response } \\
15 \text { RCTs } \\
(n=4,832)\end{array}$} & Pain type & $\begin{array}{l}\text { DPN } \\
\text { PHN } \\
\text { DPN/PHN } \\
\text { Other }\end{array}$ & $\begin{array}{l}7(2,178) \\
2(737) \\
1(338) \\
5(1,323)\end{array}$ & $\begin{array}{l}\text { RR } 1.15 \text { (1.04 to } 1.38) \\
\text { RR } 1.80(0.82 \text { to } 4.37) \\
\text { RR } 1.70(1.22 \text { to } 2.36) \\
\text { RR } 1.15(0.93 \text { to } 1.65)\end{array}$ & 0.3636 \\
\hline & $\begin{array}{l}\text { Gabapentin } \\
\text { enacarbil } \\
\text { Dose }\end{array}$ & $\begin{array}{l}\text { Pregabalin pooled } \\
150 \mathrm{mg} / \text { day } \\
300 \mathrm{mg} / \text { day } \\
600 \mathrm{mg} / \mathrm{day} \\
150-600 \mathrm{mg} / \mathrm{day} \\
300-600 \mathrm{mg} / \mathrm{day} \\
450-600 \mathrm{mg} / \mathrm{day} \\
\\
\text { Gabapentin pooled } \\
1200 \mathrm{mg} / \mathrm{day} \\
2400 \mathrm{mg} / \mathrm{day} \\
3600 \mathrm{mg} / \mathrm{day} \\
1200-3600 \mathrm{mg} / \mathrm{day}\end{array}$ & $\begin{array}{l}15(4,832) \\
2(375) \\
5(1,035) \\
4(735) \\
10(2,963) \\
2(511) \\
1(375) \\
2(725) \\
2(384) \\
2(353) \\
2(418) \\
2(725)\end{array}$ & $\begin{array}{l}\text { RR } 1.28 \text { (1.09 to } 1.54) \\
\text { RR } 1.62 \text { (0.71 to } 4.00) \\
\text { RR } 1.22 \text { (0.90 to } 1.77) \\
\text { RR } 1.99(1.42 \text { to } 2.87) \\
\text { RR } 1.36(1.14 \text { to } 1.71) \\
\text { RR } 1.63 \text { (1.15 to } 2.26) \\
\text { RR } 0.94 \text { (0.77 to } 1.16) \\
\text { RR } 1.20 \text { (0.94 to } 1.57) \\
\text { RR } 1.16 \text { (0.88 to } 1.53) \\
\text { RR } 1.17(0.72 \text { to } 1.84) \\
\text { RR } 1.29(1.01 \text { to } 1.66) \\
\text { RR } 1.20(0.94 \text { to } 1.57)\end{array}$ & $0.8149^{a}$ \\
\hline & $\begin{array}{l}\text { Study } \\
\text { quality }\end{array}$ & $\begin{array}{l}\text { Good } \\
\text { Fair }\end{array}$ & $\begin{array}{l}2(509) \\
13(4,067)\end{array}$ & $\begin{array}{l}\text { RR } 1.13 \text { (0.78 to } 1.84) \\
\text { RR } 1.31 \text { (1.12 to } 1.58)\end{array}$ & 0.6690 \\
\hline
\end{tabular}

${ }^{a}$ p-value for interaction with study drug

DPN = diabetic peripheral neuropathy; $\mathrm{MD}=$ mean difference; $\mathrm{PHN}=$ postherpetic neuralgia; $\mathrm{RCT}=$ randomized controlled trial; $\mathrm{RR}=$ relative risk 
Table I-2. Duloxetine in NPP subgroup analyses

\begin{tabular}{|c|c|c|c|c|}
\hline $\begin{array}{l}\text { Outcome } \\
\text { Sample Size }\end{array}$ & Variable & Subgroup & $\begin{array}{l}\text { N studies } \\
\text { (sample size) }\end{array}$ & Effect Size $(95 \% \mathrm{Cl})$ \\
\hline $\begin{array}{l}\text { Pain } \\
\text { Improvement }\end{array}$ & $\begin{array}{l}\text { Duloxetine } \\
\text { Dose }\end{array}$ & $\begin{array}{l}\text { Duloxetine pooled } \\
20 \mathrm{mg} / \mathrm{day} \\
40 \mathrm{mg} / \mathrm{day} \\
60 \mathrm{mg} / \mathrm{day} \\
120 \mathrm{mg} / \mathrm{day} \\
20-120 \mathrm{mg} / \text { day } \\
40-60 \mathrm{mg} / \text { day } \\
60-120 \mathrm{mg} / \text { day }\end{array}$ & $\begin{array}{l}6(2,082) \\
1(230) \\
1(252) \\
5(1,328) \\
3(675) \\
1(457) \\
1(338) \\
3(889)\end{array}$ & $\begin{array}{l}\text { MD }-0.79(-1.10 \text { to }-0.49) \\
\text { MD }-0.45(-1.05 \text { to } 0.15) \\
\text { MD }-0.80(-1.38 \text { to }-0.22) \\
\text { MD }-0.85(-1.22 \text { to }-0.54) \\
\text { MD }-1.16(-1.64 \text { to }-0.76) \\
\text { MD }-0.90(-1.40 \text { to }-0.41) \\
\text { MD }-0.86(-1.32 \text { to }-0.40) \\
\text { MD }-0.88(-1.52 \text { to }-0.24)\end{array}$ \\
\hline $\begin{array}{l}\text { Pain } \\
\text { Response }\end{array}$ & $\begin{array}{l}\text { Duloxetine } \\
\text { Dose }\end{array}$ & $\begin{array}{l}\text { Duloxetine pooled } \\
20 \mathrm{mg} / \mathrm{day} \\
40 \mathrm{mg} / \mathrm{day} \\
60 \mathrm{mg} / \mathrm{day} \\
120 \mathrm{mg} / \mathrm{day} \\
20-120 \mathrm{mg} / \text { day } \\
40-60 \mathrm{mg} / \text { day } \\
60-120 \mathrm{mg} / \text { day }\end{array}$ & $\begin{array}{l}6(2,082) \\
1(230) \\
1(252) \\
5(1,328) \\
3(675) \\
1(457) \\
1(338) \\
3(889)\end{array}$ & $\begin{array}{l}\text { RR } 1.43 \text { (1.24 to } 1.69) \\
\text { RR } 1.59 \text { (1.08 to } 2.33) \\
\text { RR } 1.57 \text { (1.18 to } 2.07) \\
\text { RR } 1.50(1.31 \text { to } 1.80) \\
\text { RR } 1.64 \text { (1.37 to } 2.02) \\
\text { RR } 1.83 \text { (1.31 to } 2.56) \\
\text { RR } 1.62 \text { (1.27 to } 2.07) \\
\text { RR } 1.38 \text { (1.09 to } 1.79)\end{array}$ \\
\hline $\begin{array}{l}\text { Function } \\
\text { (Brief Pain } \\
\text { Inventory- } \\
\text { Interference) }\end{array}$ & $\begin{array}{l}\text { Duloxetine } \\
\text { Dose }\end{array}$ & $\begin{array}{l}\text { Duloxetine pooled } \\
20 \mathrm{mg} / \mathrm{day} \\
40 \mathrm{mg} / \mathrm{day} \\
60 \mathrm{mg} / \mathrm{day} \\
120 \mathrm{mg} / \mathrm{day} \\
20-120 \mathrm{mg} / \text { day } \\
40-60 \mathrm{mg} / \mathrm{day} \\
60-120 \mathrm{mg} / \text { day }\end{array}$ & $\begin{array}{l}6(2,082) \\
1(230) \\
1(252) \\
5(1,328) \\
3(675) \\
1(457) \\
1(338) \\
3(889) \\
\end{array}$ & $\begin{array}{l}\text { SMD }-0.31(-0.42 \text { to }-0.20) \\
\text { SMD } 0.00(-0.26 \text { to } 0.26) \\
\text { SMD }-0.18(-0.44 \text { to } 0.08) \\
\text { SMD }-0.34(-0.45 \text { to }-0.22) \\
\text { SMD }-0.46(-0.65 \text { to }-0.27) \\
\text { SMD }-0.22(-0.43 \text { to }-0.00) \\
\text { SMD }-0.20(-0.41 \text { to } 0.01) \\
\text { SMD }-0.39(-0.58 \text { to }-0.17)\end{array}$ \\
\hline $\begin{array}{l}\text { Quality of Life } \\
\text { (Euro Quality } \\
\text { of Life-5D) }\end{array}$ & $\begin{array}{l}\text { Duloxetine } \\
\text { Dose }\end{array}$ & $\begin{array}{l}\text { Duloxetine pooled } \\
20 \mathrm{mg} / \mathrm{day} \\
60 \mathrm{mg} / \mathrm{day} \\
120 \mathrm{mg} / \mathrm{day} \\
60-120 \mathrm{mg} / \text { day }\end{array}$ & $\begin{array}{l}4(1,404) \\
1(457) \\
2(791) \\
2(791) \\
3(1,006)\end{array}$ & $\begin{array}{l}\text { MD } 0.20(0.07 \text { to } 0.33) \\
\text { MD } 0.10(-0.17 \text { to } 0.37) \\
\text { MD } 0.29(0.06 \text { to } 0.52) \\
\text { MD } 0.29(0.06 \text { to } 0.53) \\
\text { MD } 0.22(0.05 \text { to } 0.38)\end{array}$ \\
\hline
\end{tabular}

Table I-3. Duloxetine subgroup analyses

\begin{tabular}{|c|c|c|c|c|}
\hline $\begin{array}{l}\text { Outcome } \\
\text { Sample Size }\end{array}$ & Variable & Subgroup & $\operatorname{SMD}(95 \% \mathrm{Cl})^{\mathrm{a}}$ & $\begin{array}{l}\text { Interaction } \\
\text { p-value }\end{array}$ \\
\hline \multirow{3}{*}{$\begin{array}{l}\text { Pain } \\
\text { Improvement } \\
5 \text { RCTs } \\
(n=1,535)\end{array}$} & Pain location & $\begin{array}{l}\text { Knee } \\
\text { Knee/Hip }\end{array}$ & $\begin{array}{l}-0.90(-1.21 \text { to }-0.66) \\
-0.50(-0.80 \text { to }-0.20)\end{array}$ & 0.129 \\
\hline & Study quality & $\begin{array}{l}\text { Good } \\
\text { Fair }\end{array}$ & $\begin{array}{l}-0.93(-1.37 \text { to }-0.63) \\
-0.57(-1.00 \text { to }-0.25)\end{array}$ & 0.197 \\
\hline & Dose & $\begin{array}{l}\text { 60mg/day } \\
60-120 \mathrm{mg} / \text { day }\end{array}$ & $\begin{array}{l}-0.77(-1.32 \text { to }-0.35) \\
-0.88(-1.36 \text { to }-0.42)\end{array}$ & 0.769 \\
\hline \multirow{3}{*}{$\begin{array}{l}\text { Pain Response } \\
4 \text { RCTs } \\
(n=1,274)\end{array}$} & Pain location & $\begin{array}{l}\text { Knee } \\
\text { Knee/Hip }\end{array}$ & $\begin{array}{l}1.41(1.24 \text { to } 1.61) \\
1.28(1.08 \text { to } 1.52)\end{array}$ & 0.457 \\
\hline & Study quality & $\begin{array}{l}\text { Good } \\
\text { Fair: }\end{array}$ & $\begin{array}{l}1.38 \text { (1.16 to } 1.64) \\
1.36 \text { (1.13 to } 1.69)\end{array}$ & 0.862 \\
\hline & Dose & $\begin{array}{l}\text { 60mg/day } \\
60-120 \mathrm{mg} / \text { day }\end{array}$ & $\begin{array}{l}1.37 \text { (1.22 to } 1.56) \\
1.35 \text { (1.05 to } 1.73)\end{array}$ & 0.903 \\
\hline \multirow{3}{*}{$\begin{array}{l}\text { Function } \\
5 \text { RCTs } \\
(n=1,535)\end{array}$} & Pain location & $\begin{array}{l}\text { Knee } \\
\text { Knee/Hip }\end{array}$ & $\begin{array}{l}-0.26(-0.45 \text { to }-0.06) \\
-0.31(-0.21 \text { to }-0.10)\end{array}$ & 0.819 \\
\hline & Study quality & $\begin{array}{l}\text { Good } \\
\text { Fair }\end{array}$ & $\begin{array}{l}-0.26(-0.53 \text { to } 0.02) \\
-0.29(-0.47 \text { to }-0.10)\end{array}$ & 0.889 \\
\hline & Dose & $\begin{array}{l}\text { 60mg/day } \\
60-120 \mathrm{mg} / \text { day }\end{array}$ & $\begin{array}{l}-0.28(-0.54 \text { to }-0.01) \\
-0.25(-0.46 \text { to }-0.05)\end{array}$ & 0.876 \\
\hline
\end{tabular}

${ }^{a}$ Response outcome is a relative risk (RR) 


\section{Neuropathic Pain}

Figure I-1. Neuropathic pain-pain pregabalin and gabapentin plot

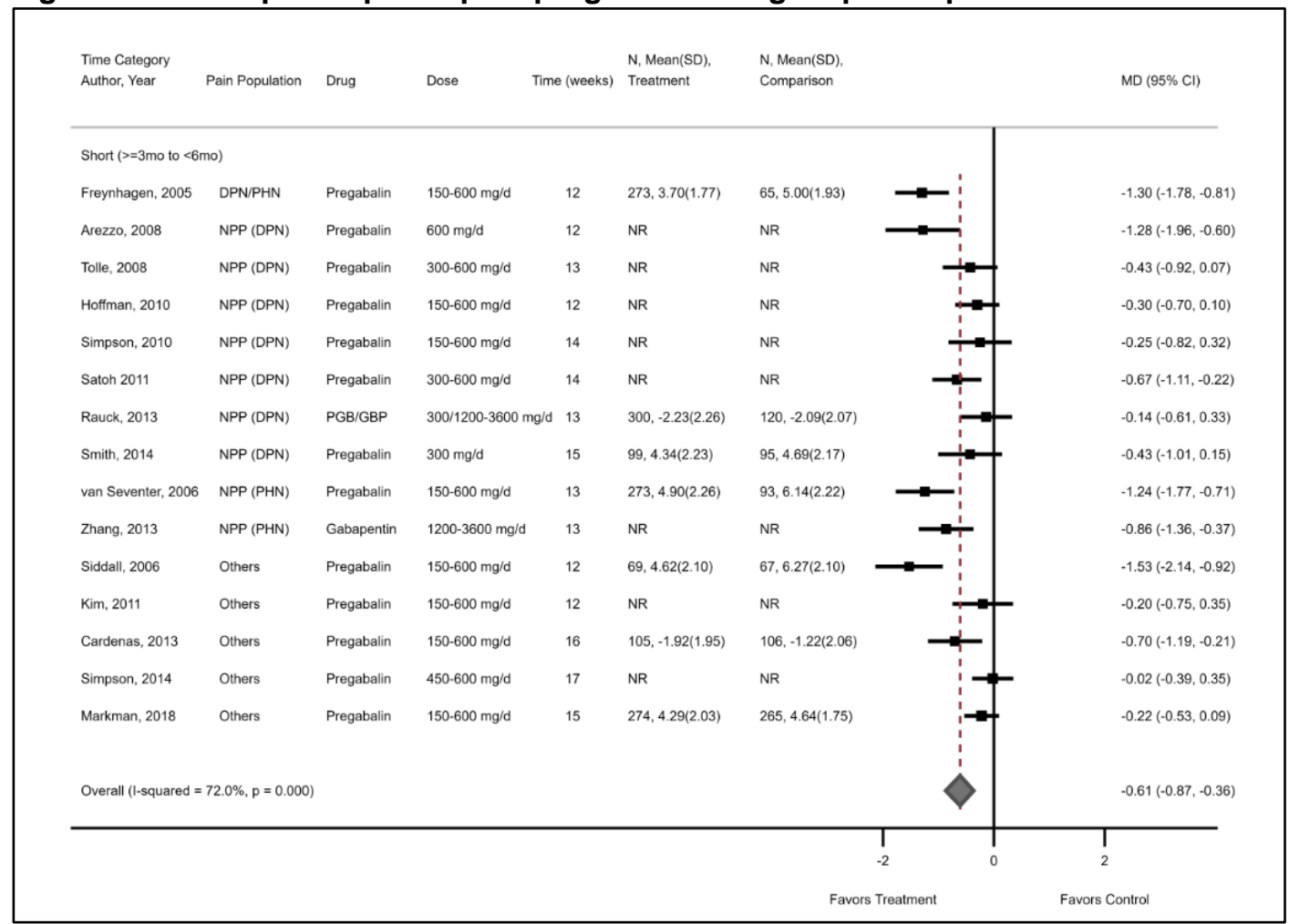

Figure I-2. Neuropathic pain-pain oxcarbazapine plot

Time Category
Author, Year Pain Population Drug
Short ( $=3$ mono to $<6 \mathrm{mo})$


Figure I-3. Neuropathic pain-pain SNRI plot

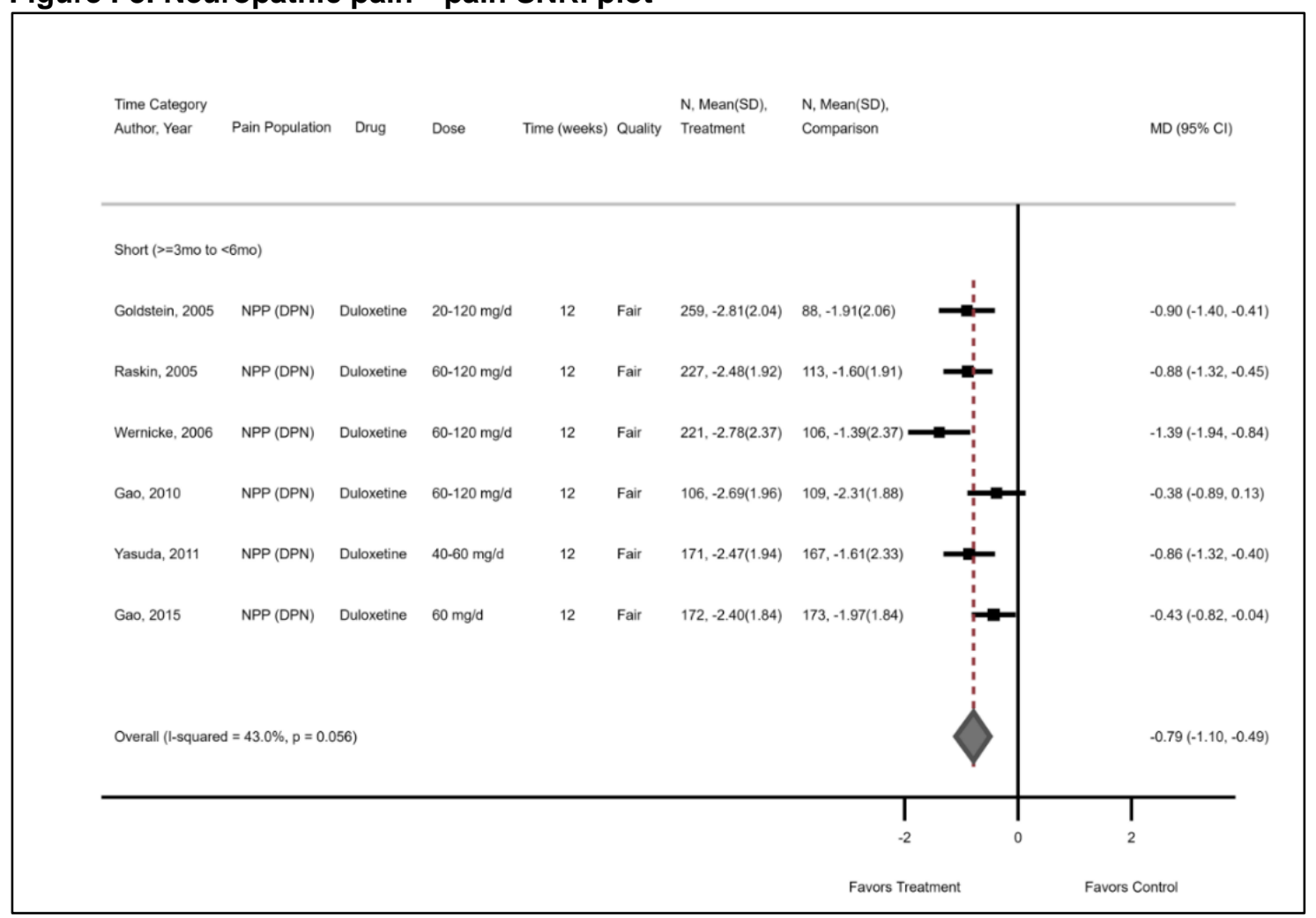

Figure I-4. Neuropathic pain-pain capsaicin plot

\begin{tabular}{|c|c|c|c|c|c|c|c|}
\hline $\begin{array}{l}\text { Time Category } \\
\text { Author, Year }\end{array}$ & Pain Populatio & Drug & Dose & Time (wee & $\begin{array}{l}\text { N. Mean(SD), } \\
\text { s) Treatment }\end{array}$ & $\begin{array}{l}\text { N, Mean(SD), } \\
\text { Comparison }\end{array}$ & MD $(95 \% \mathrm{Cl})$ \\
\hline \multicolumn{8}{|c|}{ Short $(>=3 \mathrm{mot}$ to $<6 \mathrm{mo})$} \\
\hline Backonja, 2008 & NPP (PHN) & Capsaicin & $8 \%$ & 12 & $206,-1.70(1.72)$ & $196,-1.20(1.82)$ & $-0.50(-0.85,-0.15)$ \\
\hline Webster, 2010 & NPP (PHN) & Capsaicin & $8 \%$ & 12 & $102,-1.80(2.02)$ & $53,-1.70(2.04)$ & $-0.10(-0.77,0.57)$ \\
\hline Clifford, 2012 & Others & Capsaicin & $8 \%$ & 12 & $332,4.30(2.03)$ & $162,4.52(1.81)$ & $-0.21(-0.58,0.15)$ \\
\hline \multicolumn{7}{|c|}{ Overall (1-squared $=0.0 \%, p=0.423$ ) } & $-0.33(-0.60,-0.00)$ \\
\hline & & & & & & T & \\
\hline & & & & & & Favors Treatment & Control \\
\hline
\end{tabular}


Figure I-5. Neuropathic pain-pain response pregabalin and gabapentin plot

\begin{tabular}{|c|c|c|c|c|c|c|c|c|}
\hline $\begin{array}{l}\text { Time Category } \\
\text { Author, Year }\end{array}$ & Pain Population & Drug & Dose & $\begin{array}{l}\text { Response } \\
\text { Criteria }\end{array}$ & Quality & $\begin{array}{l}\text { Treatment } \\
\mathrm{n} / \mathrm{N}\end{array}$ & $\begin{array}{l}\text { Control } \\
\mathrm{n} / \mathrm{N}\end{array}$ & $\begin{array}{l}\text { Risk Ratio } \\
(95 \% \mathrm{Cl})\end{array}$ \\
\hline \multicolumn{9}{|l|}{ Short (<26 wks) } \\
\hline Arezzo, 2008 & NPP (DPN) & Pregabalin & $600 \mathrm{mg} / \mathrm{d}$ & $50 \% \mathrm{PR}$ & Fair & $40 / 82$ & $20 / 85$ & $2.07(1.33,3.23)$ \\
\hline Hoffman, 2010 & NPP (DPN) & Pregabalin & $150-600 \mathrm{mg} / \mathrm{d}$ & $30 \% \mathrm{PR}$ & Fair & $172 / 267$ & $73 / 134$ & $1.18(0.99,1.41)$ \\
\hline Rauck, 2013 & NPP (DPN) & PGB/GBP & $300 / 1200-3600 \mathrm{mg} / \mathrm{d}$ & $30 \% \mathrm{PR}$ & Fair & $150 / 300$ & $57 / 120$ & $1.05(0.85,1.31)$ \\
\hline Satoh, 2011 & NPP (DPN) & Pregabalin & $300-600 \mathrm{mg} / \mathrm{d}$ & $50 \% \mathrm{PR}$ & Fair & $55 / 179$ & 29/135 & $1.43(0.97,2.11)$ \\
\hline Simpson, 2010 & NPP (DPN) & Pregabalin & $150-600 \mathrm{mg} / \mathrm{d}$ & $30 \% \mathrm{PR}$ & Good & $84 / 149$ & $84 / 150$ & $1.01(0.82,1.23)$ \\
\hline Smith, 2014 Study 3 & NPP (DPN) & Pregabalin & $300 \mathrm{mg} / \mathrm{d}$ & $30 \% \mathrm{PR}$ & Fair & $49 / 99$ & $45 / 95$ & $1.04(0.78,1.40)$ \\
\hline Tolle, 2008 & NPP (DPN) & Pregabalin & $150-600 \mathrm{mg} / \mathrm{d}$ & $50 \% \mathrm{PR}$ & Fair & $109 / 290$ & 28/93 & $1.25(0.89,1.76)$ \\
\hline Freynhagen, 2005 & NPP (DPN/PHN) & Pregabalin & $150-600 \mathrm{mg} / \mathrm{d}$ & $30 \% \mathrm{PR}$ & Fair & $171 / 273$ & $24 / 65$ & $1.70(1.22,2.36)$ \\
\hline Cardenas, 2013 & NPP (Other) & Pregabalin & $150-600 \mathrm{mg} / \mathrm{d}$ & $30 \% \mathrm{PR}$ & Good & $48 / 105$ & $33 / 105$ & $1.45(1.02,2.07)$ \\
\hline Kim, 2011 & NPP (Other) & Pregabalin & $150-600 \mathrm{mg} / \mathrm{d}$ & CGIC & Fair & $82 / 110$ & $72 / 109$ & $1.13(0.95,1.34)$ \\
\hline Markman, 2018 & NPP (Other) & Pregabalin & $150-600 \mathrm{mg} / \mathrm{d}$ & $30 \% \mathrm{PR}$ & Fair & $113 / 196$ & 109/187 & $0.99(0.83,1.17)$ \\
\hline Siddall, 2006 & NPP (Other) & Pregabalin & $150-600 \mathrm{mg} / \mathrm{d}$ & $30 \% \mathrm{PR}$ & Fair & 29/69 & $11 / 67$ & $2.56(1.39,4.70)$ \\
\hline Simpson, 2014 & NPP (Other) & Pregabalin & $450-600 \mathrm{mg} / \mathrm{d}$ & $30 \%$ PR & Fair & $88 / 183$ & $98 / 192$ & $0.94(0.77,1.16)$ \\
\hline Zhang, 2013 Study 3 & NPP (PHN) & Gabapentin & $1200-3600 \mathrm{mg} / \mathrm{d}$ & $30 \% \mathrm{PR}$ & Fair & $157 / 276$ & $40 / 95$ & $1.35(1.04,1.75)$ \\
\hline van Seventer, 2006 & NPP (PHN) & Pregabalin & $150-600 \mathrm{mg} / \mathrm{d}$ & $30 \% \mathrm{PR}$ & Fair & $120 / 273$ & $16 / 93$ & $2.55(1.60,4.07)$ \\
\hline Subgroup & & & & & & $1467 / 2851$ & $739 / 1725$ & $1.27(1.12,1.50)$ \\
\hline \multicolumn{9}{|c|}{$(1-$ squared $=72.0 \%, p=0.000)$} \\
\hline & & & & & & & & \\
\hline & & & & & & & & \\
\hline
\end{tabular}


Figure I-6. Neuropathic pain-pain response SNRI plot

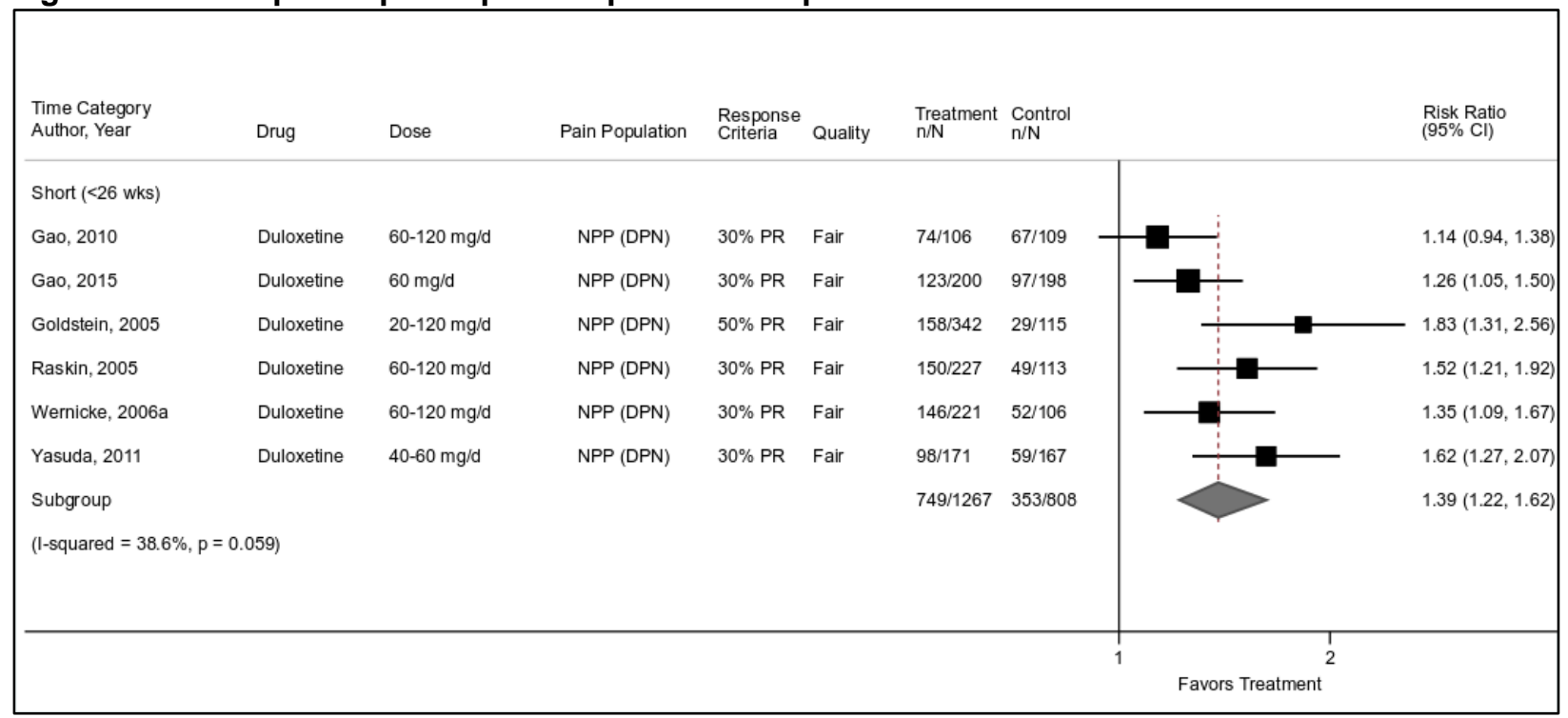

Figure I-7. Neuropathic pain-pain response capsaicin plot

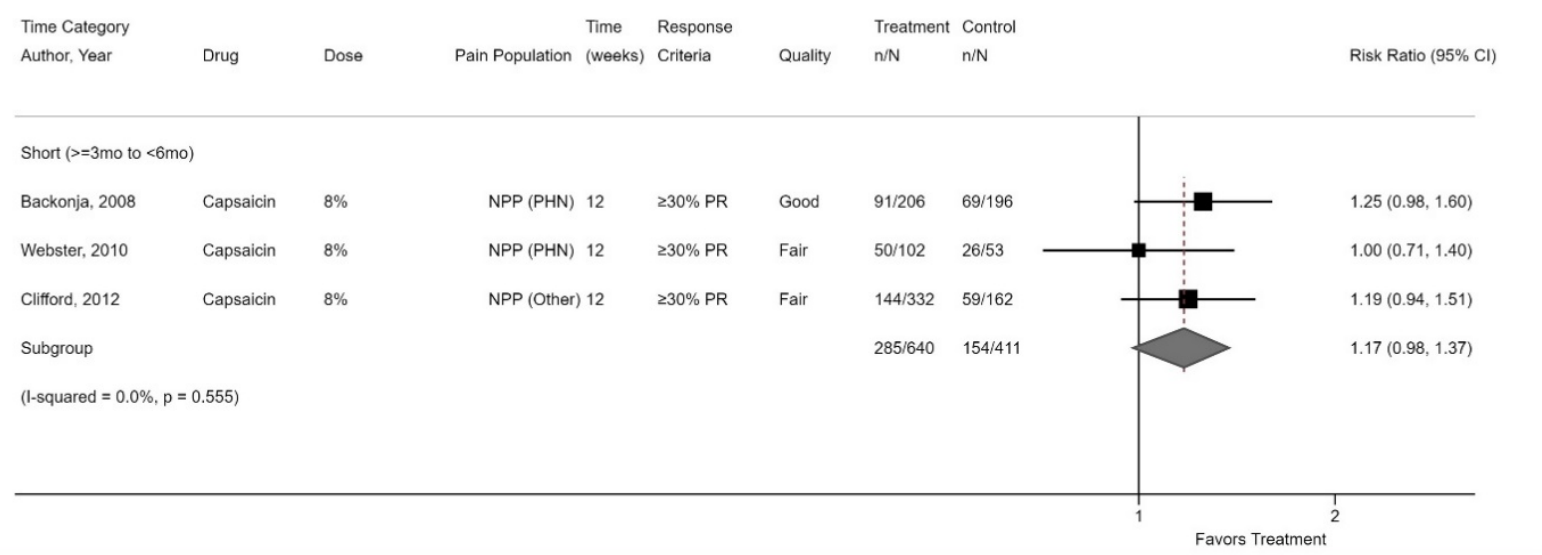


Figure I-8. Neuropathic pain-function pregabalin and gabapentin plot

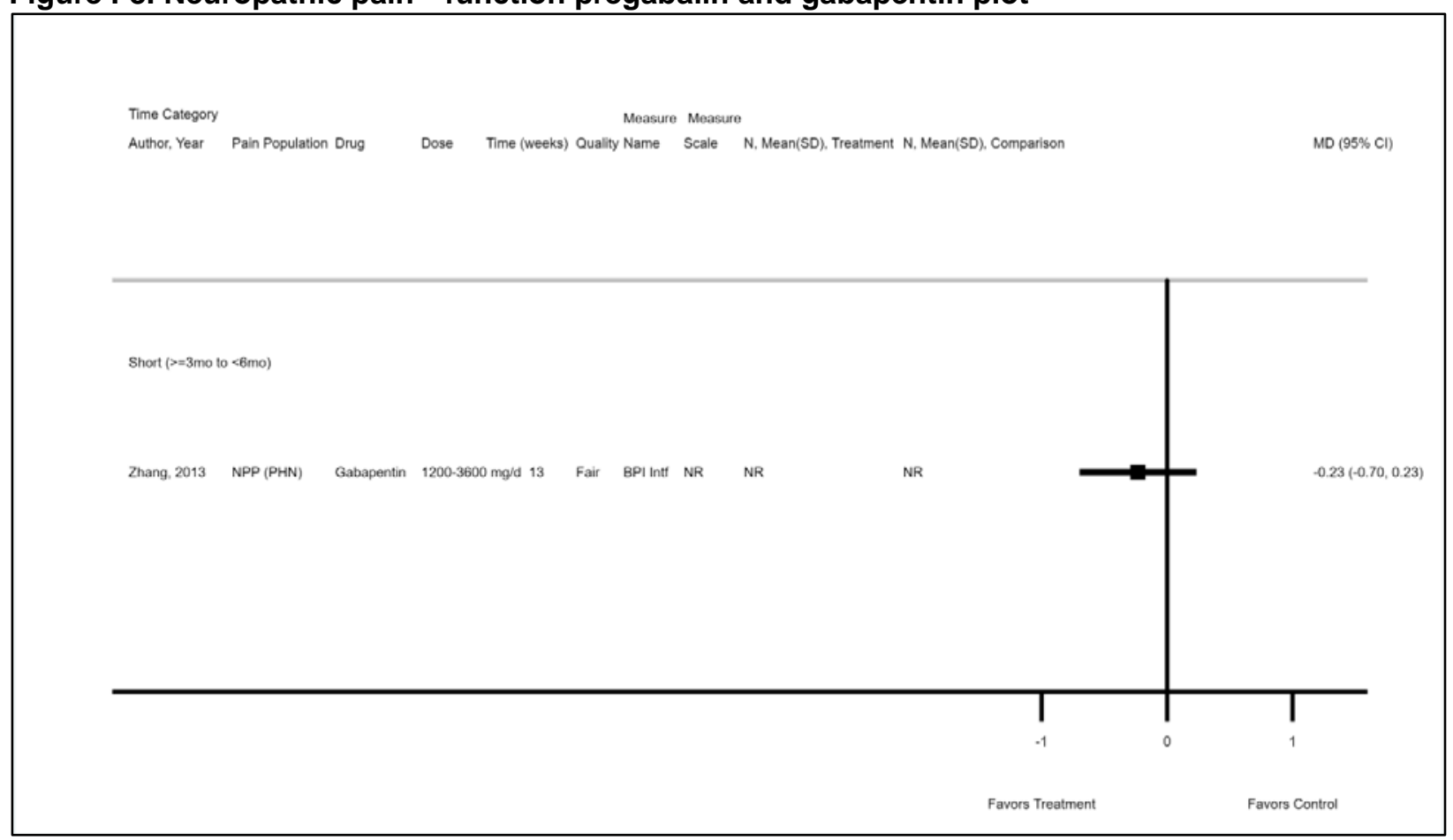

Figure I-9. Neuropathic pain-function SNRI plot

\begin{tabular}{|c|c|c|c|c|c|c|c|c|c|c|}
\hline $\begin{array}{l}\text { Time Category } \\
\text { Author, Year }\end{array}$ & Pain Population & Drug & Dose & Time (weeks) & ) Quality & $\begin{array}{l}\text { Measure } \\
\text { Name }\end{array}$ & $\begin{array}{l}\text { Measure } \\
\text { Scale }\end{array}$ & N. Mean(SD), Treatment & N. Mean(SD), Comparison & SMD (95\% Cl) \\
\hline \multicolumn{11}{|c|}{ Short $(>=3 \mathrm{mo}$ to $<6 \mathrm{mo})$} \\
\hline Goldstein, 2005 & $\mathrm{NPP}(\mathrm{DPN})$ & Duloxetine & $20-120 \mathrm{mg} / \mathrm{d}$ & 12 & Fair & BPI Intf & 0.70 & $332,-2.12(1.82)$ & $112,-1.73(1.80)$ & $-0.22(-0.43,-0.00)$ \\
\hline Raskin, 2005 & NPP (DPN) & Duloxetine & $60-120 \mathrm{mg} / \mathrm{d}$ & 12 & Fair & BPI Intt & 0.40 & $216,-2.48(1.87)$ & $109,-1.56(1.88)$ & $-0.49(-0.73,-0.26)$ \\
\hline Wernicke, 2006 & NPP (DPN) & Duloxetine & $60-120 \mathrm{mg} / \mathrm{d}$ & 12 & Fair & BPI Intf & 0.70 & $218,-2.57(1.98)$ & $104,-1.72(1.94)$ & $-0.43(-0.67,-0.20)$ \\
\hline Gao, 2010 & NPP (DPN) & Duloxetine & $60-120 \mathrm{mg} / \mathrm{d}$ & 12 & Fair & BPI Intf & 0.10 & $106,-2.28(2.16)$ & $109,-1.88(2.09)$ & $-0.19(-0.46,0.08)$ \\
\hline Yasuda, 2011 & NPP (DPN) & Duloxetine & $40.60 \mathrm{mg} / \mathrm{d}$ & 12 & Fair & BPI Intf & $0-10$ & $171,-2.04(2.22)$ & $167,-1.56(2.58)$ & $-0.20(-0.41,0.01)$ \\
\hline Gao, 2015 & NPP (DPN) & Duloxetine & $60 \mathrm{mg} / \mathrm{d}$ & 12 & Fair & BPI Intf & 0.10 & $173 .-2.42(1.71)$ & $176,-1.82(1.86)$ & $-0.35(-0.56,-0.14)$ \\
\hline \multicolumn{10}{|c|}{ Overall (1-squared $=0.0 \%, p=0.295)$} & $-0.31(-0.42,-0.20)$ \\
\hline & & & & & & & & & $T_{.5}^{T}$ & $\begin{array}{l}T \\
.5\end{array}$ \\
\hline & & & & & & & & & Favors Treatment & Favors Control \\
\hline
\end{tabular}


Figure I-10. Neuropathic pain-quality of life pregabalin and gabapentin EQ-5D plot

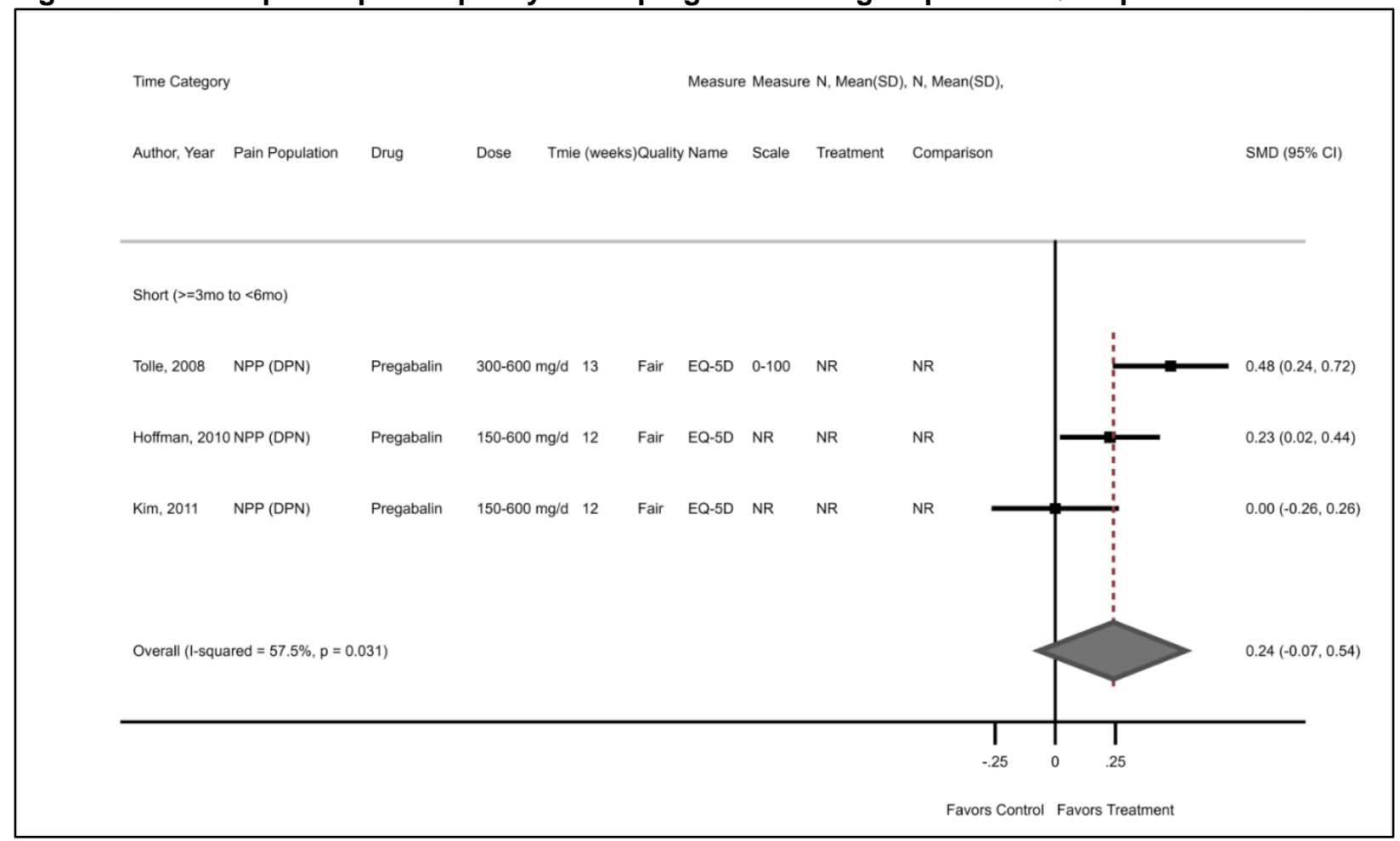

Figure I-11. Neuropathic pain-quality of life pregabalin and gabapentin SF-36 PCS plot

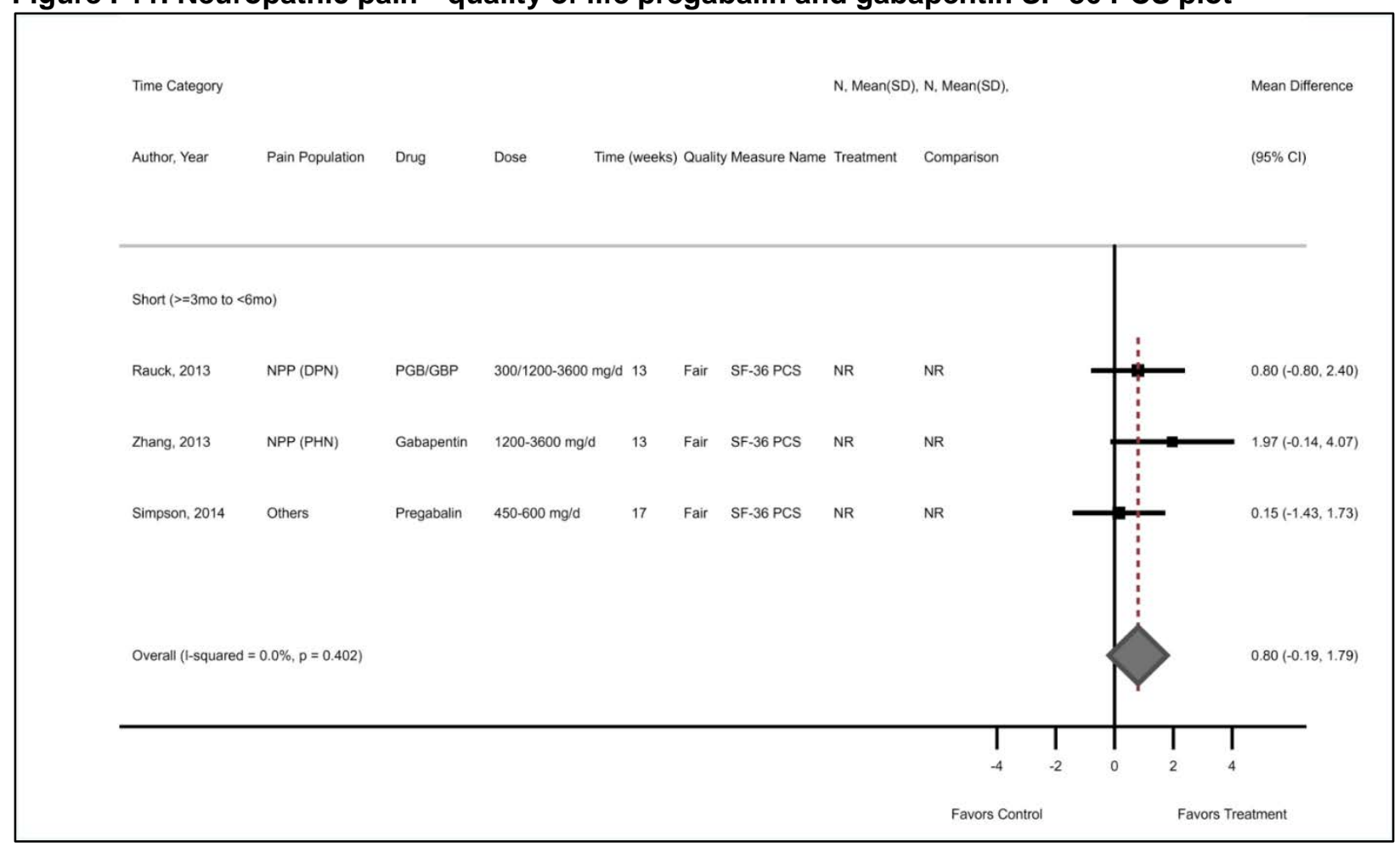


Figure I-12. Neuropathic pain-quality of life pregabalin and gabapentin SF-36 MCS plot

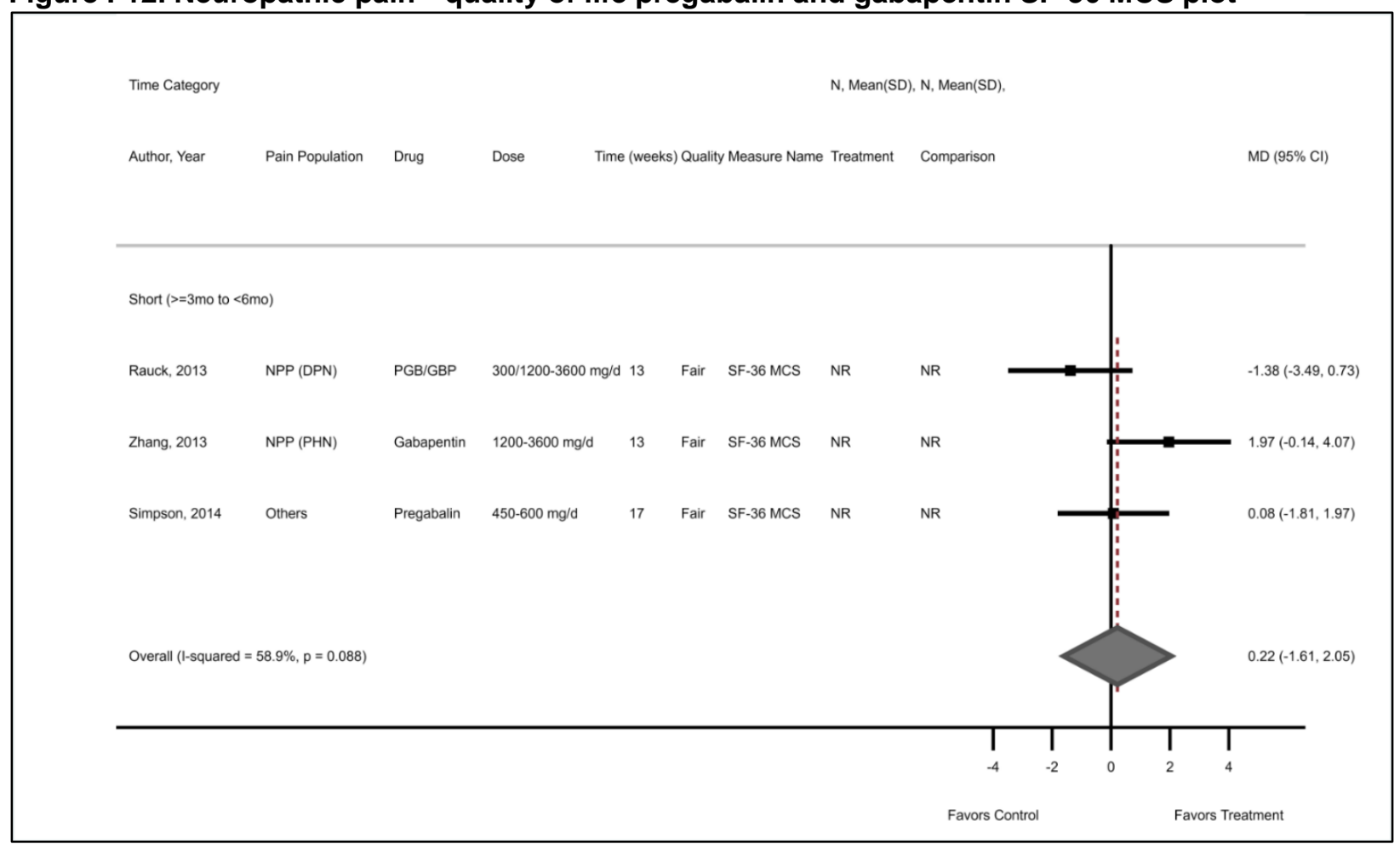

Figure I-13. Neuropathic pain-quality of life SNRI plot

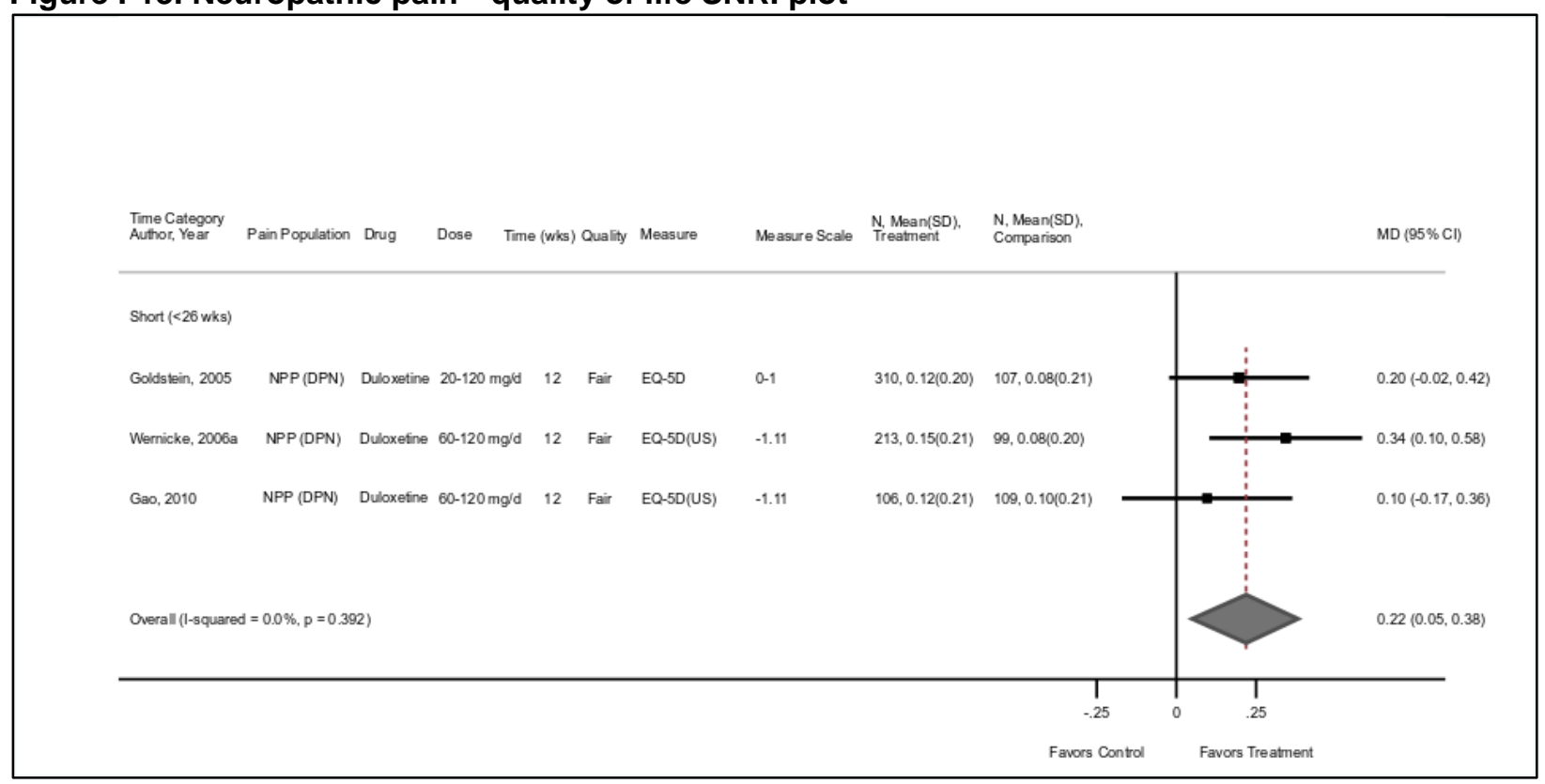


Figure I-14. Neuropathic pain-anxiety pregabalin and gabapentin plot

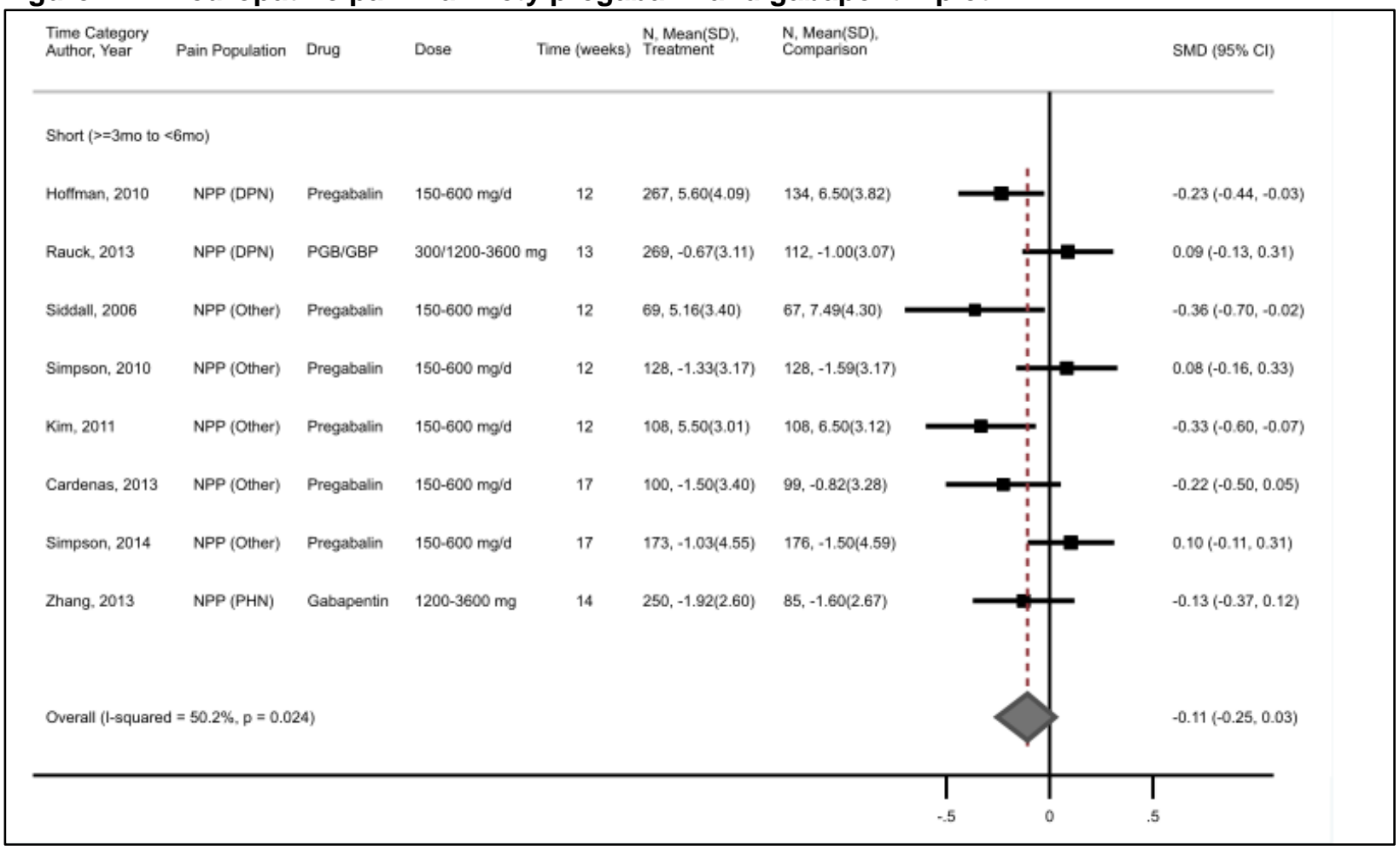

Figure I-15. Neuropathic pain-depression pregabalin and gabapentin plot

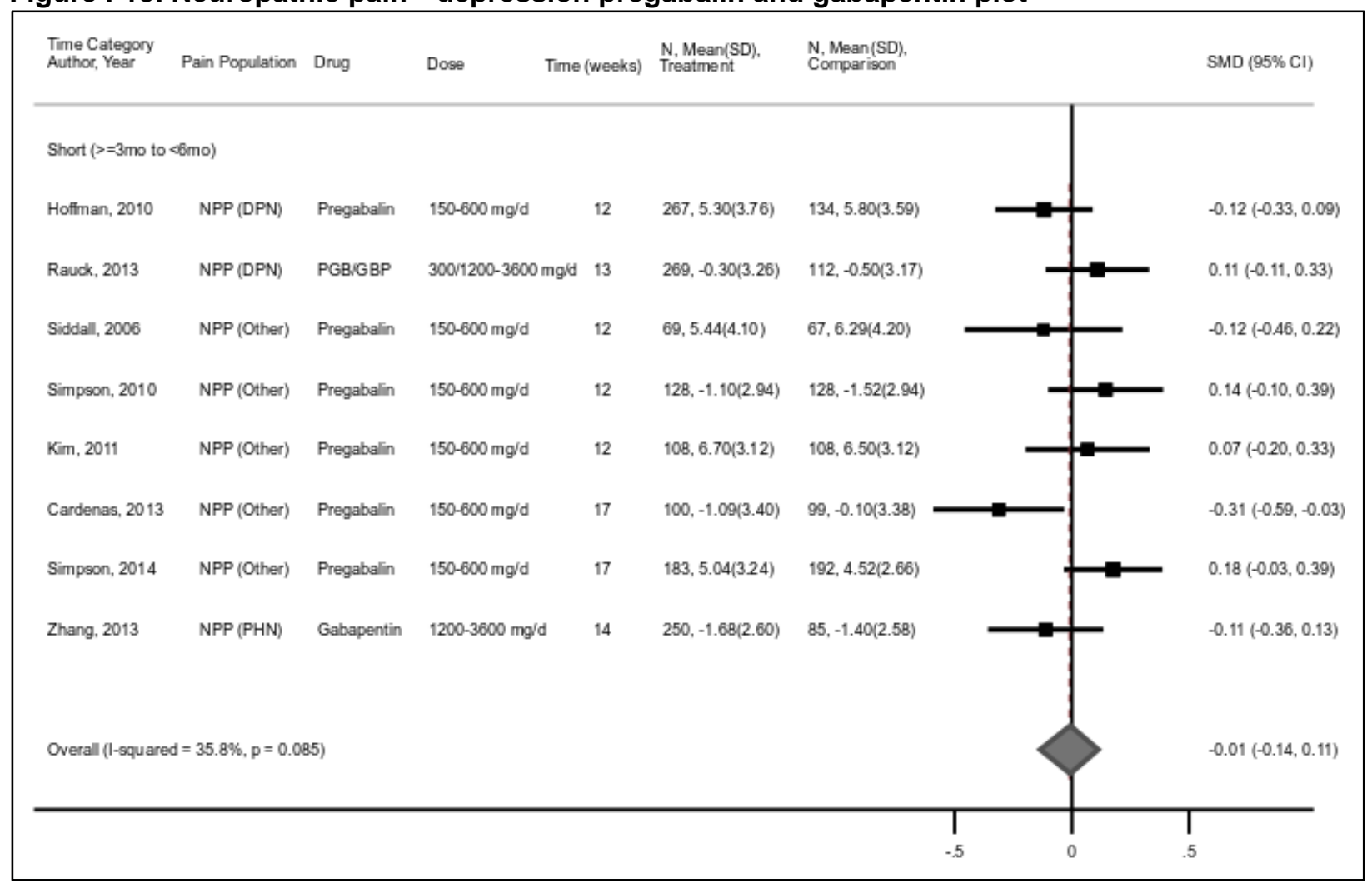


Figure I-16. Neuropathic pain-depression SNRI plot

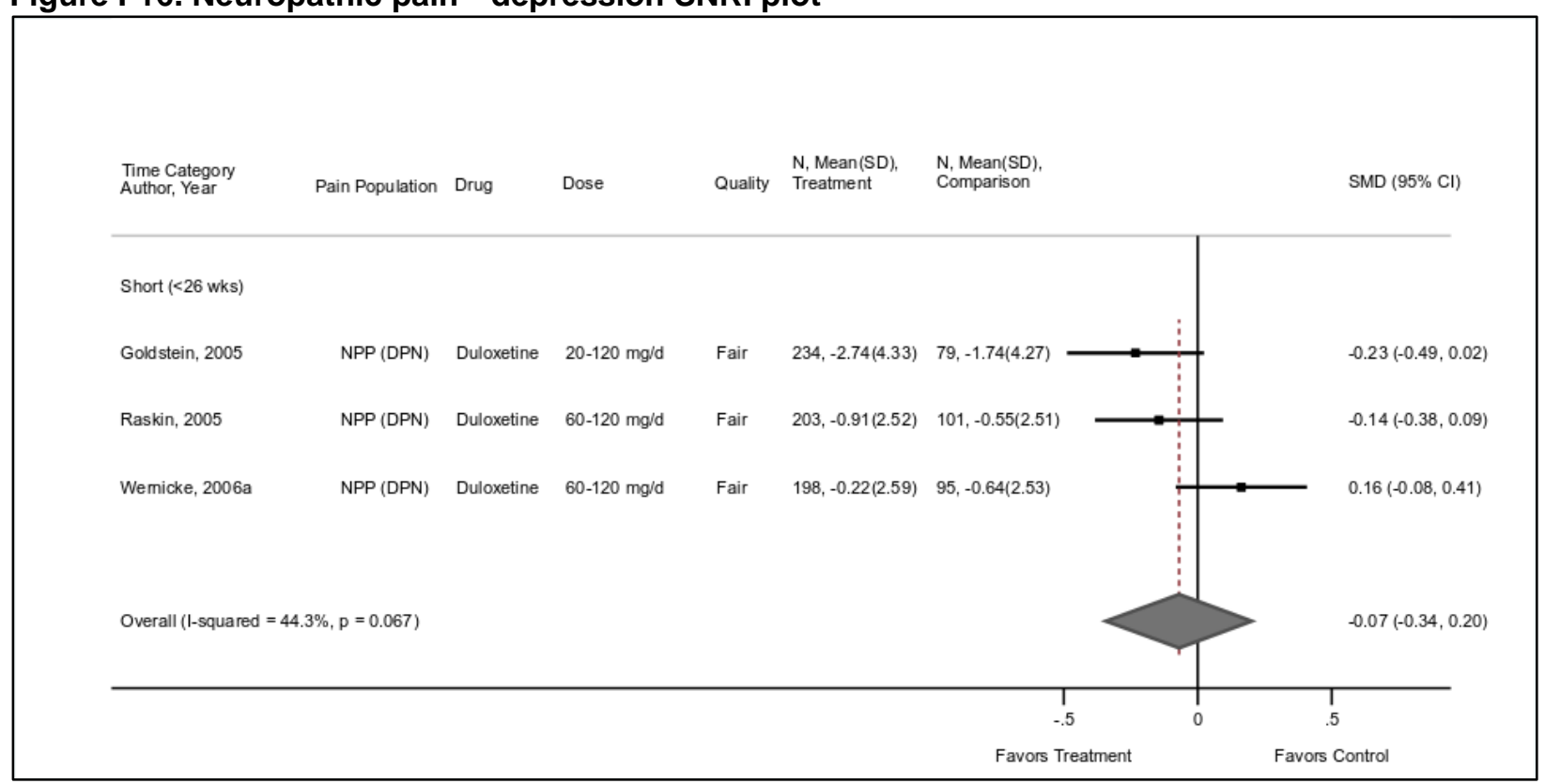

Figure I-17. Neuropathic pain-sleep pregabalin and gabapentin plot

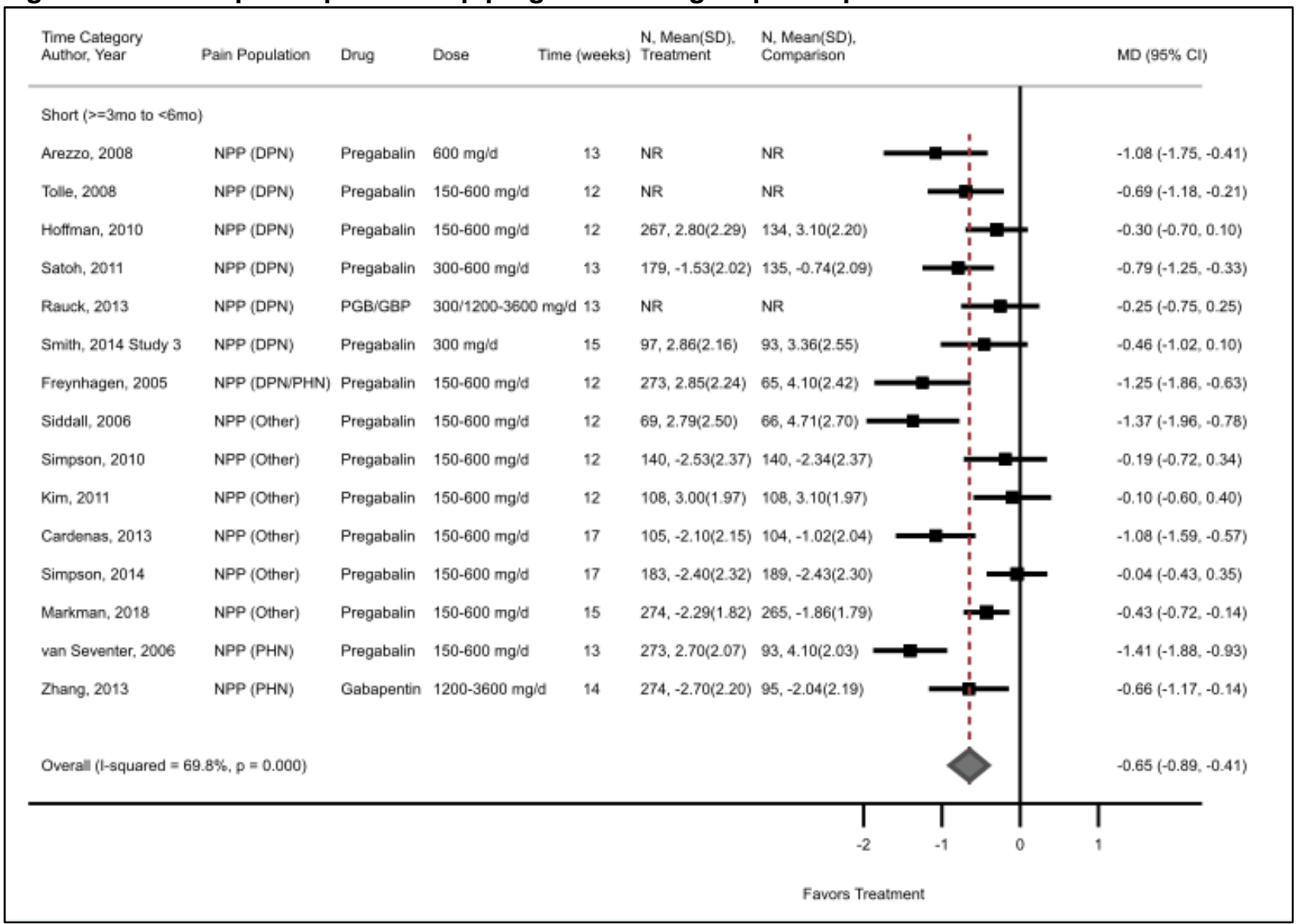




\section{Fibromyalgia}

Figure I-18. Fibromyalgia—pain pregabalin and gabapentin plot

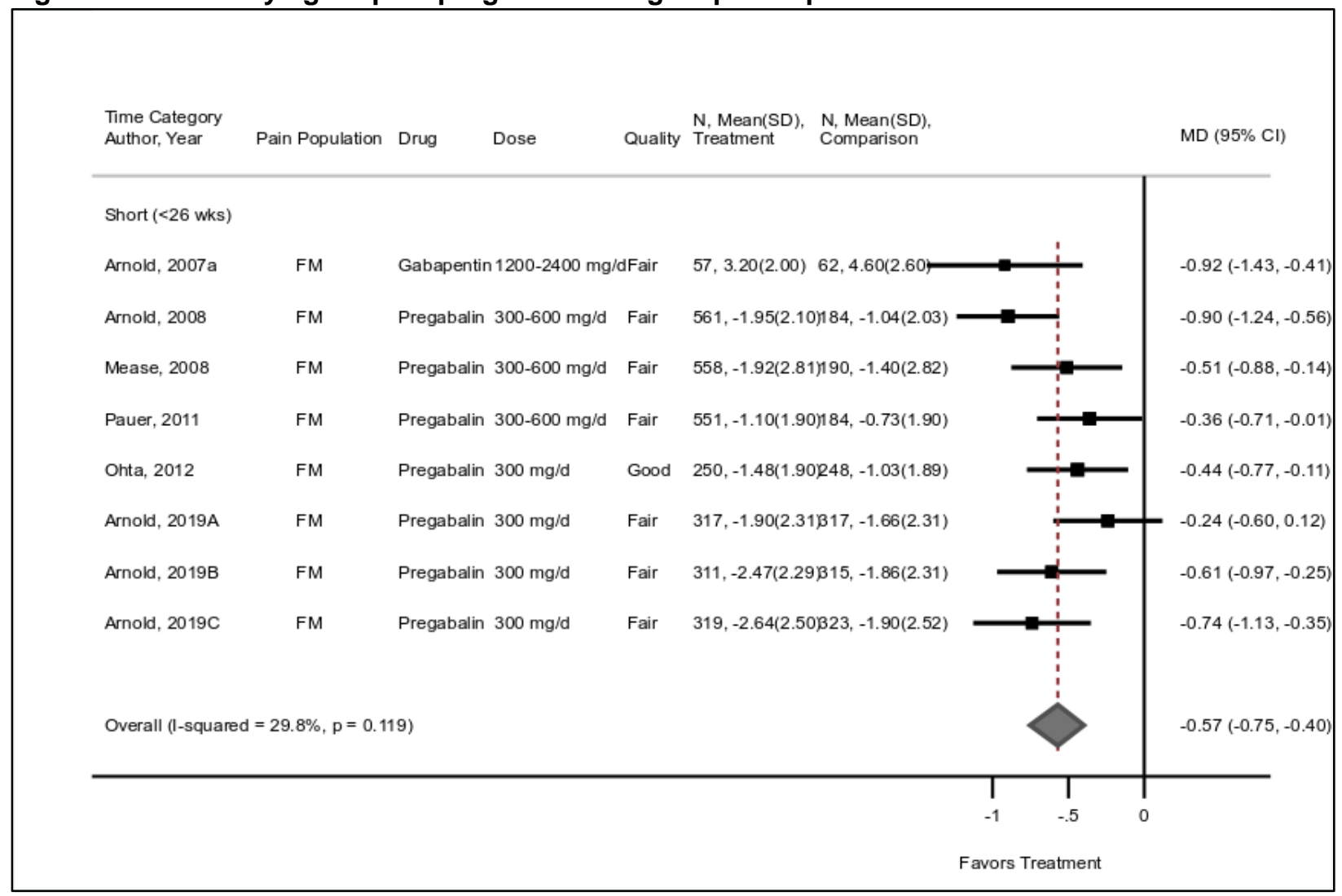


Figure I-19. Fibromyalgia-pain SNRI plot

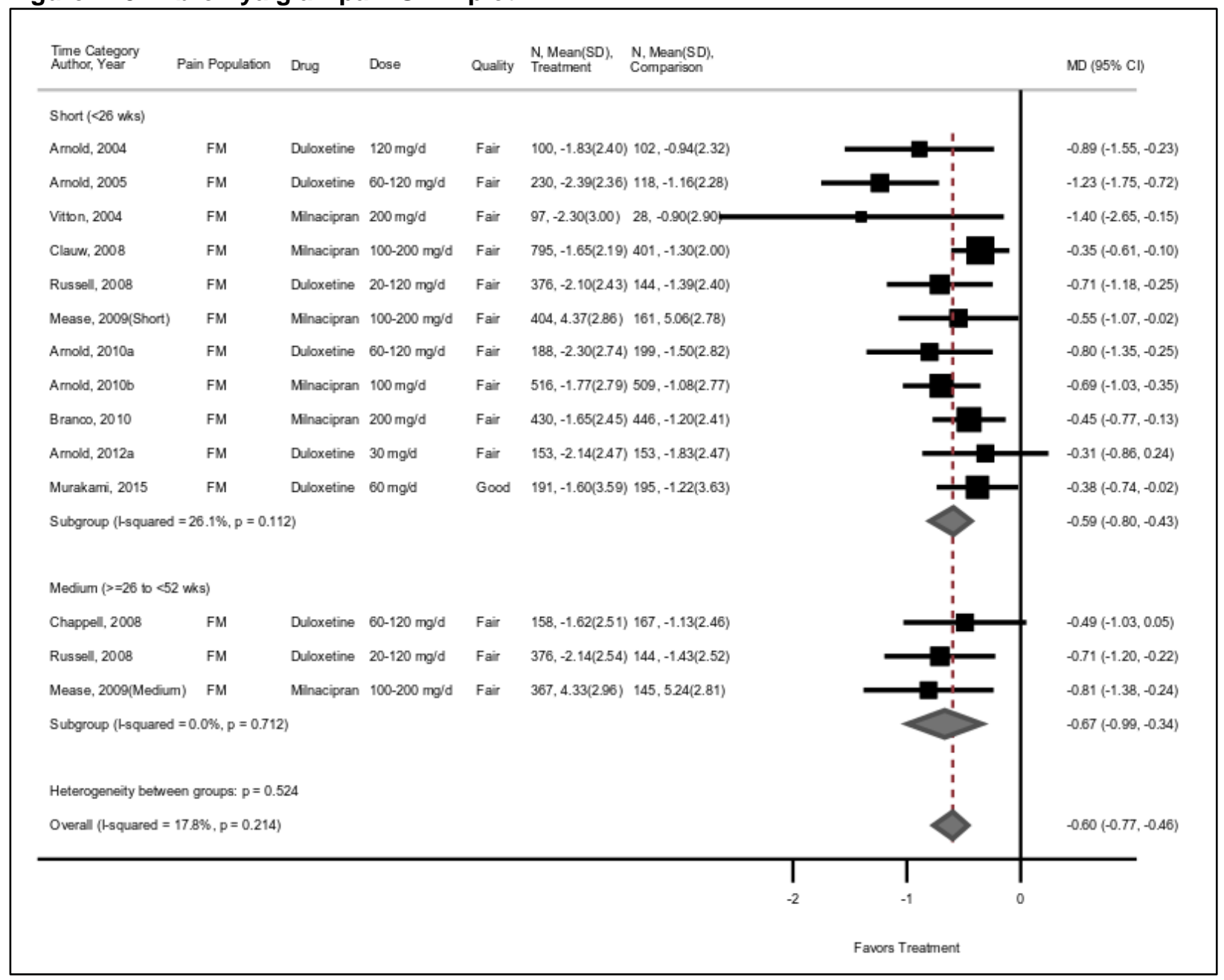

Figure I-20. Fibromyalgia_pain response pregabalin and gabapentin plot

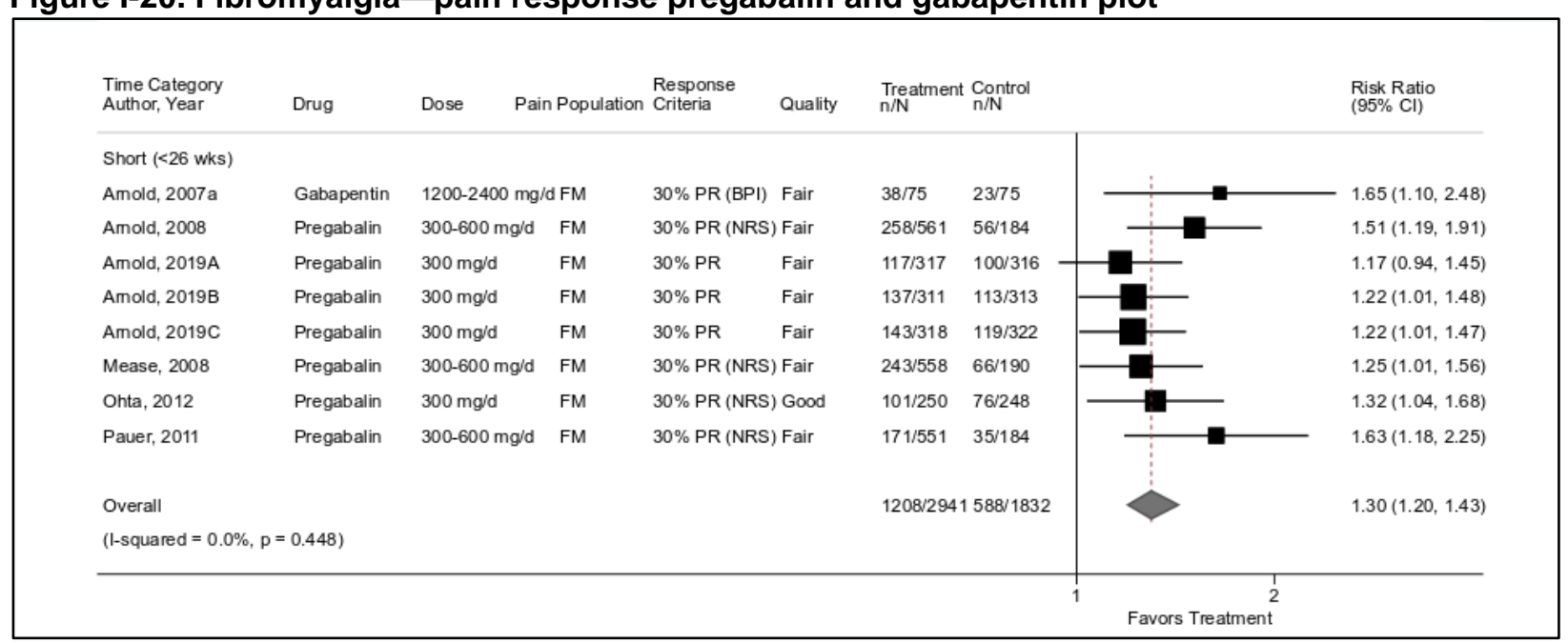


Figure I-21. Fibromyalgia-pain response SNRI plot

\begin{tabular}{|c|c|c|c|c|c|c|c|c|}
\hline $\begin{array}{l}\text { Time Category } \\
\text { Author, Year }\end{array}$ & Drug & Dose & Pain Population & $\begin{array}{l}\text { Response } \\
\text { Criteria }\end{array}$ & Quality & $\begin{array}{l}\text { Treatment } \\
\mathrm{nN}\end{array}$ & ${ }_{n \times N}^{\text {Contral }}$ & $\begin{array}{l}\text { Risk Ratio } \\
\text { ( } 95 \% \text { Cl) }\end{array}$ \\
\hline \multicolumn{9}{|l|}{ Shart (<26 wks) } \\
\hline Arnold, 2004 & Dulaxetine & $120 \mathrm{mg} / \mathrm{d}$ & FM & $30 \% \mathrm{PR}$ & Fair & $34 / 101$ & $32 / 103$ & $1.08(0.73,1.61)$ \\
\hline Arnold, 2005 & Duloxetine & $60-120 \mathrm{mg} / \mathrm{d}$ & FM & $30 \% \mathrm{PR}$ & Fair & $125 / 230$ & $39 / 118$ & $1.64(1.24,2.18)$ \\
\hline Arnold, 2010a & Dulaxetine & $60 \mathrm{mg} / \mathrm{d}$ & FM & $30 \% \mathrm{PR}$ & Fair & $119 / 249$ & $85 / 248$ & $1.39(1.12,1.73)$ \\
\hline Arnold, $2010 \mathrm{~b}$ & Milnacipran & $100 \mathrm{mg} / \mathrm{d}$ & FM & $30 \% \mathrm{PR}$ & Fair & $230 / 516$ & $156 / 509$ & $1.45(1.24,1.71)$ \\
\hline Arnold, 2012a & Dulaxetine & $30 \mathrm{mg} / \mathrm{d}$ & FM & $30 \% \mathrm{PR}$ & Fair & $77 / 155$ & $66 / 153$ & $1.15(0.90,1.47)$ \\
\hline Branco, 2010 & Milnacipran & $200 \mathrm{mg} / \mathrm{d}$ & FM & $30 \% \mathrm{PR}$ & Fair & $166 / 430$ & 134,446 & $1.28(1.07,1.55)$ \\
\hline Clauw, 2008 & Milnacipran & $100-200 \mathrm{mg} / \mathrm{d}$ & FM & $30 \% \mathrm{PR}$ & Fair & $307 / 795$ & $115 / 401$ & $1.35(1.13,1.61)$ \\
\hline Mease, 2009(short) & Milnacipran & $100-200 \mathrm{mg} / \mathrm{d}$ & FM & $30 \% \mathrm{PR}$ & Fair & $179 / 665$ & $43 / 223$ & $1.40(1.04,1.88)$ \\
\hline Murakami, 2015 & Dulaxetine & $60 \mathrm{mg} / \mathrm{d}$ & FM & $30 \% \mathrm{PR}$ & Good & $96 / 191$ & $74 / 195$ & $1.32(1.05,1.66)$ \\
\hline Vitton, 2004 & Milnacipran & $200 \mathrm{mg} / \mathrm{d}$ & FM & $30 \% \mathrm{PR}$ & Fair & $36 / 97$ & $6 / 28$ & $1.73(0.81,3.69)$ \\
\hline Subgroup & & & & & & $1369 / 3429$ & $750 / 2424$ & $1.36(1.26,1.46)$ \\
\hline \multicolumn{9}{|c|}{ (l-squared $=0.0 \%, p=0.702)$} \\
\hline \multicolumn{9}{|l|}{ Medium ( $>26$ to $<52 \mathrm{wks}$ ) } \\
\hline Chappell, 2008 & Dulaxetine & $60-120 \mathrm{mg} / \mathrm{d}$ & FM & $30 \% \mathrm{PR}$ & Fair & $60 / 158$ & $55 / 167$ & $1.15(0.86,1.55)$ \\
\hline Mease, 2009(medium) & Milnacipran & $100-200 \mathrm{mg} / \mathrm{d}$ & FM & $30 \% \mathrm{PR}$ & Fair & $171 / 665$ & $41 / 223$ & $1.40(1.03,1.90)$ \\
\hline Russell, 2008 & Dulaxetine & $20-120 \mathrm{mg} / \mathrm{d}$ & FM & $30 \% \mathrm{PR}$ & Fair & $178 / 363$ & $52 / 139$ & $1.31(1.03,1.67)$ \\
\hline & & & & & & $409 / 1186$ & 148,529 & $1.29(1.08,1.52)$ \\
\hline \multicolumn{9}{|c|}{ (1-squared $=0.0 \%, p=0.653$ ) } \\
\hline \multicolumn{9}{|c|}{ Heterogeneity between groups: $p=0.551$} \\
\hline Overall & & & & & & $1778 / 4615$ & $898 / 2953$ & $1.34(1.26,1.43)$ \\
\hline \multicolumn{9}{|c|}{ (l-squared $=0.0 \%, p=0.817$ ) } \\
\hline & & & & & & & & \\
\hline
\end{tabular}

Figure I-22. Fibromyalgia-function pregabalin and gabapentin plot

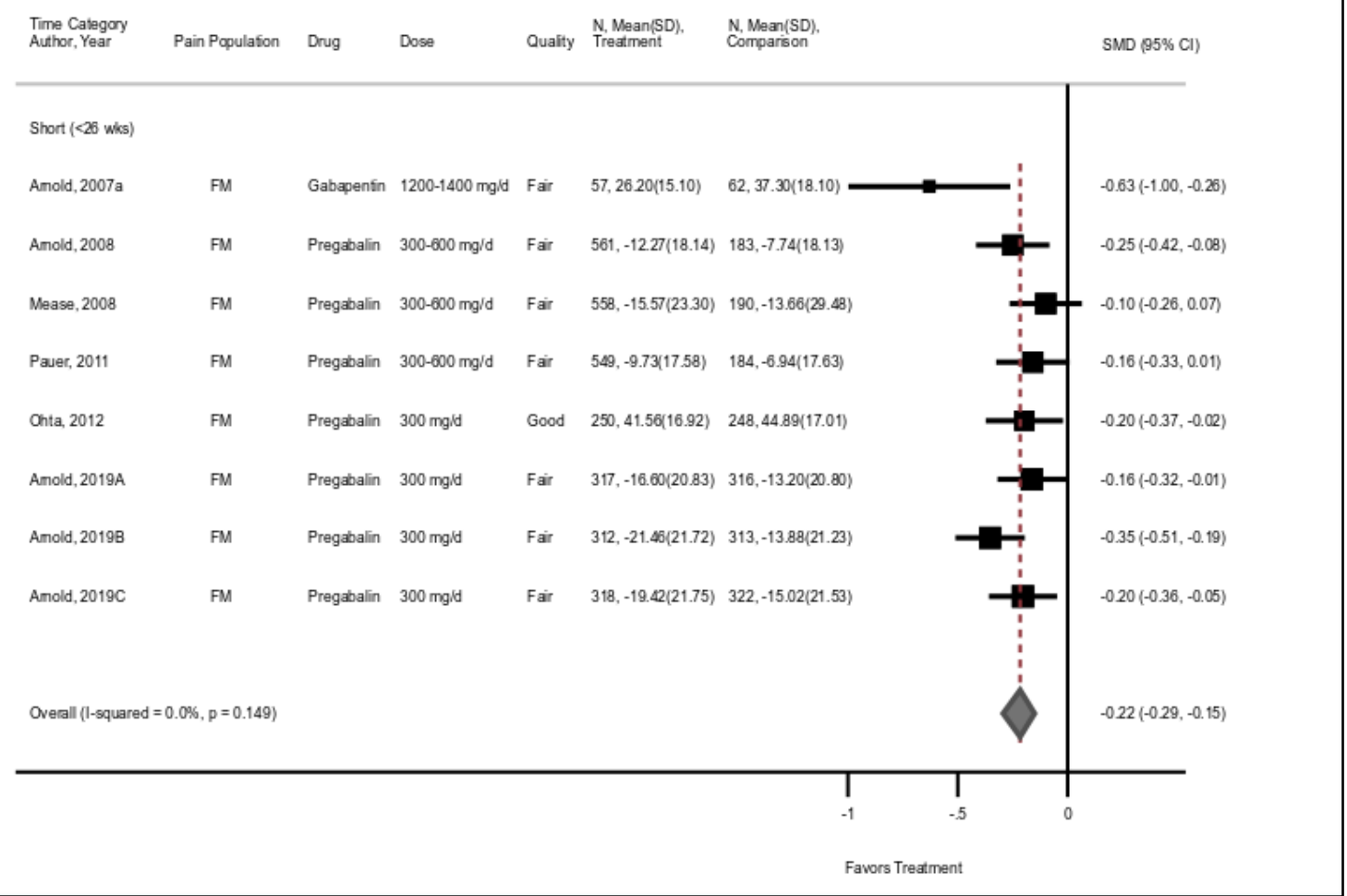


Figure I-23. Fibromyalgia-function SNRI plot

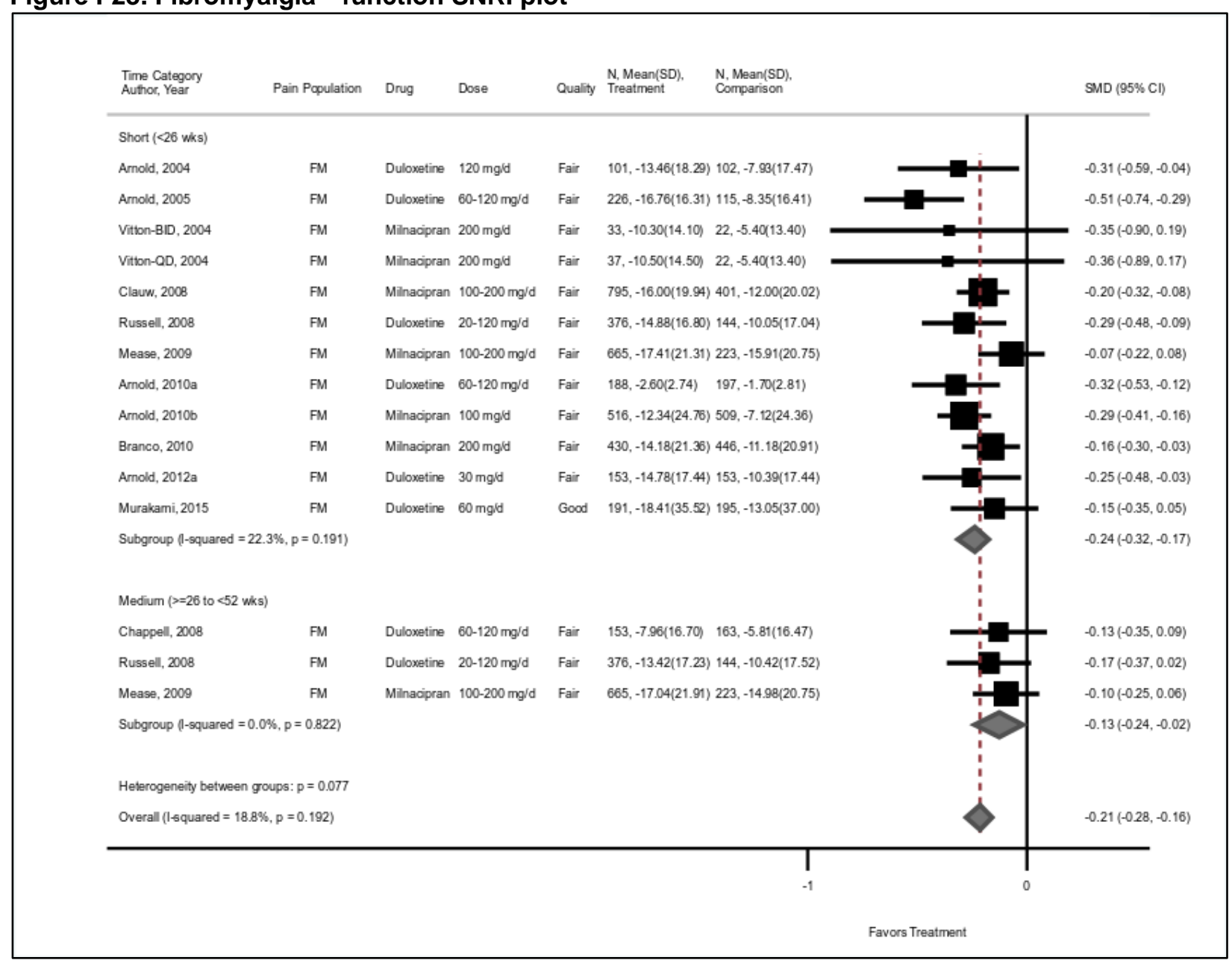


Figure I-24. Fibromyalgia—quality of life pregabalin and gabapentin plot

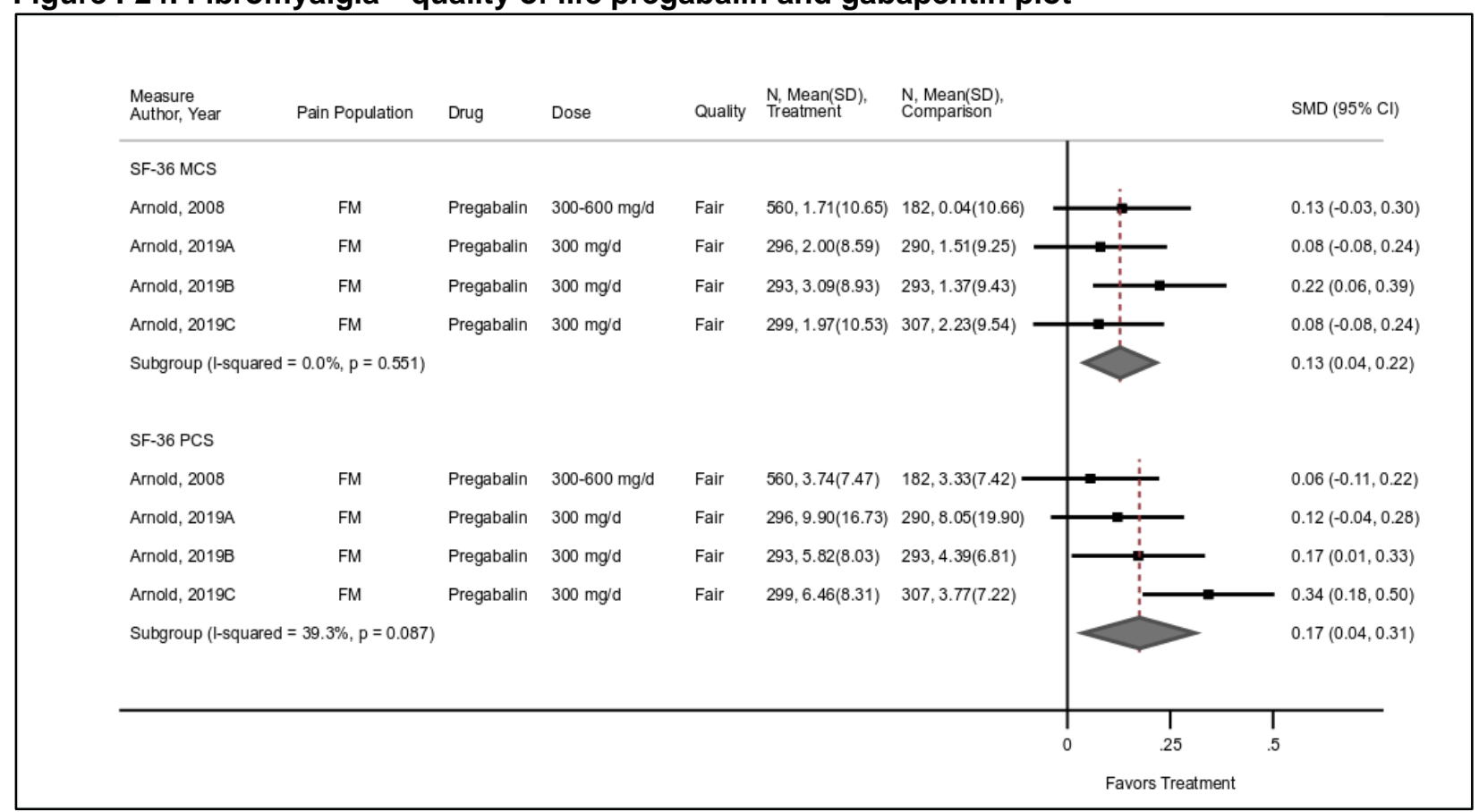

Figure I-25. Fibromyalgia-quality of life SNRI SF-36 PCS plot

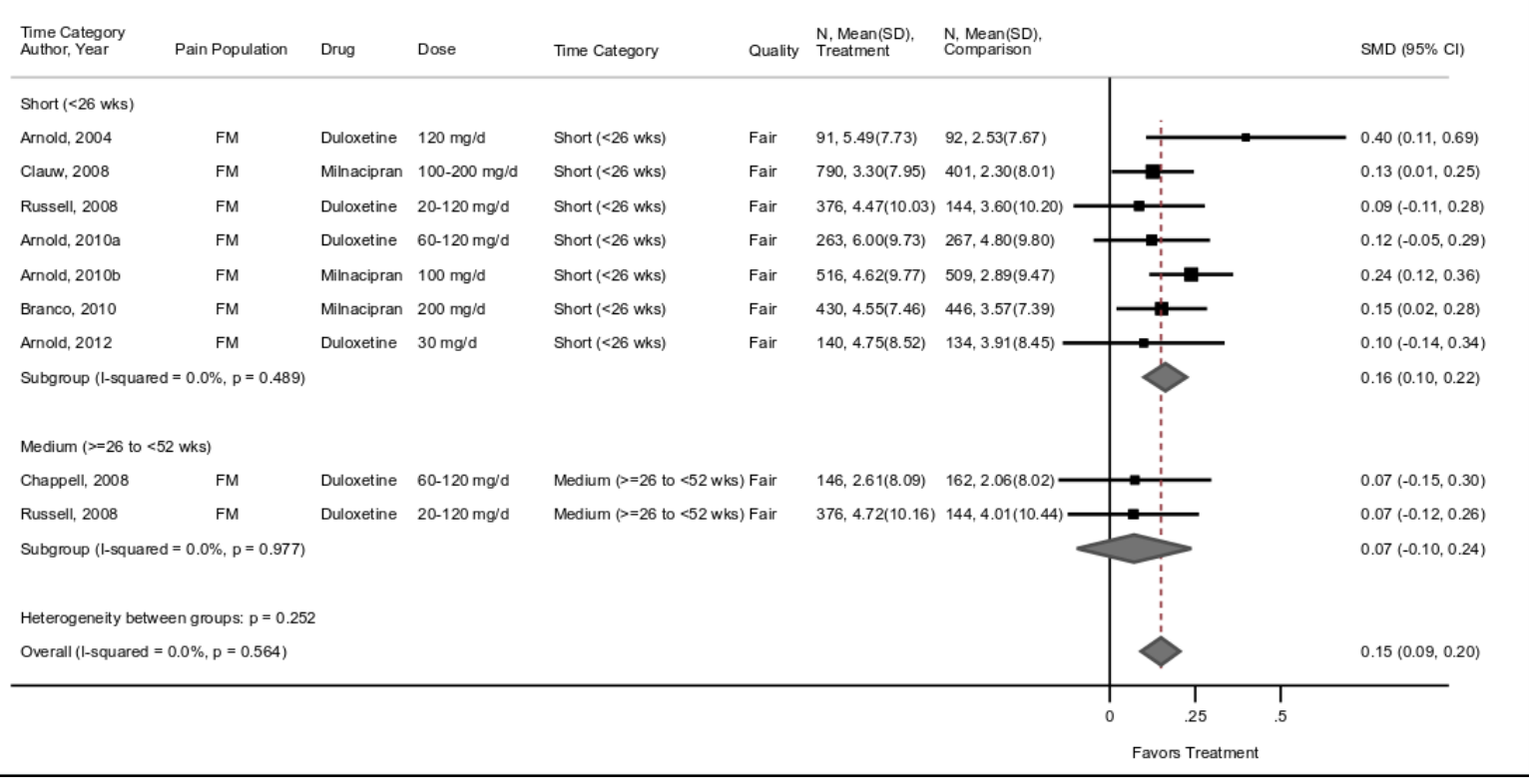


Figure I-26. Fibromyalgia-quality of life SNRI SF-36 MCS plot

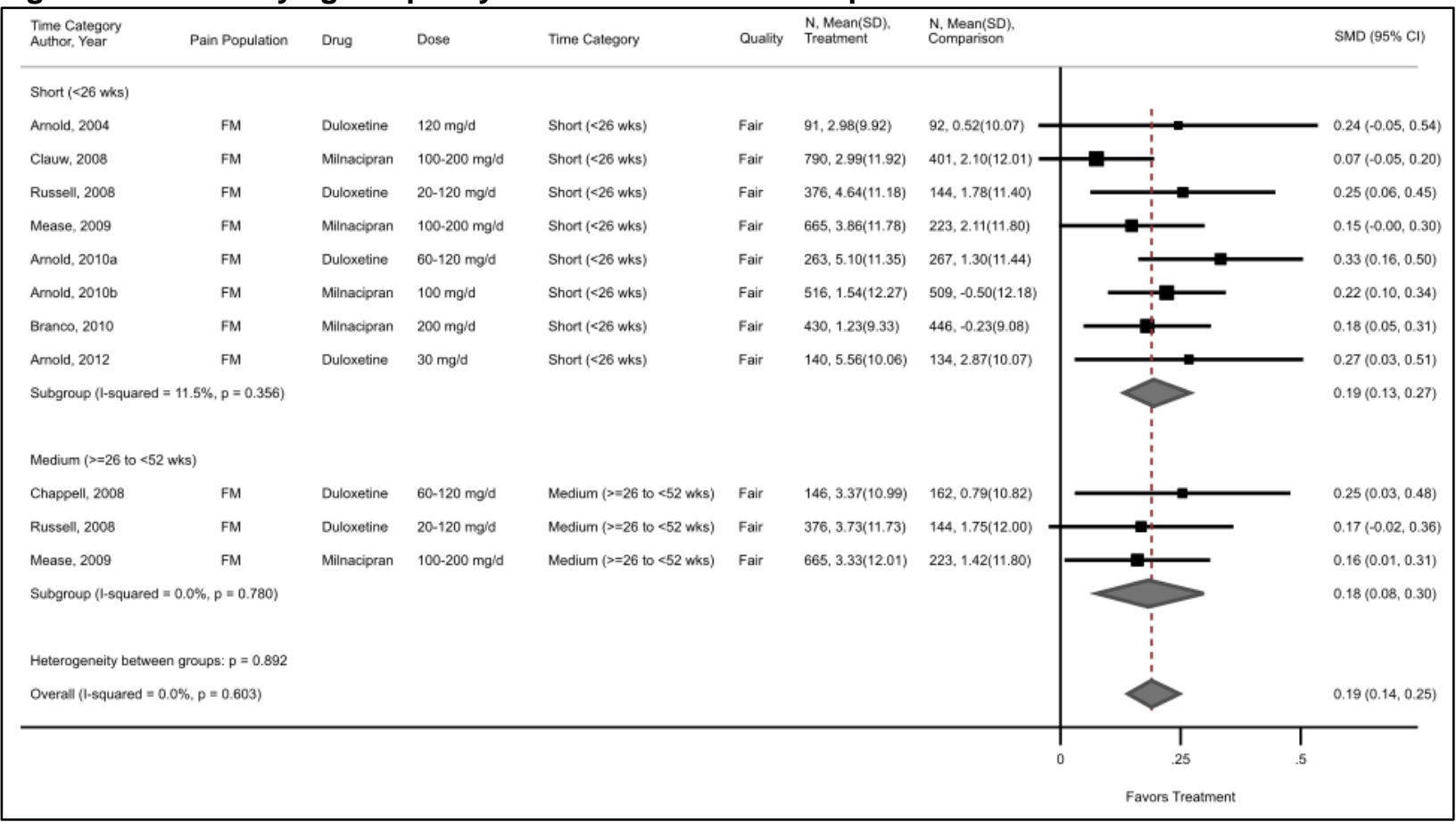

Figure I-27. Fibromyalgia-anxiety pregabalin and gabapentin plot

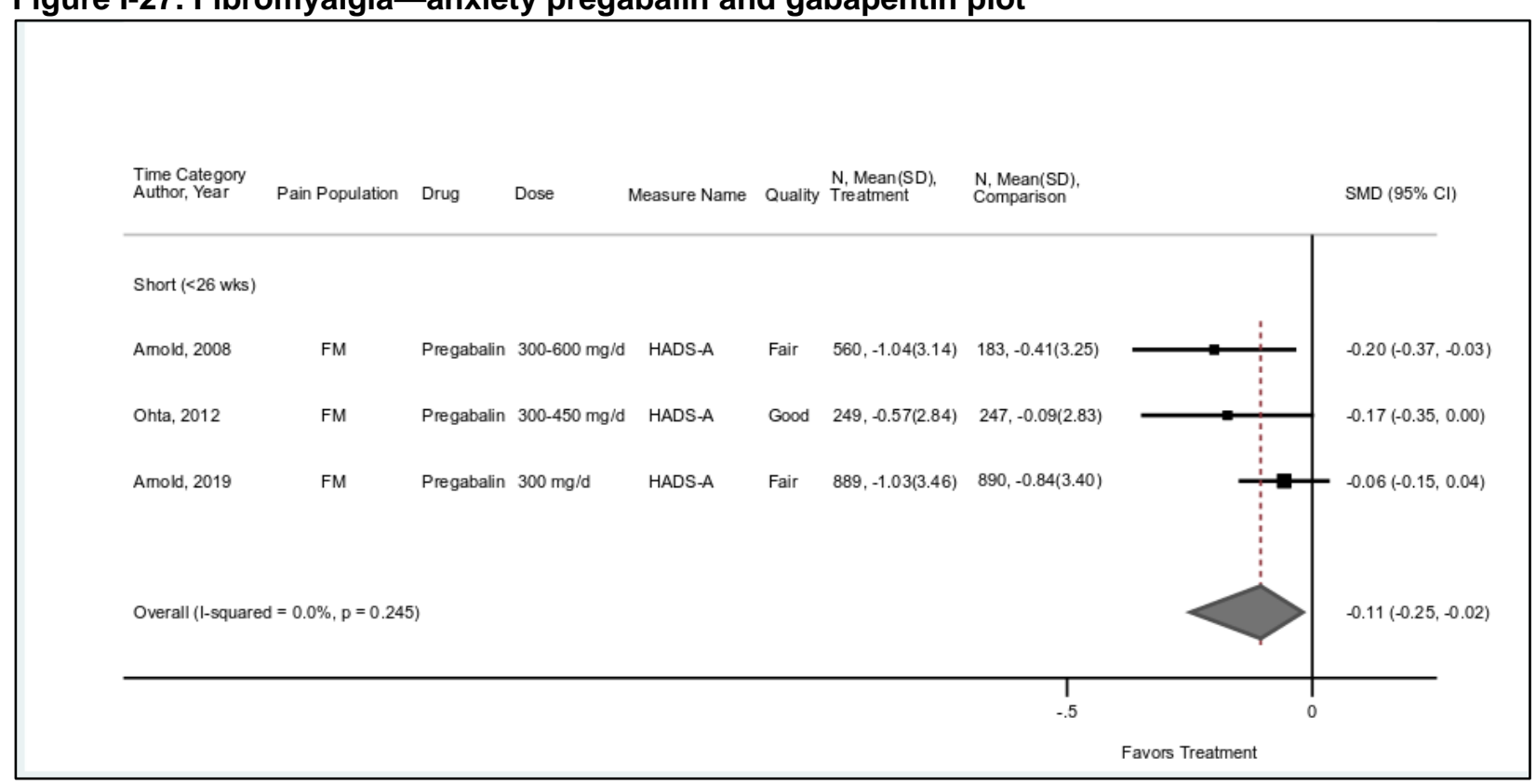


Figure I-28. Fibromyalgia-anxiety SNRI plot

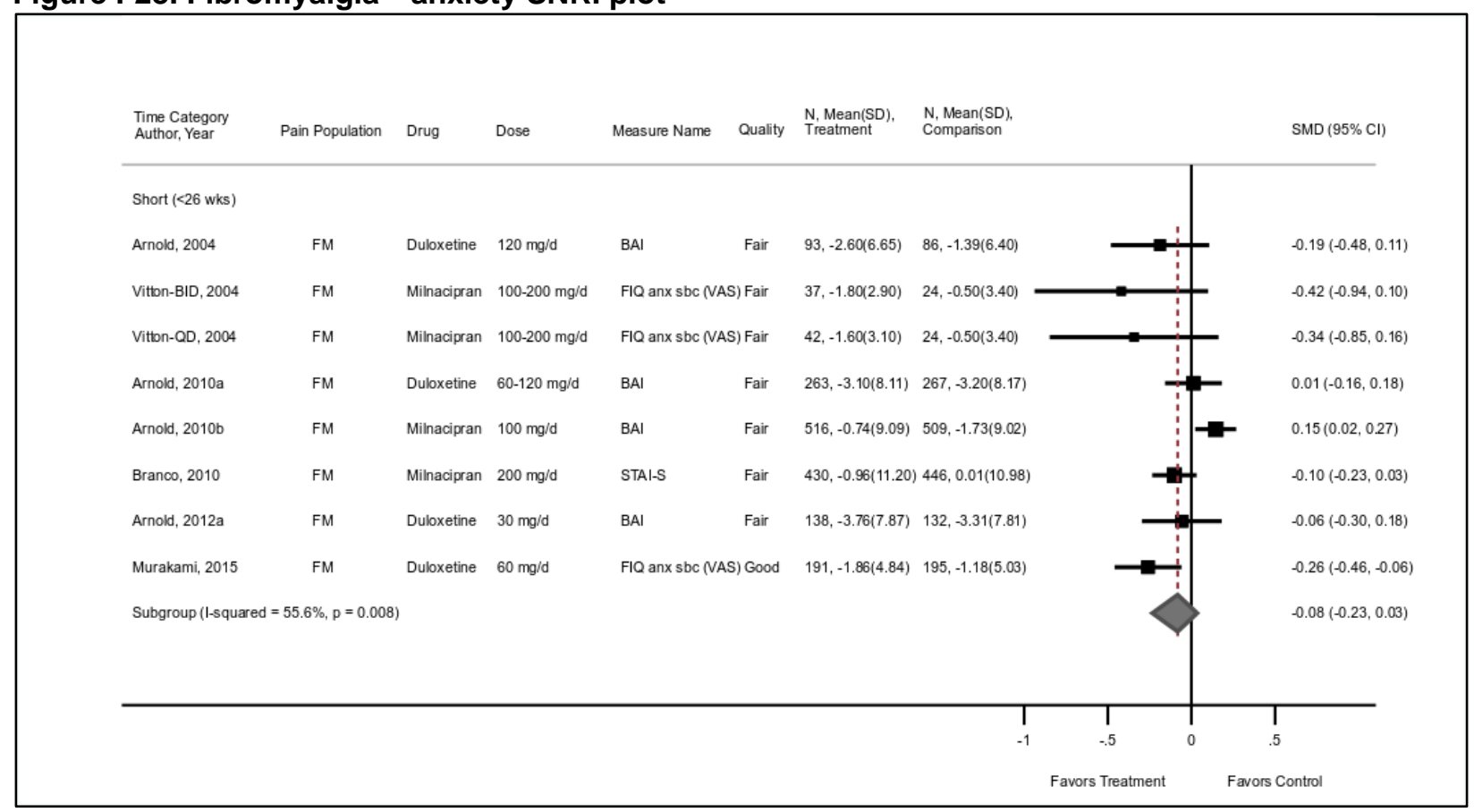

Figure I-29. Fibromyalgia-depression pregabalin and gabapentin plot

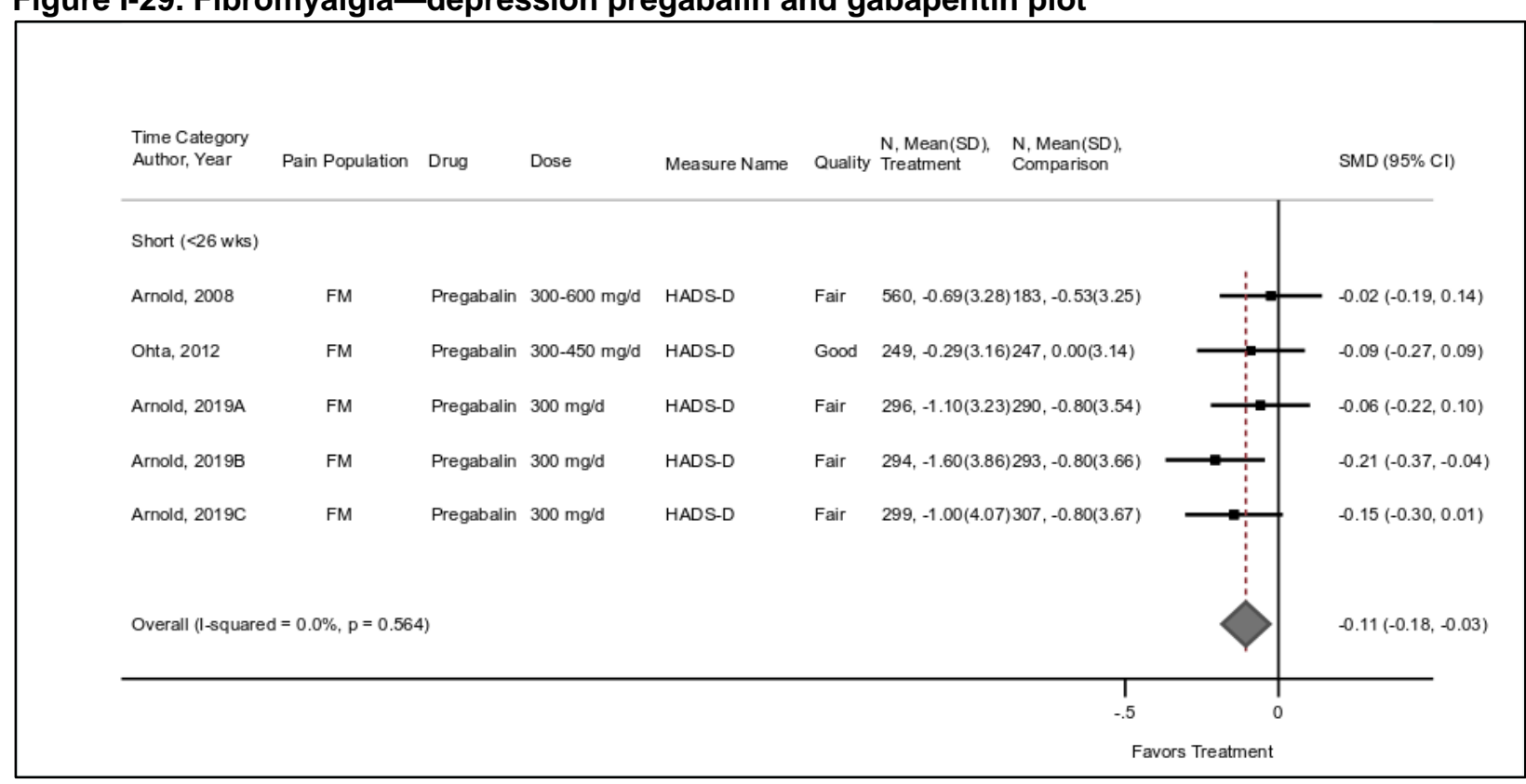


Figure I-30. Fibromyalgia-depression SNRI plot

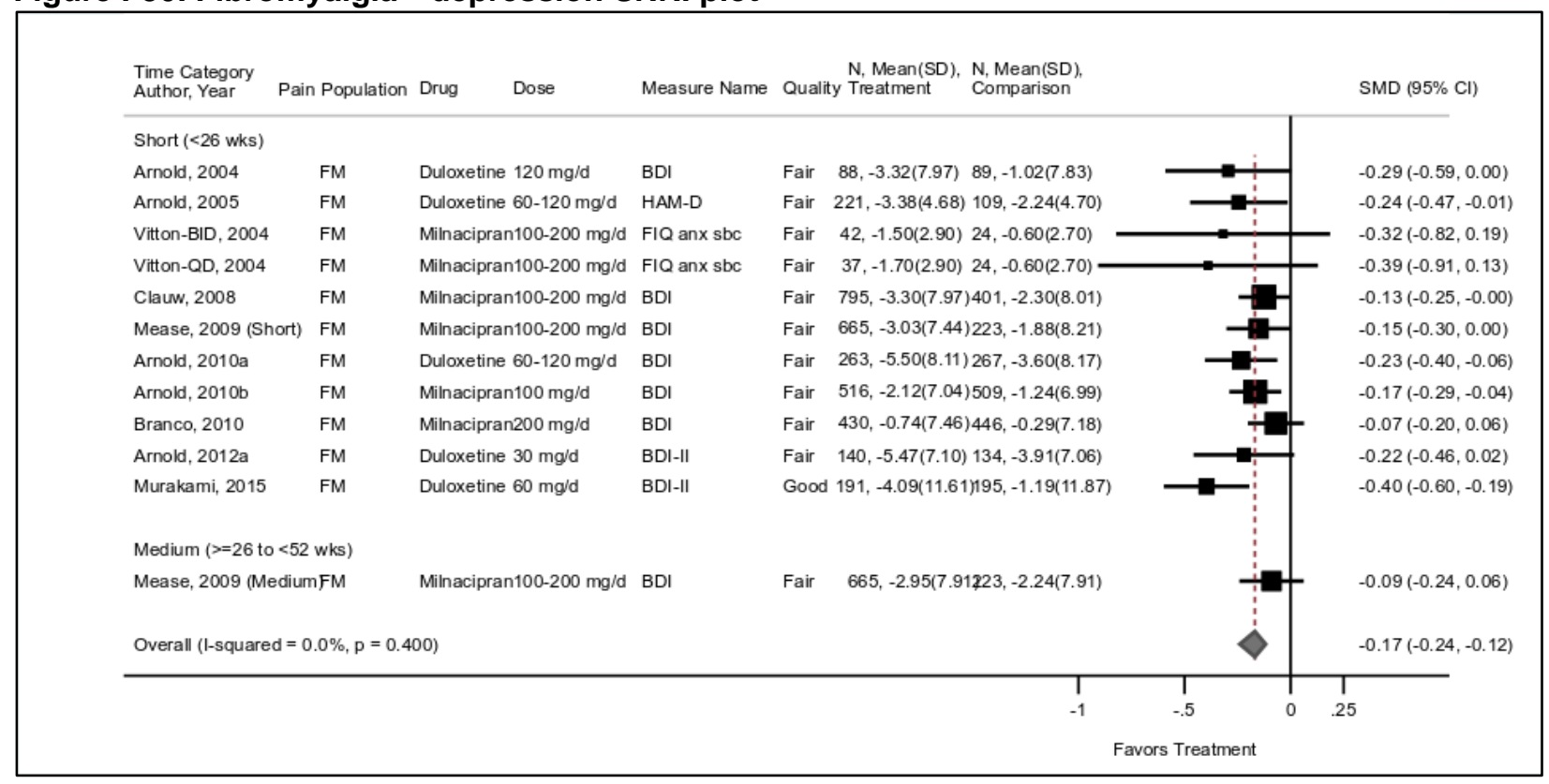

Figure I-31. Fibromyalgia-sleep pregabalin and gabapentin plot

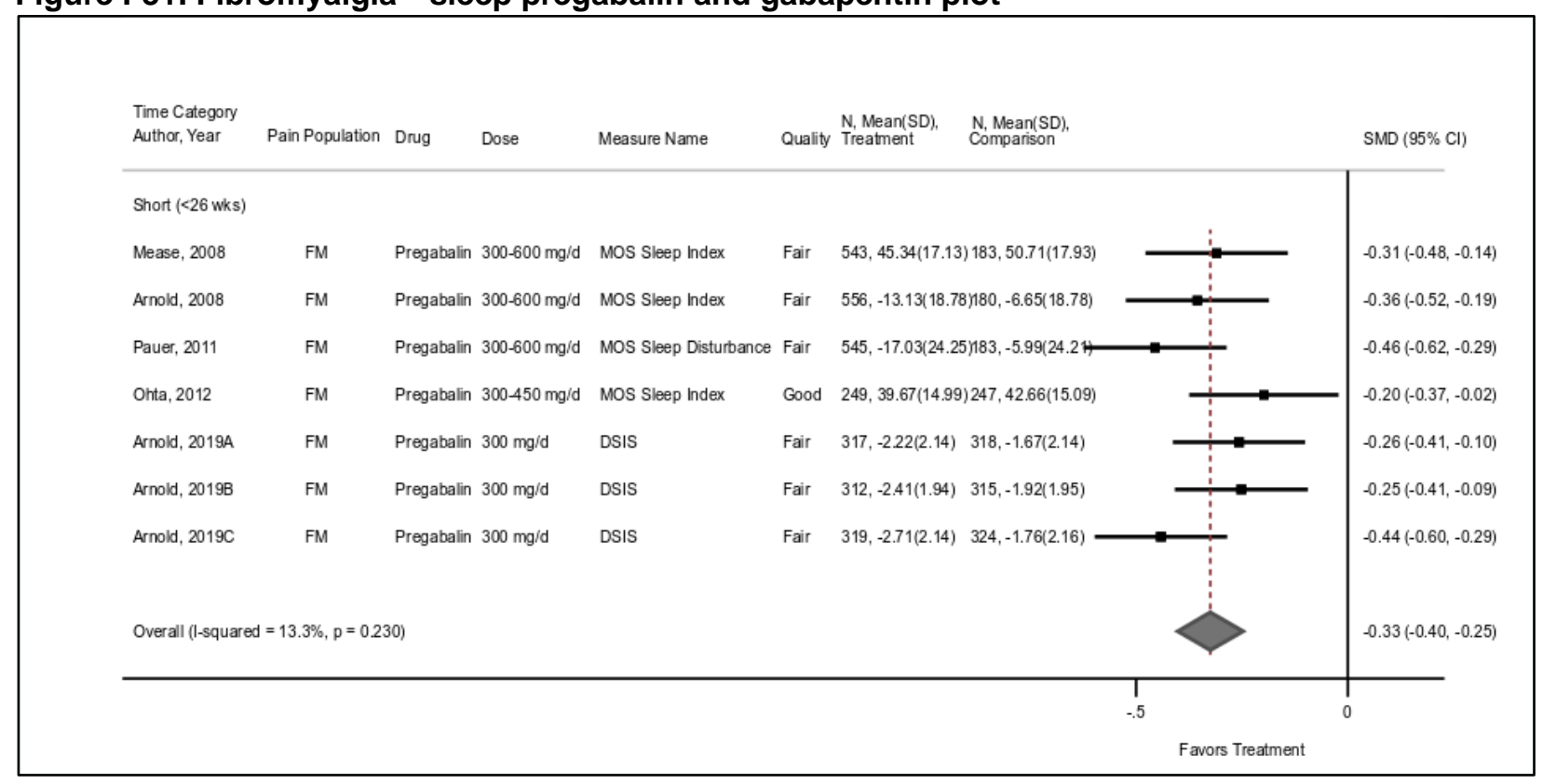


Figure I-32. Fibromyalgia-sleep SNRI plot

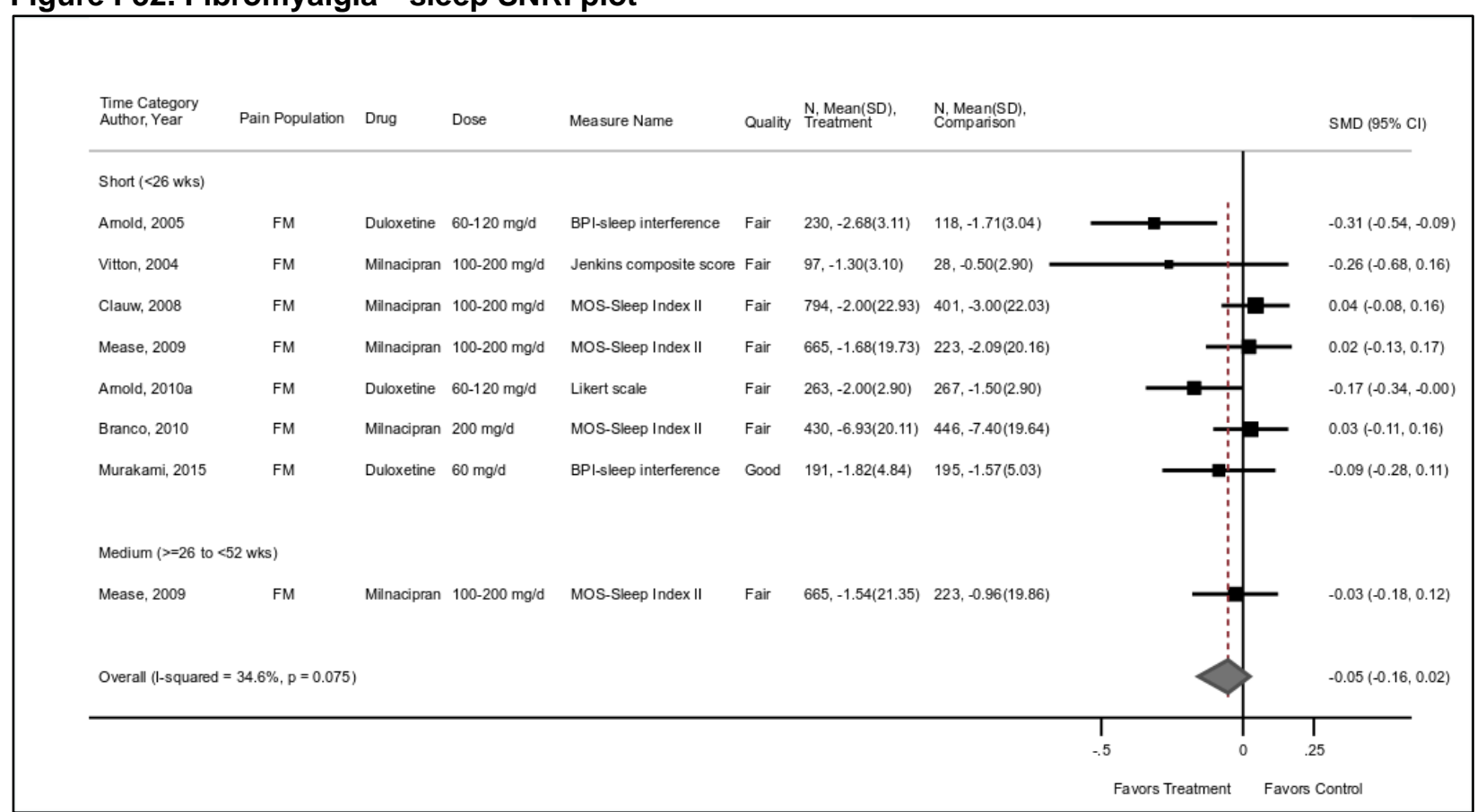




\section{Osteoarthritis}

Figure I-33. Osteoarthritis-pain NSAID plot

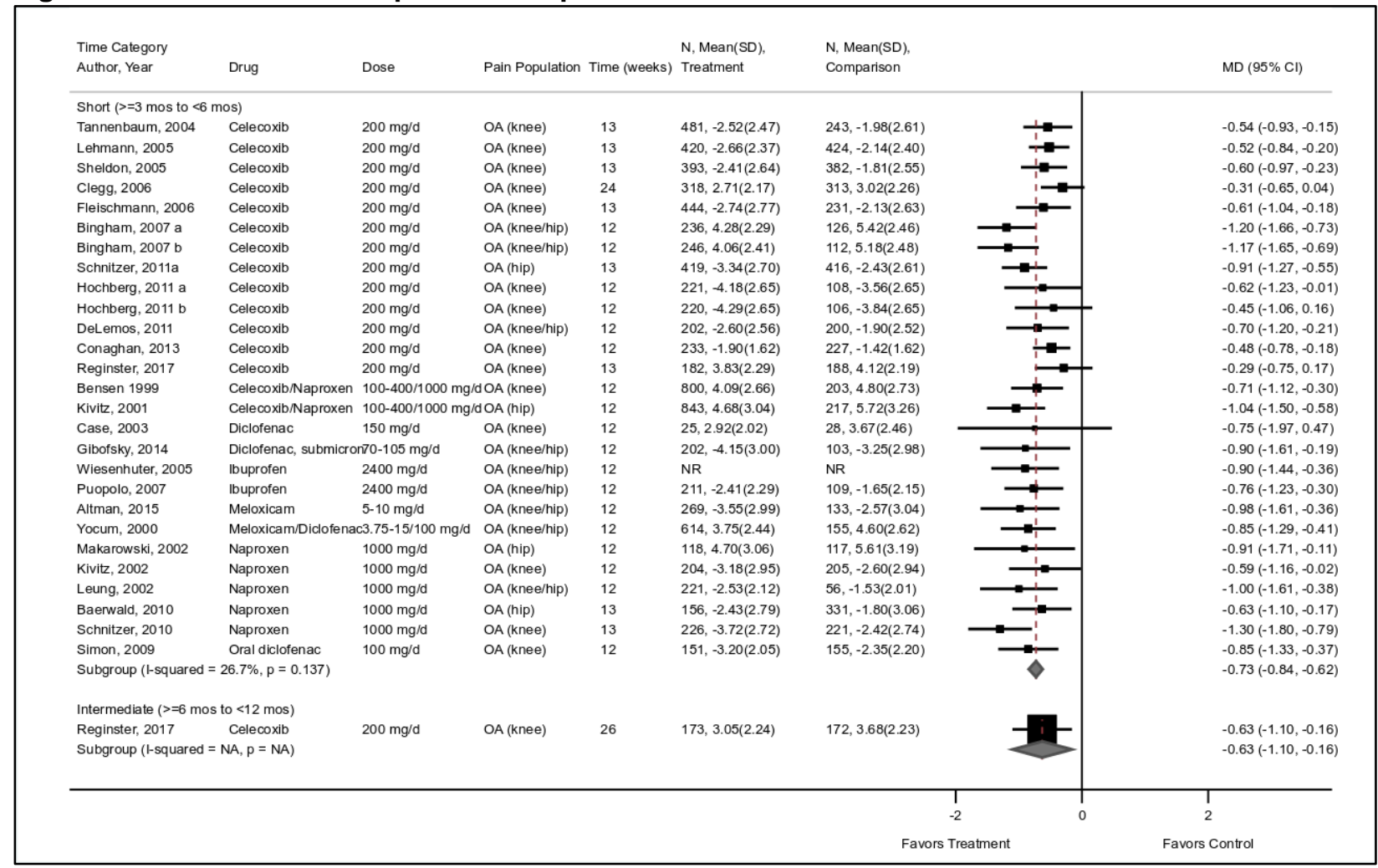

Figure I-34. Osteoarthritis-pain NSAID topical diclofenac plot

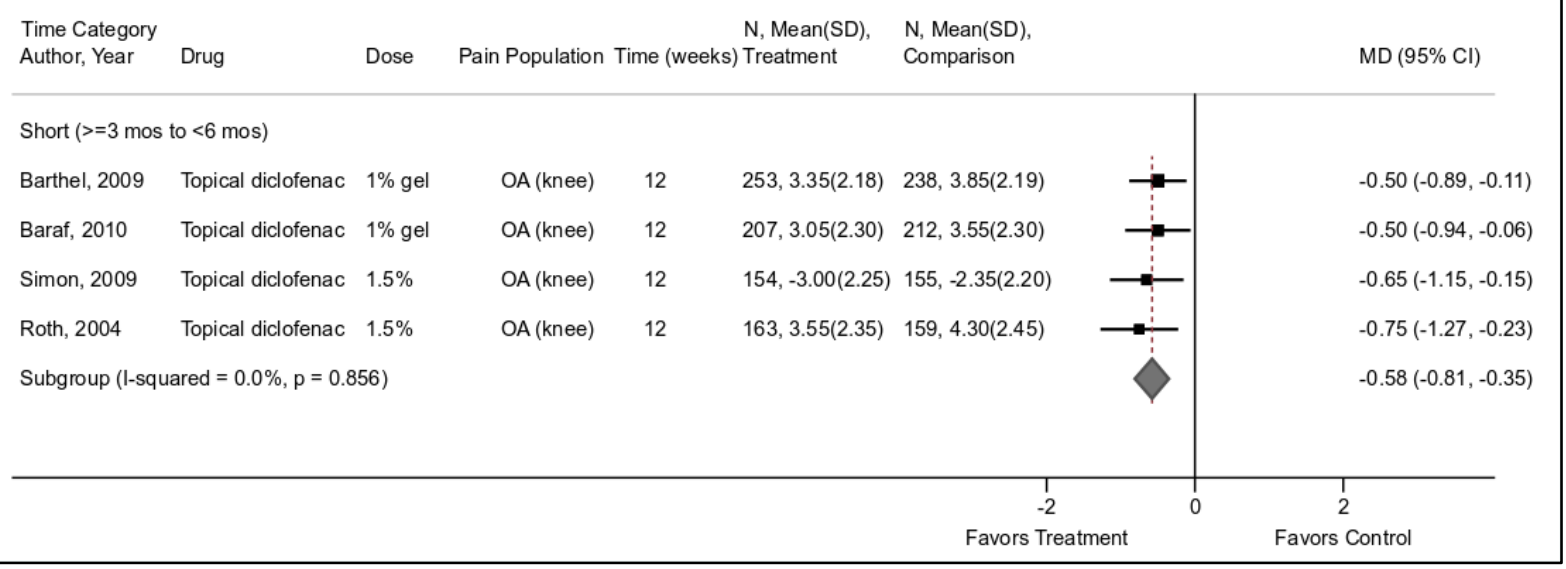


Figure I-35. Osteoarthritis-pain SNRI plot

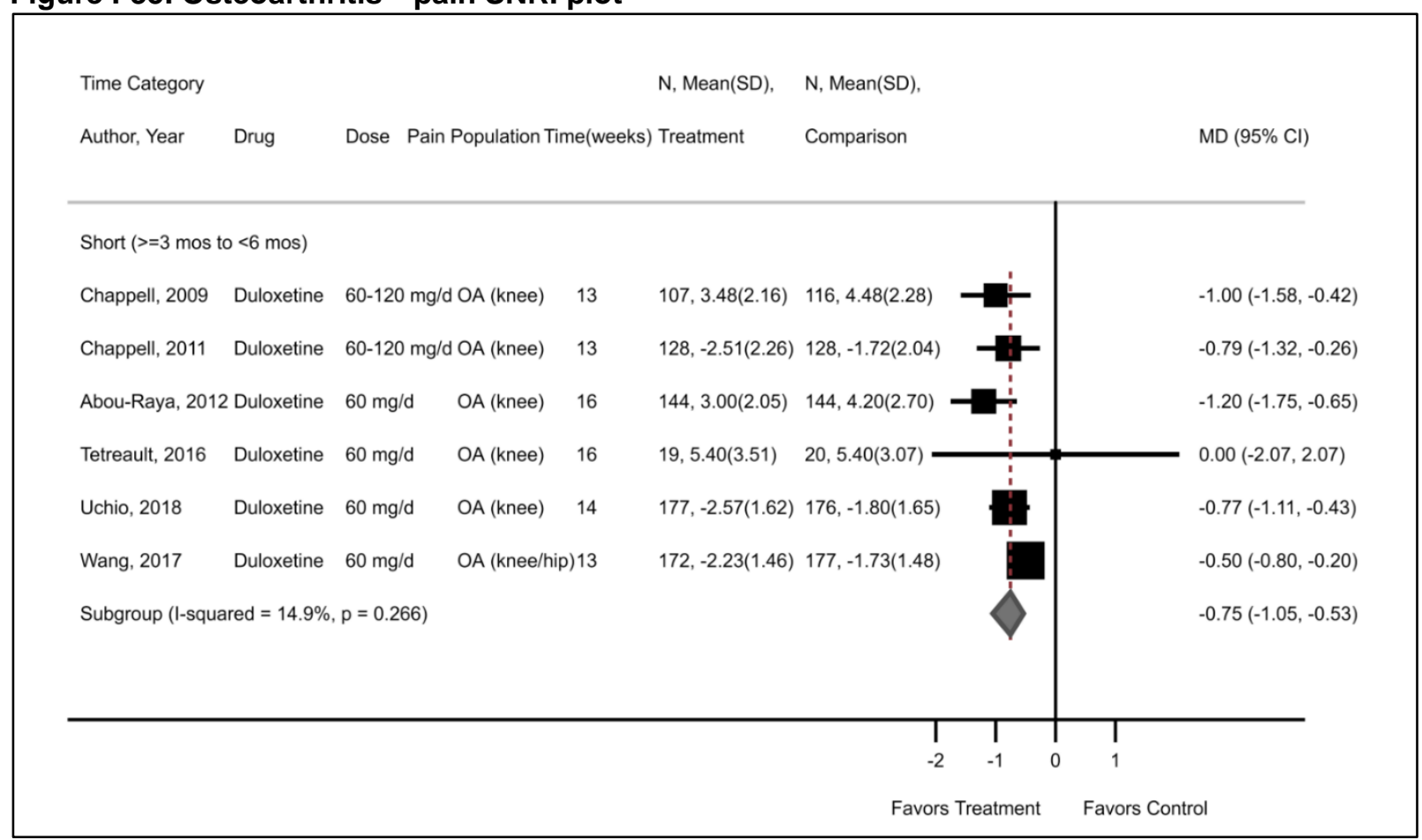

\section{Figure I-36. Osteoarthritis-pain acetaminophen plot}

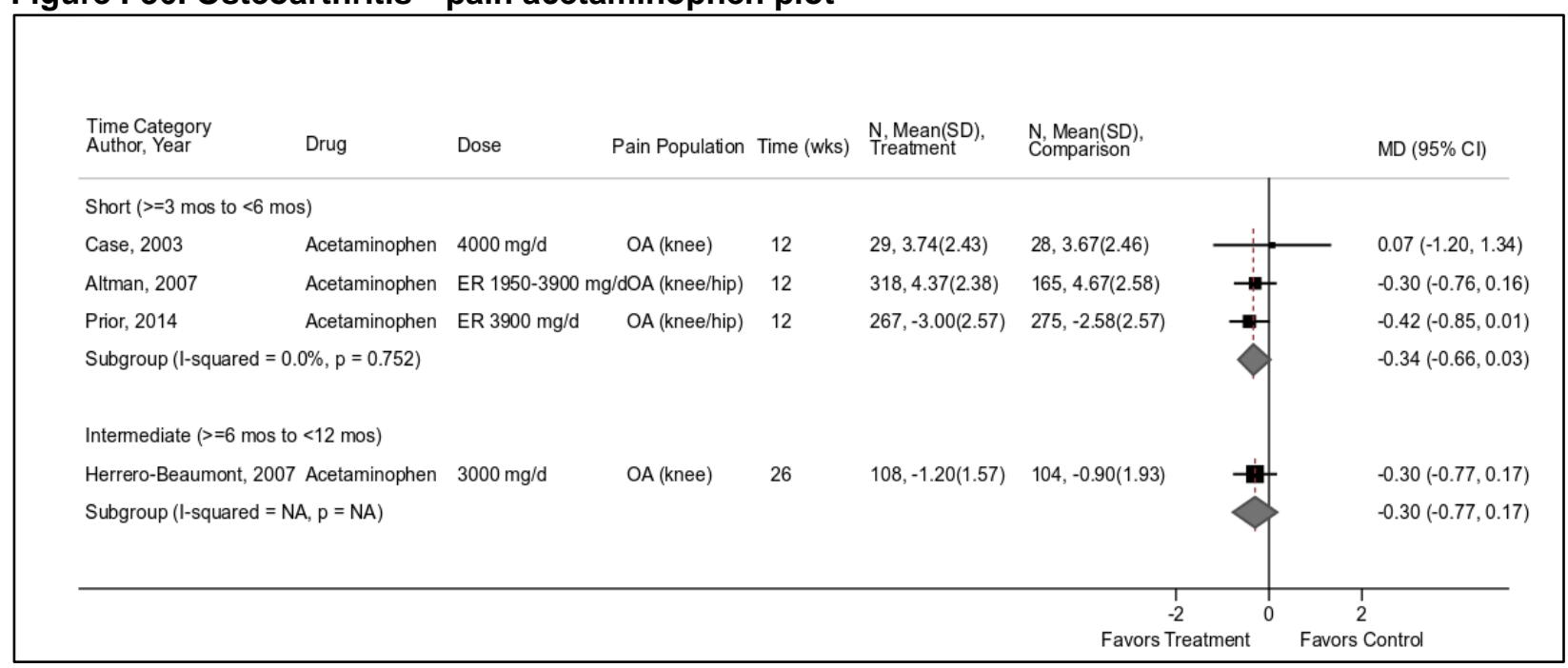


Figure I-37. Osteoarthritis-pain response NSAID plot

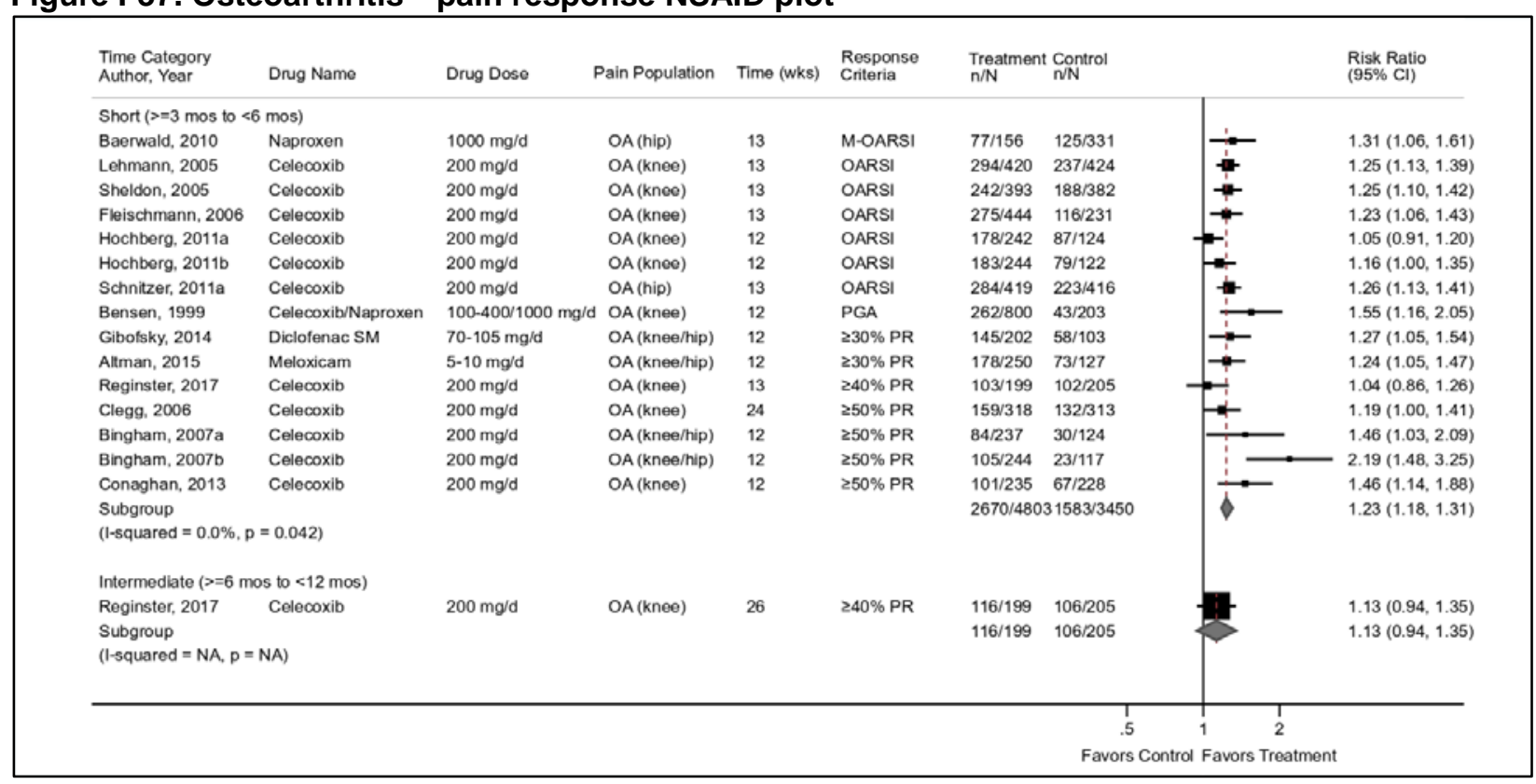

Figure I-38. Osteoarthritis-pain response NSAID topical diclofenac plot

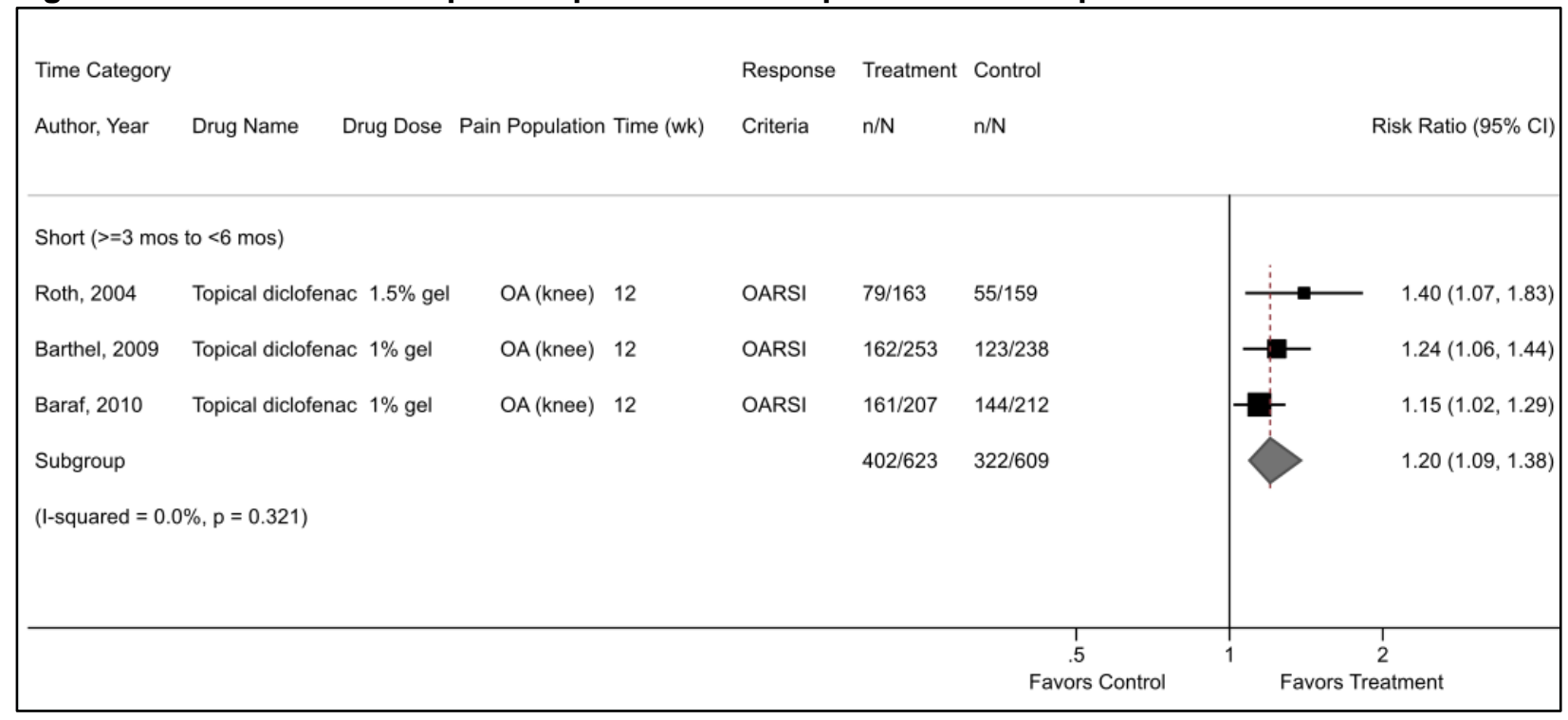


Figure I-39. Osteoarthritis-pain response SNRI plot

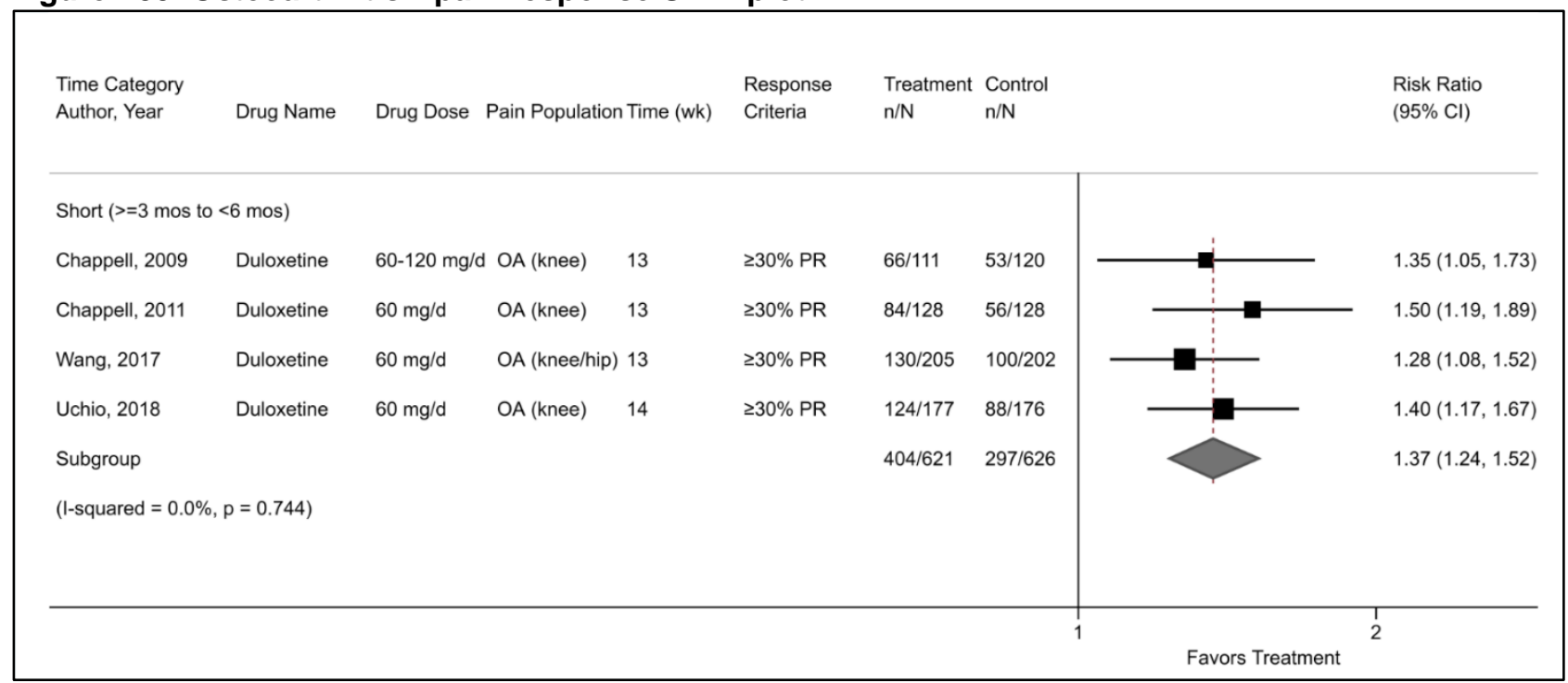

Figure I-40. Osteoarthritis-function NSAID plot

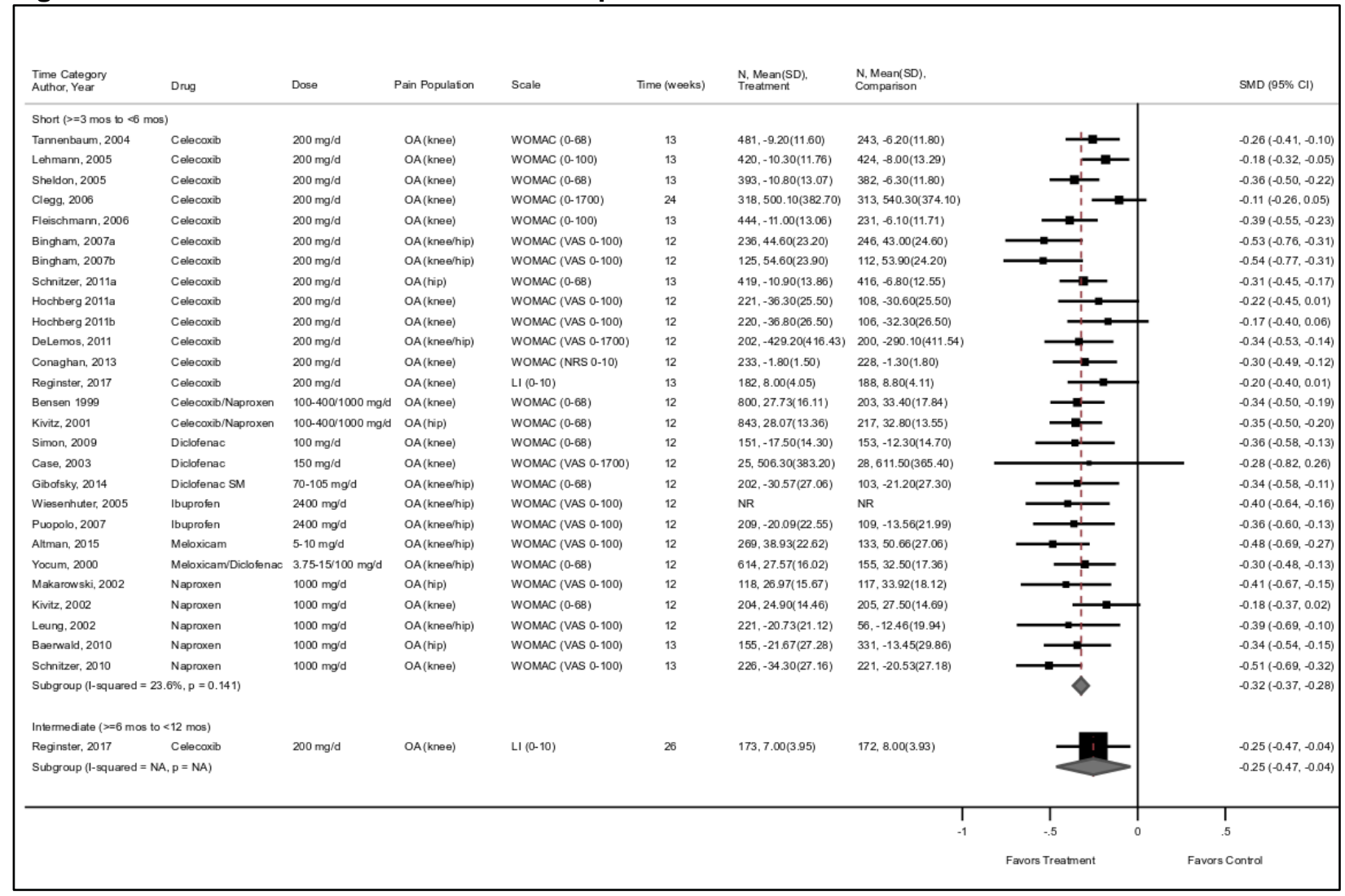


Figure I-41. Osteoarthritis-function topical diclofenac plot

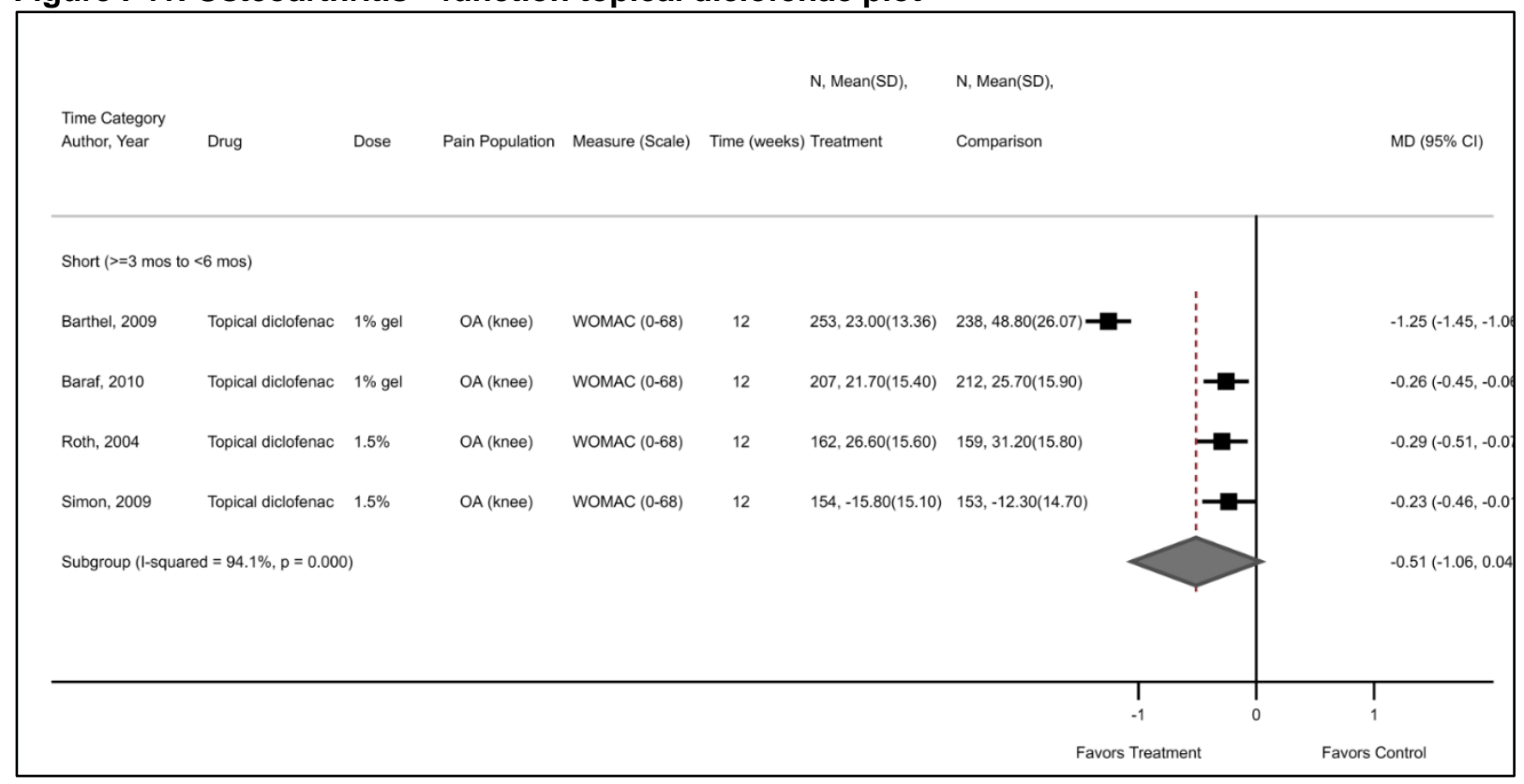

Figure I-42. Osteoarthritis-function SNRI plot

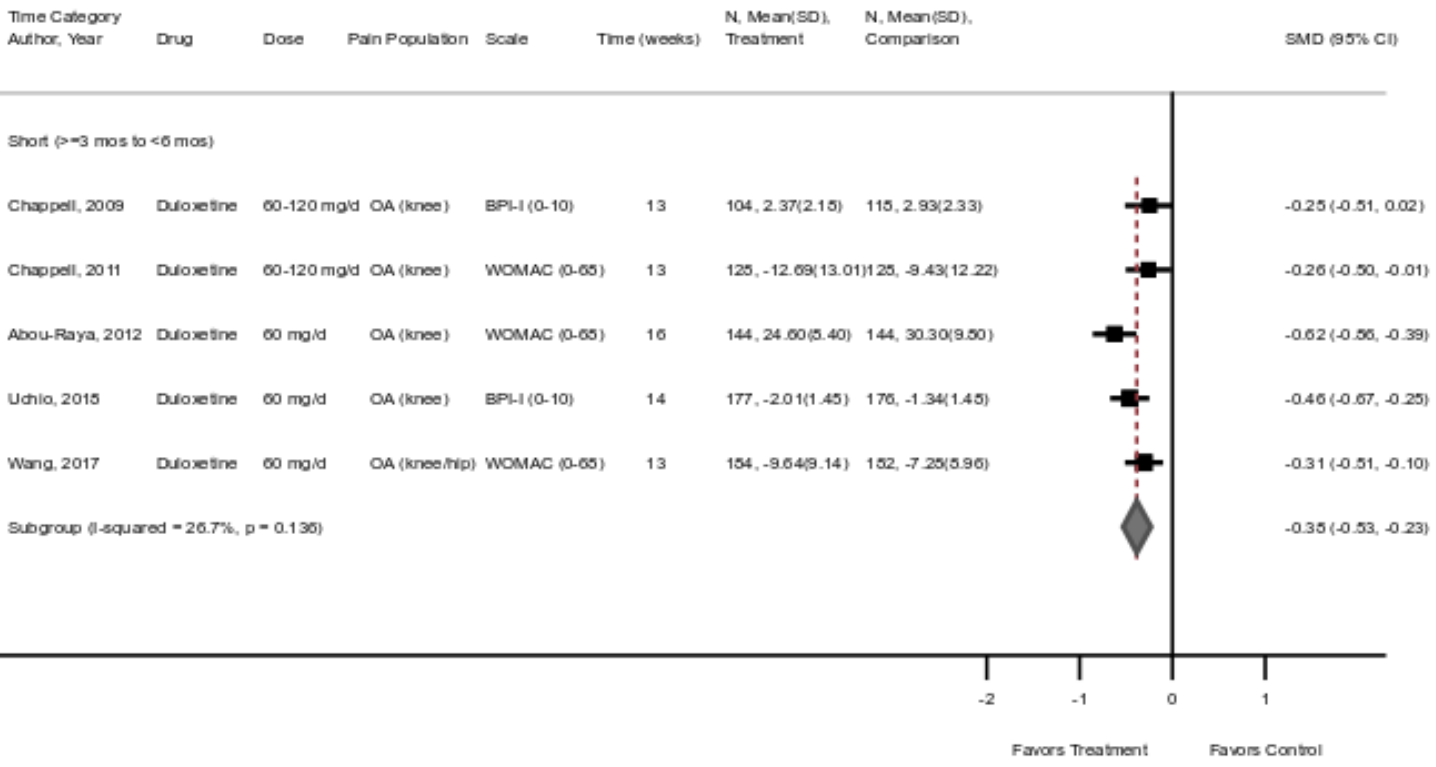


Figure I-43. Osteoarthritis-function acetaminophen plot

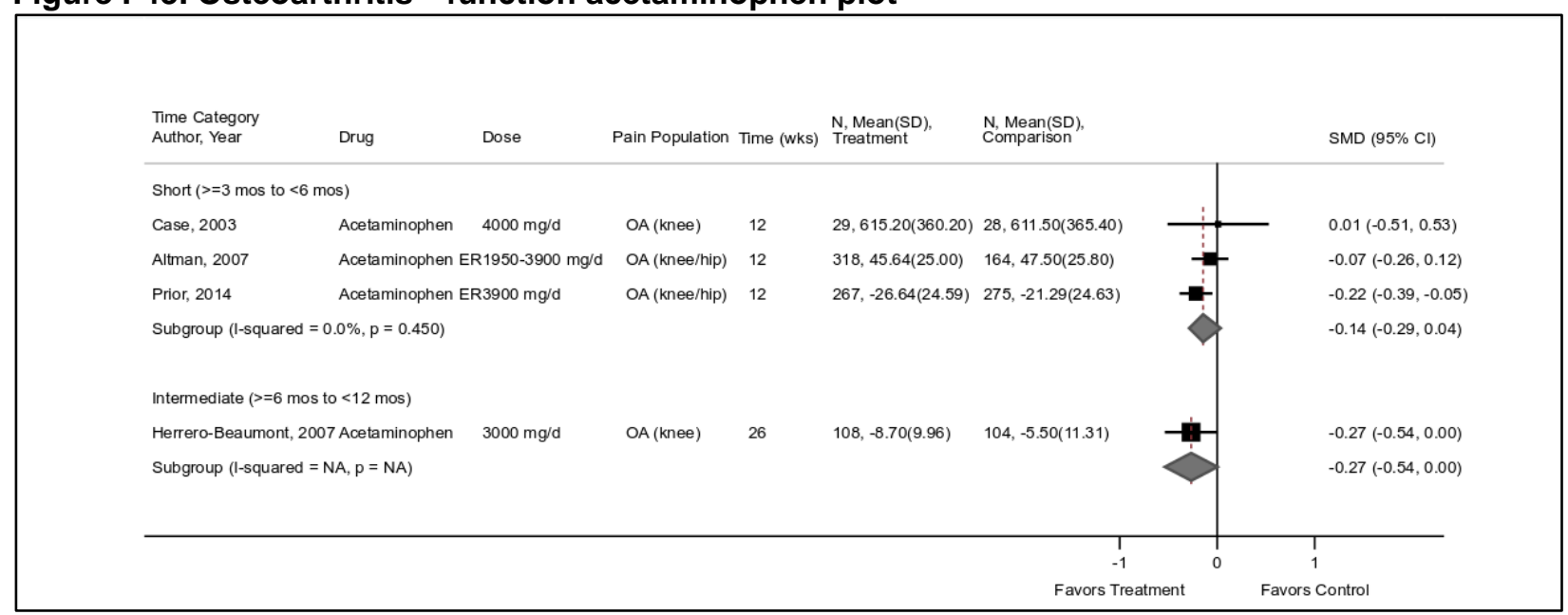

Figure I-44. Osteoarthritis-quality of life NSAID plot

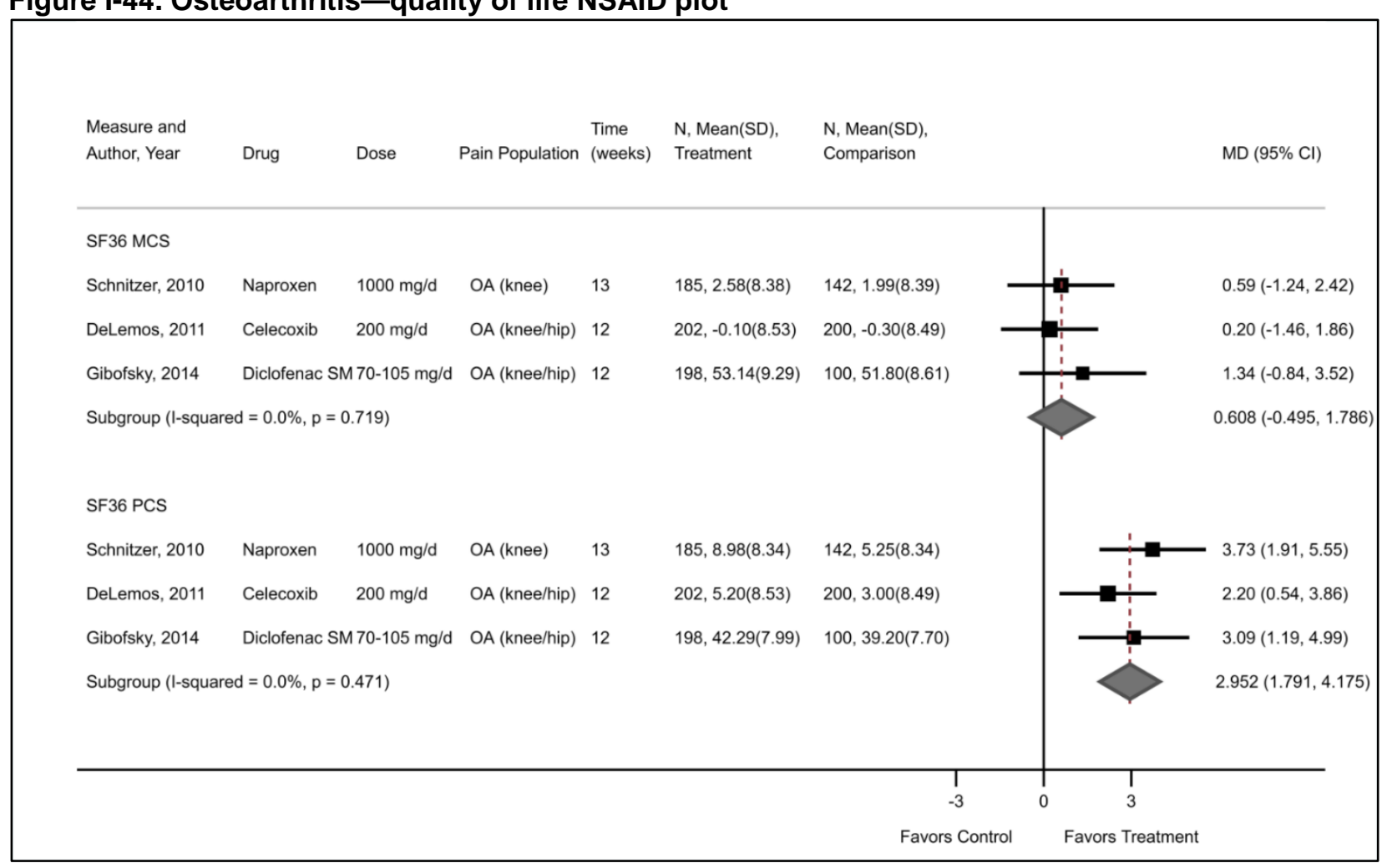


Figure I-45. Osteoarthritis-quality of life SNRI plot

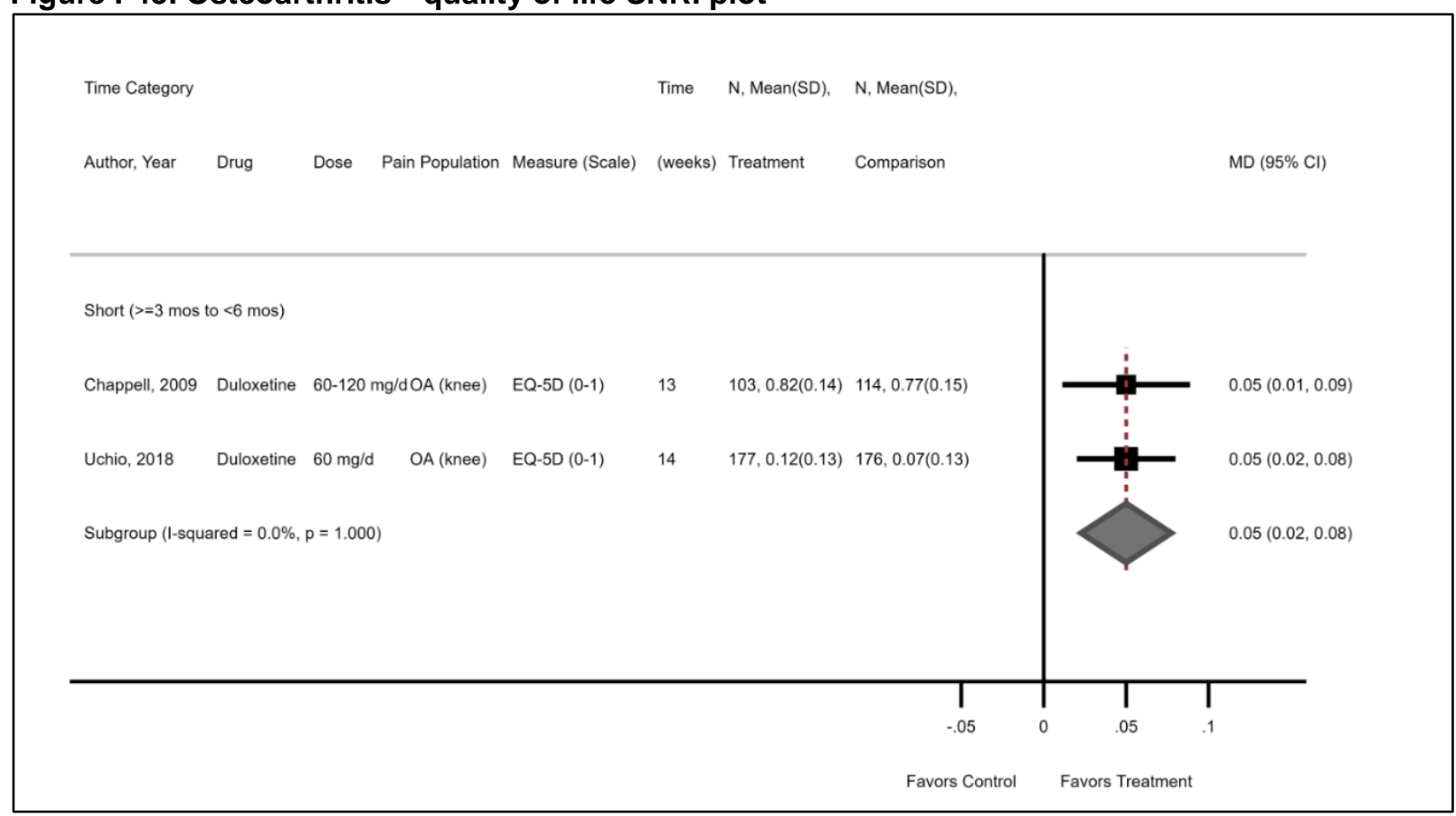

Figure I-46. Osteoarthritis-pain naproxen vs. nabumetone NSAID plot

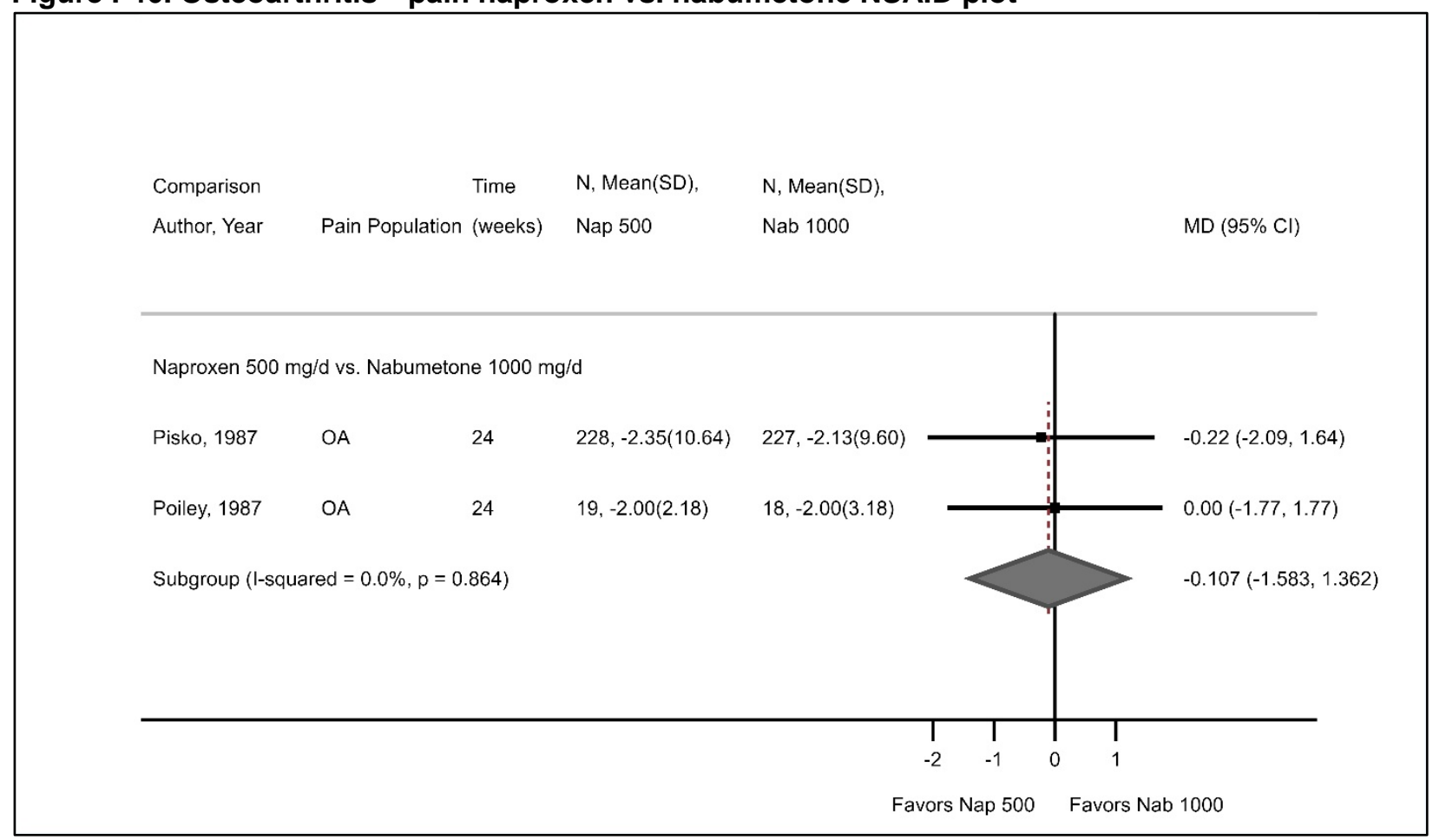




\section{Inflammatory Arthritis}

Figure I-47. Inflammatory arthritis-pain NSAID plot

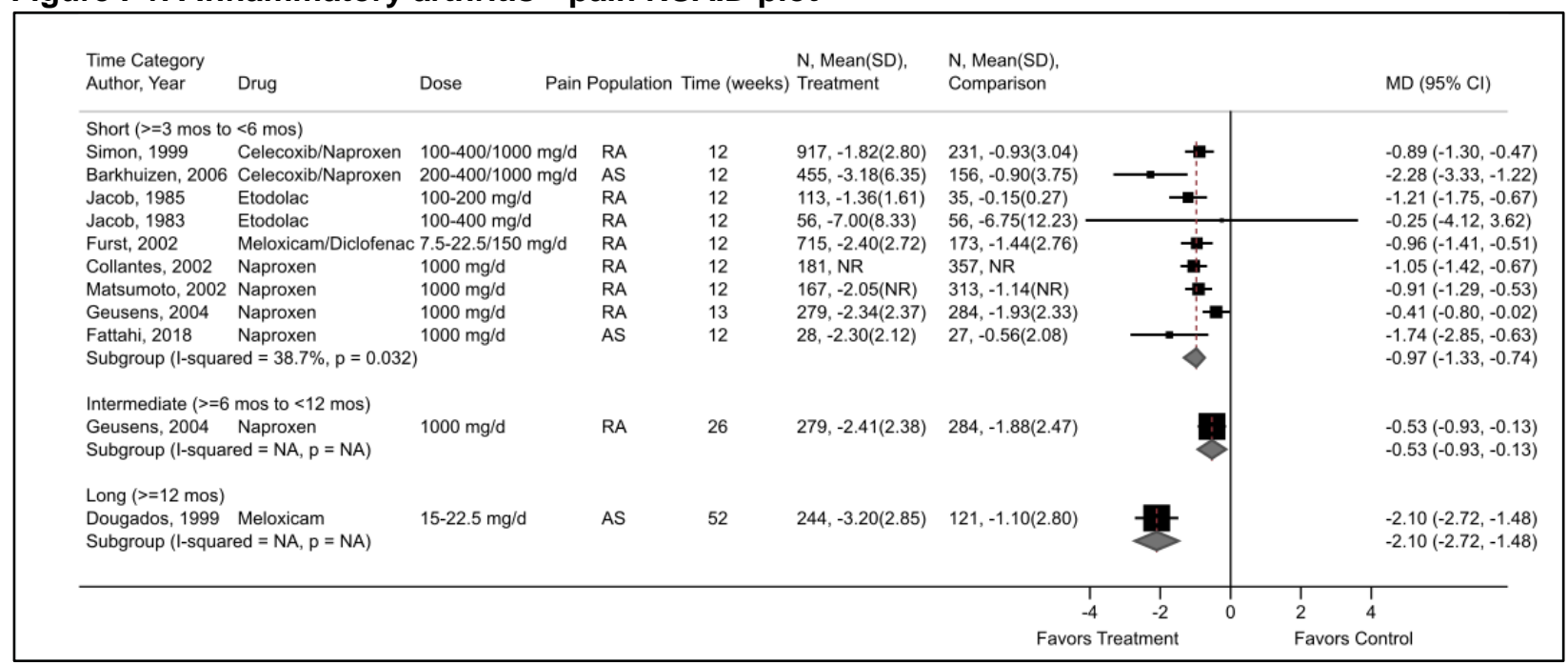

\section{Figure I-48. Inflammatory arthritis-pain response NSAID plot}

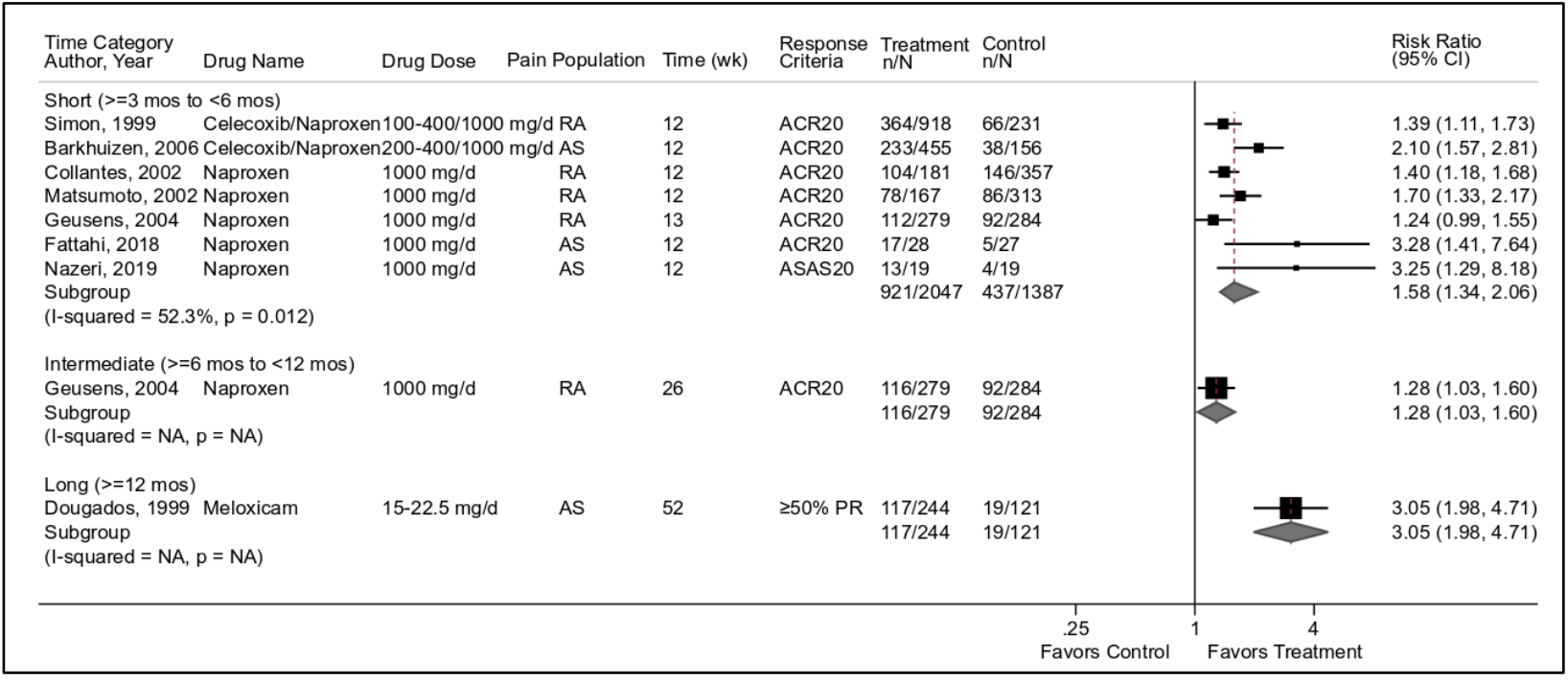


Figure I-49. Inflammatory arthritis-function NSAID plot

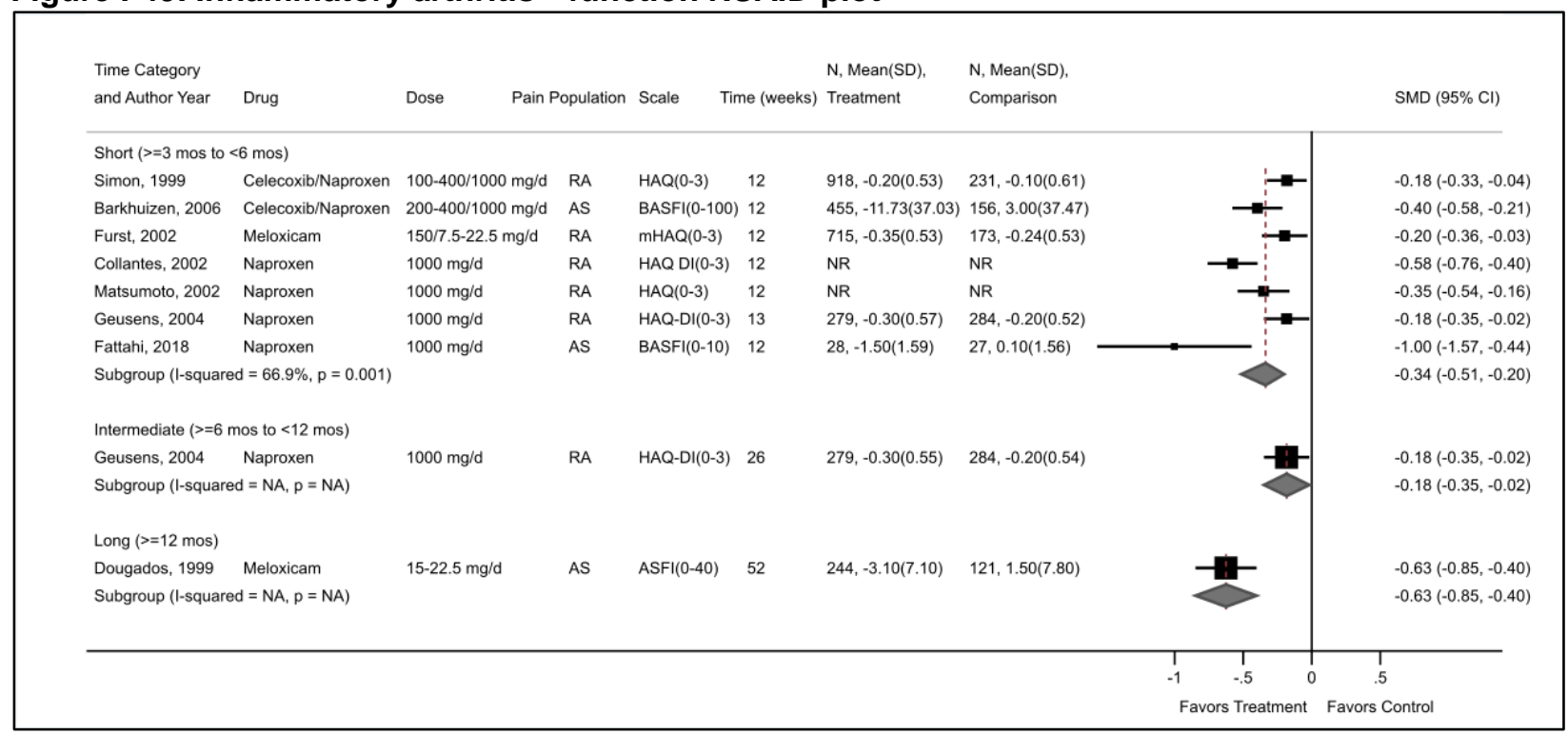

Figure I-50. Inflammatory arthritis_pain celecoxib $200 \mathrm{mg} / \mathrm{d}$ vs. naproxen plot

\begin{tabular}{|c|c|c|c|c|c|c|c|}
\hline $\begin{array}{l}\text { Comparison } \\
\text { Author, Year }\end{array}$ & Pain Population & $\begin{array}{l}\text { Time } \\
\text { (weeks) }\end{array}$ & $\mathrm{N}$ & $\begin{array}{l}\text { Cel } 200 \\
\text { Mean (SD) }\end{array}$ & $\mathrm{N}$ & $\begin{array}{l}\text { Nap } 1000 \\
\text { Mean (SD) }\end{array}$ & MD $(95 \% \mathrm{Cl})$ \\
\hline \multicolumn{8}{|c|}{ Celecoxib 200 mg/d vs. Naproxen 1000 mg/d } \\
\hline Simon, 1999 & RA & 12 & 235 & $-2.07(2.91)$ & 225 & $-1.69(2.70)$ & $-0.38(-0.89,0.13)$ \\
\hline Barkhuizen, 2006 & AS & 12 & 137 & $2.90(4.68)$ & 157 & $3.60(7.52)$ & $-0.70(-2.11,0.71)$ \\
\hline \multicolumn{2}{|l|}{ Subgroup } & & 372 & & 382 & & $-0.42(-1.16,0.20)$ \\
\hline \multicolumn{8}{|c|}{$(\mathrm{I}$-squared $=0.0 \%, p=0.677)$} \\
\hline & & & & & & $\begin{array}{cc}1 & -1 \\
\text { Favors Cel } 200\end{array}$ & $\begin{array}{lcc}0 & 1 & 2 \\
& \text { Favors Nap } 1000\end{array}$ \\
\hline
\end{tabular}


Figure I-51. Inflammatory arthritis_pain celecoxib $400 \mathrm{mg} / \mathrm{d}$ vs. naproxen plot

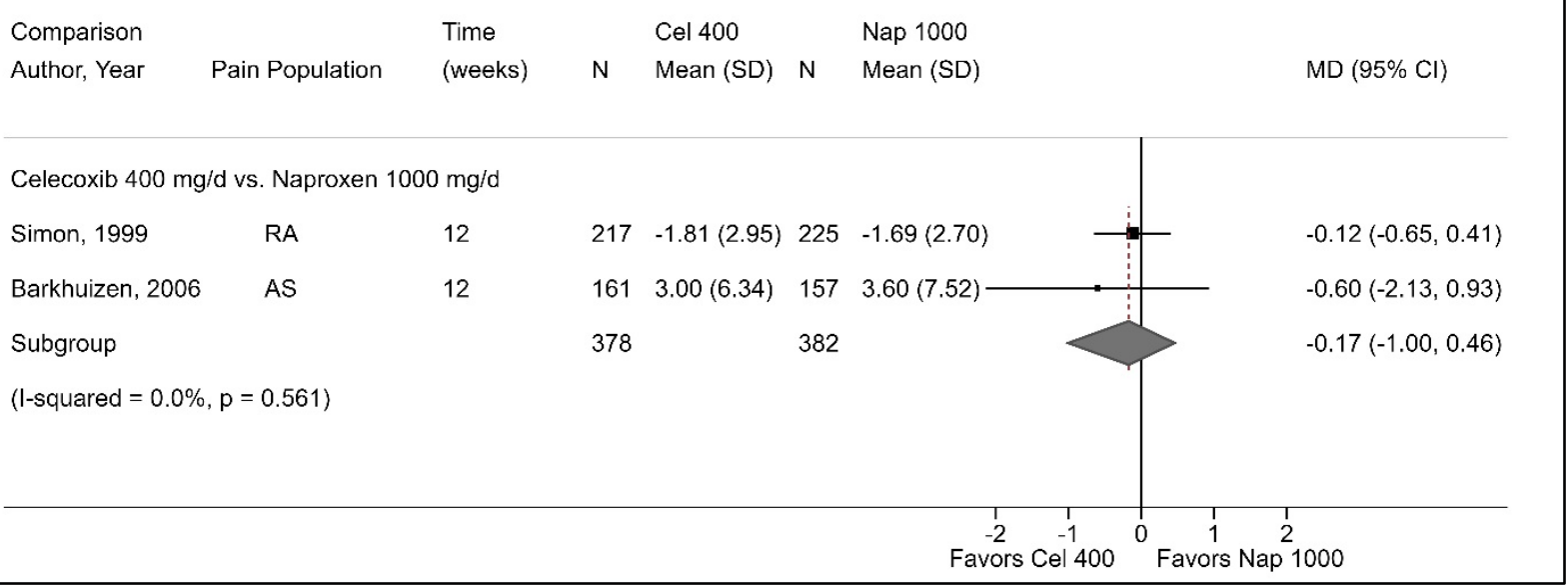

Figure I-52. Inflammatory arthritis-pain diclofenac vs. celecoxib $200 \mathrm{mg} / \mathrm{d}$ plot

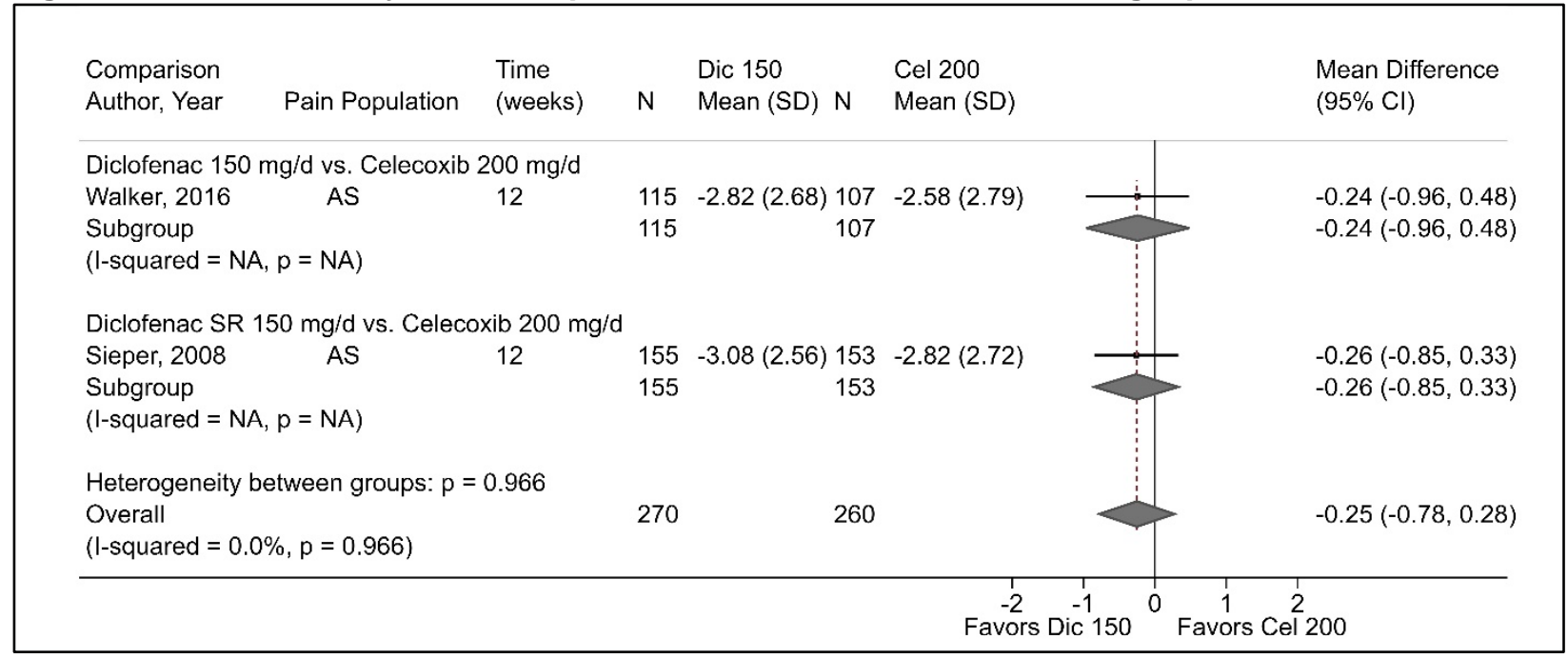


Figure I-53. Inflammatory arthritis-pain diclofenac vs. celecoxib $400 \mathrm{mg} / \mathrm{d}$ plot

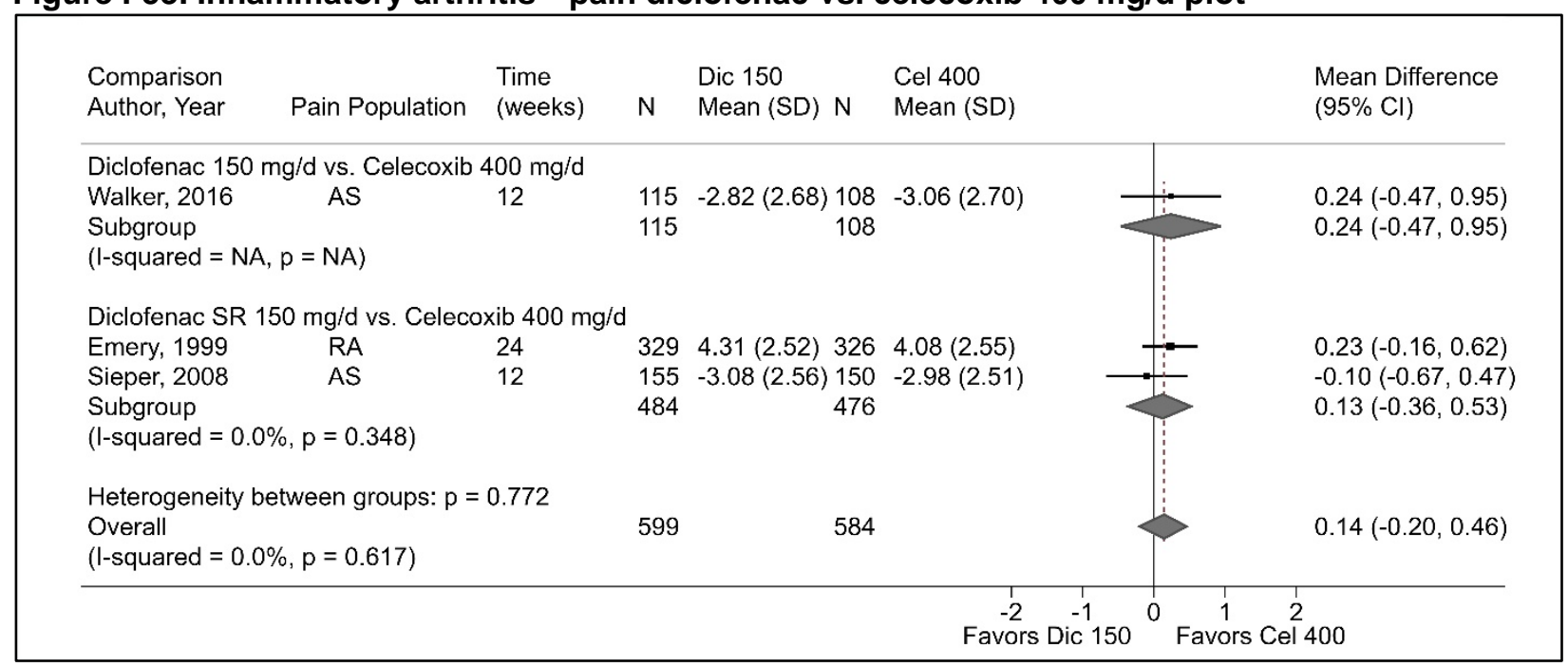

Figure I-54. Inflammatory arthritis_pain naproxen vs. nabumetone plot

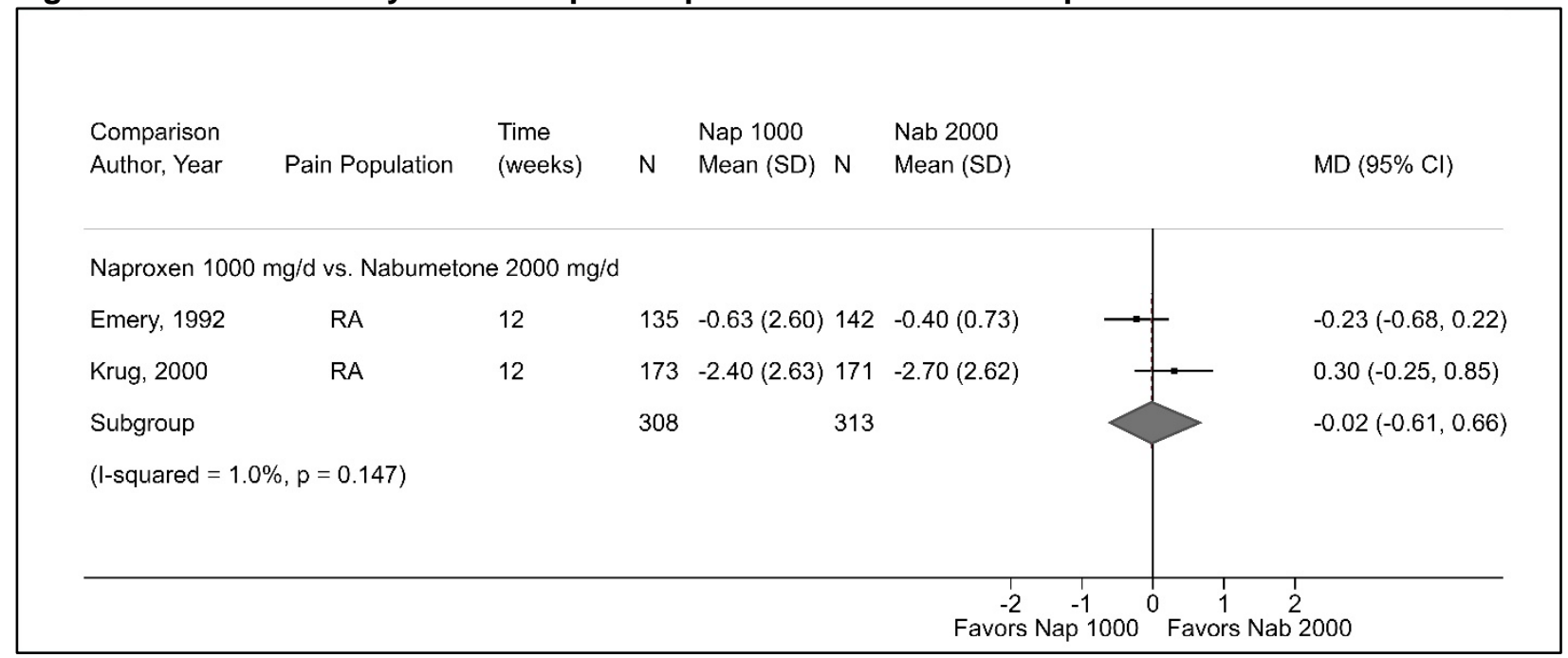


Figure I-55. Inflammatory arthritis_pain response celecoxib $200 \mathrm{mg} / \mathrm{d}$ vs. naproxen plot

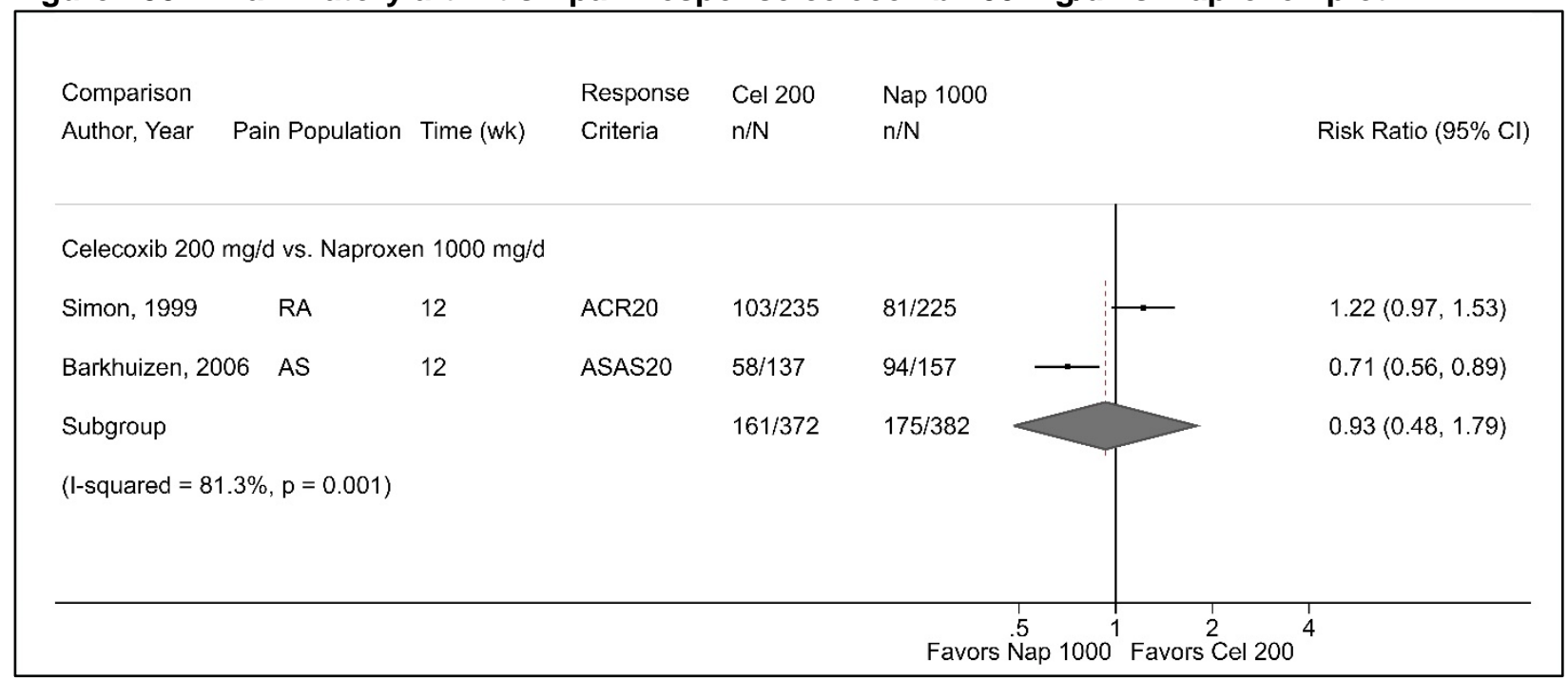

Figure I-56. Inflammatory arthritis_pain response celecoxib 400 mg/d vs. naproxen plot

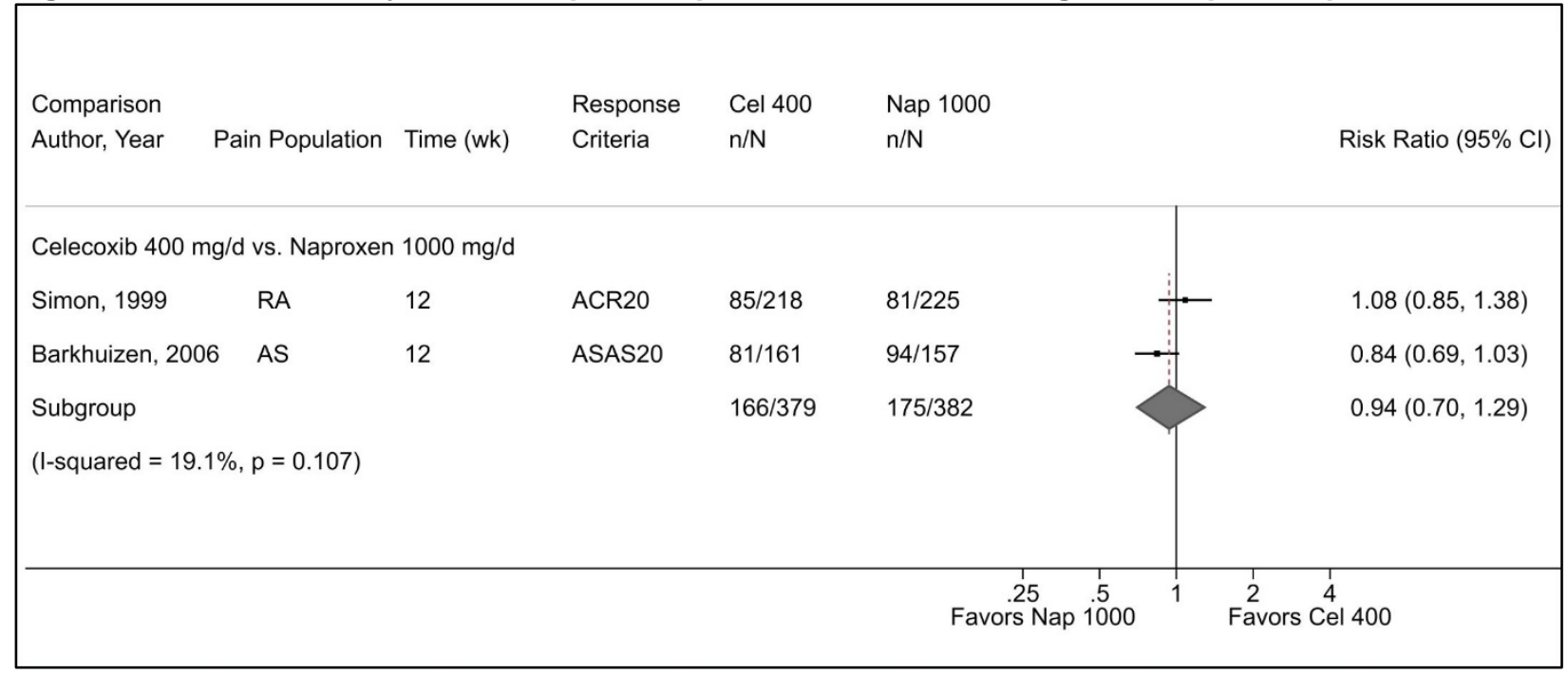


Figure I-57. Inflammatory arthritis-pain response diclofenac vs celecoxib $200 \mathrm{mg} / \mathrm{d}$ plot

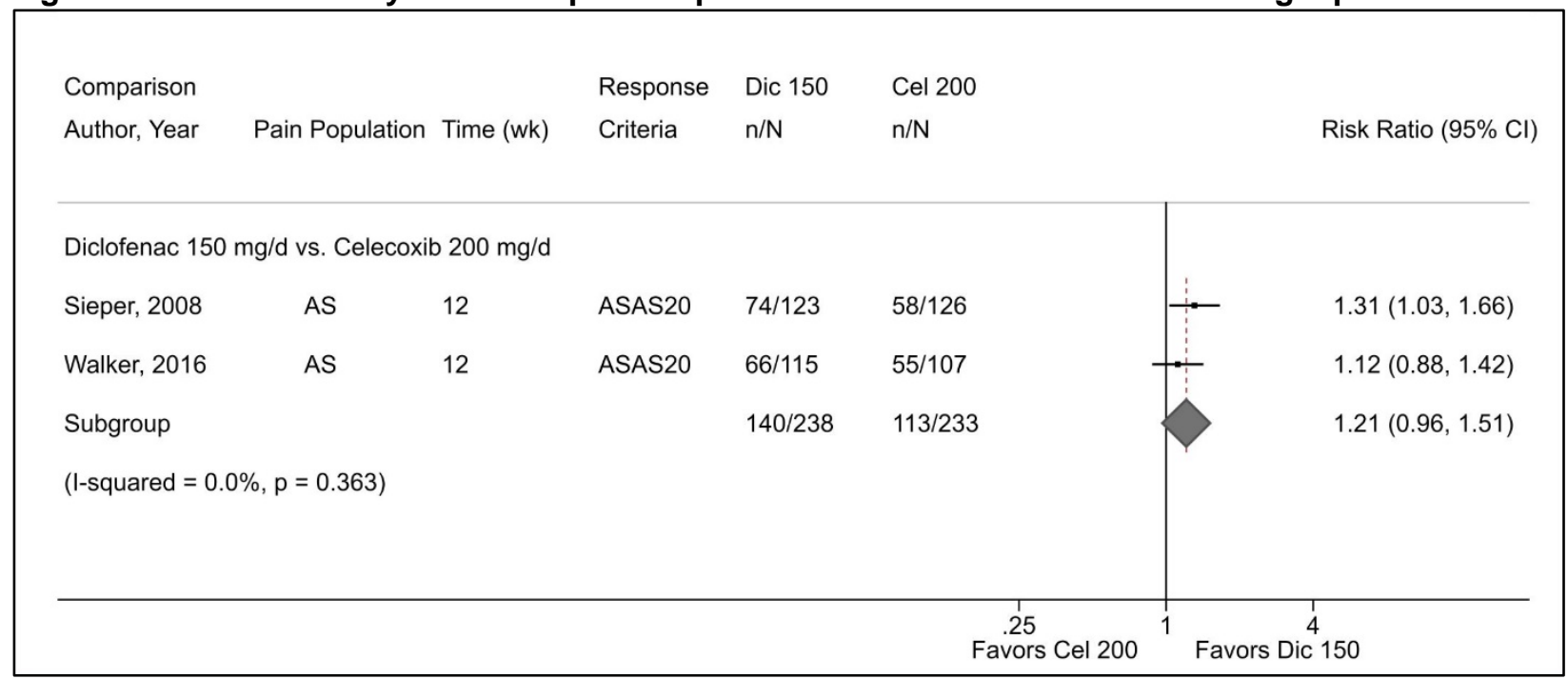

Figure I-58. Inflammatory arthritis_function celecoxib $200 \mathrm{mg} / \mathrm{d}$ vs. naproxen plot

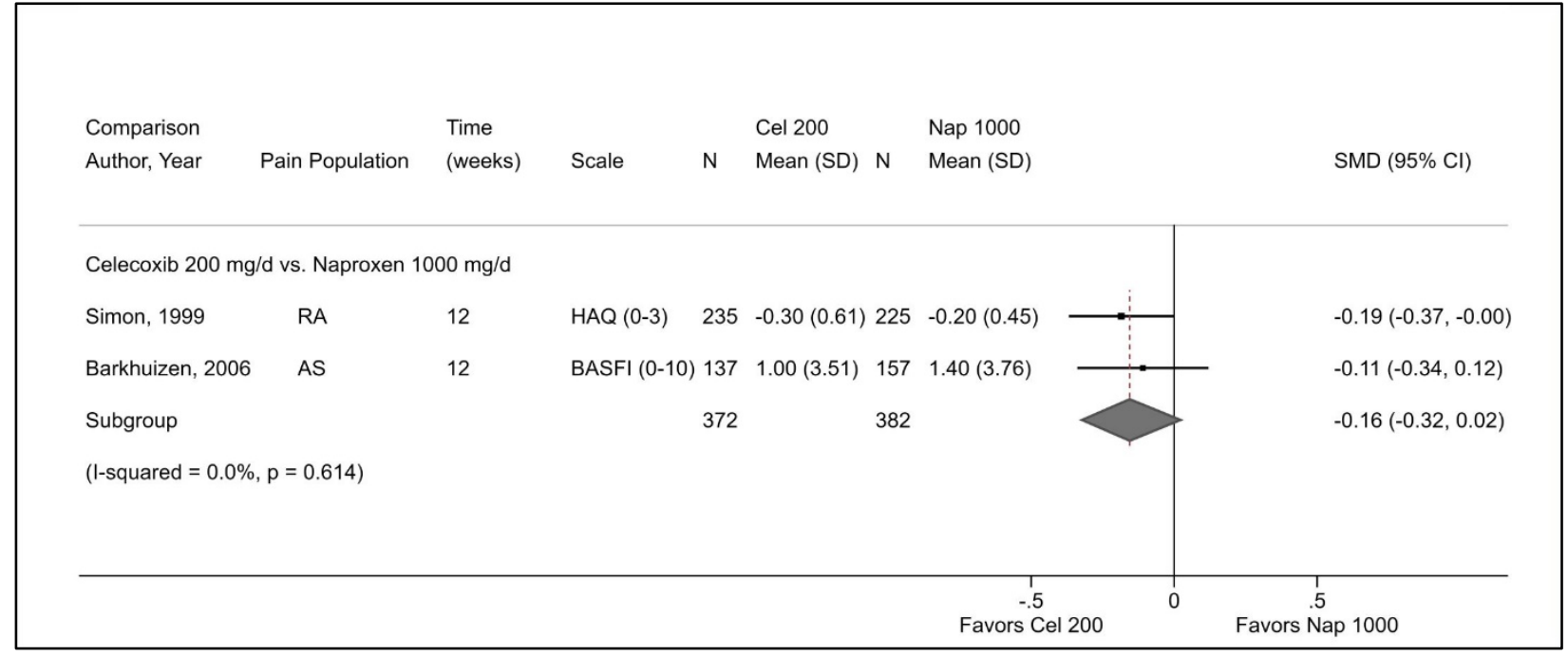


Figure I-59. Inflammatory arthritis-function celecoxib $400 \mathrm{mg} / \mathrm{d}$ vs. naproxen plot

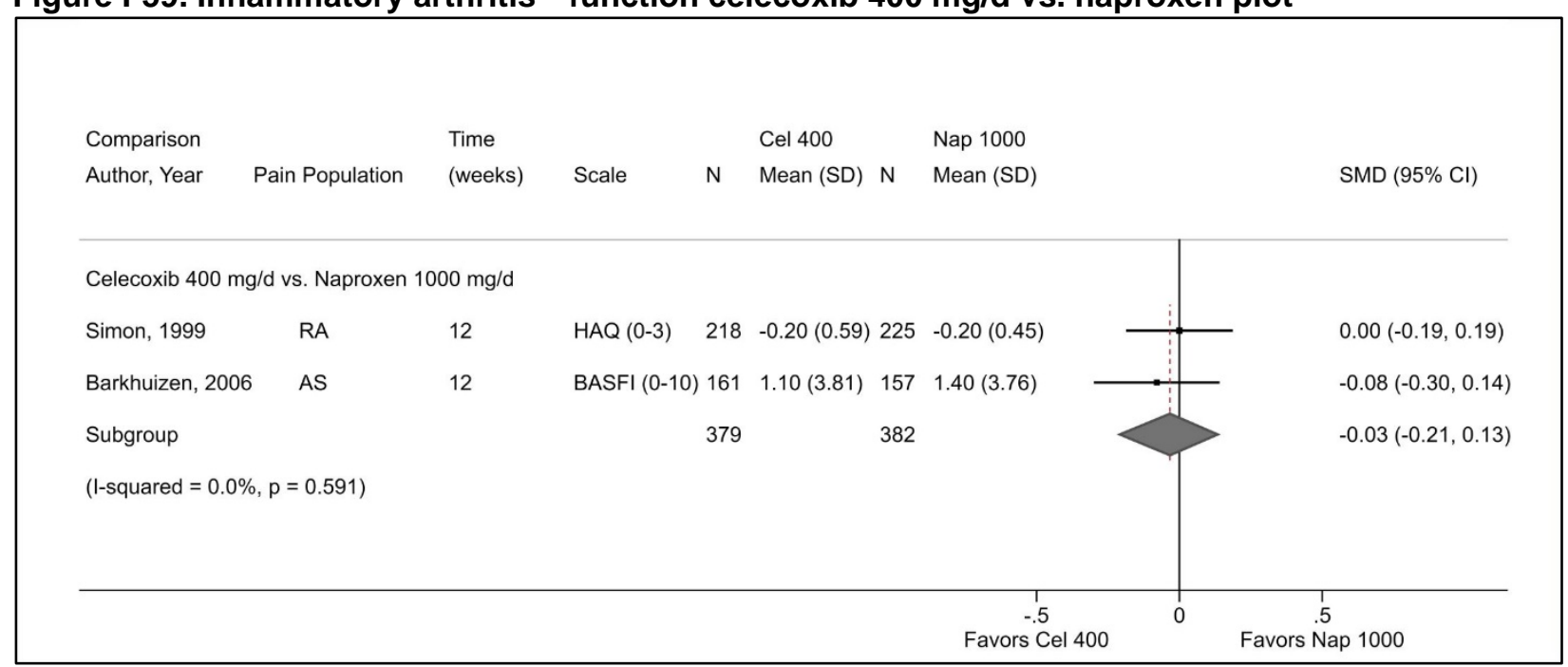

Figure I-60. Inflammatory arthritis-function diclofenac vs. celecoxib $200 \mathrm{mg} / \mathrm{d}$ plot

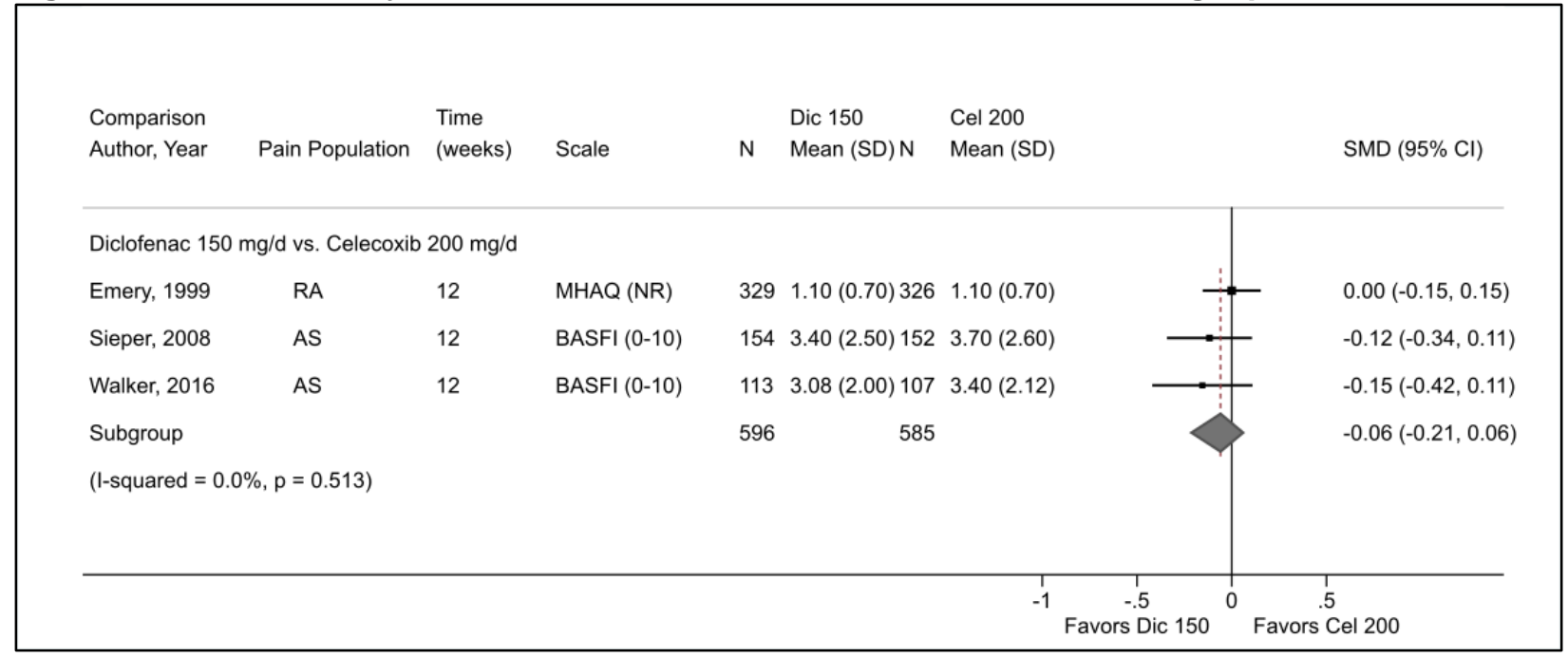


Figure I-61. Inflammatory arthritis-function diclofenac vs. celecoxib $400 \mathrm{mg} / \mathrm{d}$ plot

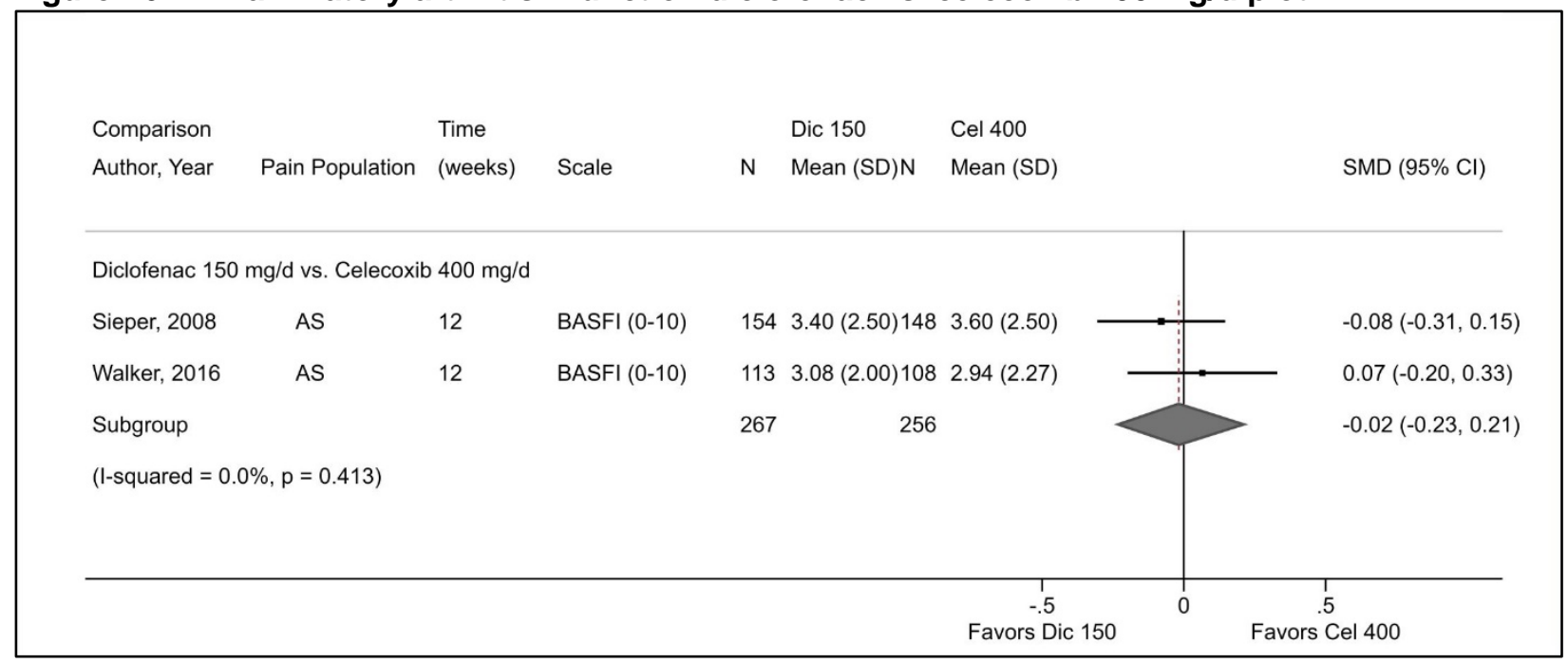

Figure I-62. Inflammatory arthritis-function naproxen vs. nabumetone plot

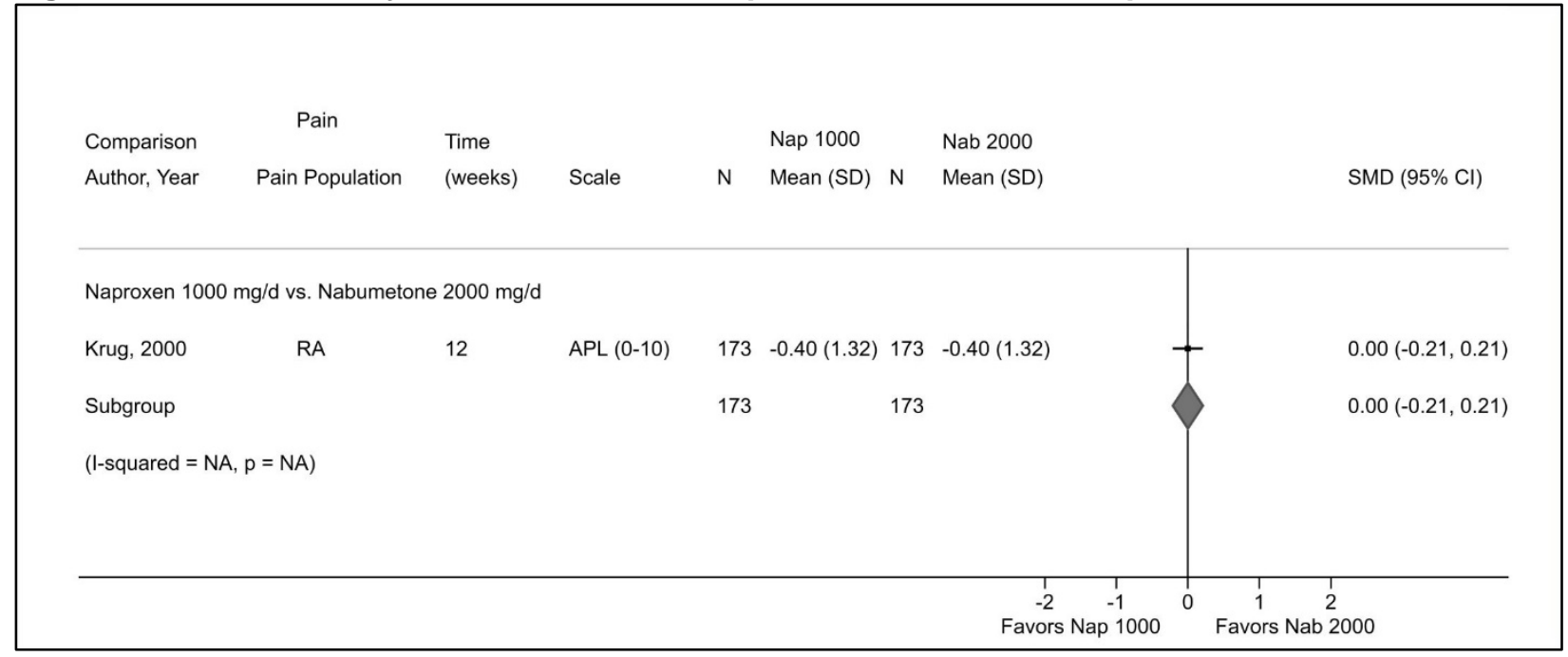




\section{Low Back Pain}

Figure I-63. Low back pain-pain SNRI plot

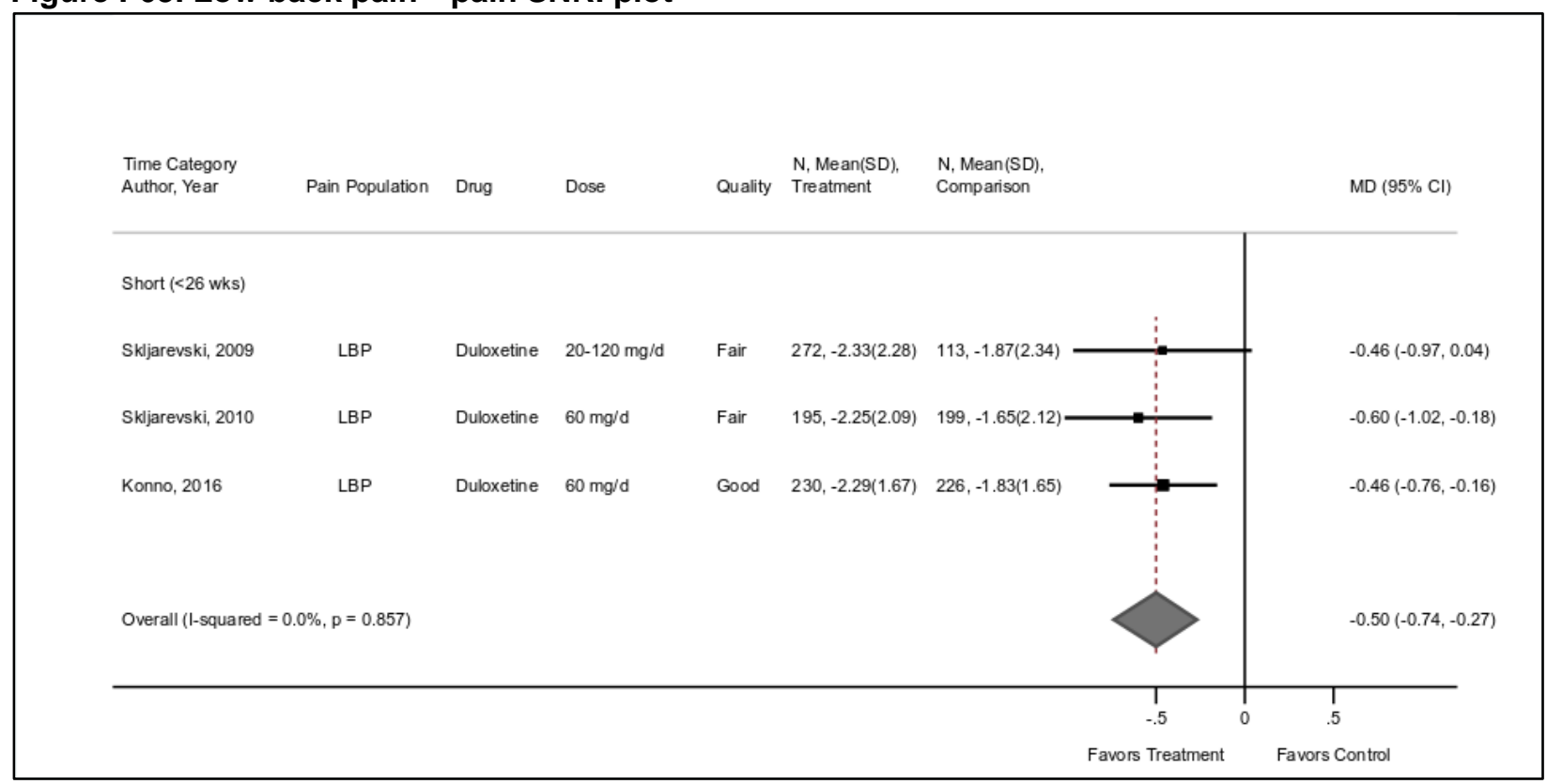

Figure I-64. Low back pain-pain response SNRI plot

\begin{tabular}{|c|c|c|c|c|c|c|c|c|c|}
\hline $\begin{array}{l}\text { Time Category } \\
\text { Author, Year }\end{array}$ & Drug & Dose & in Population & $\begin{array}{l}\text { Time } \\
\text { (weeks) }\end{array}$ & $\begin{array}{l}\text { Response } \\
\text { Criteria }\end{array}$ & Quality & $\begin{array}{l}\text { Treatment } \\
\mathrm{n} / \mathrm{N}\end{array}$ & $\begin{array}{l}\text { Control } \\
\mathrm{n} / \mathrm{N}\end{array}$ & $\begin{array}{l}\text { Risk Ratio } \\
(95 \% \mathrm{Cl})\end{array}$ \\
\hline \multicolumn{10}{|c|}{ Short (>=3mo to <6mo) } \\
\hline Konno, 2016 & Duloxetine & $60 \mathrm{mg} / \mathrm{d}$ & LBP & 14 & $\geq 30 \% \mathrm{PR}$ & Good & $158 / 230$ & $118 / 226$ & $1.32(1.13,1.53)$ \\
\hline Skljarevski, 2009 & Duloxetine & $20-120 \mathrm{mg} / \mathrm{d}$ & dd LBP & 13 & $230 \%$ PR & Fair & $146 / 272$ & 49/113 & $1.24(0.98,1.57)$ \\
\hline Skljarevski, 2010 & Duloxetine & $60 \mathrm{mg} / \mathrm{d}$ & LBP & 12 & $\geq 30 \%$ PR & Fair & $111 / 195$ & 97/199 & $1.17(0.97,1.41)$ \\
\hline Subgroup & & & & & & & $415 / 697$ & $264 / 538$ & $1.25(1.11,1.40)$ \\
\hline \multicolumn{10}{|c|}{ (I-squared $=0.0 \%, p=0.622)$} \\
\hline \multicolumn{10}{|c|}{ Favors Treatment } \\
\hline
\end{tabular}


Figure I-65. Low back pain-function SNRI plot

\begin{tabular}{|c|c|c|c|c|c|c|c|c|c|}
\hline $\begin{array}{l}\text { Time Category } \\
\text { Author, Year Pain Population }\end{array}$ & Drug & Dose & fime (wee & s) Quality & Measure & e, Scale & $\begin{array}{l}\mathrm{N}, \text { Mean(SD), } \\
\text { Treatment }\end{array}$ & $\begin{array}{l}\mathrm{N}, \text { Mean(SD), } \\
\text { Comparison }\end{array}$ & $\mathrm{MD}(95 \% \mathrm{Cl})$ \\
\hline \multicolumn{10}{|l|}{ Short (> $>3 \mathrm{mo}$ to $<6 \mathrm{mo}$ ) } \\
\hline Konno, 2016 & Duloxetine & $60 \mathrm{mg} / \mathrm{d}$ & 14 & Good & BPI Intf & $0-10$ & $209,-1.83(1.45)$ & $226,-1.70(1.50)$ & $-0.13(-0.41,0.15)$ \\
\hline Skljarevski, 2009 LBP & Duloxetine & $20-120 \mathrm{mg} / \mathrm{d}$ & d 13 & Fair & BPI Intf & $0-10$ & $272,-2.09(1.97)$ & $113,-1.61(2.02)$ & $-0.48(-0.92,-0.05)$ \\
\hline Skljarevski, 2010 LBP & Duloxetine & $60 \mathrm{mg} / \mathrm{d}$ & 12 & Fair & BPI Intf & $0-10$ & $195,-2.01(1.82)$ & $199,-1.43(1.83)$ & $-0.58(-0.94,-0.22)$ \\
\hline \multicolumn{9}{|c|}{ Overall (l-squared $=33.6 \%, p=0.115$ ) } & $-0.36(-0.73,-0.04)$ \\
\hline & & & & & & & & T & $T$ \\
\hline & & & & & & & & Favors Treatment & Favors Control \\
\hline
\end{tabular}

\section{Figure I-66. Low back pain-quality of life SNRI plot}

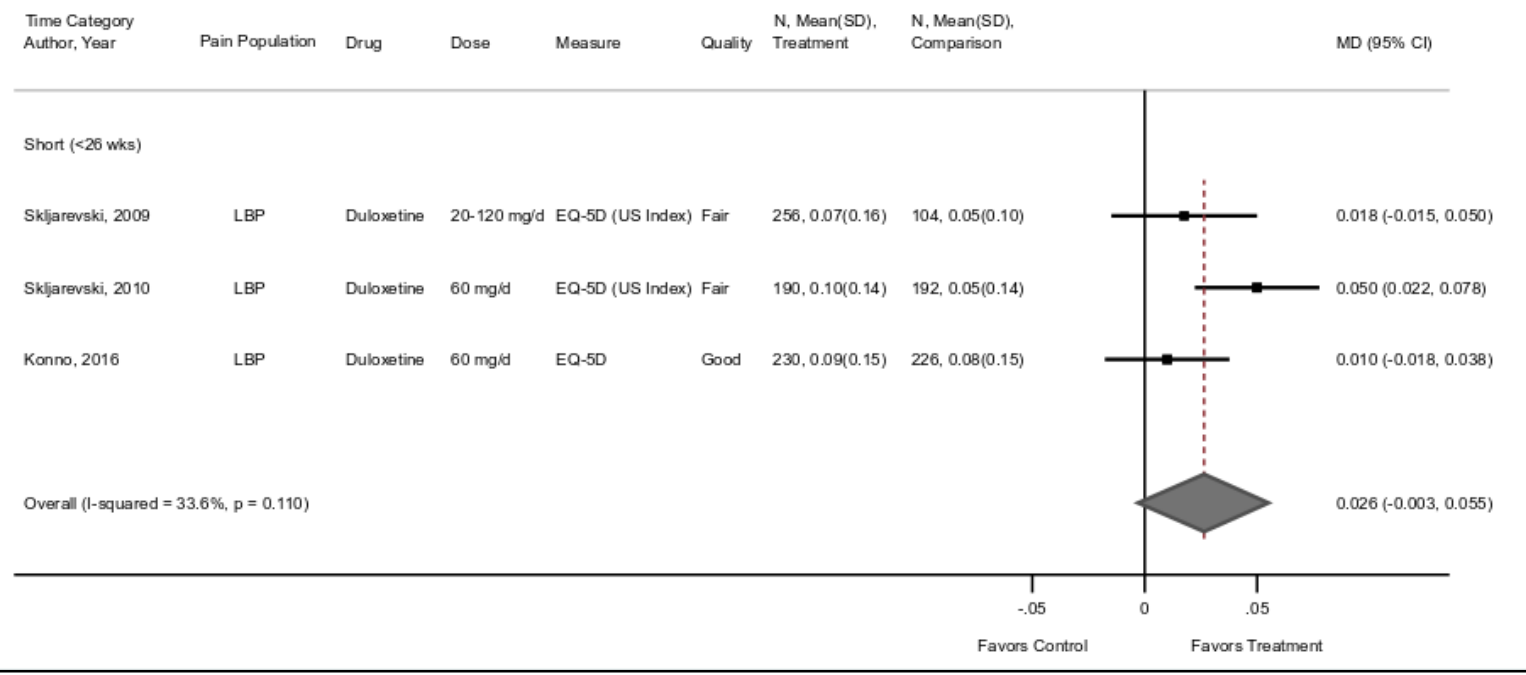




\section{Adverse Events}

\section{Figure I-67. Antidepressants-SNRI withdrawals due to adverse events plot}

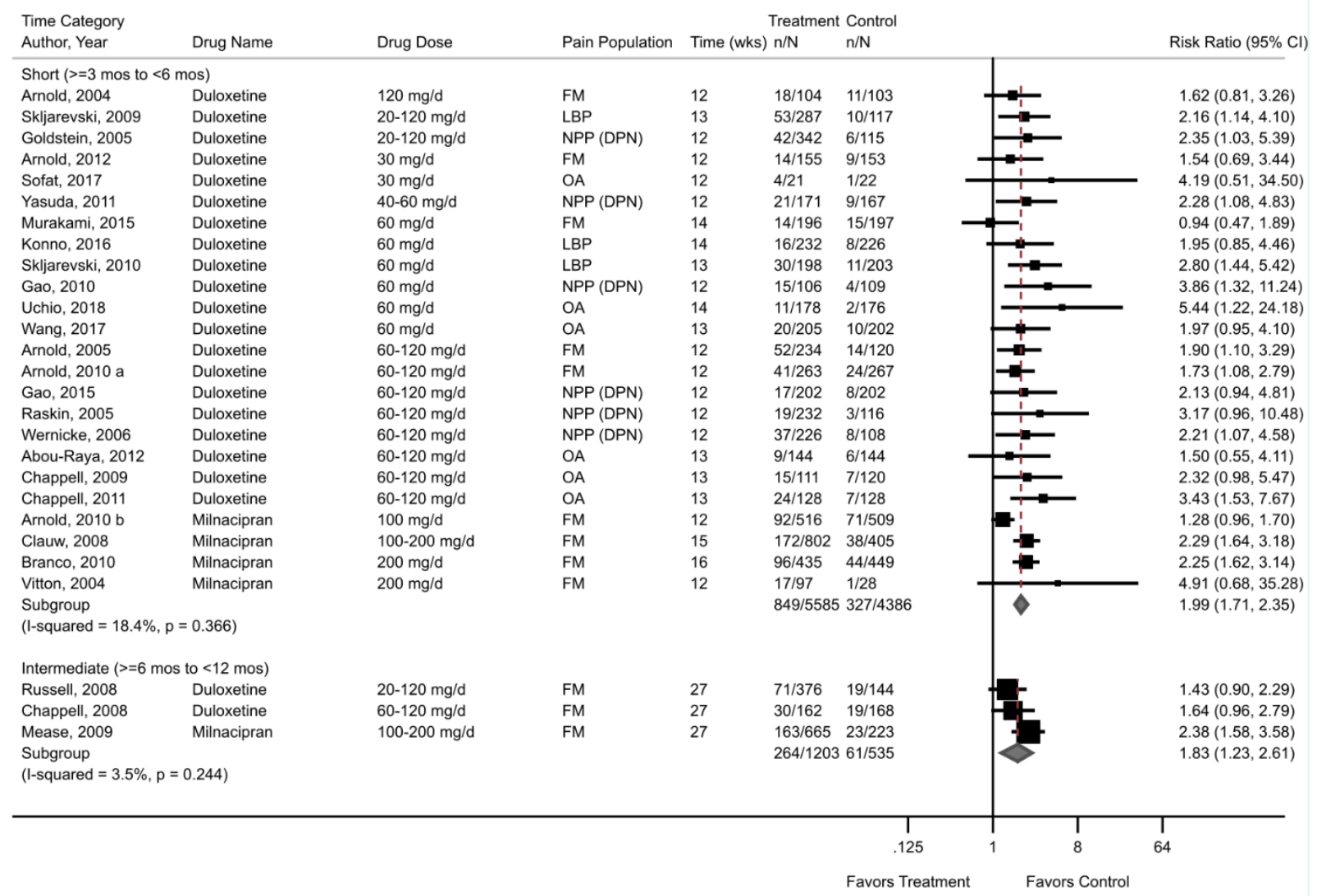


Figure I-68. Antidepressants-SNRI serious adverse events plot

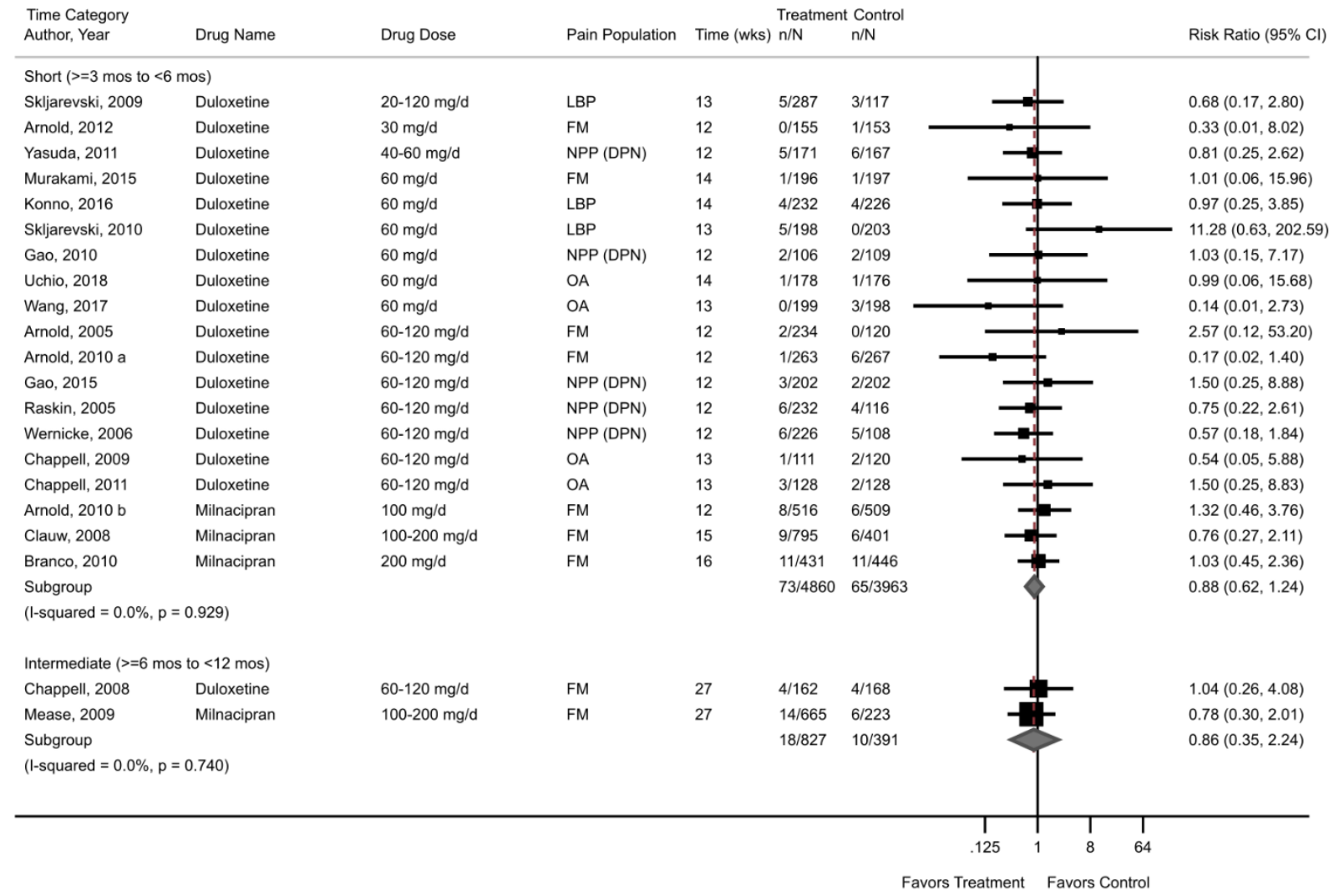

Figure I-69. Antidepressants-SNRI adverse cognitive effects plot

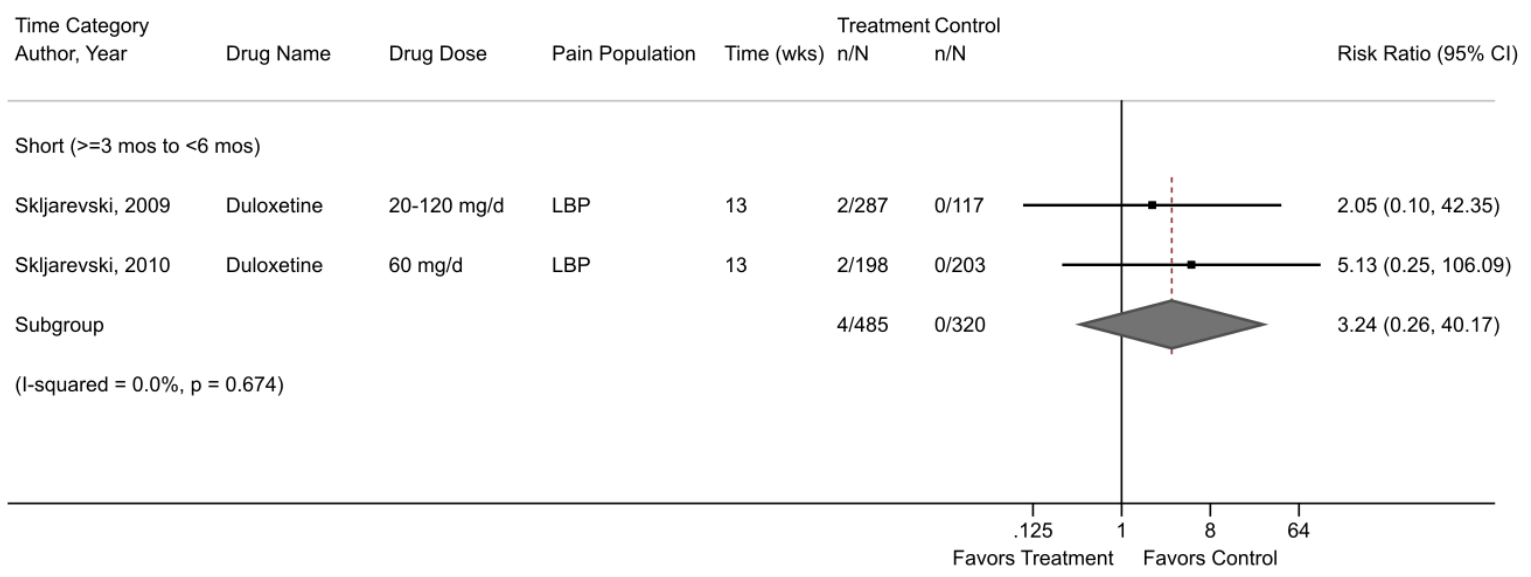


Figure I-70. Antidepressants-SNRI nausea plot

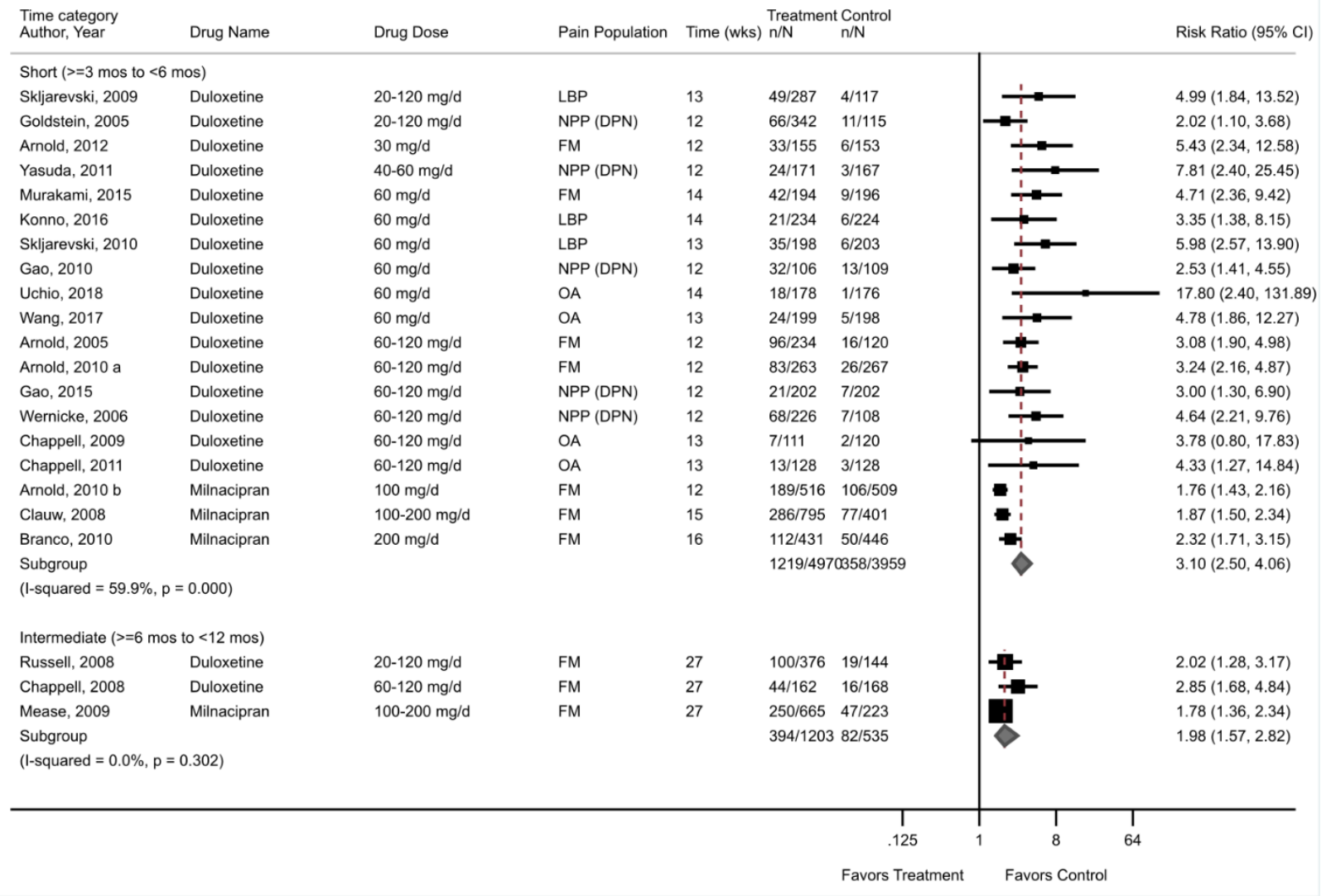


Figure I-71. Antidepressants-SNRI sedation plot

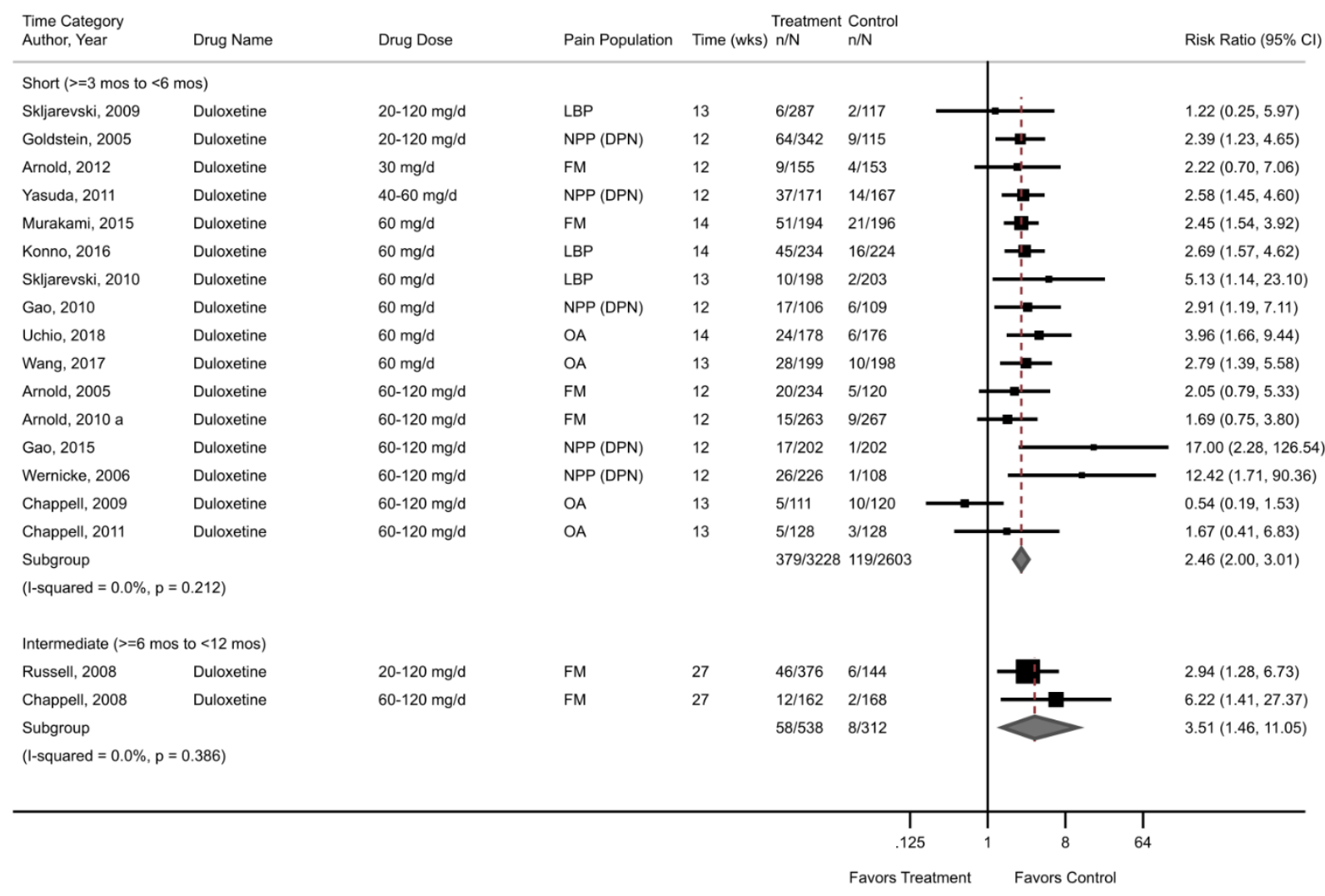

Figure I-72. Antidepressants-TCA withdrawals due to adverse events plot

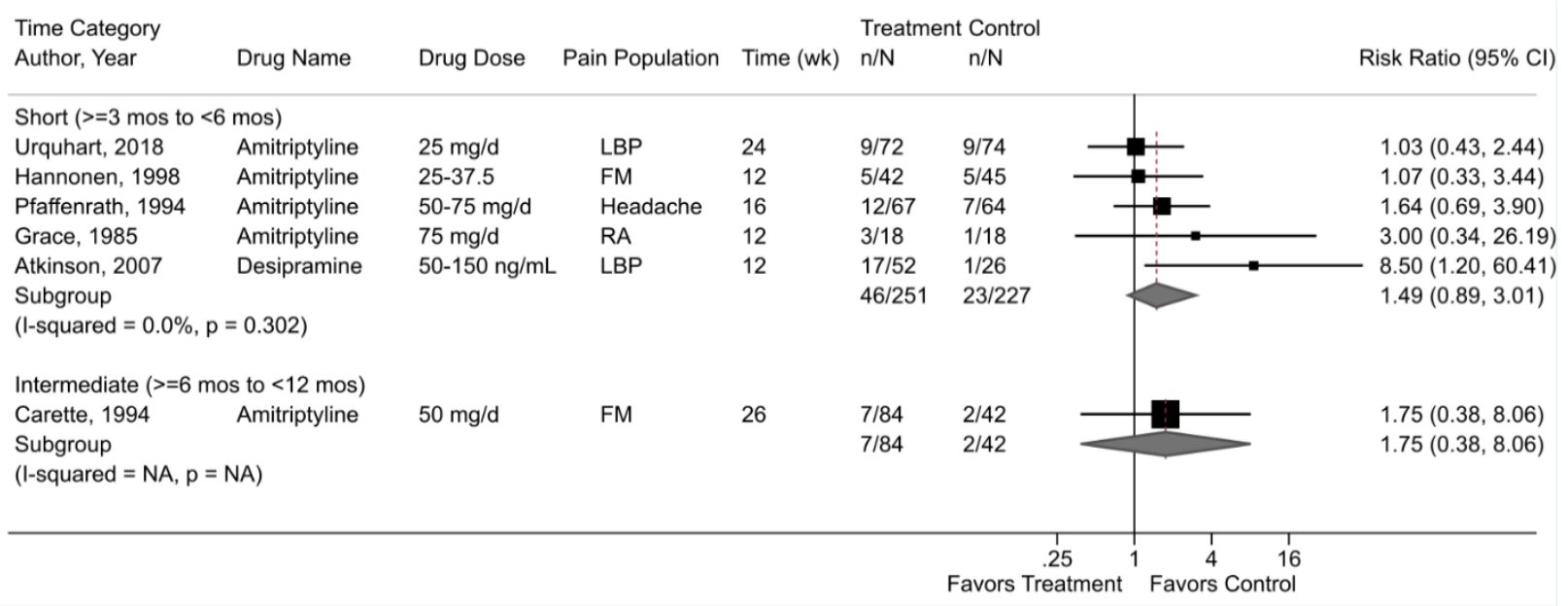


Figure I-73. Anticonvulsants-pregabalin and gabapentin withdrawals due to adverse events plot

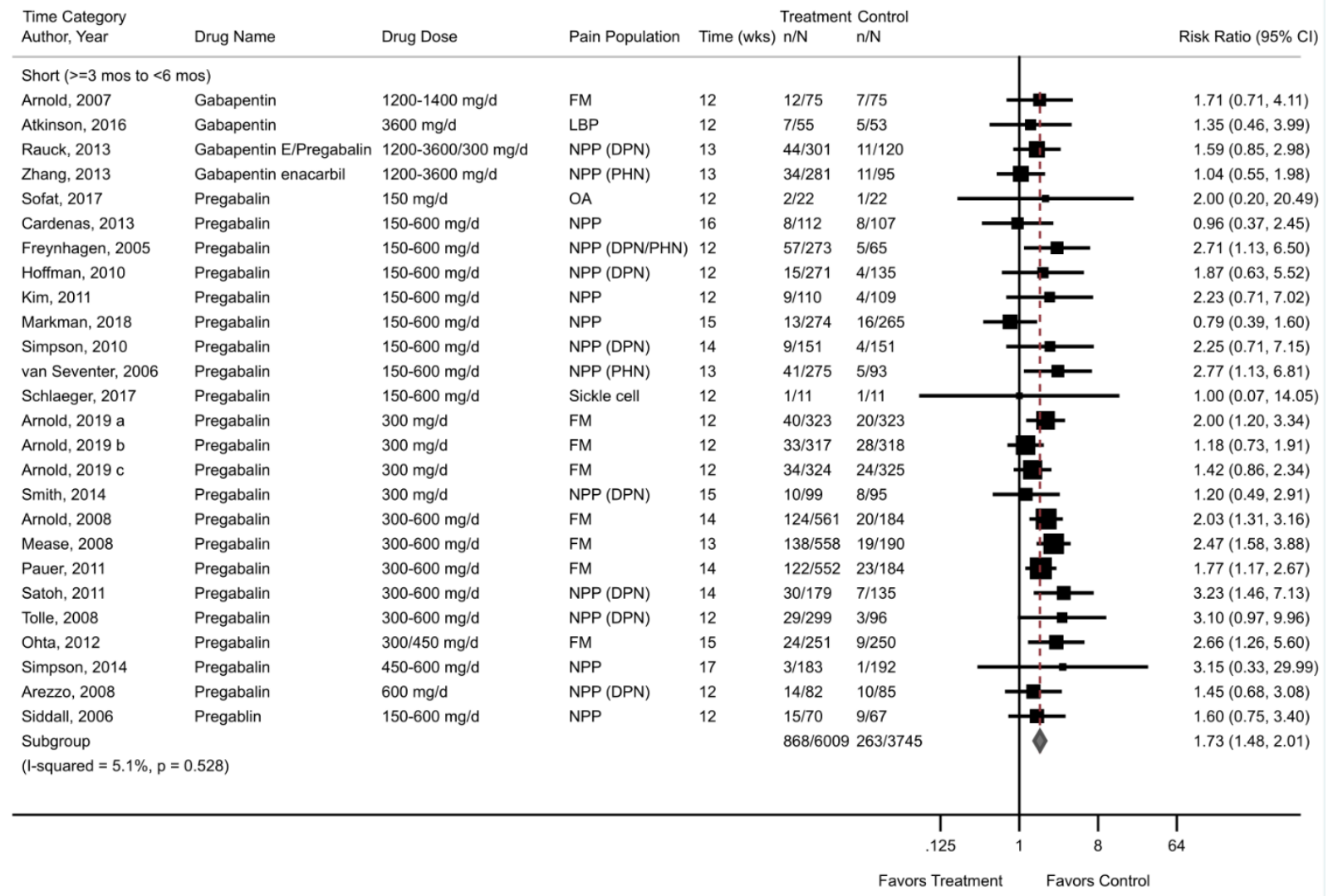


Figure I-74. Anticonvulsants-pregabalin and gabapentin serious adverse events plot

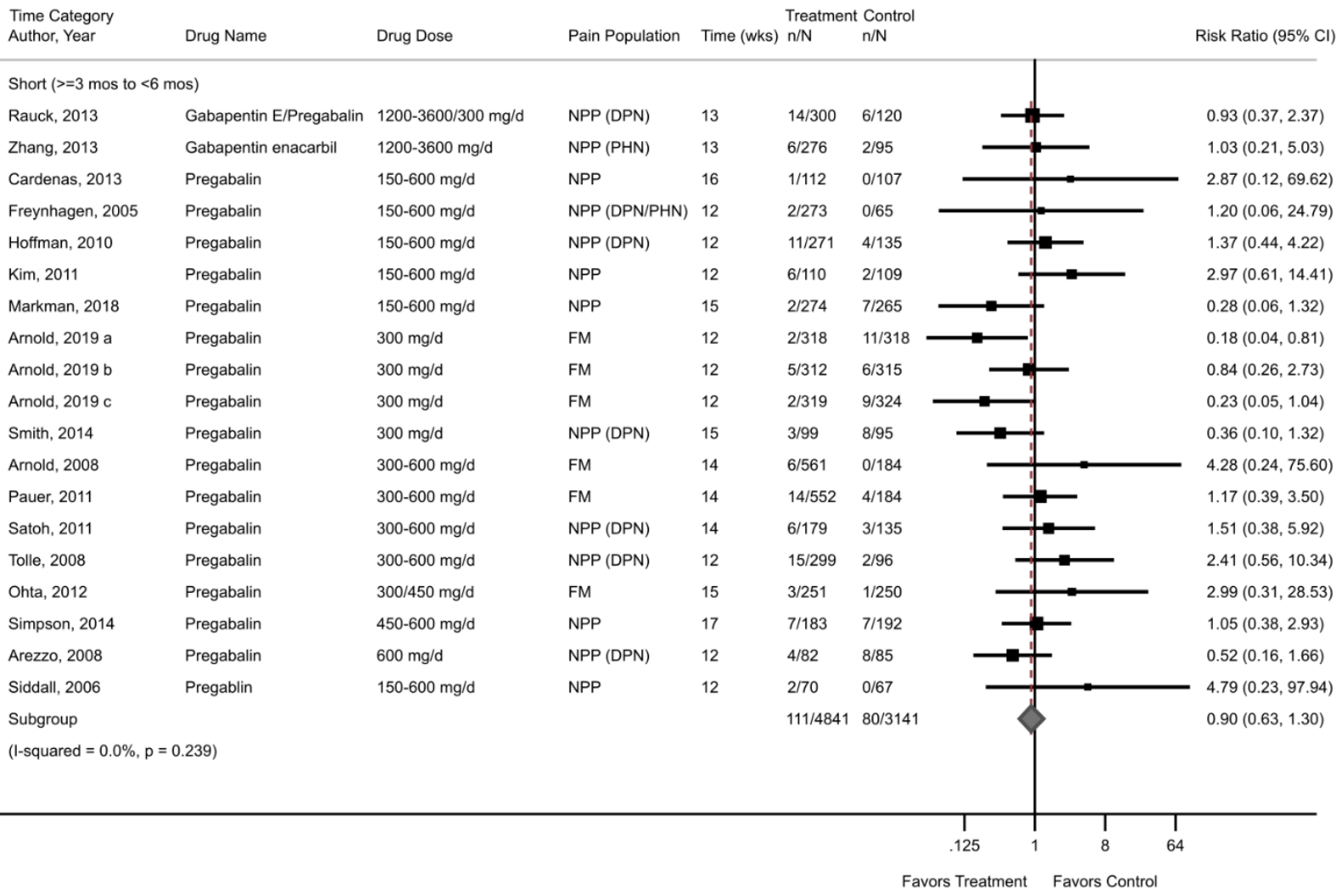

Figure 1-75. Anticonvulsants-pregabalin and gabapentin blurred vision plot

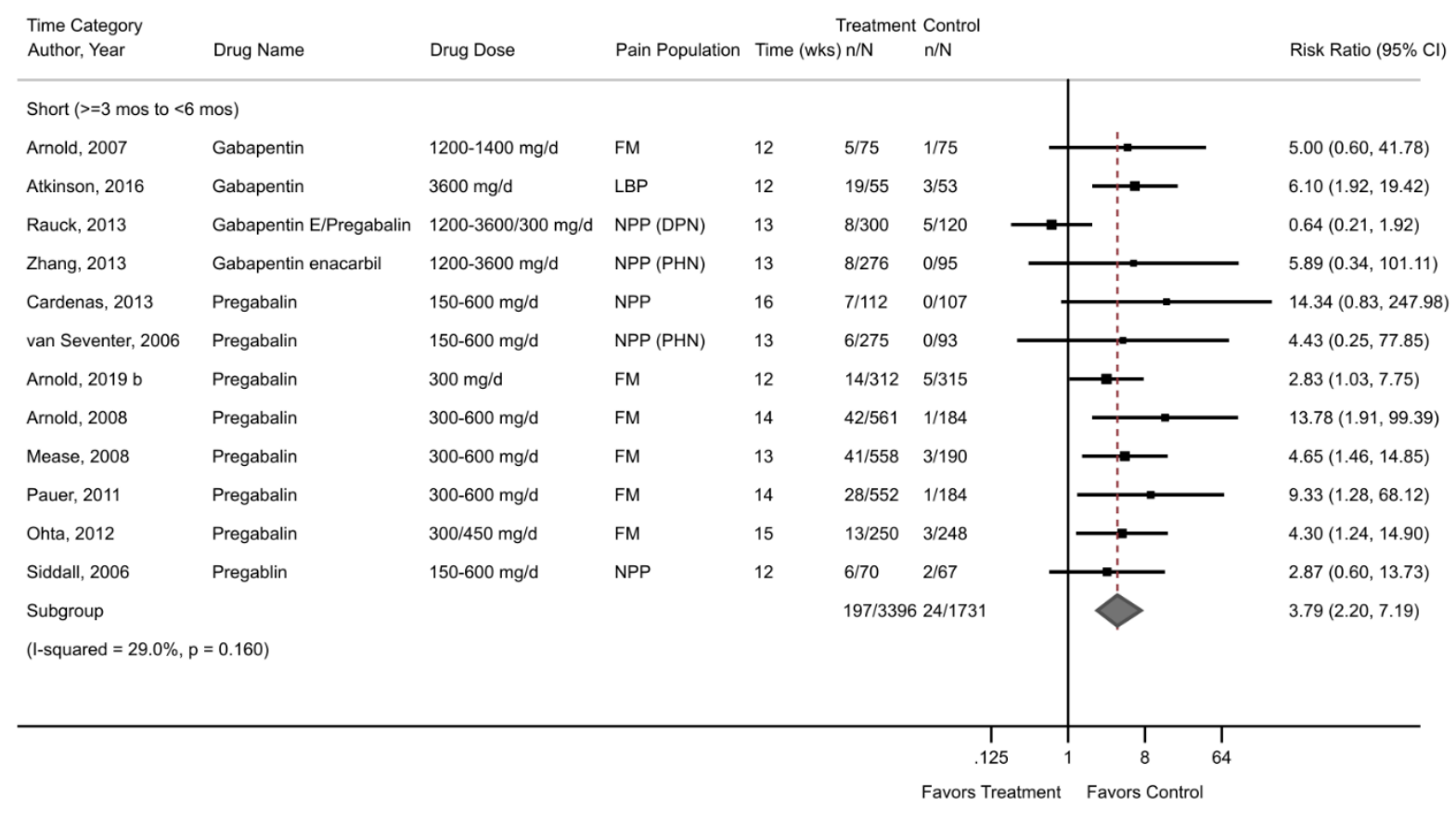


Figure I-76. Anticonvulsants-pregabalin and gabapentin adverse cognitive effects plot

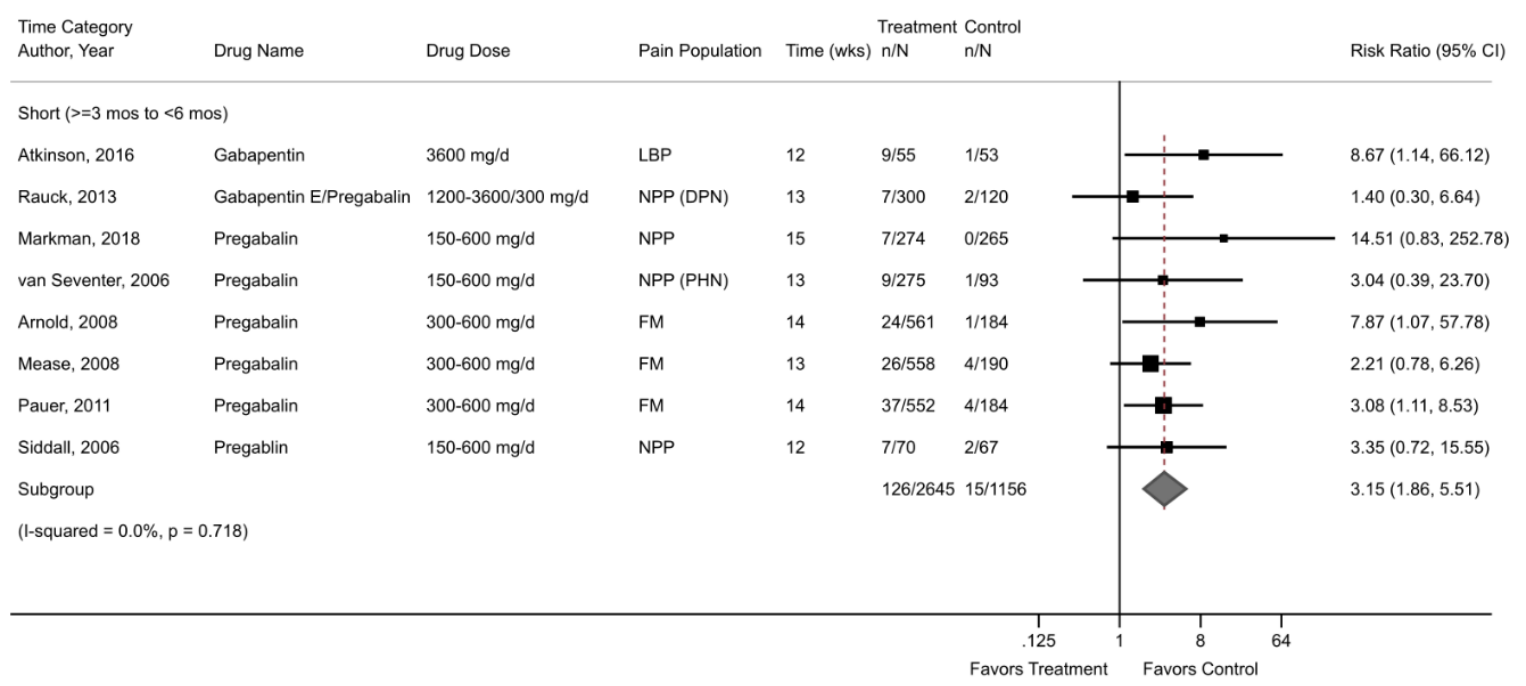

Figure I-77. Anticonvulsants-pregabalin and gabapentin dizziness plot

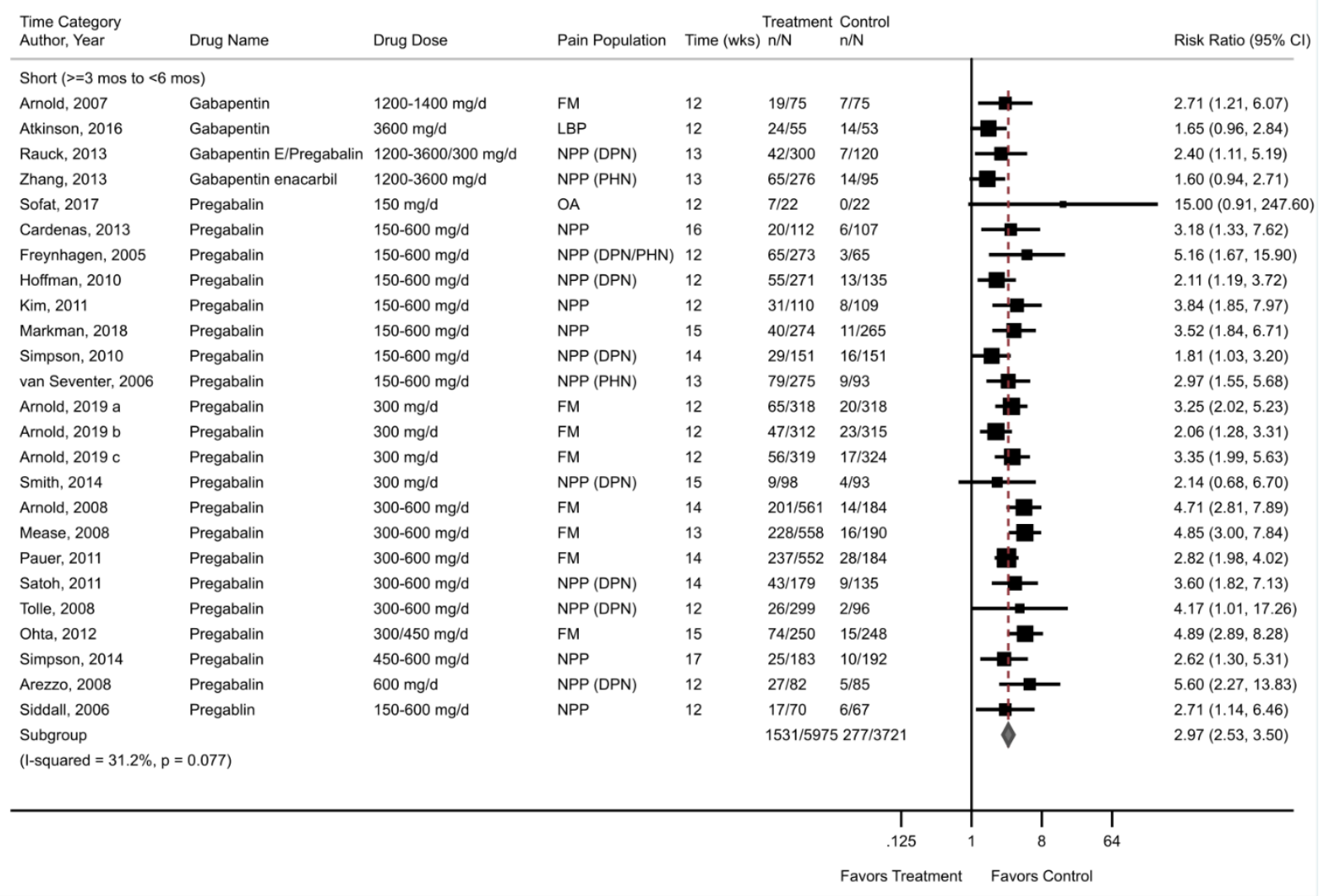


Figure I-78. Anticonvulsants-pregabalin and gabapentin peripheral edema plot

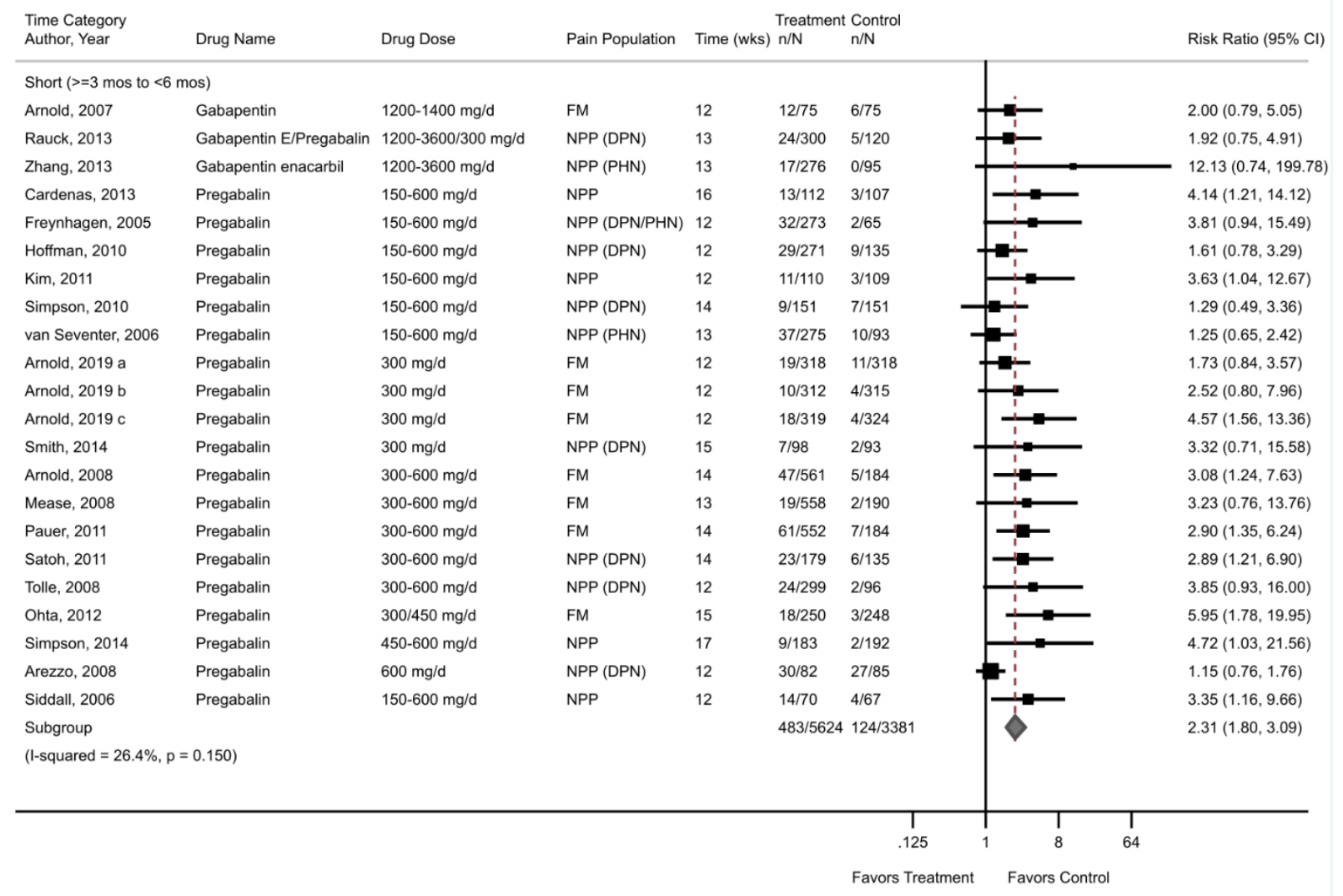


Figure I-79. Anticonvulsants-pregabalin and gabapentin sedation plot

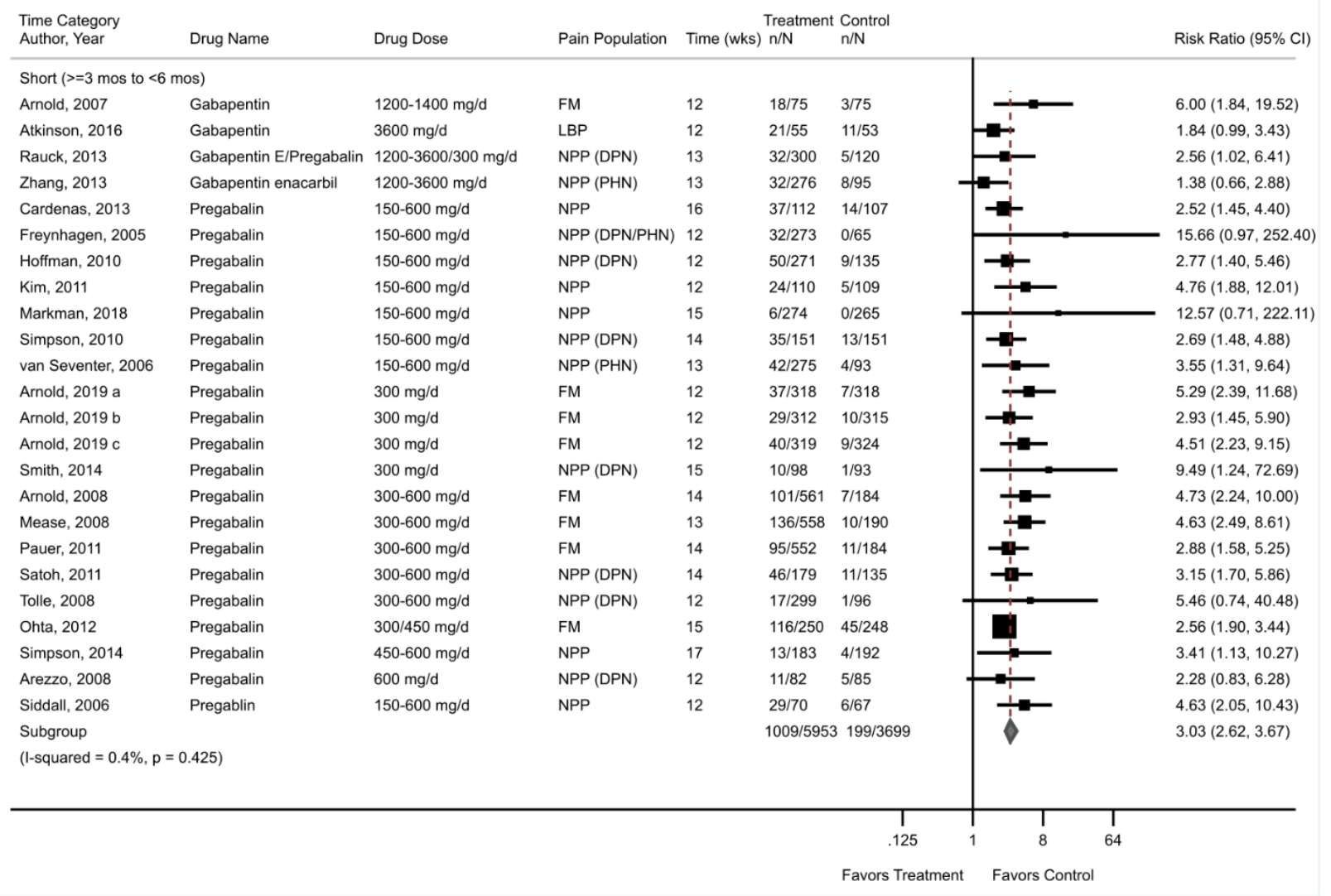


Figure I-80. Anticonvulsants-pregabalin and gabapentin weight gain plot

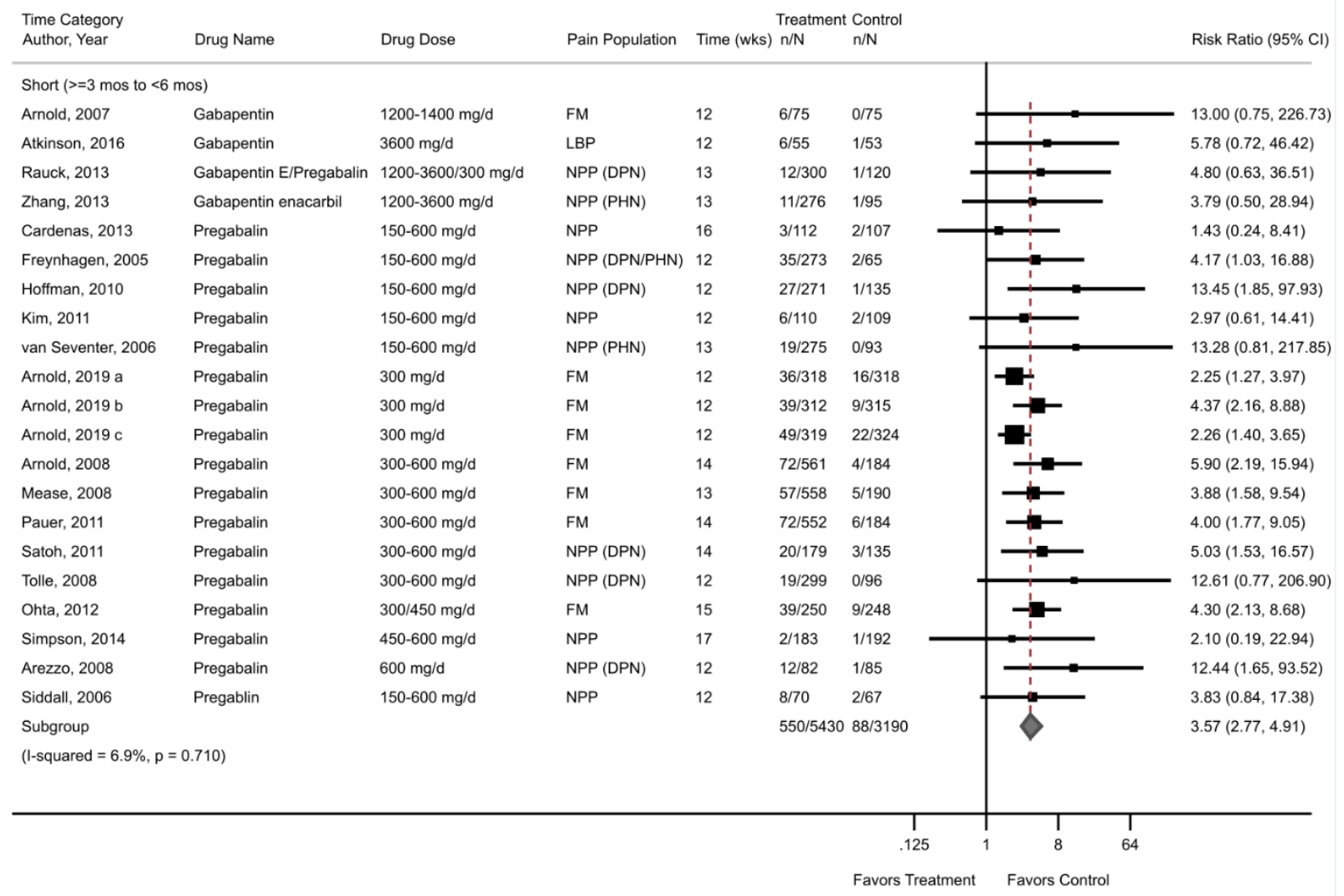

Figure I-81. Anticonvulsants-oxcarbazepine withdrawals due to adverse events plot

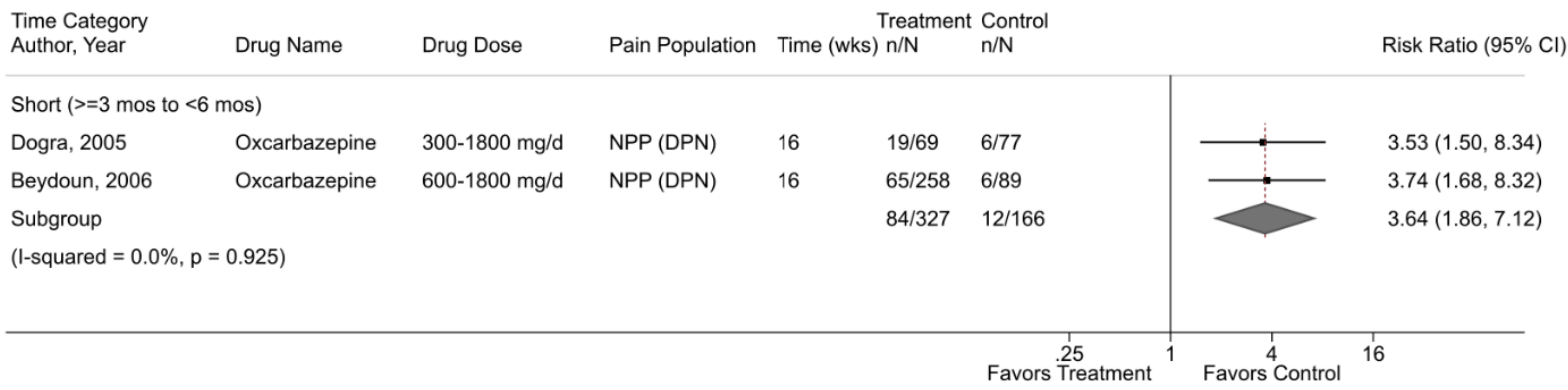

Figure I-82. Anticonvulsants-oxcarbazepine serious adverse events plot

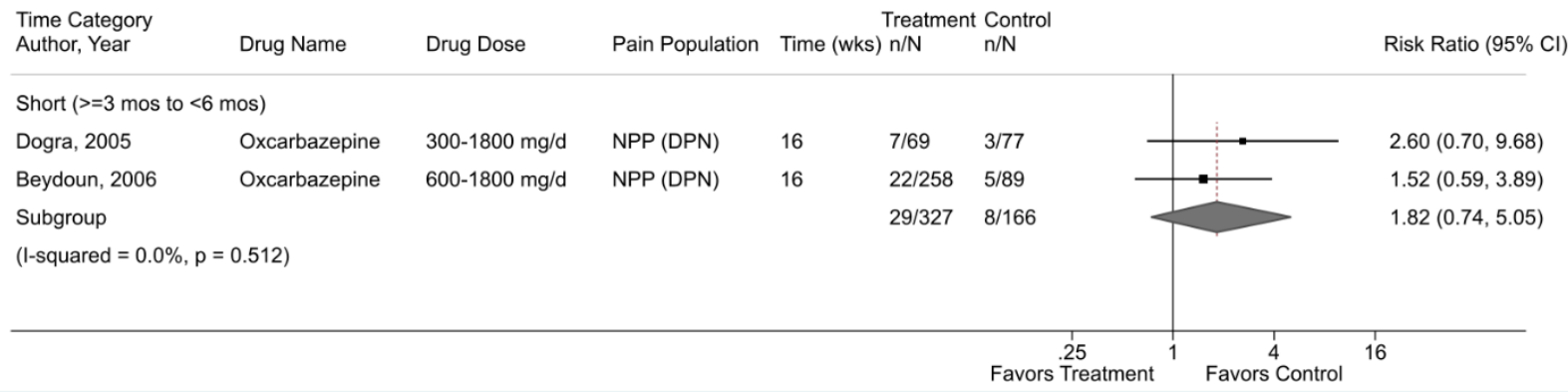


Figure I-83. Anticonvulsants-oxcarbazepine hyponatremia plot

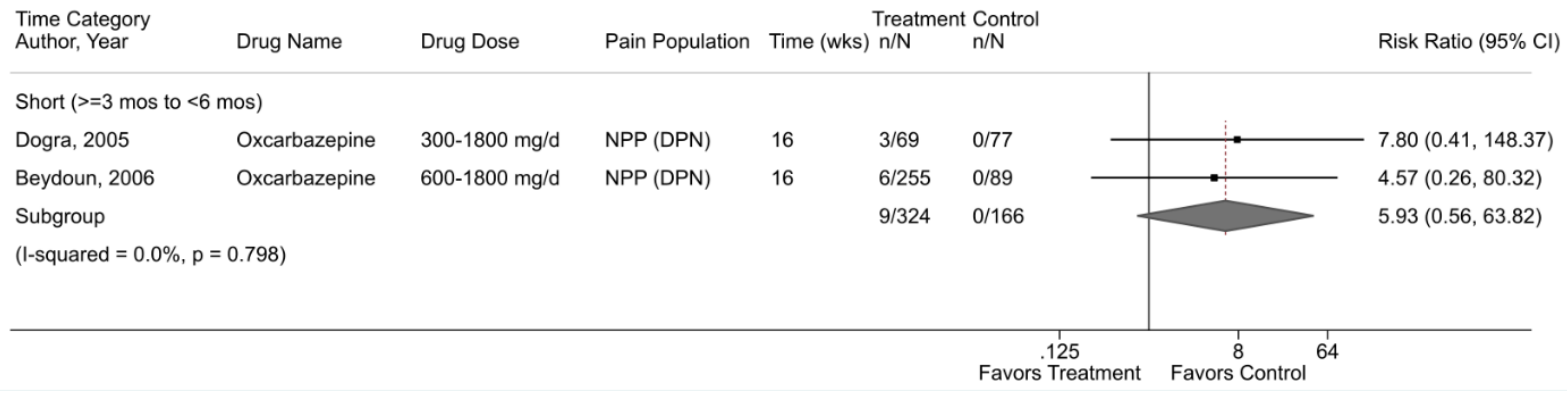

Figure I-84. Anticonvulsants-oxcarbazepine sedation plot

\begin{tabular}{|c|c|c|c|c|c|c|}
\hline \multirow{2}{*}{$\begin{array}{l}\text { Time Category } \\
\text { Author, Year }\end{array}$} & \multirow[b]{2}{*}{ Drug Name } & \multicolumn{5}{|c|}{ Treatment Control } \\
\hline & & Drug Dose & Pain Population & Time (wks) $\mathrm{n} / \mathrm{N}$ & $\mathrm{n} / \mathrm{N}$ & Risk Ratio $(95 \% \mathrm{Cl}$ \\
\hline \multicolumn{7}{|c|}{ Short ( $>=3$ mos to $<6$ mos) } \\
\hline Dogra, 2005 & Oxcarbazepine & $300-1800 \mathrm{mg} / \mathrm{d}$ & NPP (DPN) & $12 / 69$ & $2 / 77$ & $6.70(1.55,28.87)$ \\
\hline Beydoun, 2006 & Oxcarbazepine & $600-1800 \mathrm{mg} / \mathrm{d}$ & NPP (DPN) & $16 / 255$ & $3 / 89$ & $1.86(0.56,6.24)$ \\
\hline Subgroup & & & & $28 / 324$ & $5 / 166$ & $3.13(0.74,16.08)$ \\
\hline \multicolumn{7}{|c|}{$(I-$ squared $=0.0 \%, p=0.184)$} \\
\hline
\end{tabular}


Figure I-85. NSAIDs-oral NSAIDs withdrawals due to adverse events plot

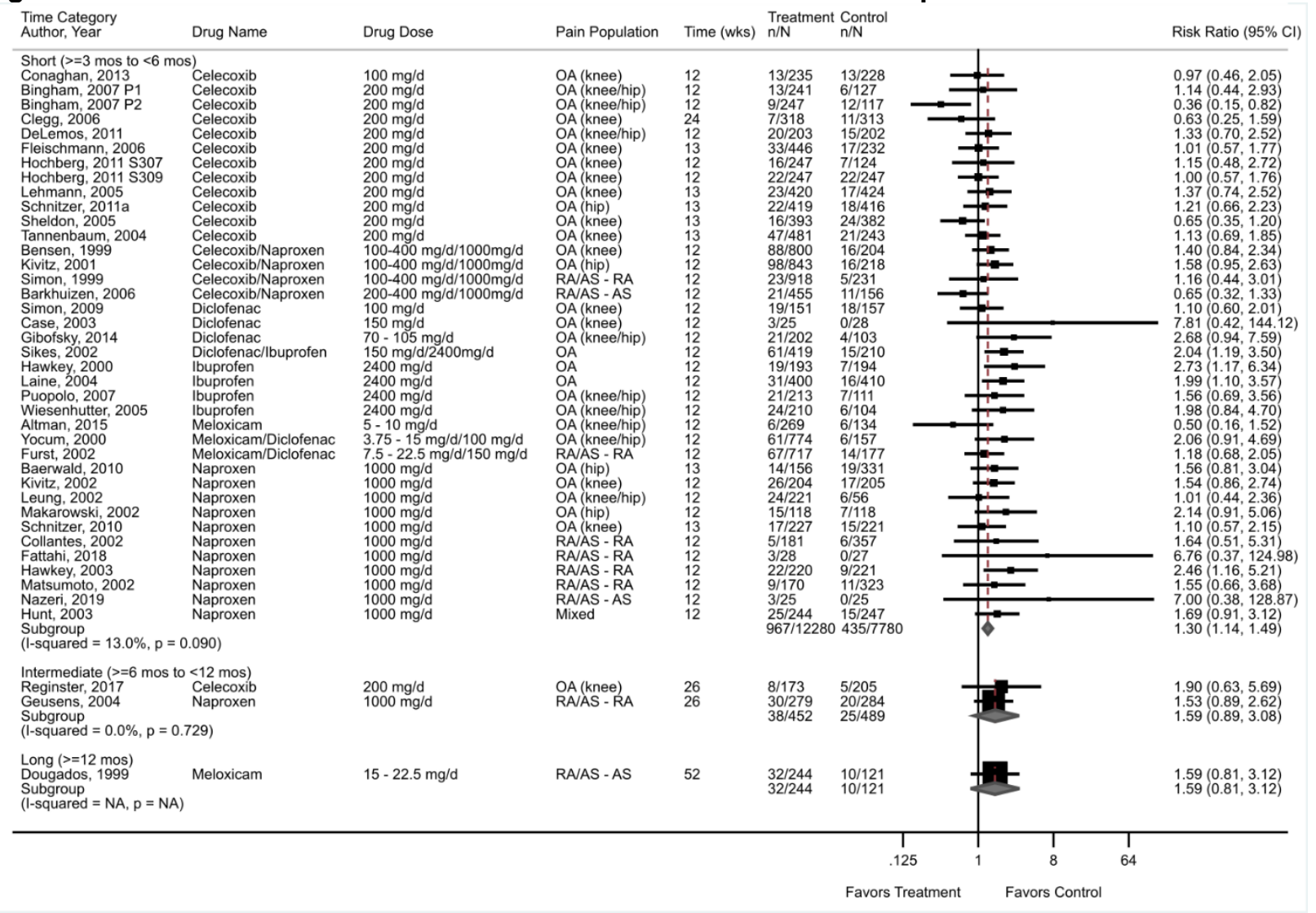


Figure I-86. NSAIDs-oral NSAIDs serious adverse events plot

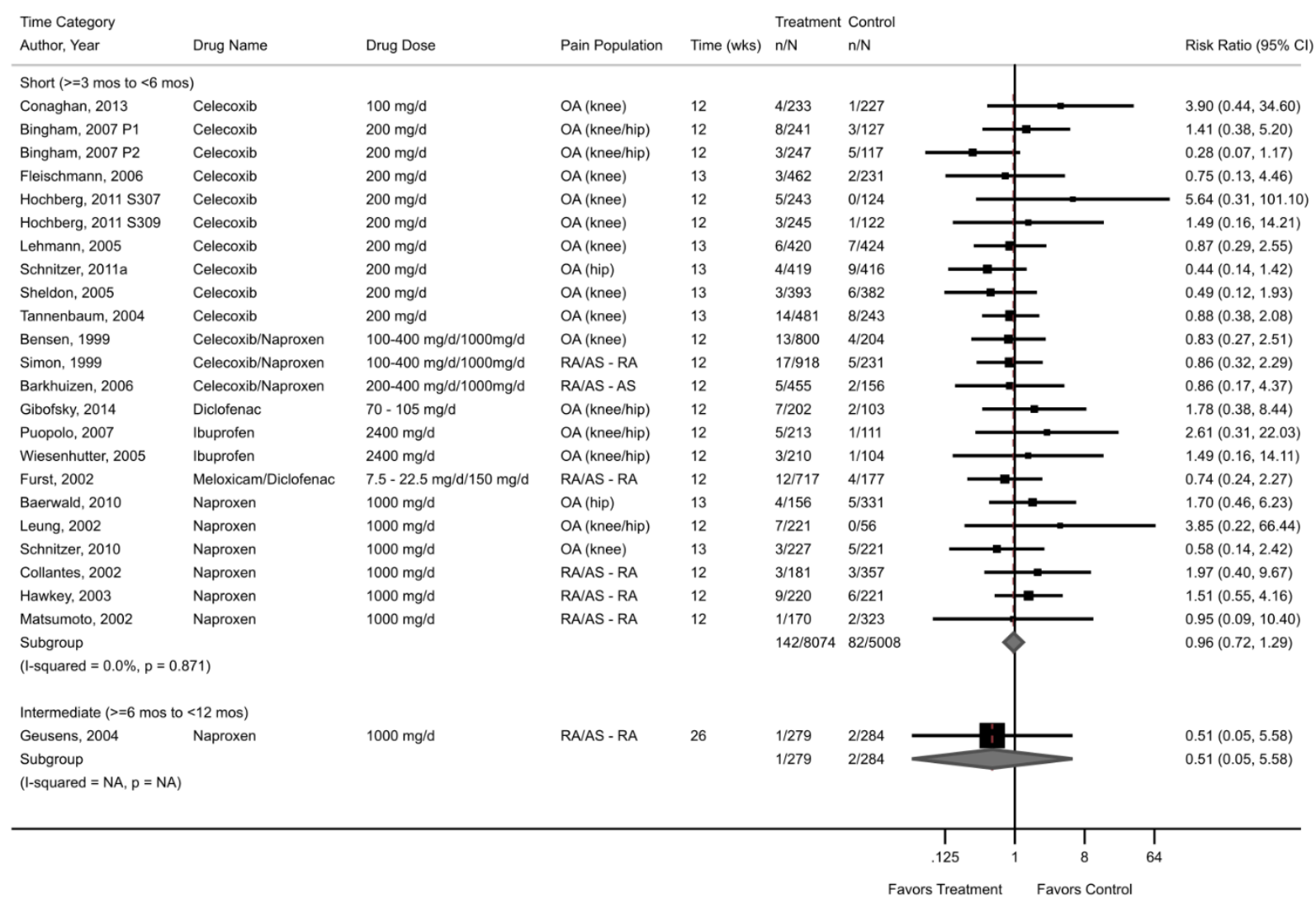

Figure I-87. NSAIDs-oral NSAIDs serious gastrointestinal events plot

\begin{tabular}{|c|c|c|c|c|c|c|c|}
\hline \multirow{2}{*}{$\begin{array}{l}\text { Time Category } \\
\text { Author, Year }\end{array}$} & \multirow[b]{2}{*}{ Drug Name } & \multirow[b]{2}{*}{ Drug Dose } & \multirow[b]{2}{*}{ Pain Population } & \multicolumn{3}{|c|}{ Treatment Control } & \multirow[b]{2}{*}{ Risk Ratio $(95 \% \mathrm{Cl}$} \\
\hline & & & & Time (wks) & $\mathrm{n} / \mathrm{N}$ & $\mathrm{n} / \mathrm{N}$ & \\
\hline \multicolumn{8}{|c|}{ Short ( $>=3$ mos to $<6$ mos) } \\
\hline Clegg, 2006 & Celecoxib & $200 \mathrm{mg} / \mathrm{d}$ & $\mathrm{OA}(\mathrm{knee})$ & 24 & $1 / 318$ & $0 / 31$ & $0.30(0.01,7.24)$ \\
\hline Fleischmann, 2006 & Celecoxib & $200 \mathrm{mg} / \mathrm{d}$ & $\mathrm{OA}(\mathrm{knee})$ & 13 & $96 / 462$ & $46 / 231$ & $1.04(0.76,1.43)$ \\
\hline Schnitzer, 2011a & Celecoxib & $200 \mathrm{mg} / \mathrm{d}$ & OA (hip) & 13 & $1 / 419$ & $2 / 416$ & $0.50(0.05,5.45)$ \\
\hline Simon, 1999 & Celecoxib/Naproxen & $100-400 \mathrm{mg} / \mathrm{d} / 1000 \mathrm{mg} / \mathrm{d}$ & RA/AS - RA & 12 & $59 / 561$ & $4 / 99$ & $2.60(0.97,7.00)$ \\
\hline Barkhuizen, 2006 & Celecoxib/Naproxen & $200-400 \mathrm{mg} / \mathrm{d} / 1000 \mathrm{mg} / \mathrm{d}$ & RA/AS - AS & 12 & $3 / 455$ & $0 / 156$ & $2.41(0.13,46.40)$ \\
\hline Sikes, 2002 & Diclofenac/lbuprofen & $150 \mathrm{mg} / \mathrm{d} / 2400 \mathrm{mg} / \mathrm{d}$ & $\mathrm{OA}$ & 12 & $50 / 371$ & $8 / 178$ & $3.00(1.45,6.19)$ \\
\hline Hawkey, 2000 & Ibuprofen & $2400 \mathrm{mg} / \mathrm{d}$ & $O A$ & 12 & $55 / 187$ & 9/182 & $5.95(3.03,11.68)$ \\
\hline Laine, 2004 & Ibuprofen & $2400 \mathrm{mg} / \mathrm{d}$ & $O A$ & 12 & $62 / 374$ & $21 / 381$ & $3.01(1.87,4.83)$ \\
\hline Furst, 2002 & Meloxicam/Diclofenac & $7.5-22.5 \mathrm{mg} / \mathrm{d} / 150 \mathrm{mg} / \mathrm{d}$ & RA/AS - RA & 12 & $9 / 717$ & $1 / 177$ & $2.22(0.28,17.42)$ \\
\hline Kivitz, 2002 & Naproxen & $1000 \mathrm{mg} / \mathrm{d}$ & $\mathrm{OA}(\mathrm{knee})$ & 12 & $18 / 183$ & $8 / 178$ & $2.19(0.98,4.90)$ \\
\hline Leung, 2002 & Naproxen & $1000 \mathrm{mg} / \mathrm{d}$ & OA (knee/hip) & 12 & $5 / 221$ & $0 / 56$ & $2.82(0.16,50.33)$ \\
\hline Hawkey, 2003 & Naproxen & $1000 \mathrm{mg} / \mathrm{d}$ & RA/AS - RA & 12 & $51 / 209$ & $6 / 209$ & $8.50(3.73,19.37)$ \\
\hline Hunt, 2003 & Naproxen & $1000 \mathrm{mg} / \mathrm{d}$ & Mixed & 12 & $57 / 244$ & $4 / 247$ & $14.43(5.32,39.14)$ \\
\hline Subgroup & & & & & $467 / 4721$ & $109 / 2541$ & $3.04(1.73,5.11)$ \\
\hline \multicolumn{8}{|c|}{$(\mathrm{l}-$ squared $=73.0 \%, p=0.000)$} \\
\hline \multicolumn{8}{|l|}{ Long (>=12 mos) } \\
\hline Dougados, 1999 & Meloxicam & $15-22.5 \mathrm{mg} / \mathrm{d}$ & RA/AS - AS & 52 & 2/244 & $0 / 121$ & $2.49(0.12,51.46)$ \\
\hline Subgroup & & & & & 2/244 & $0 / 121$ & $2.49(0.12,51.46)$ \\
\hline \multicolumn{8}{|c|}{$($ I-squared $=N A, p=N A)$} \\
\hline & & & & & & & \\
\hline
\end{tabular}

Favors Treatment Favors Control 
Figure I-88. NSAIDs-topical diclofenac withdrawals due to adverse events plot

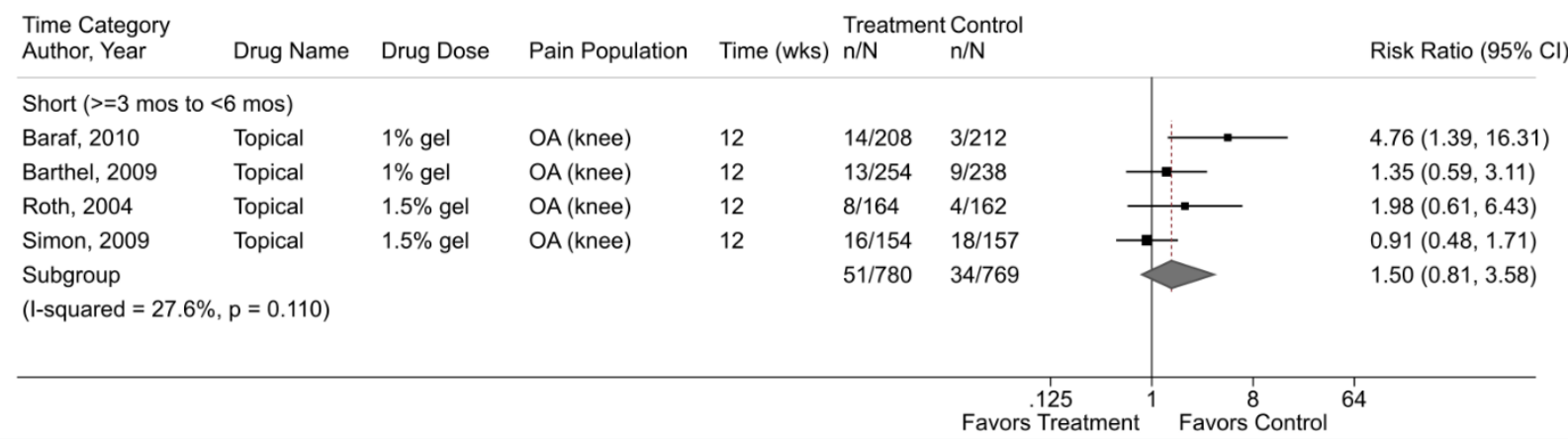

Figure I-89. NSAIDs-topical diclofenac serious adverse events plot

\begin{tabular}{|c|c|c|c|c|c|c|c|}
\hline $\begin{array}{l}\text { Time Category } \\
\text { Author, Year }\end{array}$ & Drug Name & Drug Dose & Pain Population & Time (wks) & $\begin{array}{l}\text { Treatme } \\
\mathrm{n} / \mathrm{N}\end{array}$ & $\begin{array}{l}\text { Control } \\
\mathrm{n} / \mathrm{N}\end{array}$ & Risk Ratio $(95 \% \mathrm{Cl})$ \\
\hline \multicolumn{8}{|c|}{ Short ( $>=3 \mathrm{mos}$ to $<6 \mathrm{mos}$ ) } \\
\hline Baraf, 2010 & Topical & $1 \% \mathrm{gel}$ & $\mathrm{OA}(\mathrm{knee})$ & 12 & $6 / 208$ & $0 / 212$ & $13.25(0.75,233.69)$ \\
\hline Barthel, 2009 & Topical & $1 \%$ gel & $\mathrm{OA}(\mathrm{knee})$ & 12 & $13 / 254$ & $14 / 238$ & $0.87(0.42,1.81)$ \\
\hline Subgroup & & & & & $19 / 462$ & $14 / 450$ & $1.03(0.29,27.01)$ \\
\hline \multicolumn{8}{|c|}{$(\mathrm{I}$-squared $=0.0 \%, p=0.057)$} \\
\hline
\end{tabular}

Figure I-90. NSAIDs-topical diclofenac application site pruritus plot

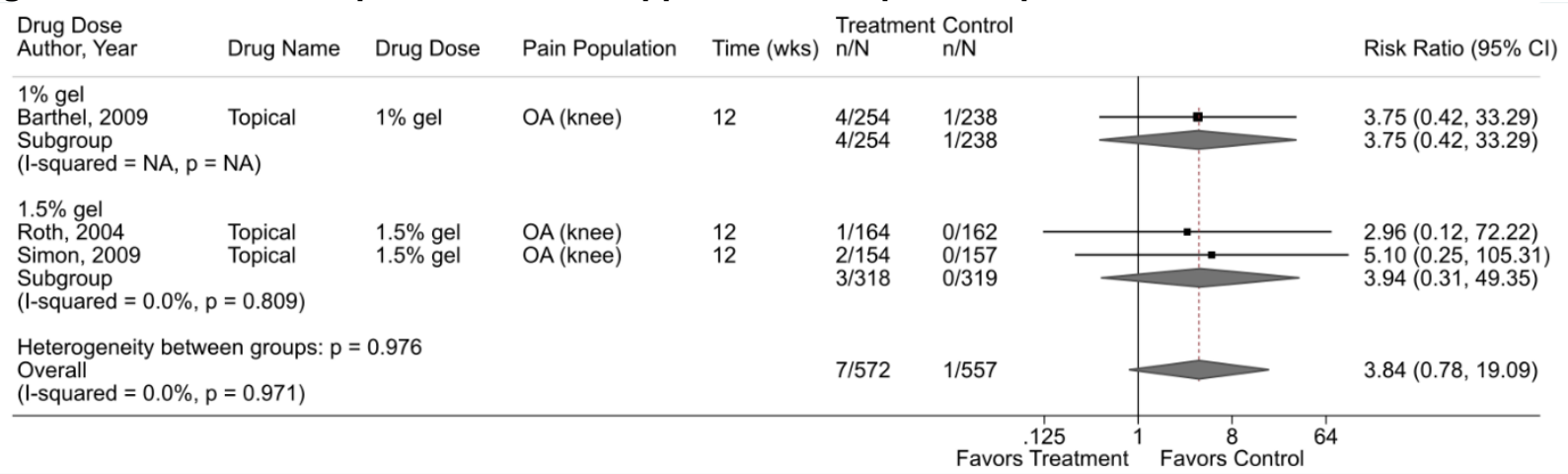

Figure I-91. Acetaminophen-withdrawals due to adverse events plot

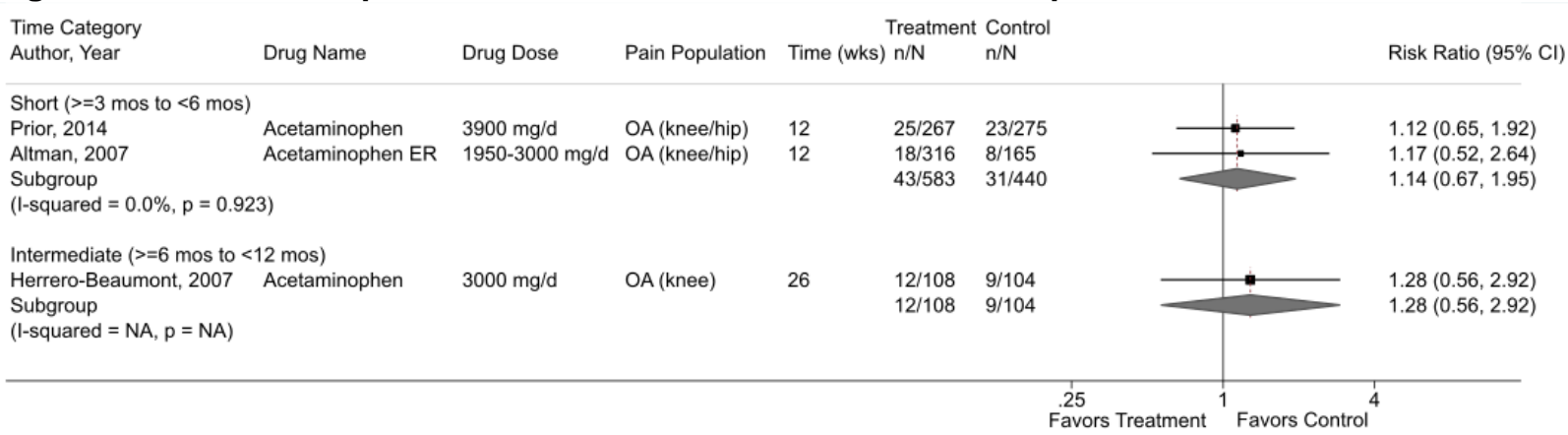


Figure I-92. Acetaminophen-serious adverse events plot

\begin{tabular}{|c|c|c|c|c|c|c|c|c|}
\hline $\begin{array}{l}\text { Time Category } \\
\text { Author, Year }\end{array}$ & Drug Name & Drug Dose & Pain Population & Time (wks) & Treatment Control & $\begin{array}{l}\text { Control } \\
\mathrm{n} / \mathrm{N}\end{array}$ & & Risk Ratio $(95 \% \mathrm{Cl})$ \\
\hline \multicolumn{9}{|c|}{ Short $(>=3$ mos to $<6$ mos) } \\
\hline Prior, 2014 & Acetaminophen & $3900 \mathrm{mg} / \mathrm{d}$ & OA (knee/hip) & 12 & $8 / 267$ & $2 / 275$ & & $4.12(0.88,19.22)$ \\
\hline Altman, 2007 & Acetaminophen ER & $1950-3000 \mathrm{mg} / \mathrm{d}$ & $\mathrm{OA}$ (knee/hip) & 12 & $6 / 318$ & 2/165 & $\rightarrow$ & $1.56(0.32,7.63)$ \\
\hline \multicolumn{9}{|c|}{$(1-$ squared $=0.0 \%, p=0.388)$} \\
\hline \multicolumn{9}{|c|}{ Intermediate ( $>=6$ mos to $<12 \mathrm{mos}$ ) } \\
\hline Herrero-Beaumont, 2007 & Acetaminophen & $3000 \mathrm{mg} / \mathrm{d}$ & $\mathrm{OA}(\mathrm{knee})$ & 26 & $5 / 108$ & $5 / 104$ & - & $0.96(0.29,3.23)$ \\
\hline \multirow{2}{*}{\multicolumn{9}{|c|}{$(I-$ squared $=N A, p=N A)$}} \\
\hline & & & & & & & & \\
\hline & & & & & & $\begin{array}{l}.125 \\
\text { Favors Treatment }\end{array}$ & $\begin{array}{c}8 \\
\text { Favors Control }\end{array}$ & 64 \\
\hline
\end{tabular}

Figure I-93. Capsaicin patch-withdrawals due to adverse events plot

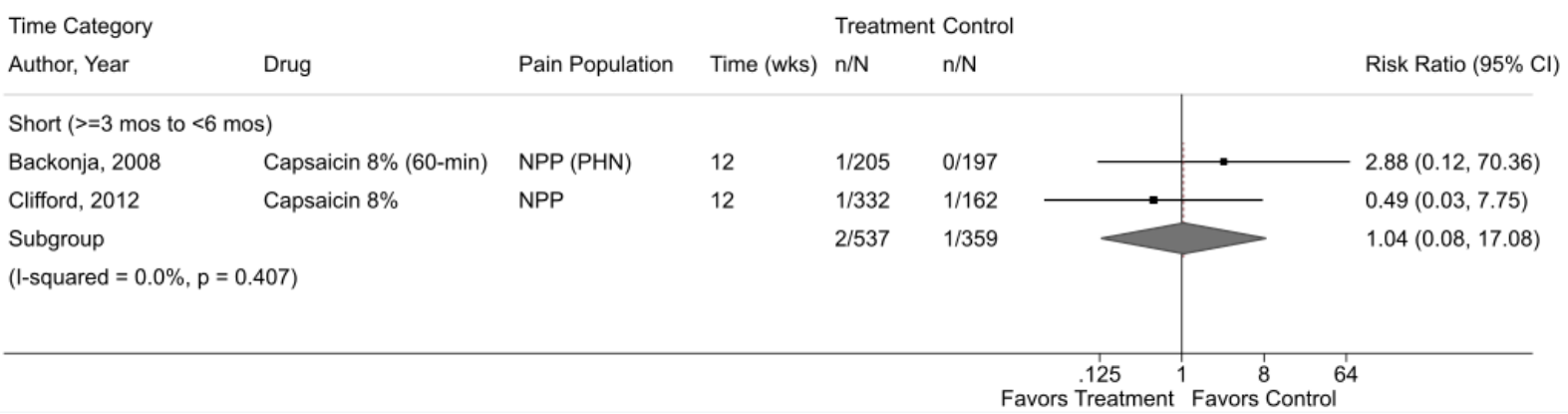

Figure I-94. Capsaicin patch-application site erythema plot

\begin{tabular}{|c|c|c|c|c|c|c|c|}
\hline \multirow{2}{*}{$\begin{array}{l}\text { Time Category } \\
\text { Author, Year }\end{array}$} & \multirow[b]{2}{*}{ Drug } & \multirow[b]{2}{*}{ Pain Population } & \multirow[b]{2}{*}{ Time (wks) } & \multicolumn{3}{|c|}{ Treatment Control } & \multirow[b]{2}{*}{ Risk Ratio $(95 \% \mathrm{Cl}$} \\
\hline & & & & $\mathrm{n} / \mathrm{N}$ & $\mathrm{n} / \mathrm{N}$ & & \\
\hline \multicolumn{8}{|c|}{ Short ( $>=3 \mathrm{mos}$ to $<6 \mathrm{mos}$ ) } \\
\hline Backonja, 2008 & Capsaicin 8\% (60-min) & NPP (PHN) & 12 & $193 / 205$ & $128 / 197$ & + & $1.45(1.30,1.61)$ \\
\hline Clifford, 2012 & Capsaicin $8 \%$ & NPP & 12 & $176 / 332$ & $58 / 162$ & \# & $1.48(1.18,1.86)$ \\
\hline Webster, 2010 & Capsaicin $8 \%(60-\min )$ & NPP (PHN) & 12 & $4 / 102$ & $0 / 53$ & : & $4.72(0.26,86.02)$ \\
\hline Subgroup & & & & $373 / 639$ & $186 / 412$ & 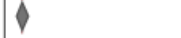 & $1.46(1.29,1.66)$ \\
\hline \multicolumn{8}{|c|}{$(I-$ squared $=0.0 \%, p=0.706)$} \\
\hline
\end{tabular}

Figure I-95. Capsaicin patch-application site pain plot

\begin{tabular}{|c|c|c|c|c|c|c|c|}
\hline \multicolumn{4}{|l|}{ Time Category } & \multicolumn{4}{|c|}{ Treatment Control } \\
\hline Author, Year & Drug & Pain Population & Time (wks) & $\mathrm{n} / \mathrm{N}$ & $\mathrm{n} / \mathrm{N}$ & & Risk Ratio $(95 \%$ C \\
\hline \multicolumn{8}{|c|}{ Short ( $>=3 \mathrm{mos}$ to $<6 \mathrm{mos}$ ) } \\
\hline Backonja, 2008 & Capsaicin 8\% (60-min) & NPP (PHN) & 12 & $114 / 205$ & $43 / 197$ & & $2.55(1.90,3.41)$ \\
\hline Clifford, 2012 & Capsaicin $8 \%$ & NPP & 12 & $274 / 332$ & $62 / 162$ & & $2.16(1.76,2.64)$ \\
\hline Webster, 2010 & Capsaicin 8\% (60-min) & NPP (PHN) & 12 & $4 / 102$ & $2 / 53$ & & $1.04(0.20,5.49)$ \\
\hline Subgroup & & & & $392 / 639$ & $107 / 412$ & $\gamma$ & $2.26(1.81,2.82)$ \\
\hline \multicolumn{8}{|c|}{$(\mathrm{l}$-squared $=0.0 \%, p=0.428)$} \\
\hline & & & & & $\begin{array}{cc}.125 & 1 \\
\text { Favors Treatment }\end{array}$ & $\stackrel{8}{8}$ & 64 \\
\hline
\end{tabular}


Figure I-96. Capsaicin patch-application site pruritus plot

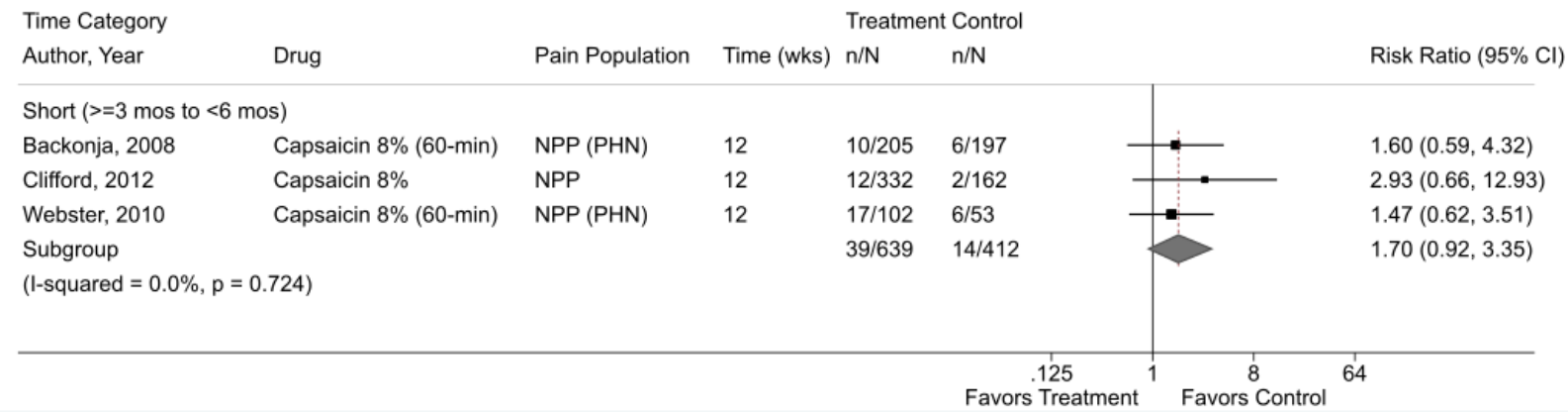

\section{Publication Bias}

Figure I-97. Osteoarthritis - Egger's test for publication bias

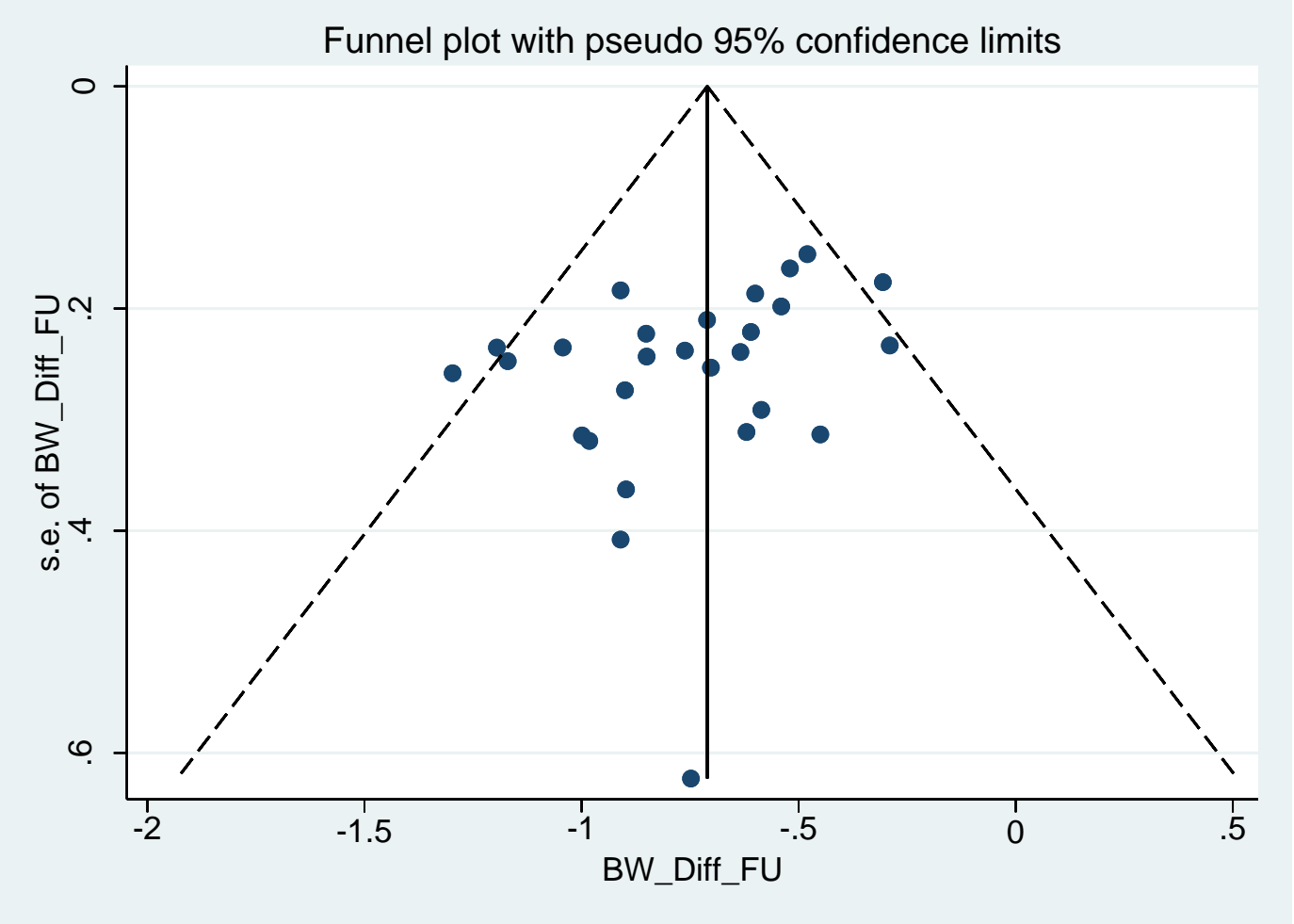




\section{Appendix J. Appendix References}

1. Methods Guide for Effectiveness and Comparative Effectiveness Reviews. AHRQ Publication No. 10(14)-EHC063-EF. Rockville, MD: Agency for Healthcare Research Quality; Jan 2014. https://effectivehealthcare.ahrq.gov/topics/ce r-methods-guide/overview Accessed February 1, 2019. PMID: 21433403.

2. Headache Classification Committee of the International Headache Society (IHS). The International Classification of Headache Disorders, 3rd edition. Cephalalgia. 2013 Jan;38(1):1-211. doi: 10.1177/0333102417738202. PMID: 29368949.

3. Skelly AC, Chou R, Dettori JR, et al. AHRQ Comparative Effectiveness Reviews. Noninvasive Nonpharmacological Treatment for Chronic Pain: A Systematic Review. Rockville (MD): Agency for Healthcare Research and Quality (US); 2018.

4. Furlan AD, Malmivaara A, Chou R, et al. 2015 Updated Method Guideline for Systematic Reviews in the Cochrane Back and Neck Group. Spine (Phila Pa 1976). 2015 Nov;40(21):1660-73. doi: 10.1097/BRS.0000000000001061. PMID: 26208232.

5. Viswanathan M, Patnode CD, Berkman ND, et al. Assessing the risk of bias in systematic reviews of health care interventions. Methods Guide for Effectiveness and Comparative Effectiveness Reviews. Rockville, MD: Agency for Healthcare Research and Quality; 2017.

6. Fu R, Gartlehner G, Grant M, et al. AHRQ Methods for Effective Health Care Conducting Quantitative Synthesis When Comparing Medical Interventions: AHRQ and the Effective Health Care Program. Methods Guide for Effectiveness and Comparative Effectiveness Reviews. Rockville (MD): Agency for Healthcare Research and Quality (US); 2008.
7. Hardy RJ, Thompson SG. A likelihood approach to meta-analysis with random effects. Stat Med. 1996 Mar 30;15(6):61929. doi: 10.1002/(sici)10970258(19960330)15:6<619::Aidsim188>3.0.Co;2-a. PMID: 8731004.

8. DerSimonian R, Laird N. Meta-analysis in clinical trials. Control Clin Trials. 1986 Sep;7(3):177-88. PMID: 3802833.

9. Higgins J, Green S. Cochrane Handbook for Systematic Reviews of Interventions.

Version 5.1. 0. The Cochrane Collaboration; March 2011. http://handbook-5-1.cochrane.org/ Accessed February 1, 2019.

10. Arezzo JC, Rosenstock J, Lamoreaux L, et al. Efficacy and safety of pregabalin 600 $\mathrm{mg} / \mathrm{d}$ for treating painful diabetic peripheral neuropathy: a double-blind placebocontrolled trial. BMC Neurol. 2008 Sep 16;8(33)doi: 10.1186/1471-2377-8-33. PMID: 18796160.

11. Cardenas DD, Nieshoff EC, Suda K, et al. A randomized trial of pregabalin in patients with neuropathic pain due to spinal cord injury. Neurology. 2013 Feb 5;80(6):533-9. doi: 10.1212/WNL.0b013e318281546b. PMID: 23345639.

12. Freynhagen R, Strojek K, Griesing T, et al. Efficacy of pregabalin in neuropathic pain evaluated in a 12-week, randomised, doubleblind, multicentre, placebo-controlled trial of flexible- and fixed-dose regimens. Pain. 2005 Jun;115(3):254-63. PMID: 15911152.

13. Hoffman DL, Sadosky A, Dukes EM, et al. How do changes in pain severity levels correspond to changes in health status and function in patients with painful diabetic peripheral neuropathy? Pain. 2010;149(2):194-201. doi: 10.1016/j.pain.2009.09.017. PMID: 20303665.

14. Kim JS, Bashford G, Murphy TK, et al. Safety and efficacy of pregabalin in patients with central post-stroke pain. Pain. 2011 May;152(5):1018-23. doi: 10.1016/j.pain.2010.12.023. PMID: 21316855. 
15. Markman J, Resnick M, Greenberg S, et al. Efficacy of pregabalin in post-traumatic peripheral neuropathic pain: a randomized, double-blind, placebo-controlled phase 3 trial. J Neurol. 2018 Dec;265(12):2815-24. doi: 10.1007/s00415-018-9063-9. PMID: 30242745.

16. Rauck R, Makumi CW, Schwartz S, et al. A randomized, controlled trial of gabapentin enacarbil in subjects with neuropathic pain associated with diabetic peripheral neuropathy. Pain pract. 2013 Jul;13(6):48596. doi: 10.1111/papr.12014. PMID: 23186035.

17. Satoh J, Yagihashi S, Baba M, et al. Efficacy and safety of pregabalin for treating neuropathic pain associated with diabetic peripheral neuropathy: a 14 week, randomized, double-blind, placebocontrolled trial. Diabet Med. 2011 Jan;28(1):109-16. doi: 10.1111/j.14645491.2010.03152.x. PMID: 21166852.

18. Siddall PJ, Cousins MJ, Otte A, et al. Pregabalin in central neuropathic pain associated with spinal cord injury: a placebo-controlled trial. Neurology. 2006 Nov 28;67(10):1792-800. doi: 10.1212/01.wnl.0000244422.45278.ff. PMID: 17130411.

19. Simpson DM, Rice AS, Emir B, et al. A randomized, double-blind, placebocontrolled trial and open-label extension study to evaluate the efficacy and safety of pregabalin in the treatment of neuropathic pain associated with human immunodeficiency virus neuropathy. Pain. 2014 Oct;155(10):1943-54. doi: 10.1016/j.pain.2014.05.027. PMID: 24907403.

20. Simpson DM, Schifitto G, Clifford DB, et al. Pregabalin for painful HIV neuropathy: a randomized, double-blind, placebocontrolled trial. Neurology. 2010 Feb 2;74(5):413-20. doi: 10.1212/WNL.0b013e3181ccc6ef. PMID: 20124207.
21. Smith T, DiBernardo A, Shi Y, et al. Efficacy and safety of carisbamate in patients with diabetic neuropathy or postherpetic neuralgia: results from 3 randomized, double-blind placebocontrolled trials. Pain pract. 2014 Apr;14(4):332-42. doi: 10.1111/papr.12080. PMID: 23692321.

22. Tolle T, Freynhagen R, Versavel M, et al. Pregabalin for relief of neuropathic pain associated with diabetic neuropathy: a randomized, double-blind study. Eur J Pain. 2008 Feb;12(2):203-13. doi:

10.1016/j.ejpain.2007.05.003. PMID: 17631400 .

23. van Seventer R, Feister HA, Young JP, Jr., et al. Efficacy and tolerability of twice-daily pregabalin for treating pain and related sleep interference in postherpetic neuralgia: a 13week, randomized trial. Curr Med Res Opin. 2006 Feb;22(2):375-84. doi: 10.1185/030079906X80404. PMID: 16466610.

24. Zhang L, Rainka M, Freeman R, et al. A randomized, double-blind, placebocontrolled trial to assess the efficacy and safety of gabapentin enacarbil in subjects with neuropathic pain associated with postherpetic neuralgia (PXN110748). J Pain. 2013 Jun;14(6):590-603. doi: 10.1016/j.jpain.2013.01.768. PMID: 23602345.

25. Dogra S, Beydoun S, Mazzola J, et al. Oxcarbazepine in painful diabetic neuropathy: a randomized, placebocontrolled study. Eur J Pain. 2005 Oct;9(5):543-54. doi: 10.1016/j.ejpain.2004.11.006. PMID: 16139183.

26. Beydoun A, Shaibani A, Hopwood M, et al. Oxcarbazepine in painful diabetic neuropathy: results of a dose-ranging study. Acta Neurol Scand. 2006 Jun;113(6):395404. doi: 10.1111/j.16000404.2006.00631.x. PMID: 16674606.

27. Goldstein DJ, Lu Y, Detke MJ, et al. Duloxetine vs. placebo in patients with painful diabetic neuropathy. Pain. 2005 Jul;116(1-2):109-18. doi: 10.1016/j.pain.2005.03.029. PMID: 15927394. 
28. Yasuda H, Hotta N, Nakao K, et al. Superiority of duloxetine to placebo in improving diabetic neuropathic pain: results of a randomized controlled trial in Japan. J Diabetes Investig. 2011 Apr 7;2(2):132-9. doi: 10.1111/j.2040-1124.2010.00073.x. PMID: 24843472.

29. Gao Y, Ning G, Jia WP, et al. Duloxetine versus placebo in the treatment of patients with diabetic neuropathic pain in China. Chin Med J. 2010 Nov;123(22):3184-92. PMID: 21163113.

30. Raskin J, Pritchett YL, Wang F, et al. A double-blind, randomized multicenter trial comparing duloxetine with placebo in the management of diabetic peripheral neuropathic pain. Pain Med. 2005 SepOct;6(5):346-56. doi: 10.1111/j.15264637.2005.00061.x. PMID: 16266355.

31. Wernicke JF, Pritchett YL, D'Souza DN, et al. A randomized controlled trial of duloxetine in diabetic peripheral neuropathic pain. Neurology. 2006a Oct 24;67(8):141120. doi: 10.1212/01.wnl.0000240225.04000.1a. PMID: 17060567.

32. Gao Y, Guo X, Han P, et al. Treatment of patients with diabetic peripheral neuropathic pain in China: a double-blind randomised trial of duloxetine vs. placebo. Int J Clin Pract. 2015 Sep;69(9):957-66. doi: 10.1111/ijcp.12641. PMID: 25939897.

33. Schimrigk S, Marziniak M, Neubauer C, et al. Dronabinol is a safe long-term treatment option for neuropathic pain patients. Eur Neurol. 2017;78(5-6):320-9. doi: 10.1159/000481089. PMID: 29073592.

34. Serpell M, Ratcliffe S, Hovorka J, et al. A double-blind, randomized, placebocontrolled, parallel group study of THC/CBD spray in peripheral neuropathic pain treatment. Eur J Pain. 2014 Aug;18(7):999-1012. doi: 10.1002/j.15322149.2013.00445.x. PMID: 24420962.
35. Backonja M, Wallace MS, Blonsky ER, et al. NGX-4010, a high-concentration capsaicin patch, for the treatment of postherpetic neuralgia: a randomised, double-blind study.[Erratum appears in Lancet Neurol. 2009 Jan;8(1):31]. Lancet Neurol. 2008 Dec;7(12):1106-12. doi: 10.1016/S1474-4422(08)70228-X. PMID: 18977178.

36. Clifford DB, Simpson DM, Brown S, et al. A randomized, double-blind, controlled study of NGX-4010, a capsaicin 8\% dermal patch, for the treatment of painful HIVassociated distal sensory polyneuropathy. J Acquir Immune Defic Syndr. 2012 Feb 1;59(2):126-33. doi: 10.1097/QAI.0b013e31823e31f7. PMID: 22067661.

37. Webster LR, Tark M, Rauck R, et al. Effect of duration of postherpetic neuralgia on efficacy analyses in a multicenter, randomized, controlled study of NGX-4010, an $8 \%$ capsaicin patch evaluated for the treatment of postherpetic neuralgia. BMC Neurol. 2010 Oct 11;10:92. doi: 10.1186/1471-2377-10-92. PMID: 20937130.

38. Schifitto G, Yiannoutsos CT, Simpson DM, et al. A placebo-controlled study of memantine for the treatment of human immunodeficiency virus-associated sensory neuropathy. J Neurovirol. 2006

Aug;12(4):328-31. doi: 10.1080/13550280600873835. PMID: 16966223.

39. Devi P, Madhu K, Ganapathy B, et al. Evaluation of efficacy and safety of gabapentin, duloxetine, and pregabalin in patients with painful diabetic peripheral neuropathy. Indian J Pharmacol. 2012 Jan;44(1):51-6. doi: 10.4103/02537613.91867. PMID: 22345870.

40. Kelle B, Yavuz F, Yasar E, et al. The efficacy of gabapentin and pregabalin in the treatment of neuropathic pain due to peripheral nerve injury. J Musculoskelet Pain. 2012;20(4):300-5. doi: 10.3109/10582452.2012.733801. 
41. Arnold LM, Lu Y, Crofford LJ, et al. A double-blind, multicenter trial comparing duloxetine with placebo in the treatment of fibromyalgia patients with or without major depressive disorder. Arthritis Rheum. 2004 Sep;50(9):2974-84. doi: 10.1002/art.20485. PMID: 15457467.

42. Arnold LM, Rosen A, Pritchett YL, et al. A randomized, double-blind, placebocontrolled trial of duloxetine in the treatment of women with fibromyalgia with or without major depressive disorder. Pain. 2005 Dec 15;119(1-3):5-15. doi: 10.1016/j.pain.2005.06.031. PMID: 16298061.

43. Clauw DJ, Mease P, Palmer RH, et al. Milnacipran for the treatment of fibromyalgia in adults: a 15-week, multicenter, randomized, double-blind, placebo-controlled, multiple-dose clinical trial. Clin Ther. 2008 Nov;30(11):19882004. doi: 10.1016/j.clinthera.2008.11.009. PMID: 19108787.

44. Vitton O, Gendreau M, Gendreau J, et al. A double-blind placebo-controlled trial of milnacipran in the treatment of fibromyalgia. Hum Psychopharmacol. 2004 Oct;19 (Suppl 1):S27-35. doi: 10.1002/hup.622. PMID: 15378666.

45. Russell IJ, Mease PJ, Smith TR, et al. Efficacy and safety of duloxetine for treatment of fibromyalgia in patients with or without major depressive disorder: results from a 6-month, randomized, double-blind, placebo-controlled, fixed-dose trial. Pain. 2008 Jun;136(3):432-44. doi: 10.1016/j.pain.2008.02.024. PMID: 18395345.

46. Mease PJ, Clauw DJ, Gendreau RM, et al. The efficacy and safety of milnacipran for treatment of fibromyalgia. a randomized, double-blind, placebo-controlled trial.[Erratum appears in J Rheumatol. 2009 Mar;36(3):661]. J Rheumatol. 2009

Feb;36(2):398-409. doi: 10.3899/jrheum.080734. PMID: 19132781.

47. Arnold LM, Clauw D, Wang F, et al. Flexible dosed duloxetine in the treatment of fibromyalgia: a randomized, double-blind, placebo-controlled trial. J Rheumatol. 2010a Dec;37(12):2578-86. doi: 10.3899/jrheum.100365. PMID: 20843911.
48. Arnold L, Gendreau R, Palmer R, et al. Efficacy and safety of milnacipran 100 $\mathrm{mg} /$ day in patients with fibromyalgia: results of a randomized, double-blind, placebocontrolled trial. Arthritis Rheum. 2010b Sep;62(9):2745-56. doi: 10.1002/art.27559. PMID: 20496365.

49. Branco JC, Zachrisson O, Perrot S, et al. A European multicenter randomized doubleblind placebo-controlled monotherapy clinical trial of milnacipran in treatment of fibromyalgia. J Rheumatol. 2010 Apr;37(4):851-9. doi: 10.3899/jrheum.090884. PMID: 20156949.

50. Arnold LM, Zhang S, Pangallo BA. Efficacy and safety of duloxetine $30 \mathrm{mg} / \mathrm{d}$ in patients with fibromyalgia: a randomized, doubleblind, placebo-controlled study. Clin J Pain. 2012a Nov-Dec;28(9):775-81. doi: 10.1097/AJP.0b013e3182510295. PMID: 22971669.

51. Murakami M, Osada K, Mizuno H, et al. A randomized, double-blind, placebocontrolled phase III trial of duloxetine in Japanese fibromyalgia patients. Arthritis Res Ther. 2015 Aug 22;17:224. doi: 10.1186/s13075-015-0718-y. PMID: 26296539.

52. Hannonen P, Malminiemi K, Yli-Kerttula U, et al. A randomized, double-blind, placebocontrolled study of moclobemide and amitriptyline in the treatment of fibromyalgia in females without psychiatric disorder. Br J Rheumatol. 1998 Dec;37(12):1279-86. PMID: 9973149.

53. Chappell AS, Bradley LA, Wiltse C, et al. A six-month double blind placebo-controlled, randomized clinical trial of duloxetine for the treatment of fibromyalgia. Int J Gen Med. 2008 Nov 30;1:91-102. PMID: 20428412.

54. Arnold LM, Goldenberg DL, Stanford SB, et al. Gabapentin in the treatment of fibromyalgia: a randomized, double-blind, placebo-controlled, multicenter trial. Arthritis Rheum. 2007a Apr;56(4):1336-44. doi: 10.1002/art.22457. PMID: 17393438. 
55. Arnold LM, Russell IJ, Diri EW, et al. A 14week, randomized, double-blinded, placebocontrolled monotherapy trial of pregabalin in patients with fibromyalgia. J Pain. 2008 Sep;9(9):792-805. doi: 10.1016/j.jpain.2008.03.013. PMID: 18524684.

56. Arnold LM, Whitaker S, Hsu C, et al. Efficacy and safety of mirogabalin for the treatment of fibromyalgia: results from three 13-week randomized, double-blind, placeboand active-controlled, parallel-group studies and a 52-week open-label extension study. Curr Med Res Opin. 2019 Jul 9:1-11. doi: 10.1080/03007995.2019.1629757. PMID: 31284771.

57. Mease PJ, Russell IJ, Arnold LM, et al. A randomized, double-blind, placebocontrolled, phase III trial of pregabalin in the treatment of patients with fibromyalgia. J Rheumatol. 2008 Mar;35(3):502-14. PMID: 18278830 .

58. Pauer L, Winkelmann A, Arsenault P, et al. An international, randomized, double-blind, placebo-controlled, phase III trial of pregabalin monotherapy in treatment of patients with fibromyalgia. J Rheumatol. 2011 Dec;38(12):2643-52. doi: 10.3899/jrheum.110569. PMID: 21965636.

59. Ohta H, Oka H, Usui C, et al. A randomized, double-blind, multicenter, placebocontrolled phase III trial to evaluate the efficacy and safety of pregabalin in Japanese patients with fibromyalgia. Arthritis Res Ther. 2012 Oct 12;14(5):R217. doi: 10.1186/ar4056. PMID: 23062189.

60. Olivan-Blazquez B, Herrera-Mercadal P, Puebla-Guedea M, et al. Efficacy of memantine in the treatment of fibromyalgia: a double-blind, randomised, controlled trial with 6-month follow-up. Pain. 2014

Dec;155(12):2517-25. doi: 10.1016/j.pain.2014.09.004. PMID: 25218600.

61. Carette S, Bell MJ, Reynolds WJ, et al. Comparison of amitriptyline, cyclobenzaprine, and placebo in the treatment of fibromyalgia. A randomized, double-blind clinical trial. Arthritis Rheum. 1994 Jan;37(1):32-40. doi: 10.1002/art.1780370106. PMID: 8129762.
62. Altman R, Hochberg M, Gibofsky A, et al. Efficacy and safety of low-dose SoluMatrix meloxicam in the treatment of osteoarthritis pain: a 12-week, phase 3 study. Curr Med Res Opin. 2015 Dec;31(12):2331-43. doi: 10.1185/03007995.2015.1112772. PMID: 26503347.

63. Baerwald C, Verdecchia P, Duquesroix B, et al. Efficacy, safety, and effects on blood pressure of naproxcinod $750 \mathrm{mg}$ twice daily compared with placebo and naproxen 500 mg twice daily in patients with osteoarthritis of the hip: a randomized, double-blind, parallel-group, multicenter study. Arthritis Rheum. 2010 Dec;62(12):3635-44. doi: 10.1002/art.27694. PMID: 20722026.

64. Bensen WG, Fiechtner JJ, McMillen JI, et al. Treatment of osteoarthritis with celecoxib, a cyclooxygenase-2 inhibitor: a randomized controlled trial. Mayo Clin Proc. 1999 Nov;74(11):1095-105. doi: 10.4065/74.11.1095. PMID: 10560596.

65. Bingham CO, 3rd, Sebba AI, Rubin BR, et al. Efficacy and safety of etoricoxib $30 \mathrm{mg}$ and celecoxib $200 \mathrm{mg}$ in the treatment of osteoarthritis in two identically designed, randomized, placebo-controlled, noninferiority studies. Rheumatology (Oxford). 2007 Mar;46(3):496-507. doi: 10.1093/rheumatology/kel296. PMID: 16936327.

66. Case JP, Baliunas AJ, Block JA. Lack of efficacy of acetaminophen in treating symptomatic knee osteoarthritis: a randomized, double-blind, placebocontrolled comparison trial with diclofenac sodium. Arch Intern Med. 2003 Jan 27;163(2):169-78. PMID: 12546607.

67. Clegg DO, Reda DJ, Harris CL, et al. Glucosamine, chondroitin sulfate, and the two in combination for painful knee osteoarthritis. N Engl J Med. 2006 Feb 23;354(8):795-808. doi: 10.1056/NEJMoa052771. PMID: 16495392. 
68. Conaghan PG, Dickson J, Bolten W, et al. A multicentre, randomized, placebo- and active-controlled trial comparing the efficacy and safety of topical ketoprofen in Transfersome gel (IDEA-033) with ketoprofen-free vehicle (TDT 064) and oral celecoxib for knee pain associated with osteoarthritis. Rheumatology (Oxford). 2013 Jul;52(7):1303-12. doi:

10.1093/rheumatology/ket133. PMID: 23542612.

69. DeLemos BP, Xiang J, Benson C, et al. Tramadol hydrochloride extended-release once-daily in the treatment of osteoarthritis of the knee and/or hip: a double-blind, randomized, dose-ranging trial. Am J Ther. 2011 May;18(3):216-26. doi:

10.1097/MJT.0b013e3181cec307. PMID: 20215961.

70. Fleischmann R, Sheldon E, MaldonadoCocco J, et al. Lumiracoxib is effective in the treatment of osteoarthritis of the knee: a prospective randomized 13-week study versus placebo and celecoxib. Clin Rheumatol. 2006 Feb;25(1):42-53. PMID: 16132165.

71. Gibofsky A, Hochberg MC, Jaros MJ, et al. Efficacy and safety of low-dose submicron diclofenac for the treatment of osteoarthritis pain: a 12 week, phase 3 study. Curr Med Res Opin. 2014 Sep;30(9):1883-93. doi: 10.1185/03007995.2014.946123. PMID: 25050589.

72. Hochberg MC, Fort JG, Svensson O, et al. Fixed-dose combination of enteric-coated naproxen and immediate-release esomeprazole has comparable efficacy to celecoxib for knee osteoarthritis: two randomized trials. Curr Med Res Opin. 2011 Jun;27(6):1243-53. doi: 10.1185/03007995.2011.580340. PMID: 21524238.

73. Kivitz A, Eisen G, Zhao WW, et al. Randomized placebo-controlled trial comparing efficacy and safety of valdecoxib with naproxen in patients with osteoarthritis. J Fam Pract. 2002 Jun;51(6):530-7. PMID: 12100776.
74. Kivitz AJ, Moskowitz RW, Woods E, et al. Comparative efficacy and safety of celecoxib and naproxen in the treatment of osteoarthritis of the hip. J Int Med Res. 2001 Nov-Dec;29(6):467-79. doi: 10.1177/147323000102900602. PMID: 11803730.

75. Lehmann R, Brzosko M, Kopsa P, et al. Efficacy and tolerability of lumiracoxib 100 mg once daily in knee osteoarthritis: a 13week, randomized, double-blind study vs. placebo and celecoxib. Curr Med Res Opin. 2005 Apr;21(4):517-26. doi: 10.1185/030079905X38196. PMID: 15899100.

76. Leung AT, Malmstrom K, Gallacher AE, et al. Efficacy and tolerability profile of etoricoxib in patients with osteoarthritis: a randomized, double-blind, placebo and active-comparator controlled 12-week efficacy trial. Curr Med Res Opin. 2002;18(2):49-58. doi: 10.1185/030079902125000282. PMID: 12017209.

77. Makarowski W, Zhao WW, Bevirt T, et al. Efficacy and safety of the COX-2 specific inhibitor valdecoxib in the management of osteoarthritis of the hip: a randomized, double-blind, placebo-controlled comparison with naproxen. Osteoarthritis Cartilage. 2002 Apr;10(4):290-6. doi: 10.1053/joca.2001.0510. PMID: 11950252.

78. Puopolo A, Boice JA, Fidelholtz JL, et al. A randomized placebo-controlled trial comparing the efficacy of etoricoxib $30 \mathrm{mg}$ and ibuprofen $2400 \mathrm{mg}$ for the treatment of patients with osteoarthritis. Osteoarthritis Cartilage. 2007 Dec;15(12):1348-56. doi: 10.1016/j.joca.2007.05.022. PMID: 17631392.

79. Reginster JY, Dudler J, Blicharski T, et al. Pharmaceutical-grade Chondroitin sulfate is as effective as celecoxib and superior to placebo in symptomatic knee osteoarthritis: the ChONdroitin versus CElecoxib versus Placebo Trial (CONCEPT). Ann Rheum Dis. 2017 Sep;76(9):1537-43. doi: 10.1136/annrheumdis-2016-210860. PMID: 28533290. 
80. Schnitzer TJ, Dattani ID, Seriolo B, et al. A 13-week, multicenter, randomized, doubleblind study of lumiracoxib in hip osteoarthritis. Clin Rheumatol. 2011a Nov;30(11):1433-46. doi: 10.1007/s10067011-1776-4. PMID: 21607551.

81. Schnitzer TJ, Kivitz A, Frayssinet H, et al. Efficacy and safety of naproxcinod in the treatment of patients with osteoarthritis of the knee: a 13-week prospective, randomized, multicenter study.

Osteoarthritis Cartilage. 2010

May;18(5):629-39. doi:

10.1016/j.joca.2009.12.013. PMID: 20202489.

82. Sheldon E, Beaulieu A, Paster Z, et al. Efficacy and tolerability of lumiracoxib in the treatment of osteoarthritis of the knee: a 13-week, randomized, double-blind comparison with celecoxib and placebo. Clin Ther. 2005 Jan;27(1):64-77. doi: 10.1016/j.clinthera.2005.01.002. PMID: 15763607.

83. Simon LS, Grierson LM, Naseer Z, et al. Efficacy and safety of topical diclofenac containing dimethyl sulfoxide (DMSO) compared with those of topical placebo, DMSO vehicle and oral diclofenac for knee osteoarthritis. Pain. 2009 Jun;143(3):238-45. doi: 10.1016/j.pain.2009.03.008. PMID: 19380203.

84. Tannenbaum H, Berenbaum F, Reginster $\mathrm{JY}$, et al. Lumiracoxib is effective in the treatment of osteoarthritis of the knee: a 13 week, randomised, double blind study versus placebo and celecoxib. Ann Rheum Dis. 2004 Nov;63(11):1419-26. doi: 10.1136/ard.2003.015974. PMID: 15020310.

85. Wiesenhutter CW, Boice JA, Ko A, et al. Evaluation of the comparative efficacy of etoricoxib and ibuprofen for treatment of patients with osteoarthritis: a randomized, double-blind, placebo-controlled trial. Mayo Clin Proc. 2005 Apr;80(4):470-9. PMID: 15819283.
86. Yocum D, Fleischmann R, Dalgin P, et al. Safety and efficacy of meloxicam in the treatment of osteoarthritis: a 12-week, double-blind, multiple-dose, placebocontrolled trial. The Meloxicam Osteoarthritis Investigators. Arch Intern Med. 2000 Oct 23;160(19):2947-54. PMID: 11041902.

87. Baraf HS, Gold MS, Clark MB, et al. Safety and efficacy of topical diclofenac sodium 1\% gel in knee osteoarthritis: a randomized controlled trial. Phys Sportsmed. 2010 Jun;38(2):19-28. doi: 10.3810/psm.2010.06.1779. PMID: 20631460.

88. Barthel HR, Haselwood D, Longley S, 3rd, et al. Randomized controlled trial of diclofenac sodium gel in knee osteoarthritis.[Erratum appears in Semin Arthritis Rheum. 2010 Aug;40(1):95]. Semin Arthritis Rheum. 2009 Dec;39(3):203-12. doi: https://dx.doi.org/10.1016/j.semarthrit.2009. 09.002. PMID: 19932833.

89. Roth SH, Shainhouse JZ. Efficacy and safety of a topical diclofenac solution (pennsaid) in the treatment of primary osteoarthritis of the knee: a randomized, double-blind, vehicle-controlled clinical trial. Arch Intern Med. 2004 Oct 11;164(18):2017-23. doi: 10.1001/archinte.164.18.2017. PMID: 15477437.

90. Bakshi R, Ezzet N, Frey L, et al. Efficacy and tolerability of diclofenac dispersible in painful osteoarthrosis. Clin Rheumatol. 1993 Mar;12(1):57-61. PMID: 7682167.

91. Morgan GJ, Jr., Kaine J, DeLapp R, et al. Treatment of elderly patients with nabumetone or diclofenac: gastrointestinal safety profile. J Clin Gastroenterol. 2001 Apr;32(4):310-4. PMID: 11276273.

92. Emery P, Koncz T, Pan S, et al. Analgesic effectiveness of celecoxib and diclofenac in patients with osteoarthritis of the hip requiring joint replacement surgery: a 12week, multicenter, randomized, doubleblind, parallel-group, double-dummy, noninferiority study. Clin Ther. 2008 Jan;30(1):70-83. doi: 10.1016/j.clinthera.2008.01.016. PMID: 18343244. 
93. Essex MN, Bhadra P, Sands GH. Efficacy and tolerability of celecoxib versus naproxen in patients with osteoarthritis of the knee: a randomized, double-blind, double-dummy trial. J Int Med Res. 2012;40(4):1357-70. doi: 10.1177/147323001204000414. PMID: 22971487.

94. Dahlberg LE, Holme I, Hoye K, et al. A randomized, multicentre, double-blind, parallel-group study to assess the adverse event-related discontinuation rate with celecoxib and diclofenac in elderly patients with osteoarthritis. Scand J Rheumatol. 2009 Mar-Apr;38(2):133-43. doi: 10.1080/03009740802419065. PMID: 19165648.

95. Hosie J, Distel M, Bluhmki E. Meloxicam in osteoarthritis: a 6-month, double-blind comparison with diclofenac sodium. $\mathrm{Br} \mathrm{J}$ Rheumatol. 1996 Apr;35 Suppl 1:39-43. PMID: 8630635.

96. Abou-Raya S, Abou-Raya A, Helmii M. Duloxetine for the management of pain in older adults with knee osteoarthritis: randomised placebo-controlled trial. Age Ageing. 2012 Sep;41(5):646-52. doi: 10.1093/ageing/afs072. PMID: 22743149.

97. Chappell AS, Desaiah D, Liu-Seifert H, et al. A double-blind, randomized, placebocontrolled study of the efficacy and safety of duloxetine for the treatment of chronic pain due to osteoarthritis of the knee. Pain pract. 2011 Jan-Feb;11(1):33-41. doi: 10.1111/j.1533-2500.2010.00401.x. PMID: 20602715.

98. Chappell AS, Ossanna MJ, Liu-Seifert H, et al. Duloxetine, a centrally acting analgesic, in the treatment of patients with osteoarthritis knee pain: a 13-week, randomized, placebo-controlled trial. Pain. 2009a Dec;146(3):253-60. doi: 10.1016/j.pain.2009.06.024. PMID: 19625125.

99. Tetreault P, Mansour A, Vachon-Presseau E, et al. Brain connectivity predicts placebo response across chronic pain clinical trials. PLoS Biol. 2016 Oct 27;14(10):e1002570. doi: 10.1371/journal.pbio.1002570. PMID: 27788130 .
100. Uchio Y, Enomoto H, Alev L, et al. A randomized, double-blind, placebocontrolled phase III trial of duloxetine in Japanese patients with knee pain due to osteoarthritis. J Pain Res. 2018;11:809-21. doi: 10.2147/JPR.S164128. PMID: 29713194.

101. Wang G, Bi L, Li X, et al. Efficacy and safety of duloxetine in Chinese patients with chronic pain due to osteoarthritis: a randomized, double-blind, placebocontrolled study. Osteoarthritis Cartilage. 2017 Jun;25(6):832-8. doi: 10.1016/j.joca.2016.12.025. PMID: 28043937.

102. Altman RD, Zinsenheim JR, Temple AR, et al. Three-month efficacy and safety of acetaminophen extended-release for osteoarthritis pain of the hip or knee: a randomized, double-blind, placebocontrolled study. Osteoarthritis Cartilage. 2007 Apr;15(4):454-61. doi: 10.1016/j.joca.2006.10.008. PMID: 17142063.

103. Prior MJ, Harrison DD, Frustaci ME. A randomized, double-blind, placebocontrolled 12 week trial of acetaminophen extended release for the treatment of signs and symptoms of osteoarthritis. Curr Med Res Opin. 2014 Nov;30(11):2377-87. doi: 10.1185/03007995.2014.949646. PMID: 25121804.

104. Herrero-Beaumont G, Ivorra JA, Del Carmen Trabado M, et al. Glucosamine sulfate in the treatment of knee osteoarthritis symptoms: a randomized, double-blind, placebo-controlled study using acetaminophen as a side comparator. Arthritis Rheum. 2007 Feb;56(2):555-67. doi: 10.1002/art.22371. PMID: 17265490.

105. Sofat N, Harrison A, Russell MD, et al. The effect of pregabalin or duloxetine on arthritis pain: a clinical and mechanistic study in people with hand osteoarthritis.[Erratum appears in J Pain Res. 2017 Dec 15;10:2843]. J Pain Res. 2017;10:2437-49. PMID: 29066930.

106. Barkhuizen A, Steinfeld S, Robbins J, et al. Celecoxib is efficacious and well tolerated in treating signs and symptoms of ankylosing spondylitis. J Rheumatol. 2006 Sep;33(9):1805-12. PMID: 16960941. 
107. Collantes E, Curtis SP, Lee KW, et al. A multinational randomized, controlled, clinical trial of etoricoxib in the treatment of rheumatoid arthritis [ISRCTN25142273]. BMC Fam Pract. 2002 May 22;3:10. PMID: 12033987.

108. Fattahi MJ, Jamshidi AR, Mahmoudi M, et al. Evaluation of the efficacy and safety of beta-d-mannuronic acid in patients with ankylosing spondylitis: A 12-week randomized, placebo-controlled, phase I/II clinical trial. Int Immunopharmacol. 2018 Jan;54:112-7. doi: https://dx.doi.org/10.1016/j.intimp.2017.11. 003. PMID: 29127910.

109. Furst DE, Kolba KS, Fleischmann R, et al. Dose response and safety study of meloxicam up to $22.5 \mathrm{mg}$ daily in rheumatoid arthritis: a 12 week multicenter, double blind, dose response study versus placebo and diclofenac. J Rheumatol. 2002 Mar;29(3):436-46. PMID: 11908554.

110. Geusens P, Alten R, Rovensky J, et al. Efficacy, safety and tolerability of lumiracoxib in patients with rheumatoid arthritis. Int J Clin Pract. 2004 Nov;58(11):1033-41. PMID: 15605667.

111. Jacob G, Messina M, Caperton E. Safety and efficacy of etodolac, once or twice a day, in the treatment of active rheumatoid arthritis. Curr Ther Res Clin Exp. 1985;37(6):1124-9.

112. Jacob GB, Hart KK, Mullane JF. Placebocontrolled study of etodolac and aspirin in the treatment of rheumatoid arthritis. Curr Ther Res Clin Exp. 1983;33(4):703-13.

113. Matsumoto AK, Melian A, Mandel DR, et al. A randomized, controlled, clinical trial of etoricoxib in the treatment of rheumatoid arthritis. J Rheumatol. 2002

Aug;29(8):1623-30. PMID: 12180720.

114. Simon L, Weaver AL, Graham DY, et al. Anti-inflammatory and upper gastrointestinal effects of celecoxib in rheumatoid arthritis: a randomized controlled trial. JAMA. 1999 Nov 24;282(20):1921-8. PMID: 10580457.
115. Dougados M, Gueguen A, Nakache JP, et al. Ankylosing spondylitis: what is the optimum duration of a clinical study? A one year versus a 6 weeks non-steroidal antiinflammatory drug trial. Rheumatology (Oxford). 1999 Mar;38(3):235-44. doi: 10.1093/rheumatology/38.3.235. PMID: 10325662.

116. Nazeri S, Jamshidi AR, Mahmoudi M, et al. The safety and efficacy of Guluronic acid (G2013) in ankylosing spondylitis: A randomized controlled parallel clinical trial. Pharmacol Rep. 2019 Jun;71(3):393-8. doi: 10.1016/j.pharep.2019.02.002. PMID: 31003148.

117. Dougados M, Gueguen A, Nakache JP, et al. Evaluation of a functional index for patients with ankylosing spondylitis. J Rheumatol. 1990 Sep;17(9):1254-5. PMID: 2290177.

118. Emery P, Zeidler H, Kvien TK, et al. Celecoxib versus diclofenac in long-term management of rheumatoid arthritis: randomised double-blind comparison. Lancet. 1999;354(9196):2106-11. doi: 10.1016/S0140-6736(99)02332-6. PMID: 10609815.

119. Sieper J, Klopsch T, Richter M, et al. Comparison of two different dosages of celecoxib with diclofenac for the treatment of active ankylosing spondylitis: results of a 12-week randomised, double-blind, controlled study. Ann Rheum Dis. 2008 Mar;67(3):323-9. doi: 10.1136/ard.2007.075309. PMID: 17616556.

120. Walker C, Essex MN, Li C, et al. Celecoxib versus diclofenac for the treatment of ankylosing spondylitis: 12-week randomized study in Norwegian patients. J Int Med Res. 2016 Jun;44(3):483-95. doi: 10.1177/0300060516628704. PMID: 26980340.

121. Lonauer G, Tisscher JR, Lim HG, et al. Double-blind comparison of etodolac and diclofenac in patients with rheumatoid arthritis. Curr Med Res Opin. 1993;13(2):70-7. doi: 10.1185/03007999309111535. PMID: 8325044. 
122. de Queiros MF. Double-blind comparison of etodolac and naproxen in the treatment of rheumatoid arthritis. Clin Ther. 1991 JanFeb;13(1):38-46. PMID: 1827613.

123. Emery P, Clarke A, Williams P, et al. Nabumetone compared with naproxen in the treatment of rheumatoid arthritis: a multicenter, double blind, randomized, parallel group trial in hospital outpatients.[Erratum appears in J Rheumatol 1993 May;20(5):924]. J Rheumatol Suppl. 1992 Nov;36:41-7. PMID: 1474534.

124. Krug H, Broadwell LK, Berry M, et al. Tolerability and efficacy of nabumetone and naproxen in the treatment of rheumatoid arthritis. Clin Ther. 2000 Jan;22(1):40-52. PMID: 10688389.

125. Wojtulewski JA, Schattenkirchner M, Barcelo P, et al. A six-month double-blind trial to compare the efficacy and safety of meloxicam $7.5 \mathrm{mg}$ daily and naproxen 750 mg daily in patients with rheumatoid arthritis. Br J Rheumatol. 1996 Apr;35(Suppl 1):22-8. PMID: 8630632.

126. Hazleman BL, Thomas PP. Single-blind comparative study of nabumetone (Relafen) versus naproxen in the treatment of rheumatoid arthritis. Am J Med. 1987 Oct 30;83(4B):60-4. PMID: 3318431.

127. Grace EM, Bellamy N, Kassam Y, et al. Controlled, double-blind, randomized trial of amitriptyline in relieving articular pain and tenderness in patients with rheumatoid arthritis. Curr Med Res Opin. 1985;9(6):426-9. doi: 10.1185/03007998509109614. PMID: 3886308.

128. Konno S, Oda N, Ochiai T, et al. Randomized, double-blind, placebocontrolled phase III trial of duloxetine monotherapy in Japanese patients with chronic low back pain. Spine (Phila $\mathrm{Pa}$ 1976). 2016 Nov 15;41(22):1709-17. doi: 10.1097/BRS.0000000000001707. PMID: 27831985.

129. Skljarevski V, Ossanna M, Liu-Seifert H, et al. A double-blind, randomized trial of duloxetine versus placebo in the management of chronic low back pain. Eur J Neurol. 2009 Sep;16(9):1041-8. doi: 10.1111/j.1468-1331.2009.02648.x. PMID: 19469829.
130. Skljarevski V, Zhang S, Desaiah D, et al. Duloxetine versus placebo in patients with chronic low back pain: a 12-week, fixeddose, randomized, double-blind trial. J Pain. 2010 Dec;11(12):1282-90. doi: 10.1016/j.jpain.2010.03.002. PMID: 20472510.

131. Atkinson J, Slater MA, Capparelli EV, et al. Efficacy of noradrenergic and serotonergic antidepressants in chronic back pain: A preliminary concentration-controlled trial. J Clin Psychopharmacol. 2007 Apr;27(2):13542. doi: 10.1097/jcp.0b013e3180333ed5. PMID: 17414235.

132. Atkinson JH, Slater MA, Capparelli EV, et al. A randomized controlled trial of gabapentin for chronic low back pain with and without a radiating component. Pain. 2016 Jul;157(7):1499-507. doi: 10.1097/j.pain.0000000000000554. PMID: 26963844.

133. Urquhart DM, Wluka AE, Van Tulder M, et al. Efficacy of low-dose amitriptyline for chronic low back pain: a randomized clinical trial. JAMA Intern Med. 2018 Nov 1;178(11):1474-81. doi: 10.1001/jamainternmed.2018.4222. PMID: 30285054.

134. Kalita J, Kohat AK, Misra UK, et al. An open labeled randomized controlled trial of pregabalin versus amitriptyline in chronic low backache. J Neurol Sci. 2014 Jul 15;342(1-2):127-32. doi: 10.1016/j.jns.2014.05.002. PMID: 24857356.

135. Pfaffenrath V, Diener HC, Isler $\mathrm{H}$, et al. Efficacy and tolerability of amitriptylinoxide in the treatment of chronic tension-type headache: a multi-centre controlled study. Cephalalgia. 1994 Apr;14(2):149-55. doi: 10.1046/j.1468-2982.1994.1402149.x. PMID: 8062354.

136. Schlaeger JM, Molokie RE, Yao Y, et al. Management of sickle cell pain using pregabalin: a pilot study. Pain Manag Nurs. 2017 Dec;18(6):391-400. doi: 10.1016/j.pmn.2017.07.003. PMID: 28843636. 
137. Hawkey CJ, Laine L, Simon T, et al. Incidence of gastroduodenal ulcers in patients with rheumatoid arthritis after 12 weeks of rofecoxib, naproxen, or placebo: a multicentre, randomised, double blind study.[Erratum appears in Gut. 2003

Dec;52(12):1800]. Gut. 2003

Dec;52(6):820-6. doi: 10.1136/gut.52.6.820. PMID: 12740337.

138. Hawkey C, Laine L, Simon T, et al. Comparison of the effect of rofecoxib (a cyclooxygenase 2 inhibitor), ibuprofen, and placebo on the gastroduodenal mucosa of patients with osteoarthritis: a randomized, double-blind, placebo-controlled trial. The Rofecoxib osteoarthritis endoscopy multinational study group. Arthritis Rheum. 2000 Feb;43(2):370-7. doi: 10.1002/15290131(200002)43:2<370::AIDANR17>3.0.CO;2-D. PMID: 10693877.

139. Hunt RH, Harper S, Callegari P, et al. Complementary studies of the gastrointestinal safety of the cyclooxygenase-2-selective inhibitor etoricoxib. Aliment Pharmacol Ther. 2003 Jan;17(2):201-10. PMID: 12534404.

140. Laine L, Maller ES, Yu C, et al. Ulcer formation with low-dose enteric-coated aspirin and the effect of COX-2 selective inhibition: a double-blind trial. Gastroenterology. 2004 Aug;127(2):395402. PMID: 15300570.

141. Sikes DH, Agrawal NM, Zhao WW, et al. Incidence of gastroduodenal ulcers associated with valdecoxib compared with that of ibuprofen and diclofenac in patients with osteoarthritis. Eur J Gastroenterol Hepatol. 2002 Oct;14(10):1101-11. PMID: 12362101.
142. Bhala N, Emberson J, Merhi A, et al. Vascular and upper gastrointestinal effects of non-steroidal anti-inflammatory drugs: meta-analyses of individual participant data from randomised trials. Lancet. 2013 Aug 31;382(9894):769-79. doi: 10.1016/s01406736(13)60900-9. PMID: 23726390.

143. Cryer B, Li C, Simon LS, et al. GIREASONS: a novel 6-month, prospective, randomized, open-label, blinded endpoint (PROBE) trial. Am J Gastroenterol. 2013 Mar;108(3):392-400. doi: 10.1038/ajg.2012.467. PMID: 23399552.

144. Nissen SE, Yeomans ND, Solomon DH, et al. Cardiovascular safety of celecoxib, naproxen, or ibuprofen for arthritis. N Engl J Med. 2016 Dec 29;375(26):2519-29. doi: 10.1056/NEJMoa1611593. PMID: 27959716.

145. MacDonald TM, Hawkey CJ, Ford I, et al. Randomized trial of switching from prescribed non-selective non-steroidal antiinflammatory drugs to prescribed celecoxib: the Standard care vs. Celecoxib Outcome Trial (SCOT).[Erratum appears in Eur Heart J. 2016 Dec 24;:; PMID: 28025195]. Eur Heart J. 2017 Jun 14;38(23):1843-50. doi: 10.1093/eurheartj/ehw387. PMID: 27705888.

146. Cryer BL, Sostek MB, Fort JG, et al. A fixed-dose combination of naproxen and esomeprazole magnesium has comparable upper gastrointestinal tolerability to celecoxib in patients with osteoarthritis of the knee: results from two randomized, parallel-group, placebo-controlled trials. Ann Med. 2011 Dec;43(8):594-605. doi: 10.3109/07853890.2011.625971. PMID: 22017620.

147. Rostom A, Goldkind L, Laine L. Nonsteroidal anti-inflammatory drugs and hepatic toxicity: a systematic review of randomized controlled trials in arthritis patients. Clin Gastroenterol Hepatol. 2005 May;3(5):489-98. PMID: 15880319. 